\title{
Dear my very problematic blood glucose meter: Adolescents' experiences self-managing type 1 diabetes and their psychosocial user requirements of medical technologies
}

Gillian M. McCarthy

\author{
A thesis submitted to Victoria University of Wellington \\ in fulfilment of the requirements for the degree of \\ Doctor of Philosophy
}

Victoria University of Wellington

2018 



\begin{abstract}
"Dear insulin pump. I love that you came into my life! You give me flexibility, confidence, and happiness."

"Dear my very problematic blood glucose meter. I wish I could let you go. I don't ask much from you, just correct readings and that you stop deciding to pack it in. You make me second guess myself and my health."
\end{abstract}

The difference between a loved and a despised medical device is stark. Adolescents with type 1 diabetes require medical devices that facilitate their self-management throughout every aspect of their lives, from school, to the sports field, to managing hypoglycaemia in the middle of the night. This research aims to describe adolescents' experiences self-managing type 1 diabetes, and identify their psychosocial user requirements of medical technologies.

Following a constructivist research paradigm, a range of predominantly qualitative and participatory design methods were employed with 16 adolescent and young adult participants with type 1 diabetes and nine health professionals. Methods included semi-structured interviews and a card-sort task to understand the psychosocial impacts of current medical devices. Cultural probes elicited: adolescents' metaphors for managing diabetes, how they would like to manage their diabetes, the best and worst features of their devices, and the relationships they have with them. Undergraduate design students used secondary research and emergent psychosocial user requirements of medical devices to design blood glucose meters, lancets, storage solutions, and insulin pumps that resembled watches, jewellery, and smartphones, could be attached to a bike, or could glow at night. I used these designs to provide additional support for adolescents' user requirements, demonstrate how they were of use to designers, and stimulate discussion with the adolescents. The adolescents with type 1 diabetes completed a participatory design workshop, designing blood glucose meters that challenged the ways in which medical devices currently draw attention. Findings were analysed using a constructivist approach to grounded theory, and psychosocial user requirements were developed. 
On average, current medical devices have positive psychosocial impacts on adolescents, with large positive impacts on users' feelings of competence, followed by increased feelings of adaptability and self-esteem. However, some adolescent requirements remain unmet. Issues include the transition of responsibility for diabetes management from parent to adolescent, managing blood glucose while participating in everyday activities such as sports, managing attention, and developing acceptance of a long-term condition. Other issues stem from devices' features, usability, reliability, and context of use. As research has indicated, the traditional health approach is about curing illness, but with diabetes, managing wellness is key. The person learns to fit diabetes around the rest-of-life.

While it is pertinent that diabetes technologies are clinically effective, they should also be designed in alignment with adolescent psychosocial user requirements, taking into account not only their physical health, but also the ways and contexts in which adolescents go about their daily lives. 


\section{Acknowledgements}

I would like to acknowledge the people who took part in this research: adolescents and young adults with type 1 diabetes, health professionals, and designers. Without your input, the research would not have been possible. It has been a privilege to hear your stories and share them throughout this research.

To my supervisors: Dr Edgar Rodríguez Ramírez and Dr Brian Robinson, for their ever-supportive academic supervision and pastoral care, hot chocolates, and homebrewed beer. I have been lucky to have such a great team.

To Mailin, my PhD buddy, who has navigated her research alongside me. It has been brilliant sharing this experience with you. To all the $\mathrm{PhD}$ and Masters students, who welcomed me to this university and shared in this experience; thank you for your support.

I would also like to acknowledge the members of staff who have helped me learn the ropes and provided me with opportunities to become a better researcher: Mailyn, Kim, Bronwyn, Eli, Stu, Michael, Lizzie, Julie, and Marisa and all the others; thank you for your help along the way. Also to Jean McDowall for her proofreading expertise.

To the University, Faculty, School of Design, School of Health, Nursing and Midwifery, and MedTech CoRE. Thank you for supporting this research and providing me with the opportunity to present my work at conferences, gaining invaluable feedback.

I would also like to acknowledge the brilliant work of some special staff members of the University of Otago, who sparked this passion for design and psychology research, and set me up with the skills I needed: Nick Laird, Mick Abbott, David O'Hare, and Jonathan Jong, your influence persists.

Finally, to my family, who have supported me on countless occasions along the way. Your home cooked meals, logic sessions, proofreading, and countless hours spent listening to me talk about research were invaluable. I can't thank you enough. 


\section{Table of Contents}

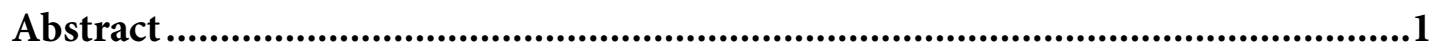

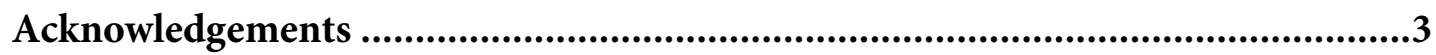

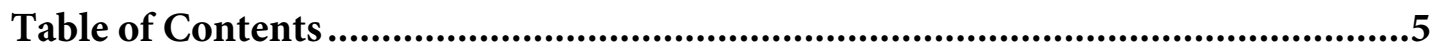

List of Figures...................................................................................................12

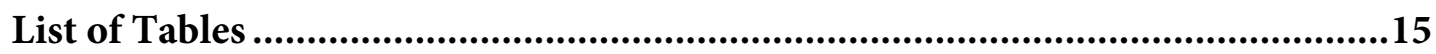

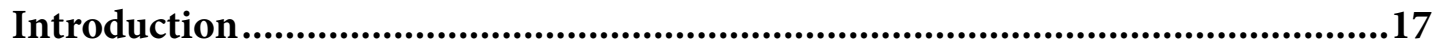

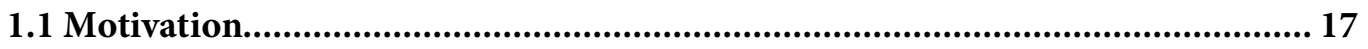

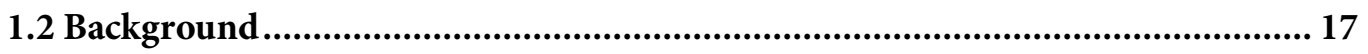

1.2.1 Adolescent development ................................................................................. 19

1.2.2 A holistic design and human factors approach .................................................... 19

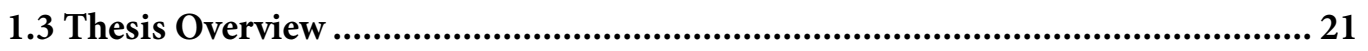

Chapter 2: Literature Review.................................................................................... 21

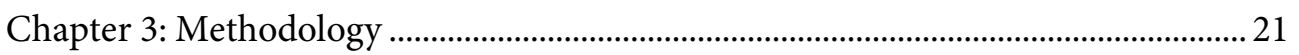

Chapter 4: Clinician Interviews ............................................................................ 21

Chapter 5: Designing Medical Technologies for Adolescents ....................................... 22

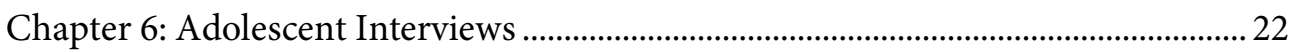

Chapter 7: Psychosocial Impacts of Medical Technologies............................................ 22

Chapter 8: Designing Medical Technologies from Adolescents' Requirements....... 22

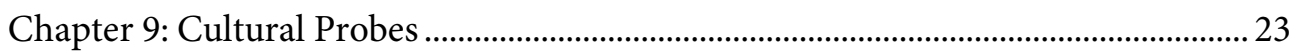

Chapter 10: Addressing Medical Device-Related Attention Through Participatory

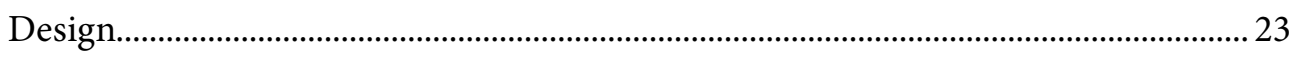

Chapter 11: Discussion .................................................................................................. 23

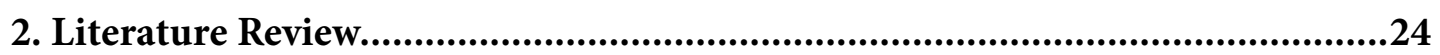

2.1 Type 1 Diabetes .................................................................................................. 24

2.2 Conceptual Approaches to Diabetes Management.............................................. 31

2.3 User Requirements of Medical Technologies.......................................................... 34

2.3.1 Access and affordability ............................................................................................ 36

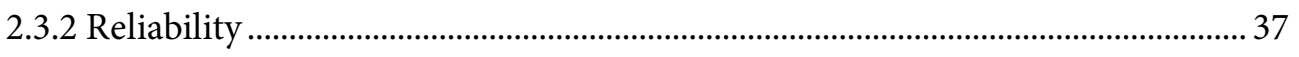

2.3.3 Usability ……………………….................................................................. 37

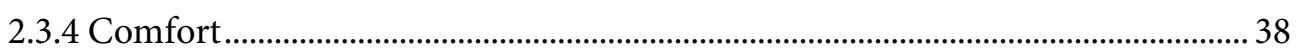


2.3.5 Suitability and desirability.

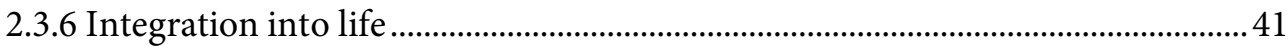

2.3.7 Provide meaningful information to facilitate decision-making............................. 42

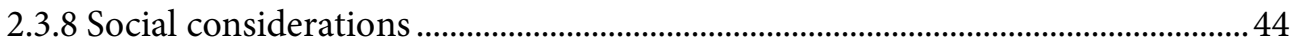

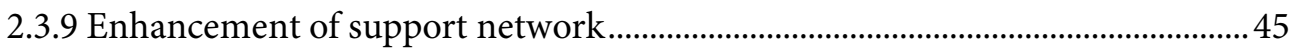

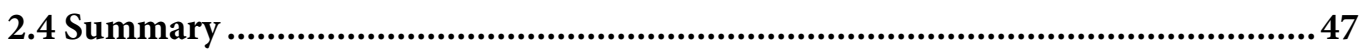

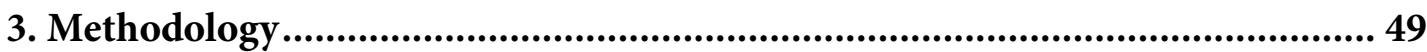

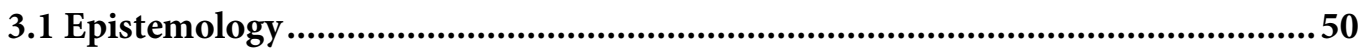

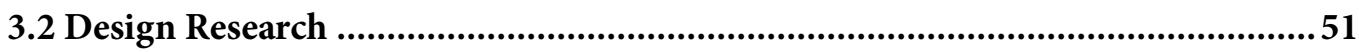

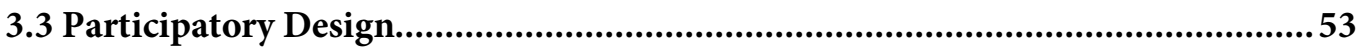

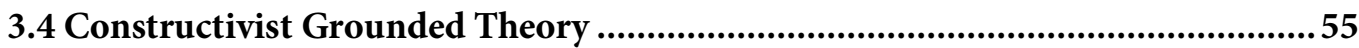

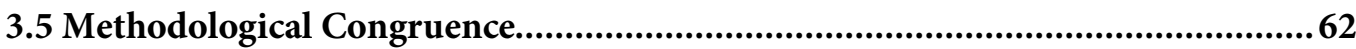

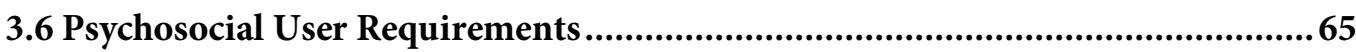

3.7 Conducting Research with Adolescents................................................................67

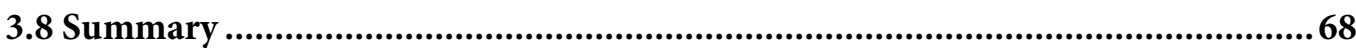

4. Experiences and User Requirements as Described by Health Professionals.... 69

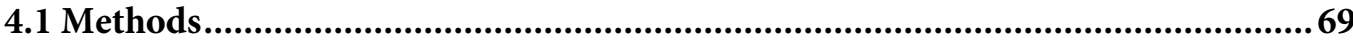

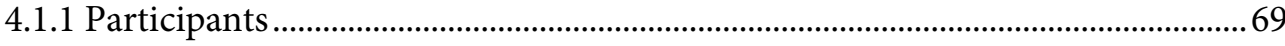

4.1.2 Procedure ………………………………………………………………………...... 70

4.2 Interview Findings................................................................................................... 71

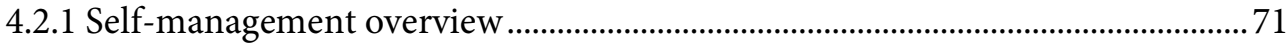

4.2.2 Conceptualising type 1 diabetes and its management ............................................ 73

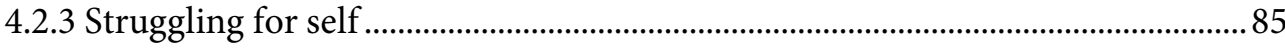

4.3.4 Managing identity ……………………………………………………………..... 93

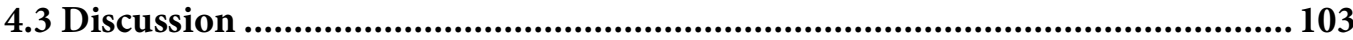

4.3.1 Psychosocial user requirements of medical technologies....................................103

4.3.2 Discussion of findings and methods.......................................................................106

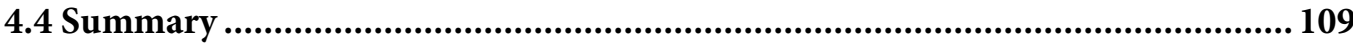

5. Designing Medical Technologies for Adolescents ........................................ 111

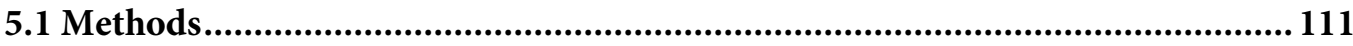

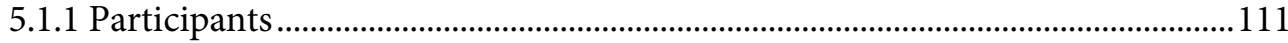

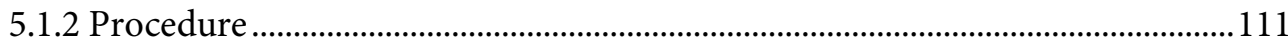

5.2 Design Outcomes and Reviews ............................................................................. 113

5.2.1 Subscope design and development.....................................................................114 
5.2.2 Subscope expert review.

5.2.3 Joya design and development......................................................................... 116

5.2.4 Joya expert review …………………………………......................................... 117

5.2.5 Armilla design and development.................................................................... 118

5.2.6 Armilla expert review....................................................................................... 119

5.2.7 Emoti-Comms design and development ........................................................119

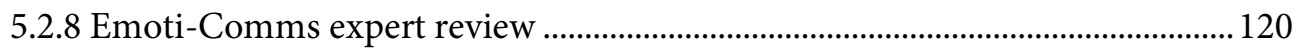

5.2.9 Simpac design and development ................................................................. 120

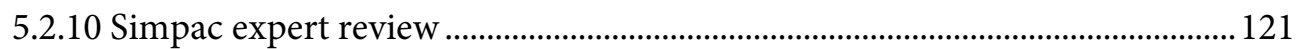

5.3 Thematic Analysis of Designers' Experiences .................................................. 122

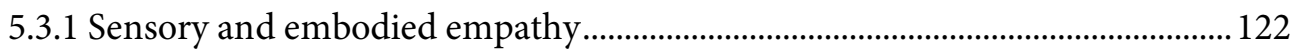

5.3.2 The inspiration and the necessary ....................................................................... 123

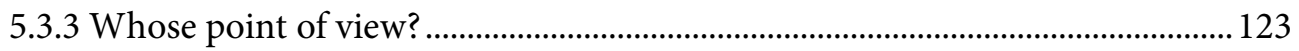

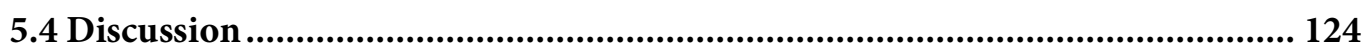

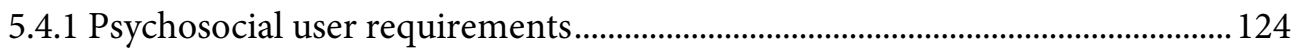

5.4.2 Approach to research and design ......................................................................... 126

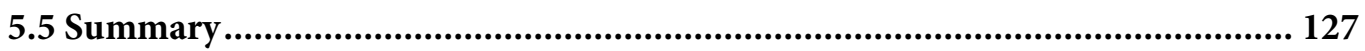

6. Experiences and User Requirements as Described by Adolescents and Young

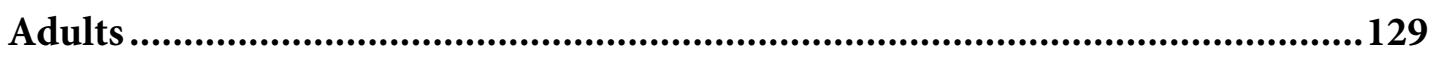

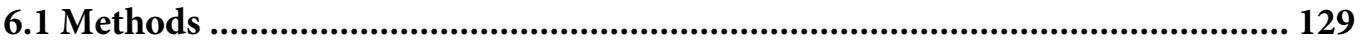

6.1.1 Participants........................................................................................................... 129

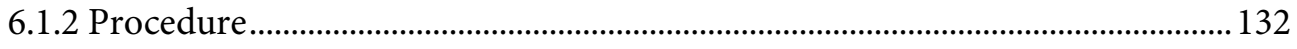

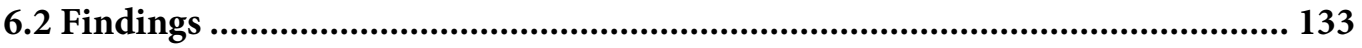

6.2.1 Self-management overview ............................................................................... 133

6.2.2 Conceptualising type 1 diabetes and its management ......................................... 137

6.2.3 Developing independence................................................................................... 151

6.2.4 Experiencing and managing interpersonal interactions...................................... 156

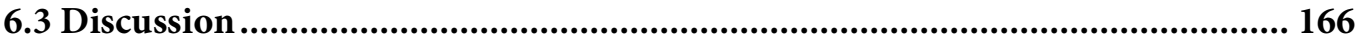

6.3.1 Psychosocial user requirements of medical technologies...................................... 166

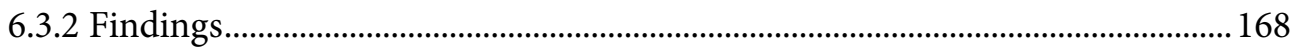

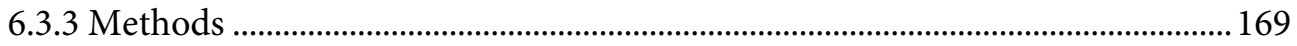

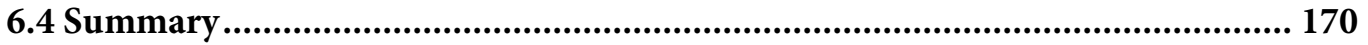

7. Psychosocial Impacts of Medical Technologies...............................................171

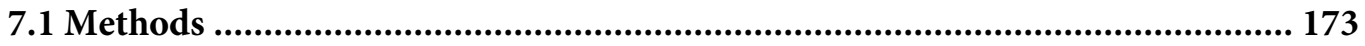




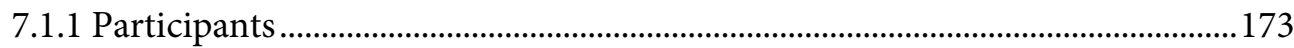

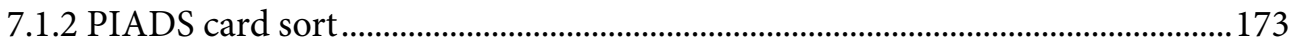

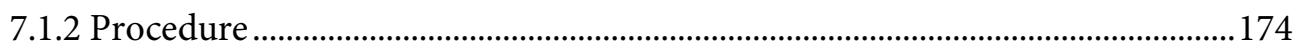

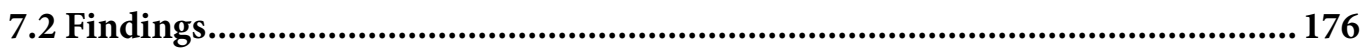

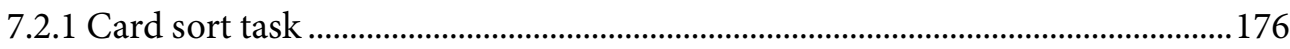

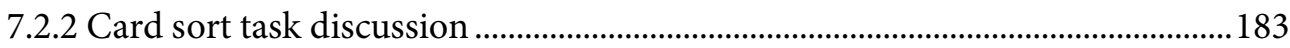

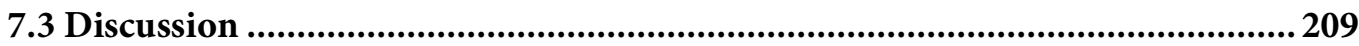

7.3.1 Psychosocial user requirements of medical technologies...................................209

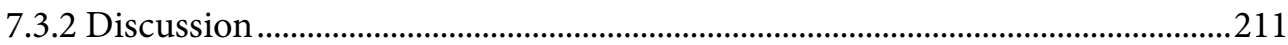

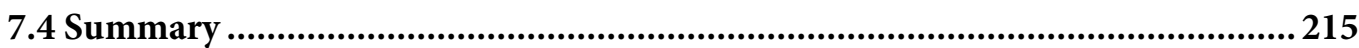

8. Designing Medical Technologies from Adolescents' User Requirements ..... 217

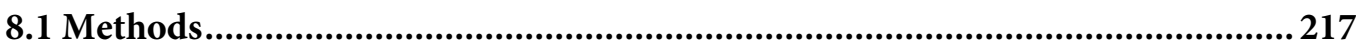

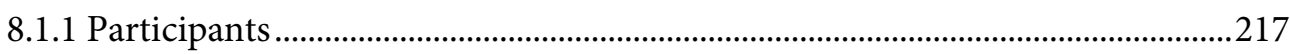

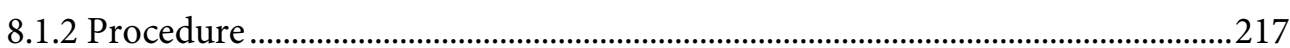

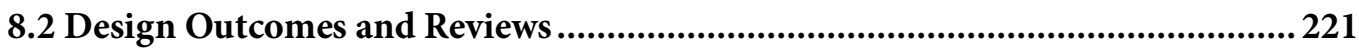

8.2.1 Blood glucose monitoring phone case and app ...................................................222

8.2.2 Blood glucose monitoring jewellery.....................................................................225

8.2.3 Blood glucose test reminder watch......................................................................226

8.2.4 Blood glucose meter test compact ...........................................................................227

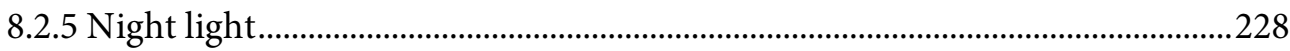

8.2.6 Continuous glucose monitoring sock ...................................................................229

8.2.7 Blood glucose testing bike attachment ...................................................................231

8.2.8 Additional design reviews .............................................................................232

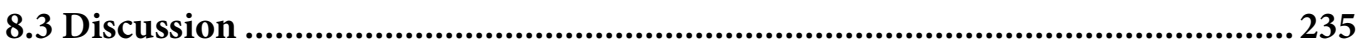

8.3.1 Psychosocial user requirements of medical technologies...................................235

8.3.2 Designing to user requirements ............................................................................237

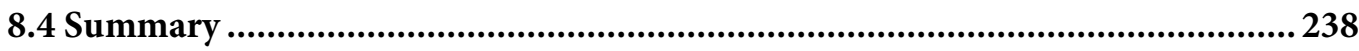

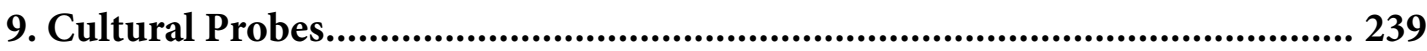

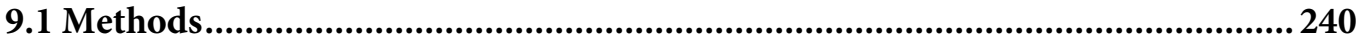

9.1.1 Participants ................................................................................................................240

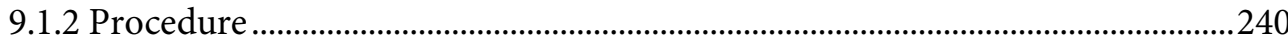

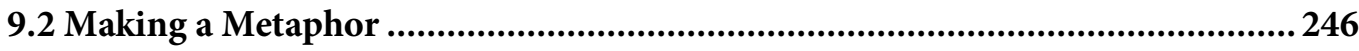

9.2.1 Managing a balancing act ........................................................................................246

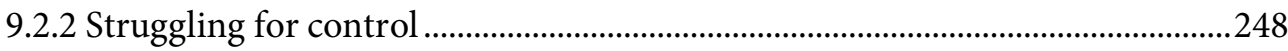


9.2.3 Rushing to beat the clock

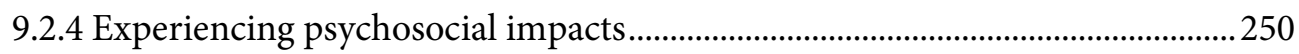

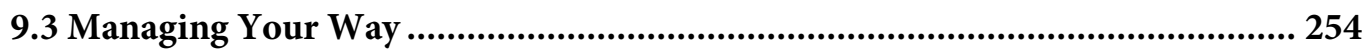

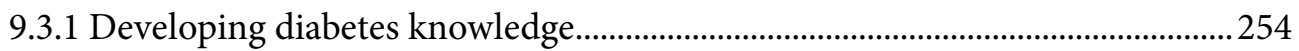

9.3.2 Balancing support and self-reliance ....................................................................25

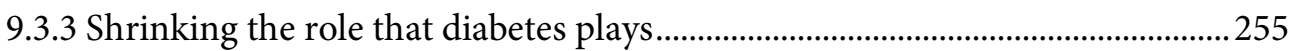

9.3.4 Focusing on positives or negatives ......................................................................256

9.3.5 Accessing reliable technology and meaningful information ..............................256

9.4 Love Letter, Break-up Letter ................................................................................. 256

9.4.1 Acquiring and changing medical devices ...........................................................258

9.4.2 Requiring convenience and practicality for everyday contexts.........................259

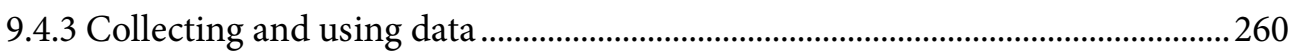

9.4.4 Corresponding with preferences and values .......................................................261

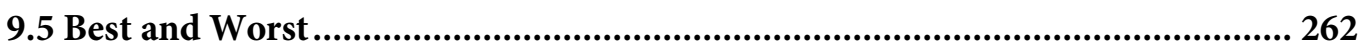

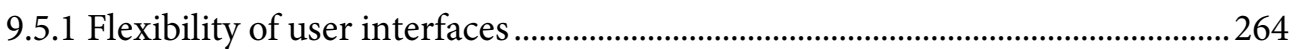

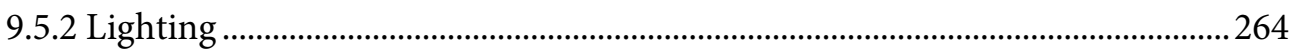

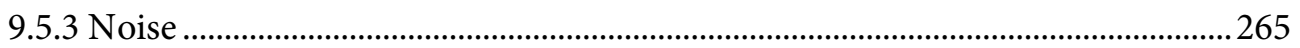

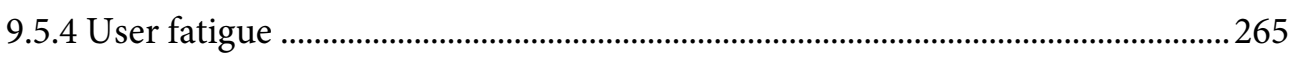

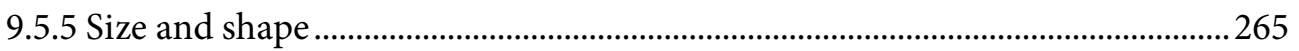

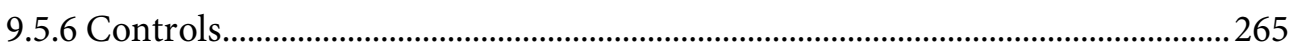

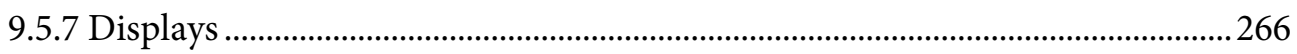

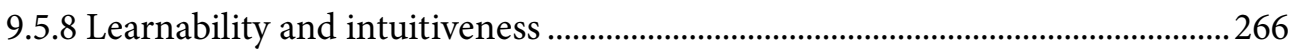

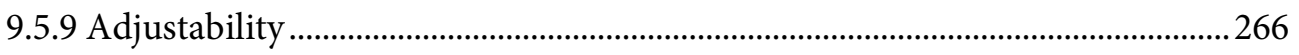

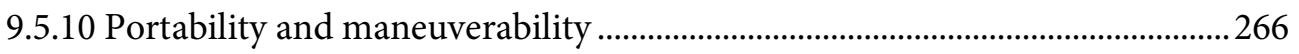

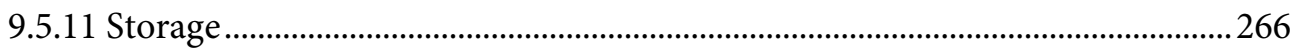

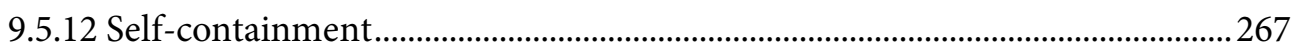

9.5.13 Freedom from calibration, maintenance, and repair ......................................... 267

9.5.14 Climate (thermal environment, humidity, airflow, pressure)..........................267

9.5.15 Power requirements ....................................................................................... 267

9.5.16 Aesthetics and unobtrusiveness.......................................................................268

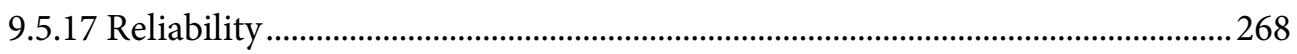

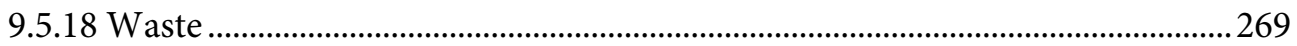

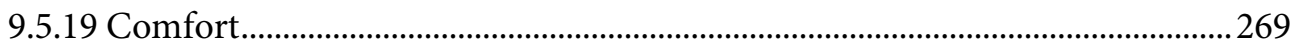

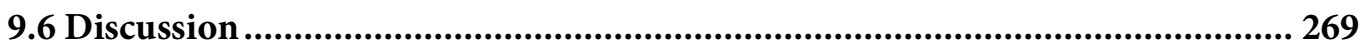

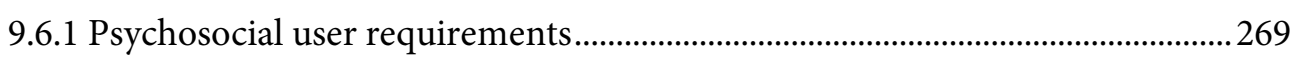


10. Addressing Medical Device-Related Attention Through Participatory Design 275

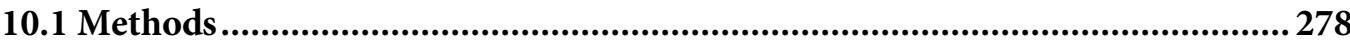

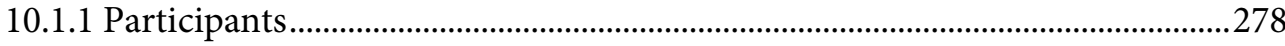

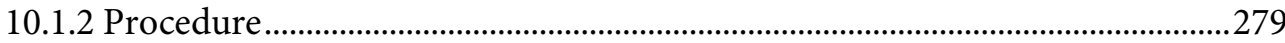

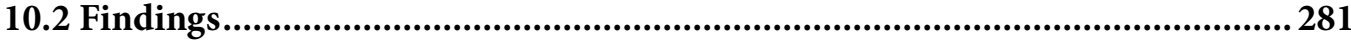

10.2.1 Exploration of strategies to address stigma .........................................................281

10.2.2 Selecting strategies to address stigma for various users........................................288

10.2.3 Design concepts and reviews for glucose monitoring.........................................289

10.3 Discussion .................................................................................................................. 299

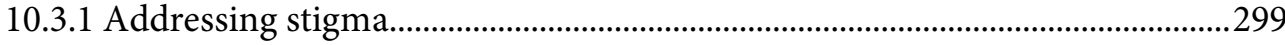

10.3.2 Participatory design approach ………………………………………………….......301

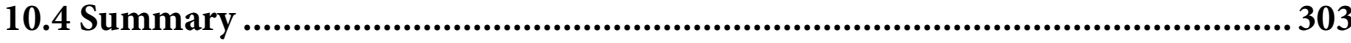

Chapter 11 - Discussion................................................................................... 305

11.1 Psychosocial User Requirements of Medical Technologies ................................305

11.1.1 Allow accessibility and affordability .....................................................................305

11.1.2 Foster trust in accuracy and reliability ...................................................................306

11.1.3 Manifest usability ...............................................................................................306

11.1.4 Affording comfort..................................................................................................306

11.1.5 Suitability and desirability..................................................................................308

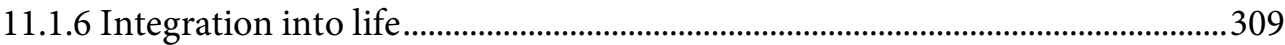

11.1.7 Enhancement of self-management......................................................................311

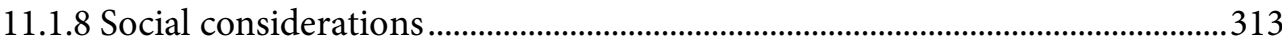

11.1.9 Enhancement of support network...................................................................314

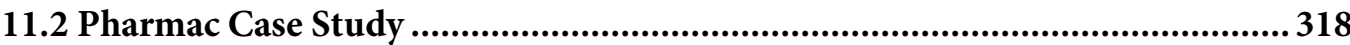

11.3 Grounded Theory Summary of Adolescent Experiences ....................................321

11.3.1 Conceptualising type 1 diabetes and its management .......................................321

11.3.2 Wanting to minimise the impact of type 1 diabetes...........................................324

11.3.3 Having individual medical technology needs .......................................................325

11.3.4 Struggling for independence ................................................................................326

11.3.5 Managing identity ..............................................................................................328

11.3.6 Receiving and providing social support .................................................................330 


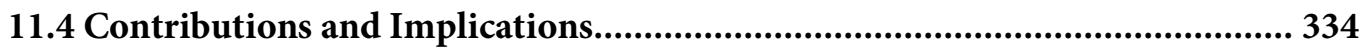

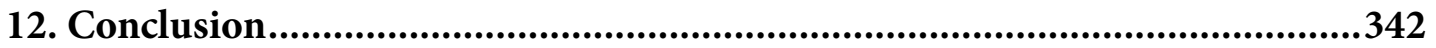

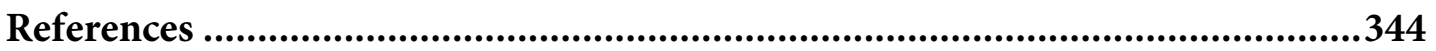

Appendix A …................................................................................................387

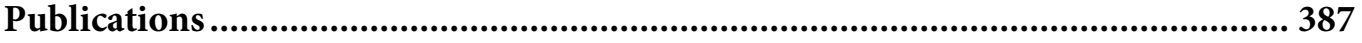

Appendix B...........................................................................................388

Information and Consent Form for Health Professionals......................................... 388

Appendix C ..............................................................................................390

Sample Information and Consent Form for Designers ............................................. 390

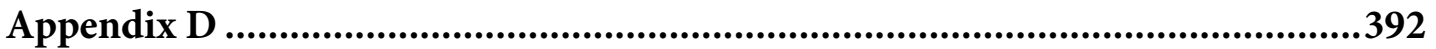

Sample Information Sheet for Adolescents and Young Adults............................... 392

Sample Assent Form for Adolescents Younger than 14 Years ................................ 395

Sample Assent Form for Adolescents 14-15 Years ................................................. 396

Parental Consent Forms for Adolescents 13 -15 Years .............................................. 397

Sample Consent Form for Adolescents and Young Adults 16 Years and Above..... 398 


\section{List of Figures}

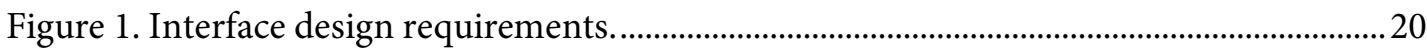

Figure 2. Blood glucose meter with test strip and lancet. ................................................................2 25

Figure 3. Continuous blood glucose monitor. …………………………………………………...... 26

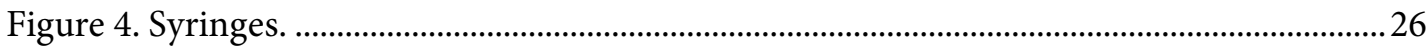

Figure 5. Reusable and disposable insulin pens.............................................................................. 27

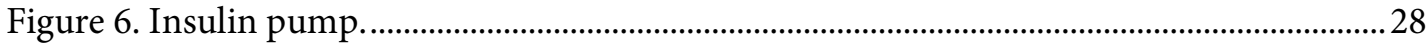

Figure 7. Test kit contents and interior photograph of a handbag, showing diabetes equipment in plastic and patterned bags.................................................................................. 29

Figure 8. Diabetes equipment stored under the bed or in drawers at home. ..............................2 29

Figure 9. Insulin stored in the refrigerator. ……………….......................................................... 30

Figure 10. Used insulin pump and blood glucose meter consumables left on a household surface, then sorted for sharps disposal, recycling, or waste..................................................30

Figure 11. Overview of participatory design approach. ....................................................................54

Figure 12. Visual representation of the grounded theory process..................................................56

Figure 13. Excerpt from an interview transcript and initial coding................................................59

Figure 14. Focused coding and development of categories in process. .......................................59

Figure 15. Excerpt from a memo written over multiple days after interviews with health

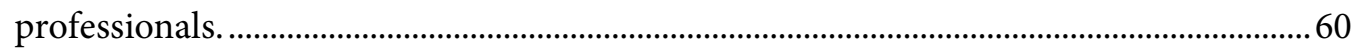

Figure 16. Digital mapping of focused codes to aid development of categories.........................61

Figure 17. Physical clustering of focused codes to aid development of categories, with colours used to represent different data sources...................................................................................... 61

Figure 18. Subscope continuous glucose monitor........................................................................115

Figure 19. Joya blood glucose monitoring jewellery....................................................................117

Figure 20. Armilla insulin pump........................................................................................118

Figure 21. Emoti-Comms buddy bracelet. ..................................................................................120

Figure 22. Simpac all-in-one blood glucose testing and insulin delivery..................................121

Figure 23. Participant recruitment flyer.....................................................................................130

Figure 24. Adolescent and young adult participant age and gender..........................................131

Figure 25. Adolescent and young adult participant age at diagnosis........................................131

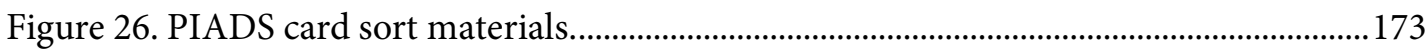

Figure 27. Psychosocial impacts of blood glucose meters. ………………………………….......177

Figure 28. Psychosocial impacts of continuous glucose meters..................................................178

Figure 29. Psychosocial impacts of insulin pens......................................................................180 
Figure 30. Psychosocial impacts of insulin pumps.

Figure 31. Average subscale scores of diabetes medical devices.

Figure 32. Infographic exploring blood glucose levels, diabetes complications, and daily selfmanagement tasks.

Figure 33. Vignettes displaying user requirement, related quotations form interviews with adolescents, and a related design concept.

Figure 34. Blood glucose monitoring phone case in dog and monster options, with accompanying mobile application.

Figure 35. Blood glucose monitoring jewellery in a choker and pendant combination........ 225

Figure 36. Blood glucose test reminder watch with twistable watch face. 226

Figure 37. Blood glucose monitoring compact with lights to prompt use of different fingers and replacement of lancets.

Figure 38. Night light and diabetes storage device.

Figure 39. Continuous glucose monitoring sock with cooling and vibration.

Figure 40. Storyboard showing the sock waking the adolescent with cooling and vibration, and completing a simple puzzle to silence the phone's alarm.

Figure 41. Blood glucose monitoring bike attachment with lever action to prick finger and shift test strip into place.

Figure 42. Subscope continuous glucose monitor.

Figure 43. Simpac all-in-one blood glucose testing and insulin delivery. 234

Figure 44. Cultural probes kit supplied to participants. 241

Figure 45. Activity card for metaphor method. 242

Figure 46. Activity card for managing your way method. 243

Figure 47. Activity card for letter-writing method. 244

Figure 48. Activity card for method choosing best and worst device features. 245

Figure 49. Diabetes management as a balancing act.

Figure 50. Diabetes management as a seesaw, with many factors affecting blood glucose levels.

Figure 51. Diabetes management as an arcade pinball machine...............................................248

Figure 52. Diabetes management as an arcade claw machine...................................................249

Figure 53. Diabetes management as rushing to work to clock in on time................................249

Figure 54. Diabetes appearing as an angel or devil on your shoulder. ......................................220

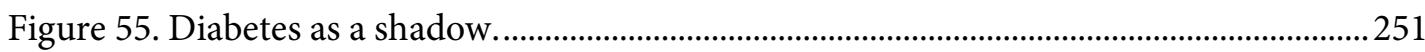

Figure 56. The negative psychosocial impacts of diabetes. ………………………………….......252 
Figure 57. What diabetes should be like: no confusion, feeling relaxed and well-rested, included, and happy......

Figure 58. Best, worst, and suggested features of medical devices.

Figure 59. Jewellery for insulin delivery.

Figure 60. A 'pimped' insulin pump.

Figure 61. Blind portraits drawn to ready participants for drawing activities and partner interaction

Figure 62. Participants sketching design concepts

Figure 63. Earring and ring designs for glucose monitoring. 283

Figure 64. Sub-cutaneous chip and phone application for blood glucose testing, showing wireless connection to a chip implanted under the skin. .284

Figure 65. Personalising lancets with colourful skins. .285

Figure 66. Lancet that borrows a 'sporty' aesthetic. 286

Figure 67. Diabetes festival with a stall with food shaped as a pancreas, syringe water slide, and blood glucose guessing competitions

Figure 68. Smartphone attachment. 290

Figure 69. Smartphone attachment and user interface. 290

Figure 70. Storyboard of functions: measuring blood glucose and ketones, displaying data and trends, and communicating with an insulin pump and health professional..........291 Figure 71. Miniature keychain blood glucose meter.......................................................................2.292

Figure 72. Keychain prototype at a larger scale. …………..........................................................2.293

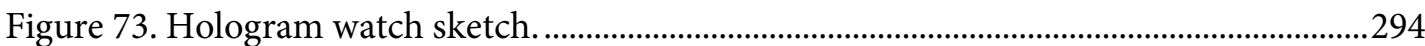

Figure 74. Hologram watch paper prototype. .............................................................................2. 294

Figure 75. Sketch of software and 3D-printed blood glucose meters..........................................296

Figure 76. Prototype of a customised blood glucose meter...........................................................297

Figure 77. Sketch of a modular test kit with core element and extra storage............................298

Figure 78. Prototype of a modular test kit. .................................................................................29

Figure 79. Mapping psychosocial user requirements to grounded theory categories.............333 


\section{List of Tables}

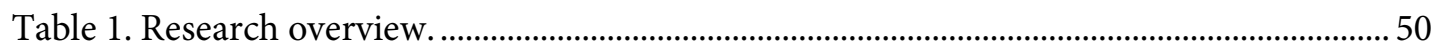

Table 2. Criteria for constructivist grounded theory..................................................................... 58

Table 3. Concepts, categories, and properties developed from interviews with health

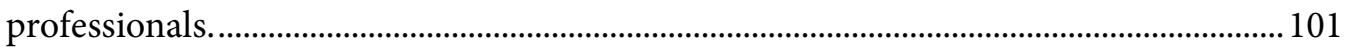

Table 4. User requirements addressed by student design concepts. ...........................................114

Table 5. Summary of concepts, categories, and properties. ........................................................... 165

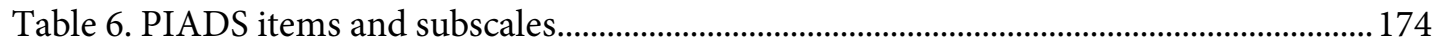

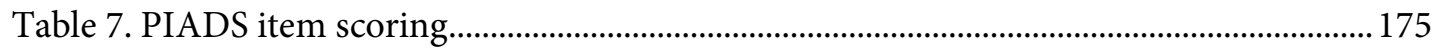

Table 8. User requirements addressed by student design concepts. ............................................222

Table 9. Medical devices reviewed by participants. ......................................................................2.

Table 10. Levels of information elicited by cultural probe activities.............................................2. 273

Table 11. Voting by participants for strategies to address stigma for different types of users.

Table 12. Development of psychosocial user requirements..........................................................316

Table 13. Comparison of psychosocial user requirements and Pharmac usability criteria. .319 


\section{Introduction}

\subsection{Motivation}

I am a human-centred designer and researcher who has brought together the Design, Psychology, and Anthropology disciplines to better my understanding of people's needs, behaviours, and decision-making. I have thrived on working in these interstitial spaces, utilising these disciplines to inform design.

I have applied these skills in the healthcare context: investigating what kinds of medical technologies adolescents with type 1 diabetes want to use, designing an app to get children engaged in their health care, and designing a system to help women manage pelvic floor dysfunctions. While the contexts vary, my empathic approach to building user engagement remains. I put people before their health conditions, and work to make solutions that fit their needs, goals, and everyday lives. As such, people-first language is used throughout my thesis (Harrington, 2008).

The design of personal medical technologies for long-term conditions requires attention to the ways in which people integrate devices into their lives. After talking with a clinician in the early phases of my $\mathrm{PhD}$, I narrowed the scope of my research to work with adolescents with type 1 diabetes as there was a clear need to elicit their experiences of diabetes management and requirements of medical technologies to facilitate improvements in their wellbeing.

\subsection{Background}

Personal medical devices are increasingly used to manage long-term conditions in a variety of contexts, by a variety of users. The World Health Organization has named chronic diseases as "the health care challenge of this century" (World Health Organization, 2002, p.8). New Zealanders are living longer, but in poorer health, with $50 \%$ of health loss attributed to disabling conditions (Ministry of Health, 2013). Further, reductions in years affected by fatality or disability are not realised for adolescents and young adults in the same magnitude as other age groups (Ministry of Health, 2016). 
Designers need to take this shift into consideration when creating personal medical devices, considering user experiences and contexts. Clinical efficacy needs to be paired with effective use: "The most carefully designed and prescribed technology is of no value if the intended user leaves it in the closet" (Polgar, 2010, p. 22). Currently, medical devices are assessed for safety (Medsafe, 2017) and can be designed in line with usability criteria (International Electrotechnical Commission, 2007), however, further considerations of how devices are integrated into daily life are not required. Establishing user requirements and incorporating them into the design process is pertinent, as this can improve device safety, usability, efficiency, and patient outcomes (J. L. Martin, Murphy, Crowe, \& Norris, 2006, p. 51) .

There is uncertainty around the causes of and best interventions to improve self-management (Williams, DiMatteo, \& Haskard, 2009). In one study, factors that affect self-management were: patient, regimen, clinical setting, disease characteristics, and provider-patient relationship (Ickovics \& Meisler, 1997). In another review that examined both assistive and medical technologies, adherence was affected by health beliefs, personal, environmental, resource, condition, intervention, and clinician relationship factors, yet only assistive devices, and not medical devices were considered as a factor affecting adherence (Wessels, Dijcks, Soede, Gelderblom, \& De Witte, 2003). This reflects the complexity of the factors that affect adherence, and also a recurring finding that literature on adherence among people with chronic disease provides only cursory mention, if at all, of the impact of medical devices on adherence. However, factors that affect the non-use of assistive devices show a strong similarity with factors that affect self-management with medical devices, and as such, the strategies used for reducing non-use of assistive devices could be applied to medical devices (Wessels et al., 2003).

When medical devices are considered, factors thought to impact selfmanagement include: discomfort, lack of discretion, inconvenience, portability, lack of training, lack of intuitiveness or usability, perceived value and device features, motivation, cultural predisposition, and aesthetics (Hgen, 2012; Turieo, 2011). Barber (1996) considers the reason many devices are not used effectively is because they are created for alleviating impairment or treating disease, rather than for the user. Hgen $(2012$, p. 3) articulated the ways in which healthcare devices affect 
adherence: "If the device enhances the user's experience, fits into the patient's life more effectively, and has less of a perceived stigma attached to it, there is a greater chance it will be used, and used properly." Almost every psychosocial and contextual aspect of a patient's life is likely to be correlated with their adherence (Williams et al., 2009).

\subsubsection{Adolescent development}

Normative adolescent development can interfere with treatment regimens for chronic diseases, and be interfered with by chronic diseases (C. Martin, Nunez, \& Royo, 2012; Warner \& Hauser, 2009). For instance, the common adolescent desire for social acceptance by peers can conflict with diabetes management (Badlan, 2006; Berge, Patterson, Goetz, \& Milla, 2007; Gjengedal, Rustoen, Wahl, \& Hanestad, 2003). Additionally, approximately $50 \%$ of adolescents with long-term conditions do not manage them effectively (KyngÄs, Kroll, \& Duffy, 2000). However, adolescence is considered a critical period for forming long-term habits. Furthermore, a change in behaviour at this stage can dramatically alter an adolescent's health trajectory (Holmbeck, 2002). Adherence can be affected by 'the rest of life': the other components that make up an adolescent's day such as school, or spending time with friends (Warner \& Hauser, 2009). Creating interventions that specifically focus on combatting factors that inhibit adolescents' self-management could result in more targeted and effective research and improved health outcomes (Warner \& Hauser, 2009).

\subsubsection{A holistic design and human factors approach}

The United States Food and Drug Administration has declared that human factors methods must be used in the development of medical devices (Wiklund, 2005a, 2005b). People who use this approach seek to design systems or objects around users, rather than asking the user to change their behaviour (Russ et al., 2013). Historically, human factors approaches have focused on functionality, usability, and reliability, but are now shifting toward a holistic approach that attunes not only to people's physical and cognitive traits, but also their affective reactions (Norman, 2002). While many human factors approaches aim simply to eliminate dissatisfaction, there is 
room for an approach that creates pleasurable design (Jordan, 1999). Norman (2002, 2004) suggests that attractive devices put people in a positive mood, helping them learn to use the device, creatively solve any problems that might arise, and better deal with the stress of using the device. Norman's (2004) three-factor model outlines interactions people may have with designed objects: 'visceral', where appearances and attractiveness are important; 'behavioural', where typical human factors principles such as usability and function exist in addition to pleasure and physical feel; and 'reflective', where people rationalise their responses towards the design, and consider how it links with their self-image, memories, ideals of beauty, and such. Walter (2010) advocates that functionality, reliability, and usability, but also pleasure, are required for effectiveness (Figure 1).

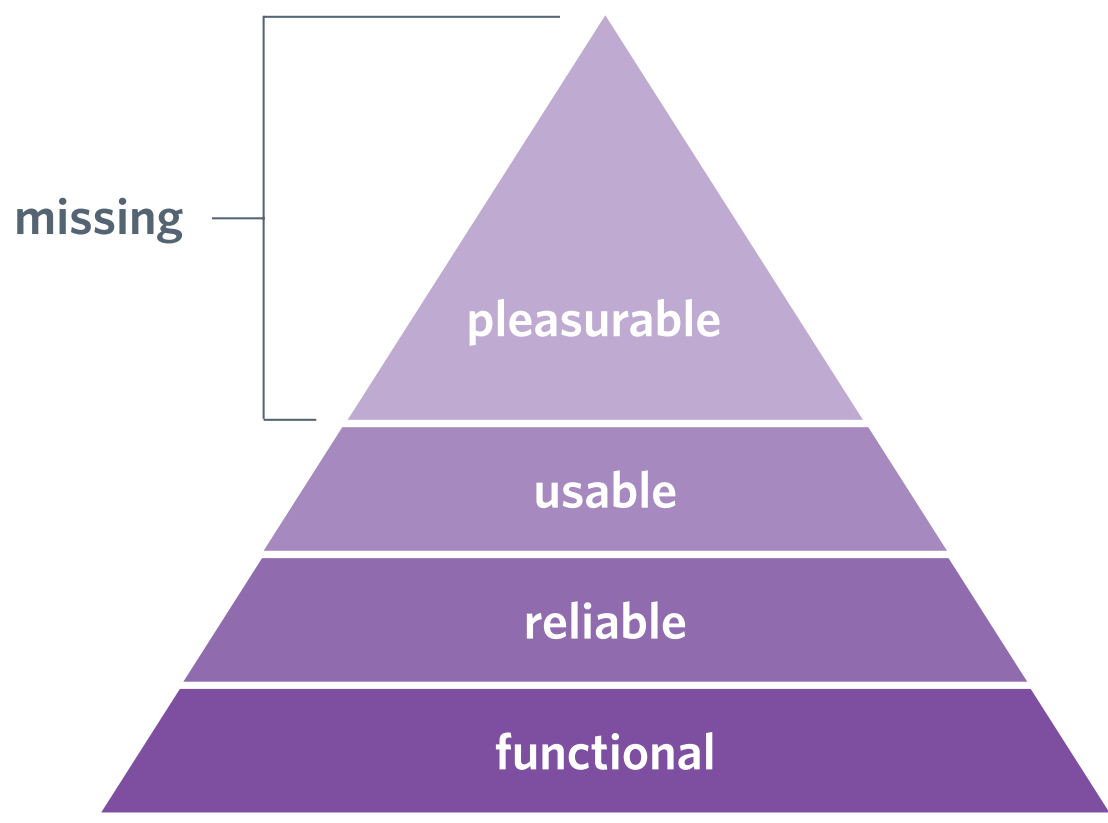

Figure 1. Interface design requirements. Reproduced from Designing for Emotion, (p. 6), by A. Walter, 2010. Copyright 2010 by New York: Book Apart. Reproduced with permission.

Although functionality and usability are important to consider, focusing too narrowly on these factors can inhibit designers from exploring the meanings their devices convey (Polgar, 2010). There is a semantic nature to medical devices, and as designers, this is something that can be manipulated in addressing self-management.

Design is uniquely positioned to address barriers to self-management. It can help people feel understood and cared for, empower them to shape their healthcare, and challenge existing practices (Sklar \& Naar, 2017). Images of people using 
personal medical devices have become prevalent on social media, adding to an ongoing conversation about living a life with devices used to treat long-term conditions, and how people choose to hide or publicise these devices and their medical condition.

\subsection{Thesis Overview}

This research aimed to answer an overarching research question: How do adolescents experience self-management of type 1 diabetes, and what psychosocial user requirements of medical technologies are relevant for them? The thesis follows the sequential nature of the research and the content of each chapter is outlined below. Additionally, peer-reviewed publications adapted from the thesis are listed in Appendix A.

\section{Chapter 2: Literature Review}

The literature review outlines type 1 diabetes and its treatment regimen, theoretical approaches to diabetes management, existing user requirements for medical technologies, common barriers to diabetes management for adolescents, and existing technologies and concepts that aim to overcome these barriers.

\section{Chapter 3: Methodology}

The methodology outlines the constructivist epistemology of the research and discussions about designing and conducting research with adolescents. I describe the methodologies I used: participatory design and constructivist grounded theory. Finally, I discuss methods for constructing user requirements. While grounded theory and user requirements are reported throughout this thesis, the final summaries of these are reported in Chapter 11.

\section{Chapter 4: Clinician Interviews}

This chapter describes semi-structured interviews conducted for the purposes of this research with health professionals who treat adolescents and young adults with type 1 diabetes. Interviews covered: impressions of self-management, developments in and impacts of medical technologies, acquiring and changing medical technologies, 
and adolescent-specific needs. Constructivist grounded theory was used to collect and analyse the interview data, and psychosocial user requirements were formed.

\section{Chapter 5: Designing Medical Technologies for Adolescents}

This chapter documents the development of adolescents' psychosocial user requirements and corresponding designs for medical technologies created by 24 undergraduate design students. A clinician reviewed the designs and user requirements. This method of using design to further develop and provide additional support for user requirements is discussed.

\section{Chapter 6: Adolescent Interviews}

This study's semi-structured interviews with 16 adolescents and young adults with type 1 diabetes covered: daily life, hobbies and interests, diagnosis period, diabetes knowledge, self-management experiences, acquiring and changing medical devices, support networks, interactions with peers, and views on ideal self-management. Constructivist grounded theory was used to collect and analyse the interview data, and psychosocial user requirements were formed.

\section{Chapter 7: Psychosocial Impacts of Medical Technologies}

This chapter focuses more narrowly on the impacts of current medical technologies. The Psychosocial Impacts of Assistive Devices Scale (PIADS) was adapted into a card sort task, and participants with type 1 diabetes sorted the scale's items, illustrating the psychosocial impacts their blood glucose testing and insulin administering technologies have on them. Descriptive statistics and grounded theory were used to analyse the data, and user requirements were formed.

\section{Chapter 8: Designing Medical Technologies from Adolescents' Requirements}

Undergraduate design students created medical technology designs in response to five psychosocial user requirements of medical technologies constructed in the previous chapters. Vignettes of the designs and user requirements were discussed with six adolescents and young adults with type 1 diabetes in individual expert 
reviews. This method of using design to further develop and provide additional support for user requirements is discussed.

\section{Chapter 9: Cultural Probes}

Participants with type 1 diabetes completed cultural probes activities at home. The activities were: drawing a metaphor for diabetes management, describing a personal view of ideal self-management, writing a love letter or break-up letter to a medical device, and pinpointing the best, worst, and desired features of current medical devices. Constructivist grounded theory was used to analyse the interview data, and psychosocial user requirements were formed.

\section{Chapter 10: Addressing Medical Device-Related Attention Through Participatory Design}

Participants with type 1 diabetes and designers took part in a design workshop to address medical technology-related attention. Participants brainstormed advantages, disadvantages, and ideas for implementing six existing strategies for addressing stigma. Participants sketched and prototyped blood glucose monitoring designs based on the most promising strategies.

\section{Chapter 11: Discussion}

The final psychosocial user requirements of adolescents with type 1 diabetes are reported and compared to the selection criteria for choosing blood glucose meters to be subsidised in New Zealand. The grounded theory concepts developed throughout the thesis are also summarised, and implications and extensions of the research are discussed. 


\section{Literature Review}

\subsection{Type 1 Diabetes}

Type 1 diabetes is an autoimmune condition with no cure, where pancreatic $\beta$-cells are destroyed. This results in a deficiency of insulin. The cause is believed to have both genetic and environmental factors. In Western countries, type 1 diabetes accounts for approximately $90 \%$ of diabetes in children and adolescents and 5-10\% of diabetes among all diagnosed individuals. Worldwide, approximately 80,000 people under the age of 15 develop type 1 diabetes annually, with the incidence rising in recent decades (ISPAD, 2014). Diagnosis rates increase from birth, peaking between 10-14 years then declining following puberty (South Island Alliance, 2013). In New Zealand, there are approximately 2500 people aged $0-18$ with type 1 diabetes (“Diabetes Youth New Zealand," n.d.). While the incidence of type 1 diabetes is lower in Māori and Pasifika than New Zealanders of European descent (Derraik et al., 2012), their metabolic control is poorer, and there may be cultural and socioeconomic differences in adolescents' requirements (P. J. Carter et al., 2008).

Adolescents must manually manage their blood glucose levels, eating to avoid hypoglycaemia (low blood glucose levels) and injecting insulin to avoid hyperglycaemia (high blood glucose levels). From diagnosis, adolescents are assigned a diabetes team consisting of health professionals such as endocrinologists, general practitioners, clinical nurse specialists, nurse practitioners, clinical psychologists, and dieticians (ISPAD, 2014). This team prescribes a treatment regimen; generally four or more blood glucose tests per day, healthy diet and exercise, and calculating and self-administering insulin three or more times per day (D'Alberton, Nardi, \& Zucchini, 2012; Strawhacker, 2001). To achieve this, adolescents use a range of medical devices. To measure blood glucose levels, adolescents commonly prick their finger with a lancet and transfer a drop of blood onto a test strip, which is inserted into a blood glucose meter. This displays a single blood glucose reading. A lancet, blood glucose monitor, and test strip are pictured (Figure 2). 


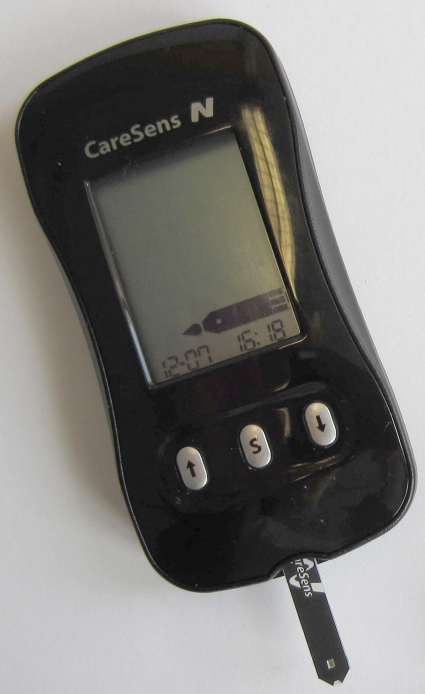

Figure 2. Blood glucose meter with test strip and lancet.

To measure glucose, adolescents may also use a continuous blood glucose monitor (CGM), where a sensor is partially inserted under the skin and intermittently transmits glucose levels to a phone or monitor. The CGM interface (Figure 3) displays a graph of glucose levels and anticipated trend. CGMs are uncommon in New Zealand as they are not subsidised by Pharmac. ${ }^{1}$ Using a CGM requires periodic calibrations against a blood glucose meter.

${ }^{1}$ Pharmac is the New Zealand Government drug purchasing and funding agency that chooses which medical devices and consumables are subsidised. In 2013 Pharmac moved to a single supplier arrangement for blood glucose meters and their consumables. 


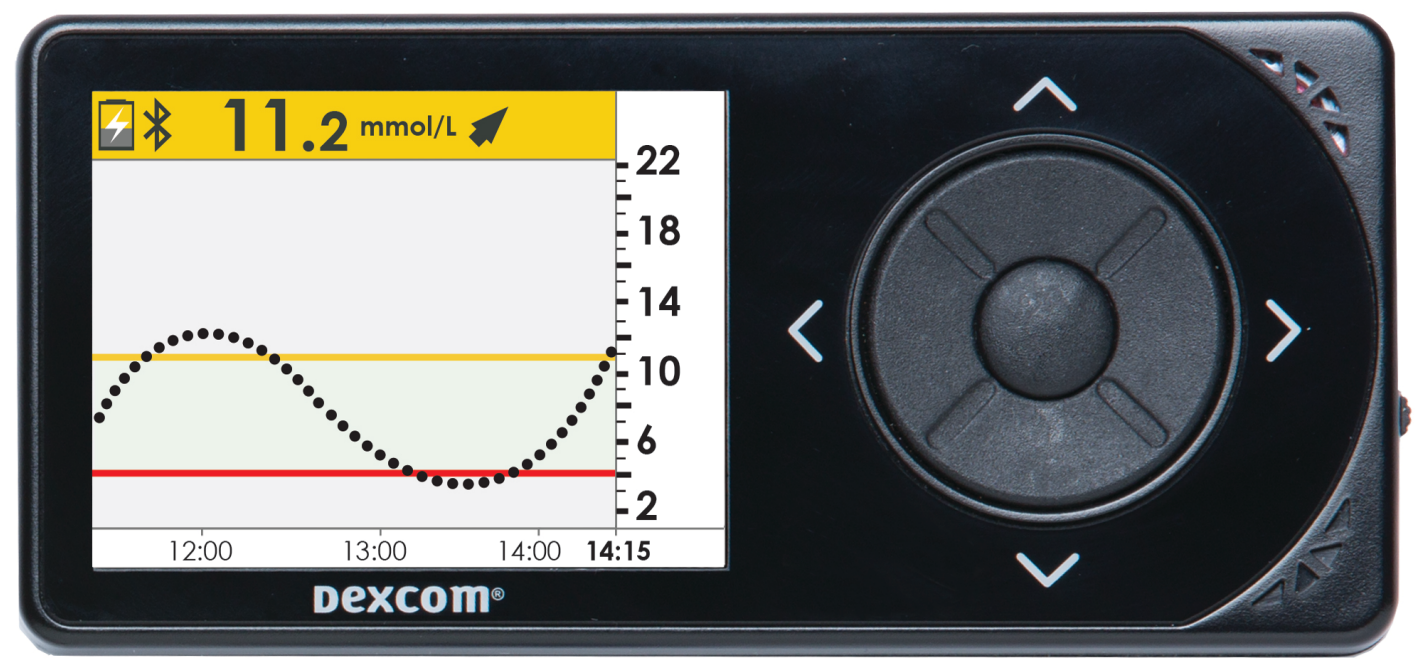

Figure 3. Continuous blood glucose monitor. Reproduced from "Images," by Dexcom, 2015, (http://www.mynewsdesk.com/uk/dexcom-europe/images). Copyright 2015 by Dexcom. Reprinted courtesy of the Copyright Holder under a Creative Commons License.

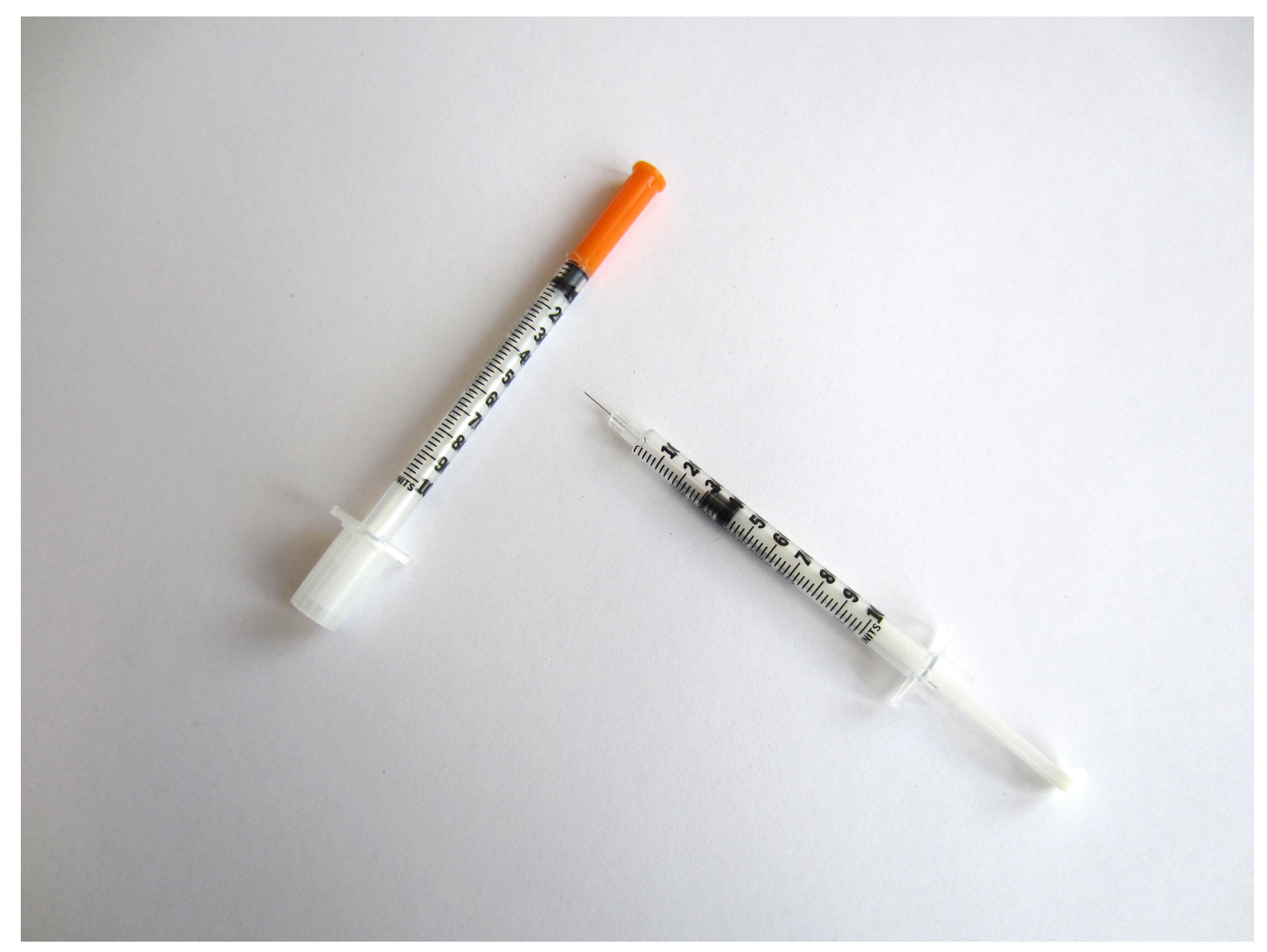

Figure 4. Syringes.

There are three options for insulin delivery: vial and syringe (Figure 4), insulin pen (Figure 5), and insulin pump (Figure 6). In Figure 5, a refillable insulin pen is pictured on the far left. Pictured to the immediate right of this device is a disposable 
pen. Once the insulin in this pen is finished, the whole pen is disposed of. The introduction of insulin pens has increased adherence, reduced dosage errors, needle fear, and embarrassment in comparison to syringe and vial combinations (Pfützner, Asakura, Sommavilla, \& Lee, 2008).

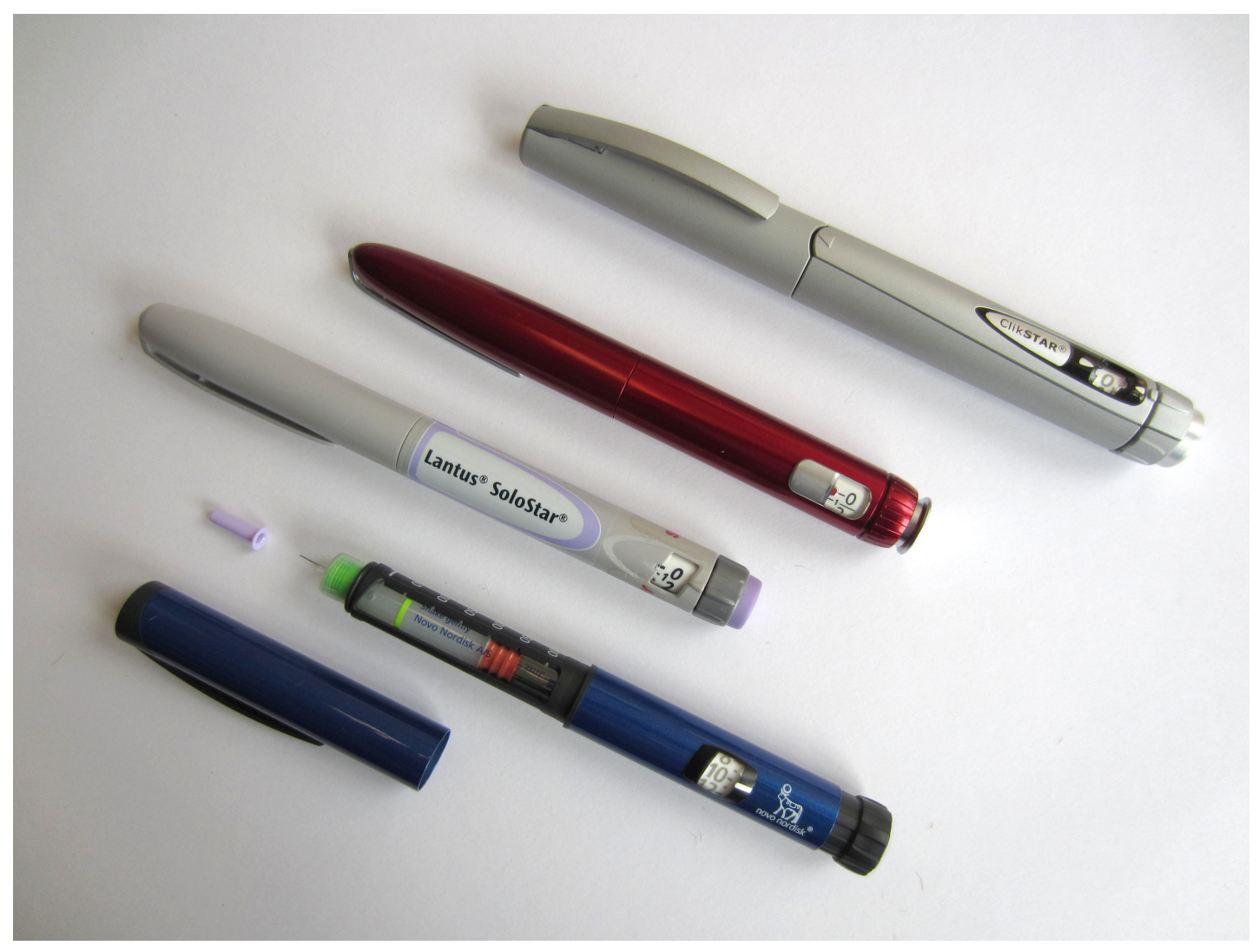

Figure 5. Reusable and disposable insulin pens.

Insulin pumps are usually connected via tubing to an invasive site, where rapidacting insulin is intermittently pumped as required. The site remains in place for approximately three days, minimising the number of invasive procedures required. 


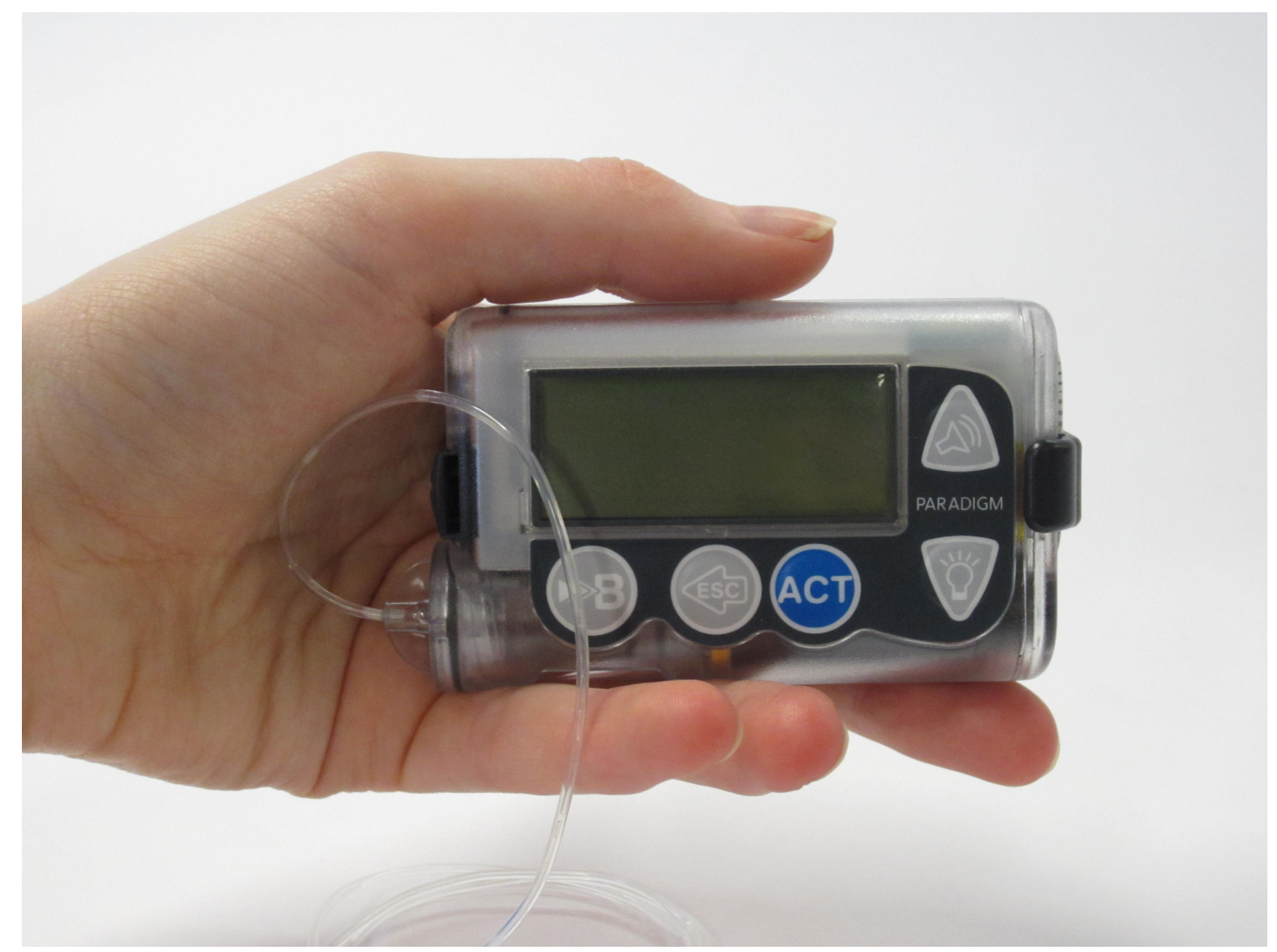

Figure 6. Insulin pump.

Adolescents use combinations of these devices, consumables (e.g., needles, test strips), carbohydrate sources, and accessories. Below are photographs of adolescents' medical equipment stored in-situ (Figures 7-10). These images were collected during a research activity reported on in Chapter 9. 

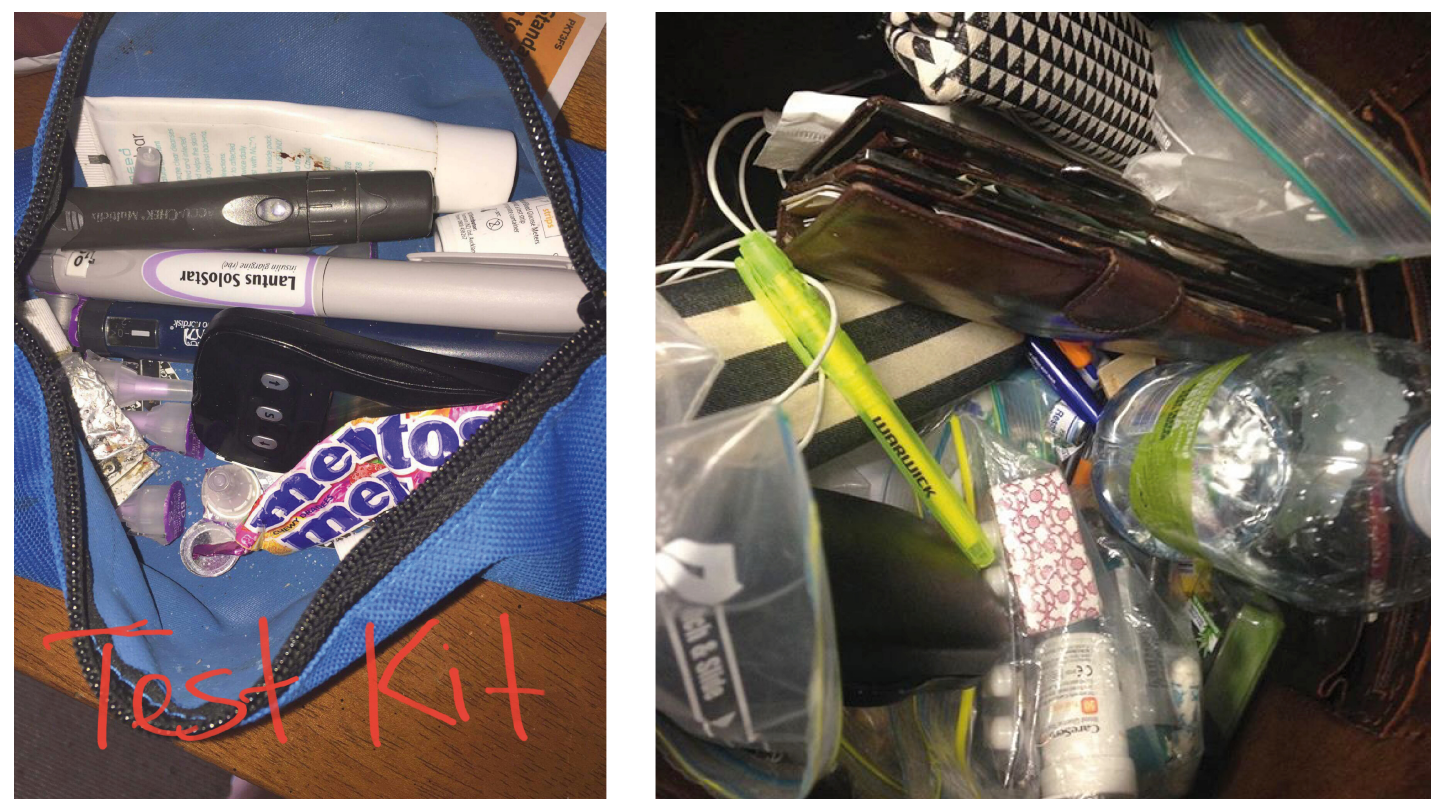

Figure 7. Test kit contents and interior photograph of a handbag, showing diabetes equipment in plastic and patterned bags.
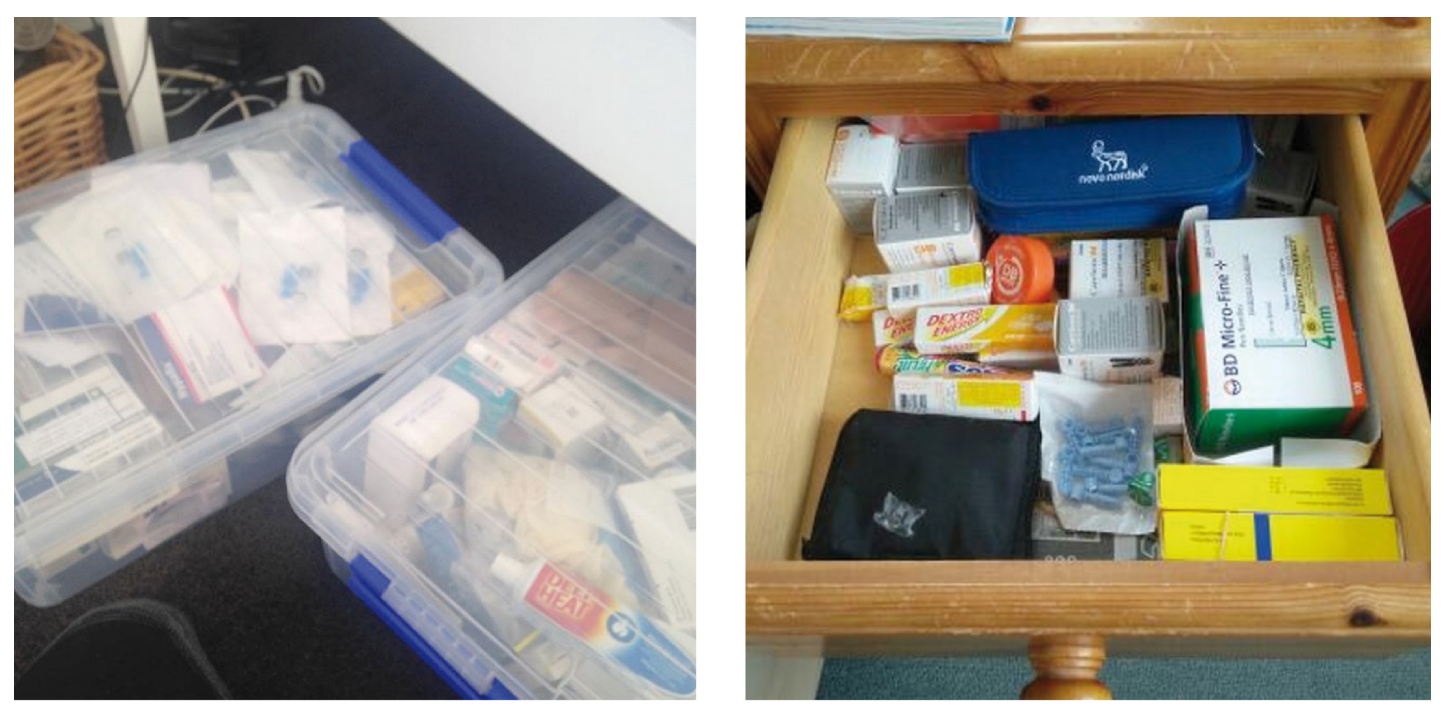

Figure 8. Diabetes equipment stored under the bed or in drawers at home. 

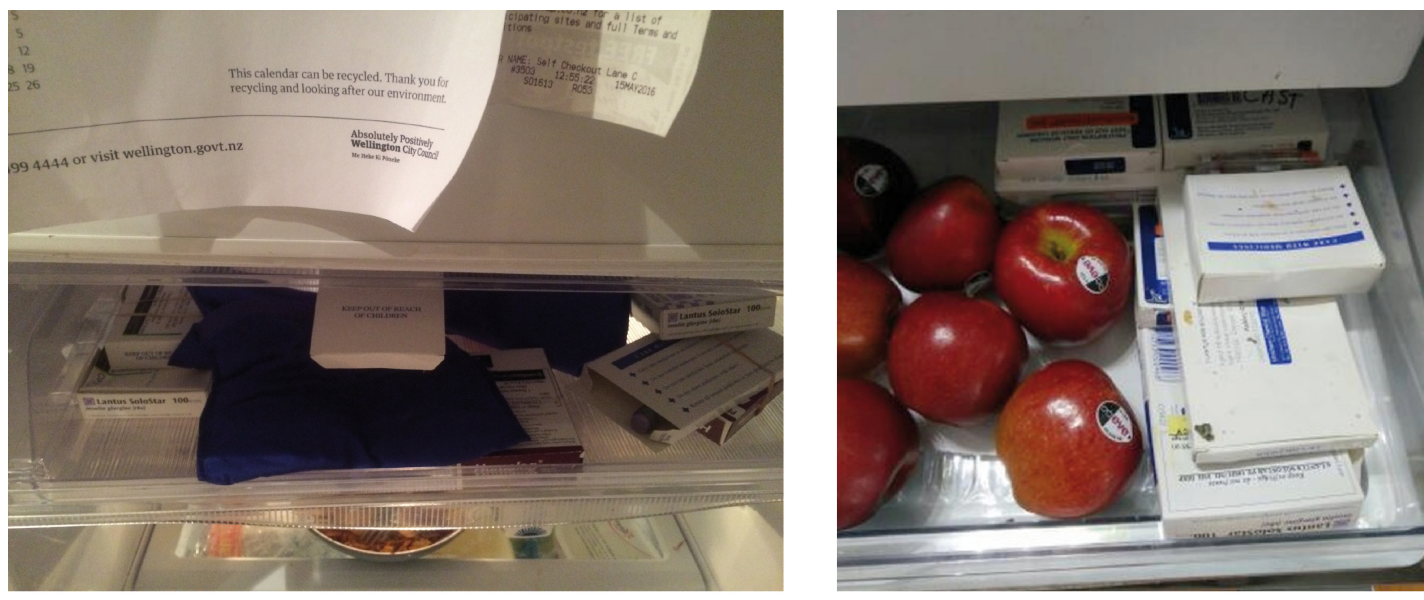

Figure 9. Insulin stored in the refrigerator.
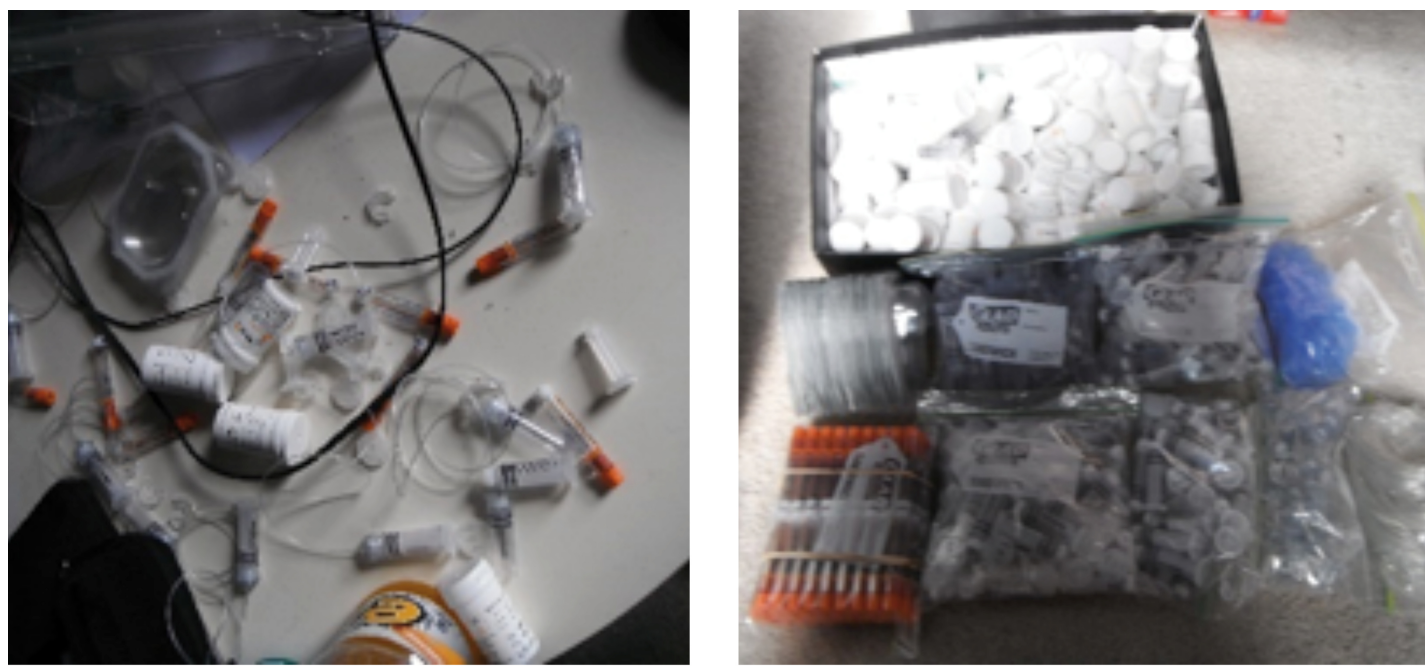

Figure 10. Used insulin pump and blood glucose meter consumables left on a household surface, then sorted for sharps disposal, recycling, or waste.

This intensive treatment regimen of using an insulin pump or three or more insulin injections per day, in addition to frequent blood glucose monitoring, delays the onset and reduces the progression of diabetes complications such as diabetic retinopathy (eye damage) and nephropathy (kidney damage) in adolescents (DCCT Research Group, 1994). Additionally, there is a bi-directional relationship between adherence and glycaemic control, in which an initial decline in glycaemic control resulting from pubertal development worsens levels of adherence to the treatment regimen, which in turn predicts declining glycaemic control among adolescents (Du PasquierFediaevsky, Chwalow, \& Tubiana-Rufi, 2005).

Concentrations of glycated haemoglobin (HbA1c) and number of blood glucose tests per day are used as proxy measures for adherence to a type 1 diabetes 
treatment regimen. In previous studies, approximately one-fifth to one-third of adolescents meet HbA1c targets or the required four blood glucose tests per day. (Hilliard, Wu, Rausch, Dolan, \& Hood, 2013; Wood et al., 2013). Adolescents with type 1 diabetes have less adherence to treatment regimens than other paediatric or adult groups (Borus \& Laffel, 2010), despite greater diabetes knowledge than children (B. J. Anderson, Auslander, Jung, Miller, \& Santiago, 1990; Berlin et al., 2006; A. M. Thomas, Peterson, \& Goldstein, 1997). Accordingly, diabetes knowledge or awareness of $\mathrm{HbA1c}$ results do not necessarily translate into better glycaemic control (Heisler, Piette, Spencer, Kieffer, \& Vijan, 2005). As such, deteriorating metabolic control during adolescence is relatively common, with diminishing acceptance of medical advice and adherence to treatment regimens (Cameron, 2006). When adolescents commonly have hyperglycaemic levels, this is referred to as 'running high' (Beshyah, 2009). While it is clinically important for adolescents to use medical technologies frequently to manage their health and decrease their diabetes complications, many do not.

\subsection{Conceptual Approaches to Diabetes Management}

This thesis focuses on self-management of adolescents with type 1 diabetes.

Terminology surrounding the management of medical conditions varies significantly within health literature and medical practice. Since the 1980s, researchers have been critical of 'compliance' and 'adherence' (Glasgow \& Anderson, 1999). These terms grew out of the traditional patient-clinician model used in the treatment of acute illnesses (R. M. Anderson \& Funnell, 2000) and were seen as measures of a person's success in adhering to a prescribed treatment regimen (Haynes, Taylor, \& Sackett, 1979). Adherence can consist of actions such as taking medications at certain times, and attending scheduled appointments, with nonadherence leading to poor health outcomes, more hospitalisations, unused prescriptions, and a diminished quality of the patient-clinician relationship (Haskard, DiMatteo, \& Williams, 2009). However, a significant power imbalance and negative, authoritarian connotations exist within this relationship, whereby patients who fail to comply may be viewed as disobedient, forgetful, or lazy (Delamater, Patino-Fernandez, Pulgaron, \& Daigre, 2012b; Haskard et al., 2009; Julius, Novitsky, \& Dubin, 2009), though some feel adherence implies a 
more active and collaborative role for the patient in achieving desirable health outcomes (Delamater et al., 2012b). Further, the views of health professionals and patients can differ, with clinicians focused on management within a clinical context and patients focused on management in a real-world context, balancing the impact of the regimen on their life and condition (Hunt, Arar, Larme, Rankin, \& Anderson, 1998).

While approaches such as adherence and compliance have fallen from favour, several other approaches have emerged, with 'patient activation' and 'patient engagement' gaining traction from 2013 (Menichetti, Libreri, Lozza, \& Graffigna, 2014). This suggests a shift has occurred, from patients being viewed as passive recipients of health care to engaged, active partners, reflecting a systems view of healthcare.

Engagement is an umbrella term that houses: 'empowerment', a person's capacity and whether they feel empowered to take action; 'partnership' or 'collaborative care', the relationship and development of an individual approach between a patient and health professional; 'activation', a person's understanding of their role in managing their health and being capable of taking on that role, typically used to refer to actions within a patient-doctor consultation; and 'self-management education', going beyond teaching patients about disease and facilitating healthrelated problem-solving (R. M. Anderson \& Funnell, 2012; Bodenheimer et al., 2002; Hibbard et al., 2004; Menichetti et al., 2014; Rozenblum, Miller, Pearson, \& Marelli, 2015).

Importantly, in addition to patients, health care professionals have a role in patient engagement, referred to as 'reciprocal tasks'. Both parties are expected to take an active role in developing engagement (Coulter, 2011). This partnership extends beyond patient-clinician consultations, with patients able to give input at all levels of the healthcare system (Bellows, Kovacs Burns, Jackson, Surgeoner, \& Gallivan, 2015; S. M. Brown et al., 2015). While defining engagement is challenging, there is a need for shared expectations and understandings to facilitate and achieve engagement (Gallivan, Kovacs Burns, Bellows, \& Eigenseher, 2012).

Engagement can be defined as "a co-constructed process and state. It incorporates a process of gradually connecting with each other and/or a therapeutic 
program, which enables the individual to become an active, committed and invested collaborator in healthcare" (Bright, Kayes, Worrall, \& McPherson, 2015, p. 650). While engagement may be seen as a continuum, it is unclear how much engagement is necessary and whether 'full engagement' is feasible or desirable (Bright et al., 2015). Examples of engagement in the literature include patients' actions such as accessing services, asking questions, and sharing information with health professionals (Bright et al., 2015; Franklin, Greene, Waller, Greene, \& Pagliari, 2008).

Engagement is a core component of 'patient-centred care', which seeks to move away from the power imbalances of compliance (Rozenblum, Miller, Pearson, \& Marelli, 2015). It is defined as "care that is respectful and responsive to individual patient preferences, needs and values, and ensuring that patient values guide all clinical decisions" (Briere, 2001, paragraph 19). In a meta-review of patient-centred care definitions, the six concepts that were evident across multiple sources were:

- "Education and shared knowledge;

- involvement of family and friends;

- collaboration and team management;

- sensitivity to nonmedical and spiritual dimensions;

- $\quad$ respect for patient needs and preference; and

- the free flow and accessibility of information." (Cronin, 2004, paragraph 33)

While there is much discussion in health literature about these approaches, they can be problematic in their implementation, with clinicians still describing the 'control' they have over patients and the 'control' they expect patients to have over their medical conditions, requiring a change in thinking and strategies to shift towards a self-management approach (Mudge, Kayes, \& McPherson, 2015). Further, for patients there can be both barriers to (e.g., resource scarcity, involvement fatigue) and benefits of engagement (e.g., improved understanding, developing and being valued for expertise) (Kovacs Burns, Bellows, Eigenseher, \& Gallivan, 2014).

At a simpler level, 'self-care behaviours' can also be discussed. These are actions carried out by patients or people who support them, such as checking blood glucose levels. Looking at specific behaviours can be helpful as they may not correlate 
with other self-care behaviours and can pinpoint areas for intervention (Delamater, Patino-Fernandez, Pulgaron, \& Daigre, 2012a).

This research is guided by the literature around engagement, recognising diabetes self-management as situated within an adolescent's life. Further, this research recognises that individuals require different approaches to suit their lives. In this thesis, engagement in self-management is used to describe adolescents considering or carrying out self-care actions related to type 1 diabetes in a manner that works for their lifestyle.

\subsection{User Requirements of Medical Technologies}

This section outlines relevant, existing user requirements of medical technologies that signal possible adolescent user requirements, and reported advantages and barriers of medical technologies. Eleven existing sets of user requirements for medical technologies were identified through a snowball search of predominantly health and human computer interaction literature (fewer requirements were found in the Design literature). Terms used within the literature were inconsistent, with searches for 'user requirements' and 'design guidelines' returning few results and making a more structured search strategy unfeasible. Additionally, much of the literature described a single medical device, making general search terms such as 'medical device' impractical, requiring searches for individual devices or brands. Finally, recent literature was predominantly about complex technologies such as insulin pumps and CGMs, however adolescents in New Zealand predominantly use older technologies such as insulin pens and blood glucose meters, as access and affordability differ between countries. Through comparing and contrasting the lists of user requirements, commonalities among them were identified. The following seven requirements were identified in at least two sources.

Minimise impact on daily life and facilitate living a full life. Medical technologies that are convenient to use in various daily contexts, lighten the load of selfmanagement and are designed around living life, rather than solely managing a disease (Cafazzo, Casselman, Hamming, Katzman, \& Palmert, 2012; Glasemann \& 
Kanstrup, 2011; Kanstrup, 2014; Kanstrup, Bertelsen, Glasemann, \& Boye, 2008; J. L. Martin, Norris, Murphy, \& Crowe, 2008).

Facilitate extended engagement. Medical technologies designed to encourage continued, long-term use (Lang, 2012; J. L. Martin et al., 2008).

Facilitate support network and connective services. While adolescents are the primary medical technology users, designers must consider adolescents' relationships with others, including family, health professionals, technicians, and peers with diabetes, and consider how these relationships shift during adolescence. Medical technology should facilitate relationships with health professionals and peers through features such as optional data sharing or chat functions, and facilitate the safe transition of responsibility from parents. This includes alerting parents of extremes in data and whether the adolescent has been testing blood glucose regularly, but not providing full access to daily data (Cafazzo et al., 2012; Glasemann \& Kanstrup, 2011; Kanstrup et al., 2008; Lang, Martin, Sharples, Crowe, \& Murphy, 2012; Machniak, 2013; J. L. Martin et al., 2008; Nordfeldt, Hanberger, \& Berterö, 2010; Toscos, Connelly, \& Rogers, 2011, 2012).

Provide meaningful information. Medical technologies turning data into appropriate, reliable, and useful information to enable adolescents to learn about diabetes and make health-related decisions. This information should be easy to access and visualised in a meaningful manner, providing feedback and prompts to aid healthy behaviour changes (Cafazzo et al., 2012; Kanstrup, 2014; Kanstrup et al., 2008; Lang, 2012; Machniak, 2013; Nordfeldt et al., 2010).

Usability. Intuitive, flexible, and easy use that supports the effective uptake of medical technologies (Lang, 2012; Machniak, 2013).

Affording choice and personalisation. Medical technologies allowing for individual preferences, personality, and identity, and accommodating age-appropriate choices, 
customisation, and personalisation (Glasemann \& Kanstrup, 2011; Lang, 2012; Machniak, 2013).

Accommodate social considerations and attention preferences. Adolescents require control over their identity, therefore medical technologies should be able to be used discreetly in social contexts if desired, and not contribute to embarrassment. Disguising these technologies could help to achieve this (Cafazzo et al., 2012; Kanstrup, 2014; Kanstrup et al., 2008; Lang, 2012).

Provide security. Medical technologies integrating data from relevant sources while maintaining users' control over privacy and sharing (Kanstrup et al., 2008; Machniak, 2013).

In addition to these explicit requirements, there was also literature available on adolescents' barriers to using current medical technologies. While this information is not in the ideal form for designers, barriers can be used to inform user requirements. These barriers are clustered into nine areas below, which mirror the user requirements described in Chapter 11.

\subsubsection{Access and affordability}

Health literature often ignores barriers to access for medical technologies (Prendiville, 2017), yet these are important for adolescents. In a meta-analysis and systematic review of randomised control studies, insulin pump therapy was more effective for glycaemic control than multiple daily injections (syringe or insulin pen) (Pańkowska, Blazik, Dziechciarz, Szypowska, \& Szajewska, 2009). However, insulin pumps are costly and only subsidised in New Zealand when criteria are met, such as four severe hypoglycaemic episodes in the previous six months, a HbA1c between 53 and $90 \mathrm{mmol} / \mathrm{mol}$, and adherence to an injection regimen for six months (Ministry of Health, 2017). Health professionals advocate for lowering the costs of medical devices and consumables for adolescents with type 1 diabetes, and giving them greater access to services such as psychological therapy (Tanenbaum et al., 2017). 


\subsubsection{Reliability}

Adolescents require reliable medical technologies to provide correct information on which to base decisions and precise actions, particularly in the wake of tightening regulations around medical devices (Bateman \& Jacoby, 2017). A bolus advisor feature that calculated suggested insulin dose provided users confidence about dosage and reduced worries about hypoglycaemia (Barnard, Parkin, Young, \& Ashraf, 2012). However, one CGM has been identified as unreliable, producing many false alarms during the night (Chase et al., 2010). While a closed-loop insulin pump and CGM system reduced anxiety and produced stable blood glucose levels, leaving users feeling safe, it also had calibration and battery issues, causing mistrust (Barnard et al., 2014). Finally, in a study of 18 adolescents with type diabetes, 16 had returned their insulin pump for repair or replacement due to malfunctions. These adolescents were concerned about damaging the expensive pump in the course of daily life (Low, Massa, Lehman, \& Olshan, 2005). A design that embodied this requirement was the 'Insulin Angel' tracker and app, that allows people to know whether their insulin has been exposed to unsuitable temperatures (Treuhaft, 2015).

\subsubsection{Usability}

In addition to reliability, medical technologies also need to be usable. Examples of increasing usability were the removal of the procedure to check codes on batches of test strips before testing blood glucose levels, and developing lancets to be used on multiple parts of the body (Perfetti, 2013). Studies of insulin pens illustrate the attention to individual features of medical technologies required to improve usability. For example, features that affect usability include the ability to set and read a dose, correct a dose if over-dialled, auditory feedback, effort to inject, cartridge size, and ability to check how much insulin has been injected (Haak, Edelman, Walter, Lecointre, \& Spollett, 2007; Hanas, de Beaufort, Hoey, \& Anderson, 2011). 


\subsubsection{Comfort}

\subsubsection{Allow comfortable and non-invasive use}

Medical technologies should be comfortable or non-invasive where possible.

Approximately a quarter of people with various types of diabetes have anxiety about self-injecting. Using an insulin pen, automatic injection device, or ultrafine needles rather than syringe can ease social anxieties about injecting, needle anxiety, and pain, and improve self-management (Davis \& Renda, 2006; Hanas et al., 2011;

Korytkowski, Niskanen \& Asakura, 2005; Perfetti, 2013). Advantages of insulin pumps include having fewer invasive injection procedures, portability, and improved quality of life due to the reduced fear of hypoglycaemia and increased lifestyle flexibility. Disadvantages include high costs, the site being difficult to change and painful, increased blood glucose testing, unwieldiness during exercise, constant invasive presence and visibility, and risk of the insulin not being delivered (Lindholm Olinder, Kernell, \& Smide, 2007; Perfetti, 2013; Seereiner et al., 2010).

Wearable medical devices could be improved by making them smaller and more comfortable to wear (Barnard et al., 2014). CGMs could also be improved by making them less painful to insert (Chase et al., 2010). While it could be beneficial for blood glucose monitoring to be non-invasive, the technology to achieve this is not yet accurate enough (Tura, Maran, \& Pacini, 2007). Here, it is evident that user requirements may be contradictory in some instances, with comfort and reliability at odds until reliable non-invasive technologies are developed.

Concepts in development for non-invasive glucose monitoring include a device that checks eyes rather than finger-pricking (Inventor Spot, n.d.), devices using infrared spectroscopy (Turner, 2015b; Yoon, Wang, Ramachandran, \& Gunhee Lee, n.d.), 'Gluos', a device worn on the earlobe (Tran, 2009), 'Glucowise', a device used between the thumb and finger (Glucowise, n.d.), a temporary tattoo-like glucose monitor (American Chemical Society, 2015), a disposable CGM patch (Nemaura Medical, 2016), watch-like bands (Designbuzz, 2012a, 2012b), and Google's contact lenses for measuring glucose through eye fluids (Ingraham, 2014). The 'Genteel' vacuum-based lancet also aims to reduce pain (Genteel, n.d.). Products for non-invasive insulin delivery include 'Afreeza' inhalable insulin and insulin 
patches (Tenderich, 2015) and 'Insujet' jet insulin infusion (“InsuJet," 2017). The 'TickleFlex' injection accessory overstimulates nerve endings and gathers the skin for a less painful injection (Noe, 2016).

\subsubsection{Minimise psychological distress}

While physical comfort is important, so is a reduction in psychological distress caused by medical technologies. One positive example was a night-time closed-loop system that reduced anxiety about nocturnal hypoglycaemia (Barnard et al., 2014). Another was an insulin pump that reduced stress and improved quality of life and flexibility (Barnard, Speight, \& Skinner, 2008), though not all studies have found insulin pumps to reduce worries or improve life satisfaction (McMahon et al., 2005).

In a study of insulin pump users, those with lower HbA1c levels felt greater normalcy, flexibility, and ease when discussing the pump, whereas those with higher levels felt more negatively about the pump. Further, females were more likely to voice concern about the pump negatively affecting their body image. These feelings seemed to stem from whether the user considered the pump as separate to them, or as almost a part of their body (Ritholz et al., 2007).

Accordingly, some medical technologies already alleviate the psychological distress of diabetes management, whereas others contribute to it. Designs to reduce distress include the 'GlucaShield' kit for parents and teachers, making it easy for them to counter hypoglycaemia (Bluelarix, 2010), 'Diabetes Sentry', a wristband that alerts the wearer of nocturnal hypoglycaemia (Neugent, 2015), and 'Blood Buddy', a blood glucose meter disguised as a whimsical monster (Turner, 2012a). The 'Didget' blood glucose meter also connects to a Nintendo gaming system, awarding points and unlocking games for consistently testing blood sugar levels and meeting bloodglucose targets (Boyle, 2010).

\subsubsection{Suitability and desirability}

\subsubsection{Inform realistic expectations}

Medical technologies can be complex, and users' expectations of these devices prior to initiating use are not always in alignment with the capabilities of the device. 
Insulin pump users who viewed the device as a tool took an active role in their diabetes management, whereas those who viewed the pump as a 'miracle', took a more passive approach to management, and were less confident in using the device's features (Ritholz et al., 2007). In a study of adolescents initiating insulin pump therapy, unrealistic expectations about the benefits of insulin pumps could be counterproductive and disappointing. While many initiated insulin pump use for better glycaemic control, not everybody achieved this, and improved flexibility became their most preferred aspect of using the device (Low et al., 2005).

In addition to having realistic expectations of medical technologies, adolescents may not always have realistic expectations of glycaemic control. Recommendations for achieving more realistic expectations using blood glucose meters include users annotating test results with contextual information and shaping parents' expectations of blood glucose levels (Toscos, Connelly, \& Rogers, 2013).

\subsubsection{Align with values and preferences}

Personal use medical devices should align with users' values and preferences. When reviewing insulin pens, people's preferences were based on features such as design, style, and tactility, and while these features do not affect the clinical performance of the device, they do affect satisfaction (Haak et al., 2007). These aesthetic elements of devices can be improved to match user preferences. Current designs include an elegant blood glucose kit (Shogo, 2017), clutches (“Stylish Diabetes Clutch,” n.d.), medic alert bracelets (Vieira, 2015), and colourful diabetes equipment that look like consumer technology (van Voorden, 2013).

\subsubsection{Provide choices, customisation or personalisation}

As medical device users differ, manufacturers should offer options for choice, personalisation, or customisation to ensure their suitability. In a mobile carbohydrate counting game, children and adolescents desired ways of customising the game, wanting to adapt or create their own characters (Glasemann, Kanstrup, \& Ryberg, 2010). In another gaming study for adolescents with diabetes, the authors suggested that games must be responsive to the needs of individuals and allow for personalisation (Kamel Boulos et al., 2015). 
Some manufacturers currently offer options for customising non-clinical device features. For instance, Medtronic provide users with a choice of colours for their insulin pumps and allows users to choose or create their own custom skin for their pump. Additionally, a phenomenon accompanied by the hashtag \#wearenotwaiting is occurring, where users of medical devices and their family members are adapting existing medical devices for increased functionality (One News, 2014; O’Kane, 2016; “ROUND II,” n.d.; “"We Are Not Waiting' = Diabetes Data Innovation Now!," n.d.).

\subsubsection{Integration into life}

\subsubsection{Enhance convenience}

Diabetes management requires considerable time and effort from adolescents, so where possible diabetes technology should minimise the effort required for selfmanagement and fit into the day-to-day life of the user. Though people with type 1 diabetes have described the convenience, flexibility, portability, and freedom that the insulin pen and pump facilitate, female adolescents found the insulin pump cumbersome, ugly, and difficult to wear with skirts and dresses, and pump failures and site changes required significant effort (Lindholm Olinder et al., 2007). While there were positive aspects, a closed-loop system was criticised for being too large and uncomfortable to wear (Barnard et al., 2014). Further, blood glucose meters were criticised for users needing to wash their hands before using them (which half of users do not do) and having to clean the meter regularly (Bergenstal et al., 2000).

However, there may be unintended side effects of increasing convenience. For example, while insulin pump users experienced more flexibility around eating, and bolused insulin when eating snacks, their diet also worsened (Low et al., 2005). Designs to improve convenience include the 'Genesis' and 'iLet' artificial pancreas (Brand, 2016; A. Brown \& Wolf, 2015), 'Dibkit', 'Gluco', and 'Dario' integrated lancet, test strip and blood glucose meters (Neithercott, 2014; Pathi, 2011; Turner, 2012b), a smartphone with built-in blood glucose monitoring (Katz, 2010), the 'Glycount' and 'Glucowear' blood glucose measuring watches (Turner, 2015a, 2016), and 'Turtyl', a waterproof CGM case (Turtyl, 2014). 


\subsubsection{Facilitate spontaneity and participation in everyday activities and}

minimise impact on life

Where possible medical technologies should minimise the impact of diabetes management on daily life and allow for spontaneity. Insulin pens are compact, portable and can be disposable, allowing them to be used flexibly throughout daily life (Korytkowski, Niskanen, \& Asakura, 2005). Female adolescents using insulin pumps found they could deliver insulin and eat more flexibly, and could sleep in more easily (Lindholm Olinder et al., 2007). In another study with adolescents, insulin pumps improved flexibility around eating and going out (Barnard, Speight, \& Skinner, 2008).

Hypoglycaemia can be problematic during and after exercise, and traditional blood glucose testing can be impractical during sports. CGMs have been proposed as means to monitor blood glucose levels without halting participation, allowing for proactive rather than reactive actions to be taken (Iscoe, Campbell, Jamnik, Perkins, \& Riddell, 2006). Designs to facilitate participation in physical activity include the 'Move' and 'Flex' insulin pumps that shape to the body, facilitating movement, comfort, and discreetness (May, 2009; Turner, 2013).

\subsubsection{Facilitate reliable and efficient night-time management}

Adolescents need to ensure their blood glucose levels are normoglycaemic overnight, however, they also require sleep. Medical technologies should be comfortable, safe, and efficient to use during the night. A closed-loop system used at night-time improved the sleep of users, as they did not need to test at night or in the early hours of the morning, and were less worried about their blood glucose levels (Barnard et al., 2014). However, another closed-loop system gave false alarms that disrupted sleep (Chase et al., 2010).

\subsubsection{Provide meaningful information to facilitate decision-making}

Data are collected, shared, and used to make diabetes-related decisions however, translating these data into meaningful information is desirable. Although data collection can bring advantages, users can also feel overwhelmed by the presentation of data (Snell-Bergeon, 2015). When presented effectively, such as displaying blood 
glucose trends rather than only individual data points with a CGM, technologies can prevent hypoglycaemia (Riddell \& Milliken, 2011). Additionally, blood glucose meters that store and share data with apps can facilitate discussion between users and health professionals and cater for adding contextual information (e.g., meals eaten) (Perfetti, 2013).

Some insulin pens have a memory function that displays the size and time of the previous insulin dose, allowing users to check whether they have already administered their insulin, and avoid stacking doses (Hanas et al., 2011). Additionally, some parents attempt to increase reflection and awareness by creating games around blood glucose testing, or collecting contextual information alongside blood glucose tests (Toscos et al., 2012). While there are tensions in data sharing between adolescents and parents, one study suggested that parents were more concerned about whether their adolescent had tested or not, rather than what their blood glucose levels were. As such, novel displays could be developed to show a broad representation of the adolescent's testing patterns (Toscos et al., 2011).

Information has to be meaningful for adolescents, for example, a description of a small glass rather than an exact amount, 200ml. Further, there are tensions between making tasks easier with automated technology, on which adolescents may come to rely, versus educating them to solve problems in everyday situations (Glasemann et al., 2010).

Translating data to meaningful information currently happens with some medical technologies, but could be better employed. Further, issues of data sharing must be confronted. Designs to provide information include 'Timsulin', an insulin pen attachment that displays the duration since last injection (Timesulin, n.d.) and 'KiCoPen', an insulin pen that tracks dosage (Cambridge Consultants, 2014), 'Sugar', a watch-like CGM that displays trends and syncs to a mobile app (Turner, 2014), 'iHealth' and 'iBGStar' smartphone attachments that graph blood glucose to improve insights (iBGStar, 2012; Sullivan, 2014), and a system that sends self-management data to a mobile phone, prompting reflection on actions and results by young adults and their clinicians (Farmer et al., 2005). Additionally, edutainment services and toys such as Jerry the Bear help children practically learn about diabetes management tasks after diagnosis (Aoki et al., 2004; Boise, 2017). These examples align with the 
field of consumer health informatics, which analyses users' information needs and preferences and develops services that respond to these, increasing the effectiveness of information transmission while quantifying the effect on health outcomes (Eysenbach, 2000).

\subsubsection{Social considerations}

\subsubsection{Help to comfortably disclose and explain type 1 diabetes when appropriate}

Disclosure to friends and having their support is important for self-management (Wysocki \& Greco, 2006). To not disclose a health condition can put adolescents at greater risk of poor self-management and psychosocial adjustment (Thompson Jr. \& Gustafson, 1996; Warner \& Hauser, 2009). If adolescents anticipate negative reactions from peers or friends, they are likely to struggle to self-manage in social situations and have increased stress and poorer metabolic control (Hains et al., 2007). However, adolescents do limit their disclosures to people they trust. They recognise the importance of having others know they have diabetes in case of an emergency, however, they do experience teasing and uneducated comments about diabetes that limit their disclosures (Y. -L. Wang, Brown, \& Horner, 2010). Accordingly, while it is often advantageous for adolescents to disclose their diabetes to peers, medical technologies should facilitate them doing so when they feel it is appropriate, rather than taking this choice from them.

\subsubsection{Minimise or eliminate feelings of stigma or embarrassment while using medical technology}

Medical technologies should not cause stigma or embarrassment for users in social contexts. Adolescents can be embarrassed or annoyed by onlookers asking them questions, watching them test blood glucose levels or administer insulin (Dickinson \& O'Reilly, 2004; Y. -L. Wang et al., 2010). To avoid these situations, adolescents may skip blood glucose tests, keep their devices hidden, or go to a bathroom to inject or test, learning to 'not be different' (A. E. Carroll et al., 2007; Y. -L. Wang et al., 2010, p. 261). 
Insulin pens are more discreet to use in public spaces than syringes, and may reduce social anxiety (Korytkowski et al., 2005). Using colourful pens or 'skins' on these devices may reduce embarrassment, in addition to ensuring the correct insulin pen is used, however, these coverings are predominantly designed for children (Hanas et al., 2011; Perfetti, 2013). Insulin pumps, allow some adolescents to deliver insulin in public spaces more freely (Barnard, Speight, \& Skinner, 2008), but other adolescents and young adults described the visibility of insulin pumps as a primary reason for not initiating insulin pump use or ceasing use (Seereiner et al., 2010).

For adolescents who do use insulin pumps, the majority were comfortable wearing and explaining the pump, and felt less stigma and burden, with little impact on their body image. However body image was negatively affected for some, yet while some adolescents were tired of explaining the device, or avoided questions about it, most were not embarrassed by it (Low et al., 2005; Ritholz et al., 2007). Device alarms can also cause embarrassment. Adolescents thought an alarm that sounded to remind them to test would be embarrassing and call attention, while their parents thought it would be a positive feature (Carroll, Downs, \& Marrero, 2007).

Multiple authors caution concealment of medical and assistive devices, as this may be a signifier of shame and not address issues of stigma. Instead, they aim to create devices that highlight the person behind the condition, counter stereotypes, and facilitate acceptance and pride (Bispo \& Branco, 2011; Pullin, 2009; Sansoni, Speer, Wodehouse, \& Buis, 2016). However, this approach may not be desirable to all users, and not all users are bothered by social factors around device use (Sansoni et al., 2016). Designs that respond to this requirement include fashionable clothing that allows access to injection sites (Balmain, 2017), and blood glucose meters that pass as a smartwatch (Lai, 2013) or consumer technology (Sbarigia, 2009).

\subsubsection{Enhancement of support network}

\subsubsection{Support transition of responsibility}

Diabetes technologies may be able to better facilitate transitions of responsibility from clinicians and parents to adolescents. Adolescents and their parents commonly experience tensions about blood glucose monitoring and hyperglycaemia. 
Adolescents strive for independence, which can be facilitated by devices such as insulin pumps, as they do not require parental supervision (Barnard, Speight, \& Skinner, 2008). However, some adolescents do not test if they think their levels are high or low because they expect a negative parental reaction (Carroll et al., 2007). Adolescents are motivated to improve their self-management due to their parents' love and concern, however, this can lead to problematic actions. One adolescent described eating extra food to calm others' worries about hypoglycaemia, and another shared only selective information about her school-time diabetes management with her mother to prevent her worrying (Y. -L. Wang, et al., 2010).

Data sharing was another area of tension, with parents and health professionals wanting access to data. While some adolescents thought this may reduce parental nagging, older adolescents viewed sharing data directly with parents negatively. Parents were willing to settle for summaries of adolescents' results, not needing full real-time data if their adolescent objected to this. However, both parties wanted a feature for emergency notification when experiencing a dangerously high or low blood glucose level (Carroll et al., 2007). As such, data sharing requirements between parents and adolescents change as the adolescent nears an emergency situation, and independence and privacy become less prioritised.

Rather than relying on what adolescents typically view as supportive in the transition of responsibility for diabetes management, adolescents' individual preferences should be considered. Actions by families that were supportive of selfmanagement included support around meals, glucose testing, and insulin delivery. While emotional support was unrelated to adherence, it may be useful in other dimensions, including improving quality of life and minimising depression (Greca \& Bearman, 2002).

Adolescents were also negotiating responsibility with health professionals. In discussing a hypothetical phone-based blood glucose meter, adolescents wanted to send data to health professionals and were in favour of receiving instructions for regimen changes directly from health professionals, and possibly via this technology, though parents were wary of being less involved and unsure if younger adolescents had sufficient diabetes knowledge to understand regimen changes (Carroll et al., 
2007). Diabetes technologies may be able complement the traditional patientclinician relationship.

The Glucophone begins to address this transition of responsibility, with results from a mobile phone-integrated blood glucose meter sent to a clinician, who makes periodic suggestions in response via text messaging, and a web portal that adolescents and their parents can access. Adolescents and parents reviewed the system positively, considering it to minimise parental worry and burden and benefit health. However, the system was faulty, requiring further development (Carroll, DiMeglio, Stein, \& Marrero, 2011).

\subsubsection{Facilitate interaction with diabetes peers and friends}

For optimal social support, medical technologies should facilitate positive interactions between adolescents and their friends and peers with diabetes. Adolescents currently experience conflicts between wanting to socialise with friends and having to complete self-management tasks (Warner \& Hauser, 2009). However, friends can be a source of emotional support for adolescents with type 1 diabetes, and some adolescents desire peer support from other young people ( $\mathrm{Lu}$ et al., 2015; Wysocki \& Greco, 2006).

In one mobile carbohydrate counting game for older children and adolescents, participants at a diabetes camp interacted with each other and competed while using the educational game, motivating them to repeat it (Glasemann et al., 2010). Medical technologies may be able to facilitate interactions with diabetes peers and friends; the 'HelpAround' app responds to this requirement, allowing people to get diabetes advice from others with diabetes (HelpAround, n.d.).

\subsection{Summary}

Adolescents with type 1 diabetes are required to engage in intensive selfmanagement to minimise the complications of their condition. To achieve this, they must use medical devices regularly. However, adolescents are not managing at a level that achieves optimal health outcomes. Further, new medical technology offerings have not significantly improved health outcomes as anticipated. As such, this research seeks to investigate adolescents' experiences of self-management and their 
psychosocial user requirements of medical technologies. While medical technologies are not the only factor to influence adolescents' self-management, it is likely they have an effect and could be improved, though there is very little literature available about their psychosocial impact.

No comprehensive set of psychosocial user requirements of medical technologies was found specifically for adolescents with type 1 diabetes, though specific requirements do exist for adolescents with type 1 diabetes, particularly in transitioning responsibility from adult to adolescent. There is plentiful research available about adolescent experiences, but this research tends to be reported as barriers, which could be translated into user requirements to better inform designers. While it may seem logical to solely identify and address barriers to use of current medical technologies, exploring adolescents' experiences also allows me to investigate areas where technology may be useful, but is not currently utilised. Also, there is very little literature reported by designers in this area, perhaps because this information is commercially sensitive. Designers are well-positioned to tackle issues of medical technology use, investigate lived experiences and requirements, and complement the well-established biomedical approach.

Finally, theoretical approaches to managing long-term conditions vary. While the current research is aligned with an engagement approach, definitions of what this means in practice are limited. Further investigation of what is involved in being and becoming engaged in self-management is required. 


\section{Methodology}

This research sought to answer a two-part question: How do adolescents experience self-management of type 1 diabetes, and what psychosocial user requirements of medical technologies are relevant for them? The constructivist research paradigm applied combines several methods for eliciting adolescents' experiences and user requirements. These predominantly qualitative methods fall under the umbrella of participatory design, and are implemented and analysed through constructing grounded theory and user requirements.

Each method and its findings are documented in detail in the relevant chapter. However, the findings are also combined to produce a substantive grounded theory and list of user requirements in Chapter 11. In this chapter, epistemology, characteristics of design research, participatory design, grounded theory, methodological congruence, user requirements construction, and the process and challenges of conducting research with adolescents with type 1 diabetes are discussed.

In Table 1, an overview of the research is presented. As no comprehensive list of adolescent psychosocial user requirements of medical technologies for type 1 diabetes was identified in the literature review, this research fills this gap. I not only explored adolescents' interactions with their medical technologies, but also their broader experiences of managing diabetes. Therefore, the research examines not only the impact medical technologies currently have based on their existing features, but which additional or alternative functions could address adolescents' requirements. 
Table 1

Research overview.

\begin{tabular}{|c|c|c|c|}
\hline $\begin{array}{l}\text { Research } \\
\text { question }\end{array}$ & \multicolumn{3}{|c|}{$\begin{array}{l}\text { How do adolescents experience self-management of type } 1 \text { diabetes, and } \\
\text { what psychosocial user requirements of medical technologies are } \\
\text { relevant for them? }\end{array}$} \\
\hline Aims & \multicolumn{2}{|c|}{$\begin{array}{l}\text { To describe adolescents' experiences of self- } \\
\text { managing type } 1 \text { diabetes }\end{array}$} & $\begin{array}{l}\text { To construct } \\
\text { psychosocial user } \\
\text { requirements for the } \\
\text { design of medical } \\
\text { technology for } \\
\text { adolescents' self- } \\
\text { management of type } \\
1 \text { diabetes }\end{array}$ \\
\hline Objectives & $\begin{array}{l}\text { Elicit adolescents' } \\
\text { experiences of self- } \\
\text { management, and the } \\
\text { opinions of other } \\
\text { stakeholders }\end{array}$ & $\begin{array}{l}\text { Elicit adolescents' } \\
\text { medical technology- } \\
\text { related experiences } \\
\text { and preferences }\end{array}$ & $\begin{array}{l}\text { Develop and provide } \\
\text { additional support } \\
\text { for psychosocial user } \\
\text { requirements of } \\
\text { medical technology } \\
\text { that address self- } \\
\text { management for } \\
\text { adolescents with } \\
\text { type } 1 \text { diabetes }\end{array}$ \\
\hline Methods & $\begin{array}{l}\text { Clinician interviews } \\
\text { Adolescent interviews } \\
\text { Student design work } \\
\text { Psychosocial impacts o } \\
\text { Cultural probes } \\
\text { Participatory design wo }\end{array}$ & tive devices card sort & \\
\hline
\end{tabular}

\subsection{Epistemology}

The point of view of the adolescents is essential to this research, and as such a participatory approach was required. As the individual and subjective points of view of adolescents were to be elicited from a broad starting point, a positivist 
epistemology is inappropriate, as measurable variables have not yet been established. Alternatively, a fully subjectivist approach would bring into question the validity of the analysis of the data gathered, given that the goal of the research is to inform the design of medical devices that can be applied to a broader population that just the individual.

Constructionism and constructivism call for a balance between the subjectivity of the researcher and the participants, and the ability to generalise within limits. In constructivism, the relationship between a person and the 'reality' to be discovered is both person- and context-specific. Transactions that take place between people and 'reality' are subjective, and mediated by factors including prior experiences, religious beliefs, gender, social status, cultural values, and nationality. While some constructivists argue for a more individualistic stance, constructivist grounded theory, used in this research, aligns well with contemporary views of social constructionism (Charmaz, 2014; Rockmore, 2005). As such, knowledge is not discovered, but constructed, and is specific to the context in which it was created (Lincoln, 2013). Further, in a research setting, participants and researchers coconstruct understandings (Hatch, 2002). Throughout the thesis, participants and I co-construct meaning. Following this constructivist approach, the findings of this thesis are specific to the people and context in which they were constructed.

\subsection{Design Research}

Design research is a relatively young discipline, with academics still debating what constitutes good design research (Frankel \& Racine, 2010). Archer (1981, p. 31) proposed a broad definition of design research: "Design research is systematic enquiry whose goal is knowledge of, or in, the embodiment of configuration, composition, structure, purpose, value, and meaning in man-made things and systems." The key here is in describing the process as systematic, which may differ from design practice in some instances (Swann, 2002). Building on Archer's (1981) work, Cross (2006, p. 102) defined the characteristics of good research, which are embodied in this research:

- "Purposive - based on identification of an issue or problem worthy and capable of investigation 
- Inquisitive - seeking to acquire new knowledge

- Informed - conducted from an awareness of previous, related research

- Methodical - planned and carried out in a disciplined manner

- Communicable - generating and reporting results that are testable and accessible by others."

Design research commonly deals with the interactions between people and designed artefacts or systems, moving the discipline towards the uncertainty and complexity of social sciences (Swann, 2002). As such, designers no longer focus solely on objects and designed outcomes, but shift towards listening, understanding, and provoking reflection on possible futures (Muratovski, 2016). This approach to research for design provides designers the information and implications needed to inform design outcomes (Frankel \& Racine, 2010). This shift is partially due to the application of design to 'wicked problems' such as diabetes management, where countless factors impact any design intervention, opening designers to a more constructivist, subjective approach (Bratteteig \& Wagner, 2014; Crouch \& Pearce, 2013; Kolko, 2012; Rittel \& Webber, 1973).

While there are many commonalities between design and research practices, they are not always aligned (Pedgley \& Wormald, 2007). In this research, new knowledge is communicated from purposive and systematic research. This includes documenting and discussing design activity that has taken place, along with other research methods.

As with much design research, aspects of research for, about, and through design are incorporated. Research for design can be considered research that enables design. Research about design deals with aspects such as design history, aesthetics, and analyses of design activity. Finally, research through design is a method of designing as research (Downton, 2003; Frankel \& Racine, 2010; Schneider, 2007). As such, while much of the research is applied in the context of diabetes management, there is also knowledge developed about design methods and produced through designing. 


\subsection{Participatory Design}

This research uses a participatory design methodology. Participatory design, originating in Scandinavia in the 1970s, has been defined as "A methodology that argues in favour of the possibility, the significance, and the usefulness of involving research partners in the knowledge production process" (Bergold \& Thomas, 2012, paragraph two). Participatory design is not tied to particular methods, but characterised by three phases: an initial exploration of users and their environments, discovery of users' goals, values, and desired outcomes, and prototyping of artefacts (Spinuzzi, 2005). Commonly used methods include workshops, probes, mapping, prototyping, and ethnography (Velden \& Mörtberg, 2015). In working with participants, researchers must develop tools that enable effective communication and facilitate the co-construction of knowledge (Robertson \& Wagner, 2013). Participants have many roles, taking on the roles of user, designer, and evaluator (Robertson \& Simonsen, 2013; Shah \& Robinson, 2007). However, within a project there can be different depths of participation at varying stages (Bratteteig \& Wagner, 2014).

Participatory design is founded on constructivism and recognises that knowledge can be tacit. It is evident in artefacts, practices, and interactions, is essentially interpretive, and unable to be decontextualised. Participants' knowledge and perspectives are considered invaluable in the design research process (Spinuzzi, 2005). Participatory design 'gives voice' to end users, though researchers need to develop approaches to hear and understand these voices (Kanstrup, 2012). Participants not only have a voice in the design process, but also develop visions of possible futures and technologies, sometimes redesigning or replacing existing technologies (Velden \& Mörtberg, 2015).

In this research, the participatory design methodology housed methods including semi-structured interviews, card sorting, cultural probes, and a design workshop. Participants were repeatedly invited to take part in subsequent stages of the research, able to participate in aspects of the research that interested them. Figure 11 shows an overview of the participants, research methods and outputs of the thesis. Elements of the participatory design approach are displayed in coloured blocks 
chronologically from the top of the diagram to the bottom, covering a ten-month period and showing the overlap between different research phases. Parentheses are used to indicate the chapter in which each stage is discussed, and lighter tints of a colour indicate the span of time between initial and final participants taking part in that stage of the research.

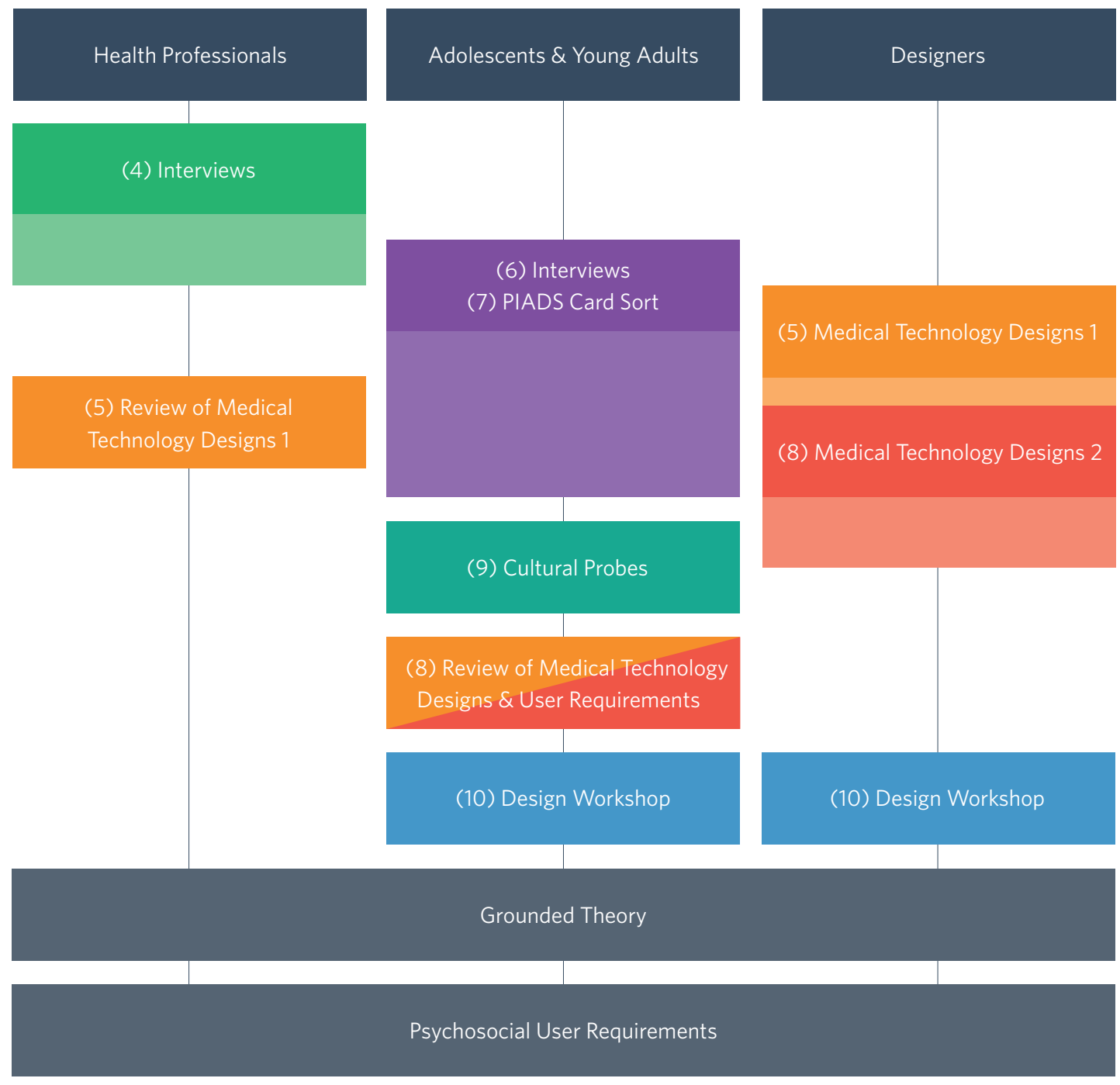

Figure 11. Overview of participatory design approach. 


\subsection{Constructivist Grounded Theory}

The research was conducted and analysed in alignment with Charmaz's (2014) constructivist grounded theory. This is a process that spans data collection and analysis. While interviews are commonly used, grounded theory has no prescribed data collection methods. However, analysis is carried out concurrently with data collection, guiding the research. Data analysis begins with inductive open coding, where data are coded line-by-line, focusing on gerunds and processes rather than themes. Next, data are coded again using focused coding, whereby the connections between codes and sources become more apparent and the level of analysis is raised, producing conceptual categories. Where appropriate, theoretical coding can also be applied, drawing on existing theory to elevate the level of analysis. However, there is a tension here, between the construction of grounded theory in an inductive manner and the application of existing codes. Throughout this entire data collection and coding process, memos are written and codes and theory are developed through theoretical sampling, whereby data collection is shaped by the emerging theory. A technique of constant comparison is also employed throughout, comparing data with data and codes with codes. The final grounded theory product takes varying forms, with some aiming for formal and fully saturated theory, substantive theory, simply reporting categories, or elevating a single category to a theoretical concept. This research aims for the reporting of categories, and in some instances, substantive theory underpinned by theoretical concepts. Substantive theory seeks to interpret or explain a problem in a particular area, in this instance, the self-management of adolescents with type 1 diabetes (Charmaz, 2014). Figure 12, adapted from Charmaz (2014), illustrates the constructivist grounded theory process. 


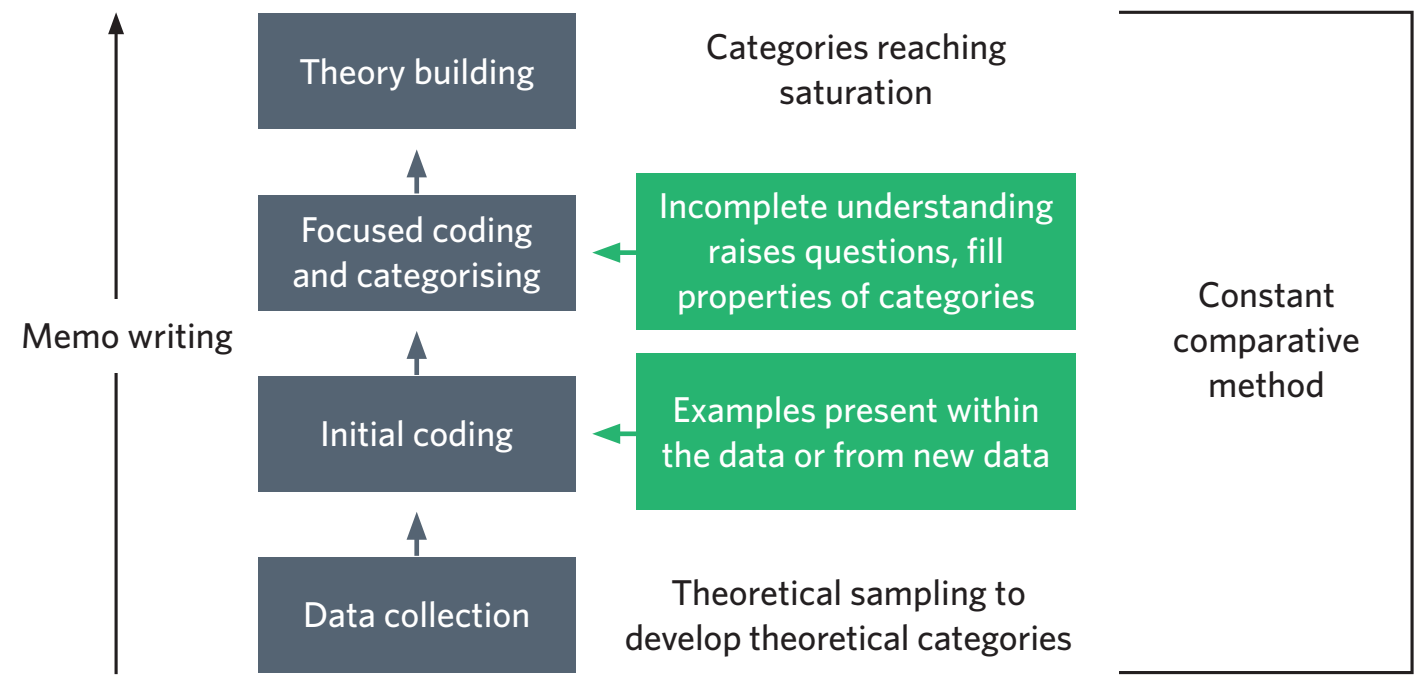

Figure 12. Visual representation of the grounded theory process. Adapted from Constructing Grounded Theory (p. 18), by K. Charmaz, 2014, London, United Kingdom: Sage. Copyright 2014 by Sage.

The term 'grounded' recognises that theory emerges from research grounded in data collected from a range of sources (Leedy \& Ormrod, 2013). Theory has been defined as such: "A theory states relationships between abstract concepts and may aim at either explanation or understanding" (Thornberg \& Charmaz, 2012, p. 41).

Grounded theorists have differing views of what constitutes theory, however, interpretive theories "aim to understand meanings and actions and how people construct them" (Charmaz, 2014, p. 231). These theories acknowledge the subjectivity of participants and researchers. Accordingly, the role of the researcher is to construct an impression of the world as they view it (Ratner, 2008).

Constructivist grounded theory builds on earlier positivist approaches by Glaser and Strauss (1967) and post-positive developments by Strauss and Corbin (1990). Charmaz and Bryant (2010, p. 406) set forth the ways in which it differs from the original:

1. "is founded on a relativist epistemology,

2. views the method as interactive as well as comparative and inductive,

3. takes into account the multiple and shifting standpoints of the researcher and the researched,

4. builds on the method's pragmatist heritage, and

5. emphasizes reflexivity." 
To be reflexive, researchers consider the impact of their assumptions on the research process and output, scrutinising experiences and decisions. They also state what areas have been emphasised, de-emphasised, or omitted (Charmaz, 2006). While some other theses about long-term conditions have been authored by a researcher with first-hand experience of the disease, I do not have this experience, and so while guided by literature, I remained open-minded to participants' experiences, having little preconception of what these might be. Where early literature investigations were not compatible with participants' accounts, I used the primary data to help guide searches for more relevant literature, and to develop lines of questioning, for instance in shifting from a framework of compliance to engagement, and in beginning to investigate the role of goal-setting.

In conducting the research I was aware that being a young female may make my presence less intimidating, and that I might be able to elicit honest accounts as I was not likely to be viewed in the same category as a health professional. Similarly, the designers chosen to contribute to the research were all in their twenties, likely to remember their own adolescent experiences and be approachable to adolescents. A common issue in design research is the unwillingness of participants to critique designs created by the researcher. I was able to circumvent this by facilitating the design process, yet letting designers and adolescents create the design outputs described and critiqued in this thesis.

Another issue is the designer's tendency to seek out problems and desire to change something about the participant's context, rather than simply understand it. To counter this, I endeavoured to identify aspects of self-management that currently worked well, in addition to aspects that could be changed.

Charmaz (2014 p. 236) also sets out criteria that define a constructivist approach to grounded theory (Table 2). These criteria are adhered to throughout this thesis. 
Table 2

Criteria for constructivist grounded theory.

\begin{tabular}{ll}
\hline Foundational & Assumes multiple realities \\
assumptions & Assumes mutual construction of data through interaction \\
& Assumes researcher constructs categories \\
& Views representation of data as problematic, relativistic, \\
& situational, and partial \\
& Assumes the observer's values, priorities, positions, and actions \\
& affect views \\
& Views generalisations as partial, conditional, and situated in time, \\
& space, positions, action, and interactions \\
Objectives & Aims for interpretive understanding of historically situated data \\
& Specifies range of variation \\
& Aims to create theory that has credibility, originality, resonance, \\
& and usefulness \\
Acknowledges subjectivities throughout data analysis \\
Views co-constructed data as the beginning of the analytic \\
direction \\
Engages in reflexivity throughout the research process \\
Seeks and (re)presents participants' views and voices as integral \\
to the analysis \\
\hline data analysis
\end{tabular}

Figures 13-15 give an overview of how the grounded theory process worked in practice. These figures show excerpts from the initial coding of transcripts, focused coding and categorising process, and iterative writing of memos. 
Q: How do you feel about other people's reactions to your condition?

A: It's tough. Like I said friends and family are generally supportive but then there are like people who don't really understand it or who make the comment like, "But you're not overweight," or "You're not this or that." It's like it's not how it works.

I think that's with any medical condition. Like a big struggle I have been having recently actually is I get a disability allowance through Study Link because can't get a student allowance but I can get that. Basically it's kind of interesting the amount of battles I have with them.

How it works is you make claims; so basically you will fill out a form and you will say, "Because of my illness I need this, this, this and this." And then you will probably have receipts as proof of said cost. Often there will be, "Why do you need this?" and they won't include it because they don't.

Obviously a lot of the staff aren't medically trained so they don't understand the condition. Often I will have to go to [name] and get them to write a supporting letter to back what I am saying. Often they just won't take on board that these things may not look like a cost to them but they actually are. But that's like a kind of thing.

I'm a bit lucky because a lot of my friends are nurses but for the ones like people who don't understand conditions they kind of attach these weird stigmas or opinions to them and it's like well that's not it. Or it's like, "Why did you allow that to happen to you?" and I was like, "Well I didn't, it was genetically based, I didn't do anything."|

So I guess that's kind of a struggle. It doesn't make me sad, just makes me annoyed. Obviously I am not expecting everybody to be medically proficient but try not to be too ignorant if you know what I mean. It's tough.
Friends and family being supportive

Others not understanding

Making uninformed comments - not overweight

Battling for a disability allowance

Having to make claims for expenses, fighting for why they relate to a disability

Staff not being medically trained - not understanding Having to get nurse to write a supporting letter

Lots of friends working/studying in health (nurses)

People who don't understand attaching stigmas and opinions, blaming

Bit of a struggle

Not making her sad but annoyed

Not expecting everyone to have great knowledge, but to not be ignorant

Figure 13. Excerpt from an interview transcript and initial coding.

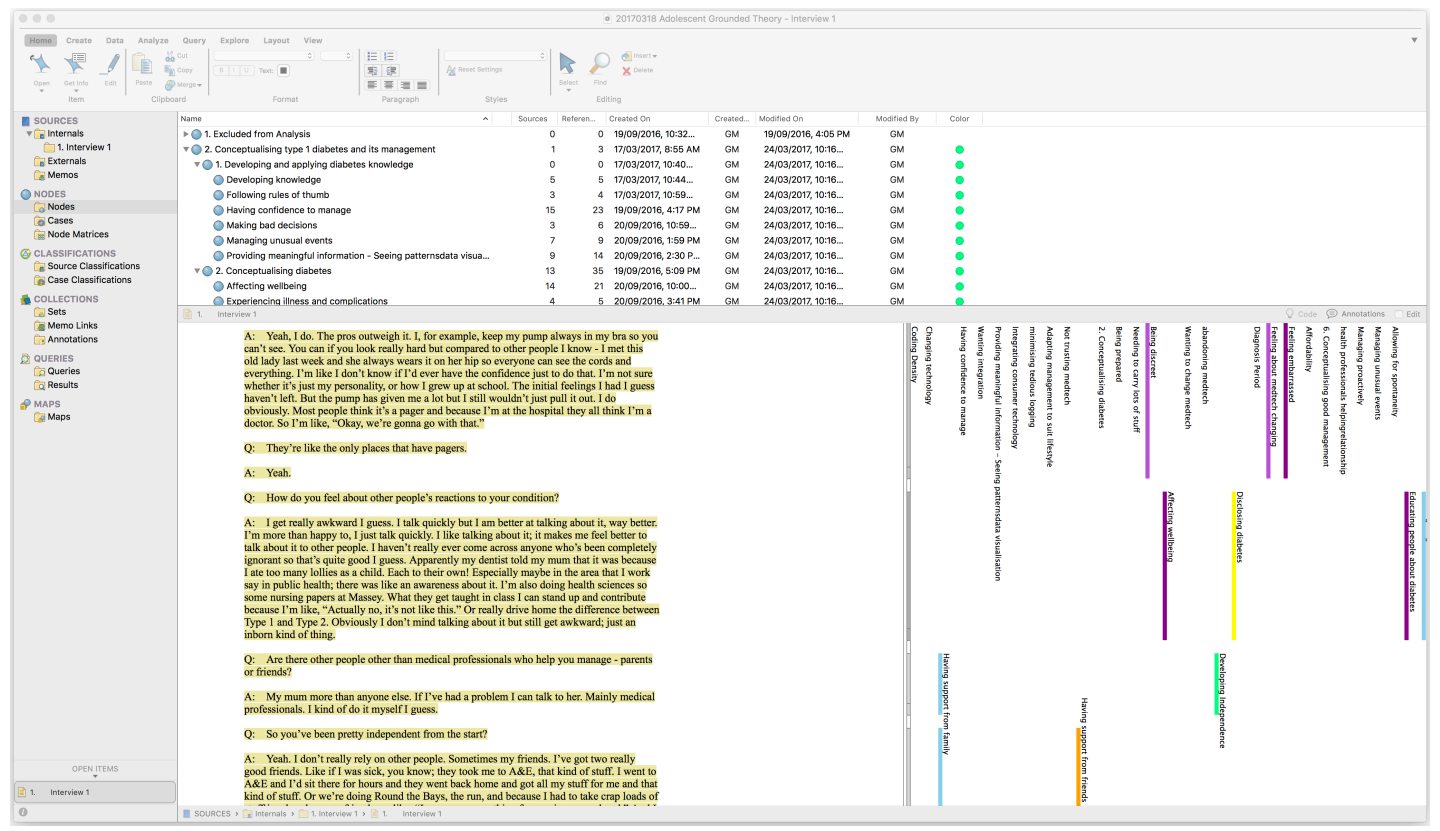

Figure 14. Focused coding and development of categories in process. 
Memo - Self-Management vs. Engagement vs. Adherence vs. Compliance

05/08/15: Adherence seems like trying to achieve best practice (idealistic), but even clinicians will prescribe a lesser treatment plan (e.g., two injections per day) if the likelihood of it being carried out is more realistic. So perhaps 'adherence' or 'compliance' are not as simple as they might seem with other conditions (e.g., antibiotics). Perhaps it is about adherence to the prescribed regimen, whether this is best practice or not.

06/08/15: Self-management, see CNS2 interview - they want people to be proactive, (e.g., change their insulin if the amount isn't working). In this respect the exact values aren't always prescribed by the clinician. So the regimen to be adhered to is 'being proactive', not delivering a certain dose of insulin. It seems adherence and compliance are too simplistic for this highly-changeable regimen, where the 'patient' makes many of the decisions. Self-management and engagement seem necessary in this case.

26/08/15: Clinicians still refer to quantifiable aspects of management (e.g., HbAlc measures), and these are important to them in measuring how well their clinic is performing. However, when discussing individuals they tend to use language more congruent with engagement, and are flexible with regimen. They do, however, express uneasiness around prescribing a regimen that might not be medically ideal. They seem to lie between engagement and adherence approaches, and the tension of this is evident.

Figure 15. Excerpt from a memo written over multiple days after interviews with health professionals.

While these steps are not commonly outlined formally in the grounded theory process, Figures 16-17 demonstrate the types of tools used to conduct grounded theory analyses. I used both physical and digital tools to aid my search for connections and between codes, categories and data elicited through different research methods. 


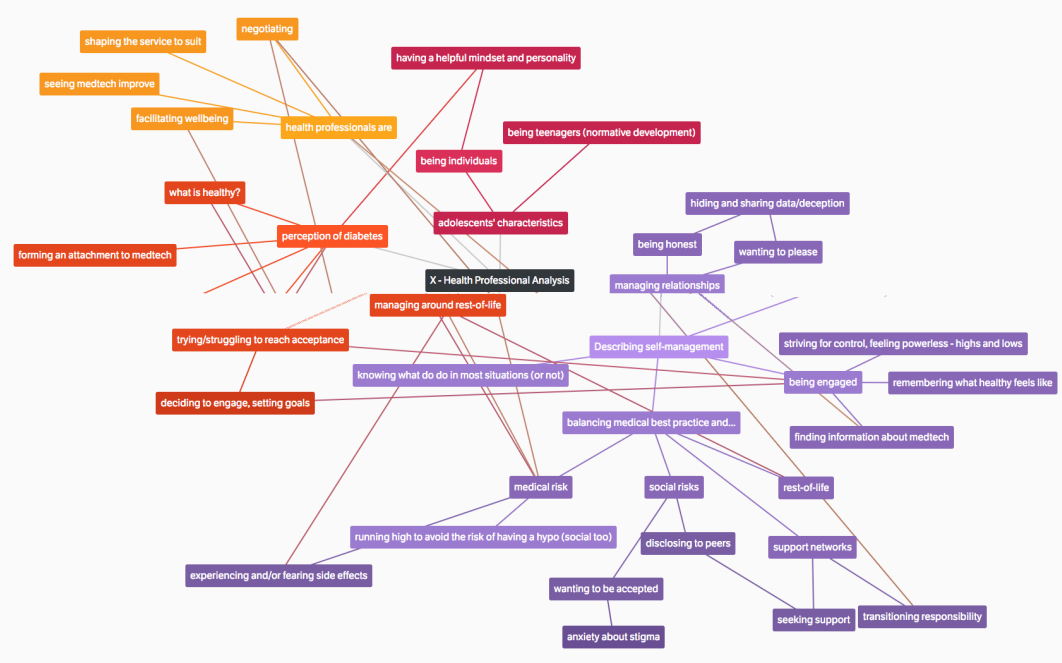

Figure 16. Digital mapping of focused codes to aid development of categories.

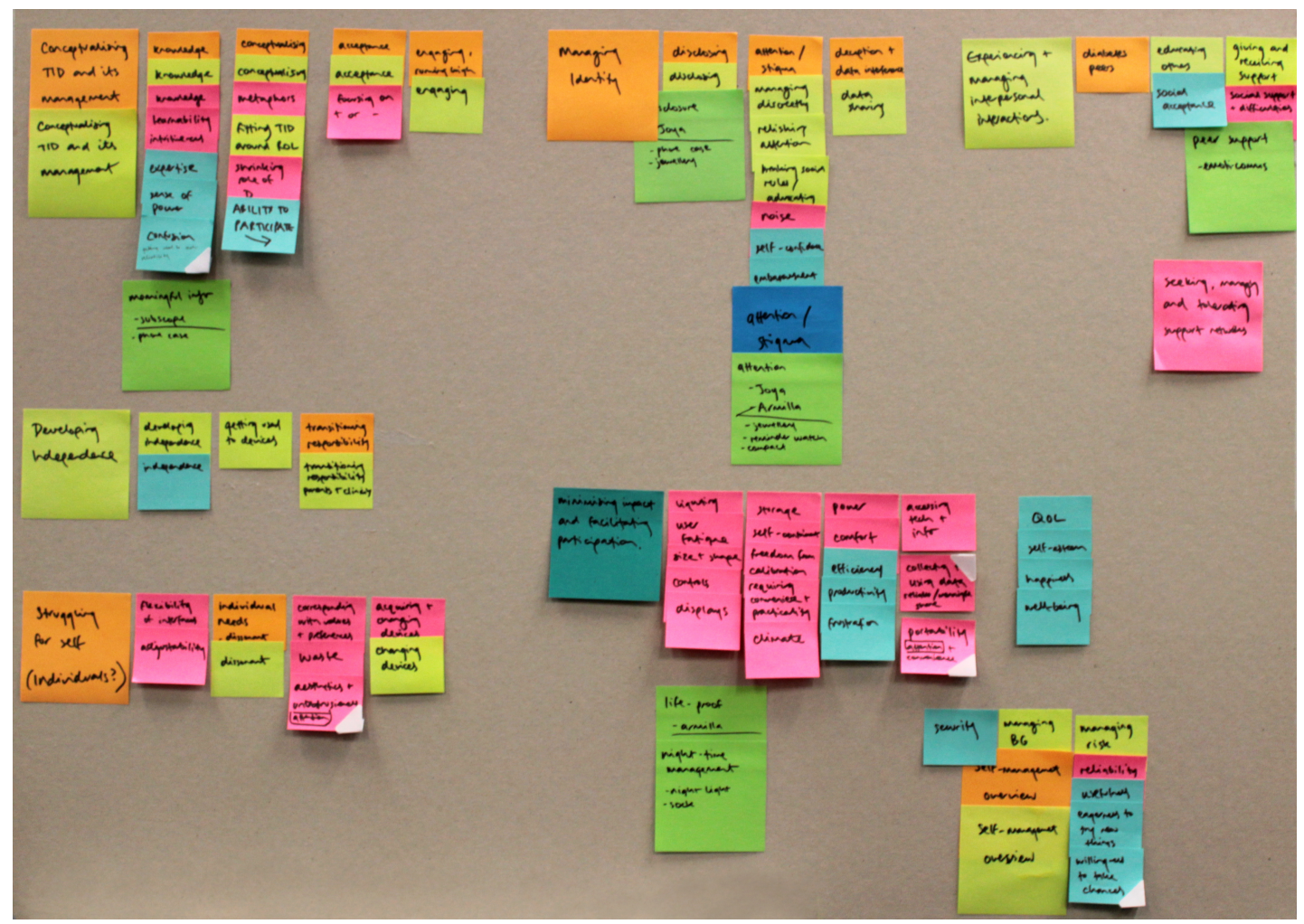

Figure 17. Physical clustering of focused codes to aid development of categories, with colours used to represent different data sources. 


\subsection{Methodological Congruence}

The methods and methodologies used throughout this thesis are numerous and perhaps unexpected in their combination. Grounded theory is not commonly used in design research, though it is found more commonly in health and human computer interaction disciplines. Participatory design and grounded theory both share a constructivist epistemology, and are highly congruent. In addition to their shared views on the co-construction of knowledge and involvement of the researcher, Table 2 above alludes to two other areas of congruence: data being constructed through interaction and participants' voices being integral. While grounded theory and participatory design commonly go about these in different ways, for instance, interviews versus design workshops, and reporting extended quotes versus participants being given roles as designers, the values that drive these decisions are shared. At their core, participatory design and constructivist grounded theory share a deep respect for the participant. They both champion and give voice to the experiences and ideas of participants.

Contrastingly, grounded theory and participatory design are also divergent, and combining them provides a complementary opportunity. Through using methods associated with participatory design, such as cultural probes or designing prototypes, researchers can elicit different types of information from participants than by solely using interviews, which is commonly done in grounded theory studies. They can also vary methods of communication (e.g., talking, writing, drawing) so that different participants might each be catered for. Further, a series of methods was designed not only for its intended research output, but also for the engagement, enjoyment and retention of adolescent participants. Alternatively, in practice, designers commonly use synthesis methods such as affinity diagrams created by inductively clustering sticky notes on walls (B. Martin, 2012). While these have a similarity to grounded theory in clustering information and examining the similarities and differences between data points, grounded theory offers a more rigorous approach, particularly through the writing of memos and constant comparison.

While grounded theory methods are commonly applied to interview transcripts, its proponents are unequivocal that this methodology may be applied to 
a variety of data sources and do not prescribe what these must be. This research takes this principle and explores it in practice, pairing constructivist grounded theory with traditionally paired methods such as interviews, and many methods rarely paired such as cultural probes, designing objects, and a participatory design workshop. I considered this combination of methods to be important in providing the various levels of information required by the two-part research question. The question requires high-level information about self-management experiences, and more specific information about psychosocial user requirements of devices.

This thesis builds on the work of Lang (2012), who used grounded theory in combination with multiple methods: interviews with clinicians; a workshop about current medical devices with adolescents; and interviews with adolescents with cystic fibrosis using design vignettes as prompts for discussion. These various methods were treated as case studies and analysed separately and a substantive grounded theory was not reported. While Lang (2012) explicitly outlined an approach to grounded theory, detailed findings were often reported as themes and displayed using Venn diagrams. Finally, a co-design project was reported, where the researcher created a design specification and worked alongside one participant to design a medical device concept that met these specifications. Although grounded theory was not used in this case study, through the process of design and evaluation the researcher created additional user requirements.

In another instance combining grounded theory and creative research methods, researchers used grounded theory to analyse their reflections on working with adolescent participants across a series of creative studies and form guidelines for future researchers. A number of creative methods were used in the work, including diaries, story-making, mind-mapping, paper-based design activities, and focus groups and workshops. However, grounded theory analysis was only used to analyse researchers' reflections of working with these participants, and not to analyse the outputs of these methods directly (Fitton et al., 2016)

As such, there are existing instances of grounded theory being used in partnership with creative, participatory or co-design methods. However, these instances do not commonly result in substantive theory through expanding and refining theory through sequential methods. In this thesis, grounded theory is used 
throughout, with the knowledge constructed from each method combining to form a single, substantive grounded theory in the Chapter 11. Though participatory design and grounded theory are highly congruent, there did lie a tension between producing theory and design outputs. This thesis aims to construct grounded theory to a level that will be of use to designers of medical technologies, and to complement this with psychosocial user requirements that will be actionable by designers and examples of products designed to meet them.

In contrast with typical approaches, this thesis is structured around individual research methods, with preliminary grounded theory categories reported in each chapter and the substantive grounded theory reported in whole in Chapter 11 (Muratovski, 2016). While this structure makes the reporting of grounded theory more challenging, it better illustrates the type of data produced by each research method. This was particularly important because of the broad range of methods used and the specific and varied outcomes of each method. The main body of this thesis demonstrates how the theory was developed sequentially, by seeking further information through additional research methods to saturate categories.

In instances throughout this thesis, quotes have been edited to increase readability, while changing their meaning as little as possible. This approach is in line with editing criteria for length, relevance, readability, comprehensibility, and anonymity (Emerson, Fretz, \& Shaw, 2011; Glesne, 2011).

Due to the breadth of this research, each grounded theory category and the connections between them are not saturated to the level of a complete, formal grounded theory. Instead, further research could be completed to saturate each category. Similar patterns and stories were elicited repeatedly, however, full saturation for grounded theory requires that the researcher finds no new properties of categories, that these properties account for patterns in the collected data, and that the relationships and variation between categories have been examined (Charmaz, 2014).

Although approaches to validation such as triangulation and memberchecking have been criticised as following realist traditions (Rolfe, 2006; Sandelowski, 1993), strategies to establish rigour have been used in this research to further data collection and understanding of emergent categories. This approach has 
been described as 'subtle realism', and is used in an attempt to ensure the research, while context-specific, is meaningful (Angen, 2000).

Credibility with participants was ensured by conducting member-checks, particularly through using design vignettes to aid discussions of proposed psychosocial user requirements (Sandelowski, 2008). While results are not intended to be widely generalised, transferability is facilitated through descriptions of participants and context, allowing other researchers to judge whether results are relevant to their context. Further, interviews were conducted with health professionals to gain a view of the full spectrum of self-management behaviours of adolescent patients. As reliability is not appropriate given the constructivist epistemology, dependability was fostered through accounting for changing contexts. For instance, the medical technologies provided to adolescents in New Zealand differ from other countries, and have changed substantially over the past twenty years. Additionally, many participants were involved in multiple stages of the research over a period of up to ten months. Finally, confirmability was demonstrated through describing the research methods in detail, and through triangulating data sources and methods within the current research and existing literature (T. Farmer, 2006; Rothbauer, 2008).

\subsection{Psychosocial User Requirements}

Although the medical device industry has been slow to incorporate user requirements into the design process, the benefits of incorporating these requirements include:

- "Improve safety of devices

- Improved usability of devices

- Reduce device recalls

- Limit need for ad hoc modifications

- Improved efficiency of users

- Improve patient outcomes and satisfaction

- Assistance with obtaining development grants." (J. L. Martin et al., 2006, p. 51) 
As such, the development of user requirements is important for medical technologies (Shah, Robinson, \& AlShawi, 2009). The requirements developed in this thesis are psychosocial; they refer to how medical technologies interact with both intra and interpersonal factors in varying environments (Jutai \& Day, 2002).

Many methods are available for informing and testing user requirements for medical devices. Scoping methods such as contextual inquiry, focus groups, and ethnography can be used to determine user requirements while evaluative methods, such as usability tests and cognitive walkthroughs determine the success of a design in meeting these requirements (J. L. Martin et al., 2006). These various methods elicit different types of data. For instance, usability tests can elicit detailed requirements, whereas focus groups can elicit contextual requirements (Garmer, Ylven, \& Karlsson, 2004). As such, there is a growing emphasis on face-to-face methods with multiple stakeholders, and use of multiple methods (Kanstrup, Madsen, Nøhr, Bygholm, \& Bertelsen, 2017).

While previous researchers have constructed user requirements for medical technologies and reported on associated methods of semi-structured interviewing, questionnaires, and usability tests, and developed themes, created grounded theory, or deployed prototypes that inform these requirements, they often do not elaborate on exactly how the user requirements were constructed (Consolvo, Everitt, Smith, \& Landay, 2006; Lang, 2012; Milewski \& Parra, 2010; Owen, Buchanan, \& Thimbleby, 2012; Shah \& Robinson, 2006). Sometimes, the requirements are linked back to elements of the research, which elucidate their origins. For example:

The system shall provide visualizations and/or testimonials of the negative effects that uncontrolled diabetes may have on the body. We include this requirement because 1) our participants did not use information to understand their disease because the information was "irrelevant" to their condition, and 2) our participants desired some way to help them take the disease more seriously. (Milewski \& Parra, 2010, p. 416)

Alternatively, examples of current devices that meet these requirements are provided: “The design of a medical device should afford the adolescent user 
opportunity to integrate 'identity' and the device. E.g. Physical customisation or choice and tailoring of feedback options. Insulin pen sheaths provide option for customisable appearance" (Lang, 2012, p. 291).

As such, in this research user requirements will be reported with examples, showing instances where the requirements are currently met or unmet and demonstrating aspects of the research that led to the creation of the requirement. Multiple methods will be used to inform user requirements, including semistructured interviews, a card sort task, design vignettes, cultural probes, and a design workshop.

\subsection{Conducting Research with Adolescents}

Two ethics submissions were approved for this research. The first, ID 20993 assessed by Victoria University of Wellington, allowed for designers and design students to take part in semi-structured interviews and design activities, and for health professionals to be interviewed. The second submission, ID 15/CEN/21/AM01, was approved by the Health and Disabilities Ethics Committee. This application covered the research that took place with adolescents and young adults with type 1 diabetes. This required a study protocol, Māori consultation, and different information, consent, and assent forms for participants aged 13, 14-15, and 16 and above. While parents who accompanied adolescents to research activities attended in the role of a support person and were not consented, they did facilitate their child's contribution through providing context, and as such, their remarks are included in the thesis on these occasions.

Locality approvals were also required from District Health Boards (DHBs) to conduct research with patients, or to be present for recruitment at diabetes clinics. Locality requests were approved for the Capital \& Coast and Hutt Valley DHBs, covering five clinic locations. A locality request submitted to another DHB was denied by the Clinical Lead. A further locality request was discussed with MidCentral DHB, but was not formally requested.

The locality request process was different at each DHB, sometimes being processed by an individual or committee, requiring a health professional within the DHB to lend their name in support of the research, or requiring ethics approvals, 
study protocol, DHB-specific forms, or a memorandum of understanding drafted between the DHB and the university. Participant recruitment for individuals with type 1 diabetes across the five diabetes clinics took 12 months.

Researchers are often reluctant to include adolescent participants due to difficulties navigating the ethics process, and therefore, adolescents remain underrepresented in research (B. Carter, 2009; Hester, 2004). Further, medical devices are commonly designed for children or adults, resulting in adolescents using devices that do not meet their requirements, possibly affecting their selfmanagement (Rados, 2004; Suris, Michaud, \& Viner, 2004). While there are barriers to working with adolescents (Money et al., 2011), there has been a shift towards not only conducting research about adolescents, but increasing their participation in the research, working in a more participatory style (Druin, 1999; Waller, Franklin, Pagliari, \& Greene, 2006). Benefits of participatory design include gaining a better understanding of users' needs and experiences, and improved usability, functionality, and overall quality of device design (Shah \& Robinson, 2007). While there are challenges to conducting research with adolescents, this approach is valuable as adolescents make important contributions to research and design (Lang et al., 2012).

\subsection{Summary}

This research employs a constructivist research paradigm and follows Cross's (2006) principles for good design research. Both participatory design and constructivist grounded theory methodologies are used throughout, with grounded theory and user requirements constructed in alignment with Charmaz's (2014) criteria for constructivist grounded theory and examples of user requirements reporting (Lang, 2012; Milewski \& Parra, 2010). Methods used throughout the research are described in detail, along with related findings, throughout the thesis. 


\section{Experiences and User Requirements as Described by Health Professionals}

This chapter describes interviews conducted with clinicians who had professional experience treating adolescent and/or young adult patients with type 1 diabetes. The aims were to:

- Develop a better understanding of the type 1 diabetes treatment regimen and the medical technologies used.

- Identify patterns of adolescent self-management of type 1 diabetes, and the range between individuals.

- Identify health professionals' impressions of adolescents' psychosocial user requirements of medical technologies.

While this chapter focuses on the thesis' overarching research question and aims (see Chapter 3), it is the only primary research chapter to focus on the views of stakeholders other than adolescents and young adults. Interviews with health professionals were conducted first as they have experience working with adolescents, can observe behaviours common among groups of adolescents rather than individual viewpoints, and can discuss aspects of adolescent behaviour that adolescents themselves may not admit. The insights of these health professionals shaped the next stage of the research, conducted with adolescents, which in turn identified appropriate areas for further investigation.

\subsection{Methods}

\subsubsection{Participants}

A purposive snowball sampling method was used to recruit participants (Devers \& Frankel, 2000; Jupp, 2006). Initial contact was established through a clinical nurse specialist. An email was sent to possible participants outlining the aim of the interview and required time. Upon meeting, many participants suggested further colleagues who would be suitable to participate, or circulated the interview request throughout their diabetes colleagues. Nine health professionals participated in the 
individual interviews. Health professionals in a range of roles commonly included in a patient's diabetes team were interviewed to establish a variety of viewpoints.

Participants' roles were:

- Clinical Nurse Specialist - Diabetes (three; CNS);

- Nurse Practitioner (two; NP);

- Specialist Endocrinologist (two; SE);

- General Practitioner (one; GP);

- Clinical Psychologist (one; CP).

\subsubsection{Procedure}

Interviews lasted 45-60 minutes and were audio recorded and transcribed. Field notes were written after each interview. Locations included clinic rooms, private offices, staff meeting areas, and a café, with one interview conducted via a video calling service. At the beginning of the interview I gave an overview of the research and a definition of 'medical technology', to ensure participants considered medical devices but also logbooks, mobile apps, and such. Each participant was given an information sheet and consent form (Appendix B). The content of each interview varied due to the use of thematic sampling, however, the broad areas covered in the interviews consisted of:

- current role and prior experience;

- impressions of adolescent and/or young adult self-management;

- developments in medical technology;

- impacts of medical technology on self-management;

- choosing and changing medical technologies;

- adolescent-specific needs.

Constructivist grounded theory was used to collect and analyse the interview data, and psychosocial user requirements were formed. This approach is described in Chapter 3. 


\subsection{Interview Findings}

This section begins with an overview of adolescents' self-management of type 1 diabetes. Following this, three grounded theory theoretical concepts and their constituent sub-categories are discussed. At times, health professionals recounted their impressions of adolescents' perspectives. As such, the adolescent perspectives reported below are based on second-hand reflections.

\subsubsection{Self-management overview}

Health professionals described what they considered to be ideal self-management and the range of actual self-management practices they saw among their patients. Ideal self-management included testing blood glucose levels regularly (4-12 times per day), particularly before meals, two-to-three hours after each meal, or when exercising or driving, giving insulin regularly throughout the day, carbohydrate counting, correcting hyperglycaemia, managing hypoglycaemia, eating healthily, exercising, and having an $\mathrm{HbAlc}$ between $40-60 \mathrm{mmol} / \mathrm{mol}$. Clinicians recognised the ideal treatment regimen had become progressively more intensive over time, and also as people shifted from childhood to adolescence (Hoey, 2009).

Health professionals described an 'extreme' range of actual self-management practices, with some adolescents self-managing as described above and being happy and healthy. At the other end of the spectrum, adolescents were 'surviving' this period. Additionally, adolescents' self-management practices transform from childhood to young adulthood, with many adolescents putting little effort into management during adolescence but improving as they enter their twenties.

Clinicians spoke empathically about adolescents not self-managing sufficiently and were conscious of the impact of pressures from 'the rest of life', such as being busy, living away from home, wanting to fit in socially, struggling with acceptance of diabetes and mental health, and dealing with risky behaviours such as drinking and drug taking (Warner \& Hauser, 2009). Aspects of poor selfmanagement included not testing blood glucose levels frequently or at all, taking insulin randomly or after eating, minimising insulin dose to manage weight, not using medical devices during school hours due to social distress, hypoglycaemia 
from administering too much insulin, running high, eating unhealthy foods, and attempting to conceal these behaviours from parents and clinicians. When transitioning into the young adult service at approximately 18 years of age, some adolescents had HbA1cs between $80-100 \mathrm{mmol} / \mathrm{mol}$.

NP1: I've realised the majority of our patients probably test twice a day, several times a week. . There's a couple who are testing really frequently and doing it properly, but the majority of them are not. . They don't even test if they feel low. They often just take something and don't follow it up with a blood test.

Access and affordability to a variety of medical technologies were also problematic. Subsidised devices and consumables were commonly used; however, some families chose to purchase unsubsidised equipment. This included importing medical devices not currently available in New Zealand. Many adolescents found the only brand of blood glucose meter that was subsidised was unreliable, and as such, some families purchased another meter and consumables. CNS3: “The blood sugar machines don't look bad, but we've had terrible trouble with accuracy and we've only got the one on the market, which is terrible. We've got people who are paying for strips 'cause they just don't trust them.”

Health professionals also wanted adolescents to have access to existing meters with extra functionality, such as dual blood glucose and ketone testing devices, and meters that sync with smartphones. There was a sense of inequality, in that choice was dependent on disposable income. NP2: "I think it's like anything with healthcare, if you have the money you can get what you want and have more choice."

The proportion of people using insulin pumps had risen since these were subsidised. However, criteria had to be met for individuals to procure and retain access. Some health professionals argued that giving adolescents increased flexibility and access to medical devices could improve self-management, and this would be more affordable for the health system in the long-term.

Three concepts are explored below, each of which includes multiple categories that explore aspects of the concept. These categories were constructed through the grounded theory process, then related back to existing theoretical 
concepts and literature. The aim was to create substantive theory. The three concepts explored are:

- conceptualising type 1 diabetes and its management;

- $\quad$ struggling for self;

- managing identity.

\subsubsection{Conceptualising type 1 diabetes and its management}

Conceptualising diabetes is about how adolescents come to understand diabetes and its place in their lives. This concept has several properties, including developing and applying diabetes knowledge, conceptualising diabetes and its management, developing acceptance, and engaging in self-management. Each of these processes are described, along with how the processes vary between adolescents. While much of this section describes health professionals' opinions on how adolescents conceptualise diabetes, it also describes how health professionals customise regimens to suit adolescents.

\subsubsection{Developing and applying diabetes knowledge}

Health professionals described a process whereby people with diabetes begin by seeking knowledge, then gain confidence that they have sufficient knowledge to manage in most situations and experiment with changes in their regimen, such as making changes to insulin dosage. On the far end of this continuum, some adolescents thought they knew more than was possible, telling health professionals they knew their blood glucose levels accurately without needing to test, whereas health professionals disagreed, conceding they may have a sense of being high or low, but not with the necessary accuracy for intensive management. As adolescents grew confident in their diabetes knowledge their goals shifted from a stricter ideal of management; getting levels absolutely right, to a more 'blasé' approach; reducing their anxiety, wanting to get through appointments quickly, and asking fewer questions.

Health professionals viewed the adolescent cohort as either having appropriate or a variety of levels of diabetes knowledge. Levels of knowledge differ by age of diagnosis. While some adolescents had been living with diabetes since infancy, 
others were diagnosed recently, during adolescence. For those diagnosed as children, education was aimed primarily at their parents, and the ways in which information was transitioned from parent to child varied widely between families.

Medical technology played a role in the development and application of diabetes knowledge. There was a broad consensus that a positive feature of medical technology was its ability to visualise data to increase health professionals' and adolescents' understanding of their blood glucose levels and the impacts of diet, exercise, and insulin doses. This was illustrated in a study where visualising cornerstones of diabetes management (food intake, insulin dose, physical activity, and blood glucose levels) when logging data increased adolescents' knowledge and understanding of type 1 diabetes management (Frøisland, Årsand, \& Skårderud, 2012). Visualisations can transform ever increasing amounts of data into meaningful information (Visocky O'Grady \& Visocky O'Grady, 2008, p. 16) "highlighting the signal from the noise" (Sklar \& Naar, 2017, p. 384). Information design facilitates 'edification'; personal enlightenment from data, through collecting, processing and presenting information to increase understanding (Jacobson, 1999, pp. 1-10). Examples of technology that visualise data include mobile apps that graph blood glucose trends in colour and CGMs that show adolescents the action of their insulin, reinforcing that they need to administer it.

Remaining barriers to medical technologies included difficulties downloading and sharing data, patients using a wide variety of mobile applications, and difficulties in compiling data from numerous devices. While medical technologies that reduced tedious logging were described positively, one health professional heeded that by writing down their results adolescents engage with the data, and using automation may decrease this.

Finally, numbers attached to blood glucose levels could hold positive and negative meanings for adolescents. One health professional encouraged patients to see their data simply as numbers to help them make decisions, rather than attaching value judgements to them. NP2: "I often say 'it's just numbers, it's just information'.”

Health professionals stated that there was a mediating process in which knowledge did not always translate into corresponding actions. Adolescents who had good knowledge about diabetes management did often apply it. However, diabetes 
knowledge was not always a positive factor in terms of medically recommended selfmanagement, as it was sometimes manipulated for conflicting goals such as weight loss, whereby predominantly female adolescents would run high, taking minimum insulin to lose weight while not becoming ketotic.

\subsubsection{Conceptualising diabetes and its management}

Health professionals described how they customise regimens and a process where adolescents are conceptualising both diabetes and its management, in particular, the role diabetes plays in daily life.

The language used by both adolescents and clinicians tended towards metaphor and personification. CNS2: "It will come back to bite you. And sometimes it will bite you hard straight away." One health professional also used adolescents' own metaphors with them.

CP1: With teenagers if there's a metaphor that fits for them, like 'I feel like I'm in prison because I can't do what I want to do.' Then we might say, 'Well, how can you get out of this prison?'

Language played an important role, with the labels 'diabetes' and 'diabetic' being problematic for some adolescents due to their seriousness, and the connotation that something was wrong with them. This could be more overwhelming than empowering in terms of taking on self-management. Health professionals are conscious about their choices of language. When describing self-management, they deliberately used words such as 'influence' rather than 'control', to not set unrealistic expectations about what impact adolescents could have on the condition.

\subsection{Fitting diabetes around rest-of-life}

A diabetes management goal described by clinicians was getting adolescents to manage diabetes around their lives, rather than placing diabetes at the centre. Previous research has demonstrated that the greater adolescents perceive the impact of diabetes on their day-to-day life, the poorer their wellbeing is (Skinner, John, \& Hampson, 2000). This is a shift from traditional thinking and is mirrored in the way diabetes is currently treated, particularly with modern insulins and insulin pumps 
that make it easier to respond to unplanned food and exercise, facilitating spontaneity and flexibility.

CP1: The narrative that they are usually are given and take on board is, 'You've got this thing that's going to dominate your life for the rest of your life,' and so we're looking at an alternative narrative 'Actually, you can take control'. It's an empowerment thing. 'There is going to be some degree to which there's always going to be the need for your testing and your insulin injections, but if you do that in a consistent sort of way then you're not going to go blind when you're middle aged or have your feet amputated'.

However, for diabetes to not significantly impact daily life (e.g., through common episodes of hyperglycaemia), adolescents needed to self-manage at a sufficient level. Only then could diabetes take more of a back-seat role. Although the conceptualisation of managing diabetes around the rest of life seems straightforward, in reality many aspects of adolescents' lifestyles did interact negatively with diabetes management. These aspects included decreasing input from parents, living away from home for the first time, eating unhealthy foods or having little control over what foods were available, experimenting with alcohol and drugs, having rigid or unstructured timetables with school, work, or university, playing sports, and dealing with mental health issues such as anxiety and depression.

Conversely, diabetes also had an impact on daily life, with hyperglycaemia and hypoglycaemia making adolescents tired, making learning difficult, and requiring adolescents to miss classes and educational content to manage diabetes. Additionally, adolescents were sometimes scared of breaking expensive equipment and some devices only worked in narrow temperature ranges, requiring adolescents to spend time warming or cooling them before use.

A recurring suggestion for minimising the impact of diabetes on everyday life was to minimise tedious elements. Clinicians cited glucose meters that automatically sync to smartphones and closed-loop glucose monitoring/insulin delivery systems as technologies able to reduce the management burden. However, these technological aids were currently unaffordable and unavailable to many. While free mobile apps 
are available for carbohydrate counting and tracking and visualising blood glucose levels, they have a limited uptake as information is input manually, and this was considered 'tedious.' An app that could accurately suggest insulin dose to be administered would be effective for some people, particularly those who struggled with the mathematical calculations. Many health professionals identified blood glucose testing as the most important aspect of self-management that many adolescents were not currently engaged in to a level that would significantly improve their health.

CNS3: The biggest barrier to self-management is the testing... A lot of them will give insulin blind because they don't test. They guess where they're at and what they'll need... The only way you can test is by a drop of blood. It takes a few minutes to get your machine out, wash your hands and test, then see what your blood sugar is, then you decide how much insulin you're gonna have, what you're gonna eat, what you're gonna be doing after lunch. It's a rigmarole.

Another suggestion was to combine multiple devices and consumables, such as the blood glucose monitor, lancet, and test strips, into one multipurpose device to minimise the equipment being carried.

\subsection{Customising the treatment regimen}

Clinicians had targets for their patients, which some were reaching, but clinicians were also willing to negotiate and customise the regimen based on the needs and goals of the individual, as is advised (Perfetti, 2013; Sawyer \& Aroni, 2005). However, the tensions between an adherence approach reflected in measurements such as average clinic $\mathrm{HbA1c}$ levels, and an engagement approach were evident (Mudge et al., 2015).

An adapted regimen might include taking an insulin injection after school, rather than with lunch, or administration of fewer injections by parents temporarily. Health professionals felt a responsibility to review these practices periodically to facilitate long-term health. Although intensive self-management was beneficial for 
health outcomes, health professionals were balancing the risk of being too demanding with maintaining long-term goals for patients, and acting as a safety net.

Self-management is viewed as interacting with a phase of normative adolescent development, characterised by physical, psychological, and social changes through which the individual must progress (Michaud, Suris, \& Viner, 2007). This framing of an 'adolescent phase' is particularly used for those whose behaviour is challenging. The health professionals' expectations of these adolescents differed from those who self-manage at a medically optimal level. Adolescents struggled to consider long-term impacts, and thus clinicians did so on their behalf, balancing current, individual needs with their knowledge about the importance of an intensive regimen for years of ongoing metabolic benefit.

Adolescents who were trying to self-manage but not getting positive outcomes felt challenged to continue, questioning 'what's the point?'. In these situations there is a question of whose goals and wishes should be prioritised. Some adolescents do not wish to improve their self-management, but their parents do. With the adolescent's brain still developing, adults may feel responsible to think about long-term complications while adolescents have not prioritised these, in case the adolescent comes to regret their decisions later when the consequences are evident. This is particularly problematic with the loose coupling between everyday self-management behaviours and long-term complications.

One health professional discussed working towards patient-selected goals, as adolescents were already committed to achieving these (Delamater, 2006). Goals were small enough for change to be assessed at the next appointment. Health professionals accepted that other aspects of patients' lives that were important to them could be in conflict with their diabetes management, and that these things could be done with care (e.g., eating party food), as there was an emerging holistic view of health.

CP1: Maybe occasionally going out and eating what your mates is going to be important as long as they don't go way over the top, because you don't want them to feel totally deprived. They've got to look at their mental health and their social wellbeing as well as their physical [health]. We have all our little divisions; doctors look at this 
aspect and the psychologist looks at that aspect and the dietician looks at that aspect. But overall it's the young people who have this holistic life where they look at all these different aspects and make decisions based on all these things.

The clinician notes that by the nature of different roles within the diabetes team, health professionals may be focused on specific areas, such as mental health or nutrition. The adolescent themself has the best holistic view of their broader goals and how diabetes is managed alongside these. At times, health professionals explicitly prompted adolescents to improve their self-management.

GP1: I give them a gentle sort of 'maybe you should try a bit harder.' I try really hard not to lecture them about how bad diabetes is and what happens if you don't manage, because they know all that stuff and they don't need to hear it again. If they are doing well then they get a lot of encouragement.

However, health professionals thought adolescents often have a feeling of invincibility; that diabetes complications would not happen to them.

GP1: It's just youth. I think they really believe that it's not going to happen to them, all those horror stories of people losing their legs and going blind and the kidneys failing. They think 'well that happens to someone else, it's not happening to me.'

Some adolescents did not want to interact with health professionals as they wanted to feel 'normal;' appointments shattered this illusion. When adolescents did attend medical appointments, some were honest about their previous or intent for selfmanagement, whether it was medically successful or not, and others would say that they would manage as instructed, then not do so. Clinicians also recognise the need for behaviour change to be driven by the adolescent.

NP2: It's really, really hard to watch, and sometimes you think 'what am I doing, why can't I get through to this person?' and often it's just waiting for them to be ready to ask for help. 


\subsubsection{Developing acceptance}

Acceptance of type 1 diabetes and its required management was conceptualised as a process and a state, where from diagnosis onwards acceptance could be worked towards and a state of acceptance could finally be achieved. In this state, the person could become engaged in their self-management. However, not everybody reaches a state of acceptance and there may be regression, with hurdles such as workload and social problems. It was easier to get back on track if good groundwork had already been laid.

SE2: It's [diabetes] there in the background the whole time. So it's coping with that chronic disease; something that's not going to go away and something that's going to be part of their life forevermore... Some people are very okay with that concept, some people become ok with that concept overtime, and some people probably never get the hang of that concept.

There was also a trend for people to manage well initially after diagnosis, and then begin struggling after a few months when they fully understood the long-term nature of diabetes management.

CNS1: People come into hospital and they're sick and you can make them better quite quickly by giving them insulin, and so they feel better and they'll do the jobs you require of them. Then at three to six months it suddenly dawns on them that this is forever and often they'll hit the wall. They'll stop testing and often that's when insulin becomes a bit more random and then they're not going to tell because they know they'll be in trouble, and then they come to clinic and it's all revealed.

Two health professionals hypothesised that a diagnosis after adolescence may result in an easier process to acceptance, as opposed to a diagnosis as a child or adolescent. Diagnosis during adolescence was particularly problematic for acceptance, with a developing awareness of the unfairness of having to self-manage diabetes when peers did not. 
CP1: At 12, 13, 14, they're saying, 'Hold on, why do I have to do this?

Why can all my friends just pig out on chippies and fizzy drink and I

have to watch my diet?'.

This perspective relates back to normative adolescent development, with the growing importance of the peer group and the developing capacities towards adulthood, thinking not only about current situations and needs but also about the long-term future and health consequences (Michaud et al., 2007). The perspective also relates to the progression through Goffman's (1963) 'moral career' discussed later in Section 4.2.4.2.

Family also played a role in facilitating acceptance; how parents felt about the condition affected the adolescent's own feelings. Parents who were proactive, rather than feeling overwhelmed - almost grieving for the adolescent - tended to fare better. Health professionals also attempted to shift people along the continuum to acceptance. Rather than repeatedly warning patients about possible complications of poor diabetes management, health professionals tried to get adolescents to give voice to their need to manage.

CP1: I'm working with them to identify for themselves some consequences that they don't want and that they're prepared or motivated to work towards. Because often they come up with excuses, 'I've got exams or tests and I've got this big sports event coming up and I can't focus on the diabetes now.' And then we look at, 'Well, when that's over won't there be other things?', and, 'When will there be a time in your life when you will be able to focus on the diabetes?' The reality is usually there's no better time than the present.

On the opposite side of the continuum to acceptance is adolescents feeling invincible, as per normative development, characterised by immature brain development (O’Donohue, Benuto, \& Tolle, 2013).

CNS2: There's a large proportion who think they're fairly bulletproof and are much more keen to just fit in with their peers and turn a blind eye to their diabetes... Sometimes it will bite you hard straight 
away... But for some it'll be more gradual and it will be complications 10, 15, 20 years down the line, which is always hard to tell them at the time, because that happens to other people, it's not going to happen to them.

Another barrier of developing acceptance can be a sense of identity conflict from having a long-term condition, whereby a young person who has previously been fit and healthy struggles to balance the existing self-image of healthiness with also having type 1 diabetes (Charmaz, 1995). This brings broader questions around what 'healthy' means to someone with a long-term condition.

\subsubsection{Engaging in diabetes management}

Similarly to acceptance, engagement is described as both a process and a state (Bright et al., 2015). The process of becoming engaged was predominantly described in one of two ways. The first was a gradual process that aligned with the individual gradually maturing, and the second was a defining moment where some external factor resulted in an epiphany. Defining moments are incidents or turning points that can affect daily life and impact on people's sense of identity (Denzin, 1992; Strauss, 1969), sometimes experienced in response to medical diagnoses and management (Beard, 2004). One health professional thought engagement typically happened in late adolescence for females and a few years later during young adulthood for males. CNS1: "It's like suddenly they've grown up, and the dots have connected and they've gone 'I've got to do something about this', and they do." SE2: "I don't know what happens, but a sort of switch is activated."

Reasons health professionals recalled for a conscious choice to engage were the adolescent meeting somebody new, seeing someone suffer or die from diabetes complications, an impactful remark, the threat of losing funding for an insulin pump, and wanting independence from parents. The excerpt below describes the impact of a health professional's behaviour on one adolescent's choice to engage.

NP2: It [blood glucose test] came back at 27, and the HbA1c was 106 or something, and the doctor said 'so what should I do?' . . So he called her... and then the next day she was there and was like 'I want 
to work on my diabetes'. She said it was happening anyway, but she thought that phone call just really brought it home to her; 'we're really worried about you'.

Being engaged in self-management involved being proactive (Bellows, Kovacs Burns, Jackson, Surgeoner, \& Gallivan, 2015). For instance, one health professional contrasted a reactive approach where an adolescent's blood glucose may be high in the afternoon so they take more insulin, with a more proactive approach where the adolescent realises their blood glucose levels have consistently been high in the afternoon, so they adjust their insulin in the morning to prevent the high from happening.

NP2: It's sort of like how psychologically you look at it, like are you going to be passive to this disease, or are you going to be aggressive and take control of it?

This can be seen in adolescent populations with type 1 diabetes, where 'active coping' is associated with improved glycaemic control and life satisfaction, whereas 'emotion-focused coping' (e.g., disengagement) is associated with poorer glycaemic control and life satisfaction (Graue, Wentzel-Larsen, Bru, Hanestad, \& Søvik, 2004).

Managing long-term disease presents a considerable challenge to patients, family members, and clinicians. The traditional passive role for patients that is established within the healthcare community is ineffective. Instead, having the patient take an active, empowered role in their own management is encouraged (Levich, 2007). Health professionals perceive part of their role as helping people shift from a passive state of feeling something unfair has happened to them to a proactive state with the tools to take action.

CP1: I'll often say, 'Yes, it is unfair, nobody else got it but you, you happen to be the one in your family who got it, and you can choose to get stuck in the unfairness of it or you can say, okay, this is the reality of my situation and I can still have a full and meaningful life provided I manage it.' 
This shift from passivity to proactivity links back to developing acceptance, where with acceptance of diabetes, adolescents can choose to engage.

While health professionals spoke about allowing adolescents to be spontaneous, many also felt having structure in their lives made self-management easier. This is supported by prior research, where parents found routine improved management and ability to participate, though it could be at odds with spontaneous participation (Quirk, Blake, Dee, \& Glazebrook, 2014). Weekend routines were often in stark contrast to weekday routines for adolescents. For some, having some consistency in meals, particularly breakfasts and lunches, made it easier to calculate their required insulin dosage. For health professionals, facilitating engagement was crucial before pushing patients for optimal management.

\subsection{Running high}

Clinicians talked about a common tendency for adolescents to run high. There were a variety of reasons for this. Some were afraid of hypoglycaemia, and would deliberately elevate their levels to avoid this possibility. While this avoided the shortterm risk of hypoglycaemia, it increased the longer-term risk of diabetes complications, putting the feelings of short-term safety in contradiction to long-term health. An example of this was one adolescent who experienced bouts of anxiety after experiencing hypoglycaemia. Although his levels were still above the recommended range, he had managed to bring them down slightly for the first time in seven years and was happy with this effort, while not ready to bring his levels into the normoglycaemic range.

For others there was a social aspect to running high, avoiding the risk of hypoglycaemia in public settings, as they worried about losing control and what others might think. One clinician recounted a young woman having a seizure due to hypoglycaemia at a public gathering, and seeing how 'devastating' this was for her.

Alternatively, running high could be the result of the adolescent forgetting whether they had administered insulin. If in doubt, people often chose not to take a dose, in case of stacking doses, which may result in hypoglycaemia. Often this resulted in them having high levels as they had not actually taken a dose. Some medical devices, though, were helping with this issue. For instance, one Novo 
Nordisk insulin pen has a memory feature that displays the size of the last dose and how long ago it was taken. These proved popular, though there were a limited supply to be distributed free of charge.

Others who were less engaged in diabetes management would take sufficient insulin to avoid being acutely unwell. With persistently high levels, having normoglycaemic levels made them feel as if they were hypoglycaemic, with their body acting like a thermostat. Some adolescents were doing this to avoid testing or injecting in public places such as school, not wanting to be singled out as different. Others were almost giving up, when their efforts were not reflected in their results.

CNS2: They can get really disappointing results that make them feel unwell despite their very best efforts, and that leads to a lot of frustration and that in turn can lead to 'what's the point?', they throw their hands up in despair and stop testing and stop taking insulin because when they were trying they weren't getting anywhere so you do get that attitude a lot, quite possibly magnified in adolescence.

This attitude was again magnified by normative adolescent development. In an attempt to look like their peers, adolescents can avoid disclosure and selfmanagement in social settings (Michaud et al., 2007).

\subsubsection{Struggling for self}

Struggling for self represents adolescents' struggle for independence and recognition of their individual needs. Various stakeholders including adolescents, their parents, and health professionals undertake the processes described in this section. This concept has two properties: the first is about being an individual and requiring the diabetes service and technologies to suit your needs, while the second is about asserting independence and taking control.

\subsubsection{Recognising individual needs}

In addition to normative adolescent development, some characteristics that affect adolescents' self-management can be more individual. While adolescents are a small subset of people with type 1 diabetes, they are "extremely heterogeneous" (SE2). 
Personality and intelligence were raised as factors that may influence how an adolescent manages diabetes. Diabetes management requires challenging skills of reasoning, analysis and mathematics. Further, adolescents who are organised and like structure tend to manage better. Information about personality is increasingly being used to tailor technology offerings (Anagnostopoulou et al., 2017; Mattheiss, Hochleitner, Busch, Orji, \& Tscheligi, 2017; Orji, Nacke, \& Di Marco, 2017; Thomas, Masthoff, \& Oren, 2017). Health professionals were divided in whether they observed gender differences in self-management. While some saw no differences, others were split between whether males or females might take better to selfmanagement.

In response to these individual characteristics, health professionals were customising their approaches with adolescents to deliver information and set selfmanagement expectations in a targeted and realistic manner for the individual. This links back to discussion of how health professionals conceptualise their role in Section 4.2.2.2.2; negotiating and customising the regimen for individual patients in response to medical best-practice and individual requirements.

\subsection{Responding to dissonant technology}

When examining the interactions between adolescents and their medical technologies, individuals have varying compatibility with these devices. While adolescents are heterogeneous, there are limited choices and adaptability in current medical technology. Where there was dissonance between adolescents and their medical technologies, two processes that alleviated frictions were described: rejecting technology or changing technology and behaviours.

When there are issues of compatibility between adolescents and medical technologies, some adolescents opt to reject medical devices. A health professional recalled instances where adolescents had been taken off insulin pumps to rectify a mismatch between person and technology:

SE2: I've got a few patients who have come off pumps . . at 14, 15, 16 sort of age. I think one of the difficulties of pumps is they actually add a few levels of complexity to management. That's often misunderstood. They do require a significant amount more 
understanding and responsibility than simply injecting insulin

does... Some people cope very well with that, some people don't cope

very well.

The health professional's impression is that adolescents' expectations of insulin pumps can be misaligned with their reality. The pump needs to be managed closely, with more frequent blood glucose testing, and the associated data needs to be interpreted accurately to make sophisticated calculations and reap the possible rewards (Cogen, Streisand, \& Sarin, 2002). While some adolescents accomplish this, others eliminate their initial dissonance with medical technology by discarding the item rather than adapting their behaviours or diabetes knowledge. This misalignment between insulin pump expectations and realities has been reflected in previous work, whereby those who viewed the pump as a tool rather than a panacea took a more active role and achieved better glycaemic control (Ritholz et al., 2007). In another study, while $89 \%$ of adolescents were pleased with their insulin pumps, outcomes were less positive for those who had unrealistic expectations of the pump (e.g., the pump as a 'cure-all') (Low et al., 2005, p. 25). As such, it is important that users of medical technologies have realistic expectations of the capabilities and behaviours required for devices they intend to use.

In addition to aligning with expectations, appropriateness of medical technology was also in relation to broader values. One clinician recalled changing prescriptions from disposable insulin pens to reusable insulin pens because adolescents voiced dissonance with the device because of their environmental ideologies. Their values were impacting their feelings about medical device use, and changing devices to better suit their values appeased this. Here, communication between the adolescent and health professional can help in choosing appropriate technologies and alleviating frustrations. A health professional described the importance of having the right fit between individuals and medical technology. NP2: "It's the marriage of all the equipment, and the marriage of their life situation."

The second response to experiencing dissonance with medical technology is for adolescents to change medical technologies and demonstrate a resulting change in behaviour. A health professional recalled an instance where an adolescent began 
an insulin pump regimen, and because of a newly found harmony between his personal characteristics and the medical technology, his self-management behaviours improved significantly.

SE1: So one guy, he was just shocking and didn't really engage with his diabetes, but he's a real gadget guy and when he got a pump all of a sudden his control was perfect and he just loved it. He was always playing with it, doing all the stuff that enabled him to get good selfcontrol, and that just worked for him.

In this instance, the adolescent's response to the technology resulted in better selfmanagement. The health professional sees the adolescent's 'gadget guy' personal characteristics as being in alignment with the medical technology. This alignment results in a positive behaviour change. A similar pattern emerged in the phenomenon of 'running high' discussed in Section 4.2.2.4.1. While adolescents were initially afraid of stacking insulin doses and the resultant hypoglycaemia, by switching to an insulin pen with a memory function, their anxiety was appeased and they delivered their insulin injections confidently, lowering their blood glucose levels, as they were not afraid that they had already administered them. Again, these processes rely on effective communication between the adolescent and the health professional. The health professional needs to understand the adolescent's individual characteristics and behavioural patterns to decide which technology is in alignment with the adolescent's requirements, in partnership with them. This relates to a subset of engagement, described as an active partnership where patient goals and values are communicated and the individual is respected (Brown et al., 2015). Moreover, there are possibilities for increasing the array of choices in medical technology and introducing aspects of personalisation.

Adolescents having choices about which medical technologies to use arose as a factor that is currently limited by affordability and clinically driven decisions, but could be important to self-management. Having choice could help the adolescent establish control, an important aspect of the normative adolescent process of developing freedom and independence and pushing back against authority (Michaud et al., 2007). 
The phrase 'one size doesn't fit all' was used by multiple clinicians, although none were aware of any diabetes medical technologies designed specifically for adolescents. Research has suggested patients preferred insulin pens over syringes as they were easier to use, more convenient, less painful, socially accepted, and increased their self-efficacy (B. J. Anderson \& Redondo, 2011), and that insulin pen use led to a higher adherence rate and fewer adverse health outcomes (Goldstein, 2008). This suggests that fulfilling some medical technology preferences may result in improved health outcomes, and that this relationship should be further considered (B. J. Anderson \& Redondo, 2011).

While medical technology was thought to play a role in adolescents' selfmanagement, health professionals also stated that more suitable technology alone would not overcome all barriers, as the individual's characteristics also shape their self-management. One health professional spoke about modern technology and how this could be used to create personalised medical technology, where the technology is adapted to the needs of the individual. Current options such as stickers were aimed at younger children. Another commented that adolescents should have the opportunity to love their medical technology as they would a favourite consumer device. The possibility of trialling a medical technology before committing to it was also raised.

Therefore, for a better fit between person and technology we must design medical technology that meets the needs of individuals, whether their differences stem from personality, IQ, values, or other aspects. By introducing more choice to adolescents, we may increase their sense of control.

\subsection{Shaping the service}

Health professionals were shaping the adolescent-specific diabetes service to suit their patients; developing a website, holding diabetes clinics in the city centre and at a local university in addition to hospital locations, having a diabetes nurse specialist that cared for 13-25-year-olds, ensuring continuity of care, using text messaging rather than phone calls, and incorporating sexual health, drug, and alcohol education and risk-management into care. They were looking to improve their web and app services and careful to use language that was appropriate for adolescents' knowledge 
level and preferences. Additionally, they were advocating on behalf of adolescents for free services such as eye screening, dental and podiatry appointments, disability allowance access, special exam facilities, and help with job seeking. Health professionals also organised coffee groups and beer tasting sessions to facilitate diabetes peer networks and teach adolescents about managing alcohol. They also suggested adolescents' needs would be better met through greater clinical psychology support, as referring a patient to the clinical psychologist was currently viewed as abnormal. In summarising the adolescent diabetes service, one clinician explained their role was to help adolescents address having a long-term condition, and fitting that into their life, particularly focusing on unique adolescent experiences.

\subsubsection{Transitioning responsibility}

Health professionals strived to facilitate adolescents' independent self-management, but also their support network. CNS1: "The whole aim is to get people to be independent with their diabetes management, but still maintain support from family or parents or the clinic."

For those diagnosed as children or younger adolescents, there was commonly a transition of responsibility for diabetes management that took place during childhood and adolescence from parent to adolescent. This was an age-specific aspect of managing diabetes, with adolescents who were diagnosed at an older age not affected by this process.

Under the age of ten, most diabetes education was aimed at parents. While some children were interested to take on management activities from diagnosis, most were not. From 11 and 12 years onwards, clinicians would include the child in the education much more and start to assign them tasks such as blood glucose testing. Some young people enjoyed this independence. Slowly, the adolescents became independent with other tasks, such as giving injections, though often they would check the dose with a parent. By their late teens it was uncommon for adolescents to not be administering insulin. For a small number of adolescents, their parents, typically mothers, regularly or occasionally administered their insulin. Typically, administrative tasks such as arranging transport to appointments and collecting prescriptions were taken on last. Variables in the process included the age of 
diagnosis, parental style, diabetes team approach, and the adolescent's individual characteristics. This process of transitioning responsibility relates back to the process of engagement described in Section 4.2.3.4, whereby the adolescent becomes more involved, often consciously, in their diabetes management.

Characteristics of a successful transition strategy included beginning at a young age rather than waiting until the adolescent left the family home, making the process gradually, and making the adolescent aware that they could ask for help whenever required. There is no agreed upon age for this transition of responsibility in the literature, however, if the transition takes place too soon, there is likely to be poor disease management and difficulty with psychological adjustment (Greca, 1990; Ingersoll, Orr, Herrold, \& Golden, 1986).

SE2: Some people manage things exceptionally well in an independent fashion, some with the help of their parents, and some people manage things awfully irrespective of who's involved...

Health professionals hypothesised that experience and maturity were factors that helped to improve self-management, as over time adolescents became used to managing largely by themselves. While transitioning responsibility for management was crucial, often the standard of management slipped during and after this process. Accordingly, health professionals attempted to identify the barriers to selfmanagement for adolescents and explore how they could overcome them. These aspects included hormones, struggling for independence and testing boundaries, social pressures to be accepted, struggling with long-term thinking, dealing with highly structured school or work environments, or conversely, unstructured university environments.

SE1: Younger adolescents can sometimes use diabetes as a weapon, and as a way to exert independence.

When adolescents were not managing well, however, often it was hidden from their parents. Parents were often shocked and upset when they became aware of this. Health professionals reassured parents that this behaviour was common, though aimed to improve self-management. Health professionals facilitated this transition of 
responsibility, sometimes helping to negotiate adolescent and parent roles and boundaries.

CNS: Parents have a hard job... They've received the education and they've managed that child... and then they can't quite step back and they still try to micromanage and then you get a lot of conflict and the teenager can cause a lot of distress by not testing and not giving insulin because they know it'll wind mum and dad up.

This clinician took a 'bird's eye view' of the situation, knowing that responsibility had to be transitioned and management had to be safe, and would help negotiate parent and adolescent roles, although some parents did not like this. Often, an adolescent experiencing hypoglycaemia a few times after letting management slip would not enjoy this, and it would spur them to take responsibility.

CNS3: You often say to people you know if you write it in the book, or left your machine somewhere where mum can have a look, she'd be off your back. So as long as mum can see you're doing ok she won't be asking you 'Have you tested? Have you given your insulin?'; it drives them nuts.

This 'nagging' was not appreciated by adolescents, and parents had to develop strategies and roles for managing this transition effectively. The transition can also result in medical technology changes.

CNS1: I've taken quite a few teens off pumps because mum and dad decided the pump was a good idea when they were children, and then when they've become adolescents they suddenly don't want to be attached to the pump anymore and they're not very good at running it, cause mum and dad have always run it for them.

While support from family typically decreased as the adolescent became more independent, this was not always the case. In rarer, temporary instances, independence decreased. 
CP1: I've got a 13-year-old that I'm working with at the moment and he was diagnosed at the age of seven and immediately wanted to take responsibility for his diabetes... What came out was, 'I've been managing my diabetes for all these years and I'm sick and tired of doing it.' So what we've negotiated is that he will continue to take responsibility at school, but when he's at home mum has overall responsibility... There are other factors - he's the oldest in a family of four kids... So he's saying, 'I've got enough responsibility without having to take responsibility for my testing at home... So my role here is just facilitating something that works for them.

Negotiating a family setup where the adolescent decreased their responsibility was referred to as a 'diabetes holiday.' While diabetes was constant, the adolescent could have a break from personally managing aspects of it, and rely more on their parents. Often, within the space of weeks adolescents were ready to manage again, frustrated by their parents' invasion of their privacy and independence.

\subsubsection{Managing identity}

Managing identity was the third concept constructed in the grounded theory analysis. While many of the adolescent self-management processes described to this point have been intrapersonal, this concept examines the ways in which adolescents seek to manage their identity in relation to others. Aspects include disclosing diabetes, managing attention, embarrassment and stigma that can arise from having and managing diabetes, interacting with peers who also have type 1 diabetes, and attempting deception to convince parents and clinicians of 'good' self-management practices. In this sense, adolescents are managing their identities both as regular adolescents and as 'good' diabetics.

\subsubsection{Disclosing diabetes}

Disclosing diabetes was a challenging aspect of diabetes management, with adolescents wary of being negatively judged (Schabert, Browne, Mosely, \& Speight, 2013). While initial disclosure varied widely based on the age of diagnosis, adolescents were intermittently placed in situations, such as changing schools, 
making new friends, or joining sports teams, where they could choose whether to disclose their diabetes. Health professionals stressed the importance of informing teachers, peers, or co-workers of diabetes in case of an emergency. However, they recognised that adolescents may want to disclose selectively and maintain their privacy. Interestingly, it was using medical technologies in public that often signalled the adolescent had a health condition. Health professionals helped adolescents form strategies for disclosure at an important transitional stage by bringing in Year 9 students (13-year-olds) to talk to Year 8 students (12-year-olds) about how they told their new high school friends about diabetes. Again, adolescents are characterised by their heterogeneity in needs and preferences.

CNS1: Some kids will be really upfront, other kids will just let people discover it when they see them testing or giving insulin, and other people are completely private and don't want anybody to know, and then when it's exposed they're mortified.

There was also a psychological benefit of disclosure. Not disclosing illness puts adolescents at a high risk for non-adherence and poorer psychosocial adjustment (Badlan, 2006; Thompson Jr. \& Gustafson, 1996). Adolescents with long-term conditions demonstrate a strong desire to live a normal life, sometimes minimising their differences from other healthy adolescents and showing a reticence to disclose their illness to their peers, fearing it might attract attention to their 'differentness', and as such affect them being socially accepted by peers (Berge et al., 2007; Gjengedal et al., 2003). They choose whether to disclose by balancing increased safety in possible emergencies with the possibility of negative social reactions (Y.-L. Wang et al., 2010). However, if adolescents disclosed and discussed diabetes, this became the norm for them.

One health professional recalled how a patient felt that an insulin pump could aid disclosure. Although she did not currently test her blood glucose levels, she was considering applying for an insulin pump, which would require frequent testing. The impetus for this decision was that she found it challenging to disclose diabetes to potential romantic partners. With an insulin pump she could choose to show it to a partner and wear it discreetly at other times, and use it to help explain her diabetes 
management. The health professional contrasted this with other patients, some of whom wore their insulin pumps discreetly and others who wore them obviously, outside their clothing.

\subsection{Peer knowledge}

Adolescence is a time of increasing peer influence. As diabetes is managed throughout the day, often peers at school, university, or in the workplace may be aware of the condition. Close friends commonly knew about the condition and its management, but other peers had little knowledge. Peers asking questions about diabetes management sometimes distressed adolescents. CP1: "What does it mean that you've got diabetes?' and, 'What can you eat and what can't you eat?' and, 'Why do you have to do these blood tests?"”

Some schools let the adolescent or a health professional brief their class on diabetes at the beginning of a new school year, making sure peers were aware in case of an emergency and allowing the adolescent the status of being an authority on diabetes. Other adolescents did not want their peers to know they had diabetes and were embarrassed by it. There also arises a safety aspect of who the adolescent is socialising with when not in the care of their parents. In some cases, there can be issues socialising with preferred peers because they, or their parents, are not equipped to deal with diabetes emergencies.

\subsubsection{Managing attention, embarrassment, and stigma}

Feeling different from peers was problematic for adolescents. Some, as discussed in Section 4.2.2.3, questioned why they had to take insulin, monitor their food intake, check they had their medical devices with them, and such, when their friends did not have to. In some social situations where they did not want to feel different from their friends, adolescents would skip a self-care activity, and if this had no immediate consequence, this would become a recurring behaviour. Some adolescents resisted special treatments, such as separate exam facilities, while others welcomed these.

Health professionals described instances of adolescents feeling too embarrassed to wear medic alert jewellery or use medical technologies to selfmanage in social contexts. Resultantly, adolescents would not test blood glucose 
levels or administer insulin at recommended times due to social considerations. CNS2: "They're still medical devices that label them as 'there's something wrong with you ...', so there's still that stigma." There were advantages and disadvantages to both insulin pumps and insulin pens in terms of use in public spaces. While insulin pumps were constantly attached they could be discreetly used, being easily 'passed off as consumer technology. While insulin pens were not attached constantly, they looked more medical as the pen metaphor broke down at the point of use.

Adolescents who are overweight, may also experience stigma as people conflate type 1 and type 2 diabetes and blame them for having diabetes. Further, there was stigma around the use of medical devices, particularly for drawing blood and injecting insulin. Stigma refers to a social response to a discrediting attribute. In his seminal description on stigma, Goffman (1963) described two types of stigmatised people: the discredited and the discreditable. The discreditable, such as those with diabetes, are those whose differentness is not immediately evident. A 'moral career' describes the process of recognising and dealing with being stigmatised:

- learning what 'normal' is;

- discovering your stigma and its consequences;

- learning to cope with the reactions of others;

- learning to conceal the stigmatising attribute;

- acceptance.

In some aspects, Goffman's moral career mirrors the gradual process of coping and acceptance of having type 1 diabetes. Medical devices can be used to minimise symptoms, however, in doing so the medical device can become a stigma symbol, drawing attention to an otherwise invisible disease, leading people to possibly reject the device (Goffman, 1963). Whether individuals view their device as a tool to aid them or as a visible, stigmatising sign of their disease can affect their use of the device (Polgar, 2010). This intense awareness of others' perceptions is a major characteristic of meta-cognition development during adolescence (Huebner, 2009), the preoccupation characterised as the 'imaginary audience' (Elkind, 1998). 
One clinician noticed that children were more amenable to initiating insulin pump use than adolescents. Adolescents did not want to initiate use of a device attached to them if peers might notice it. While many children who had moved onto insulin pumps were happy to continue using them through adolescence, there were also some adolescents coming off insulin pumps to assert independence, and because they did not know how to manage the pump independently. Older adolescents and young adults began to give others' opinions less weight, and prioritise their selfmanagement over perceptions of what others may think.

Although many adolescents felt that their peers may judge them negatively both for having diabetes and for carrying out self-management activities, their peers did not commonly react negatively when they did find out. The adolescents' expectations of how others may react was enough to negatively influence their selfmanagement behaviours, irrespective of whether there was an enacted stigma. This reflects the importance of constructing meaning, whereby perception is important. Finally, in some cases, the extra attention garnered from managing diabetes in public settings was welcomed.

\subsection{Making medical technologies socially acceptable}

Making medical technologies socially acceptable could help some adolescents to better self-manage their diabetes. Health professionals described two strategies to achieve this: medical technologies being discreet, and medical technologies being cool or rewarding. The first strategy gives adolescents a choice about disclosure, letting them manage in public privately or choosing to disclose. The second strategy would continue to draw attention, but ideally in a more positive form, by not looking clinical. Currently some medical technologies can 'pass' as consumer technologies, and some adolescents changed devices based on aesthetic preferences.

Clinicians suggested providing choices about device colour, using modern technologies, having cooler device names, small, slim devices, desirable features, and syncing with mobile devices. Clinicians felt that these features may also be desirable to adults, signalling that an 'extreme user' approach to design, whereby products are designed for users with unusual needs (adolescents), may result in technologies that are suitable for a wide range of users (Holmquist, 2006). 
One clinician suggested that technology could shift from a compensatory approach to a game-based reward for self-management. He described a concept for a mobile game where normoglycaemic blood glucose levels could be rewarded, where people could benchmark their levels against an average, and where friends could give kudos. This may also allow adolescents to develop an understanding of how they are doing compared to others. Digital technologies have the potential to convert selfmanagement activities into more relevant experiences, improving adherence to treatment regimens (Bainbridge, Bevans, Keeley, \& Oriel, 2011; Gerling, Schild, \& Masuch, 2010; Tanaka et al., 2012).

Another strategy suggested was broadly normalising health-monitoring behaviours, as we are beginning to see with more sports-focused devices such as smart watches and Fitbits. This trend of monitoring and deliberately improving health, and using technologies in public spaces both physically and digitally, is helpful in normalising diabetes management (Fotopoulou \& O'Riordan, 2017).

\subsubsection{Interacting with diabetes peers}

Some adolescents choose to socialise with peers with type 1 diabetes. Initially after diagnosis there was a reticence to meet others with the condition, but eventually, meeting peers with type 1 diabetes was usually seen as a positive.

CNS1: Most people at diagnosis don't want to meet anybody else, they're very much 'I'm the only person in the world with this and it's awful' and then they kind of unbend from that and often are ready to meet someone a couple of months down the track.

CNS3: We did a pump class and we couldn't get them out of the room when they were finished because they were so intensely talking with each other because some of them had never met another with type 1 diabetes.

At times, health professionals asked for a patient to be a 'buddy' to another who was struggling with self-management, recognising that the adolescents could provide a different type of support than a clinician. Much of this socialising between people with type 1 diabetes was handled by youth support groups and camps. These services 
normalised self-care behaviours and allowed adolescents to compare their treatment regimen and technology to other adolescents'. These interactions often resulted in lasting friendships, and some adolescents asked to change to medical technologies that their peers were using. Older adolescents who had no contact with diabetes peers had little interest to meet new people. While one of the diabetes services created a Facebook forum, adolescents preferred to chat and arrange events organically and privately.

\subsubsection{Attempting deception and interfering with data}

Adolescents were requested to monitor their blood glucose levels regularly and supply physical or digital logs of these tests to their health professionals, to be discussed during appointments, as this can improve glycaemic control (Deacon \& Edirippulige, 2015). Some adolescents used paper logbooks, others used mobile apps, and some downloaded data directly from their medical devices at home or in a health professional's office. Clinicians felt that data could be stored on mobile phones because adolescents always had them nearby, and because the phone functioned as a reminder for blood glucose testing.

However, health professionals struggled to attain complete data. Some adolescents used multiple blood glucose meters and rarely brought them all to appointments, some were not provided with a cable for downloading data at home, and some logs stored only blood glucose data, without insulin dose or contextual information. Nevertheless, glucose meters can act as a catalyst for sharing personal stories of balancing medical requirements and daily life (Prendiville, 2017).

Adolescents who do not regularly test their blood glucose levels, or whose levels fall outside the recommended range, can feel reluctant to share data that substantiates this with health professionals (R. M. Anderson \& Funnell, 2000; Gabbay \& Durdock, 2010). When a request for this information is made, the adolescent must decide whether to be truthful or not. In cases of the latter, two adolescent strategies for alleviating tensions were described by health professionals: attempting deception and interfering with data. 
NP1: I saw this young man and his HbA1c is very high, in fact, it's gotten worse, it's over 100. He's sitting there rattling off these numbers of his tests and stuff and they [the other health professionals in attendance] ask that he come back to see me in two weeks and to bring his meter. So he brought his meter and it didn't even have the right date or time in it but it had two tests in it. So I realised he's just making it up, and I was like 'you know there's no point doing that. If you're not testing just say you're not testing'.

CNS1: I rumbled someone in clinic last week, I didn't actually mean to but it just happened, and he was relying on us not being able to download his meter..., We've had huge problems with the software but suddenly it's working. So he handed over his meter, which I downloaded, and then the numbers were all in red, which meant they were really high. And he just said to me, 'that's not my meter', and I said, 'but it is, because you gave it to me, and there it is, it's plugged in', and he completely denied it.

The above adolescent's reticence to share data when not self-managing adequately also extended to hiding his lack of self-care behaviours and hyperglycaemic levels from his parents. Although honest communication results in the most accurate treatment response and customisation of the regimen, the adolescent's own diabetes identity and their relationship with the health professional are challenged in these moments. In these situations, health professionals endeavour not to reprimand, but to encourage honesty.

In similar situations to those outlined above, some adolescents choose to bolster their attempt at deception with fabricated data, or by interfering with the transmission of data to health professionals (e.g., adding false data to a logbook or tampering with a CGM). Where it was historically easier to supplement a fabrication with a falsified physical logbook, adolescents' deceptions are now being exposed by the presence of digital data, which can be harder to alter. This deception relates to Goffman's (1978) defensive practices in the presentation of self, where the adolescent lies in an attempt to present an ideal version of themselves to their health 
professional, yet the failed deception can lead to a cycle of concealment and discovery.

Medical technologies are currently a factor in revealing adolescents' attempted deceptions. Adolescents with type 1 diabetes, their parents, and health professionals need to establish acceptable approaches to sharing data, and make sure these expectations are in place prior to medical appointments. Further, while health professionals have stated their preference for honest communication from adolescents regardless of their level of self-management, some adolescents still prioritise presenting a favourable version of self rather than open sharing. While sharing data may infringe on adolescents' independence and privacy, it may also reduce deception and be of aid in emergency situations. Medical technologies could use flexible settings so that adolescents and their parents can customise the technology to support the transition of responsibility.

These concepts and their categories and properties are listed below (Table 3).

Table 3

Concepts, categories, and properties developed from interviews with health professionals.

\begin{tabular}{|c|c|c|}
\hline Concept & Category & Properties \\
\hline $\begin{array}{l}\text { Self-management } \\
\text { overview }\end{array}$ & & $\begin{array}{l}\text { Ideal and actual management } \\
\text { Access and affordability }\end{array}$ \\
\hline \multirow[t]{2}{*}{$\begin{array}{l}\text { Conceptualising type } \\
1 \text { diabetes and its } \\
\text { management }\end{array}$} & $\begin{array}{l}\text { Developing and applying } \\
\text { diabetes knowledge }\end{array}$ & $\begin{array}{l}\text { Developing knowledge and } \\
\text { confidence } \\
\text { Visualising information } \\
\text { Manipulating knowledge }\end{array}$ \\
\hline & $\begin{array}{l}\text { Conceptualising diabetes } \\
\text { and its management }\end{array}$ & $\begin{array}{l}\text { Fitting diabetes around rest-of-life } \\
\text { Diabetes and technologies } \\
\text { impacting daily life } \\
\text { Customising the treatment } \\
\text { regimen } \\
\text { Impacts of normative adolescent } \\
\text { development } \\
\text { Prioritising goals and wishes }\end{array}$ \\
\hline
\end{tabular}




\begin{tabular}{|c|c|c|}
\hline & & $\begin{array}{l}\text { Prompting to improve self- } \\
\text { management }\end{array}$ \\
\hline & Developing acceptance & $\begin{array}{l}\text { Relationship with diagnsosis } \\
\text { Facilitating acceptance } \\
\text { Identity conflict }\end{array}$ \\
\hline & $\begin{array}{l}\text { Engaging in diabetes } \\
\text { management }\end{array}$ & $\begin{array}{l}\text { Gradual engaging and defining } \\
\text { moments } \\
\text { Acting practively } \\
\text { Running high }\end{array}$ \\
\hline \multirow[t]{2}{*}{ Struggling for self } & $\begin{array}{l}\text { Recognising individual } \\
\text { needs }\end{array}$ & $\begin{array}{l}\text { Personality, intelligence, and } \\
\text { gender } \\
\text { Responding to dissonant } \\
\text { technology } \\
\text { Match with expectations } \\
\text { Values } \\
\text { Having choices } \\
\text { Shaping the service }\end{array}$ \\
\hline & Transtioning responsibility & $\begin{array}{l}\text { Successful strategies } \\
\text { Negotiating roles } \\
\text { Providing strategies } \\
\text { Changing technology } \\
\text { Decreasing independence }\end{array}$ \\
\hline \multirow[t]{4}{*}{ Managing identity } & Disclosing diabetes & $\begin{array}{l}\text { Psychological benefits } \\
\text { Peer knowledge }\end{array}$ \\
\hline & $\begin{array}{l}\text { Managing attention, } \\
\text { embarrassment, and } \\
\text { stigma }\end{array}$ & $\begin{array}{l}\text { Experiencing stigma } \\
\text { Perceptions of negative } \\
\text { judgements influencing behaviour } \\
\text { Making medical technologies } \\
\text { socially acceptable }\end{array}$ \\
\hline & $\begin{array}{l}\text { Interacting with diabetes } \\
\text { peers }\end{array}$ & \\
\hline & $\begin{array}{l}\text { Attempting deception and } \\
\text { interfering with data }\end{array}$ & $\begin{array}{l}\text { Attempting deception } \\
\text { Fabricating data }\end{array}$ \\
\hline
\end{tabular}




\subsection{Discussion}

\subsubsection{Psychosocial user requirements of medical technologies}

From the grounded theory analysis above, 18 psychosocial user requirements of medical technology were constructed. These are developed throughout the thesis, and reported in full in Chapter 11. The sub-categories in which each requirement was present are listed in italics.

1. Allow accessibility and affordability: Fitting diabetes around rest-of-life Affordable and accessible medical technologies that ensure access to adolescents with minimal resources. An example of this was the subsidising of blood glucose meters, whereas CGMs were not accessible for many.

2. Foster trust in accuracy: Running high, Self-management overview Accurate medical technologies; fostering trust in reliability so adolescents can make informed decisions without added stress. Examples of not meeting this requirement include blood glucose meters that give inaccurate results in everyday settings.

3. Minimise psychological distress: Developing acceptance, Transitioning responsibility

Medical technologies not causing psychological distress, and where possible, facilitating adolescents' development of acceptance. Examples of medical technologies contributing to distress were identity conflict between being a young, fit person and managing a chronic condition, and in tensions about data sharing with parents, where digital records could reveal undesirable habits and result in family arguments. Alternately, sharing logbooks with parents could ease 'nagging' and distress.

4. Communicate to inform realistic expectations: Responding to dissonant technology

Medical technologies and supporting services clearly communicating their advantages and disadvantages, so adolescents have clear expectations when initiating use of a new device. An example where expectations often differ from reality is in initiating insulin pump therapy, where adolescents expected the pump to do the work for them. 
5. Align with values: Responding to dissonant technology

Medical technology aligning with adolescents' values. An example is being able to choose reusable insulin pens over disposable insulin pens to align with ideals about environmental sustainability.

6. Employ language and communication methods relevant to adolescents: Shaping the service

Health professionals are making effort to adapt their communication style to suit adolescents. An example is sending text messages rather than making phone calls.

7. Provide choices, customisation or personalisation: Responding to dissonant technology

Having choices of medical technologies or being able to personalise or customise devices. This affords adolescents more control in the decision-making process, and clinicians predicted this may improve self-management. An example is being able to choose insulin pump colour and accessories.

8. Perform in a life-proof manner: Fitting diabetes around rest-of-life Medical technology being life-proof, allowing confident and convenient selfmanagement. Examples include water-resistant devices. Glucose meters failing to function over a broad range of temperatures is another example of this requirement not being met.

9. Enhance convenience: Fitting diabetes around rest-of-life

Medical technology minimising tedious aspects of management, such as manually inputting data from glucose meter to app and allowing opportunities for users to engage with data and information. An example is glucose meters that sync automatically to smartphones and graph data.

10. Provide meaningful information: Developing and applying diabetes knowledge, Responding to dissonant technology

Medical technology providing meaningful information that is accurate, appropriate, and actionable to facilitate proactive self-management. Examples include data visualisations, pattern recognition, prompts (e.g., levels high in the afternoon three days in a row), and clear state awareness (e.g., insulin dose of $x$ amount delivered $y$ hours ago)

11. Couple behaviours with consequences: Running high 
Medical technology strengthening the link between behaviours and consequences. An example is continuous blood glucose monitors, which show a trace of blood glucose levels, allowing users to see the impact of food, exercise, and insulin.

12. Facilitate daily structure while allowing for spontaneity: Engaging in diabetes management, Recognising individual needs

Medical technology introducing daily structure to make self-management easier, and allowing for spontaneity. Eating similar breakfast and lunch foods daily to minimise the carbohydrate calculations, while allowing for a range of dinner foods was described positively in this study. Being engaged in self-management involved being proactive. For instance, where an adolescent realises their blood glucose levels have been consistently high in the afternoon, they adjust their insulin in the morning to prevent hyperglycaemia.

13. Facilitate realistic goals and improved management: Developing and applying diabetes knowledge, Customising the treatment regimen Medical technologies should facilitate improved self-management through appropriate goals. Health professionals recognised that adolescents' goals may differ from the ideal treatment regimen, that they may have conflicting non-diabetes related goals, and that they may not fully consider the long-term implications of their actions. Patient-selected goals that were small enough to assess between appointments were successful for behaviour change.

14. Make disclosure optional: Disclosing diabetes

Medical technology allowing for disclosure of diabetes, while not making this inevitable when used in public spaces. This happens sometimes with insulin pumps, which are mistaken for pagers and mobile phones.

15. Not being embarrassing or stigmatising: Managing attention, embarrassment, and stigma

Medical technology not drawing negative attention. Instead, devices could be discreet or draw positive attention through being 'cool'. The shift from traditional syringes to insulin pens has minimised stigma.

16. Normalising devices and managing health: Making medical technologies socially acceptable 
Visible medical technology can help to normalise managing health and connect with broader trends for health informatics and wearables. An example of normalising health tracking is the Fitbit. Games could also be designed to provide an engaging and non-distressing way of facilitating self-management.

\section{Support transition of responsibility: Transitioning responsibility, Attempting} deception and interfering with data

Devices supporting the transition of responsibility by fostering clear expectations about information and data sharing between adolescents, clinicians, and parents, with adjustable settings. An example where this was not currently happening was adolescents being unprepared for health professionals to access their blood glucose logs during appointments. While access may be helpful for adjusting the plan for self-management, expectations about data sharing should be clear in advance. 18. Facilitate interaction with diabetes peers: Interacting with diabetes peers Medical technology facilitating interaction between adolescents and their peers. Facebook is currently used to maintain friendships and share diabetes-related thoughts and information.

\subsubsection{Discussion of findings and methods}

\subsubsection{Findings}

The grounded theory largely complemented existing literature. Diabetes management was framed in association with normative adolescent development, in particular, social pressures and the 'imaginary audience', bodily changes, risky behaviours, feeling invincible, establishing control and independence, and lack of long-term thinking (Elkind, 1998; Huebner, 2009; Michaud et al., 2007; O’Donohue et al., 2013; Warner \& Hauser, 2009). Many of these factors resulted in adolescents running high, and prioritising short-term and social factors (Beshyah, 2009).

Difficulties around social acceptance and disclosure featured prominently, with health professionals recognising the medical and psychological benefits of disclosure (Badlan, 2006; Thompson Jr. \& Gustafson, 1996), but the social difficulties of doing so (Berge et al., 2007; Gjengedal et al., 2003; Y. -L. Wang et al., 2010).

Goffman's (1963) 'moral career' of recognising and dealing with stigma mirrored the gradual process of coping and acceptance of having type 1 diabetes, 
with medical technologies often being the component that induces stigma, thereby negatively affecting self-management (Polgar, 2010). However, health tracking trends could normalise health monitoring (Fotopoulou \& O’Riordan, 2017).

Although no clinicians were aware of any diabetes technologies designed specifically for adolescents, they recognised that adolescents had medical technology preferences, particularly for accurate, discreet, or cool devices. Previous research suggests that clinicians should consider the relationship between device preference and self-management (B. J. Anderson \& Redondo, 2011; Goldstein, 2008). Additionally, expectations of medical devices, particularly insulin pumps, could be unrealistic and affect self-management (Cogen et al., 2002; Low et al., 2005; Ritholz et al., 2007). As such, it is important that users of medical technologies have realistic expectations of the devices they intend to use. When implemented well, medical technologies could provide meaningful information to improve decision-making.

There was broad consensus that a positive feature of medical technology was its ability to visualise and communicate data to increase health professionals' and adolescents' understanding of their blood glucose levels and the impacts of their diet, exercise, and insulin doses, though this did not commonly occur to its potential (Deacon \& Edirippulige, 2015; Frøisland et al., 2012; Prendiville, 2017; Sklar \& Naar, 2017). There was also discussion about adolescents needing to take a proactive, engaged approach to management to improve glycaemic control (Bellows et al., 2015; Bright et al., 2015; Graue et al., 2004; Levich, 2007). The process of becoming engaged was predominantly described in two ways: a gradual process that aligned with the individual maturing and a defining moment where some factor external to the person resulted in an epiphany. Adolescents also strove, on the advice of health professionals, to fit diabetes around their daily lives so it did not prevent them from participating in daily activities. However having some structure, balanced with spontaneity, was beneficial (Quirk et al., 2014). Previous research has demonstrated that the greater adolescents perceive the impact of diabetes on day-to-day life, the poorer their wellbeing (Skinner et al., 2000).

Characteristics of a successful transition of responsibility from parent to adolescent included beginning at a young age, but making the process gradual (Greca, 1990; Ingersoll et al., 1986). However, there were tensions around transition 
and data sharing, particularly when self-management was poor, with tendencies to act defensively and provide misinformation (R. M. Anderson \& Funnell, 2000; Gabbay \& Durdock, 2010). If used successfully, data sharing through blood glucose meters can prompt the sharing of personal stories and challenges (Prendiville, 2017), and improve glycaemic control (Deacon \& Edirippulige, 2015). The deception described relates to Goffman's (1978) defensive practices in the presentation of self, where the adolescent presents an ideal version of themselves.

Finally, while advocating for an intensive self-management approach (Delamater, 2006; Hoey, 2009), health professionals were also customising the regimen (Perfetti, 2013; Sawyer \& Aroni, 2005) and balancing adherence and engagement approaches (Mudge et al., 2015). Exploring the relationships between medical device use and personality may be a promising avenue for further research (Orji et al., 2017; Thomas et al., 2017).

While the research uncovered new information on user experiences and requirements, it largely supported existing literature. However, there were two areas that were surprising. The first was that many adolescents chose to change from insulin pumps back to multiple daily injections. This goes against growing trends of insulin pump users, with approximately $20 \%$ of paediatric patients using insulin pumps in one New Zealand city (“Starship Insulin Pump Program,” n.d.). A clinician hypothesised that this may be an outlet for adolescents' sense of control and decision-making, possibly because the initial decision to move to the pump was their parents', because they did not like being attached to a device, or because they could not use the device as effectively as their parents had done.

The second observation that arose was that some adolescents were requesting refillable insulin pens, as the disposable insulin pens clashed with their values around environmental sustainability. Here, there was a dissonance between the technology and the adolescent's values.

\subsubsection{Methods}

While clinicians described their roles, they also provided their interpretations of adolescents' feelings and behaviours. While their analyses and accounts may differ from those of adolescents, conducting interviews with health professionals gives 
access to information about a wide range of adolescents, some of whom may not be inclined to take part in research. Clinicians described a broad continuum of adolescent self-management. The use of proxies is common in medical research with adolescents (B. Carter, 2009; Lang, Martin, Sharples, \& Crowe, 2010; J. L. Martin et al., 2006), which has demonstrated that proxies are accurate with quality of life ratings, and that when there are mismatches health professionals err towards reporting more problems (Essen, 2004). However, to explore a different viewpoint, the following chapters explore adolescents' first-hand accounts.

Further interviews could have been conducted with other health professionals not represented in this sample, such as dieticians and podiatrists. However, the sample was limited to the commonly available diabetes teams adolescents had access to, and clinical psychologists, as much of the research grappled with psychological issues such as adolescent development and conceptualisation of managing health.

Using semi-structured interviews resulted in a broad range of types of information and analysis, from high-level concepts to specific examples about the benefits and disadvantages of individual medical devices. Interestingly, clinicians suggested that features they thought adolescents would benefit from would also suit other users, demonstrating the value of an 'extreme user' approach (Holmquist, 2006). Employing thematic sampling, I adapted the content of the interview to explore emergent, grounded theory categories. The concepts explored in this chapter informed the areas of focus of the following chapters.

\subsection{Summary}

The interviews with health professionals largely supported the literature and uncovered novel areas, such as adolescents' willingness to change medical devices based on concerns about environmental sustainability. Three theoretical concepts were identified: conceptualising type 1 diabetes and its management, struggling for self, and managing identity. While these concepts were built through grounded theory, they also relate back to existing theory. The analysis describes processes adolescents undergo while self-managing, and identified health professionals' impressions of adolescent user requirements, resulting in 18 psychosocial user 
requirements of medical technologies. Additionally, as per the aims of this chapter, these interviews provided an overview of the nuances of the treatment regimen for type 1 diabetes, medical devices commonly available in New Zealand, and patterns of self-management and the range between individuals. 


\section{Designing Medical Technologies for Adolescents}

In this chapter, undergraduate students developed psychosocial user requirements and responding medical technology designs for adolescents with type 1 diabetes. The study built on secondary research and a preliminary analysis of interviews with health professionals in Chapter 4. A Diabetes Nurse Specialist reviewed the designs and user requirements, providing additional support for and aiding development of the user requirements. The aim of this study was to further develop adolescents' psychosocial user requirements, investigate methods for validating these, and explore methods of designing for adolescents with type 1 diabetes, informing the methods to be used in Chapters 8 and 10 .

\subsection{Methods}

\subsubsection{Participants}

Participants were 24 undergraduate students enrolled in Tangible Interactions Design at Victoria University of Wellington. Students were predominantly industrial designers, however, some had different areas of design or psychology expertise. I facilitated the design process along with an Industrial Design Associate Professor and a Teaching Fellow. One clinician (a Clinical Nurse Specialist in diabetes) gave formative feedback and reviewed the designs. Sample information and consent forms are in Appendix C.

\subsubsection{Procedure}

The research through design study was conducted over eight weeks (Schneider, 2007). To initiate the project, I delivered a lecture communicating insights from interviews with health professionals (Chapter 4) and secondary research, outlining type 1 diabetes treatment regimens and common adolescent self-management challenges. Students were provided with literature on managing diabetes, along with a collection of videos and websites created by people with type 1 diabetes and organisations that represent them ("Diabetes New Zealand," n.d.; "Diabetes Youth 
New Zealand,” n.d.; “Six Until Me,” n.d.; McCarthy, 2014; “Type 1 Diabetes Network," n.d.). These resources explained diabetes and common treatment regimens, demonstrated medical technologies in use, and described experiences, habits, and emotions from the point of view of adolescents, children, parents, sports coaches, teachers, and friends (e.g., a typical day in the life of an adolescent with type 1 diabetes).

Students were instructed to address an aspect of diabetes self-management (e.g., blood glucose monitoring or insulin delivery) and use the resources provided, along with independent secondary research to identify a psychosocial user requirement of medical technologies. While the students primarily found inspiration from non-academic sources, I checked these requirements against those identified in Chapter 4 and existing literature. The resulting requirements are described alongside each design concept. Many students utilised emerging technologies for their concepts, such as near-infra red blood glucose monitoring. While these technologies are not commercially available, they allowed students to ideate based on possible futures.

Students created a persona of an adolescent with type 1 diabetes (Goodwin, 2009), scenarios depicting issues the adolescent may encounter when self-managing in various contexts (J. M. Carroll, 1995; Schwartz, 1992), and used methods for ideation, including sketching, prototyping, and acting out scenarios (Houde \& Hill, 1997; Stickdorn \& Schneider, 2011; Warfel, 2009) to create dozens of design concepts. The clinician reviewed the design concepts in an informal group discussion with the students, giving them feedback and making them aware of relevant devices currently or previously available. Next, the design concepts with the best potential to address the user requirements were selected through confidential student voting and staff review, and the concepts were developed in small groups.

At the culmination of the study, the Diabetes Nurse Specialist reviewed images, videos, and prototypes of the students' designs in relation to the user requirements they addressed in an individual expert review (Wilson, 2014). The nurse was selected for her 'product genre expertise' (experience with products related to those being developed), 'domain expertise' (knowledge of specific concepts and practices), and frequent contact with young people with type 1 diabetes. Aspects 
discussed in the review included first impression, desirability, usefulness, appropriateness, and security. Feedback from the review is described in relation to each design outcome. While the review is illustrative of a clinician's view, it is not comprehensive. If a review had been conducted with adolescents, it is possible that other responses may have been elicited.

Students also evaluated the design process in semi-structured group interviews, which were analysed using thematic analysis (Braun \& Clarke, 2006), to identify insights to shape the methods used in further design tasks in Chapters 8 and 10 .

\subsection{Design Outcomes and Reviews}

Five of the designs, the psychosocial user requirements they respond to, and their review by a clinician are described. All user requirements developed with students, except for 'facilitating spontaneity and participation in everyday activities' and 'facilitating comfortable and non-invasive use', were evident in Chapter 4. 
Table 4

User requirements addressed by student design concepts.

\begin{tabular}{|c|c|c|c|}
\hline Design & User requirements addressed & Chapter 4 & Literature \\
\hline \multirow[t]{2}{*}{ Subscope } & $\begin{array}{l}\text { Facilitate spontaneity and participation in } \\
\text { everyday activities }\end{array}$ & $x$ & $\checkmark$ \\
\hline & $\begin{array}{l}\text { Provide meaningful information to } \\
\text { facilitate decision-making }\end{array}$ & $\checkmark$ & $\checkmark$ \\
\hline \multirow[t]{3}{*}{ Joya } & $\begin{array}{l}\text { Minimise or eliminate feelings of stigma } \\
\text { or embarrassment while using medical } \\
\text { technology }\end{array}$ & $\checkmark$ & $\checkmark$ \\
\hline & $\begin{array}{l}\text { Comfortably disclose and explain type } 1 \\
\text { diabetes when appropriate }\end{array}$ & $\checkmark$ & $\checkmark$ \\
\hline & Allow comfortable and non-invasive use & $x$ & $\checkmark$ \\
\hline \multirow[t]{2}{*}{ Armilla } & Perform in a life-proof manner & $\checkmark$ & $\checkmark$ \\
\hline & $\begin{array}{l}\text { Minimise or eliminate feelings of stigma } \\
\text { or embarrassment while using medical } \\
\text { technology }\end{array}$ & $\checkmark$ & $\checkmark$ \\
\hline Emoti-Comms & Facilitate interaction with diabetes peers & $\checkmark$ & $\checkmark$ \\
\hline \multirow[t]{2}{*}{ Simpac } & $\begin{array}{l}\text { Facilitate spontaneity and participation in } \\
\text { everyday activities }\end{array}$ & $x$ & $\checkmark$ \\
\hline & Enhance convenience & $\checkmark$ & $\checkmark$ \\
\hline
\end{tabular}

\subsubsection{Subscope design and development}

People with type 1 diabetes sometimes stop exercising to check their blood glucose, as hypoglycaemia frequently occurs during and after vigorous exercise, and fear of hypoglycaemia is a barrier to exercise (Iscoe et al., 2006; Roberts \& Taplin, 2015). 
This can disrupt participation and performance and draws unwanted attention. Trends in blood glucose provided by CGMs, paired with effective procedures for taking on carbohydrates, can prevent hypoglycaemia from occurring during exercise (Riddell \& Milliken, 2011). Medical technologies should communicate data and information to facilitate decision-making and participation in everyday activities, including exercise.

Subscope (Figure 18) responds to these requirements, pairing a small subdermal implant with a watch-like CGM. The O-LED wristband changes through a spectrum of colours as the wearer's glucose levels rise and fall, using blue to denote a safe level and red a dangerously high or low level. The band uses animations to alert wearers that they need to take immediate action to address their blood glucose level. Designed specifically for swimmers, the band is waterproof, durable and draws from the aesthetics of wearable sports devices. It pairs with a mobile app to track and visualise data. While they were initially designing the product for swimmers, the designers also suggested the possibility of everyday wear.
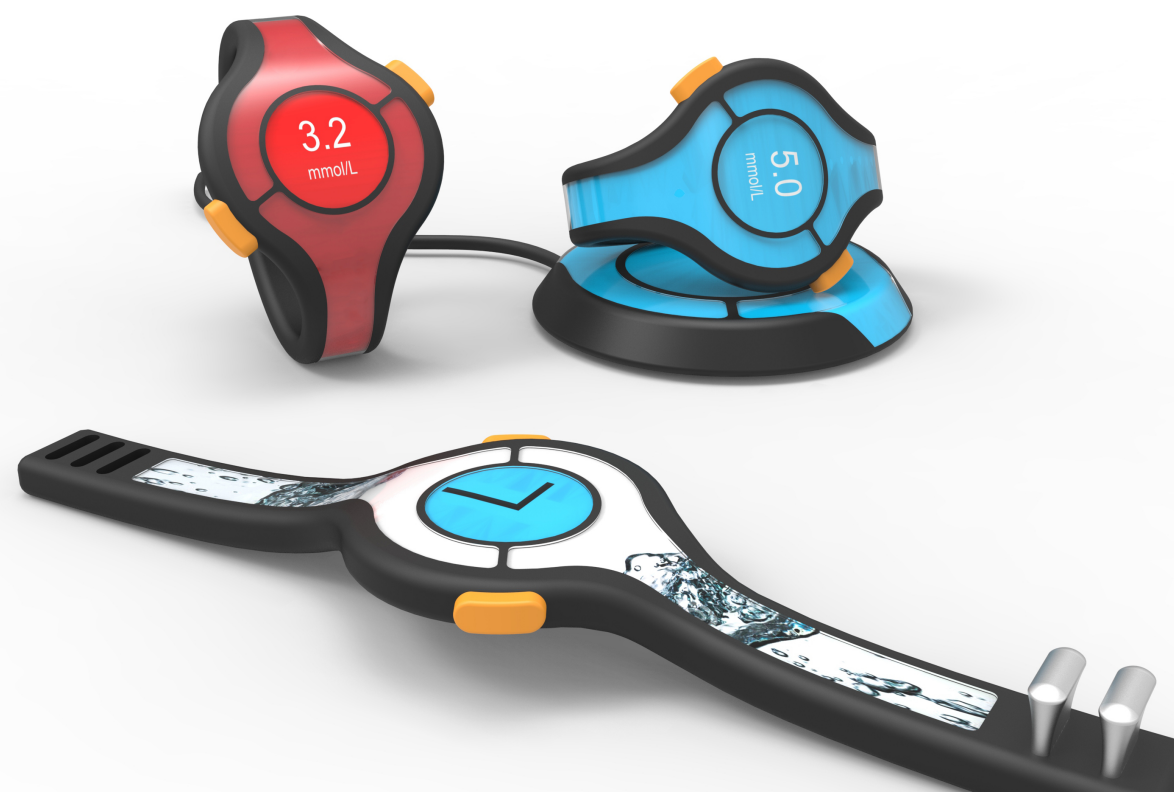

Figure 18. Subscope continuous glucose monitor. 


\subsubsection{Subscope expert review}

The clinician verified that the non-invasive, waterproof design would be quick and discreet to use, minimising current frustrations and increasing engagement. The concept could be improved by using different colours to distinguish low and high blood glucose levels. Further, having not just an indication of whether the user's levels were falling or rising, but the speed at which they were changing, would be important. This could help people predict when they would need to take action, helping them to be proactive rather than reactive. The design was selected as a New Zealand James Dyson award finalist, as it sought to address an identified problem.

\subsubsection{Joya design and development}

Adolescents who make negative attributions about peers' or friends' reactions to their self-management are likely to struggle with glycaemic control and selfmanagement in social situations, and have increased stress (Hains et al., 2007). Disclosure to friends is important for self-management, allowing friends to support the individual with diabetes (Wysocki \& Greco, 2006). Additionally, female adolescents can be embarrassed by onlookers asking them questions, or watching them test or administer insulin, making non-invasive methods preferable (Dickinson \& O’Reilly, 2004; Tura et al., 2007). Medical technologies should minimise or eliminate feelings of stigma or embarrassment, be comfortable and non-invasive where possible, and allow adolescents to comfortably disclose and explain type 1 diabetes when appropriate.

Joya (Figure 19), is a locket and earring combination for non-invasive blood glucose monitoring. Activated by opening the locket, the earring sends a nearinfrared light through the earlobe, measuring blood glucose concentrations and displaying the result on a small screen within the locket. 


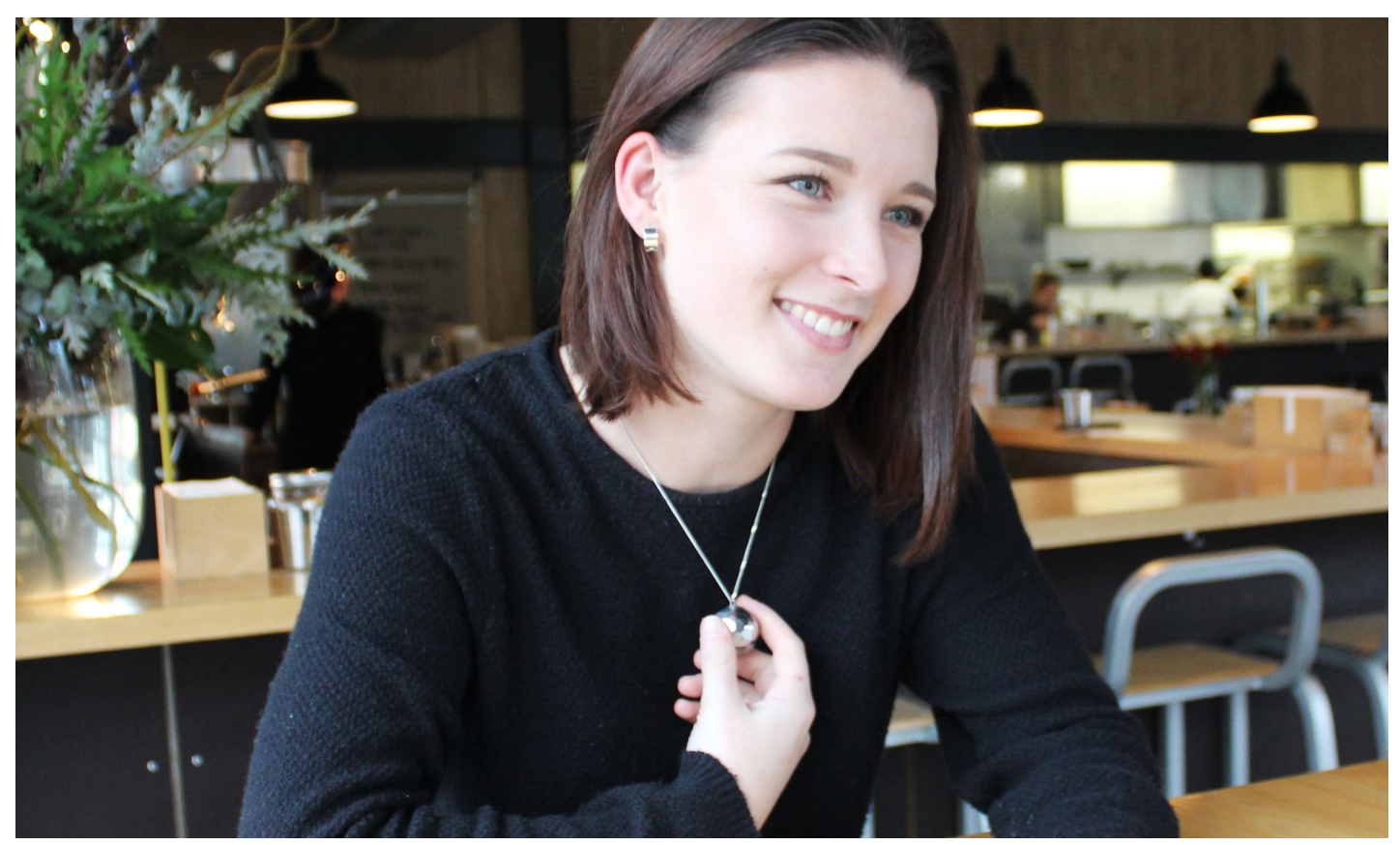

Figure 19. Joya blood glucose monitoring jewellery.

The system makes blood glucose testing discreet, fast and non-invasive, aiming to ease the social pressures of testing in public. However, while many designs for discreet medical technology hide the device, this design creates a desirable object. The intention was to create a device that allowed the wearer to choose whether to disclose diabetes, sharing the jewellery's hidden function with onlookers, without sending a message that the device ought to be hidden or ashamed of. While medical technology can be stigmatising, it can also be a tool for communication and education when the wearer chooses.

\subsubsection{Joya expert review}

The clinician verified that many people do not like to test their blood glucose levels in public. Further, because this design concept is not overtly medical, it might be successful at removing barriers to blood glucose testing, improving glycaemic control. Introducing a choice for disclosure was reviewed positively. The clinician suggested a range of designs for various users, including options for men could also be developed. Additionally, this device could suit not only adolescents, but many people with type 1 or type 2 diabetes. 


\subsubsection{Armilla design and development}

Many adolescents are reluctant to try and will discontinue using insulin pumps due to concerns about the pump being unwieldy, overly visible, and bothersome during exercise (Seereiner et al., 2010). Further, many adolescents return insulin pumps for repair or replacement due to malfunctions, and are concerned about damaging the pump in day-to-day life, particularly through physical activity, sand, or water (Low et al., 2005). Medical technologies should function in a life-proof manner while not being embarrassing or stigmatising to the user. Armilla (Figure 20) drew from the appearance of jewellery and wearable, digital sports devices. The designers adapted the form of an existing insulin pump and created a durable, waterproof armband to be worn over it, envisaging overcoming challenges of using an insulin pump at the beach.

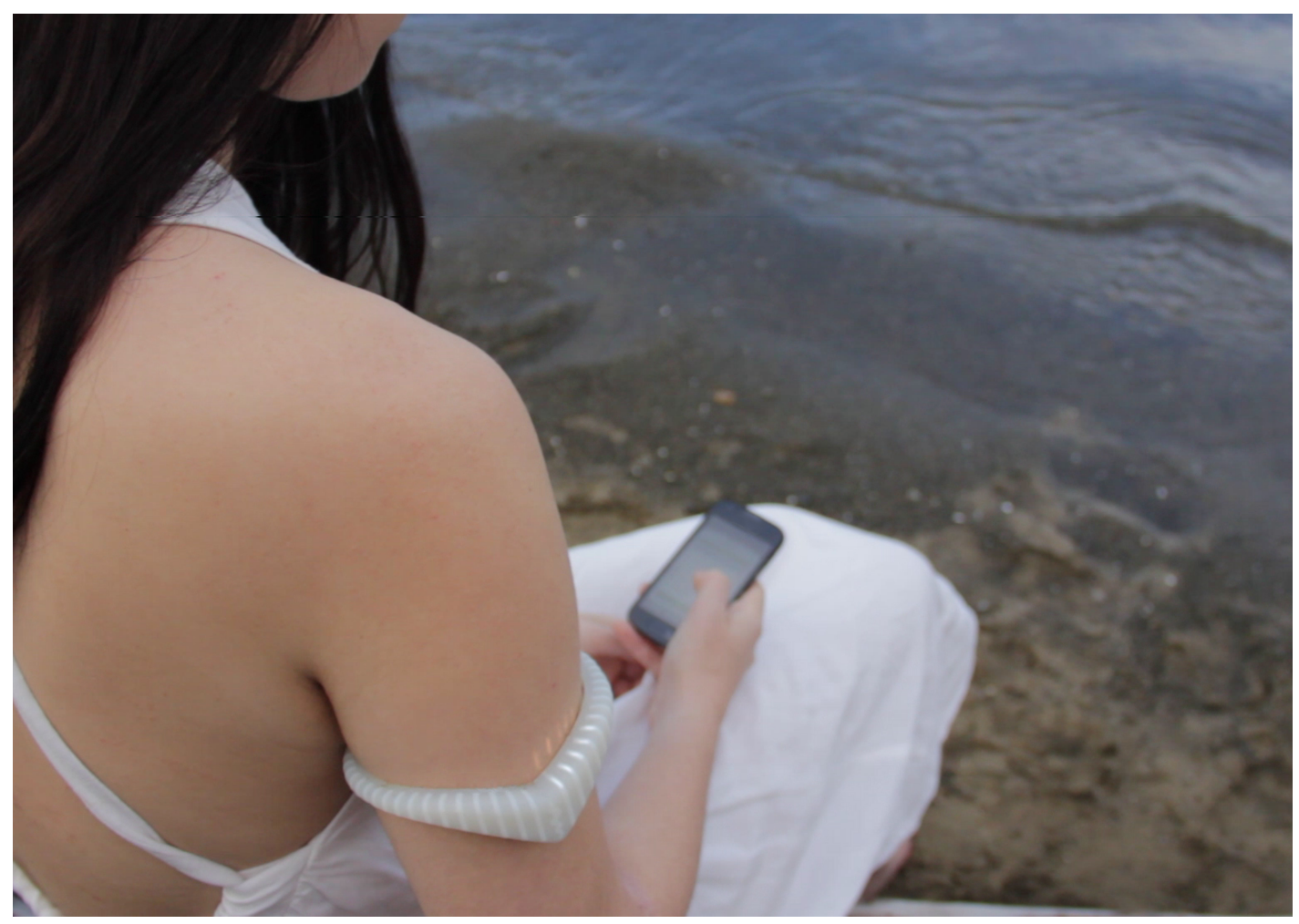

Figure 20. Armilla insulin pump.

While an original criterion for this concept was to look desirable, the designers realised there are instances in which the device needs to be identified as medical (e.g., going through security checkpoints, being noticed by paramedics). The team added a 
subtle medical symbol to the device to balance the psychosocial barriers with these practical implications.

\subsubsection{Armilla expert review}

While this concept was designed in response to the embarrassment of wearing an insulin pump in public and attempting to hide it under clothing, and a fear that the expensive equipment might get broken, the clinician felt that these issues were not common. Although some people struggled to find a place to wear a pump with certain clothes, people tended to quickly become comfortable wearing insulin pumps. While the team had considered how an adolescent may need to pass through security checks with the device, they also needed to consider the digital security of the device in response to tampering.

\subsubsection{Emoti-Comms design and development}

Some adolescents struggle to accept having and managing type 1 diabetes and desire peer support (Lu et al., 2015). Higher levels of acceptance are correlated with better metabolic control, while an avoidance coping style is associated with less frequent blood glucose monitoring and feelings that glucose monitoring is a burden (Richardson, Adner, \& Nordstrom, 2001; Weijman et al., 2005). As such, medical technologies could facilitate peer support. The designers focussed on creating a tangible takeaway from diabetes camps to help young adolescents maintain new friendships. The kit produced (Figure 21) allows each young person to make a customised touchscreen friendship bracelet with a partner. The users swipe different parts of their own bracelet, and the corresponding part of their buddy's bracelet illuminates, sending gestures such as happy or sad faces and custom signs. 


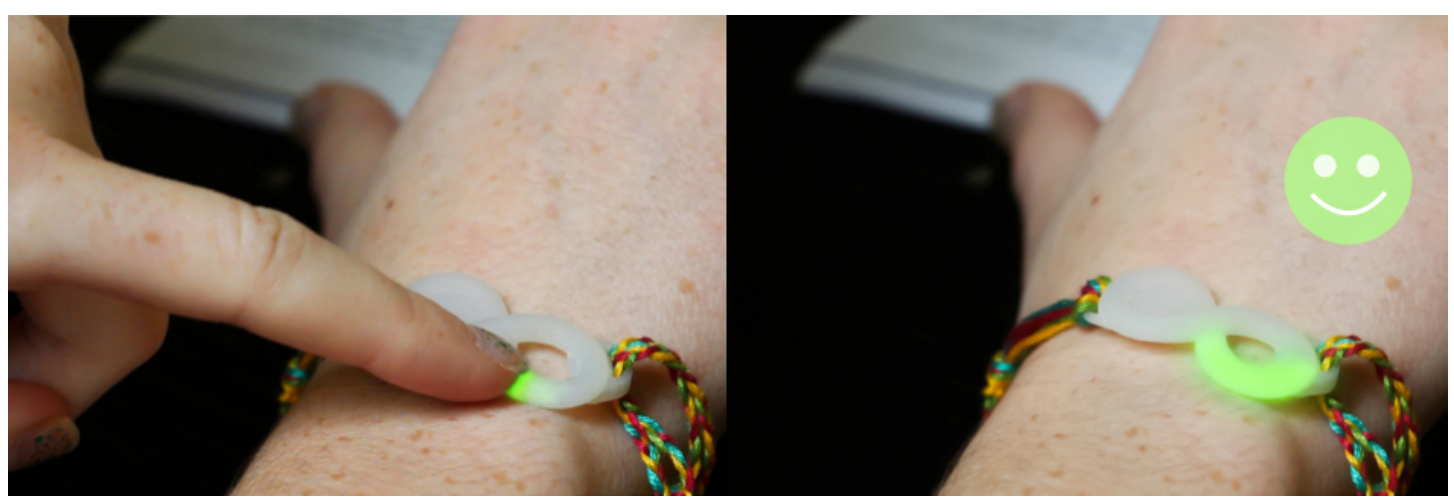

Figure 21. Emoti-Comms buddy bracelet.

The students initially thought that interactions should focus solely on selfmanagement tasks such as blood glucose testing, however, they realised that the support provided using the bracelet could be emotional rather than exclusively practical, and that this would facilitate engagement in self-management. This is reflected by research showing friends largely provide emotional support to adolescents with type 1 diabetes, more so than the practical support families provide (La Greca et al., 1995; Wysocki \& Greco, 2006).

\subsubsection{Emoti-Comms expert review}

The clinician verified adolescents' feelings of isolation and the importance of diabetes camps for letting them meet others with the same condition, for normalising the experiences of and technology used in self-management, and for building peer support. Customising and using the bracelets would be helpful in strengthening these peer support networks, and lending emotional support. However uptake was questioned, as many adolescents currently use other digital media, such as texting and Facebook, to communicate.

\subsubsection{Simpac design and development}

The challenge that prompted the design of Simpac (Figure 22) was the conflict between adolescents' desire to socialise with their peers and the need to self-manage responsibly (Warner \& Hauser, 2009). Some adolescents struggle with spontaneous events, where they must check they have essential supplies with them to be able to participate. There can be a large amount of medical equipment to be carried. Medical technologies should allow for spontaneity and convenient, portable use. Simpac is a 
credit-card-sized backup blood glucose testing kit with two prefilled insulin syringes. It allows adolescents to twist open the device cover, swiftly clean their finger, test their blood glucose level, and inject an insulin dose. The electronic component is reusable and snaps onto the disposable portion of the product.

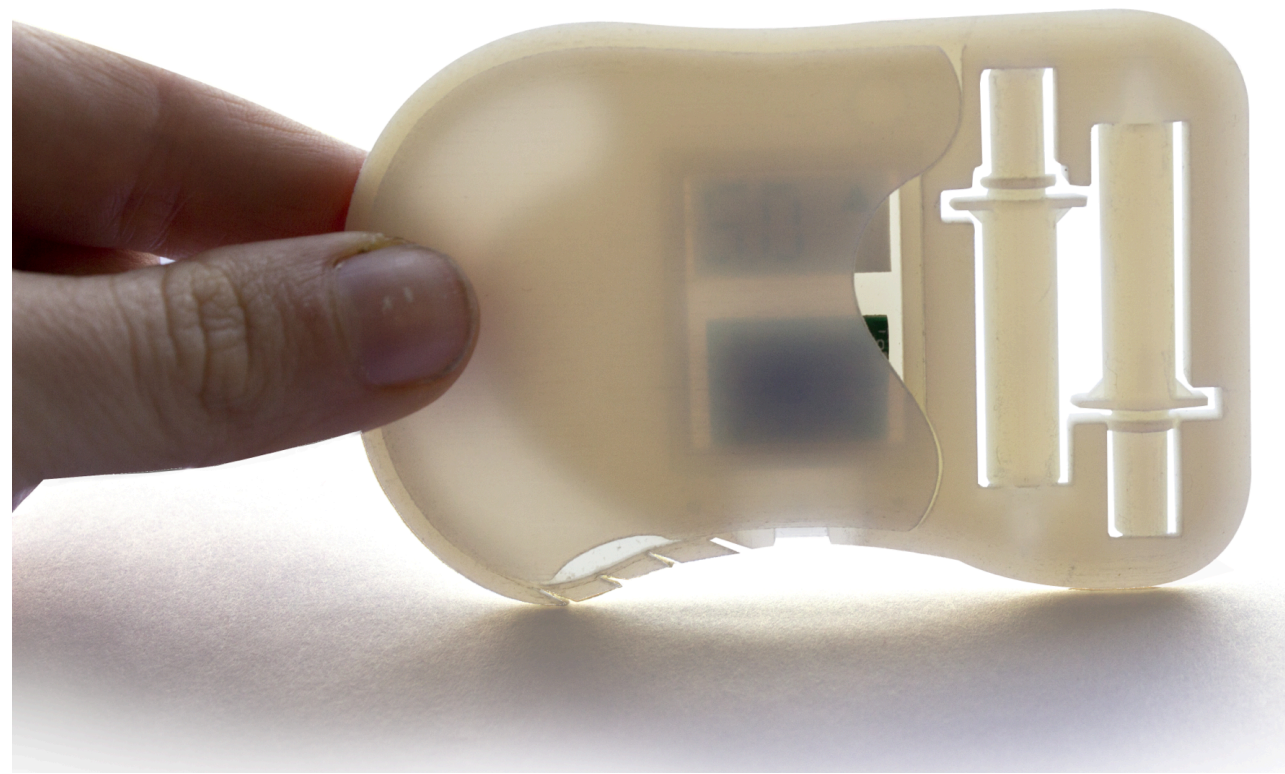

Figure 22. Simpac all-in-one blood glucose testing and insulin delivery.

The designers adapted their design to finger cleaning, as skipping this step may result in inaccurate blood glucose measurements (Bergenstal et al., 2000). They came to this realisation by walking through the processes that the adolescent would have to undergo when using the device.

\subsubsection{Simpac expert review}

Carrying backup equipment is important (e.g., replacement insulin, a syringe or pen, an infusion set, and a replacement cartridge), and reducing the bulkiness of this equipment would be beneficial. However, as the device is still invasive, it would only partially resolve barriers to self-management.

Requiring the user to push their finger onto the lancet, rather than using a controlled depth lancet, may also be a considerable barrier to use. Further, many ecologically and environmentally aware young people object to the amount of diabetes-related waste that is produced, so minimising waste would be important. A 
system for identifying how long the device had been out of the fridge for, and whether it had been exposed to heat, would be beneficial.

\subsection{Thematic Analysis of Designers' Experiences}

The designers were interviewed at the culmination of the study to gain insights into the design methods used, and how they could be improved for other design studies in Chapters 8 and 10. A thematic analysis of interviews resulted in three themes: 'sensory and embodied empathy', 'inspiration versus the necessary', and 'whose point of view?'.

\subsubsection{Sensory and embodied empathy}

An important part of the design process was developing an understanding and empathy for the self-management experiences of adolescents with type 1 diabetes. To do this, the students used digital platforms for secondary research. The students reported that watching videos documenting adolescents' first-hand experiences helped them to achieve greater empathy, understanding, and inspiration to design. One student commented, "I'm not going to empathise as much if I'm reading some writing, but if I can see someone and the expression on their face, then it really gets you.”

These digital platforms were time-efficient and rich with examples of day-today life, along with reflections on past events and opinions about how to realistically manage diabetes. While the content of these digital platforms was not reflective of all adolescents' experiences, it helped the designers to narrow their area focus and design for a specific persona. Some students used a series of videos or blog posts by an individual, developing respect for the way the individual faced self-management challenges.

Many students thought about how they would feel were it them in the situation. Interestingly, most students were a similar age to those they were designing for, so found it helpful to reflect on their past preferences and behaviours. There was a tendency, however, to underestimate the level of capability and aesthetic sophistication of adolescents. 
Some designers had first-hand experiences managing medical conditions that used similar technology. While the nurse was present, one student requested to try using a blood glucose meter and insulin pen. A careful balance is necessary when using 'empathic modelling' to develop a sense of what an adolescent with type 1 diabetes might experience, without assuming to completely understand what this must be like (McDonagh \& Thomas, 2010).

\subsubsection{The inspiration and the necessary}

The designers found some sources of information helpful for inspiring them to design while others furnished their projects with constraints and procedural details. Designers found adolescents' user requirements a helpful starting point, though balanced these with the devices' clinical requirements. For some, beginning by exploring the experiences of adolescents with type 1 diabetes was helpful, and could be linked directly to individual design concepts. For instance, one designer created a digital insulin buddy, inspired by videos where people named their insulin pumps and spoke of them like friends. Others found inspiration by looking at current medical devices and seeking ways to improve them.

While the designers had to do considerable research to develop an understanding of type 1 diabetes, its treatment regimen, and the technology used for treatment, they also used this novice status to their advantage. They found it easy to ideate when they were not overly constrained by the complexity of self-management.

\subsubsection{Whose point of view?}

Designers recognised that it would be beneficial to have contact with adolescents with diabetes and other stakeholders, to validate ideas, test prototypes, or participate in the design process. "Although we can empathise to a certain extent, none of us have actually had the experience; none of us have really had to deal with even to a limited extent the sort of things that diabetes entails." However, they were also cautious about contacting individuals with type 1 diabetes, anxious that they may cause distress. For these projects, the students did not have ethical approval to conduct primary research with adolescent participants. 
Some students narrowed the scope of the research by identifying an area that they would enjoy designing for, aiming for a design outcome that satisfied the brief, but also developed their skillset and portfolio of work in a particular area, such as sportswear. Findings from these interviews and reflections on the expert review process helped to shape the design process for the second design project and participatory design workshop in Chapters 8 and 10.

\subsection{Discussion}

\subsubsection{Psychosocial user requirements}

Students were successful in developing psychosocial user requirements and designing responding technologies. Their designs were respectful of adolescent users and their requirements. By showing the designs to the Diabetes Nurse Specialist, the advantages and disadvantages of each design became clear. However, as there was no explicit discussion of user requirements, the method did not contribute substantially to developing user requirements. Making and evaluating these designs aided the construction of two new user requirements:

1. Allow comfortable and non-invasive use

Non-invasive technologies that are accurate, safe, and able to be integrated into daily life. Currently, adolescents may be reticent to test blood glucose in public spaces. Joya addressed this by offering a jewellery-inspired, non-invasive testing method that females could easily incorporate into daily life.

2. Facilitate spontaneity and participation in everyday activities Medical technologies being fast and easy to use during everyday activities. For example, adolescents currently must stop swimming to test blood glucose, aware that exercise may induce hypoglycaemia, and that this could be mistaken for physical exertion. Carrying and using medical equipment can also inhibit adolescents' spontaneity, setting them apart from peers. Subscope was an attempt to reduce impact on participation, by letting adolescents see their blood glucose levels at a glance, so they only need to stop exercising briefly, if necessary. 
Additional support for six of the other user requirements described in Chapter 4 was provided and the requirements were developed further through this study:

\section{Perform in a life-proof manner}

While the Armilla insulin pump design was the only item to respond directly to this requirement, the design was not evaluated positively. The feedback from the clinician was primarily about a lack of stigma and embarrassment associated with insulin pump use, so it is unclear whether this user requirement was applicable. Medical technologies should be designed robustly for challenging, yet common, environments.

\section{Enhance convenience}

Medical technology can enhance convenience through reducing bulk, for better portability. Currently, life goals can be in conflict with diabetes management goals. As became clear when reviewing Simpac, this must be balanced with attention to sustainable use of materials for those who value this, comfort, and safety.

3. Provide meaningful information to facilitate decision-making Medical technologies communicating information such as blood glucose trends so that adolescents can act proactively, rather than reactively. Having state awareness for insulin, such as duration unrefrigerated or exposed temperature range, would also ensure insulin efficacy.

4. Help to comfortably disclose and explain type 1 diabetes when appropriate Disclosure can be psychologically beneficial for adolescents. Medical technologies that are not recognisable provide users a choice whether to disclose diabetes. The Joya design, where the creation of a desirable object may stimulate discussion and avoid connotations of shaming or hiding the product, is similar to the design of prosthetic limbs (Sansoni et al., 2016).

5. Minimise or eliminate feelings of stigma or embarrassment while using medical technology

Adolescents who make negative attributions about peers' or friends' reactions to their self-management are likely to struggle with self-management. Blood glucose monitoring can be embarrassing and inconvenient, though insulin pumps were not thought to be embarrassing. Medical technologies may reduce embarrassment and 
increase engagement by being desirably designed. However, designs should be appropriate for users' characteristics (e.g., a jewellery alternative for males).

6. Facilitate interaction with diabetes peers

Diabetes peer relationships are important for normalising experiences and technology use, and for emotional support. However, adolescents currently have access to communication channels that are desirable to them.

The final description of each user requirement is presented in Chapter 11.

\subsubsection{Approach to research and design}

From this study, I tested the process of designing from psychosocial user requirements for adolescents with type 1 diabetes, and devised ways to improve this process for the following design activities in Chapters 8 and 10.

Firstly, the importance of designers finding empathy and inspiration by seeing and hearing adolescents' experiences in engaging formats was clear. The designers had limited access to adolescents, relying primarily on secondary sources. The abundance of shared experiences on digital platforms has great implications for research with groups that are difficult to access. The designers relied primarily on these non-academic sources for inspiration, though the experiences contained within these sources were also evident in more traditional research publications and validated by the clinician. While this approach did not fully conform to an empathic design approach (Mattelmäki, Vaajakallio, \& Koskinen, 2013; J. Thomas \& McDonagh, 2013), the students' empathic view of diabetes management did increase. Further, a designer applying 'empathic modelling' by using the diabetes equipment needed to be carefully balanced, not assuming to completely understand what this must be like (McDonagh \& Thomas, 2010). Going forward, the designers will be able to read first-hand quotes describing adolescents' experiences, and finally will take part in a design workshop, meeting adolescents with type 1 diabetes and working with them to design and review medical technologies. This approach may also help to overcome designers' tendencies to design for their own interests.

Secondly, designers needed to balance these inspiring and empathy-building resources with more technical information to understand the basic elements of 
managing type 1 diabetes and the devices used to do so. Going forward, I will ensure the designers have further access to both types of information.

As discussed in Chapter 4, the use of proxies is common in medical research with adolescents. Relevant proxies include healthy adolescents, parents, caregivers, and health professionals (Lang et al., 2010; J. L. Martin et al., 2006). The clinician was the proxy for reviewing the designs. While there is remarkable similarity in the user requirements developed based on insights from health professionals and adolescents in the following chapters, these are not completely aligned, and as such, adolescents should be included in the design and review process where possible, as their opinions may differ from proxies. Though a single user reviewer was unlikely to identify all issues with the designs, this review was appropriate as a tool before access was granted to adolescent participants, and as a method of providing additional support for user requirements in tandem with other triangulated sources. Although the clinician did not have first-hand knowledge of adolescents with diabetes, she did have knowledge of a wide range of medical devices that were currently or previously available, from which insights could be derived. Further, the clinician's feedback that the designs may be relevant for a broader range of people with diabetes, and not only adolescents, parallels an 'extreme user' approach, as aforementioned (Holmquist, 2004). It is analogue to an 'extreme characters' technique, yet is based on real people rather than fictional characters (Djajadiningrat, Gaver, \& Fres, 2000).

The design method predominantly used aspects of research for and research through design, with applied diabetes knowledge produced through designing and eliciting discussion. The design review was structured around reviewing the designs, and the user requirements were not formally stated and reviewed. As such, much of the focus of the review was on individual design concepts, and not whether the psychosocial user requirements from which they stemmed were accurate. Accordingly, in a following design review in Chapter 8, user requirements are explicitly stated.

\subsection{Summary}

This study led to the construction and initial support of eight psychosocial user requirements of medical technologies: to allow comfortable and non-invasive use, 
facilitate spontaneity and participation in everyday activities, perform in a life-proof manner, enhance convenience, provide meaningful information to facilitate decision-making, help to comfortably disclose and explain type 1 diabetes when appropriate, minimise or eliminate feelings of stigma or embarrassment while using medical technology, facilitate interaction with diabetes peers. The study illustrated how designers can use digital platforms and healthcare professionals as proxies in the design of medical technologies for a group of users that is difficult to access. While involving adolescents in the design process would have been beneficial, this approach was useful in the early stages of the design research process. Further, the purpose of the design activity was not to create viable medical devices, but to provide additional support for and populate the psychosocial user requirements and to garner insights about design and review methods, thereby informing Chapters 8 and 10 . 


\section{Experiences and User Requirements as Described by Adolescents and Young Adults}

This chapter describes interviews conducted with adolescents and young adults with type 1 diabetes. The aim of this chapter was to develop the grounded theory analysis of adolescents' experiences and psychosocial user requirements. Adolescents and young adults with type 1 diabetes were interviewed, as they have first-hand knowledge of their experiences and requirements, though interviews were delayed until ethical approval was sought. Adolescents are often excluded from research and medical technology design processes, and their opinions consequently unrepresented. Insights from health professionals helped to shape the direction of the interviews and pinpoint areas for further research.

\subsection{Methods}

\subsubsection{Participants}

A purposive, convenience sampling method was used to recruit participants (Devers \& Frankel, 2000; Jupp, 2006). Inclusion and exclusion criteria were established prior to recruitment to help health professionals aiding recruitment to establish a sampling frame, using patient databases, although I did not have access to these patient lists.

Inclusion criteria:

- diagnosed with type 1 diabetes at least 12 months ago;

- 13-24 years old;

- resides in the Wellington region for most of the year;

- registered with and attends a diabetes clinic or primary health organisation for management of diabetes.

Exclusion criteria:

- diagnosed mental health disorder;

- significant morbidity that affects daily living from another health condition (e.g., Crohn's disease, cancer); 
- $\quad$ any other reason that would be practicably difficult.

Two clinical nurse specialists sent details of the study to patients who met the criteria, informed some patients of the study in-person, and had flyers and posters available for waiting areas. I also recruited participants in waiting rooms in three hospitals after clinic appointments. Clinical Nurse Specialists approved each patient before I made contact. I also presented an overview of the research to a Diabetes Youth meeting and distributed flyers with a description of the research (Figure 23).
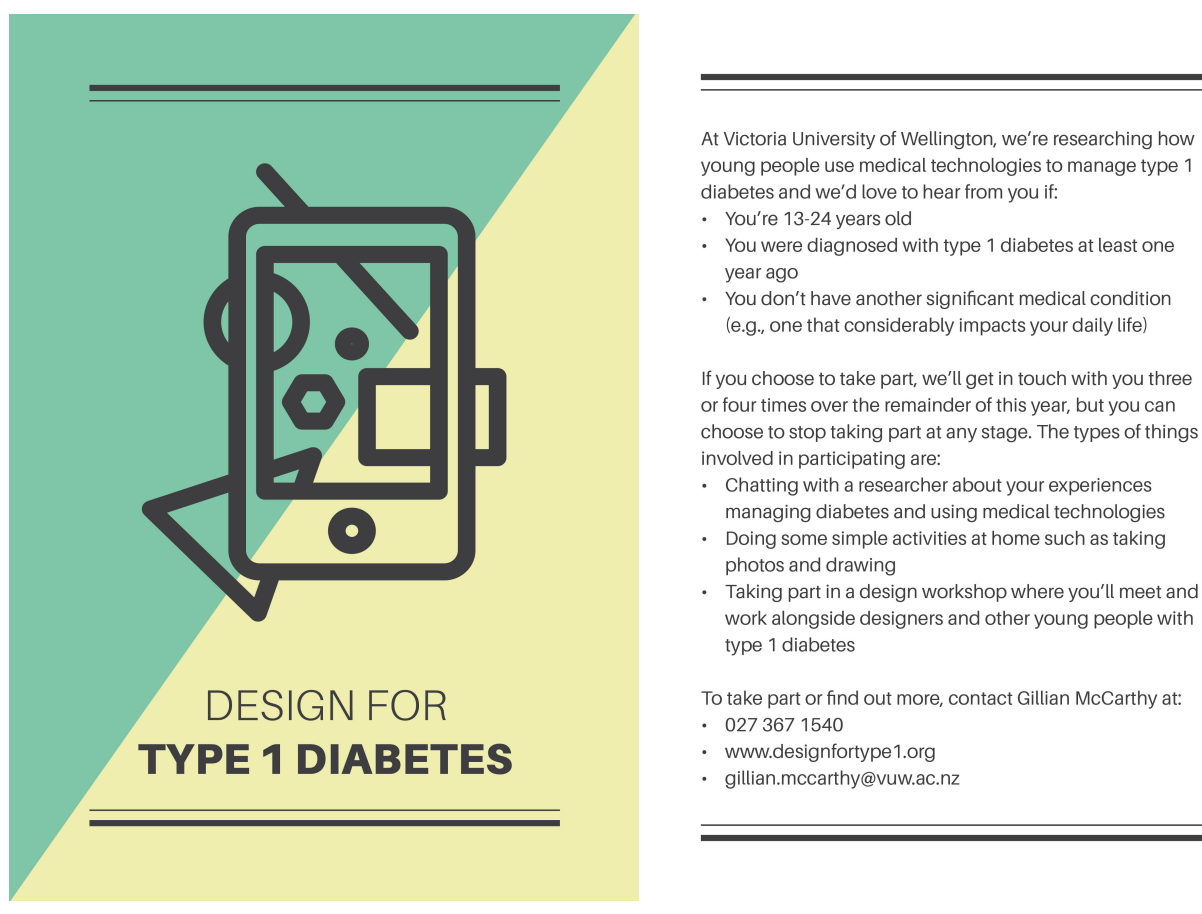

Figure 23. Participant recruitment flyer.

Participants were 16 adolescents and young adults aged 13-24, with nine females and seven males (Figure 24). Fourteen identified as New Zealand European/Pakeha, one English, and one Chinese. Three participants had a parent present at the interview. Definitions for the adolescent age range vary. In the local health service, patients are transferred into the adolescent service when they begin Year 9 at high school (aged 12-13), and between 16 and 18 are transferred to the young adult service until they reach 25 , when they transition into the adult service. Comparatively, the World Health Organization classifies adolescents as 10-19 years of age (World Health Organization, 2017). Throughout the thesis, I refer to adolescents as aged 13-18 and 
young adults aged 19-24. Participants throughout this thesis are coded to indicate whether they are an adolescent (A) or young adult (YA).

I included young adults in the research as they could reflect on their time as adolescents. There is a common participation bias in health research, where only those managing well choose to take part. As such, including young adults who were now managing well but had struggled during their adolescent period provided insight into their adolescent experiences and factors that improve self-management.

3

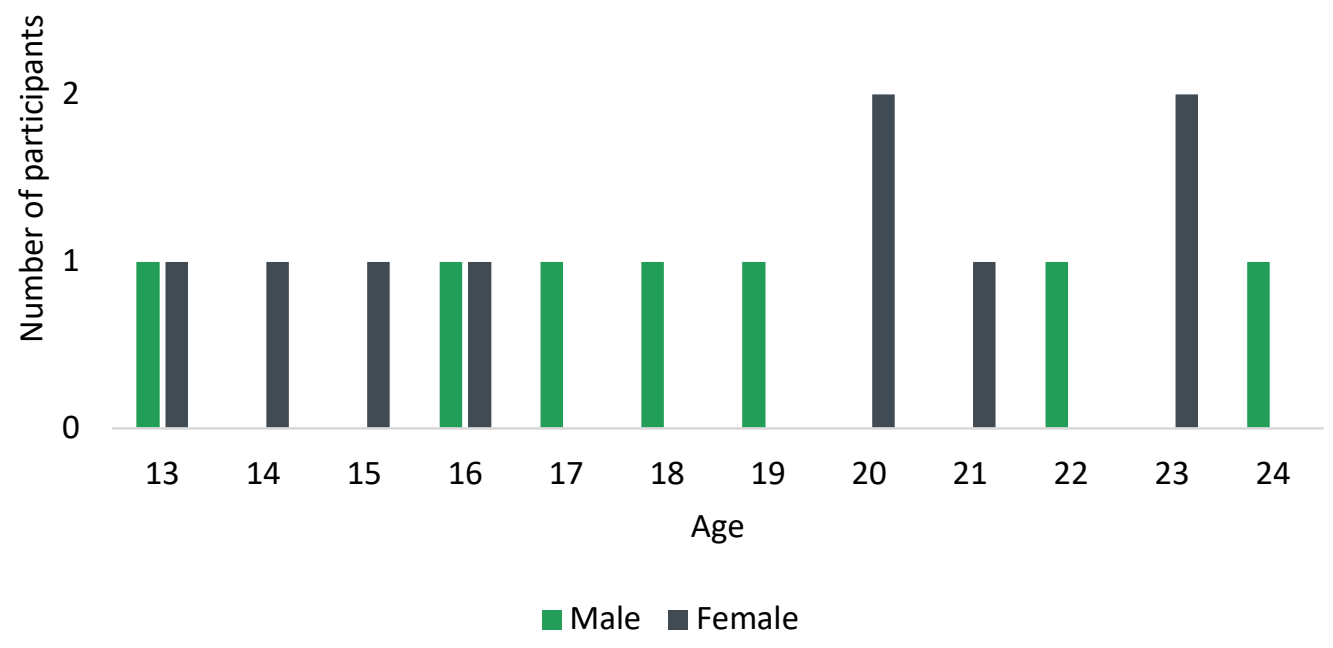

Figure 24. Adolescent and young adult participant age and gender.

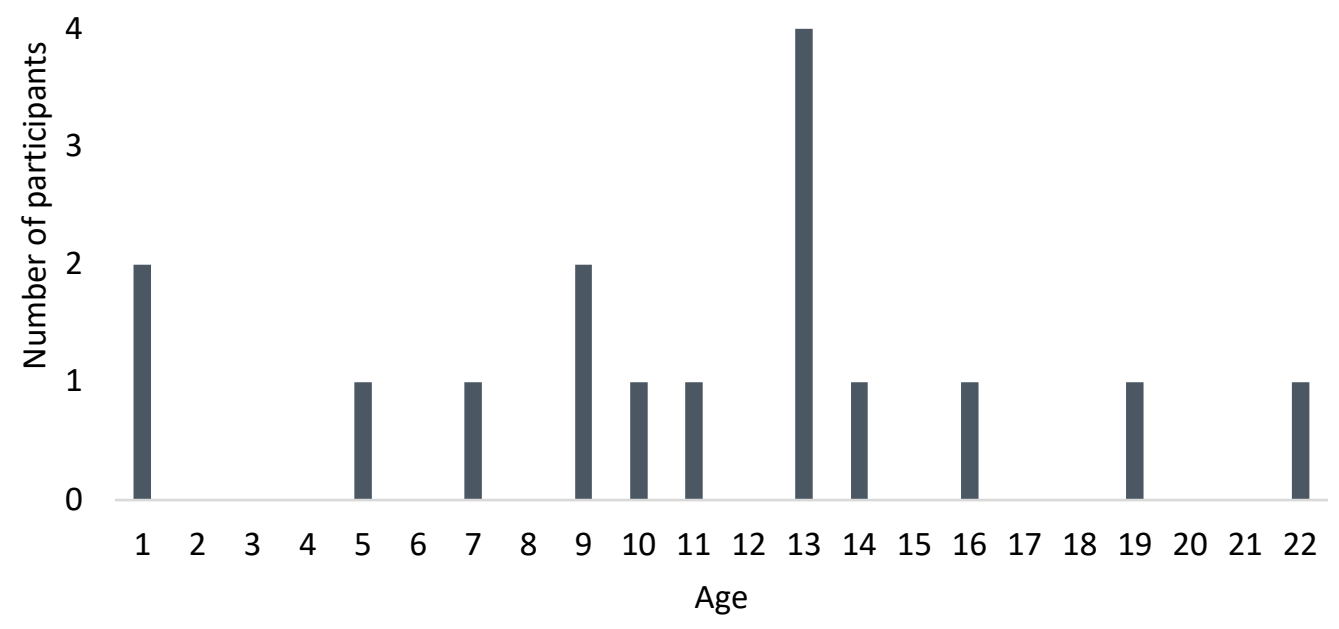

Figure 25. Adolescent and young adult participant age at diagnosis. 
Participants were diagnosed with diabetes between one and 22 years (Figure 25) and had been diagnosed with diabetes for four months to 20 years. One participant did not fit the inclusion criteria of being diagnosed for 12 months or longer, but was contacted by the clinical nurse inadvertently and wished to partake in the research, so was included. Inclusion criteria of 12 months is standard across much diabetes research, and ensures the research does not conflict with early phases of developing knowledge and acceptance.

\subsubsection{Procedure}

Appointments were made by text message or email, with reminders sent to participants. Interviews were conducted in private rooms and hospital locations were not used, as participation was confidential to health professionals. Semi-structured interviews focused on participants' experiences of self-management and use of medical technology. The content of each interview varied due to the use of thematic sampling, however, the broad areas covered in the interviews were:

- daily life, hobbies, and interests;

- diagnosis period;

- diabetes knowledge;

- self-management experiences;

- changing medical devices;

- support networks;

- interactions with peers;

- views on ideal self-management.

Interviews lasted 20-45 minutes, and were audio recorded and transcribed. At the beginning of the session, participants were given information and consent forms (Appendix D) and were offered a drink. One participant was offered food to treat hypoglycaemia during the session. Interviews were followed immediately by a card sort task, described in Chapter 7. 


\subsection{Findings}

The interview findings commence with an overview of adolescents' self-management of type 1 diabetes. Following this, three theoretical concepts and their constituent sub-categories are discussed in an aim to create substantive theory:

- conceptualising type 1 diabetes and its management;

- developing independence;

- experiencing and managing interpersonal interactions.

\subsubsection{Self-management overview}

This overview describes how adolescents manage blood glucose levels, manage risk, respond to dissonant technologies, and change between medical devices.

\subsubsection{Managing blood glucose levels}

While adolescents strove to self-manage diabetes effectively, they struggled to maintain normoglycaemia and largely accepted this. Their acceptance was similar to a health professional's views in Chapter 4, where she talked about influencing, rather than controlling, diabetes.

Factors affecting glycaemic control were illness, moving out of the honeymoon period where some insulin is still naturally produced, changes in lifestyle, prioritising other aspects of life, school or work, stress, not factoring in incidental exercise, miscalculating carbohydrates, not administering insulin for snacks, mental health, and not testing. Participants varied in how often and when they tested their blood glucose levels, with common frequencies ranging between 4-7 times per day, with a maximum of 25 times.

Forgetting to test blood glucose levels or inject insulin was a common feature of adolescent self-management. However, from diagnosis habits were formed, and there was less forgetting. Reminder alarms were not effective, and in inconvenient moments, such as while in bed, adolescents may choose not to test, rather than forget to test.

Many adolescents experienced nocturnal hypoglycaemia, leaving them tired and grumpy the following day. Nocturnal hypoglycaemia has been found in 
individuals with type 1 diabetes on an average of $8.5 \%$ of nights, with no relation to age or method of insulin delivery (Juvenile Diabetes Research Foundation Continuous Glucose Monitoring Study Group, 2010). Participants were frustrated by these events, but were confident in their ability to detect hypoglycaemia and correct the situation. For some, parents still played a role in nocturnal blood glucose testing.

\subsubsection{Managing risk}

Adolescents are commonly described as risk-takers, with brain development, importance of social situations, and reducing parental supervision playing a role. Risk-taking behaviours peak in early adulthood, in alignment with the peak in poor self-management (Wasserman, Anderson, \& Schwartz, 2017). However, illnessspecific risk-averse behaviours were also evident when adolescents managed blood glucose levels, particularly taking risk-averse actions for short-term issues such as hypoglycaemia, rather than long-term issues such as running high.

Some were hesitant to exercise in case of hypoglycaemia, but many found strategies to reduce risk. One participant adapted the way he managed risk as he gained experience in self-management.

A8: Previously I would have thought, 'I'll make sure my blood sugar's really high before I go kayaking and drink some orange juice'. But I've realised now that that would be unnecessarily making yourself go high just to feel safe, when you could just bring some glucose tablets in case you need them.

This participant's initial and more recent approaches contrast thinking about short and long-term risk. To make decisions about risk and management, adolescents need to trust the accuracy of their medical devices. Many did not trust the accuracy of a particular blood glucose meter, their only subsidised option.

YA1: The blood glucose meter over the past two days has said I've been really high, over 25. I corrected and half an hour later I'm at 10, and still have two and a half hours of insulin left. That was obviously a wrong reading and it's truly frustrating... You can't really trust it, 
so I'm overly cautious, which means I spend time higher than I need to be which has long-term impact.

When adolescents did not trust the accuracy of their blood glucose meter, some would take less insulin than recommended for the blood glucose level, some would test again shortly afterwards and their confidence would increase if the two levels were similar, and some would use their bodily awareness of whether they felt hyperglycaemic, normoglycaemic, or hypoglycaemic to decide about insulin dosage. Adolescents reported that differences between successive readings ranged by up to 18 $\mathrm{mmol} / \mathrm{L}$, a clinically significant difference. When medical technologies are trusted, they can improve confidence in dosage and reduce fears of hypoglycaemia (Barnard et al., 2012). To minimise risk, some adolescents carried backup insulin pens or site changes, particularly when away overnight, balancing possible risk with the inconvenience of carrying equipment.

\subsubsection{Responding to dissonant technology}

Medical devices needed to give clear, context-appropriate feedback to adolescents, to facilitate their self-management. At times, this did not happen. For instance, one insulin pump required four steps for priming, and if the last step was missed it remained in priming mode for hours, not delivering insulin, without alerting the user. This was contrasted with a CGM that had an effective alarm that gradually increased in intensity. However, based on context (e.g., driving), this alarm could again be frustrating. A positive example of device feedback working was an insulin pen with an inbuilt memory feature that showed the last time it had been used, and what insulin dosage was administered. If the user could not remember whether they had injected or not, this gave them clarity.

Adolescents were also concerned about device functions, as some functions were unnecessary and others were missing. For instance, one blood glucose meter recorded contextual information and had an alarm function, but these functions were already available on the insulin pump used alongside it, and it did not have a light or make noises to give feedback for button pushes, making it hard to use at night. 
There were also times in which medical devices did not function as they should, for example, a lancet that did not prick through the skin. Alternately, devices were not functioning effectively in particular contexts. One example was a lancet with a barrel of needles, which could be rotated for a new needle. While this was a positive feature, the barrel would often rotate while being stored in a bag, wasting needles. In another case, a blood glucose meter did not function in cold temperatures, making one adolescent miss out on social time while sitting near a heater for ten minutes to warm it, or putting it in the microwave. There were also physical inconveniences, such as washing hands before testing, or pump tubing being caught or knotted around door handles and other objects. If a pump was not delivering insulin, adolescents had to investigate why and troubleshoot.

\subsubsection{Changing medical devices}

Many adolescents changed devices. A common transition was to an insulin pump from syringes or insulin pens. Similarly to existing findings, adolescents chose the insulin pump because they preferred the steady release of insulin and thought it would improve their management, and that these factors outweighed being attached to a device (Low et al., 2005). While some adolescents initially disliked the idea of being attached to the pump, this was overcome if the benefits were obvious.

YA4: At first I didn't want to go on the pump at all. I really hated the idea that something was attached to me full time... But on the pump I wasn't constantly worried about going low or high or testing so much because I just knew that I had something keeping me going in the background. I felt a lot more at peace.

Nevertheless, changes between medical devices were not always positive. One young adolescent rejected his insulin pump after three months, frustrated by pump sites not staying in place while active. Others with diabetes have described initial experiences with a pump as like recalling the 'trauma' of initial diagnosis, needing to re-learn how to manage the new device and the psychological distress that can accompany this (Ritholz et al., 2007). 
Some participants were also aware of technologies such as CGMs and closedloop systems, which were currently unavailable or unaffordable. They thought these systems would be better for showing blood glucose trends, correcting high or low levels, and minimising the effort needed for management.

For some adolescents, changing medical devices was not their choice. Sometimes health professionals presented a change as a logical next step, and some changes were forced, coming about through subsidy changes, a device being discontinued, moving countries, or a device malfunctioning. One adolescent had used four blood glucose meters over six years, being affected by a subsidy change, dropping one meter and having another run over by a truck. One adolescent upset by the subsidy change for blood glucose meters wrote to Pharmac to express concern. Because of this change, many adolescents now required two meters, one for blood glucose and another for ketones. One of the devices that was no longer available had options for personalisation with stickers, which one participant missed. Adolescents suggested that having one meter that tested blood and ketones and integrating it with a smartphone would minimise the equipment to be carried, or a closed-loop system could minimise effort. It was reasonably common for adolescents to also use differently branded blood glucose meters and lancets. Paying for unfunded lancet consumables was affordable, as needles were not replaced often. Lancets were chosen for their comfort and effectiveness.

\subsubsection{Conceptualising type 1 diabetes and its management}

Conceptualising diabetes and its management is a concept comprised of many processes: developing and applying diabetes knowledge, developing acceptance, conceptualising current and 'good' diabetes management, becoming engaged, and attempting to fit diabetes around other aspects of their life.

\subsubsection{Developing and applying diabetes knowledge}

Adolescents were developing knowledge about managing diabetes and becoming more confident, though unusual situations were still challenging. Medical technologies play a role in collecting, visualising, and sharing data and information, which affects adolescents' decision-making. 


\subsection{Developing knowledge}

Adolescents have varying knowledge of diabetes when diagnosed. Some had no previous knowledge of diabetes, while others cited knowledge from high school biology or having a family member with the condition. There was a rapid learning experience that happened immediately from diagnosis. Initially, adolescents were reliant on health professionals for every insulin dose recommendation, but this dependence quickly reduced.

Knowledge was developed via the diabetes team and through first-hand experience. The diabetes team was an important part of this process and provided on-going support, particularly by text messaging, whenever an adolescent was unsure about what action to take.

\subsection{Creating and following guidelines}

Adolescents described a variety of guidelines for self-management. Examples were eating sweets to treat a blood glucose level below $3.5 \mathrm{mmol} / \mathrm{L}$ and fruit to treat a level between 3.5 to $4 \mathrm{mmol} / \mathrm{L}$, or only having a couple of alcoholic drinks and eating before bed to offset alcohol's hypoglycaemic effects.

Adolescents had individual guidelines for what constituted 'good' blood glucose levels and when to correct. Generally, a level between 5 and $9 \mathrm{mmol} / \mathrm{L}$ made adolescents happy, between 4 and $14 \mathrm{mmol} / \mathrm{L}$ was acceptable, and $12-14 \mathrm{mmol} / \mathrm{L}$ or higher was accompanied by disappointment and a correction. Health professionals' guidelines were delivered to adolescents and their parents gradually, which could be frustrating in certain instances.

YA5: It was not until I was about 17 or 18 that someone actually said approximately $80 \mathrm{~g}$ of carbohydrates is about the maximum you want to eat for a meal... unfortunately that meant that I got into reasonably bad eating habits.

In this instance, it was moving to a new city and seeing a different health professional that sparked this sharing of a guideline. 


\subsection{Having the confidence to manage}

Adolescents felt they had confidence to manage their diabetes in most situations. Some had sufficient confidence to make significant changes to their treatment regimen independently. For example, one young adult had transitioned to a carbohydrate-free diet and adjusted her long-acting insulin dose from 24 to four units by two units per time, and entirely cut out her fast-acting insulin. She had not consulted her diabetes team, but hoped they would approve of her actions.

However, there were times when self-management was challenging, as a considerable range of variables could affect blood glucose levels. Adolescents were learning from instances when actions and devices did and did not work, and discovering patterns by reflecting on similar occurrences. Some adolescents had strategies to minimise the effect of these events, such as embedding routine into daily life and carrying backup equipment and glucose. However, greater confidence in diabetes knowledge did not always align with ideal management, as described in Chapter 4. Adolescents became more 'blasé' with testing, trusting their bodily feelings and knowledge of the effects of different foods. They were also 'reading through the lines' when it came to data, sometimes not correcting a high blood glucose level if they anticipated it would resolve itself shortly, due to calculations about glucose and insulin on-board. As such, they were not simply managing based on current data, but expected trends.

\subsection{Managing unusual events and making mistakes}

While adolescents had confidence to self-manage in everyday contexts, unusual situations proved challenging. Examples included moving from the family home into a hall of residence for university and resultantly having a change of diet, eating during the Christmas period, and managing while ill. In these instances, adolescents managed reactively. For some unusual situations adolescents took the advice of health professionals, and at other times they disagreed, based on their personal experience.

A2: I went on a school tramp and they said, 'You should put your basal rate down 80\%.' .. We were going 'that's ridiculous, they're 
wrong'... I started down $10 \%$ and I was low, so I went to 15 and I

was low, I went to 30 and I was low, I went to 40 and I was low, so I went to 50 and ended up at 80. Should have listened.

Adolescents also made errors stemming from decision-making, communication with parents, and both forgetting to take insulin and whether insulin had already been administered. For instance, when one adolescent's mother instructed her to 'bolus six', the mother meant six carbohydrates, while the adolescent inferred six units, resulting in an extra 18 units of insulin being administered.

\subsection{Requiring meaningful information}

Medical technologies such as insulin pumps, blood glucose meters, logbooks, and digital software for sharing blood glucose logs were used for recording data. While data could be helpful for adolescents and health professionals seeing patterns if stored, shared, and visualised effectively, this did not commonly occur. While manually logging data could be helpful for comprehension, it also proved tedious to the point of abandonment. Physical logbooks have traditionally been used, but their data are increasingly being stored digitally. Three participants particularly liked using physical logs and regularly recorded blood glucose levels and insulin doses, occasionally noting meals or activity.

To attain meaningful information, adolescents had to collect or record data first. Some participants specially logged information for a three-week period before appointments, to share with health professionals. However, others found that health professionals could access a digital log of blood glucose measurements and/or insulin doses from their blood glucose meter or insulin pump, so felt that there was no point making an additional analogue log even though it may contain contextual information. Conversely, some participants did not always respect the advice of health professionals, arguing that they did not understand the contextual circumstances around the data.

YA8: One of the downsides about going to someone for ten minutes every three months is that they have no idea what happened and 
they're just looking at numbers whereas I've been through it and I can

tell I had a bad day that day because this happened.

Additionally, while health professionals often had access to data and the means to download and visualise trends, adolescents had less access to meaningful information garnered from their own data.

Participants found meaning in different ways: by hypothesising about how stress affects blood glucose levels, by using technology to calculate insulin doses in a straightforward way, and through data visualised as graphs. Suggestions were made for a mobile app where blood glucose, insulin, and exercise information could be automatically synced, help provided with carbohydrate counting New Zealand foods, and information able to be shared with health professionals, streamlining the technologies.

\subsubsection{Developing acceptance}

Adolescents were developing acceptance of their diagnosis and of self-management required. Boss (2009, p. 21) likened diagnosis to “ambiguous loss”. Adolescents' adjustment to their diagnosis has both direct and indirect impacts on their health outcomes (Greca et al., 1995). This adjustment is also affected by the adolescent's perception of control. If they feel that this experience is out of their control, they will be more inclined to develop 'learned helplessness', which can lead to poor motivation, negative emotions, and an unlikelihood to respond well to future events (Seligman \& Maier, 1967). Alternatively, if the individual perceives their situation as controllable, then there is an increased likelihood that they will behave adaptively in future (Bandura, 2001). Coping efforts to manage this process can take an approach or avoidance course (Suls \& Fletcher, 1985). Approach behaviours include searching for information, gathering social support, and problem-solving. Avoidance includes cognitive strategies such as denial and behaviours such as disengagement (Stanton, Revenson, \& Tennen, 2007). One young adult described a defining moment for developing acceptance in his early teen years.

YA7: There's definitely been a point in my life where I didn't want to deal with it. My friends didn't have to worry about doing a blood test, 
checking what level you're at if we're doing an activity. They didn't have to take insulin and injections and they didn't have to worry about any of that stuff. I just got sick of it. I tried to tell myself I wasn't a diabetic. That didn't work. I got quite ill and that's when I had my wakeup call, 'cause my father's best friend passed away when he was quite young because he neglected his diabetes... my father said to me, 'Do you want to end up just like him?... Because that's where you're heading if you don't change'. It got to me. I was angry with him, but I was thinking about it and from that point, I decided that I'm gonna look after myself'cause someday I want children, I want a family, I want a secure job. I want to live my life and get out there and prove that even though you've had a pretty crappy background in health, it doesn't have to stop you.

Over time participants accepted the negative aspects of management, such as callouses on their fingers from thousands of tests. Some adolescents tried to put diabetes in perspective, acknowledging the severity, but considering how others have their own challenges to deal with.

Three of the adolescents, diagnosed at two, five, and seven years of age, hypothesised that being diagnosed at a young age, where they had little memory of life prior to diagnosis, made developing acceptance easier. Another participant, who had both Asperger's Syndrome and diabetes, felt entirely differently about the two conditions. While Asperger's was a core component of her identity, diabetes was more tangential.

YA5: I consider myself an autistic person with diabetes, because to me, I would never want to cure my autism. It's a part of me and it's a part of my way I think, but I would quite happily get rid of my diabetes any day.

Adolescents learning that they would not have to significantly alter their lifestyle made acceptance easier. However, there was a sense of vulnerability that contrasts theories about normative adolescent development, where individuals feel invincible (Huebner, 2009; KyngÄs et al., 2000). 


\subsubsection{Conceptualising diabetes and current management}

Managing a long-term condition is vastly different from traditional medical treatments; there is no cure and a period of self-management that requires a lifetime, yet does not guarantee a future without complications.

YA1: I think medical professionals want you to be like a medical professional all the time. A lot of diabetes nurses, though, understand that you've got to live your life and diabetes has to come second... Medicine is not about how to make a person well; it's about what can go wrong... With chronic conditions, though, it's about how to manage wellness rather than cure illness. Managing wellness is the key to diabetes.

This relates to the construct of liminality, which can be used to describe a state between health and illness (Murphy, 1987; Polgar, 2010). For people with diabetes, they may spend much of their life in a liminal state, unable to be cured but also with good glycaemic control, not encountering severe symptoms. Sometimes it is the treatment of illness, achieved through medical devices rather than the disease itself, that ultimately places people in a liminal state where they might feel isolated.

Significant ways of conceptualising diabetes that adolescents reported included: diabetes being about how to manage wellness rather than cure illness; diabetes 'control' being hard to grasp, like a 'slippery fish', and diabetes management playing a background role in adolescents' lives, with managing blood sugars becoming much like brushing your teeth, particularly when things were going smoothly.

The role of diabetes diminished over time, as negative feelings dissipated and adolescents gained experience. One young adult struggled with guilt, particularly early after her diagnosis, wondering whether she was to blame for developing diabetes after having an eating disorder, which may have compromised her immune system.

Some adolescents reported positive associations to conceptualising diabetes. Two participants, aware that others thought being connected to machines was a negative, leveraged the social power of their insulin pump or continuous glucose 
monitor, referring to themselves as 'cyborgs'. Others also experienced social benefits to having diabetes. A2: "I went to diabetes camps and I made a lot of new friends ... I think that's probably the biggest impact diabetes has had; just bringing new people into my life."

Meanwhile, some adolescents felt as though diabetes had changed them, and the way others saw them. One participant described how her priorities changed significantly in her first year of managing diabetes, which was also her first year at university.

YA2: I had to reprioritise things and learn to live with the fact that I was possibly not going to be able to perform as well in exams necessarily as I would have before. It has changed my perspective. Now I have to take care of myself before doing other things that would have been more important to me before.

While some adolescents attributed moods to hypoglycaemia and hyperglycaemia, external to their personal characteristics, they recognised that others saw these moods as part of who they were. In terms of understanding the real and possible severity of diabetes, a participant who was already experiencing retinopathy and required laser surgery was philosophical about this. He did not regret his past actions and these complications, as they solidified for him the necessity for good diabetes management and his compassion towards others managing their health.

\subsubsection{Conceptualising 'good' management}

Participants conceptualised what good management of diabetes meant for them individually. There were four areas that their responses covered: following diabetes guidelines, prioritising diabetes or the rest-of-life, judging effort or results, and feeling physically and mentally healthy. The difference between medical best-practice and the reality of self-management is one of the core tensions in diabetes management, along with tensions between the individual and their interactions with others (Kanstrup, 2014). 


\subsection{Following diabetes guidelines}

Two participants thought that following diabetes guidelines provided by their health professionals would constitute good management, though they admitted that they did not currently adhere strictly to these. For example, one participant felt he ought to test more frequently, particularly before meals. Another participant commented that while knowing what to do was important, so was understanding why it needed to be done. Research shows adolescents perceive self-management in both positive and negative ways, as something which ensures their long-term health, yet as something they feel obliged to do and feel passively about, prioritising other things such as time with friends over their treatment regimen. When they felt obliged to adhere, adolescents had feelings of guilt and stress (Kyng ̈̈s \& Barlow, 1995; KyngÄs, Kroll, \& Duffy, 2000).

However, three other adolescents were more comfortable to stray from health professionals' guidelines, objecting to only doing exercise with blood glucose levels above $7 \mathrm{mmol} / \mathrm{L}$ and requests to test more often, preferring to use instinct and experience to calculate insulin doses over 'tedious' carbohydrate counting.

\subsection{Prioritising diabetes or the rest-of-life}

Participants varied in how they thought that diabetes and other aspects of daily life should be prioritised. There can be a conflict between diabetes goals and behaviours and other rest-of-life goals and behaviours. While the experience of managing diabetes and conceptualising its role varies greatly between individuals, the management routine must be integrated into daily life in a manner that the person finds acceptable to maximise psychological wellbeing in addition to physical health (Watts, O’Hara, \& Trigg, 2010).

Three participants felt that diabetes must be prioritised, yet this came with compromises. For the first, though tasks such as finger pricking were manageable, they were a constant reminder of not being able to have a 'normal' day. For the second, he had to make his lifestyle more structured to successfully manage diabetes. And for the third, she had substantially changed her diet, limited alcohol intake, and avoided experimenting with smoking and drugs. She felt her recent management leant too far towards prioritising diabetes, and that she needed to recapture a 
balance, having a set amount of energy per day, some of which could be allotted to diabetes, and some of which could be allotted to other aspects of daily living. Contrastingly, one participant lay at the other end of the spectrum, feeling that other aspects of life ought to be prioritised over diabetes.

Four participants spoke about the importance of finding an acceptable balance between self-management and other aspects of daily life. For one participant, that meant having blood glucose levels between 4 and $8 \mathrm{mmol} / \mathrm{L}$ and being able to participate in sports. Another participant quoted a health professional when explaining how she finds a balance, with the balancing point also changes at times.

YA5: I think my doctor puts it really well; it's about having your blood glucose as best as possible without it having too much of an impact on day-to-day life.

Making improvements, and making the best effort she felt capable of at that time, were also factors in her feeling satisfied with her management.

\subsection{Judging effort or results}

Three participants spoke about the importance of trying your best, and accepting that this effort was good enough, rather than focusing on the result of these efforts. There was an element of acceptance by these individuals; that effort was not always correlated with results. This embodies a 'growth mindset', whereby adolescents are not naturally good or bad at self-management, but through effort, can improve their management (Dweck, 2008). On the other end of this continuum, one participant who had been diagnosed only four months earlier felt that managing well meant that the results of management had to be perfect. For her, good management included a range of levels from $4-8 \mathrm{mmol} / \mathrm{L}$, but ideally $5-6 \mathrm{mmol} / \mathrm{L}$. Another aimed for 'perfect' numbers, but as she took on more responsibility she struggled to manage within the tight range that her mother had previously achieved, and there were tensions around this transition. Conversely, in relation to 'good numbers', and 'good management', one participant (YA1) added: "I don't like the word 'good'. Good implies that there's bad.” 


\subsection{Feeling physically and mentally healthy}

For three participants, an ideal form of management meant feeling both physically and mentally healthy. This meant avoiding hyperglycaemia and hypoglycaemia, as this could leave them feeling physically unwell and limit their participation in daily life. Further, it meant having a level of acceptance and a buffer around the emotional aspects of managing diabetes, contributing to better mental health. While some adolescents reacted emotionally to their blood glucose level, describing frustration, annoyance, and happiness, others used it simply as data to aid decision-making. They were not focused solely on trying to keep their numbers in range, but recognised that they felt physically and emotionally better when this was the case. Again, the psychological aspect of diabetes management was discussed as being just as challenging, if not more so, than the physical aspects.

\section{YA1: I think the mental, emotional, and social aspects are actually} harder than the medical because they're easy. You just put them in a box.

These psychological distresses are common and caused by a sense of failure from disappointing blood glucose levels, frustration associated with changing habits, and fear of complications, hypoglycaemia, and hyperglycaemia. Positive coping strategies, such as focusing on the present or choosing to exercise, were more beneficial for glycaemic control than negative coping strategies such as avoidance or 'all-or-nothing thinking' (Ho, Berggren, \& Dahlborg-Lyckhage, 2010). It was suggested that physical aspects are the ones that health professionals can easily monitor and advise on, but the lived experience and psychosocial challenges can be harder to understand for those who have not experienced diabetes first-hand.

\subsubsection{Engaging in diabetes management}

Engagement has been described as both a process and a state, with a continuum from tolerating management to a more active, committed collaboration (Bright et al., 2015). As part of the process of engagement, experiencing a defining moment was developed. Additionally, further aspects of engagement, including being proactive and setting goals, were discussed. 


\subsection{Experiencing a defining moment}

Some participants described a defining moment that prompted them to engage in their self-management. Moments included being thrown into responsibility at a diabetes camp or a blood glucose meter displaying ' $\mathrm{HI}$ ', too high for the meter to accurately read.

A4: I did my first injection at diabetes camp when I was $10 \ldots$ My camp counsellor said, 'Right. I'm not going to do your injection for you'. So I had to do it by myself. . . I've been doing them ever since.

Health professionals in Chapter 4 also described an array of these defining moments. While they were common, it was hard to predict what they might be for individuals.

\subsection{Managing proactively}

Being engaged was also about taking an active role, managing proactively rather than reactively. For one participant this meant being able to do anything in life, but recognising he first needed to manage his diabetes. For another, having a CGM might help her act proactively based on blood glucose trends. She was testing up to 25 times per day to visualise trends.

\subsection{Working towards goals}

For some, part of being engaged was working towards explicit goals. Two of the participants described having blood glucose ranges they strived to stay within daily, and how they gave this regular thought. The insulin pump was helpful for achieving this, as users could input the desired level and calculate corrections to match. Others had a goal for maintaining or reducing their HbAlc (an average of blood glucose levels for the previous three months), which was tested at appointments with health professionals. Having this explicit goal for $\mathrm{HbA1c}$ encouraged one adolescent who was frustrated by her high levels, who administered insulin to minimise their impact on her goal. She would eat to counter lows, not using these purposefully to balance highs.

In contrast, others were less explicit about goals, thinking about reducing levels and increasing exercise, but not fully committing. More effective goal-setting 
strategies may be necessary for these adolescents (McPherson, Kayes, \& Kerston, 2014). Finally, while one participant had no interest in setting goals, another explained he did not need to set goals as his current self-management was effective.

\subsubsection{Fitting diabetes around rest-of-life}

While the diabetes regimen was intensive, there was a compelling narrative about managing diabetes around the rest-of life, rather than the reverse. This meant that adolescents could live their lives the way they wanted, whether it be taking part in activities or eating desired food, and adjust diabetes management to suit. An example was having any meal and then matching insulin to the meal, rather than having to match a meal to a standard dose of insulin. Parents of adolescents discussed how the diabetes team endorsed this, and their relief at realising this was possible.

A1 Parent: The best thing we ever heard at hospital was 'we can wrap diabetes around your lifestyle; so you can just live normally. You can eat everything you normally eat. You can carry on with life and we will adjust the doses to suit'.

Nevertheless, adolescents made lifestyle changes to accommodate diabetes management, including changes to diet (e.g., minor changes to a 'common sense' diet, or major changes such as significantly reducing their carbohydrates intake), making sure they had necessary technology and food on hand, testing and getting blood glucose levels to a comfortable level before exercise, and not eating unhealthy foods with peers, or eating them but covering them with insulin. Adolescents could participate with their peers, but this often required prior preparation.

YA7: I could go out with my friends, I could drink when I was old enough to... But my friends could just leave home and go out and have a really rapid night. I couldn't; I had to prepare myself. I had to take Mentos with me. I had to have money on me, especially for food. I had to be aware of how I'm putting the alcohol in because of how it affects diabetes. 
There was often preparation necessary for events such as the evenings out described above, but ultimately the adolescents could participate if they desired. However, this preparation took time where adolescents were not fully participating in activities, and this was challenging for spontaneity.

A8: The other day me and my friends felt like some donuts in town and they were already digging in eating them and I had to sit there and inject which would have taken maybe three minutes to take my blood sugar and do everything and inject or get a new needle on ... So, you feel like you're missing out a bit.

Furthermore, this adolescent described feeling left out when he needed to manage his diabetes during exercise. While he was managing successfully, he was impacted socially. Another example was leaving class early to test before school break times, and choosing to miss class rather than social break times. Socially, there could also be pressure to keep up with the school timetable, rather than managing when necessary. Eventually, spontaneity was more achievable as adolescents' confidence in the management abilities grew, or as they changed to a medical device such as an insulin pump that better facilitated this.

YA1: Before the pump I used to take a long-acting insulin at night and it would last 24 hours. With the pump you don't have that; you just have the short acting insulin ... My friend will call me ask, 'Oh, do you want to go somewhere?' and then you're like, 'Yeah'. You just reduce the insulin... It gave me a lot more flexibility, spontaneity, and confidence as well.

Blood glucose levels were more stable on days including exercise, however, managing glycaemia during exercise was difficult, especially as feelings of exertion could be mistaken for hypoglycaemia. Also, adolescents were sometimes required to exercise with supervision as a safety precaution, eliminating individual pursuits such as orienteering. Managing and anxiety about hypoglycaemia negatively affected adolescents' participation in exercise. 
YA2: Initially I was really scared to do exercise with the diabetes... I

used to go jogging up the hills, but then when I was diagnosed I

started just walking them instead... I was also scared to do anything

more intensive than that because I didn't want to end up having

hypo's halfway through the night.

The balance between diabetes management and other aspects of life could shift.

Changes came about through life transitions such as moving out of the family home, and fluctuating mental health. While adolescents partook in the narrative of managing diabetes around life, many did make lifestyle changes. However, adolescents also chose to take part in activities rather than follow diabetes guidelines when the two were in conflict.

\subsubsection{Developing independence}

Adolescents with type 1 diabetes were developing independence as per normative adolescent development, in addition to their diabetes management (Michaud et al., 2007). There were processes of getting accustomed to medical devices, developing independence, and transitions of responsibility from health professionals and parents to adolescents. While health professionals described this process in Chapter 4 , because many of the adolescent participants were diagnosed at age nine or older, their experiences differed from those described.

\subsubsection{Developing independence}

Adolescents recounted their initial experiences using medical devices; many not wanting to, but doing so regardless. This often occurred in a hospital setting if they were old enough to begin self-management at diagnosis. Some adolescents used the 'popper' or 'rocket' for injections to ease this process. These are devices that can be placed around insulin pens, syringes, and infusion sets. They hide the needle from view and use a button push to administer the injection. Many participants described fearing needles initially, however, they became accustomed to them. For others the experience was more positive, and there were bribes and presents when initially using devices. 
Some adolescents felt independent from diagnosis, whereas for others, this feeling developed over time. One young adult described feeling that she lost some of her independence at diagnosis, but with years of experience, had gained independence.

YA1: The way that I lived my life changed... I felt a lot less independent; much more reliant than other people my age on my parents to do stuff for me... It took me a good four to five years to actually feel completely comfortable doing what I did on a day-to-day basis ... Having the confidence to be like 'this is who I am, stick it'. Just being confident in my decision-making as well.

Independence was viewed as an important part of self-management of a long-term condition. YA3: "It's about teaching people to manage their own condition rather than you doing it for them."

Changing medical devices could also impact on independence. For instance, when one adolescent began using an insulin pump, he felt emancipated. It gave him confidence and allowed him to participate in activities with peers more so than on injections, though there was a learning period involved.

A5: When I first got [diabetes] my parents were still heavily involved but I think they were much quicker to respond to my independence with the insulin pump... There's much more flexibility with it and with that comes independence and being able to hang out with friends and partake in more of what they are doing.

\subsubsection{Transitioning responsibility from health professionals}

Adolescents recounted how reliant they were on health professionals soon after the diagnosis. Over time they began to take on responsibilities, and have more input during medical appointments. There could be tensions, however, between the personal, lived experience of the adolescent and the health professionals' guidelines.

YA7: As you get older, you don't just attend your diabetic appointments, you actually speak. You try to understand where this 
expert is coming from because they've studied it . . I didn't see eye-toeye with them, when they tried to tell me what I could and couldn't do. I asked them, 'Do you have it? Do you live with diabetes? This might work for the general diabetic community, what you're trying to get me to do, but it's not working for me'.

\subsubsection{Transitioning responsibility from parents}

Four of the 16 participants were diagnosed between one and seven years. For these participants, their parents were initially the primary managers of diabetes. For other participants diagnosed at older ages, some took responsibility immediately, and some transitioned more minor responsibilities. One parent admitted that initially, she could not envisage the day when her daughter would be responsible for diabetes, or able to be left alone. However, over years they have transitioned responsibility, and the parent has built trust in her daughter's independence and decision-making.

For some the transition of responsibility started at a young age, with tasks gradually being added. One recounted the moment he was told he would have to start taking on the management tasks as he prepared to start primary school.

Early on, parents were still doing many tasks for their children, including calculating insulin doses, testing blood glucose levels during the night, and coming to school to do blood glucose tests. For two of the adolescents, getting an insulin pump sparked a step up in taking on responsibility.

For some of the younger participants, still aged 16 and below, their parents were reasonably involved in management. Tasks parents helped with included transportation to and attendance at medical appointments, arranging and collecting prescriptions, asking whether adolescents had tested their blood glucose levels, recording blood glucose levels in a logbook, and reviewing them to plan changes to the regimen. In one family, the parents would draw up their daughter's syringe for her long-acting insulin to be administered in the morning. While the adolescent carried out the injection herself and was capable of drawing up the syringe, the parents felt they were reducing her burden. In another family, the parents would sometimes ask to test the young adolescent's blood glucose levels, but he would refuse. 
For one adolescent whose mother had been largely responsible for managing her diabetes for many years with great success, she found it was easier to ask her mother to make decisions rather than making these herself (e.g., how many carbohydrates in a meal and the correlating insulin dose). While she needed to learn herself, having such a knowledgeable mother made her less likely to do so at times.

Other adolescents were further along the transition process, at a stage where they kept 'in touch' with their parents about diabetes, but were largely responsible for management themselves.

A8: I am in contact with them. It's not like they're doing things for me. It's not like they're controlling it saying, 'Oh, take your blood sugar now. Go and inject'. I have a lot of independence with it ... I always tell them if I'm having a hypo so they can make sure I am doing the right thing or just be there in case something happened if it was really low.

These adolescents were communicating with their parents, particularly about their blood glucose levels, but were not sharing their data regularly. Instead, a parent might ask whether their adolescent had tested, or what their levels were, without requesting to see their blood glucose meter or logbook. Parents were helpful, particularly when there was an unusual event or issue with management.

A5: My parents, they are very much the cavalry. When things are getting really bad they're the ones that go, 'Alright, we need to sort this out'. So while I still manage myself, doing my blood tests and doing my corrections, if my blood glucose has been high for a really long time I will tell them, because with high blood glucose comes an issue of forgetfulness... They will say ridiculously obvious things but they're things that because of the symptoms of diabetes I will have forgotten.

As adolescents progressed from childhood, having fewer people involved in the management process could also reduce the hassle of decision-making. One adolescent now managed by herself or with her mum, whereas previously information looped between herself, a teacher aide at school, and her mum, making 
every conversation more complex. Parents and adolescents were also negotiating tensions around responsibility. Sometimes input parents gave was viewed as 'nagging' or 'harassing'. This links back to Chapter 4, where a health professional recounted an adolescent saying his mother was the worst aspect of diabetes management, with health professionals playing a role in negotiating this transition of responsibility. Though adult support is effective for diabetes management, it can feel forced upon adolescents (Dickinson \& O’Reilly, 2004).

Some parents, though, were advocating for their adolescent's independence, for instance, when they were in the care of others at school camps.

A2: When I go on camps dad sometimes talks to the teachers; the main thing is 'just don't interfere with her-she's got it. If she passes out, however, or if she needs to stop while hiking... let her stop. But the rest of the time she just doesn't need you to say, 'Have you done that? Do you need to do this?'. . I know they're worried because they don't want me to die or anything, but I can do it myself. Dad has taken to writing on the health sheet 'No effort required' or 'She's got it, let her do her thing'.

The parent above also described the importance of his daughter taking on responsibility in terms of safety, as only she had full knowledge of her actions that may affect glycaemia and may not always want to share these with her parents, particularly as she moved into later adolescence.

A2 Parent: She has a much better idea of how much food she actually ate ... including the carbohydrates in the chocolate bar which might not have been mentioned to anybody... Alcohol would be a perfect example. You don't want to be trying to manage for her when she's the one who is going to have to make those decisions when she's had a couple of beers. We haven't got there yet but we are on the edge of that. 


\subsubsection{Experiencing and managing interpersonal interactions}

Diabetes self-care actions take place throughout daily life, in a variety of contexts. Accordingly, adolescents were experiencing and shaping interpersonal diabetesrelated interactions. These interactions involved disclosing diabetes and educating others about it, managing wanted and unwanted attention, breaking social rules to manage diabetes effectively, giving and receiving support, and choosing whether to share blood glucose data.

\subsubsection{Sharing blood glucose data}

Many adolescents were sharing their blood glucose data or talking about it with their parents or health professionals. Some gave paper logs to health professionals and others relied on software for data to be downloaded from a device. Some adolescents were hesitant to share data, but the majority who participated in the research were comfortable with this.

A5: I'm happy to be honest that I've actually been really bad with my testing over the last couple of months, I've been really stressed out... Doctors are the people that anyone with a condition should be honest with, because they can help.

While some adolescents were logging their data primarily for their clinicians' benefit, some also found it helpful to review their recent management. Further, they had the contextual experience to understand more about the data than was recorded on the page. One young adult with a girlfriend also described how she would fill in his logbook at times for him, and remind him to test.

Adolescents' parents also had various levels of access to blood glucose data. In one family, the parents would look directly at their son's blood glucose data, but decided it was his responsibility to make decisions about how to manage. In another family, the adolescent shared her blood glucose levels with her parents often, mainly for their peace of mind. When some families were experiencing challenges, such as persistent hyperglycaemia, they would look at the glucose log together to troubleshoot. Parents were acting as advisors in these cases. In another family, the 
parents did not directly view data, instead asking if their son had tested and what his levels were, or if he needed any help, trusting he would relay information honestly.

\subsubsection{Disclosing diabetes}

Adolescents were choosing whether to disclose diabetes in many circumstances. For some, their diagnoses were more recent, and they had initially disclosed their condition recently. For others, they had had the condition since childhood, and they or their parents had handled initial disclosure years prior, but as they shifted social circles they had to choose whether to disclose their condition to new people. There were ranging feelings about and techniques for disclosure, and a variety of reactions in response. Adolescents were advised to disclose their condition for safety reasons. One young adult took a proactive approach to disclosure.

$$
\begin{aligned}
& \text { YA7: If I meet a person, it won't be the first thing I say but if I know } \\
& \text { I'm going to be seeing them regularly I tend to say, 'Hey, I'm a diabetic } \\
& \text { so if you see me sitting down and getting what looks like a pencil case } \\
& \text { out, I'm just doing a blood test' and 'If I'm sitting down and eating } \\
& \text { lollies, trust me, I'm not just wasting your time, I'm trying to get my } \\
& \text { sugars back up'. }
\end{aligned}
$$

For many other adolescents, however, disclosure was not handled so proactively. There could be social anxiety about disclosing and being seen as different (Warner \& Hauser, 2009). Some adolescents disclosed to people they thought essential, such as close friends or a teacher, but rarely discussed it with peers, not wanting to be seen as different or excluded from activities. Developing strategies of knowing when to disclose and how to manage this socially developed over time, with the adolescent balancing the risk of emergencies with not wanting to disclose.

Many would not bring up the condition as there could be some enacted social stigma, however, they would carry out self-management tasks in public places, and wait for onlookers to ask them about what they were doing (Schabert et al., 2013). The medical devices were a prompt for disclosure, allowing an onlooker to ask questions if they felt comfortable. Adolescents were experimenting with Goffman's 
(1963) 'moral career,' learning whether to hide or expose medical technologies in public.

A5: I will make it obvious I'm doing a blood test. I will sit there and they go, 'What's that for?' and that's when I will explain it; so kind of inviting them to have an interest in it ... for me that's definitely the preferred way of doing it.

This strategy was chosen by one young adult so as not to 'burden' people with his diabetes. In some cases, diabetes nurses had a large role in helping to disclose diabetes, as they might make a presentation or screen educational videos at patient's schools. One participant found this very helpful, while another wishes she could have opted out.

For others, however, disclosing diabetes was much easier, and had what I likened to a 'moral status' (Charmaz, 2002). YA2: "I told everyone. I was never taught that it was something that should be hidden." Disclosure could be easier if there was somebody at the same school who had diabetes, as pupils had greater knowledge. However, once adolescents left school, there were fewer built-in times for disclosure (e.g., start of school year), and nobody did it on their behalf, as had often happened at school. For one young adult, although disclosing made her nervous, she felt it was psychologically beneficial to talk about diabetes.

Adolescents' peers, families, and colleagues had differing reactions to disclosure. Some reactions were positive. Schools or workplaces could be supportive, learning basic information about diabetes and flexibly catering to the adolescent's needs. Reactions could also be quite neutral, and were often triggered by noticing medical devices or medical alert jewellery. On the other end of the continuum, some adolescents dealt with negative reactions from people who did not understand type 1 diabetes, and conflated it with type 2 diabetes.

A8: They'd say things like, 'Oh, did you just eat heaps of sugar?' or, 'How could you get it? You're so skinny,' ... I didn't want people thinking that I was some real unhealthy eater, which definitely wasn't me, since I do lots of exercise. 
These misinformed judgements could clash with the adolescent's self-identity. Adolescents were managing changes in identity post-diagnosis, experiencing impairment, making trade-offs between bodily requirements and identity, and adapting identity to include diabetes and health management (Charmaz, 1995).

Previous research has shown people to be relatively knowledgeable about type 1 and 2 diabetes, though there can be some confusion between the two conditions (Anderson-Lister \& Treharne, 2014). This can lead to stigmatisation, making the individual feel abnormal, fear peer rejection, limit disclosure, and dislike questioning, even from friends (Buchbinder et al., 2005; Elissa, Bratt, Axelsson, Khatib, \& Sparud-Lundin, 2017). Disclosure is important, as attempts to conceal the condition can result in avoiding self-management practices in attempt to be socially accepted (Buchbinder et al., 2005; Schabert et al., 2013). Finally, it was tedious having to repeatedly disclose and explain diabetes to new people throughout a lifetime.

\subsubsection{Educating people about diabetes}

Two participants felt awareness of diabetes was improving, however, many recounted instances where people conflated type 1 and type 2 diabetes, assigned blame to adolescents for developing the condition, and questioned their food choices. While some ignored these comments, others used them as a catalyst for education, explaining the difference between the conditions. However, these interactions could leave young people feeling frustrated and negatively judged.

$$
\begin{aligned}
& \text { YA2: My grandmother has type } 2 \text { diabetes so she has been given this } \\
& \text { long list of things that she should not eat. Every time I ate something } \\
& \text { she would be like, 'Should you really be having that? I don't think you } \\
& \text { should be having that'. We had a bit of an argument over the holidays } \\
& \text { because I snapped and was like, 'Nan you have type } 2 \text { and not type } 1 \\
& \text { and they're very different things. I know what I can and can't do so } \\
& \text { please just leave this one'. }
\end{aligned}
$$

Some acquaintances of adolescents learned about type 1 diabetes and its management. One adolescent was more likely to manage when with her friend, as the friend's mother had developed diabetes knowledge. 
However, while teachers received education about diabetes, they did not always understand basic principles of diabetes management. One parent discussed this in relation to the importance of letting the adolescent take charge on school trips, and not to let teachers make diabetes-related decisions.

\section{A2 Parent: We did discover that her primary school teachers at the end of having had people come every year and talk to them about it didn't understand whether they would be giving sugar when she was high or low. Essentially, they understood absolutely nothing.}

Finally, some friends and peers asked questions about diabetes, and wanted to understand what to do if their friend needed medical assistance. They also provided practical and emotional support. Before diagnosis, one adolescent and his friends used to joke about getting diabetes when they ate sugary foods. After his diagnosis, this joking ceased.

\subsubsection{Relishing attention and managing social currency}

While diabetes-related attention often was unwanted, at times some adolescents did relish the attention. One adolescent abashedly admitted that she thought having diabetes made her more interesting. When she received a new medical device, she expected her friends to respond excitedly. For another adolescent, she enjoyed getting her own hospital room with a television at diagnosis at 13-years-old and relished the attention. Another 13-year-old did not want to feel different to her peers, but had found the lift pass she was given at school to help her avoid hypoglycaemia while walking up six flights of stairs gave her social currency (Ware, Hopper, Tugenberg, Dickey, \& Fisher, 2007).

\subsubsection{Managing discreetly}

Diabetes creates a social risk for patients that has to be managed in their daily lives (Holdich, Gillibrand, \& Rodriguez, 2016). Some adolescents managed diabetes in public spaces and had no qualms about this, or developed more confidence to do this over time. YA6: "I do it in restaurants, cafés, clubs, wherever I need to eat ... If I start hiding it, I hide myself and I would feel ashamed, and that's not who I am.” 
For some adolescents, storing their medical equipment in a pencil case helped it to blend in. One adolescent took the social aspect of testing further, and had created a game where she and her friends would guess her blood glucose levels, and see who got the closest.
A2: I have got this really good game whenever I do a test, which actually makes me want to do tests. We play the guessing game which means everyone takes a guess at what I am going to be and whoever gets the closest wins. You would think that would be really unfair because I would always know what I am going to be, but a lot of the time I guess 17 and then someone says you're 11 and I'm a $12 \ldots$ I don't have to go away to do it.

Other adolescents chose to move to a private space to test or administer insulin, not wanting to do this in public settings. Some tested or injected in bathrooms for privacy, though private spaces were sometimes hard to find, resulting in delayed management. For some, managing discreetly, or waiting until they were out of an environment such as a classroom was more about not distracting their peers with noises or needles, rather than feeling embarrassed. They would try to manage during break times if possible, or discreetly during classes if necessary.

Some chose to manage in public, but had developed techniques for doing this discreetly, by doing it under a desk or holding their devices in their lap. Some adolescents wished their medical devices were more discreet, and had reservations about using them in public spaces. Visual and auditory aspects of the devices stopped them from being discreet, particularly in contexts such as exams, or at the movies.

YA1: I didn't want the pump for three or four years because it was quite visible, quite intrusive and it's always there, always connected to you. It makes you feel less normal. That's a bad word, but when you're using pens or syringes you can just put them in your bag and don't think about it ... I keep my pump in my bra so you can't see it . . I met this old lady last week and she always wears it on her hip so everyone can see the cords and everything. I don't know if I'd ever 
have the confidence to do that. I'm not sure whether it's just my

personality. I guess the initial feelings I had haven't left.

Some adolescents thought that having a device that looked like a consumer device could also make things easier, drawing less attention, though this was problematic, when the device was mistaken for a consumer device such as a phone that was not allowed in that context.

YA7: I had a bit of a run of reliever teachers who tried to take it from me because it beeped. I was just holding it, doing insulin at the desk and trying to be quiet and he came up from behind, just grabs it and then pulls on it. He looked at the cord 'cause he thought it was headphones. He said, 'Give me that phone and those head phones'. I said, 'That's not what it is.' I jumped up pretty quickly and then I went, 'Look. Attached, medical'. He very quickly apologised.

Sometimes, adolescents experienced negative reactions to public self-management. Some onlookers had needle phobias and did not like to see medical devices used in public spaces. A1: "This new friend that I have at school, she hates injections and needles heaps and she won't be in the room when I do it." This was socially difficult, but for one adolescent she found this judgement by others did not make her feel unhappy; instead, she thought it reflected badly on them.

\subsubsection{Breaking social rules and advocating for needs}

In some instances when adolescents were managing diabetes they were breaking, or appearing to break, social rules. In these situations, where management was necessary, adolescents often had to advocate for themselves. At high school in particular, rules to be broken, included eating or using phones in classes.

Sometimes onlookers also confused injecting insulin for illicit drug use. A1: "Last week, there was one girl who saw I was injecting drugs and she's like: 'Bad drugs?' 'No it's not that, don't worry'." This supports previous research, where people with diabetes perceived others to judge them negatively and discriminate against them, possibly mistaking them for drug addicts or having caused their condition by overeating. Resultantly, many of these people did not disclose their 
condition in the workplace, and chose to inject or only at home or in bathrooms when in public (Shiu, Kwan, \& Wong, 2003).

At times adolescents had to advocate for their needs, and were supported by parents and health professionals. This included access to disability allowances and countering teachers when mobile phone use was necessary.

\subsubsection{Giving and receiving support}

Adolescents were receiving support from friends and peers with diabetes, and were sometimes also providing support to others with diabetes. Support could be practical or emotional.

\subsection{Receiving support from friends}

As discussed previously, participants were disclosing diabetes to their friends, in case of emergency situations. Some friends were happy to learn the basics about diabetes and to provide practical forms of support.

YA1: We're doing Round the Bays, the run, and because I had to take crap loads of stuff in a bumbag, my friend said, 'I can carry something for you in my armband'. And I was like, 'Oh, that's really nice'. It's nice to have that backup. It's not like 'help me' but if it all turns to custard they know what to do... You need that social support.

For some people with diabetes, their friends were patient when they were feeling unwell, or needed to manage, identifying early symptoms and making management suggestions. However, two of the participants spoke about getting too much input from friends at times. While friends were trying to be emotionally supportive, their actions were not always appreciated. YA3: "It did get bloody annoying; it was too much.” A combination of family and peer support is associated with greater wellbeing among adolescents with type 1 diabetes, with peers being associated largely with emotional support (Greca et al., 1995; Skinner et al., 2000). 


\subsection{Giving and receiving support from people with diabetes}

Having peer support from others with type 1 diabetes was important for some of the participants. Making friends helped, both in terms of having extra friends, but also being able to talk about diabetes.

YA1: One of my good friends and I joke about it quite a bit. We have our complaints to each other but just in a funnier kind of way that I don't think really anyone else would kind of understand . . Making light of the situation... Have a bad day you can just flick a text.

Alternately, meeting others with type 1 diabetes could be perceived as embarrassing. Eventually, young adults adolescents found it gratifying to give back through diabetes groups and camps.

YA4: Every year I go as a leader on the children's camps and help the kids. I actually attended the camps as well so was quite nice doing a bit of a role reversal... It's one of my favourite weeks of the year. I've got a few other good friends that go as leaders as well so it's really good... I learn quite a bit still.

Analogously, being able to pass on knowledge, but also demonstrate from experience the consequences of poor management, was important for one young adult with a younger sister with diabetes.

YA7: I'd been where she'd been, unhealthy. I did not want her to have what was happening to me... She's got no diabetic friends at her school so she feels outcast at times. I try to remind her it's as easy as 30 seconds and you've done your test and your jabs and you've written it down. Just do it. Trying to keep on her 'cause I've had so much to go through and now I've got the eye problems already and I should not be experiencing eye problems at 22. Now there's evidence of protein in my kidneys... It's just stuff that gets on my mind but I don't regret having been unhealthy because it lets be aware of what can happen... I know everyone's going to make their own mistakes and you can't just drill something into someone and expect them to do it. 
Adolescents recognised that their feelings about diabetes and their management needs and abilities differed from their peers'. Some mentioned that they were grateful they were aware of hypoglycaemia, could count carbohydrates, had reasonably stable levels due to a honeymoon period, didn't have negative thoughts as much as others, and were in a habit of testing often. On the other hand, one was aware that the blood glucose meter his brother was trialling was not yet available to him. Ultimately, it was about understanding your individual needs.

YA3: I think part of management is really just being aware...

Different people need different things, so knowing your own personal needs and doing what you need to do to make sure it happens.

The categories described in the chapter are outlined below (Table 5).

Table 5

Summary of concepts, categories, and properties.

\begin{tabular}{|c|c|c|}
\hline Concept & Category & Properties \\
\hline \multirow[t]{4}{*}{$\begin{array}{l}\text { Self-management } \\
\text { overview }\end{array}$} & $\begin{array}{l}\text { Managing blood glucose } \\
\text { levels }\end{array}$ & \\
\hline & Managing risk & \\
\hline & $\begin{array}{l}\text { Responding to dissonant } \\
\text { technology }\end{array}$ & \\
\hline & Changing medical devices & \\
\hline \multirow[t]{4}{*}{$\begin{array}{l}\text { Conceptualising type } \\
1 \text { diabetes and its } \\
\text { management }\end{array}$} & $\begin{array}{l}\text { Developing and applying } \\
\text { diabetes knowledge }\end{array}$ & $\begin{array}{l}\text { Developing knowledge } \\
\text { Creating and following guidelines } \\
\text { Having the confidence to manage } \\
\text { responsibility from parents } \\
\text { Managing unusual events and making } \\
\text { mistakes } \\
\text { Requiring meaningful information }\end{array}$ \\
\hline & Developing acceptance & \\
\hline & $\begin{array}{l}\text { Conceptualising diabetes and } \\
\text { current management }\end{array}$ & \\
\hline & $\begin{array}{l}\text { Conceptualising 'good' } \\
\text { management }\end{array}$ & $\begin{array}{l}\text { Following diabetes guidelines } \\
\text { Prioritising diabetes or the rest-of-life } \\
\text { Judging effort or results } \\
\text { Feeling physically and mentally healthy }\end{array}$ \\
\hline
\end{tabular}




\begin{tabular}{|c|c|c|}
\hline & $\begin{array}{l}\text { Engaging in diabetes } \\
\text { management }\end{array}$ & $\begin{array}{l}\text { Experiencing a defining moment } \\
\text { Managing proactively } \\
\text { Working towards goals }\end{array}$ \\
\hline & $\begin{array}{l}\text { Fitting diabetes around rest- } \\
\text { of-life }\end{array}$ & \\
\hline \multirow{3}{*}{$\begin{array}{l}\text { Developing } \\
\text { independence }\end{array}$} & Developing independence & Getting used to medical devices \\
\hline & $\begin{array}{l}\text { Transitioning responsibility } \\
\text { from health professionals }\end{array}$ & \\
\hline & $\begin{array}{l}\text { Transitioning responsibility } \\
\text { from parents }\end{array}$ & \\
\hline \multirow{7}{*}{$\begin{array}{l}\text { Experiencing and } \\
\text { managing } \\
\text { interpersonal } \\
\text { interactions }\end{array}$} & Sharing blood glucose data & \\
\hline & Disclosing diabetes & \\
\hline & $\begin{array}{l}\text { Educating people about } \\
\text { diabetes }\end{array}$ & \\
\hline & $\begin{array}{l}\text { Relishing attention and } \\
\text { managing social currency }\end{array}$ & \\
\hline & Managing discreetly & \\
\hline & $\begin{array}{l}\text { Breaking social rules and } \\
\text { advocating for needs }\end{array}$ & \\
\hline & Giving and receiving support & $\begin{array}{l}\text { Receiving support from friends } \\
\text { Giving and receiving support from people } \\
\text { with diabetes }\end{array}$ \\
\hline
\end{tabular}

\subsection{Discussion}

\subsubsection{Psychosocial user requirements of medical technologies}

From the grounded theory analysis above, 16 psychosocial user requirements of medical technology were identified. One new user requirement was constructed. The sub-categories in which the requirement was identified are listed in italics. 
1. Facilitate reliable and efficient night time management: Managing blood glucose levels, Responding to dissonant technology, Transitioning responsibility from parents

Medical technology allowing adolescents to manage confidently and efficiently during the night. Adolescents were weary of nocturnal hypoglycaemia, but also needed to manage efficiently to reduce tiredness the following day. An example that makes this challenging is blood glucose meters with no backlit screen or test strip port lights.

16 User requirements constructed in previous chapters were identified in this study:

1. Allow accessibility and affordability: Changing medical devices

2. Foster trust in accuracy and reliability: Managing risk, Responding to dissonant technology

3. Minimise psychological distress: Managing risk, Responding to dissonant technology, Developing acceptance, Conceptualising diabetes and current management, Judging effort or results, Feeling physically and mentally healthy, Giving and receiving support

4. Communicate to inform realistic expectations: Changing medical devices

5. Provide choices, customisation or personalisation: Changing medical devices

6. Perform in a life-proof manner: Responding to dissonant technology, Changing medical devices

7. Enhance convenience: Managing risk, Changing medical devices

8. Facilitate participation in everyday activities: Following guidelines, Prioritising diabetes or rest-of-life, Fitting diabetes around rest-of-life, Developing independence, Transitioning responsibility from health professionals

9. Provide meaningful information: Responding to dissonant technology, Judging effort or results, Managing proactively, Working towards goals

10. Couple behaviours with consequences: Managing risk

11. Facilitate daily structure while allowing for spontaneity: Managing blood glucose levels, Provide meaningful information, Managing proactively 
12. Facilitate realistic goals and improved management: Prioritising diabetes or the rest-of-life, Working towards goals

13. Make disclosure optional: Disclosing diabetes

14. Not being embarrassing or stigmatising: Relishing attention and managing social currency, Managing discreetly, Breaking social rules

15. Support transition of responsibility: Transitioning responsibility from health professionals, Transitioning responsibility from parents

16. Facilitate interaction with diabetes peers and friends: Receiving support from friends, Giving and getting support from peers with diabetes

These requirements are developed throughout the thesis, and reported in full in Chapter 11.

\subsubsection{Findings}

As per the aim of this chapter, the findings in this chapter complemented those of Chapter 4, helping to saturate the grounded theory, with some extensions, new ideas, and contrasting perspectives present.

While clinicians described possible alignment or dissonance between adolescents and medical technologies, this was extended further, with adolescents describing the psychological distress in re-learning how to manage diabetes with a new device (Ritholz et al., 2007). This paralleled the acceptance process, early feelings of 'ambiguous loss' (Boss, 2009), and the coping strategies of proactive or reactive emotional management (Greca et al., 1995; KyngÄs \& Barlow, 1995; KyngÄs et al., 2000; Seligman \& Maier, 1967; Stanton, Revenson, \& Tennen, 2007; Suls \& Fletcher, 1985). The concepts of liminality and identity were also introduced (Murphy, 1987; Polgar, 2010), describing this state between illness and health with the treatment of illness, achieved through medical devices rather than the disease itself, which ultimately places people in a liminal state where they might feel isolated and their self-identity challenged (Charmaz, 1995).

Goal setting was also identified, revealing adolescents had varying opinions and strategies. However, this needs to be explored in further detail. More effective goal-setting strategies may be necessary for adolescents (McPherson et al., 2014). 
One new area arose through the interviews; adolescents had varying perspectives of what constituted 'good' self-management for them individually. For some, this was following clinician-provided guidelines, for others this was about balancing the impact of diabetes management on daily life, others differed in whether they judged management on efforts or results (Dweck, 2008), and others balanced feeling physically and mentally well.

Areas that differed from Chapter 4 related to the transition of responsibility from parents to adolescents, risk-taking, and participation in everyday activities. While health professionals described a wide range of transition behaviours, particularly around deception and data sharing, most participants in this study were diagnosed at age nine or above, meaning they were responsible for many self-care behaviours from diagnosis, leaving fewer responsibilities to be transitioned. This did not conflict with health professionals' accounts, but demonstrated the importance of gathering multiple viewpoints.

In terms of risk-taking, adolescent development is commonly characterised in this way, and was described so throughout Chapter 4 and much of this chapter (Huebner, 2009; Wasserman et al., 2017). However, illness-specific risk-averse behaviours were also evident when adolescents managed blood glucose levels, particularly taking risk-averse actions for short-term issues such as hypoglycaemia, rather than long-term issues such as running high (KyngÄs et al., 2000). However, medical technologies could reduce fears of hypoglycaemia (Barnard et al., 2012).

Additionally, adolescents provided a different point of view in regards to the daily implementation of diabetes management, with barriers to participation in everyday activities and social risk featuring prominently (Buchbinder et al., 2005; Elissa et al., 2017; Holdich et al., 2016; Schabert et al., 2013). Some had explicit strategies for disclosure that utilised medical technologies, as supported by previous research (Schabert et al., 2013; Shiu et al., 2003). Occasionally, diabetes also had positive impacts, increasing social currency (Ware et al., 2007).

\subsubsection{Methods}

Through conducting semi-structured interviews, first-hand accounts of diabetes management and suggestions for improvements to medical technology were 
garnered. The user requirements elicited from adolescents were remarkably similar to those conveyed by health professionals. The additional user requirement elicited in this chapter was facilitating reliable and efficient night-time management. This requirement spoke to adolescents' experiences of managing diabetes in-situ daily life. This aspect of diabetes management is one health professionals are aware of, but do not experience directly.

In addition to adolescents, young adults were interviewed to increase the sample size and express a wider range of experiences and preferences. As predicted, most adolescents who chose to take part were managing diabetes confidently and were engaged in self-management. Some of the young adults were now engaged and managing confidently, but could reflect on their adolescent experiences where this had not been the case, and how they had developed engagement and acceptance. The inclusion of young adults was also to reduce the participation bias common to this research topic. While this inclusion was effective, the range of engagement described by participants was not as broad as that described by health professionals, thus it is likely that the experiences of adolescents at the end of the spectrum who are not engaged in diabetes management were not represented fully in these interviews. While the transition of responsibility was a major concept within Chapter 4, because 13 of the participants were diagnosed at nine-years-old or above, their experiences were different from those described previously. The following chapters build on the elicited experiences and requirements, and explore these in more depth.

\subsection{Summary}

Three theoretical concepts were constructed and developed: conceptualising type 1 diabetes and its management, developing independence, and experiencing and managing interpersonal interactions. While these concepts were built through grounded theory, they also relate back to existing theory, and to the analysis of interviews with health professionals. There were many overlaps with the previous chapters, including 16 of the user requirements. The analysis describes processes adolescents undergo while self-managing, and makes some suggestions for medical technologies. 


\section{Psychosocial Impacts of Medical Technologies}

While the previous chapter focused on adolescents' self-management experiences, this chapter describes a card sort task and subsequent discussion conducted individually with adolescents and young adults with type 1 diabetes. This was the second stage of research conducted with adolescents and young adults, and aimed to narrow the focus from broad experiences to the psychosocial impacts of current medical technologies.

Ten previous studies were identified from a search of health literature that explored the psychosocial effects of medical devices on self-management of type 1 and 2 diabetes in children, adolescents, and adults. A variety of methods have been developed and applied in these studies, including randomised trials assessing the clinical effectiveness of using the medical device (i.e., measuring glycaemic control) with follow-up questionnaires (D. H. Barrett et al., 1995; Chase et al., 2010; Lawrence et al., 2010), a randomised trial followed by a semi-structured interview and questionnaires (Barnard et al., 2014), a phone interview (Barnard, Speight, \& Skinner, 2008), multiple questionnaires (Lindholm Olinder et al., 2007; McMahon et al., 2005), focus groups (Ritholz et al., 2007), and meta-reviews (Davis \& Renda, 2006; Korytkowski et al., 2005).

Questionnaires used in the individual studies were the CGM Satisfaction Scale, Hypoglycaemia Fear Survey, Problem Areas in Diabetes Scale (versions for both parents and patients), Social Functioning Health Survey, PedsQL-Generic and Type 1 Diabetes Module, Sickness Impact Profile, Diabetes Quality of Life Instrument, Self-Efficacy for Diabetes Scale, Diabetes Technology Questionnaire, and an adapted Schedule for the Evaluation of Individualised Quality of Life. These questionnaires and surveys generally related to quality of life or wellbeing.

Other scales, such as the Modified Barriers to Adherence Questionnaire and the Paediatric Quality of Life Inventory assess barriers to adherence and quality of life respectively, but do not directly assess the impacts of medical devices (Lancaster et al., 2010; Varni, Seid, \& Rode, 1999). While the Diabetes Technology Questionnaire does measure adolescents' satisfaction, and change in satisfaction with medical devices, it was not made accessible for use. One alternate tool, the 
Psychosocial Impact of Assistive Devices Scale (PIADS), does address the limitations referred to above and was made available.

The PIADS is a self-report questionnaire that assesses the impacts of assistive devices on users' feelings of competence, adaptability, and self-esteem, using a scale from -3 to +3 . The questionnaire has internal consistency, test-retest reliability, construct validity, and is sensitive to device stigma. Initial research also suggests that the PIADS may be useful for predicting device use and abandonment (Jutai \& Day, 2002), reflect users' concerns elicited through interviews, and accurately predicts the psychosocial impacts of devices based on anticipated impacts before initiating use (Jutai, Rigby, Ryan, \& Stickel, 2000).

The PIADS has been used in populations with multiple sclerosis, stroke, macular degeneration, hearing loss, and wheelchair users (C. Barrett \& Taylor, 2010; Huber, Jutai, Strong, \& Plotkin, 2008; Kim et al., 2012; Mann, Taylor, \& Lane, 2011; Rigby et al., 2005; Saladin \& Hansmann, 2008; R. H. Wang, Mihailidis, Dutta, \& Fernie, 2011). It has also been used more recently in a medical context to elicit adults' willingness to use telehealth services (Bull, 2015; Wiklund Axelsson \& Melander Wikman, 2016).

The PIADS has three subscales (Day \& Jutai, 1996): 'Competence' measures feelings of efficacy and device-related impacts on performance and productivity, 'Adaptability' measures willingness for taking risks and trying new things and impacts of participation, and 'Self-esteem' measures device-related impacts on happiness, self-confidence, and wellbeing (Jutai \& Day, 2002). Because the PIADS has been validated and is specific to the usability of technologies, it was used in this study.

When medical devices are used effectively, diabetes self-efficacy, treatment satisfaction, lifestyle flexibility, safety in school, sleep schedule, self-care, and glycaemic control increase, and quality of life ultimately improves (Hirose, Beverly, \& Weigner, 2012). Therefore, understanding the psychosocial impacts of medical technologies on adolescents with type 1 diabetes is important for improving technologies, and consequently, quality of life and glycaemic control. 


\subsection{Methods}

\subsubsection{Participants}

Participants were 16 adolescents and young adults aged 13-24 years. These were the same participants described in Chapter 6. Three participants were accompanied by a parent.

\subsubsection{PIADS card sort}

For this study, the PIADS was adapted into a card sort task (Steinhoff \& Still, 2013). Additionally, while the scale had previously been used for assistive devices, here it was used for diabetes medical devices. Participants were given cards labelled with the original 26 items and instructed to sort them into three piles: increase, decrease, or no effect (Figure 26), with blank cards provided for participants to add extra items.

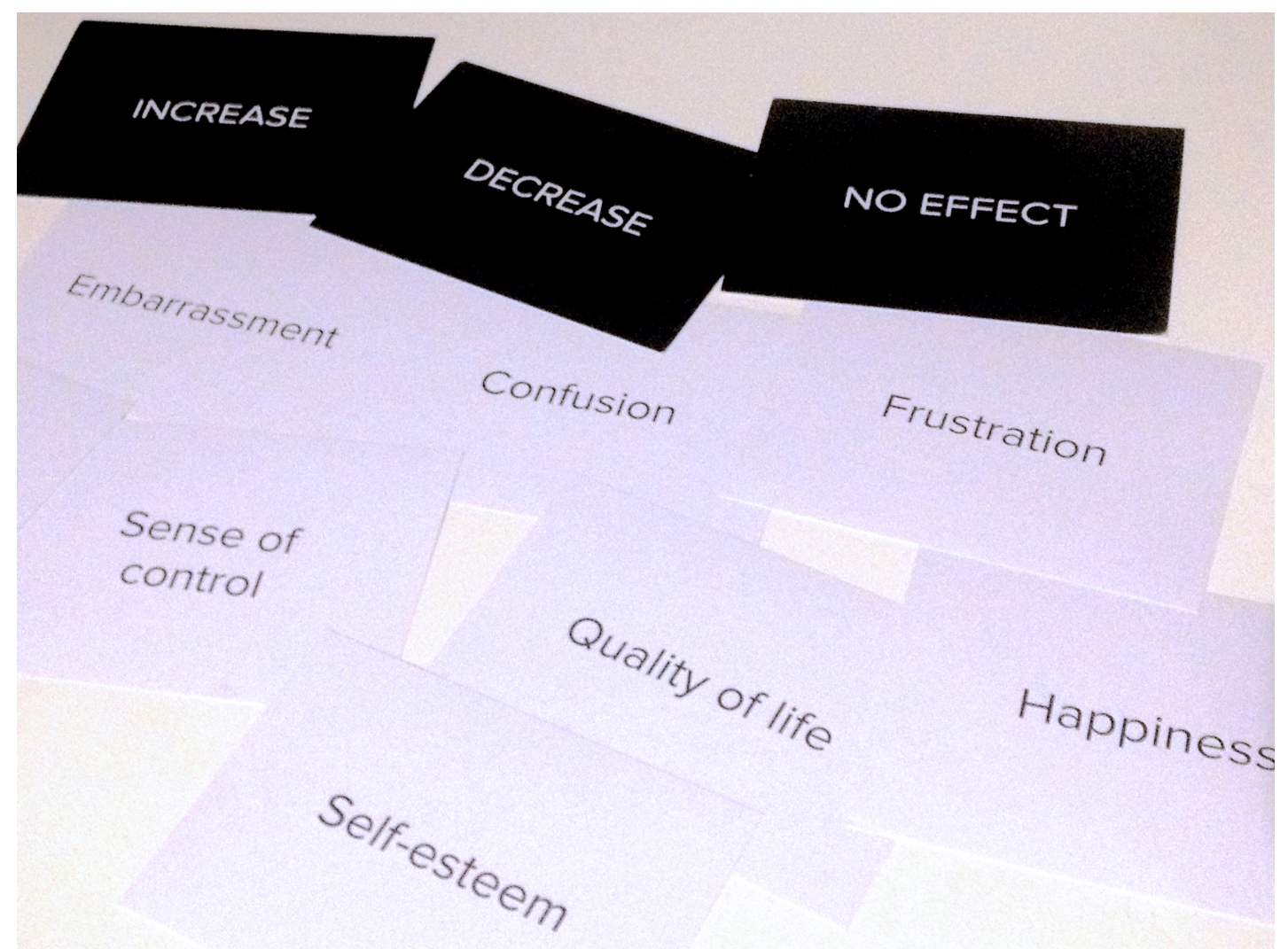

Figure 26. PIADS card sort materials. 
The card sort task retained the 26 items and three subscales of the original questionnaire (Table 6).

Table 6

PIADS items and subscales.

\begin{tabular}{|c|c|c|}
\hline Subscale 1 -Competence & Subscale 2 - Adaptability & Subscale 3 - Self-esteem \\
\hline Competence & Wellbeing & Happiness \\
\hline Independence & Willingness to take chances & Self-esteem \\
\hline Adequacy & Ability to participate & Security \\
\hline Efficiency & Eagerness to try new things & Self-confidence \\
\hline Productivity & $\begin{array}{l}\text { Ability to adapt to the } \\
\text { activities of daily living }\end{array}$ & Sense of power \\
\hline Usefulness & \multirow{7}{*}{$\begin{array}{l}\text { Ability to take advantage of } \\
\text { opportunities }\end{array}$} & Sense of control \\
\hline Expertise & & Frustration * \\
\hline Skilfulness & & Embarrassment * \\
\hline Capability & & \\
\hline Quality of life & & \\
\hline Performance & & \\
\hline Confusion * & & \\
\hline
\end{tabular}

The glossary developed with the original PIADS was available in case the meaning of an item was not understood. Responses were scored on a scale from -1 to +1 , with scores for confusion, embarrassment, and frustration reversed for their negative framing.

\subsubsection{Procedure}

Fourteen participants completed the PIADS card sort task, once for glucose monitoring and once for insulin delivery. Two participants completed the task for only glucose monitoring, and decided that their responses would be identical for insulin delivery. For these participants, the results from the first task were duplicated for the second. Seven participants completed the task for insulin delivery followed by 
blood glucose testing, and seven completed the task in the opposite order.

Participants evaluated a self-selected blood insulin delivery and glucose monitoring device. Devices reviewed were:

- blood glucose meter (15);

- $\quad$ CGM (one);

- insulin pen (eight);

- insulin pump (eight).

Though participants were instructed to sort each card into one of three piles, some participants chose to make hybrid piles (e.g., both increase and decrease) so number values and colours were assigned accordingly (Table 7). While the original PIADS forbids hybrid categories, I allowed them as it facilitated conversation about these items that were harder to categorise, and the qualitative descriptions were of high value for this study. As with the numerical values, colours (red and green) are reversed for the three negatively worded items.

Table 7

PIADS item scoring.

\begin{tabular}{ll}
\hline Response & Value and colour assigned \\
\hline Increase & 1 \\
\hline Increase sometimes, no effect sometimes & 1 \\
\hline No effect & 0 \\
\hline Some increase and some decrease & 0 \\
\hline Decrease sometimes, no effect sometimes & -1 \\
\hline Decrease & -1 \\
\hline
\end{tabular}

Following the card sort task, participants selected items of importance or interest and discussed these with myself. Each participant selected approximately six items, and 
these were generally taken from the increase, decrease, and mixed effect piles. The entire task took between 18 to 41 minutes to complete.

\subsection{Findings}

In this section, quantitative findings based on the card sort task are reported for each of the medical devices. Following this, qualitative descriptions of the PIADS items are analysed within subscales.

\subsubsection{Card sort task}

Below are graphs displaying the participants' card sort responses by medical device. For the CGM (Figure 27), a mixed impact on frustration was the only negative response. Contrastingly, for the blood glucose meter (Figure 28), at least $40 \%$ of participants felt the device had negative or mixed impacts on their feelings of embarrassment, frustration, confusion, and productivity. For the blood glucose meter, at least $50 \%$ of participants felt the device had positive or mixed impacts on their sense of control, security, ability to adapt to the activities of daily living, wellbeing, skilfulness, expertise, usefulness, efficiency, independence, and competence. The CGM had many positive psychosocial impacts and matched all the positive impacts of the blood glucose meter bar wellbeing and skilfulness, for which there was no effect. Additionally, for the CGM there were positive impacts on sense of power, happiness, ability to take advantage of opportunities, eagerness to try new things, ability to participate, confusion, performance, quality of life, capability, and productivity. For the blood glucose meter, which was reviewed by 15 participants, there was no absolute consensus on any of the items. This indicates that the psychosocial impact of blood glucose meters on adolescents is variable between individuals, and points towards the need to consider individual values and preferences when assigning and designing adolescents' medical technologies. 


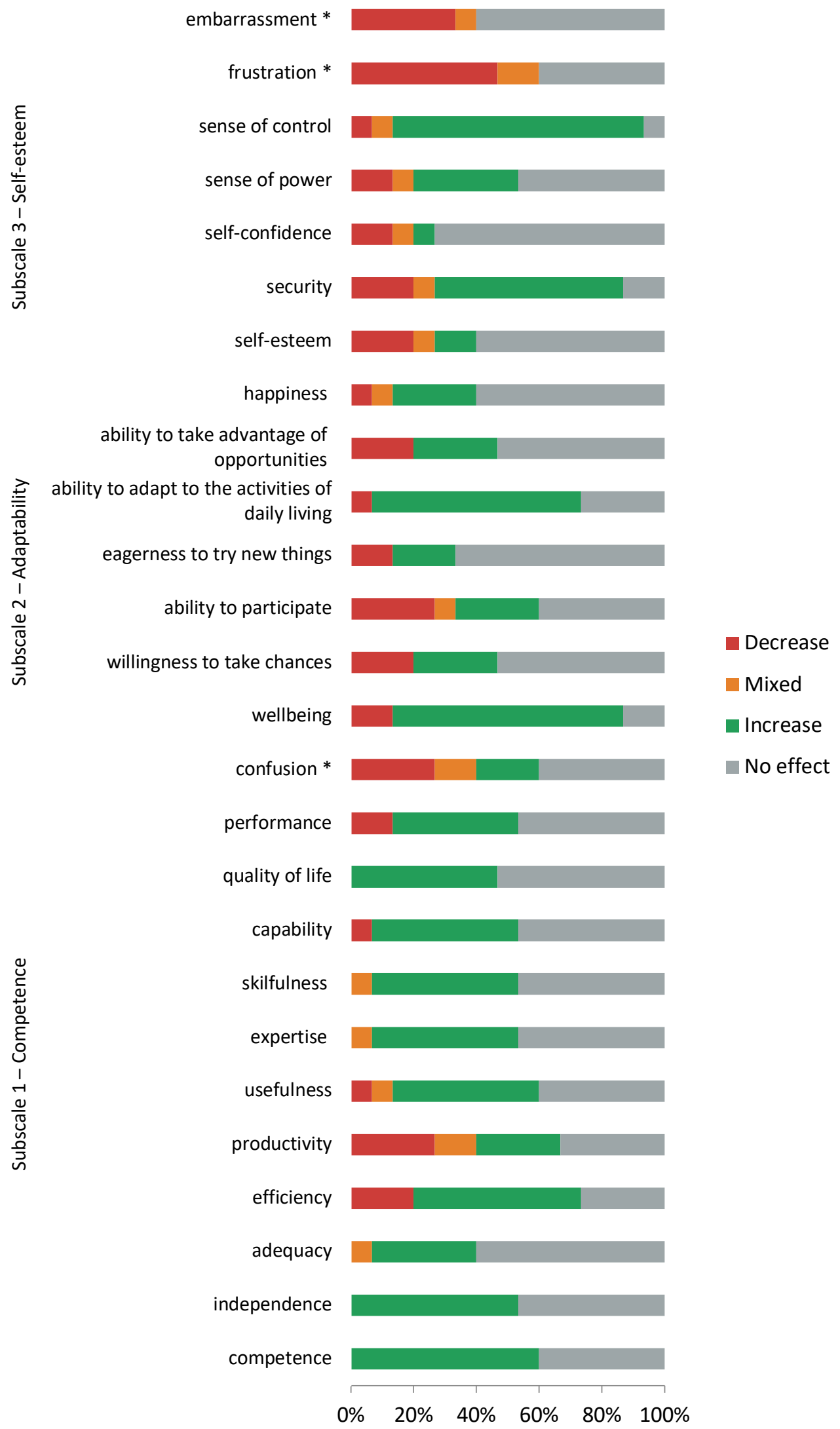

Figure 27. Psychosocial impacts of blood glucose meters. 


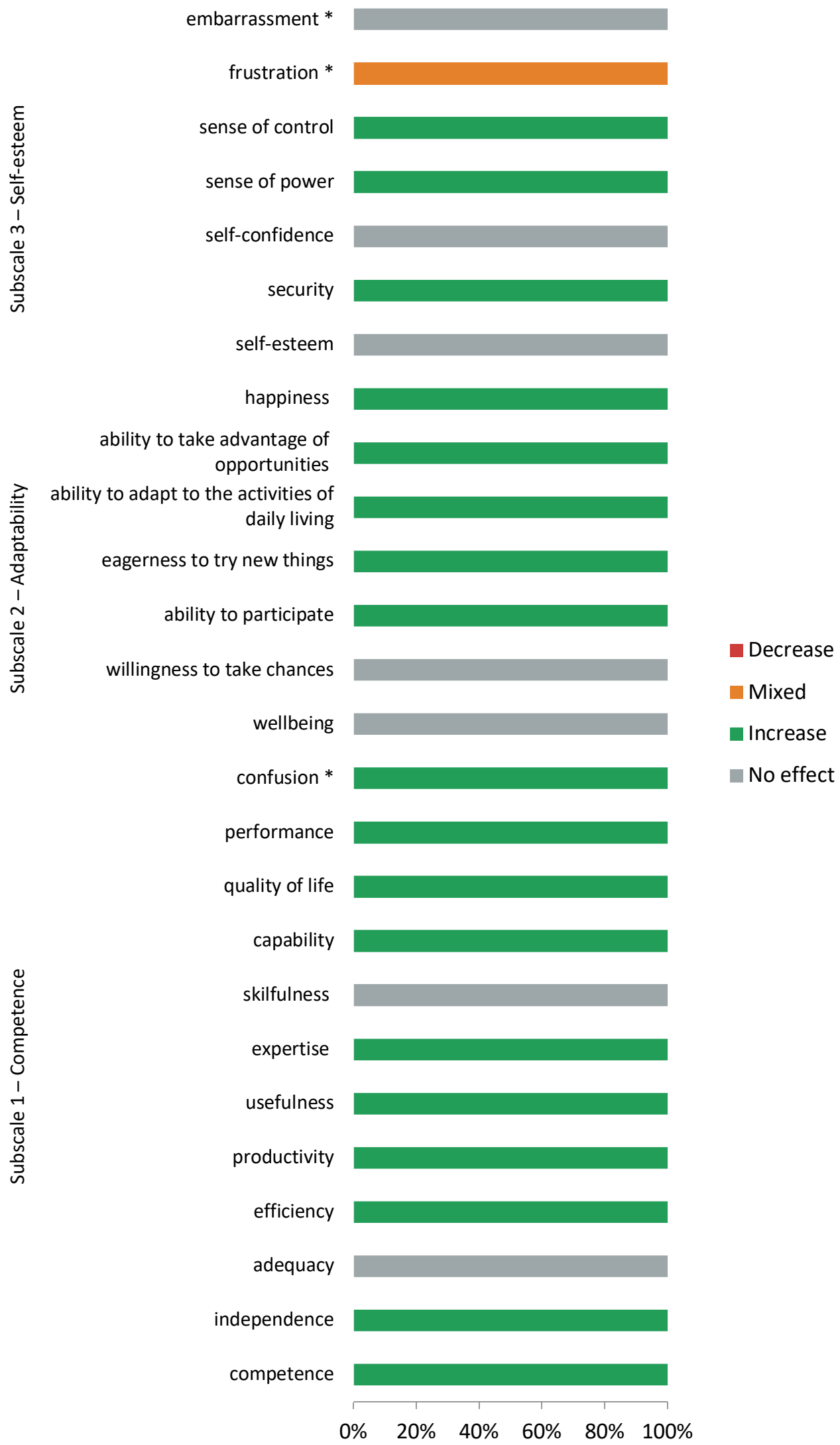

Figure 28. Psychosocial impacts of continuous glucose meters. 
When comparing the insulin pen (Figure 29) with the insulin pump (Figure 30), the pen had more negative impacts, with no mixed impacts. The most frequently reported negative impacts of the insulin pen (62.5\% of participants) were frustration and ability to participate. Twenty-five percent of participants reported a negative impact of the insulin pump on skilfulness.

Sense of control and independence were the most widely reported positive impacts of insulin pens, identified by $75 \%$ of participants. The positive impacts of insulin pumps identified were sense of control, ability to adapt to the activities of daily living, wellbeing, efficiency, and independence, reported by $87.5 \%$ or more of participants. The insulin pump has one item of consensus, with all participants reporting an increase in their ability to adapt to the activities of daily living. Again, the lack of consensus between participants identifies variation in the psychosocial impacts of personal medical devices. 


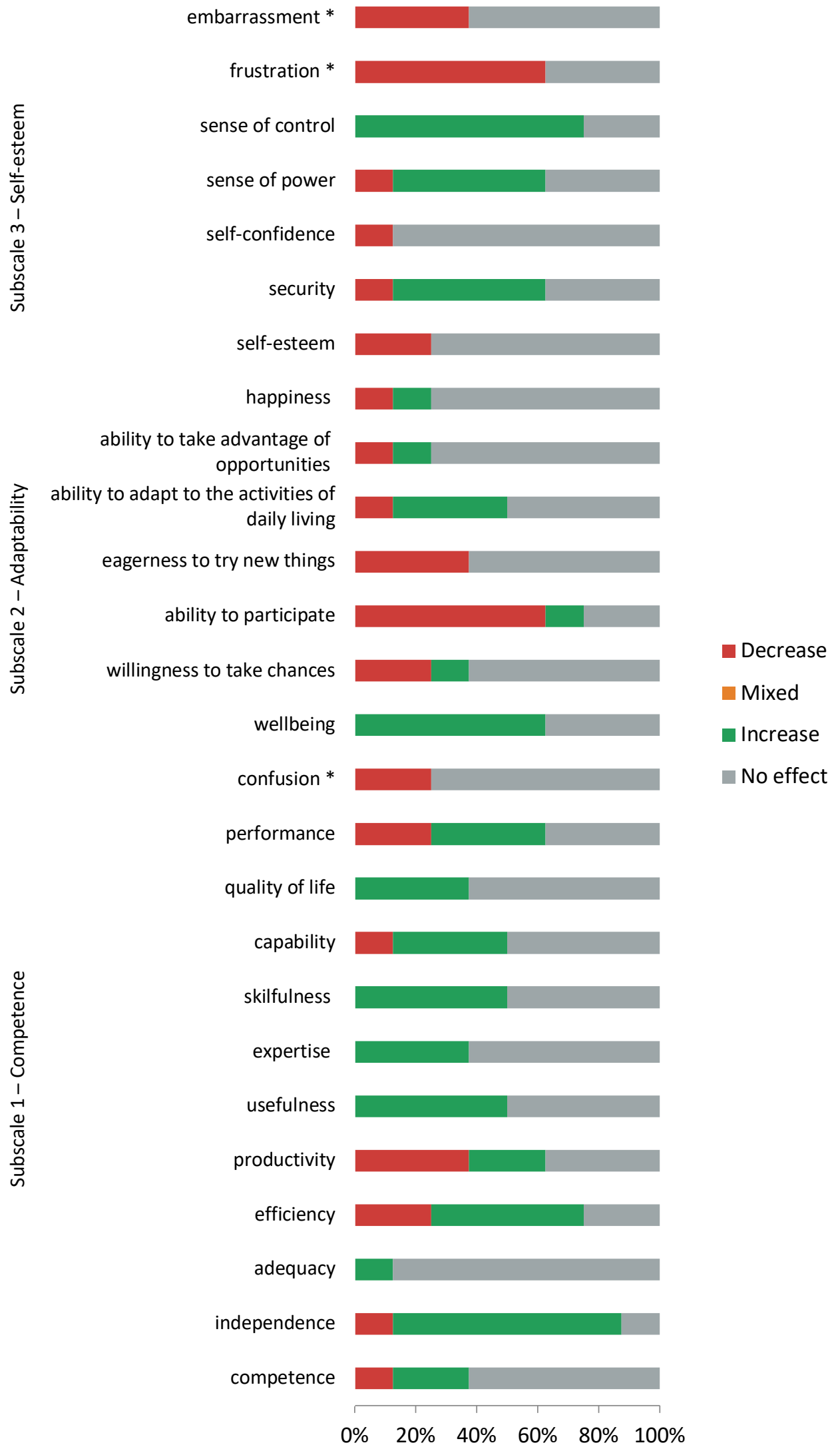

Figure 29. Psychosocial impacts of insulin pens. 


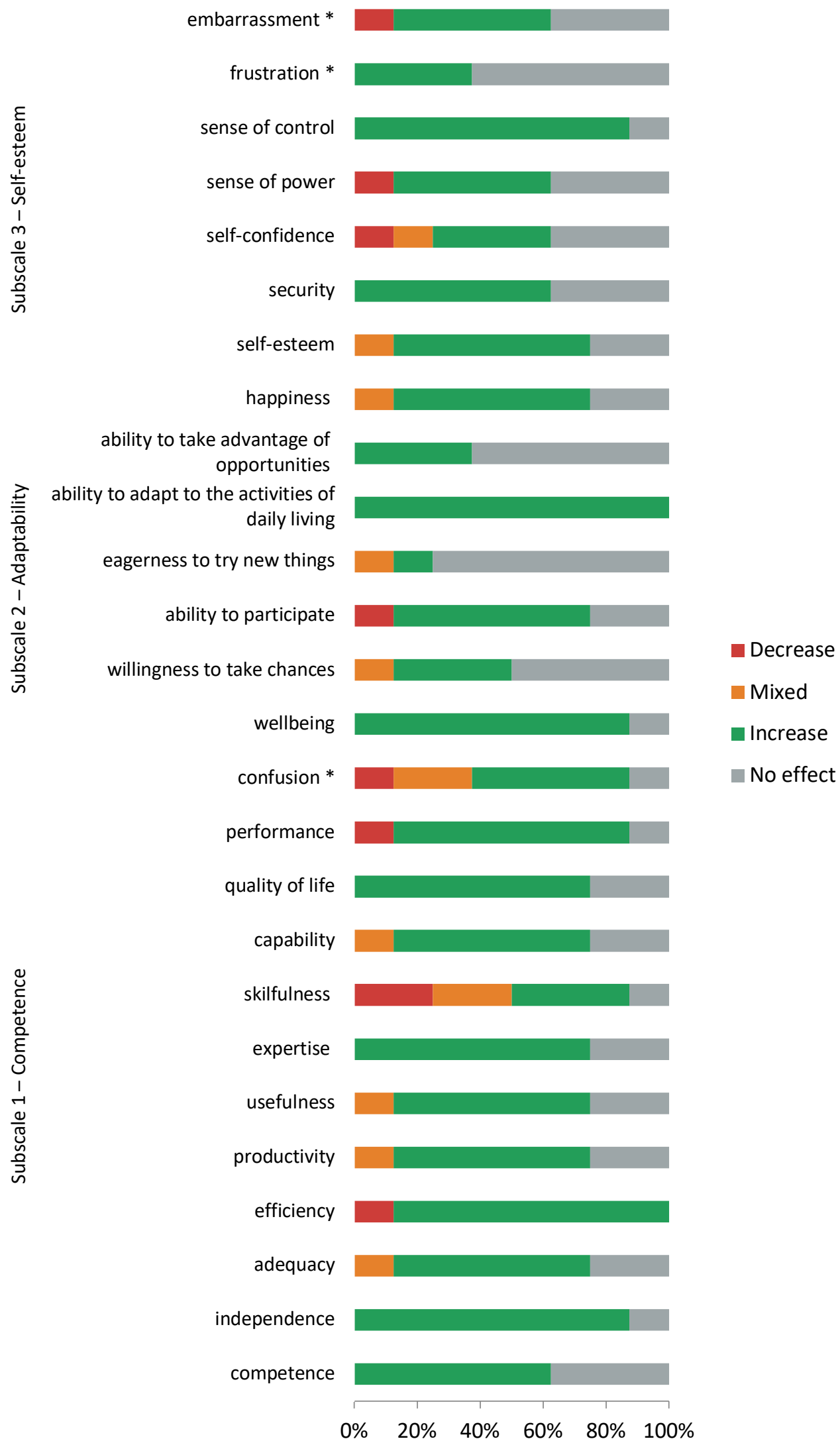

Figure 30. Psychosocial impacts of insulin pumps. 
When the results for the subscales were averaged within participants for each device, all subscales returned a positive effect (Figure 31). Thus, on average all medical devices tested improved users' feelings of competence, adaptability, and self-esteem. However, some individual participants did have negative subscale scores across all subscales for blood glucose meters and insulin pens, and for the competence subscale with insulin pumps. While the sample of participants is small, there were more positive feelings across all three respective subscales for the CGM $(M=0.83, M=$ $0.67, M=0.5)$ compared to the blood glucose meter $(M=0.34, M=0.23, M=0.08)$, and for the insulin pump $(M=0.61, M=0.54, M=0.5)$ compared to the insulin pen $(M=0.24, M=0.11, M=0.02)$. Further, positive impacts were highest for competence, then adaptability, and lowest for self-esteem across all devices.

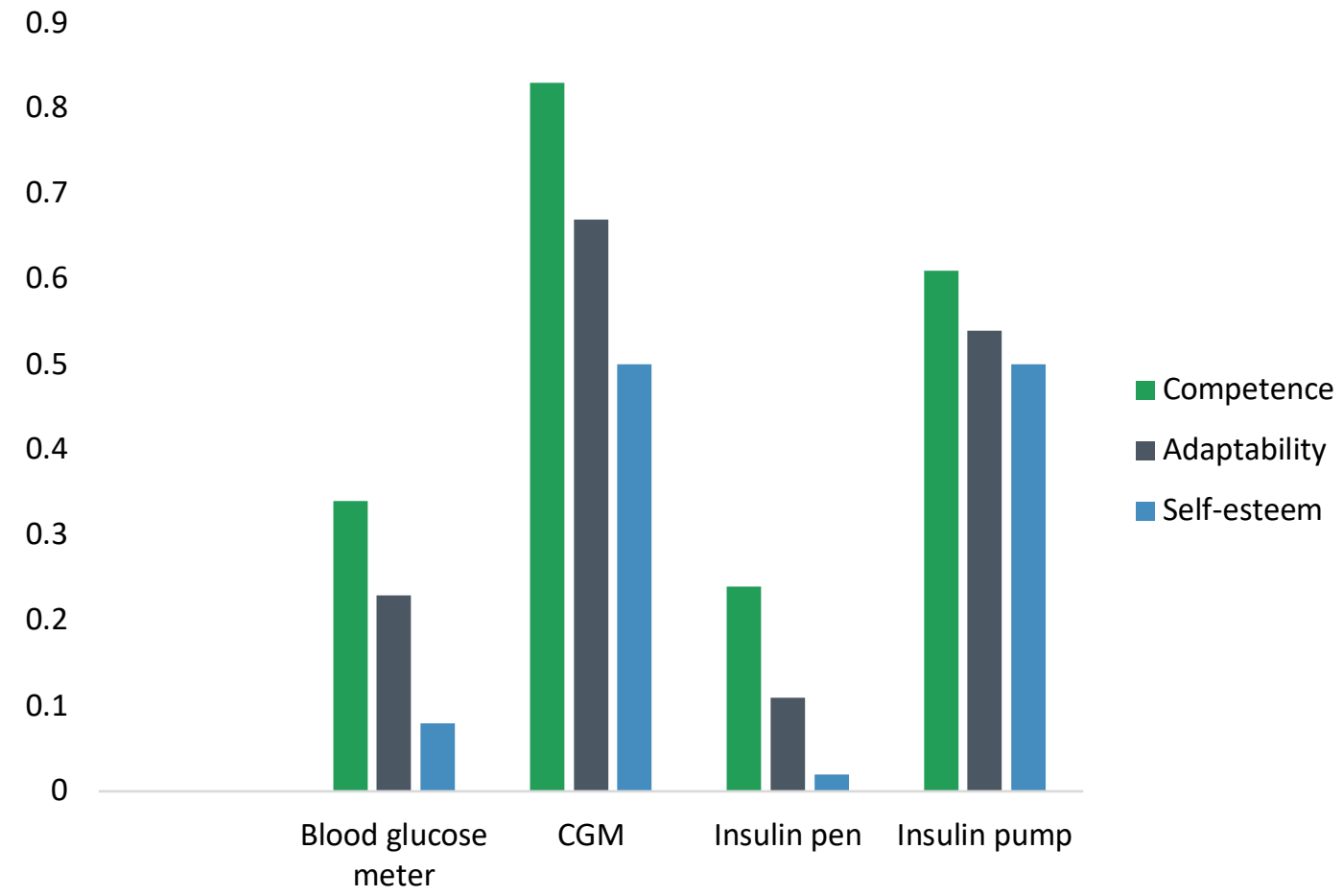

Figure 31. Average subscale scores of diabetes medical devices.

While these findings demonstrate the psychosocial impacts of medical devices on adolescents and young adults with type 1 diabetes, they do not explain why devices have these impacts. Accordingly, the following section elicits how these devices impact their users. 


\subsubsection{Card sort task discussion}

Across participants, all items (except for competence and adequacy) were selected for further discussion. Responses to each of the items are reported below and arranged within the three subscales of the PIADS. Within each item, impacts are discussed for each device in turn.

\subsubsection{Competence subscale}

\subsection{Independence}

Most participants described medical devices increasing their independence. The blood glucose meter was viewed as essential in keeping users alive. Without this, adolescents would have to rely on unreliable physiological signs. A participant described the meter as 'freeing', allowing her to go about life feeling secure; she had access to the meter if necessary. The meter also provided adolescents independence from parents and health professionals. However, there was tension evident in others viewing data and touching equipment. One participant considered the pump her life support, and treated it as a part of her body.

YA5: I am quite protective over my pump; I don't like anyone else to touch it. It would be like someone groping you in public . . My pump is the thing that keeps me alive, and if I just gave that to someone to look at and they accidentally hit a button, that could go quite badly forme.

Further, this participant thought it was best that she was independent with diabetes management, as she was the only person with first-hand knowledge of her life and diabetes. She gave an example of the manufacturer of her insulin pump warning of a safety issue. While the company's intention was that people gradually increase their insulin dose by pressing the up button, by pressing the down button, the largest dose of insulin was immediately accessible. Although the company had reportedly just become aware of this, she had been using it intentionally as a helpful device feature for correcting hyperglycaemia. While this had been convenient for the adolescent, poor interface design of infusion pumps can result in programming errors and 
mortalities (Vicente, Kada-Bekhaled, Hillel, Cassano, \& Orser, 2003). However, human factors processes can result in faster, easier, and more reliable use (Lin et al., 1998).

Insulin pumps were described as facilitating independence when compared to injections by multiple participants. They were also beneficial for unpredictable circumstances, as insulin doses could be easily adjusted. Feelings of independence and confidence garnered through self-management could also develop over years from diagnosis, and bolster more general feelings of independence and confidence in other areas of life, as supported by previous research (Dickinson \& O’Reilly, 2004), and be fuelled by a change of devices, with an insulin pump alleviating parents' anxieties about insulin delivery.

\subsection{Efficiency}

Blood glucose meters decreased efficiency as it took time to use them, preventing adolescents from engaging in other activities. While the amount of time required to test was minimal, as a participant described in Section 6.2.2.3, finding a private space in which to use medical devices could be time-consuming. While some adolescents delayed testing until convenient, others used their devices in public spaces.

YA2: Mum used to be a bit icky about doing finger-prick testing and giving myself insulin injections in public. She was always like, 'You should go to the bathroom away from everybody else and do this because you don't want to be lifting up your top in front of everyone or be stabbing yourself at a table'. She tried to sort of hurry me off to secluded places. I think if I had listened to her that would have been a decrease in efficiency. I figure if people don't like needles they can look away; which is probably not the right view.

While the insulin pen was easy and efficient to use, having to find it when easily misplaced was inefficient. Forgetting whether an injection had been administered also made for inefficient management, with one mother describing a pen with a memory function as ideal, not being aware that this device already existed and was affordable for other participants, having not been offered it. 
One participant felt his insulin pump decreased his efficiency when he had to take actions such as refilling or changing the cartridge. This could happen during school classes and be distracting, as the device would alarm. He planned to refill the device before every school exam to ensure this did not happen.

\subsection{Productivity}

The blood glucose meter improved productivity by maintaining normoglycaemia, and accordingly fostered achievement in daily life.

YA4: I can think more clearly, I can achieve more, so I can function a lot better than if I was high. Being able to test if I had a high, give insulin, and bring it down definitely allows me to achieve more.

However, time taken to use the device was a common point made about blood glucose meters, insulin pens, and insulin pumps. While the insulin pen was thought to decrease productivity, the insulin pump increased productivity, as it calculated corrections or doses, minimising the mathematics to be computed.

While the previous comments were about the user's own productivity, using the meter could be disruptive to others' activities. One of the youngest participants was conscious of how she might disrupt others, and focused on possible negative judgments about her actions. She and her mother described an instance at school after which she had completed exercise, and needed to test due to suspected hypoglycaemia, but felt she could not.

A1 Parent: You said, 'Could I first, just go into the hallway please, I think I need to eat something'. And then you got called back to do your speech before you had a chance to test. Testing then would have been great but you felt you had to do your speech; so you did that. A1: If I had a done a test, the whole class would have had to wait and would have been looking at me. They would probably wonder why I was eating lollies for medicine or why I was doing a blood test.

This reflects adolescents' preoccupation of others' perceptions of them (Huebner, 2009), and that this is strong enough to affect the adolescents' behaviour, regardless 
of whether the onlooker would actually respond in this way. This also reflects the constructivist approach to the research, recognising that perception and construction of meaning, regardless of whether these are accurate representations, have strong impacts. Adolescents may engage in 'mind reading', assuming they know what others think and can predict their actions (Nejati, Zabihzadeh, Maleki, \& Tehranchi, 2012).

\subsection{Usefulness}

Opinions were mixed about whether blood glucose meters increased or decreased usefulness. Often, participants described whether the device was useful, rather than its impact on their usefulness. One participant noted that while her current blood glucose meter was not very useful, her previous meter was.

YA3: Mine constantly tells me I am really, really low and generally you can tell when you are really, really low, and I will feel completely normal. Also sometimes they don't work when they're too hot; alternatively don't work when they are too cold. The old machines worked awesome. You can still get them. Pharmac has cut the funding for them. Their test strips are bloody expensive. . If you can't trust that what you are being told is true you can't manage as well ... I rely way more now on what my HbAlc is.

Accuracy was vital for blood glucose meters so users could trust their results and make necessary decisions. Another participant had used a blood glucose meter that synced with his smartphone, and had found this preferable, but too expensive to continue using. Also, with the lancet and test strips all contained in the same device, he was wary of losing it. Having the data on his phone was helpful, and he valued the app's reminder alerts to test as otherwise forgot to do so. Insulin pens were also useful for their portability. While they were not as convenient as an oral medication, they were sufficiently compact. 


\subsection{Expertise}

Two of the participants discussed how blood glucose meters increased their feelings of expertise in understanding blood glucose levels, and how these affected factors such as mood. Also, the meters allowed people with diabetes to pass on knowledge to their acquaintances, increasing awareness and overcoming misperceptions of diabetes.

For another participant, managing diabetes had led her to a career in health. She felt positively about health professionals asking her for her opinions, and felt she could share first-hand experiences of diabetes management with her classmates. She described herself as the 'master of judgement calls' and an expert in diabetes management.

An insulin pump increased another participant's expertise, as the device was more complex to use than insulin pens or syringes. Thus, she became an expert user, and found while this required extra effort, it improved her health. This reflects health professionals' comments in Chapter 4 about insulin pumps being mistaken as something that will manage diabetes for the user, when in fact they also require attention and effort, and when effort is put in they can be beneficial.

\subsection{Skilfulness}

Through learning how to use a new technology, one participant considered that her blood glucose meter had improved her technological skilfulness. Conversely, another participant felt his insulin pump decreased his skilfulness. While the pump was helpful, as he did not have to manually calculate his insulin dose, by not practising the necessary mathematics often he felt he had become even more 'rusty'. This became problematic when his pump was not functional and he needed to use a syringe and perform calculations manually.

Another participant reported that when exercising, the movement and placement of the insulin pump reduced his skilfulness in particular tasks such as squats, running, and karate, as it was painful when others made contact with the infusion site or the pump fell from a pocket, tugging the tubing. 


\subsection{Capability}

A few participants briefly described capability in relation to their blood glucose meters. By knowing their blood glucose levels, they could take part in activities such as exercise.

\subsection{Quality of life}

Blood glucose meters could increase quality of life, as they provided users with the means to test their levels and make corrections. However, this 'peace of mind' was only present when the meter was perceived as accurate. Users reported balancing the reading with their physiological awareness if the two did not match.

Insulin pens were positive in that they were transportable, yet they were not as convenient as oral medications. While the functionality of the device also improved quality of life, pain and marks left on the body decreased quality of life.

While some adolescents desired an insulin pump, one participant did not meet the criteria for a subsidised pump, as he would not be able to demonstrate a significant improvement in glycaemic control on the pump, as his control was already adequate, limiting his choice. Other participants with a pump found it improved their glycaemic control, and this made them feel physiologically better, improving their quality of life. The pump minimised their planning, facilitated adaptability and spontaneity, and allowed them to easily correct for extra food or not enough insulin.

\subsection{Performance}

A participant described how her blood glucose meter gave her 'peace of mind' before exercising. However, when having to stop to manage her diabetes during social exercise, she felt as though she was inconveniencing her friend to the point where she would prefer to exercise alone.

YA4: I used to do quite a bit of running with a friend. There was this particular point no matter what I did I would always crash. I don't like letting people down, so I prefer to go out and do something like 
that by myself. . . It's probably safer if I go with somebody else but I just don't like saying, 'Hey, I need to stop'

Again, participants reported that the accuracy of the blood glucose meter was important, and without trust in this accuracy, they may lose motivation to improve glycaemic control.

YA1: I just don't feel like I can trust it. Thus, it's decreased my performance in terms of my level readings, which I think are a lot more varied and not particularly accurate... I've lost that mojo to really try and tighten the control because it's like 'what's the point?'.

The one participant with a CGM found that it increased her performance by keeping her within a normoglycaemic range. When she was hypoglycaemic, her decisionmaking was impaired.

YA5: I was in the kitchen and mum walked in and looked at me and she was like, 'Do you think you should do a blood test?'. I was sitting there with one of those big utility knives chopping vegetables for dinner and I was like, 'No I just need to get this done first'. Mum was like, 'I can see your hands shaking, you are white and you are covered in sweat. Put the knife down. The next thing I can see is your fingers coming off.

This demonstrates a practical form of parental support. Another participant spoke similarly about his insulin pen, explaining that it helped him stay in the glycaemic range that allowed him to function well. Conversely, the pens could be frustrating and time-consuming to use, making adolescents miss out on other activities, particularly if they preferred managing in private.

The ways in which schools catered to diabetes management was important. One family noted that while the school and teachers were aware of the adolescent's condition, only one member of the school had spoken directly to the adolescent about it. They felt this should happen more often to foster practical ideas for supporting her, such as storing glucose in teachers' desks. 


\subsection{Confusion}

One participant reasoned that blood glucose meters were straightforward to use, and as such did not increase his confusion. Since childhood his devices now worked faster and were more legible. However, other participants felt that blood glucose meters could increase diabetes-related confusion when they were inaccurate, or when users were unsure whether readings could be trusted.

\section{YA1: The reason why it can read 25 in one test and two in another test within the space of a few minutes is confusing.}

Further, trying to pinpoint and fix the source of the malfunction in the technology could also be confusing. However, two participants felt that confusing numbers were not the fault of the blood glucose meter, but stemmed from their diabetes management (e.g., not washing hands after eating fruit) and diabetes being hard to control.

Additionally, diabetes-related numbers could be confusing themselves, especially early after diagnosis, with adolescents being informed of a blood glucose range to aim for, but not aware of the actions to take for numbers outside of this range (e.g., to take more insulin or to visit a doctor).

For another participant, the meter decreased her confusion as she knew what her levels were, but the reason for the levels was still confusing at times. If she had an unexplainable high level she was also more likely to test her blood glucose levels frequently to track her trends. Additionally, testing for ketones was confusing, with some adolescents being unsure of what they are and what their impact is. With a CGM, the numbers were less confusing, because rather than focusing on discreet data points, users could see whether levels were trending upwards or downwards.

One of the younger participants thought using a blood glucose meter in public increased other people's confusion, as they wondered what the device was and she was doing with it. This reflects adolescents' preconceptions about others' perceptions of them, as discussed previously.

Insulin pumps were described as both increasing and decreasing levels of confusion. Pumps could be confusing as there were numerous variables to control 
(e.g., basal rates, temporary rates). Alternatively, one participant felt that with the pump she could calculate more exact doses, which was beneficial.

YA1: Before it was definitely a guessing game; I didn't do any math, I just guessed. I started with the sliding scale, which is when if your blood sugar is 10 you take four, if it's eight you take three... Whereas now, it's not a set number. You do what you are minus what you want to be, divided by your ISF factor, your insulin sensitivity factor, then you get a number. It's less confusing. The numbers are confusing to some people, math, but once you know it it's a lot less confusing.

Additionally, the pump could handle much of the mathematics required for the user and had helpful functions such as temporary rates, which could be used before exercising or when ill.

\subsubsection{Adaptability subscale}

\subsection{Wellbeing}

The responses to wellbeing identified medical technologies that resulted in increased wellbeing. Blood glucose meters improved wellbeing by revealing users' current levels and illuminating what they could do to improve their health. One participant described his emotional response to blood glucose readings, feeling 'frustrated' or 'confused' by some results and 'celebrating' when achieving $6.0 \mathrm{mmol} / \mathrm{L}$.

Interestingly, he described thinking about blood glucose as a graph, focusing on trends more than data points. Further, if he had high levels but was about to be active, he would not use insulin to correct, choosing only to focus on more significant corrections. For this participant, while implementing small corrections might be beneficial to his physical health, it was detrimental to his wellbeing.

Another participant described how her insulin pen increases her wellbeing, as its precision allows accurate corrections, not leaving her feeling unwell. Finally, one participant described the sense of security and wellbeing afforded by wearing his insulin pump. A7: "It just feels like everything is going to be fine if I'm wearing it." 


\subsection{Willingness to take chances}

Participants described how blood glucose meters both positively and negatively affected their willingness to take chances. Eating without immediately taking insulin was taking a chance one participant was willing to make if her levels were not above 12. Another participant noted his willingness to take chances in general terms, but that he would not take chances in more diabetes-related ways.

YA6: I'm willing to take chances, to go out and do something in terms of networking, to try and seize initiatives and whatnot. Am I willing to take a chance of let's have this really sugary thing? Probably not.

A further participant wanted to be certain of her blood glucose levels, but this involved testing up to 25 times per day, negatively impacting her rest-of-life. She did not want to take a chance with her levels.

\subsection{Ability to participate}

Most participants discussed positive or negative impacts on their ability to participate in various activities. Blood glucose meters both increased and decreased ability to participate. By checking blood glucose levels, adolescents could remedy things if necessary and resume participation in an activity. YA3: "It doesn't have to be a barrier."

For some respondents, participation was affected by the physiological feelings of being hyperglycaemic or hypoglycaemic (e.g., feeling unwell), more so than by technology directly, however, technology could help to keep users within normoglycaemia. A participant with a CGM explained:

YA5: If your blood glucose [levels] are better in control you feel better; you're more capable of doing things. I like to say that going low is the equivalent of being strangled. Not having enough glucose in your brain is just the same as not having enough oxygen in your brain. If you go really low, then are expected to function a couple of hours later, to me that's like expecting someone who has almost drowned to be functional a couple of hours later. 
However, blood glucose meters could hinder participation. One adolescent implemented a structured schedule for eating and testing and would arrange his timetable to suit, ensuring activities did not clash with testing times when possible. Planning ahead helped to minimise these conflicts, though when they did occur, it was tempting not to test. For him, participation was important. A8: "Participating means feeling included, and that's one of the key things in life or in happiness."

While testing did delay activities, at times there was a more significant delay of approximately ten minutes that followed testing if adolescents needed to eat and wait for their levels to rise. Alternately, for one adolescent, diabetes could be used an excuse if she did not want to participate in an activity.

This contrasts with another participant, who had recently found empowerment in saying "no". She previously felt she could not participate fully in activities such as drinking alcohol and dancing with friends, however, with getting her pump and maturing she felt more in control of her diabetes and less social pressure from peers. She now said "no" when she did not want to participate in an activity, whereas previously she had said "no" because she felt she could not participate due to diabetes.

One participant discussed insulin pens decreasing her ability to participate. She currently chose to move to a private space to inject insulin, though this was not always possible. This was straightforward in some environments, but timeconsuming when she had to search for an appropriate space, and challenging as she liked to administer insulin regimentally at the same time of day.

Using an insulin pump allowed two respondents to participate more in social eating and sports, giving them more flexibility. As the pump released a gradual dose of insulin, they were not committed to the full dose if they changed their activity plans.

A5: With syringes, before a football game I would have to be very careful about the amount of insulin I was given so that I didn't crash half way through. Whereas if I pump I go, 'I'm going to turn my pump off now - awesome.' And then at half time I will do a blood test and it 
might be a little high or a little low, and I will do whatever and then I can move on.

\subsection{Eagerness to try new things}

For one participant, structure and routine were important for diabetes management, and resultantly, he was less inclined to try new things. For another participant, his insulin pen did not affect his eagerness to try new things, as he attributed this to his personal characteristics.

YA7: Just because I've got insulin on board or I've gotta take it, it's not gonna stop me from going out there and trying bungee jumping tomorrow or coming to university... It's a part of my life that I've accepted. It's something that I can maintain by myself... It's not a restrictive tool... It's an extension to my body.

Of note, this participant described his medical device as an extension to his body, as did one participant when discussing her insulin pump in Section 7.2.2.1.1. Interestingly, new closed loop devices under development are referred to as an 'artificial pancreas', also drawing from a body representation. This term is being used positively, in an empowering manner, and is promising as currently diabetes management can cause a negative body image (Shaban, 2010). Further, research has shown that adolescents' concerns about body image differ as a result of whether they consider their insulin pump as separate to them or as almost part of their body (Ritholz et al., 2007).

For some other participants, their insulin pens did decrease their eagerness to try new things. Travelling was particularly challenging, as insulin had to be carried in hand luggage when flying and could not be refrigerated. There was an uncertainty and anxiety about lack of control. Also, the hassle of having to carry and use insulin pens frequently was a barrier, though one adolescent felt 'uncertainty' and 'nakedness' if she did not have her equipment with her. If she did want to try an activity she would find a solution, such as asking someone to hold her equipment.

On the positive side, using a blood glucose meter and insulin pump increased one participant's eagerness to try new things, as knowing her levels gave her 
confidence to take part, or were a sign to raise levels before taking part. This was particularly true for new or unusual situations, such as completing a driver-licensing test.

YA4: When I went for my restricted licence and my full licence the nerves really kick in. It's usually 50/50 what happens to my blood sugars. When I get nervous I either go through the roof high or completely low. So, I elevate it a bit more so I don't risk that going low. I think being at a better level and being able to test that gives me more confidence and want to go out and try all the things.

\subsection{Ability to adapt to the activities of daily living}

Some participants reported increases or decreases to their ability to adapt to the activities of daily living. Blood glucose meters were described as increasing this ability. Although an adolescent may be unaware of events that will take place during the day, they can check their blood glucose levels and manage accordingly.

For one participant, adapting to daily life was akin to negotiations between her diabetes management and rest-of-life goals and preferences. These negotiations came about when she did not have access to her insulin pen, which would usually have increased her adaptability.

YA2: I don't often plan things around diabetes anymore, but it is something that you have to be mindful of. If you know it's a really hot day, then you are going to have to take a pen with you, but you don't have a way of keeping it cool; you might have to be like, 'Actually I can go for half an hour but then we've got to go somewhere with air conditioning.' ... There were a couple of times over Christmas where I went out with family for meals and I forgot I was diabetic and left my pen at home ... I had to request that I have vegetables instead of potatoes... You have just got to make little changes to things that you would ordinarily do without thinking. 
Finally, the insulin pump was seen as a device that increased adaptability when compared to injections, because it was easy to adjust insulin doses when a user changed their plans for the day, particularly around exercise.

\subsection{Ability to take advantage of opportunities}

Participants described how blood glucose meters decreased their ability to take advantage of opportunities. One participant felt he could not always participate spontaneously if he did not have his blood glucose meter with him. If he was going out briefly he might leave it at home, then feel some anxiety or guilt about not testing if he subsequently made other plans and was not able to retrieve his meter. While he was advised always to take it with him, he felt he could rely on physiological signs to tell if he was entering hypoglycaemia or hyperglycaemia, thought this would not be totally accurate.

For another participant, it was the time taken to complete a blood glucose test that was inconvenient. In some instances, he might take part without testing his blood glucose level, but only when he thought his levels were likely high enough or he had glucose on hand. Finally, for one participant, having an insulin pump had increased his confidence to the extent that he could take a leadership role at a diabetes camp.

\subsubsection{Self-esteem subscale}

\subsection{Happiness}

When testing using a blood glucose meter and seeing test results, one participant sometimes felt he could have done better, affecting his happiness negatively. For another participant, his happiness was significantly decreased early after his diagnosis, but now he felt there was no effect on happiness.

For insulin delivery, the pen could decrease happiness as injections could be painful. With the insulin pump, though, getting a tighter control over blood glucose levels could increase happiness by avoiding the negative moods associated with hyperglycaemia and hypoglycaemia. 


\subsection{Self-esteem}

The blood glucose meter both increased and decreased one participant's self-esteem, dependant on the results she attained.

A3: It increases self-esteem because if you get good numbers you're like, 'yes, I'm doing good', and when you have bad numbers you're like, 'oh my god I hate this, I just I can't be bothered doing this anymore'.

A few other participants described how their insulin pens decrease their self-esteem.

YA7: What do people think about it when they do see me? I'm fine with blood sugars in public, but doing injections in public, people are very quick to say 'What are you doing? What's that?'.

This participant would try to manage discreetly, under a table for instance, to avoid others reacting and to avoid distracting people. After having diabetes for many years, he questioned why there was not a device similar to a nicotine patch that would gradually administer insulin. While people did sometimes react negatively to him managing his diabetes, or misunderstand type 1 and type 2 diabetes, he made a conscious effort not to dwell on these interactions.

YA7: You don't really get offended by somebody or what they say. You let it offend you ... I guess I've just got that filter where it's not that I don't care, it's just I don't care for caring about it ... I believe that living a life with diabetes really gives you just this new appreciation.

For a second participant, the insulin pen's effect on his self-esteem also centred on social interactions. As discussed in the previous chapter, in different contexts interactions around disclosure may vary. This individual had different interactions with close friends, other friends who did not yet know he had diabetes, and other individuals at school who may enquire about his actions or stare. 


\subsection{Security}

Many participants discussed the ways in which their blood glucose meters increased their security. Having constant access to a blood glucose meter, and thus being able to know one's blood glucose level, diminished fears of exercise-induced or nocturnal hypoglycaemia, made adolescents less reliant on health professionals and physiological signs (e.g., needing to urinate or feeling hungry), and gave peace of mind when trying new foods.

For one participant, however, not trusting the accuracy of her meter meant that it decreased her feelings of security. She had changed meters after a change in government subsidies.

YA1: With the change in meter I feel a lot less secure and don't have much control... I don't really trust myself with the readings because I'm always second-guessing it ... I'll test three or four times just to get an average or see if it's different.

\subsection{Self-confidence}

Only one participant chose to discuss self-confidence. He felt uneasy being attached to his insulin pump and concerned about what others might think if they saw the pump attached to him. Also, he did not want to become defined by having diabetes, so preferred that the technology look discreet. This relates back to the challenges of integrating diabetes and identity, as discussed previously (Charmaz, 1995).

YA8: Anything to make it more discreet the better... I feel like if someone knows I've got diabetes then I'm just that diabetic kid. That's why I don't really like telling people, because you can so easily be judged.

\subsection{Sense of power}

While one participant felt her blood glucose meter increased her sense of power, another disagreed. For the first participant, the meter gave her a sense of control over diabetes in the long-term, which increased her power. For the second participant, his blood glucose meter was a reminder of his vulnerability. 


\subsection{Sense of control}

Most participants discussed their sense of control. Blood glucose meters allowed adolescents to discover their blood glucose levels, which in turn could inform their decisions about the next actions to take. Taking manual control of blood glucose levels, rather than the pancreas fulfilling this task as would happen with other people, made one participant feel more in control.

Another participant commented that he had a good level of control, as although his blood glucose levels vary, he understands why. Another felt she had good control, and appreciated that she had a blood glucose meter she felt was accurate. This use of the term 'control' in a diabetes context often refers to 'glycaemic control'.

One adolescent discussed how she had days where she tried to improve her diabetes management in addition to other aspects of her life. This naïve strategy for changing her behaviour, where she attempted to change all behaviours simultaneously, always failed. This related back to previous discussions about adolescents' need for more effective goal setting (McPherson et al., 2014).

\section{A2: After I come back from diabetes camps I often have really good tests. Sometimes I just go 'you know what, this is the day I'm going to do all the tests; going to have a quick shower in the morning, brush my teeth, have a really nice outfit, eat healthy, go on a run, walk the dog, do my homework'. Doesn't last long.}

Her father recognised that she did manage well when she was away from home, partially out of necessity, and because there were activities in which she wanted to partake and perform well. This same parent conceded that he needed to transition responsibility of management more to his daughter for her to succeed. Currently, he or her mother would bolus for their daughter when she was busy, or 'nag' to encourage their daughter to manage well. Another young adult discussed the role his parents played being more logistical. They used to transport him to appointments, though he could take the bus now. However, his parents felt their efforts were taken for granted. 
Blood glucose meters allowed adolescents to deal with challenging situations, such as eating pizza or other foods that may affect their levels, testing more frequently when trying new foods, or exercising. One adolescent described how blood glucose meters could also be used to gain rewards, such as attention and juice, saying A2: "If I want myself to be low I will do lots of tests until I'm low. If I really want that juice I can get that juice.”

One participant with a CGM described how this gave her more control than a regular blood glucose meter. She would sometimes forget to take insulin with food, and with a regular blood glucose meter there was no prompt to remind her. With the CGM, she programmed it to alarm when her blood glucose reached $12 \mathrm{mmol} / \mathrm{L}$. If she had forgotten to bolus, her pump would beep approximately 20 minutes after she had eaten, allowing her to take her insulin to correct. The CGM was also helpful for acting proactively, rather than reactively, as it visualised blood glucose trends. Seeing these trends gave more information than single data points taken from sporadic tests. It was easier to decipher the cause of blood glucose levels and patterns with the visualised data, and determine how to respond. Further, she could check her trends at any point without having to prick her finger. Also, her CGM was integrated with her insulin pump, so needing to carry fewer devices was a positive factor.

When compared to not having an insulin delivery device, the pen increased users' sense of control; letting them correct high levels, eat desired foods, and develop confidence in using ratios and making calculations for insulin. The pens were also described as "really easy to use" when compared to syringes, where the insulin had to be drawn up. They were portable and reusable, and the needle did not have to be changed with every injection.

When compared to the insulin pens, users thought the insulin pump increased their sense of control more, letting them modify their insulin administration easily. Following on from this discussion, a participant described how the role diabetes plays changes from moments when management is running smoothly, to moments of difficulty.

A2: During the rest of your life, you're you, and there's tiny little things that you've got to do that remind you that you have got 
diabetes. But when you are having a crisis like that, you do the diabetes first.

Her parent reasoned that when she was not testing, although her parents and health professionals requested her to, it was at a time when she was not worrying about diabetes, which may be beneficial for her mental health. Not completing selfmanagement tasks can allow adolescents to feel as though they do not have diabetes, though this behaviour has consequences for physical health outcomes (Dickinson \& O’Reilly, 2004).

Two other participants noted how the pump allowed them more control over taking part in activities and improving their glycaemic control. One had taken up tennis and swing dancing, and valued being able to adjust her basal insulin rate with the pump, whereas beforehand, if she decided to partake in unplanned exercise with her insulin already injected, she would need to raise her levels with a muesli bar and soft drink.

A young adult described how her sense of control and independence had increased. While she hypothesised that this partially stemmed from growing up, she also felt the insulin pump had played a role, allowing her to make decisions to participate based on her desires, rather than whether she was currently hampered by diabetes.

Finally, one participant described not necessarily wanting a cure, because with technology that worked well, the impact of diabetes on her life was mainly positive, as she valued the friendships she had formed with diabetes peers.

\subsection{Frustration}

Frustration was a common impact of medical technologies. A frequently mentioned frustration of blood glucose meters was a lack of accuracy and the side effects this could have on health and management. One participant bemoaned not having access to a more accurate meter and the implications this may have on her health. Taking inappropriate actions based on flawed data was also frustrating, for instance, experiencing hypoglycaemia after taking insulin based on an inaccurate reading. 
YA8: It's so unreliable... it sends you into a hypo, feeling crap for half an hour and that's where the frustration comes in, it really pisses you off... if it was just a number that was wrong, alright, but there's so much goes on to that that number; it determines how much insulin you have, the activities you're going to do.

Another participant found the meter to be accurate, though he thought it was 'shocking' that it was only expected to be accurate within approximately $2 \mathrm{mmol} / \mathrm{L}$. To him, the difference between a $6 \mathrm{mmol} / \mathrm{L}$ reading and a $4 \mathrm{mmol} / \mathrm{L}$ reading was significantly different. Even when the blood glucose meter was functioning accurately, the results could be frustrating.

YA7: You can see a high blood sugar [level] even after you've done so much to try and bring it down and it hasn't come down. It ticks you off... At the same time, I can feel good reading that it was 6.0. That's perfect; that's what we need to be every time.

Further, the implications of test results could be frustrating. For example, nocturnal hypoglycaemia may cause the adolescent to leave bed to eat. Adolescents had to choose how to respond to unfavourable numbers.

A3: I get frustrated when I see the number on the meter and it's not what I want it to be... If I'm low and I want to go out for a bike ride, I can't ... I cross my fingers, 'please have a good number' ... If I have bad numbers sometimes I'm like, 'okay I'm going to do good and I'm going to get my number down', but sometimes I'm like, 'I can't be bothered, I'm just going to go back to bed I don't want to deal with this right now'. It depends on my mood.

The tendency to give up was described well by a commenter on a local radio show, explaining:

Our blood glucose meter can become our worst enemy and we develop a love, hate relationship with it, and will eventually stop testing our blood glucose levels. This happens when the results that we see are continuously high, and nothing we do seems to lower them. We see 
information that we think is judging us, even information that defines us as failures, and so we give up. (Hill, 2015, 1:22)

Not meeting blood glucose targets has been associated with guilt, depression, and anxiety, perpetuating a cycle that inhibits self-management (Shaban, 2010).

Contrastingly, one participant enjoyed being able to eat freely while hypoglycaemic, provided the low did not stop her from taking part in activities. While using the blood glucose meter was frustrating, she conceded that not using it led to more frustrating results. Having to carry equipment was also frustrating as the meter was accompanied by a lancet, test strips, spare needles, glucose sources, backup equipment, and such.

On the other hand, the blood glucose meter could decrease frustration by giving adolescents peace of mind that it was accessible if necessary. Similarly, the participant with a CGM mentioned that it could decrease her frustration as she could better understand what was occurring. However, the auditory aspect of the device could be frustrating in certain contexts. At night, if she was hyperglycaemic the device would beep, and she would correct with insulin. However, the device kept beeping every hour, waking her up, until the insulin had brought her blood glucose levels down. While she previously mentioned the integration of the pump and the monitor as a positive aspect, allowing her to carry fewer devices, at night this was undesirable. Though the device could be frustrating, overall she appreciated it, and the major barrier for her using it more was the high cost, only being able to use it when out of her normal routine, such as travelling, or eating foods during Christmas holidays.

Insulin pens reduced frustration by giving adolescents some control over their blood glucose levels, and decreasing their reliance on health professionals. There were, however, many features of insulin pens that could increase frustrations. For instance, the amount of equipment that had to be carried, the waste created, and that the insulin needed to be refrigerated.

YA2: There were needle covers and little green cap things all over my bedroom floor. It was terrible; people kept stepping on them. People just stopped coming to my room. 
The device having a needle could also be hazardous, for instance, while sneezing, and the bleeding and marks injecting made on the body could be undesirable, affecting body image (Shaban, 2010). Adolescents with chronic diseases also have heightened distress about common physical changes such as weight gain or acne (Wallander \& Varni, 1995). Other frustrations included the pen being painful, not functioning as expected (e.g., running out of insulin part way through an injection), and needing to vary injection sites to stop tissue hardening.

Some participants had decreased in frustration when shifting from pens to pumps.

YA1: I just feel happier and I feel less frustrated with my life. Whereas before I felt so confined by the insulin pen delivery that I was frustrated a lot of the time. I cry less than when I was first diagnosed, so I obviously feel a lot less frustrated.

The pump gave users peace of mind, making management slightly more predictable and better utilising data. One participant thought she had fewer surprising readings and instances of exercise-induced hypoglycaemia when using the pump. Another participant felt that his frustration did not stem from the insulin pump, but rather from the nature of diabetes.

However, a couple of participants felt the insulin pump was frustrating when there were device malfunctions, resulting in insulin not being delivered. Trying to counter the malfunction was important, as adolescents did not always carry backup sources of insulin, but diagnosing the fault could be frustrating.

\section{A5: I had to replace one battery and I had three spares and it didn't} accept any of the spares and I was starting to get really worried. Then my friends and I sang it a song to co-operate and chucked the battery in and it worked. Used magic on it.

\subsection{Embarrassment}

Using medical devices could be embarrassing. One adolescent sometimes went somewhere private to test or inject, and would miss the start of her physical education lessons. However, at times she would join in rather than testing, due to 
embarrassment. For her, having to carry out a self-care activity that was different to her peers was embarrassing.

Focusing on what others may think was also problematic for adolescents when self-managing in public spaces. This is an aspect of normative adolescent development, with a strong focus on developing identity, while being highly conscious of how others perceive you (Huebner, 2009). Though one participant was concerned about what others were thinking, he also conceded that no strangers had ever come to ask him what he was doing. Sometimes an acquaintance might ask what he was doing, and he would briefly explain.

YA8: There's a bit of wondering what people would think what you're doing and then it's also thinking, 'I'm not normal, I have do this'.

A blood glucose meter made sounds that could draw attention, increasing embarrassment. It beeped when inserting a test strip and displaying a reading, and not all meters could be set to vibrate instead.

Another problematic interaction was peers judging blood glucose results and questioning the user's health. One participant (YA2) recounted: "If your results are one way or the other they'll say 'Should it be like that?' Just please go away.”

For a few participants, using blood glucose meters did not make them embarrassed. However, one of the participants would sometimes postpone testing to more socially convenient times. He had also learned to advocate for his needs as he matured.

A5: I have never had the embarrassment to the point where I don't want to say anything at all; at least not when I know that there's something desperate happening. Certainly in assembly, sometimes there's a feeling of 'I don't want to do a test now because it's quite loud and everyone is being quiet.' .. I can postpone it . . and if assembly does look like it's going to be dragging on, then I would just have to pull out my test kit.

Finally, one participant was not embarrassed by her meter, and this stemmed from a moral status. For her, managing diabetes was necessary, and thus not something to 
be ashamed of. A4: “I've never felt embarrassed by my meter because I shouldn't have to be". This discussion of 'moral status' relates to the previous chapter and the ways in which chronic illness can reduce status, whereby individuals can dwell in suffering, fight the condition, or reconstruct their identities to be inclusive of the condition (Charmaz, 2002).

Describing her insulin pen, one adolescent thought injecting increased her embarrassment, and sometimes led to her moving to a private space.

\section{A1: It's kind of embarrassing having to inject myself in front of other} people. Injecting yourself is gross for some people and that's weird.

A1 Parent: It's a bit like being told to go and breastfeed in the bathroom.

Injecting around close friends was easier, as they were accustomed to it. Others would ask questions about what she was doing. In moving to a private space, she was attempting to avoid this attention.

While another participant did not find injecting embarrassing, the association of injecting with illicit drugs could be problematic. However, she described these encounters as "funny" rather than embarrassing, confident that her actions were justified.

YA3: For a while some of my friends' parents thought I had a needle drug habit. I was sitting in the lounge and before you do your shot you dial it up and make sure the needle is not blocked. They walked in while I was doing that.

For other participants, using an insulin pump could increase or decrease their embarrassment. One of the features of the pump that could cause embarrassment was the noises it emitted, particularly while in class or at the movies. One participant would hold the pump tightly between her legs to muffle the sound when she was in a quiet space. She was not particularly embarrassed about the pump, but more about being noisy in a quiet space, breaking a social rule. For others, the visibility of the pump was embarrassing, and they would try to hide it under their clothes and avoid clothing that showed the pump. One way in which a participant overcame the 
visibility of the pump while in use was to leave social areas and bolus in the bathroom. Interestingly, she felt she could make better management decisions in private, and preferred to keep diabetes as separate as possible from the rest of her life.

YA1: We go to have lunch at a table, and one of the ladies I work with pulls out a testing kit, gets out a pen, lifts up her shirt in front of everyone eating lunch. No one else cares and I'm just like 'I have to go the bathroom first', sort it all out, push all my buttons then come and sit down and have lunch ... I think I make better decisions. I find it more comfortable and also, probably, it removes it off my everyday life. I compartmentalise. That is the whole idea, like what my nurse is saying, 'Your life comes first and diabetes comes second'. Obviously, they need to interlink and you need to inject otherwise you're not very well-managed, but it is sometimes good to keep separate decisionmaking time versus just living your life time, so that it doesn't become all consuming.

This participant also struggled with disclosure, not having told a flatmate and avoiding the word 'diabetes', calling it ' $D$ ' instead. She felt seeing the insulin pump may be scary for people as it was constantly attached. For others, they felt the insulin pump decreased their feelings of embarrassment, particularly when comparing it to injecting with syringes or insulin pens. One of the embarrassing features of injecting was having to lift up a top to inject. While the embarrassment was not centred on the device, there were body image issues caused by the pen. One participant did initially struggle with body image because of the site and the marks the pump left, but came to accept and even celebrate this.

A2: You do have an ugly patch on your skin when you wear a bikini or something; that's not so nice. I was worried about that when I first got a pump but it doesn't matter anymore; it's part of me. I've got a robotic pancreas and that's friggen awesome... In Year 8 I would be like, 'I can't wear this because it shows my site', but now it's like, 'So what?'. I suppose it draws more attention; if you walk down the corridor people are going to go, 'She's got something on her stomach,' 
but then at the moment it's like, 'Yeah look at me, damn right I look good'.

Again, this use of language is interesting, describing the technology as a part of her and referring to her 'robotic pancreas.' While the pump was attached invasively, often the site was not visible. Thus, when interacting with the pump throughout the day, many adolescents found simply having to press buttons on the pump favourable to injecting or testing in public spaces. For some, this meant that they would no longer move to a private space. In some ways, the technology blended with the consumer technology other adolescents were using. However, there were sometimes frustrating misunderstandings when others did see adolescents using their insulin pumps. One participant described an interaction with a peer from school, who enquired whether the pump was a life support device for cancer treatment. Being questioned about the technology and medical condition was tiresome. Another adolescent found she was asked about her pump frequently after moving to a new high school. One participant found she received fewer questions or issues about needle phobias about her pump than devices for injections. If she did not want to talk about the device or the medical condition in detail, she could quickly end the conversation.

YA5: Everyone freaks out about needles. It's like 'thank you for telling me that you would rather die than have to inject yourself with needles'. I guarantee you that is not the case. If you're sitting in the hospital and they tell you you have to do this or you die, you tend to do it. Hate to tell you this, but you have no idea. But to just be able to pull out a pump and they ask, 'What's that for?' it's like, 'I'm a diabetic and it keeps me alive.' That's the end of the conversation.

While insulin pumps passing as consumer technology was sometimes positive, at other times, particularly when technologies such as phones were not allowed to be used in settings such as classrooms, this was problematic, as discussed previously. Finally, it is important to note that adolescents' feelings about embarrassment and their strategies to manage it may change over time. 
A2: I definitely think that having a pump is not as embarrassing as syringes, but really, it's probably just that when I had syringes I was more embarrassed about my diabetes as a person ... and the people I am friends with know more about it.

\subsubsection{Social acceptance}

The only new item added by a participant using the blank cards was 'social acceptance.' This participant felt that using medical devices in public increased social awareness of diabetes, and that this was beneficial. Sometimes onlookers enquired about the devices, but generally did not 'make a fuss.' He felt by using his medical devices in public spaces, it normalised self-care behaviours, helping others to feel more confident about managing in public spaces too.

\subsection{Discussion}

\subsubsection{Psychosocial user requirements of medical technologies}

From the card sort task above, 18 psychosocial user requirements of medical technology were constructed and triangulated. Items from the PIADS where each user requirement was elicited are displayed in italics. Two new requirements were constructed:

\section{Usability: Independence, Sense of control}

Medical technologies being easy to use. Current examples were a blood glucose meter that was easy to use while hypoglycaemic, when decision-making may be impaired, and an insulin pen that, unlike a syringe, did not require insulin to be drawn up.

2. Facilitate responsiveness to get back on track: Quality of life, Ability to adapt to the activities of daily living, Sense of control

Medical technologies designed to be responsive and easy to recover from errors. The insulin pump was helpful for conveniently correcting highs, and letting adolescents participate in unplanned exercise. 
Existing user requirements from previous chapters that were developed were:

1. Allow accessibility and affordability: Usefulness, Quality of life, Frustration

2. Foster trust in accuracy and reliability: Usefulness, Quality of life, Performance, Confusion, Wellbeing, Willingness to take chances, Security, Frustration

3. Allow comfortable or non-invasive use: Self-esteem, Sense of control, Quality of life, Self-esteem, Frustration

4. Minimise psychological distress: Independence, Usefulness, Expertise, Quality of life, Confusion, Wellbeing, Ability to participate, Eagerness to try new things, Happiness, Self-esteem, Security, Self-confidence, Sense of control, Frustration, Embarrassment, Social acceptance

5. Perform in a life-proof manner: Usefulness, Skilfulness

6. Enhance convenience: Efficiency, Productivity, Confusion, Ability to take advantage of opportunities, Sense of control, Frustration

7. Facilitate reliable and efficient night-time management: Security, Frustration

8. Facilitate spontaneity and participation in everyday activities: Independence, Skilfulness, Capability, Performance, Willingness to take chances, Ability to participate, Ability to adapt to the activities of daily living, Security, Sense of control, Frustration

9. Provide meaningful information: Efficiency, Usefulness, Expertise, Confusion, Wellbeing, Self-esteem, Sense of control

10. Facilitate daily structure while allowing for spontaneity: Eagerness to try new things, Ability to adapt to the activities of daily living

11. Facilitate realistic goals and improved management: Ability to adapt to the activities of daily living, Sense of control, Frustration

12. Make disclosure optional: Self-esteem, Self-confidence, Embarrassment

13. Not being embarrassing or stigmatising: Efficiency, Productivity, Ability to participate, Self-esteem, Self-confidence, Frustration, Embarrassment

14. Increase social awareness and normalise self-care actions and devices: Expertise

15. Support transition of responsibility: Independence, Sense of control

16. Facilitate interaction with diabetes peers and friends: Sense of control, Embarrassment 


\subsubsection{Discussion}

\subsubsection{Findings}

This research supports previous findings about the impact of insulin pumps on embarrassment, disclosure, body image, flexibility in eating, and independence (Barnard et al., 2008; Lindholm Olinder et al., 2007; Ritholz et al., 2007). However, in contrast to previous studies, I found insulin pumps reduced frustration (Ritholz et al., 2007). My results also support previous research that found insulin pumps to have overall positive effects (Barnard et al., 2008; McMahon et al., 2005), though aspects such as the pump site visibility and pump failures can have negative effects (Lindholm Olinder et al., 2007). The study also supported the conceptual model of the possible relationships among factors influencing the adoption of diabetes technology by Hirose, Beverly and Weinger (2012).

In the research introduced at the start of the chapter, positive responses to medical devices were described or associated with terms such as decreased hassle (Chase et al., 2010), perceived safety and improved sleep (Barnard et al., 2014), quality of life and health benefits (Barnard et al., 2008; Lindholm Olinder et al., 2007) improved glycaemic control (Barnard et al., 2014, 2008; D. H. Barrett et al., 1995; Korytkowski et al., 2005), increased independence (Barnard et al., 2008), increased self-efficacy and reduction in the impact of diabetes (McMahon et al., 2005), stigma (Ritholz et al., 2007), anxiety, (Barnard et al., 2014; Korytkowski et al., 2005), and stress (Barnard et al., 2008). Positive responses were associated with increased flexibility in eating, going out, bed times, and daily living (Barnard et al., 2008; Korytkowski et al., 2005; Lindholm Olinder et al., 2007), more frequent device use in public spaces (Barnard et al., 2014; D. H. Barrett et al., 1995; Korytkowski et al., 2005), devices being a tool for disclosing diabetes (Ritholz et al., 2007), and fewer injections (Lindholm Olinder et al., 2007).

Negative responses (i.e., barriers to self-management) were associated with fearing complex technology and feeling frustrated (Ritholz et al., 2007), reduced quality of life (Lindholm Olinder et al., 2007), inconvenience and fear of judgement (Korytkowski et al., 2005), injection anxiety (Davis \& Renda, 2006; Korytkowski et al., 2005), and stigma and challenges with body image and social acceptance 
(Lindholm Olinder et al., 2007; Ritholz et al., 2007). Negative responses were also associated with actions such as being awkward to carry (Barnard et al., 2014; Korytkowski et al., 2005), experiencing negative issues with alarms and calibration (Barnard et al., 2014) or malfunctions, increased blood glucose testing, and limited clothing choices (Lindholm Olinder et al., 2007).

Both insulin pens and pumps were associated with mixed psychosocial impacts (Davis \& Renda, 2006; Korytkowski et al., 2005; Lindholm Olinder et al., 2007; McMahon et al., 2005; Ritholz et al., 2007), with insulin pumps also associated with positive (Barnard et al., 2008), and neutral effects (D. H. Barrett et al., 1995). CGMs were associated with positive (Chase et al., 2010) or neutral effects (Lawrence et al., 2010), and closed-loop systems mixed effects (Barnard et al., 2014).

A clear pattern in the card sort task was the preference of the CGM over the blood glucose meter, and the insulin pump over the insulin pen, although the CGM was viewed as unaffordable or invasive for most participants, signalling that there are further opportunities for designing based on adolescents' requirements. However, another clear finding was the conflicting nature of different adolescents' perceptions of the psychosocial impacts of their medical devices. This points to the need for choice or customisation in the medical device market, enhancing the psychosocial impacts of these devices for varied adolescent users and optimising selfmanagement.

The findings of this chapter largely support the findings of previous chapters, however more detail was developed. For instance, while normative adolescent development, particularly adolescents' preoccupation with others' perceptions of them has been discussed by previous authors in a diabetes context, I characterised this type of thinking as 'mind-reading'; adolescents assuming they know what others think and can predict their actions (Huebner, 2009; Nejati et al., 2012). This demonstrates the importance of constructing meaning. Adolescents often admitted that reactions to self-management were rarely negative, however, the perception that there may be a social risk was strong enough to influence behaviour and inhibit selfmanagement.

Disclosure and self-management as a moral status was developed further in this study (Charmaz, 2002). Some adolescents morally felt that they should not be 
embarrassed to disclose or manage diabetes, unapologetic that managing diabetes was necessary, and that they should not be negatively judged for doing so. This shielded them from negative psychological impacts.

The psychological impacts of blood glucose test numbers were also explored further. Frustration was a common response to numbers that were out of range, and this could spur adolescents to improve management, or conversely, to give up (Hill, 2015). This relates back to earlier thoughts about ideal self-management, and whether it ought to be measured by results or effort (Dweck, 2008). There is room for design improvement in the display of data, to consider the psychological impact of these measurements and how they affect actions.

A new aspect constructed was around managing body image. Traditionally, adolescents with chronic illnesses suffer from negative body image (Wallander \& Varni, 1995), and medical technologies can have negative impacts on body image (Shaban, 2010). Further, research has shown that adolescents' concerns about body image differ as a result of whether they consider their insulin pump as separate to them, or as almost part of their body (Ritholz et al., 2007). In this study, participants described medical technologies as body parts, and the 'artificial pancreas' that draws from a body representation was discussed. This signals a need for research into medical technologies that could be psychologically integrated with the body, reducing negative impacts on body image or making positive improvements.

\subsubsection{Methods}

Although the PIADS questionnaire was designed for use with adults with assistive devices, it was adapted into a card sort task and used with adolescents and young adults to describe medical devices. This is promising, as initial research suggests the PIADS may be useful for predicting device use and abandonment (Jutai \& Day, 2002), and that anticipated impacts of devices correspond to the enacted psychosocial impacts of devices after device initiation (Jutai et al., 2000; Rigby et al., 2000). Accordingly, researchers could investigate whether the PIADS could be used before device initiation, helping match adolescents with suitable medical devices.

While the initial questionnaire and card sort both elicited the psychosocial impacts of devices on participants, the discussion also allowed me to understand 
which aspects of these devices impacted participants. This extra level of 'why' information is helpful in informing the design of medical devices. Whereas the initial questionnaire used a Likert scale, and thus showed the intensity of increases and decreases of each item, the card sort did not measure intensity of feeling. Although I did not create a mixed impact category, some participants struggled to classify items without this and made their own hybrid category. While this made data analysis harder, it was worthwhile for the qualitative discussions. Currently, I cannot speculate why some devices had more mixed responses than others.

As per the aim of this chapter, the task was largely successful in focussing participants to discuss the impacts of particular medical devices, shifting the discussion from general experiences of self-management to the impacts of medical technologies. Both levels of discussion contributed to answering the thesis research question. Occasionally during the discussion period, participants would shift back to discussing self-management rather than specific medical devices.

The original PIADS includes a glossary. While most items were understood without need of the glossary, many participants did not understand the meaning of the term 'adequacy'. This may have been because they were younger than the intended users the scale was designed for. If the participant asked for the definition, the description from the glossary was read aloud to them. Although they could then sort the card into the appropriate pile, none chose to discuss this item in the following activity. I hypothesised that they may not have been confident enough in their understanding of the term to discuss the item. Competence was the only other item that was not selected for discussion.

Sense of control was one of the most commonly chosen items for discussion, possibly a popular card as 'control' is a term often used in diabetes management phrases (e.g., 'glycaemic control'). In Chapter 4, one health professional used this word, then corrected herself to use the term 'influence', communicating that realistically, adolescents should strive to influence rather than fully control their blood glucose levels.

After sorting the items, where possible, participants were asked to choose items from across different categories to discuss (e.g., increase, decrease). Confusion, Frustration and Embarrassment were selected for discussion often. This may be 
because these were the only negatively worded items in the scale. Accordingly if, for example, a participant had a positive view of a medical device, these may be the only cards categorised as 'decrease', and thus more likely to be discussed. While 14 of the participants completed the card sort task once for blood glucose monitoring and once for insulin delivery, two participants only completed the task for one device, stating their answers would be identical for both types of devices, due to the devices being viewed as a system, or feeling devices had no psychosocial impacts.

As participants were required to assess whether medical devices increased or decreased psychosocial items, they needed to compare their device use to another variable. They were not instructed what comparison to make. For example, a participant with an insulin pump might compare this experience to previously using an insulin pen or syringe, or to having no access to technology. Participants selfselected the comparisons they made to better reflect their everyday thinking about their device. The participant who felt his devices had no effect did not indicate what he was comparing this to. Impacts of blood glucose meters were compared to having to manage diabetes without access to a personal blood glucose meter (9), a previously used blood glucose meter (4), and not having to test blood glucose levels (1). The CGM was compared to a standard blood glucose meter (1). Insulin pens were compared to not having access to injection devices (3), syringes (1), not needing to inject (2), and a combination of not having type 1 diabetes and not having access to injection devices (1). Finally, the pump was compared to a syringe (1), an insulin pen (4), and both syringes and insulin pens (3). These differing reference points may go some way towards explaining why adolescents had opposing viewpoints about the psychosocial impacts of particular devices; however, they reflect the comparisons each participant naturally made.

As with Chapter 6, one adolescent experienced hypoglycaemia during this task, and had to stop to test blood glucose levels and eat. The participant was willing to continue with the task once her blood glucose level stabilised.

\subsection{Summary}

A card sort task and discussion based on the items in the PIADS questionnaire were conducted with adolescent and young adult participants. The task revealed that while 
all medical devices tested had positive psychosocial impacts on average, the increase to competence was the largest, followed by adaptability and self-esteem. Further, the CGM was rated more positively than blood glucose meters, and the insulin pump more positively than insulin pens. The discussion elucidated the ways in which these medical devices have psychosocial impacts on adolescents, providing a different form of information than the original scale. There were many overlaps with the previous chapters, including 16 of the user requirements, and with existing literature on the psychosocial impacts of diabetes devices. Additionally, this method narrowed attention from broad experiences down to specific psychosocial impacts of current medical technologies. 


\section{Designing Medical Technologies from Adolescents' User Requirements}

This chapter describes a design project conducted with undergraduate industrial design students. The students developed a series of designs to visualise what medical technologies could be like if they addressed selected psychosocial user requirements developed in Chapters 4-7. These visualisations were shown to adolescents to initiate discussion about the validity of the user requirements and to investigate each of the requirements in greater depth.

\subsection{Methods}

\subsubsection{Participants}

Participants in the design experiments were 28 students enrolled in the Bachelor of Design Innovation from Victoria University of Wellington. Six female and two male participants with type 1 diabetes aged 13-24 who had been diagnosed with type 1 diabetes for between 4 months and 15 years, and who had taken part in research described in previous chapters, reviewed the designs and user requirements.

\subsubsection{Procedure}

Five of the psychosocial user requirements of medical technologies constructed in Chapters 4-7 informed a design brief: to comfortably disclose and explain type 1 diabetes when appropriate, communicate data and information to facilitate decisionmaking, minimise or eliminate feelings of stigma or embarrassment while using medical technology, facilitate daily structure while allowing for spontaneity, facilitate reliable and efficient night-time management, and facilitate spontaneity and participation in everyday activities. A limited number of requirements were chosen to make the task manageable for the design students while they were learning about diabetes management, and so they could explore the requirements in greater depth. The requirements were chosen in discussion with supervisors. These requirements were based on findings from the first six participants and reflected the necessity of managing diabetes in various contexts, and fostering a broader psychosocial 
understanding of the impacts of medical technologies. While the requirements were constructed from primary research, they also relate to existing theories and requirements identified in the literature review. Over six weeks, design students were required to design an object that facilitated adolescents' management of type 1 diabetes through addressing at least one of the user requirements, with regular feedback from myself and an Industrial Design Associate Professor.

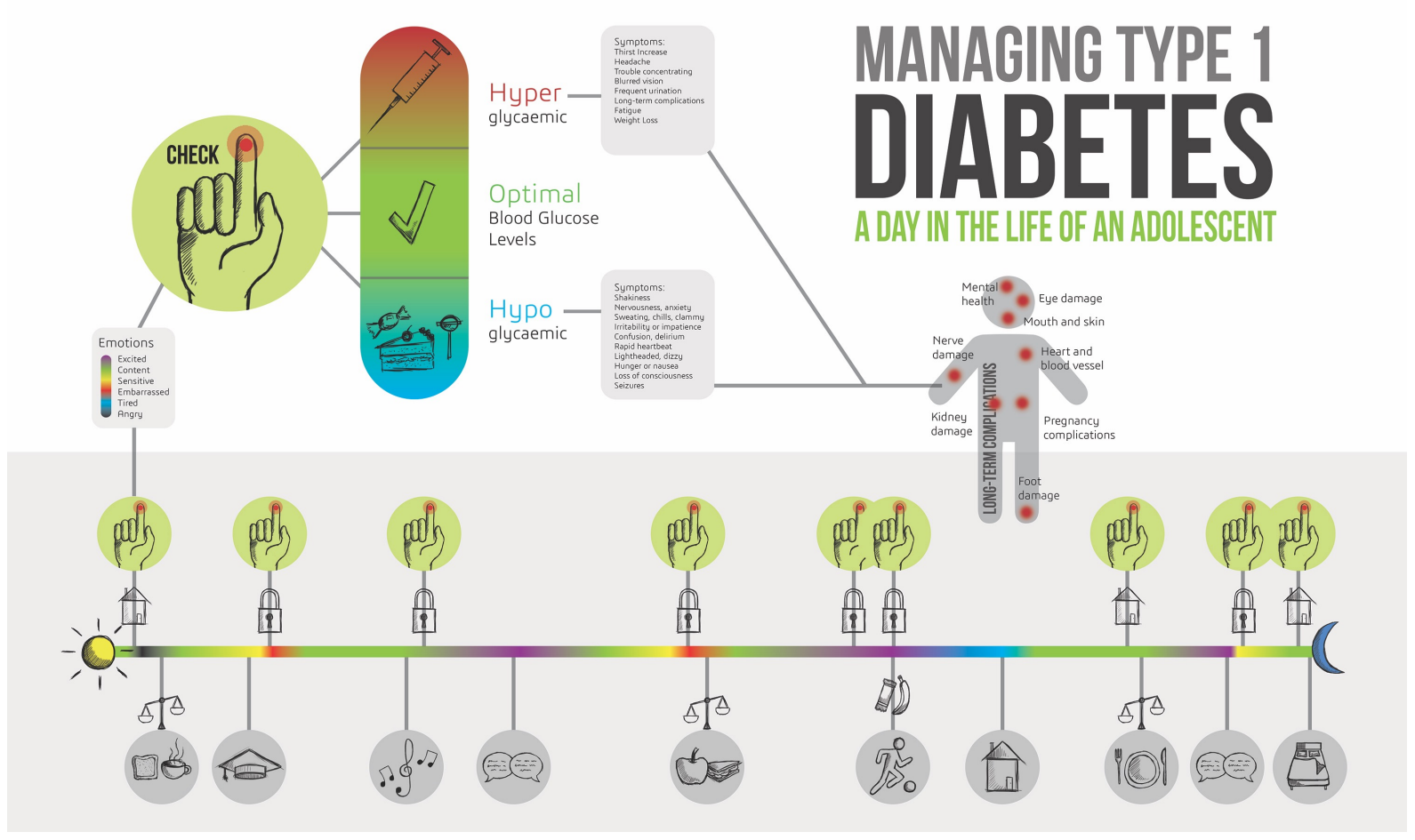

Figure 32. Infographic exploring blood glucose levels, diabetes complications, and daily self-management tasks. This student's early focus on finger pricking informed her design in Figure 37.

In week one, I delivered a lecture describing type 1 diabetes, how it is self-managed, and concepts around adherence and engagement. Students created infographics about type 1 diabetes to aid their understanding (Figure 32). In a second lecture, I introduced the selected user requirements and two personas (Goodwin, 2009). Based on the initial interviews, these personas were a sporty 15 -year-old girl using insulin pens and a blood glucose meter, and an 18-year-old female university student who was living away from home and using an insulin pump and blood glucose meter. Students selected a persona and one of the user requirements, chose an everyday context where this requirement would be important (e.g., while playing football), and sketched a scenario of how diabetes would currently be managed in this situation 
and a second scenario of how the new design could fulfil the user requirement (J. M. Carroll, 1995; Schwartz, 1992).

In week two I delivered a third lecture describing current strategies for addressing stigma through medical and assistive device design semantics. Examples included miniaturising hearing aids to a size that made them less visible but impacted on functionality, and disguising hospital equipment such as MRI and CT scanners as pirate and space ships to reduce children's anxiety and increase the accuracy of scans (GEreports, 2009; Pullin, 2009). Students designed concepts using a variety of methods for ideation, including sketching, prototyping, and acting out scenarios to create dozens of design concepts (Houde \& Hill, 1997; Stickdorn \& Schneider, 2011; Warfel, 2009). Students presented concepts based on the brief individually in week three, then were assigned groups of 2-4 members to continue developing the most successful concepts identified by anonymous student voting and academic staff review. For the following two weeks, students iteratively developed the designs.

Seven of the 11 total designs were reviewed by adolescents and are described in this chapter. Designs excluded from the review were a waist belt for carrying an insulin pump, a tubeless insulin pump with a dispersed area and low profile, a redesigned all-in-one blood glucose meter, and a smart keyring system for locating a diabetes kit. These designs were excluded as they were inadequately completed, did not correspond with the user requirements, or did not provide conversational prompts for checking support for user requirements or gaining further information.

Adapted vignettes (Figure 33) containing user requirements, quotations from prior interviews, and the designs that responded to them were presented to the adolescents with type 1 diabetes in an individual expert review (Wilson, 2014). 
"I don't really like telling people. If they have

to know, I will but... I think, I probably should;

in case I go really low, collapse or whatever."
"I told everyone. I guess I was never sort of taught that it was something that should be hidden. I have talked to a couple of people around my age with diabetes and a couple of them are like, "We pretend that we don't have it."

How might we design medical technologies that allow young people to comfortably disclose and explain type 1 diabetes to peers, teachers, friends, etc when they feel this is appropriate?

"I still struggle to tell people about it, especially new

"I like talking about it; it makes me feel people. I still haven't told my new flatmate. I've been better to talk about it to other people."

living with her now for a month. It's hard. How do you

bring it up in conversation?... It's really hard to go like, "Hey, I'm Chris and I have diabetes."

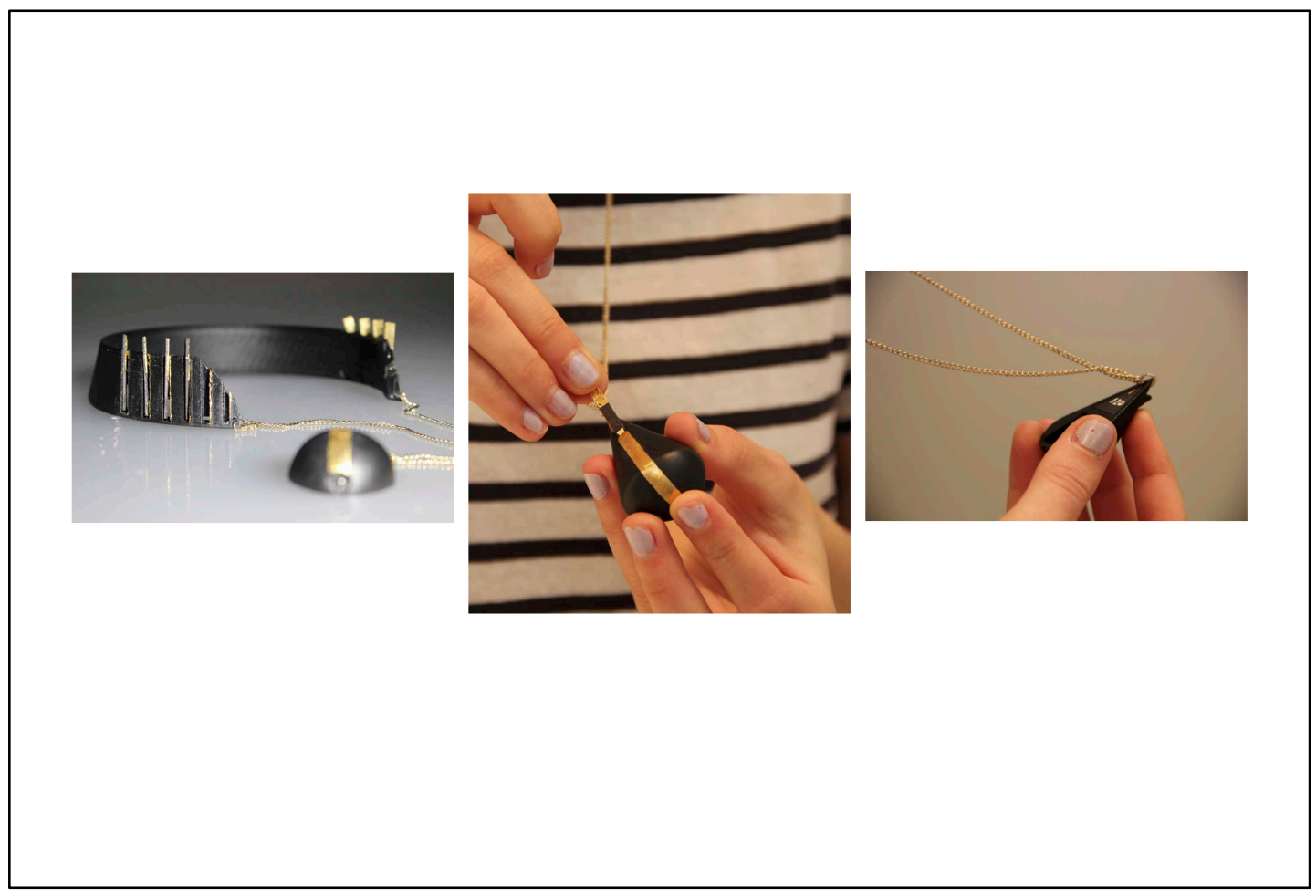

Figure 33. Vignettes displaying user requirement, related quotations form interviews with adolescents, and a related design concept.

These vignettes were based on work by Lang (2012) when conducting research with adolescents with cystic fibrosis. However, rather than including feature descriptions in the vignettes, I included the user requirements and quotes from adolescents that 
related to each requirement, and described the features of the design verbally. Vignettes portraying design concepts and stories in interviews with adolescents can prompt discussion about sensitive topics and medical devices (Barter \& Renold, 2000; Lang, Martin, Sharples, \& Crowe, 2013). Further, they contribute to an ongoing discussion about the best means to disseminate design research, whereby designed objects can be an effective means of communicating knowledge (Downton, 2003; Haseman \& Mafe, 2009; Vaughan, 2017).

The adolescents were asked to comment on whether these requirements were accurate, and on the merit of the design concepts. This was to both provide additional support for the initial analysis of the interviews and card sort task and prompt further discussion about experiences with and preferences for medical technologies.

\subsection{Design Outcomes and Reviews}

Seven of the designs, the user requirements they respond to and participants' reactions to the designs are described (Table 8). 
Table 8

User requirements addressed by student design concepts.

\begin{tabular}{|c|c|}
\hline Design & User requirements addressed \\
\hline $\begin{array}{l}\text { Blood glucose monitoring } \\
\text { phone case }\end{array}$ & $\begin{array}{l}\text { Comfortably disclose and explain type } 1 \text { diabetes when } \\
\text { appropriate } \\
\text { Communicate data and information to facilitate } \\
\text { decision-making }\end{array}$ \\
\hline $\begin{array}{l}\text { Blood glucose monitoring } \\
\text { jewellery }\end{array}$ & $\begin{array}{l}\text { Minimise or eliminate feelings of stigma or } \\
\text { embarrassment while using medical technology } \\
\text { Comfortably disclose and explain type } 1 \text { diabetes when } \\
\text { appropriate }\end{array}$ \\
\hline $\begin{array}{l}\text { Blood glucose reminder watch } \\
\text { for testing }\end{array}$ & $\begin{array}{l}\text { Facilitate daily structure while allowing for spontaneity } \\
\text { Minimise or eliminate feelings of stigma or } \\
\text { embarrassment while using medical technology }\end{array}$ \\
\hline $\begin{array}{l}\text { Blood glucose monitoring } \\
\text { compact }\end{array}$ & $\begin{array}{l}\text { Facilitate daily structure while allowing for spontaneity } \\
\text { Minimise or eliminate feelings of stigma or } \\
\text { embarrassment while using medical technology }\end{array}$ \\
\hline Night light & Facilitate reliable and efficient night-time management \\
\hline Blood glucose monitoring sock & Facilitate reliable and efficient night-time management \\
\hline $\begin{array}{l}\text { Blood glucose monitoring bike } \\
\text { attachment }\end{array}$ & $\begin{array}{l}\text { Facilitate spontaneity and participation in everyday } \\
\text { activities }\end{array}$ \\
\hline
\end{tabular}

\subsubsection{Blood glucose monitoring phone case and app}

This design responded to two requirements: to help comfortably disclose and explain type 1 diabetes when appropriate, and communicate data and information to facilitate decision-making. Students designed a mobile phone case that contained blood glucose measuring equipment (Figure 34) and an app that displayed and recorded aspects of management, including automatically synced blood glucose data, exercise details, and insulin administration records. The designers addressed disclosure by creating a playful interaction, with the lancing motion appearing as a 
dog licking the user's finger, or a monster biting the user. They hoped to draw attention to the action in a playful manner.
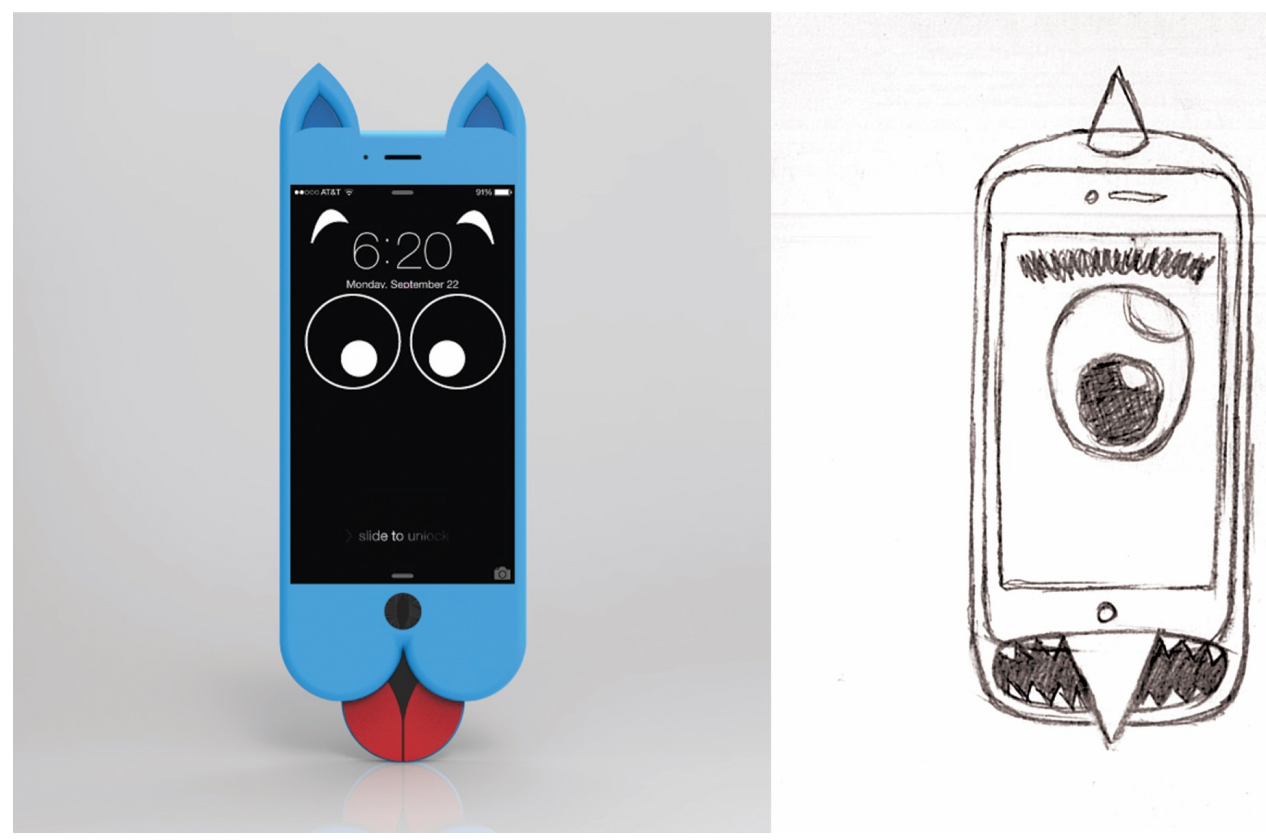

Figure 34. Blood glucose monitoring phone case in dog and monster options, with accompanying mobile application.

Most participants considered the phone "funny", and some gave feedback that it would not be stigmatising and instead might draw positive attention, allowing them to joke about blood glucose testing with friends and making the device and diabetes itself more approachable for others. This would provide an opportunity to disclose and explain type 1 diabetes. However, the design was more suitable for children, and adolescents would prefer a simpler aesthetic. Using a phone was convenient, though safety would need to be considered as some adolescents borrow each other's phones. A game could be developed where players looked after a digital pet, creating a playful reminder to test blood glucose levels.

In discussing this design participants repeated similar experiences from earlier chapters, including medical devices eliciting attention, prompting others such as peers or (occasionally) strangers to ask questions about the devices. Participants had mixed feelings about disclosure. One participant thought it was straightforward, because she is not at fault for developing type 1 diabetes. Another participant described a friend being uncomfortable when she was in the same room as the 
participant testing blood glucose as she was afraid of needles, and another had not disclosed to a peer who she had been friends with for over a year. In contrast, another participant tested openly amongst peers and described attempting to trick new members of her sports team, trying to convince them they would have to test their blood glucose levels as part of their sports training. Adolescents felt that having a choice whether to disclose diabetes was preferable, particularly as people with little understanding of type 1 diabetes could be judgmental or ask frustrating questions.

When giving feedback on the meaningful information aspect of the design, participants positively described automatically syncing data to the phone. Where current blood glucose meters did not facilitate visualising information, and only health professionals could download the data from some devices, the design would allow adolescents to graph and visualise trends and share these with a clinician if desired. Two participants described how their parents currently logged their blood glucose results manually and tried to get them to engage with the subsequent logs or graphs. Many participants had stopped using logbooks, and felt that viewing previous levels was only helpful when trying to make a significant change.

One participant felt that by logging blood glucose levels, she paid too much attention to the numbers and had started to develop obsessive thinking. For her, focusing on visualisations and trends was better for her mental health than seeing individual numbers. Another who was transitioning responsibility from her mother struggled to get the same results as her mother had achieved, and would not test if she thought she may get 'bad' numbers, thinking her mother may get mad. However, she recognised this was not productive, and that numbers should be thought of as a tool to inform insulin intake.

A3: We have a joke 'if you don't tell me I'll go check your meter'; 'no'; I run and grab it ... It should be more about treating the bad numbers, and if you have good ones that's good. More about just dealing with the number than judging it. Because of the transition it's trickier. My mum knew exactly what to do and I had to take over.

When looking for patterns in blood glucose data, context was important. For instance, one participant only tested when he felt high or low, so his numbers were 
rarely in range. However, identifying patterns in management could be helpful, and undesirable results and patterns could spur him to act.

\subsubsection{Blood glucose monitoring jewellery}

This design - a blood glucose monitor in the form of jewellery (Figure 35) responded to the requirements of minimising or eliminating feelings of stigma or embarrassment while using medical technology, and facilitating disclosure where appropriate. Disguising the technology as jewellery gives users the choice of disclosing their diabetes and makes it wearable, so it need not be carried. While this design may draw attention, it might be a positive form of attention.

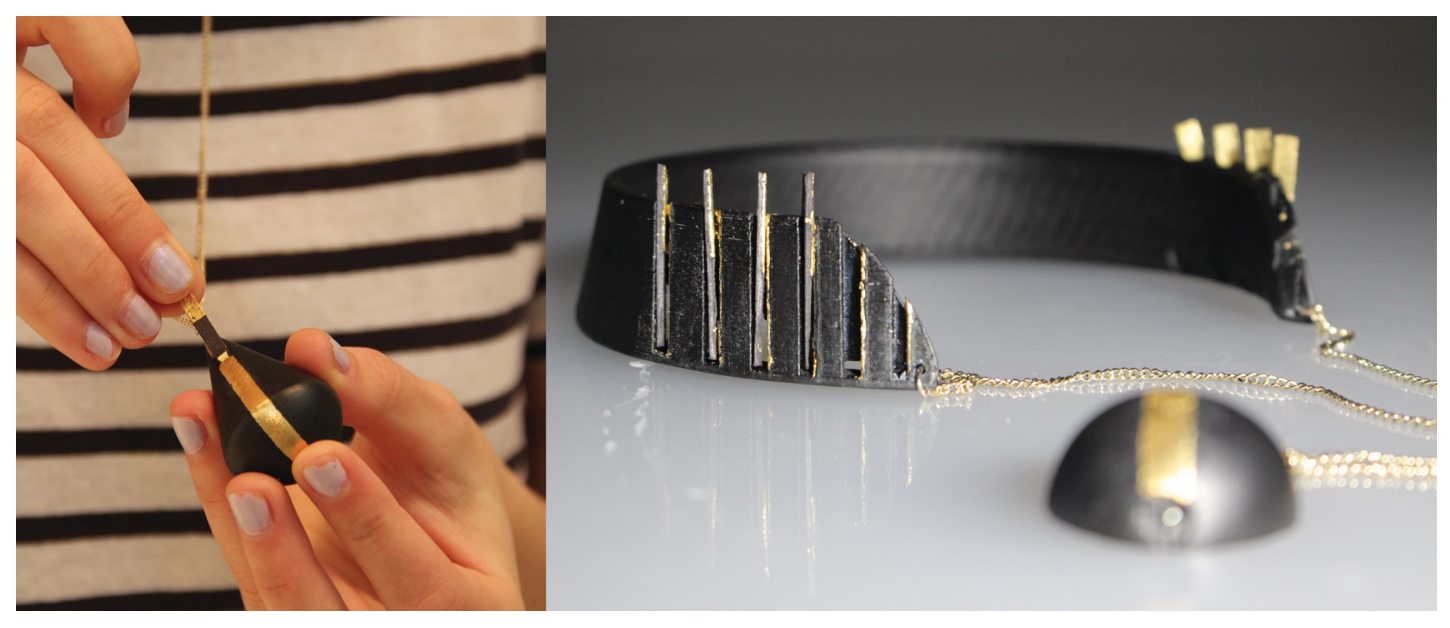

Figure 35. Blood glucose monitoring jewellery in a choker and pendant combination.

The participants recognised that a jewellery-based design would give them the choice to conceal the device as jewellery or to explain its purpose, stimulating discussion. They thought they might be prouder of the device and more likely to explain its purpose if somebody complimented it. Some participants thought this design was 'cool' and liked its aesthetics, while others thought simplifying its aesthetic, as with medical alert jewellery, would allow it to be worn in more situations. A benefit was the device being harder to lose as it was wearable, although concerns included the secure storage of affordable test strips, having sufficient memory capacity, and not being designed for males.

While participants thought having a device that did not look medical could be helpful, there were mixed practices for disclosing diabetes. Some were disclosing 
widely in case of an emergency, while others were more selective. While some wore medic alert jewellery, they may take it off in certain contexts, such as during hospitality work, to avoid being questioned about it.

\subsubsection{Blood glucose test reminder watch}

This design responded to two requirements: to facilitate daily structure while allowing for spontaneity, and to minimise or eliminate feelings of stigma or embarrassment while using medical technology. The designers noted that while some young people forgot to test their blood glucose levels, they were also reticent to use a device with alarms or vibrations that may draw unwanted attention (A. E. Carroll et al., 2007). This design concept (Figure 36) explored the use of a subtle reminder where a watch face could be twisted to set a reminder hand, with the user to take a test when the silver hour hand met the white reminder hand.

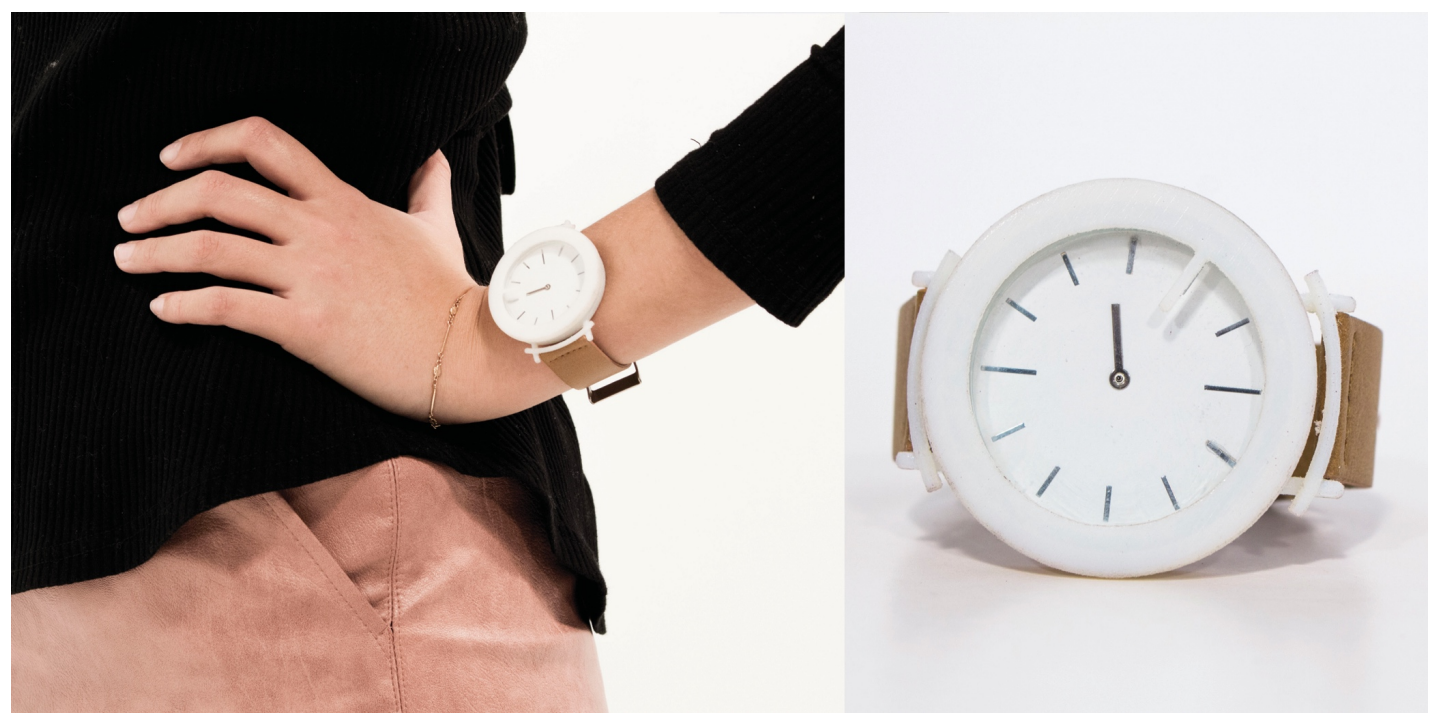

Figure 36. Blood glucose test reminder watch with twistable watch face.

Most participants preferred subtle reminders, rather than feeling pestered or having alarms drawing attention, though some noted that this watch would only be useful for a select group of people who do forget to test but were motivated to test more. While some participants had previously set reminder alarms, they now found these either unnecessary or ineffective. While many participants noted they liked the aesthetics of the watch, only two felt they currently required a reminder to test their blood glucose levels. 
While for many of the user requirements participants had varying opinions and experience, there was consensus about routine. All the participants felt that having routine embedded into their days improved their self-management, though there was still a desire to be spontaneous at times. The structure of the high school schedule was commonly used to structure diabetes management (e.g., test at morning tea, lunch, and after school).

\subsubsection{Blood glucose meter test compact}

This design responded to two requirements: to facilitate daily structure while allowing for spontaneity, and minimise or eliminate feelings of stigma or embarrassment while using medical technology. This blood glucose meter concept (Figure 37) was intended to encourage users to use multiple fingers for their blood glucose tests and to change lancets frequently. It also afforded some privacy and adopted a disguise strategy by using the visual language of a makeup compact. The designers used a series of orange lights to visually convey how many times each lancet had been used, and a blue light to encourage a certain finger to be used.

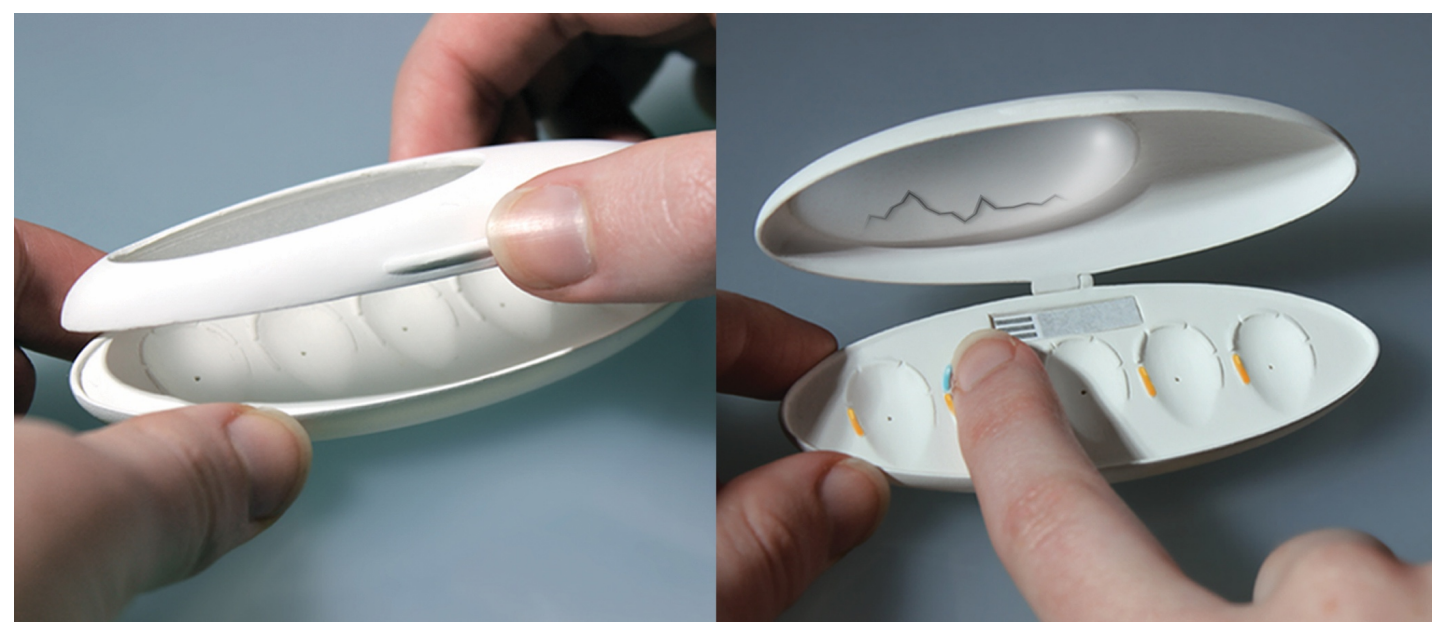

Figure 37. Blood glucose monitoring compact with lights to prompt use of different fingers and replacement of lancets.

Participants agreed that the design looked like a makeup compact. While some felt this disguise approach could maintain their privacy, it might be disadvantageous if somebody picked it up and hurt themselves, or stole the device. Important considerations were maintaining the small size of the device and being able to change 
the lancets quickly. Uniquely, one participant said she would try using all the lancets simultaneously at least once. There were several suggestions to improve the device, including indicating whether the left or right part of each finger was due to be tested, better facilitating left and right hand use, and minimising the number of lancets to coordinate with the fingers adolescents used for testing. Participants were encouraged by health professionals to use a variety of fingers for testing blood glucose levels, but all had 'favourite fingers'. Despite this, many recognised using a wider variety of fingers may be beneficial.

Participants currently used their pinkies and ring fingers on their nondominant hand predominantly, because these fingers were easy to angle the device towards, bled the best, hurt the least, were easy to stop bleeding, and were less likely to be painful during following activities (e.g., holding a pencil). While most did not use their thumbs, this was one participant's most reliable way to get blood. Many reported they did not change the lancet often, and some were unaware of how often it was supposed to be changed, and would wait until it felt blunt. In terms of stigma, multiple participants spoke about the marks the lancet left on their fingers, with one describing his fingers as looking "destroyed" and like "craters" after being in water.

\subsubsection{Night light}

This design aimed to facilitate reliable and efficient night-time management. Students noted that adolescents experiencing nocturnal hypoglycaemia or hyperglycaemia needed to use medical devices, not all of which are equipped with lights. In response, they designed a storage container for essential diabetes equipment to be placed on the bedside that gently alit when tilted (Figure 38). 


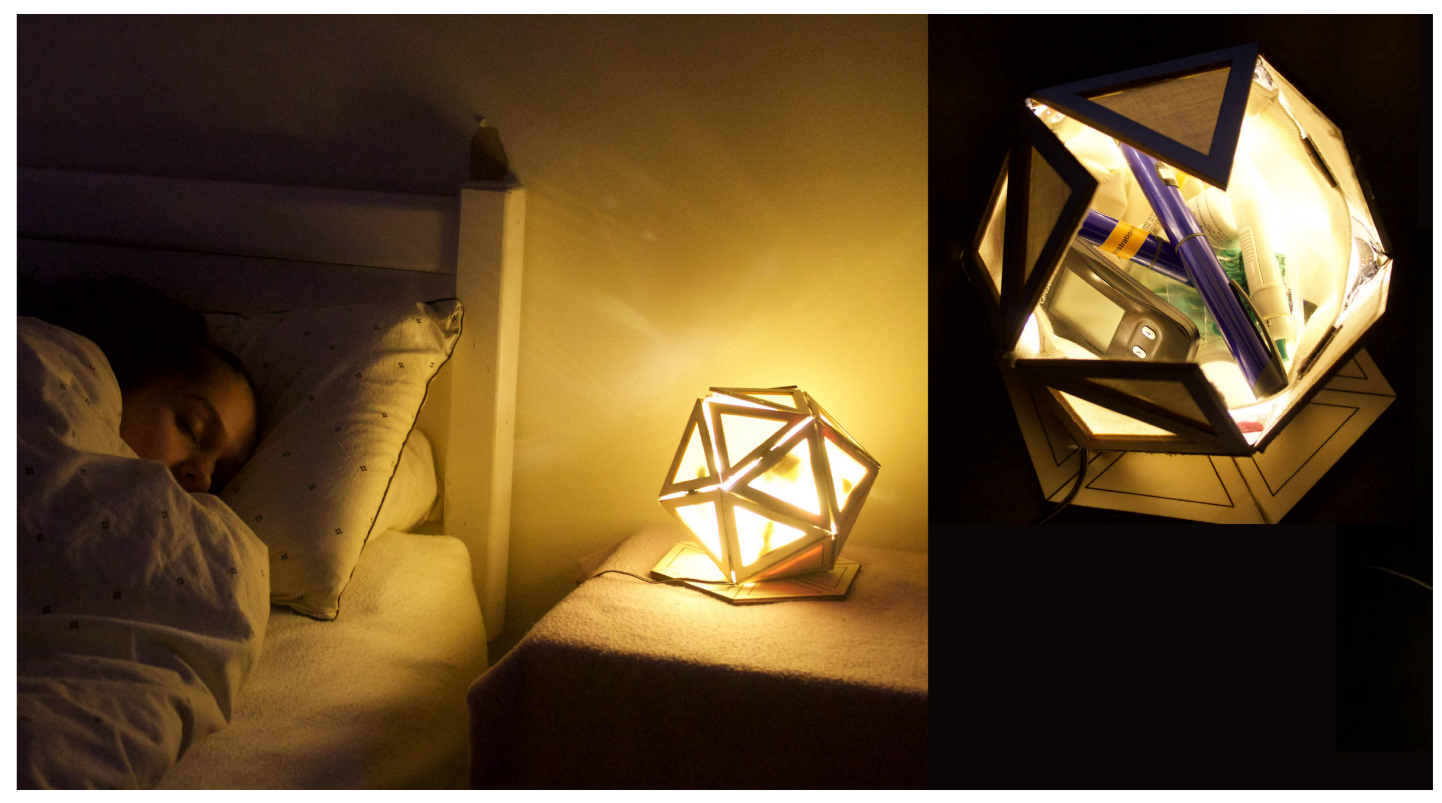

Figure 38. Night light and diabetes storage device.

Positive aspects of the design were its visual appeal, dim light, and that it would decrease bedside clutter. Concerns included insulin and blood glucose meters needing to be kept at particular temperatures, and the likelihood of spilling the contents of the object when the user was drowsy.

While most participants did not commonly experience high or low glucose levels during the night, they valued efficient management in instances where this did occur. When younger, some parents checked their child's blood glucose levels regularly during the night. Now, some participants turned on their bedroom lights to test; one had a bedside lamp and three used the light from their smartphone or another medical device to see where to insert the test strip on their meter, as although some devices were backlit, there was no light near the port. Some kept food to treat hypoglycaemia near their bed, and others would go to the kitchen to find food.

\subsubsection{Continuous glucose monitoring sock}

This design aimed to facilitate reliable and efficient night-time management. The designers employed infrared technology for glucose monitoring, currently in development. This infrared light would be sent between the toes intermittently to test levels (Figure 39). 


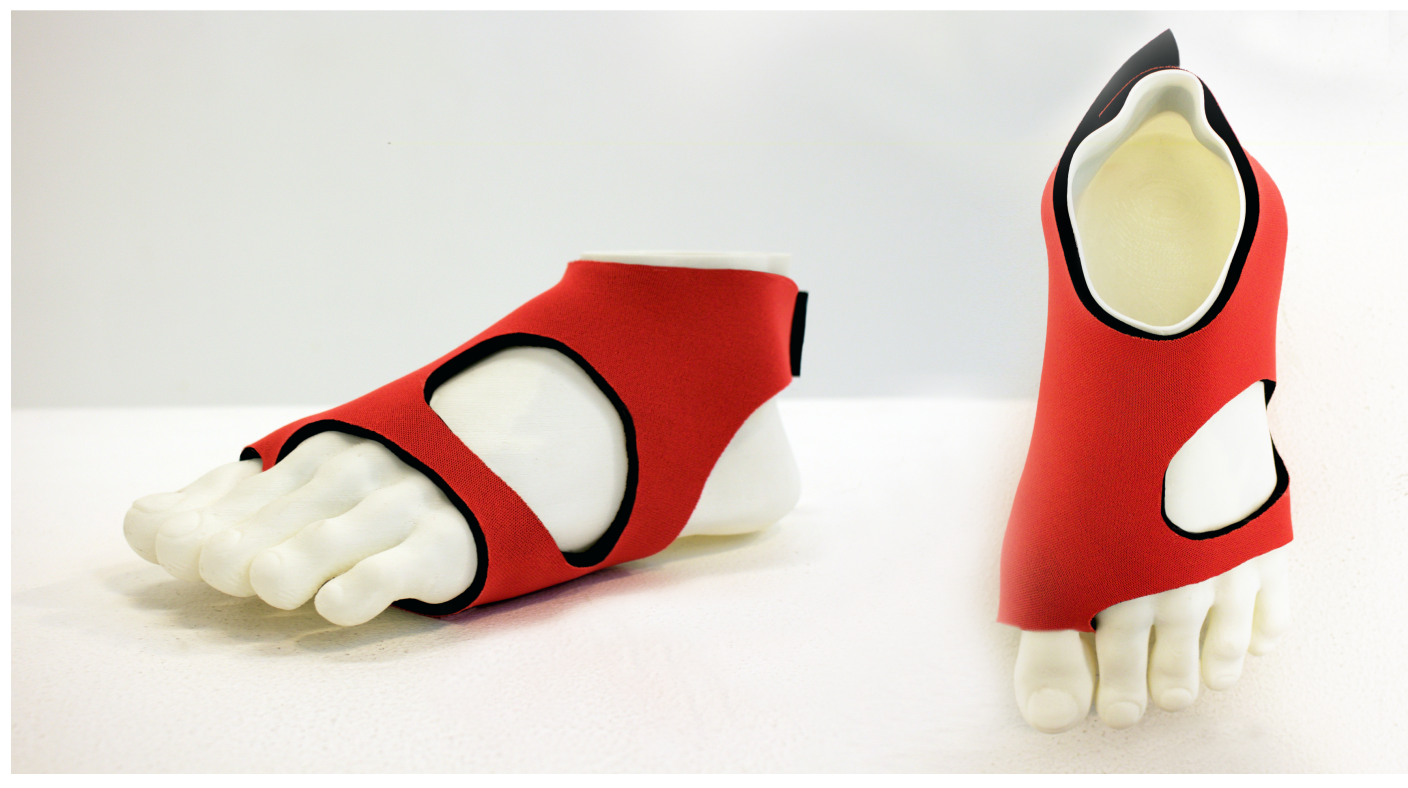

Figure 39. Continuous glucose monitoring sock with cooling and vibration.
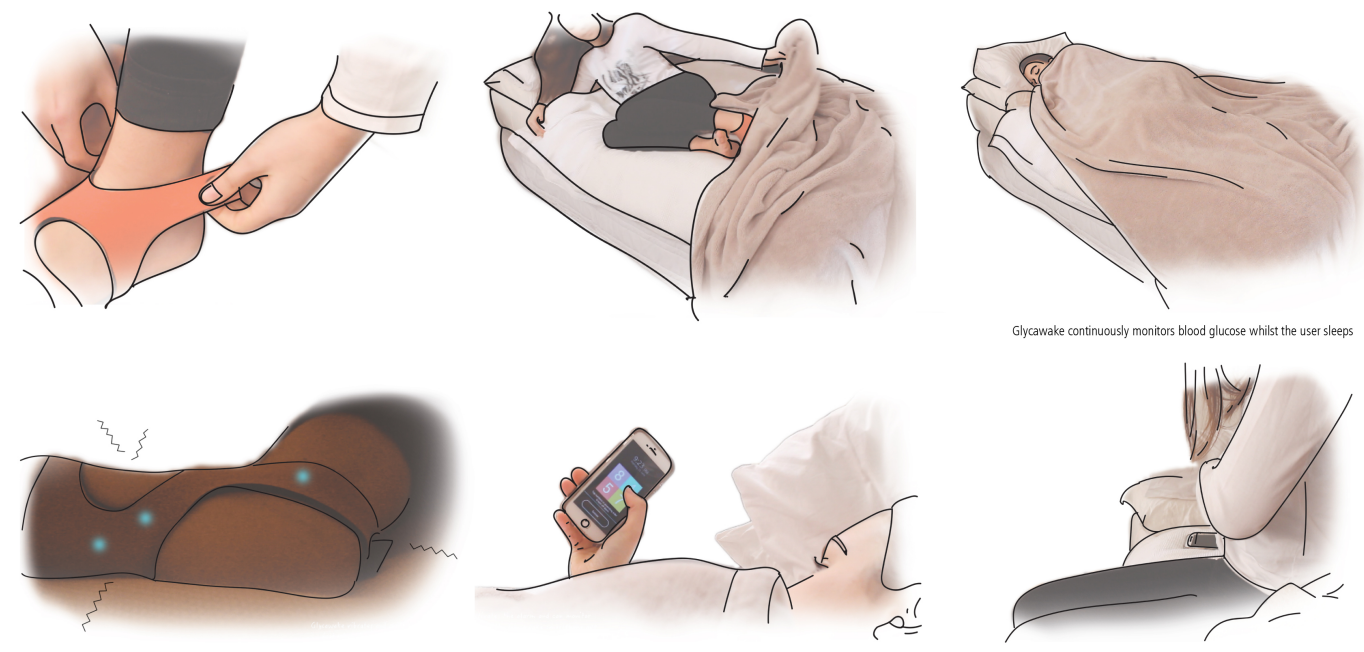

Figure 40. Storyboard showing the sock waking the adolescent with cooling and vibration, and completing a simple puzzle to silence the phone's alarm.

This storyboard (Figure 40) demonstrates that if the wearer's levels were dangerously low or high, the sock would cool and vibrate to wake the wearer. Upon waking, the wearer could use a mobile app to silence an alarm by completing a simple mathematical puzzle, then view their blood glucose level and trends. The designers were concerned that if adolescents were drowsy they may fall asleep before treating, thus they thought the puzzle would help to wake them to a level where they could make decisions. 
None of the participants currently had trouble waking when hyperglycaemic or hypoglycaemic, but some were aware of others who did struggle with this, or had struggled with this in the past. Measuring overnight could be helpful to better understanding the actions of insulin and blood glucose levels. Suggested improvements included the device preventatively alerting the wearer before the hypoglycaemic episode occurred, using a bracelet instead of socks, speeding the wake-up process rather than using slow cooling, and the app instructing people how much to eat, so they did not have to calculate this while drowsy.

Two mentioned that the puzzle was dangerous, preventing wearers from viewing their blood glucose levels unless it was successfully completed. While the designers thought adolescents ought to be completely awake to manage, many adolescents felt differently. Most were happy to manage drowsily, especially as they grew more confident, experienced, and independent, only sometimes fully waking themselves up more if they were very low.

\subsubsection{Blood glucose testing bike attachment}

This design was developed to facilitate spontaneity and participation in everyday activities. Exercise was frequently mentioned as an activity where young people with diabetes needed to stop participating to manage their blood glucose levels. The design was a blood glucose meter affixed to a bike, with a one-handed gear-like lever that triggered a lancet and shifted a new test strip into place, so users could test their blood glucose levels while cycling (Figure 41). 


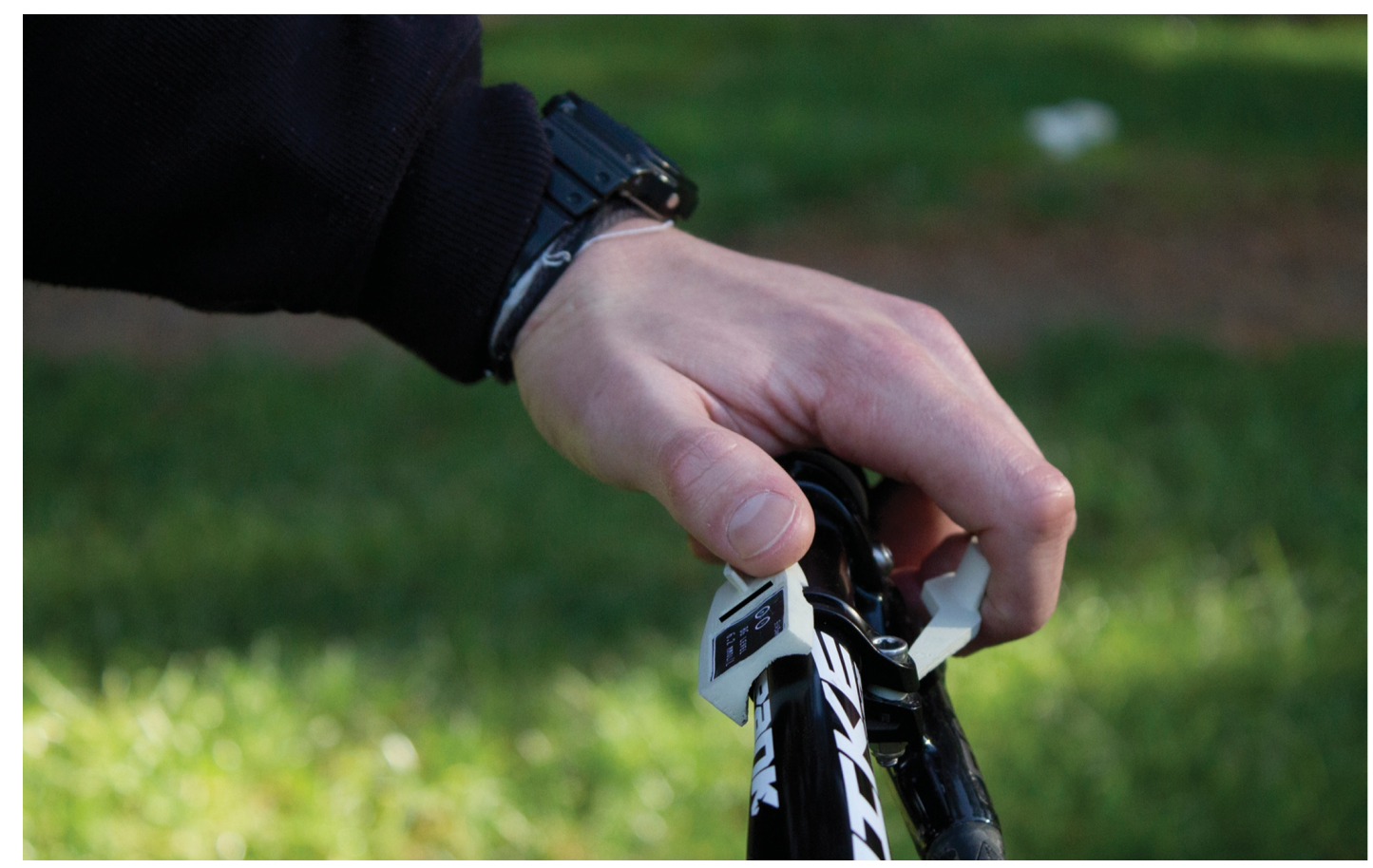

Figure 41. Blood glucose monitoring bike attachment with lever action to prick finger and shift test strip into place.

Many of the participants thought testing blood glucose levels without having to stop riding would be great, though noted this would have to be done with careful coordination. Also, most participants did not commonly use their thumbs to draw blood, and suggested a re-design of the device might allow for ambidextrous use. While participants were weary of introducing more medical devices to keep track of, this item would be fine because it could stay attached to the bike.

In reviewing the user requirement relating to participation in daily activities, participants noted the importance of exercising, finding glucose levels steadier on days including exercise, but that testing and eating needed to be fine-tuned to achieve stable levels. Others emphasised the need to test to distinguish hypoglycaemia from regular feelings of exertion during exercise, requiring adolescents to momentarily stop participating.

\subsubsection{Additional design reviews}

Two designs from Chapter 5 were also reviewed by the adolescents. These designs were chosen as they reflected the user requirements in this brief, were substantially different to the current designs, and were reviewed positively by the Diabetes Nurse Specialist. Both designs aimed to facilitate spontaneity and participation in everyday 
activities, and the Subscope watch also aimed to communicate data and information to facilitate decision-making.

\subsubsection{Subscope review}
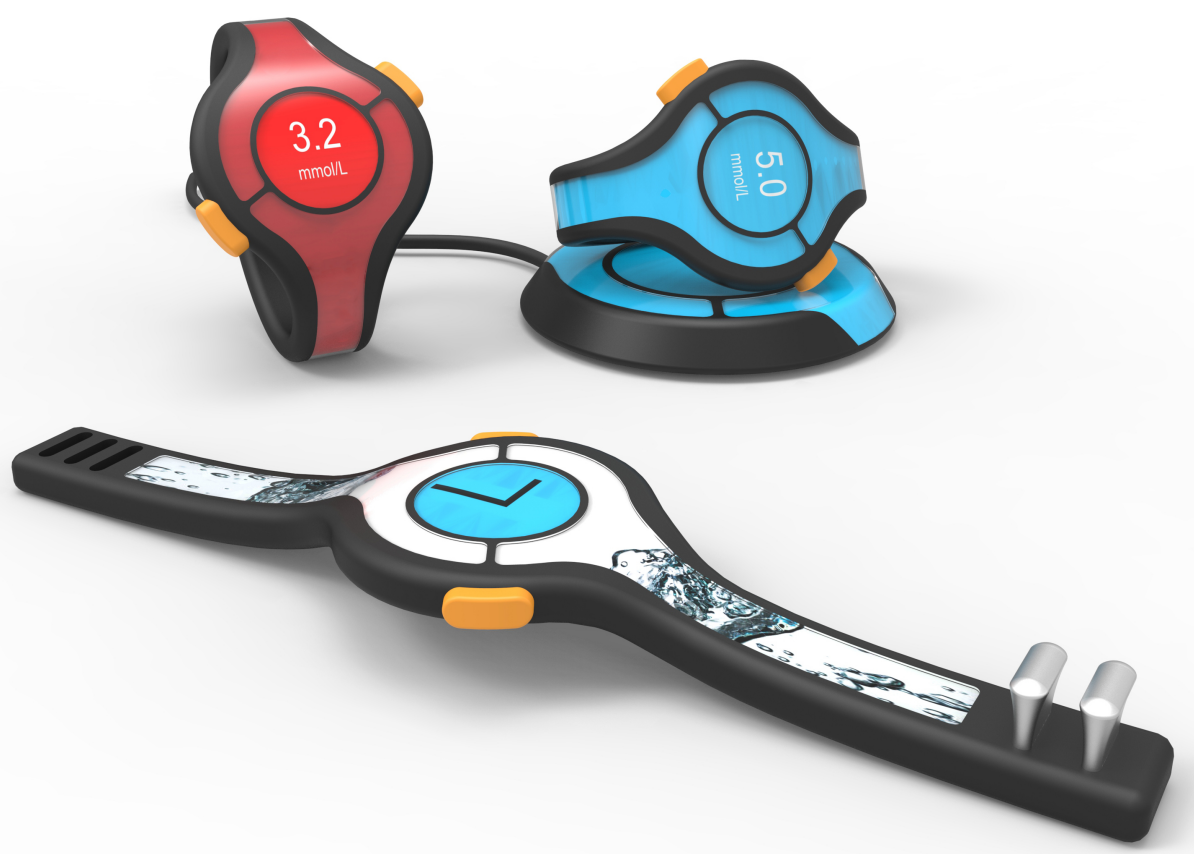

Figure 42. Subscope continuous glucose monitor.

The Subscope sports watch (Figure 42) measured glucose levels and changed colours accordingly to represent these levels. Viewing blood glucose levels and trends at a glance would mean adolescents would not need to stop participating unless necessary, and could address hypoglycaemia before it became serious. Many participants commented that they would wear this throughout the day, though one thought the aesthetic was not appropriate for a non-sports context. For many, however, the device's 'sporty' aesthetic was positive, and while divided over whether it might draw attention, the diabetes-related information provided by the watch would not be understood by others, ensuring the user's privacy. Participants also preferred the concept of using the chip implant and watch to current testing methods. 


\subsubsection{Simpac review}

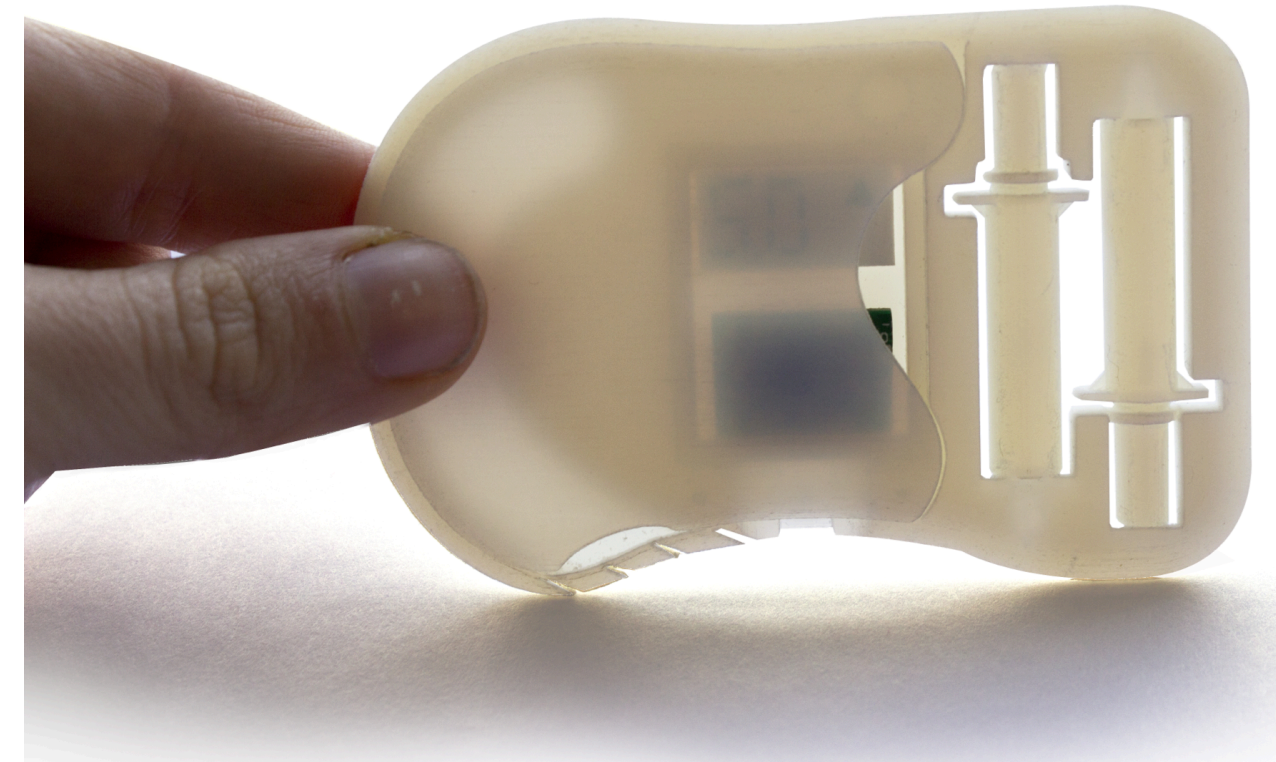

Figure 43. Simpac all-in-one blood glucose testing and insulin delivery.

Simpac (Figure 43) is a credit card-sized blood glucose testing kit with two prefilled insulin syringes and finger cleaning wipes. Some participants thought this all-in-one medical device could replace current devices, while others felt it could be a good backup to carry in a wallet for spontaneous events, emergency situations, or going for a walk, where glucose and medical devices currently needed to be distributed throughout pockets or were left at home.

Adolescents preferred small or integrated devices, as they currently had to carry many pieces of equipment and could get 'roasted' by friends for constantly carrying a backpack, although small devices could be easily lost. Suggested improvements to the design included adding a cooling feature to keep insulin in better condition, improving the appearance and materials, adding more test strips, creating a version that focused only on testing for people who use insulin pumps, and removing the cleaning wipes, as many did not wash their hands before testing and found this adequate. 


\subsection{Discussion}

\subsubsection{Psychosocial user requirements of medical technologies}

Completing these design projects and having them evaluated by participants with type 1 diabetes helped to develop and provide additional support for six user requirements. Developing user requirements as a product of the design process is discussed below.

1. Facilitate reliable and efficient night-time management While the designs addressing night-time management were not reviewed favourably, this requirement was further developed. The designers had presumed adolescents needed to be woken completely to self-manage, but many adolescents woke naturally and preferred to manage drowsily, especially as they grew more confident with experience. Automatically measuring blood glucose levels overnight could be helpful for developing a better understanding of the actions of insulin and blood glucose levels. Though nocturnal hypoglycaemia was not common among participants, when it does occur, medical technologies should allow adolescents to respond efficiently. Dim lights, backlights, straightforward information to treat lows, and the ability to act proactively could be facilitated.

2. Facilitate spontaneity and participation in everyday activities Participants noted the importance of exercising, finding that glucose levels were steadier on days that included exercise, but that testing and eating need to be finetuned to achieve stable levels. Medical technologies should minimise selfmanagement-related interruptions to daily activities. This is particularly true of exercise-based activities, where adolescents may stop to test blood glucose levels when confusing feelings of exertion with hypoglycaemia, and struggle to carry devices on their person. While designers used a 'sporty' aesthetic, this may not be appropriate for non-sports contexts, but integrated and wearable devices had potential and could increase proactive management (e.g., correcting before hypoglycaemia, carrying spare equipment).

3. Communicate data and information to facilitate decision-making 
Focusing on visualisations and trends rather than individual data points could be positive for mental health. Medical technologies should store, sync, and be able to share and visualise data in a meaningful manner that facilitates decision-making (particularly around significant changes to management) and acknowledges context. The focus should not be on 'good' or 'bad' numbers, but trends, pattern recognition, and facilitating decision-making and memory.

4. Facilitate daily structure while allowing for spontaneity Medical technologies should facilitate structure and habits, which adolescents agreed improved their diabetes management. However, they should also allow for spontaneity. Through the design and review process, it became clear that subtle prompts rather than feeling pestered or drawing attention are preferable in some contexts. The need for prompting blood glucose testing varies between adolescents. Also, adolescents largely did not adhere to guidelines on changing lancets and pricking different fingers; however the angle of the device, access to each finger and the context still need to be considered (e.g., needing to hold a pencil after pricking finger).

5. Help to comfortably disclose and explain type 1 diabetes when appropriate Medical technologies should give adolescents the choice whether to disclose their condition, not reveal it by default. As with previous chapters, disclosure could have a moral status, or adolescents could struggle with it. Technologies can act as prompts for the adolescent to engage with peers about diabetes when they feel it appropriate. 6. Minimise or eliminate feelings of stigma or embarrassment while using medical technology

Participants repeated similar thoughts from earlier chapters, whereby medical technologies currently elicited negative attention, embarrassment for some users, and body image issues. Technologies should not invoke negative connotations of drug paraphernalia. Opinions about the value of disguising the device varied, though the appearance of the device should be appropriate for the contexts in which it is used. Users ranged widely in whether they felt embarrassed, and in which contexts they felt embarrassed, illustrating the differing importance of user requirements for individuals. 


\subsubsection{Designing to user requirements}

In this study, students designed medical technologies from a brief based on psychosocial user requirements constructed in earlier interviews and a card sort task with adolescents. With guidance from an Industrial Design Professor and myself, students were mindful of these requirements and created designs that responded to one or more of them.

In Chapter 5, where designs were shown to a Diabetes Nurse Specialist, it was primarily the designs and not the user requirements that were reviewed. In this study, the user requirements and accompanying quotes from earlier research phases were displayed as vignettes along with the designs. Resultantly, there was more discussion of the user requirements than in the previous chapter, although conversation was still weighted more towards reviewing designs. This was better aligned with the purpose of this design research, which was to elicit adolescents' selfmanagement experiences and provide additional support for medical technology user requirements, rather than review possible designs for development.

Designers had limited access to adolescents, relying primarily on insights from my research conducted with adolescents. They were limited in not being able to directly ask this population questions. For instance, in designing the blood glucose monitoring compact, designers were unsure about which fingers adolescents used for testing, but in showing the design to participants after the design process, I could elicit specific information that would be helpful for following iterations. Interestingly, one participant had already seen one of the design concepts as it had been shared by a user of an online diabetes forum.

Through the study, the range in adolescents' preferences became clear. There is no design to which all participants responded positively or negatively. As might be expected, though they all have type 1 diabetes, they have individual needs and preferences. Additionally, not all user requirements developed in the research are applicable to all adolescents with type 1 diabetes. Issues around embarrassment and stigma are particularly divided, whereby some adolescents describe these as barriers to their self-management, and others did not experience these issues or chose not to adapt their behaviour. 


\subsection{Summary}

As per the aims of this chapter, this study illustrated how medical technologies can be designed to respond to user requirements, and how a designing and reviewing process can be used to stimulate discussion, and develop and provide additional support for user requirements with young participants. The psychosocial user requirements for medical technologies supported were to: help to comfortably disclose and explain type 1 diabetes when appropriate, communicate data and information to facilitate decision-making, minimise or eliminate feelings of stigma or embarrassment while using medical technology, facilitate daily structure while allowing for spontaneity, facilitate reliable and efficient night-time management, and to facilitate spontaneity and participation in everyday activities. Through these design experiments, I explored how designers can move beyond clinical efficacy and create technology that responds to adolescents' psychosocial needs. 


\section{Cultural Probes}

While this chapter sought to answer the overarching research question of the thesis, it also sought to answer four sub-questions. These sub-questions aided thematic sampling, after developing grounded theory categories that needed further saturation after completing research in Chapters 4-8. The sub-questions were:

- How do adolescents conceptualise self-management of type 1 diabetes?

- How do adolescents want to manage diabetes?

- What relationships do adolescents have with medical devices?

- What are the best and worst features of current medical devices, and what features do they lack?

Cultural probes were chosen to investigate both current and desired experiences and requirements. This method was chosen over a traditional observation method, as I had identified that my presence may intensify embarrassment and stigma, causing distress. Probes rely on self-documentation, with participants taking an active role in data collection. They are characterised by being exploratory, and focusing on the participant's personal context and perceptions (Mattelmäki, 2006). This aligns with the constructivist epistemology used throughout the research. Probes often consist of activities to be completed using resources such as maps, diaries, disposable cameras, and worksheets, and can represent a participant's personal narrative of their illness (Burrows, Mitchell, \& Nicolle, 2015; Mawson et al., 2014; Melles \& Freudenthal, 2017; Rich, Lamola, Gordon, \& Chalfen, 2000).

Probes are commonly used for: design inspiration, collecting information, facilitating design and research participation, and facilitating dialogue (Mattelmäki, 2006). In this study, probes were used to contribute to information, participation, and dialogue. Alternately to the original probes approach, probes that seek to develop empathy with participants are commonly followed by interviews, allowing the researcher to check and further their interpretation of data with the individual who created it (Boehner, Vertesi, Sengers, \& Dourish, 2007; Gaver, Dunne, \& Pacenti, 1999; Gaver \& Dunne, 1999; Mattelmäki, 2006). This participant 
involvement is akin to a participatory design approach, though analysis of the materials ultimately lies with the researcher (Mattelmäki, 2006).

Cultural probes have been used in existing research with children and adolescents with type 1 diabetes. Insights from these studies include probes being an effective way of collecting health data in private settings, adolescents not providing emotional information unless specifically prompted, and eight activities being too many (Hassling, Nordfeldt, Eriksson, \& Timpka, 2005; Tsvyatkova \& Storni, 2014). As such, I devised a letter writing method to prompt emotional information rather than a diary study, and limited the number of activities.

\subsection{Methods}

\subsubsection{Participants}

There were ten participants (four male, six female) aged 13-24 years who had been diagnosed with type 1 diabetes for between six months and 15 years. These were a subset of participants from previous chapters. Most participants completed all activities, while some completed two or more. The number of participants who completed each activity were:

- making a metaphor (eight);

- managing your way (nine);

- love letter, break-up letter (nine);

- best and worst (nine).

One participant chose not to complete the letter writing activity, describing it as "too awkward". Cultural probes were also sent to seven adolescents who had agreed to undertake the activities, but these were not returned, and no reasons were given. A 13-year-old participant had a parent present during the interview.

\subsubsection{Procedure}

Participants were given cultural probes packs to complete at home, containing instructions, activity cards, stationery, and an envelope for returning the activities (Figure 44). Participants were advised to return the probes within two weeks, and 
that activities could be completed in any order. Digital activities could be emailed or shared privately through Instagram. Some participants kept the probes for a longer duration.

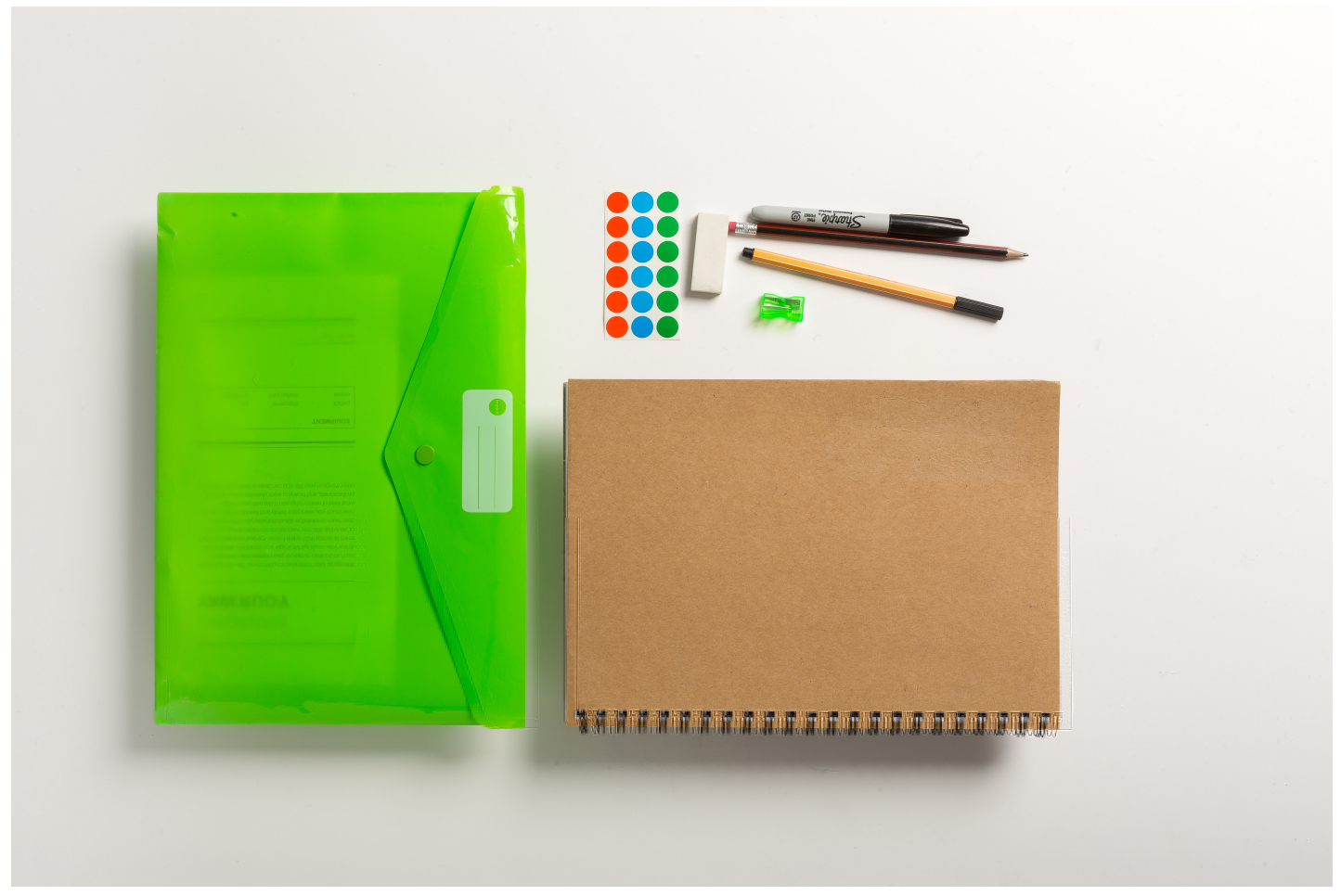

Figure 44. Cultural probes kit supplied to participants.

The probes were followed up with an interview to uncover further information, and assess whether my comprehension and interpretation of the data aligned with the participants' interpretations (Thoring, Luippold, \& Mueller, n.d.). Two participants chose to complete some of the activities during the follow-up interview rather than independently beforehand. Four activities are described below.

\subsubsection{Making a metaphor}

The aim was to elucidate how adolescents conceptualise diabetes management. Through an activity card, participants were instructed to draw a diabetes metaphor, considering what diabetes means to them, how they manage it, and the role diabetes plays in their life (Figure 45). 


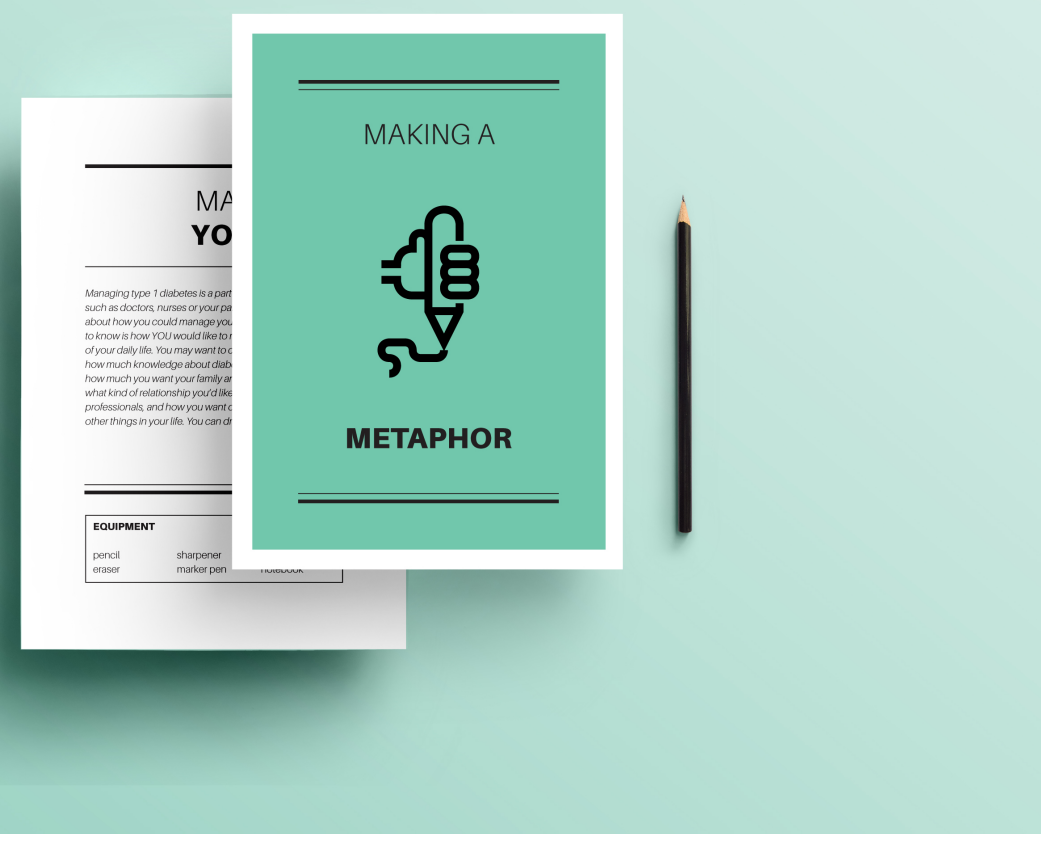

Figure 45. Activity card for metaphor method.

Health professionals use metaphors for diabetes management, which are effective educationally (Whaley, Stone, Brady, \& Whaley, 2014). Their metaphors for diabetes management include mechanical metaphors of equilibrium, the bloodstream as a river with pollutants, insulin as a key that opens a lock, bodily inner workings as transport networks, and the patient as on the frontline fighting complications (Hagey, 1984; Rossi, 2016; Whaley et al., 2014). Researchers, businesses, and the media also use metaphors, such as charting a course through choppy sea (Youngson, Cole, Wilby, \& Cox, 2015), diabetes as a monster in an app (“mySugr.com," n.d.), military metaphors, work and the inability to take time off, and a flood that could not be stopped (Hanne \& Hawken, 2007).

People with various types of diabetes have conceptualised self-management as a journey, competition, fighting a battle with various weapons, vicious disease, full-time job, balancing the 'out of control', and as a part of self-identity, with one person describing themselves as a yo-yo diabetic with up-and-down blood glucose levels, and another as having diabetes, rather than diabetes having them (Hagey, 1984; Harris \& Porkony, 2012; Huttlinger et al., 1992; Youngson, Wilby, Cole, \& 
Cox, 2015). However, it is unclear which metaphors adolescents with type 1 diabetes use to describe self-management.

Metaphors and analogies are also used in the design process: metaphors to enhance understanding during early problem framing stages, and analogies during concept generation to map ideas from different domains to the current problem (Hey, Linsey, Agogino, \& Wood, 2008). Metaphors can help restructure design problems and fuel creativity, improving design quality (Casakin, 2012).

\subsubsection{Managing your way}

Participants were instructed to write or draw how they wanted to manage diabetes as part of their everyday life, and were prompted to consider aspects including diabetes knowledge, involvement of family, friends and health professionals, and how diabetes fits into other aspects of daily life (Figure 46). These prompted areas were developed from common aspects of patient-centred care (Cronin, 2004).

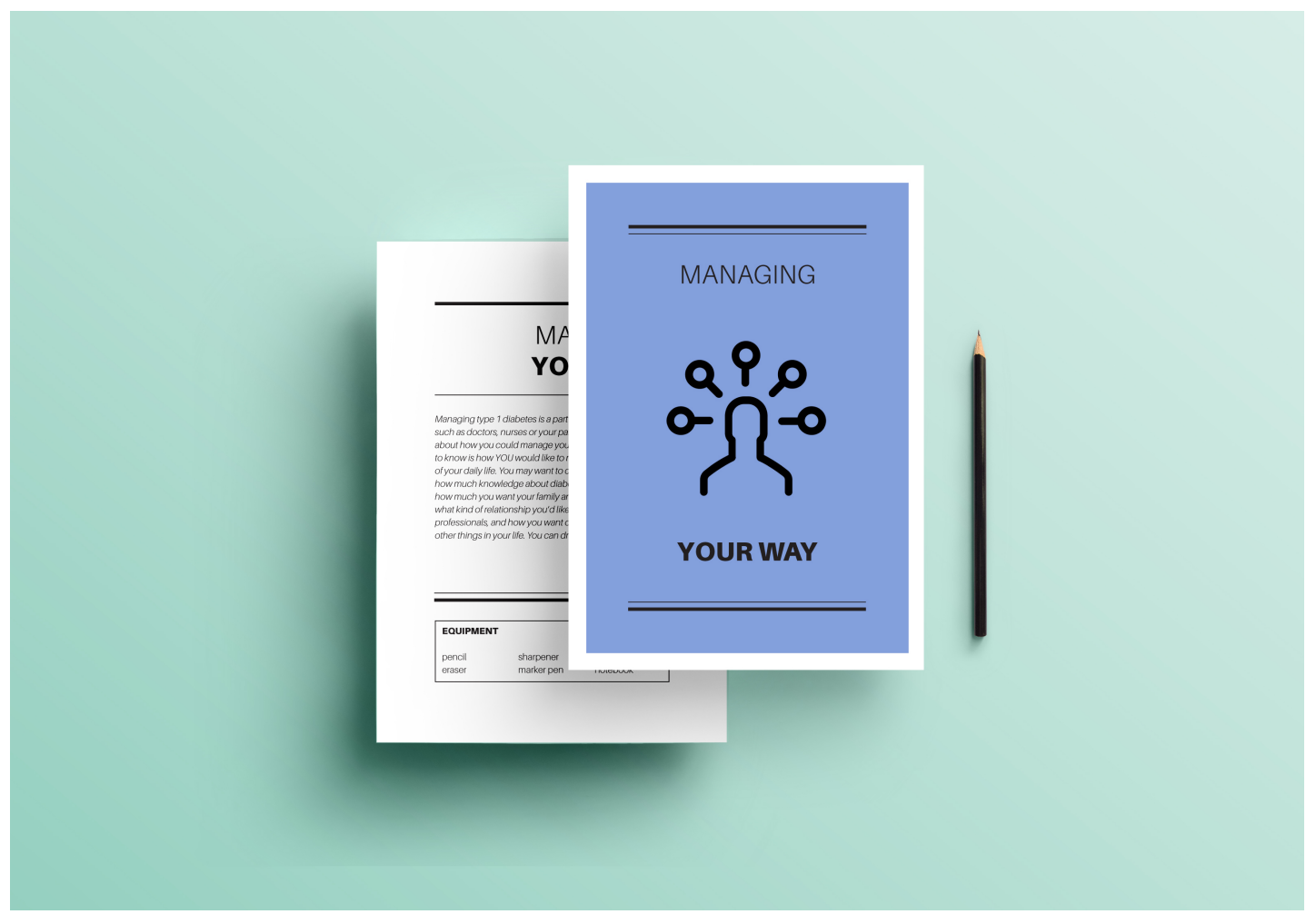

Figure 46. Activity card for managing your way method. 
This activity was also based on worksheets to identify the space diabetes currently takes up in a patient's life, how much space they wish it took, and which life and selfmanagement characteristics people are motivated to change (DAWN, 2015a, 2015b).

\subsubsection{Love letter, break-up letter}

The aim was to understand what relationships adolescents have with medical devices, particularly their expectations and experiences (Figure 47).

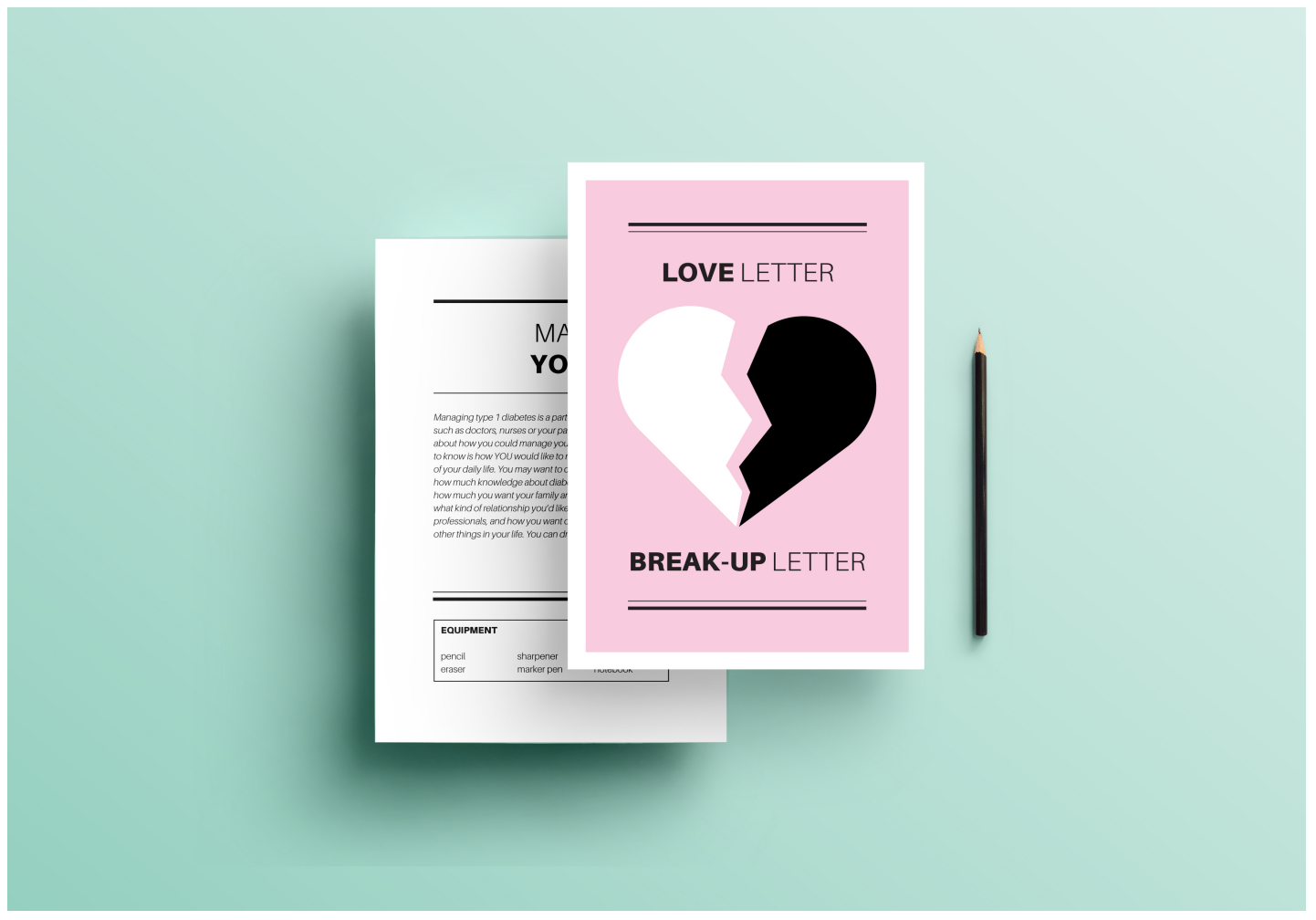

Figure 47. Activity card for letter-writing method.

Participants self-selected a device, as previous research points towards the existence of adolescent user requirements that can be generalised across devices and medical conditions (Lang, 2012). Adapted from a Smart Design group workshop method, participants were requested to write either a love letter or a break-up letter to a diabetes technology of their choice (Smart Design, 2010). This letter-writing method has not been previously documented or used to inform the design of medical technologies, but was used instead of a diary approach to prompt discussion of emotional impacts and interactions, which other probes methods do not always achieve (Hassling et al., 2005). 


\subsubsection{Best and worst}

The aim was to identify the best and worst features of current medical devices, and what features they lack (Figure 48). Adolescents were instructed to place on their devices red stickers to indicate bad features, green to indicate good features, and blue to indicate desired features. They then captioned photographs, explaining their choices. The approach was informed by work using bananas and basic materials to inform the design of mobile phones for elderly users (Gheerawo \& Bichard, 2010).

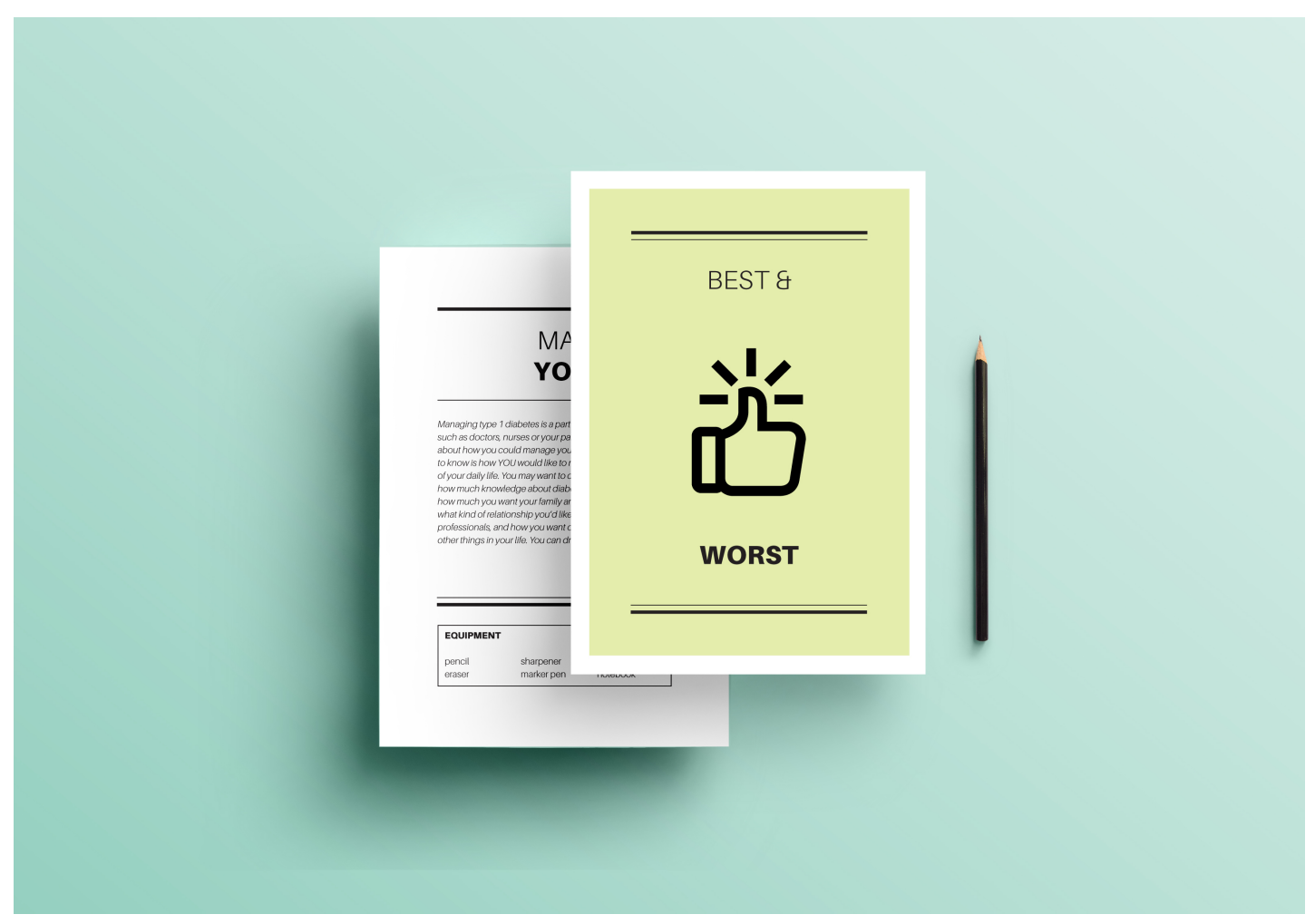

Figure 48. Activity card for method choosing best and worst device features.

Two additional cultural probes activities are not described in this chapter. These were to photograph diabetes equipment in context (some images are included in Chapter 2) and to photograph a day in the participant's life. The first activity intended to give insight into which technologies were currently used and how they were stored and transported, and the second to give insight into how adolescents managed diabetes around their daily lives, and to contribute to the development of personas. While these activities were useful, they did not significantly contribute to answering the research question. 
The probes and follow-up interviews were analysed together to inform grounded theory and user requirements. Additionally, the four-layer Needs and Aspirations of Design (NADI) model was used to evaluate the type of information elicited through each activity. The model classifies insights at four levels: solutions the products people want; scenarios - the interactions people want; goals - why people want these interactions; and themes - the values that drive people's goals (Van Der Bijl Brouwer \& Dorst, 2014). The deeper levels, such as themes and goals, are helpful for reframing design problems and broadening solutions, while scenarios and solutions are helpful for incremental innovation and refining solutions (van der Bijl-Brouwer, 2016).

\subsection{Making a Metaphor}

Eight metaphors for self-managing diabetes were created and used to construct four grounded theory categories: managing a balancing act, struggling for control, rushing to beat the clock, and experiencing psychosocial impacts.

\subsubsection{Managing a balancing act}

Two participants created seesaw metaphors for diabetes management (Figures 4950). Their objective was to keep the seesaw level by keeping their blood glucose levels within a narrow range. If the seesaw tipped it could result in hypoglycaemia and death on one end, or hyperglycaemia, sickness, and hospital visits on the other. While aspects such as food and insulin had an impact on the seesaw, so did heat, exercise, inaccurate test results, pump failures, miscalculations for insulin delivery, insulin not being delivered, emotions, stress, and illness. 


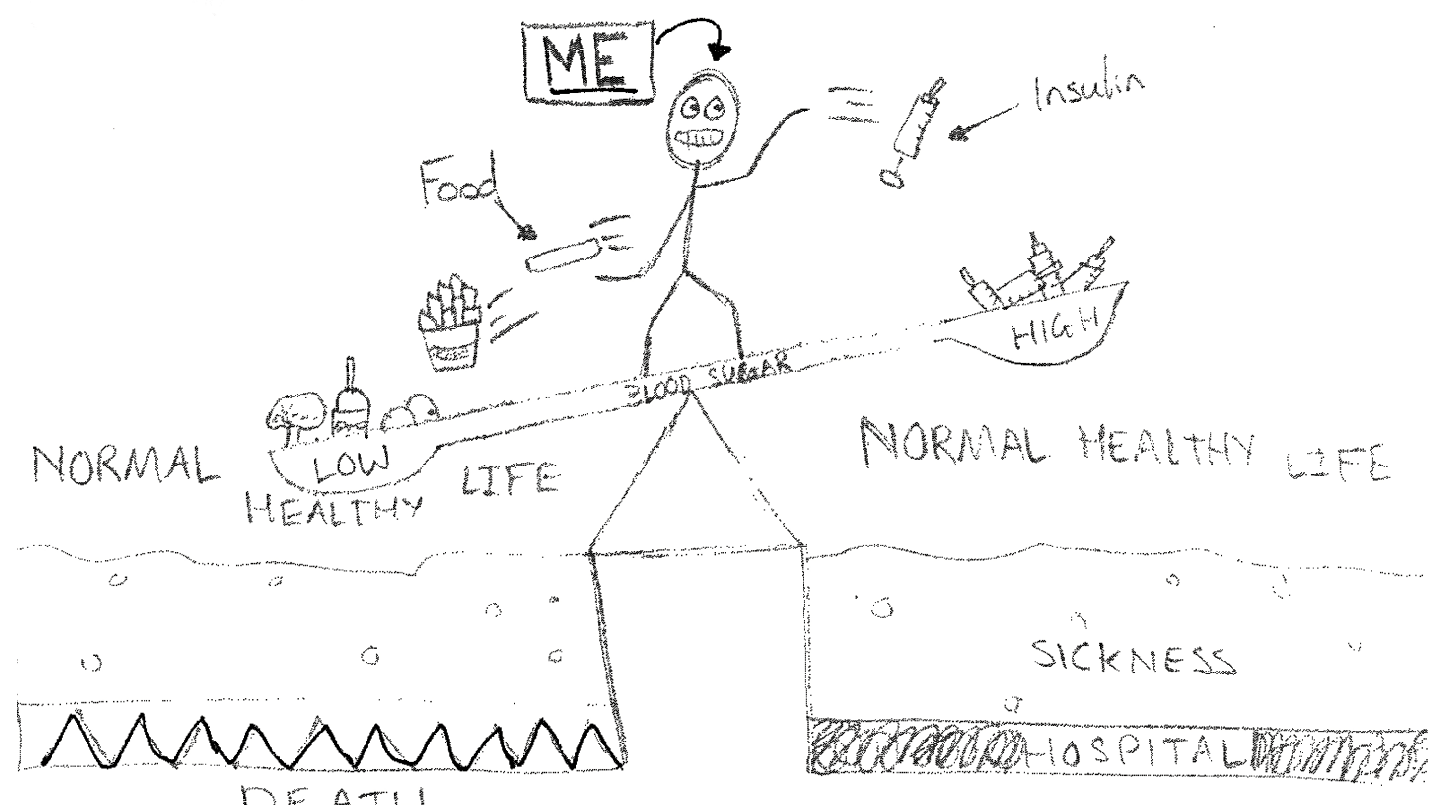

Figure 49. Diabetes management as a balancing act.

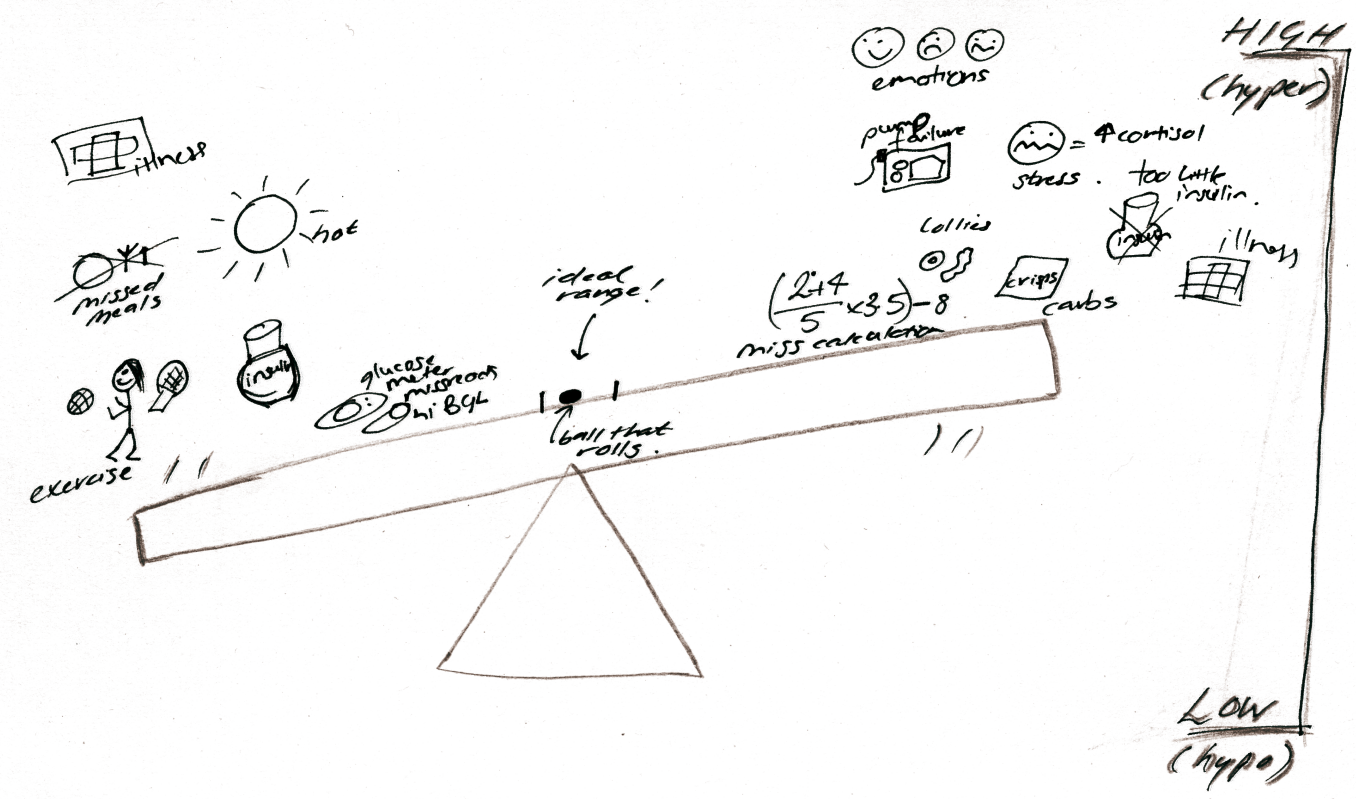

Figure 50. Diabetes management as a seesaw, with many factors affecting blood glucose levels.

It was challenging to stay within normoglycaemia, however, with years of experience, it became easier to predict how blood glucose levels may respond to a variety of factors or understand why levels were different than anticipated. Further, there was greater emotional capacity for handling temporarily high or low blood glucose levels, not letting them greatly impinge on daily life. Persistent levels outside of 
normoglycaemia could trigger emotional responses and thoughts about long-term consequences, in addition to symptoms where adolescents felt physically unwell.

\subsubsection{Struggling for control}

Two participants used metaphors based on arcade games to describe their struggle to control blood glucose levels. The first drew a pinball machine, likening operating the levers to blousing and eating (Figure 51). Keeping the ball in the air represented maintaining normoglycaemia. She had to determine the correct action in a timely manner to keep the ball in the air.

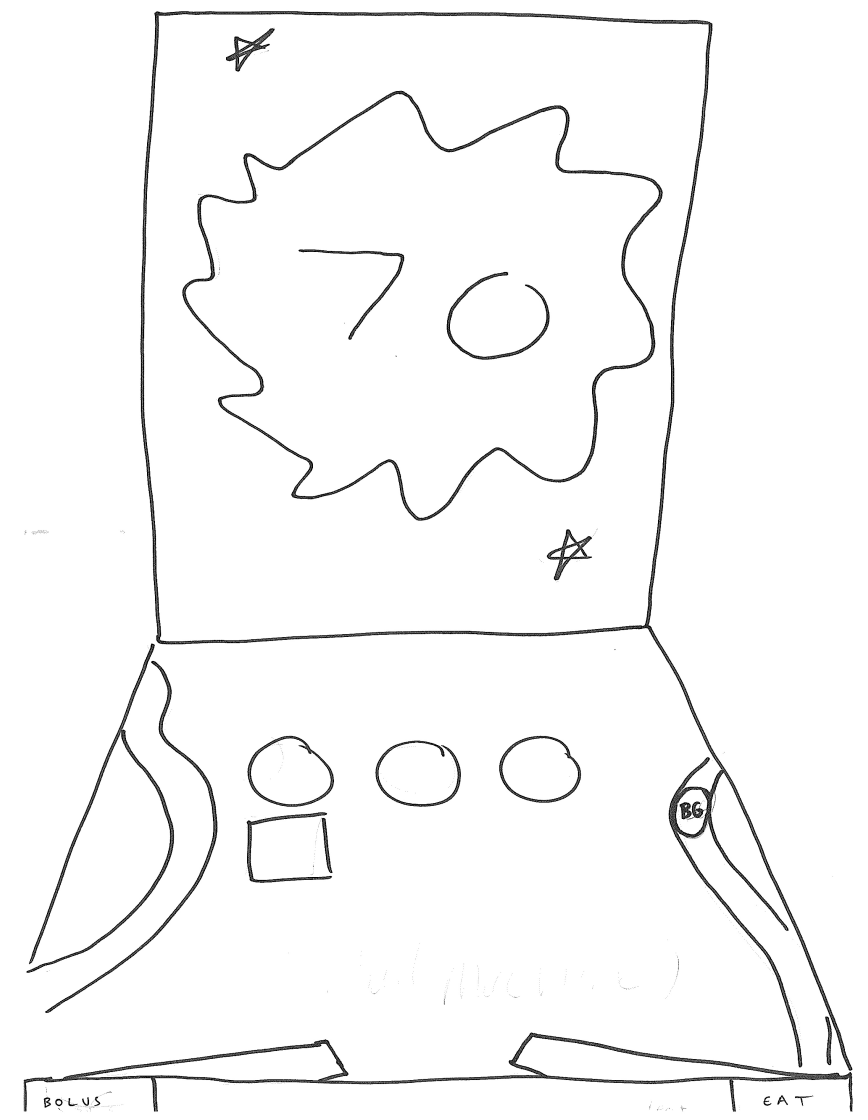

Figure 51. Diabetes management as an arcade pinball machine.

The second drew a claw machine, explaining that sometimes you appear to be in control of the machine, and in other instances it is programmed to function randomly (Figure 52). Sometimes her diabetes management was easy, and at other times she could not explain her blood glucose levels. She had learned to accept that diabetes was not completely controllable. This reflects previous chapters, where 
terminology discussing 'influencing' blood glucose levels rather than 'controlling' them was discussed, as absolute control was thought to be unachievable.

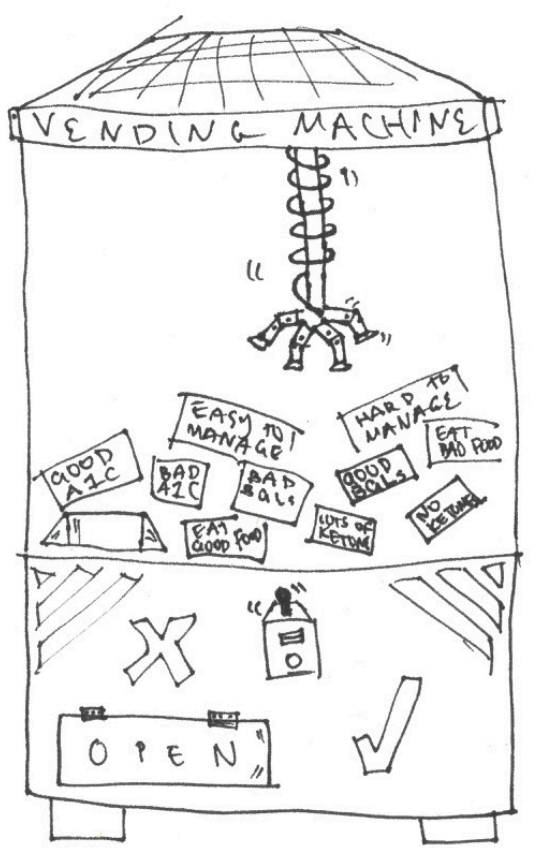

Figure 52. Diabetes management as an arcade claw machine.

\subsubsection{Rushing to beat the clock}

For one participant, there was a focus on the timeliness of diabetes management intersecting with other aspects of daily life. Her metaphor for managing type 1 diabetes was rushing to clock into work on time (Figure 53).
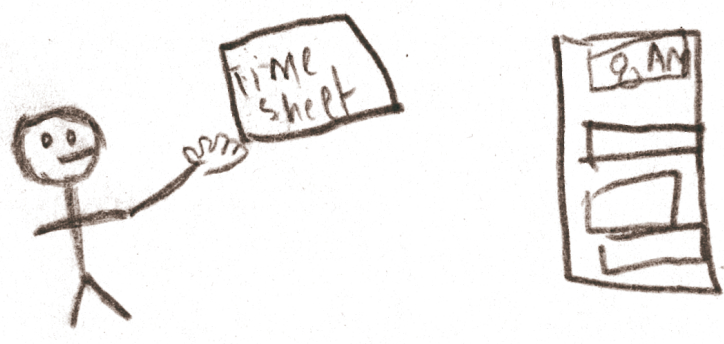

Figure 53. Diabetes management as rushing to work to clock in on time. 
With diabetes, like a full-time job, being on time was important, and there were obstacles that throw you off schedule. Insulin injections, eating, and other tasks need to be completed on time to facilitate blood glucose levels that allow for productivity. As diabetes tasks interact, if one task is not completed on time, it can create a snowball effect. This participant tries to adapt to these obstacles, such as being stuck in a long class and getting back on track where possible, but recognises that she cannot always prepare for these events. Further, her level of control varies with the time of day, being able to correct levels during the day but not being in control during nocturnal hypoglycaemia or hyperglycaemia. Thus, she tries to understand the impacts of her actions and ensure tasks are completed in a timely manner, to facilitate healthy, nocturnal blood glucose levels.

\subsubsection{Experiencing psychosocial impacts}

Finally, three participants described psychosocial impacts of diabetes and its management. The first used a metaphor of having an angel or devil on his shoulder, influencing his behaviour (Figure 54). The angel is characterised by normoglycaemia, allowing him to function and communicate as desired. The devil is characterised by rapidly changing blood glucose and is embodied as disagreement and irritation that he tries to suppress.
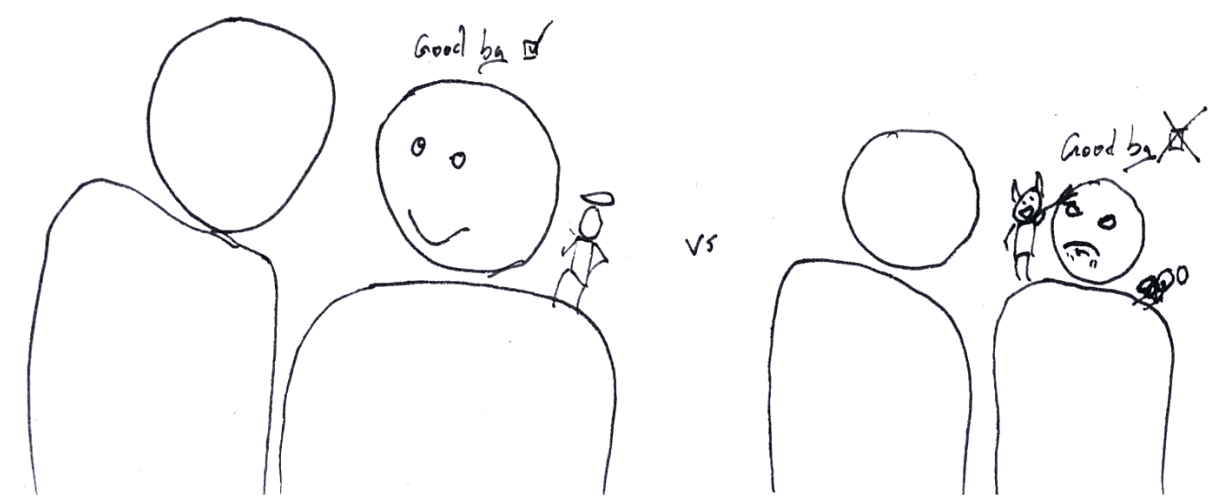

Figure 54. Diabetes appearing as an angel or devil on your shoulder.

The transition between moods can happen quickly, before noticing other symptoms. A5: "It's like you take a leash off a wild dog and it's in my head running around. I'm 
trying to catch it and have a civil conversation at the same time." Other participants also discussed how the symptoms of blood glucose levels could be underestimated.

Another participant described diabetes as her shadow (Figure 55). On some days its presence looms larger than others, but it does not define her. Like a shadow, it is always with her, but not always visible to others. While using medical technologies can make diabetes visible, devices can also pass for consumer items.

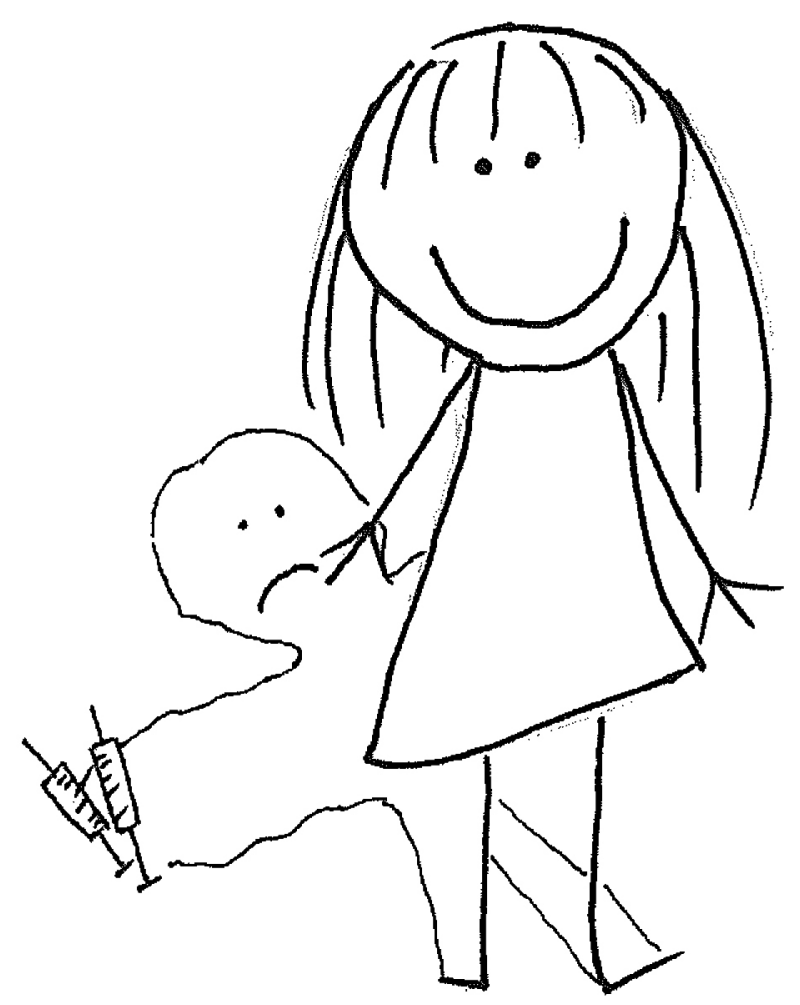

Figure 55. Diabetes as a shadow.

The final participant drew four thought bubbles to describe the current psychosocial impacts of self-management and the opposite effects she desired (Figures 56-57). She described these starting from the bottom right bubble and moving anti-clockwise. 


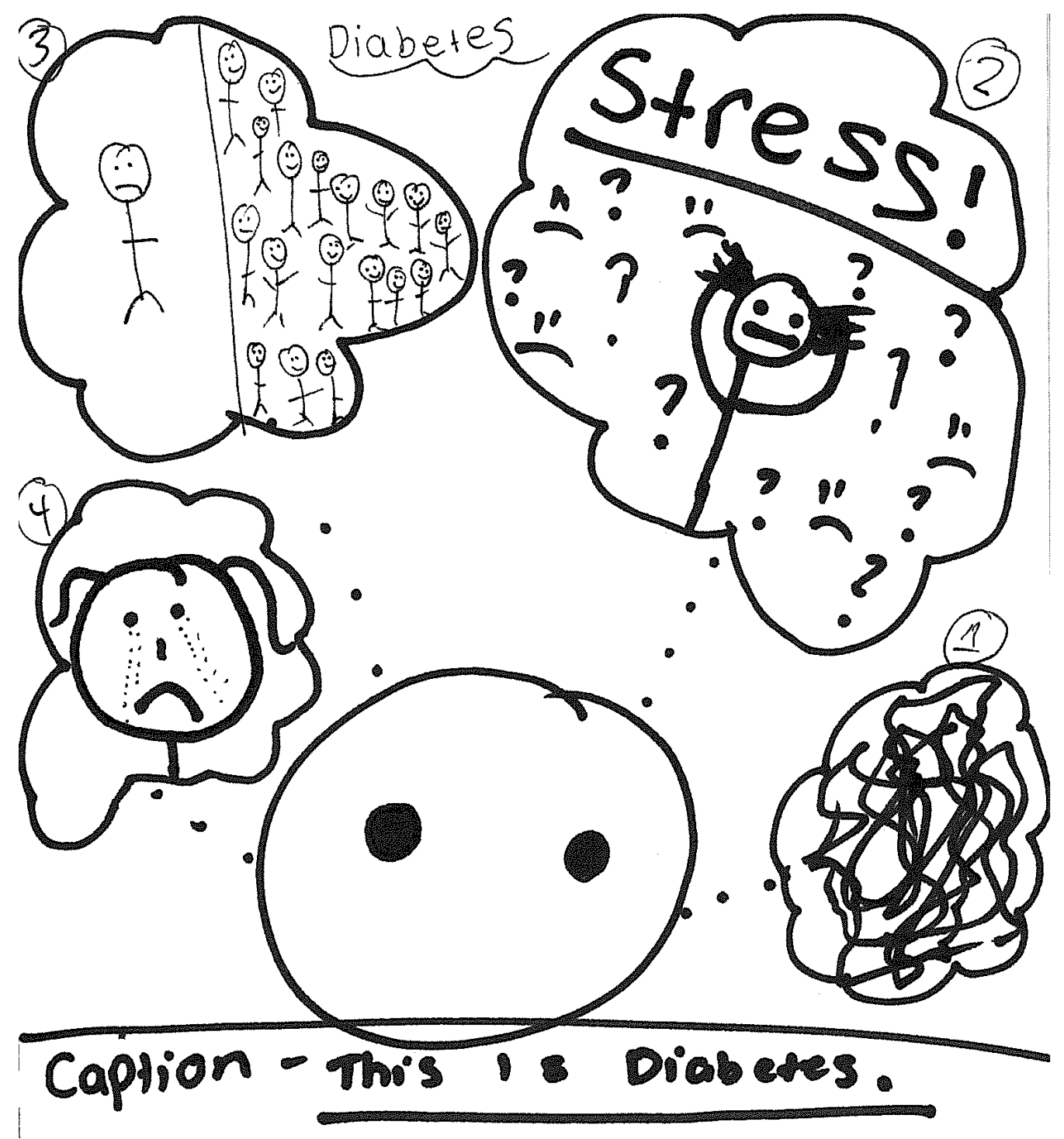

Figure 56. The negative psychosocial impacts of diabetes. 


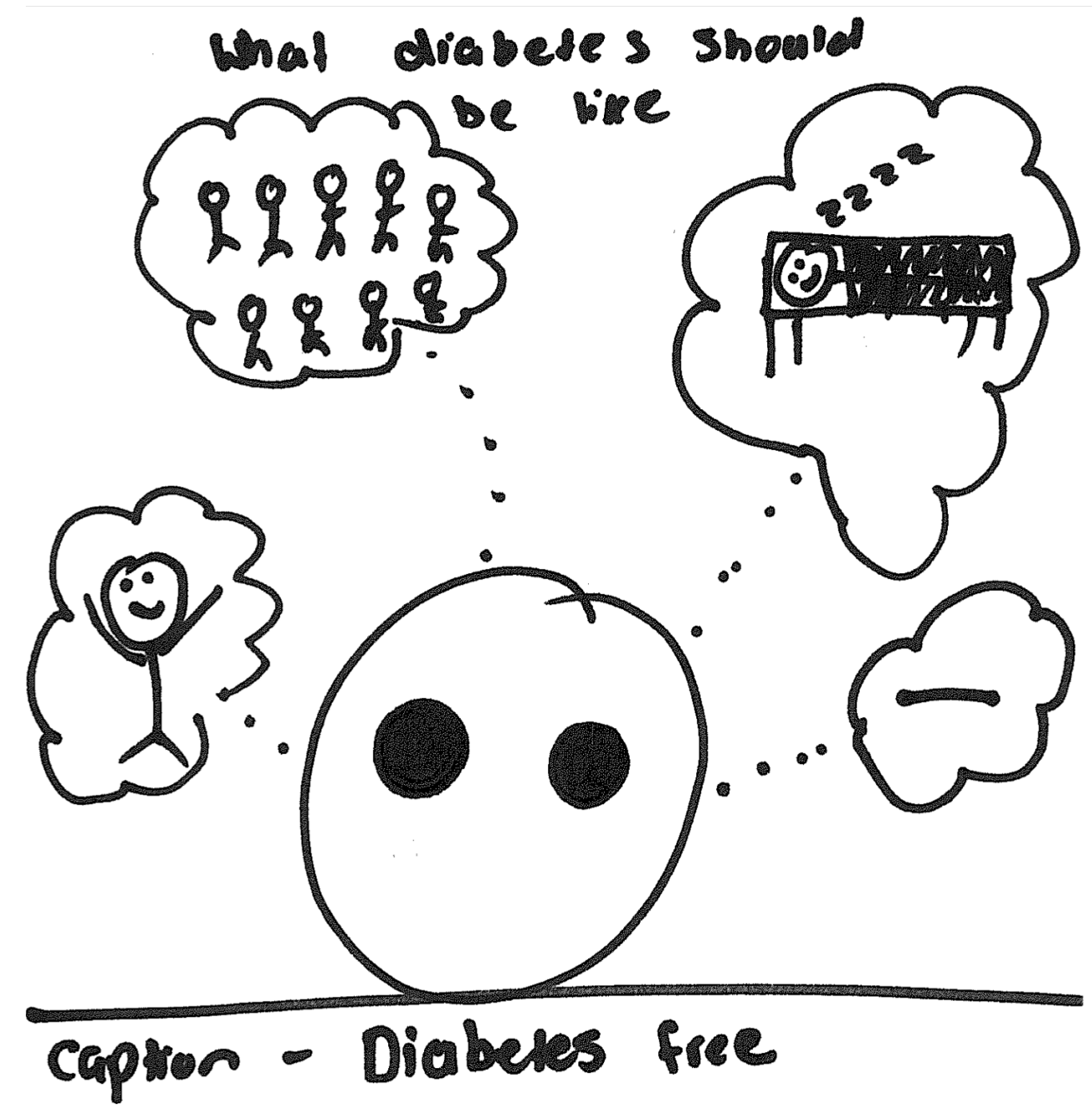

Figure 57. What diabetes should be like: no confusion, feeling relaxed and wellrested, included, and happy.

The first aspect was confusion that stemmed from having many factors to consider, such as food intake, basal levels, boluses, blood glucose test results, and exercise. The second aspect was feeling stressed and worried about everything related to type 1 diabetes, particularly attempting to communicate with health professionals when they did not recognise her point of view. The third aspect was feeling left out and different from peers. This was compounded by actions meant to support her. For instance, her mother coming to her school, teacher aides appearing in school photographs, isolating her to administer injections, or stopping her from participating in activities, and a primary teacher displaying her blood glucose levels on the class whiteboard. Consequently, she had become more secretive, and continued activities with peers regardless of blood glucose levels. Although support is 
important, it can feel forced, with adolescents tethered to adults whilst desiring independence (Dickinson \& O’Reilly, 2004). The final aspect was hating diabetes. Responsibility for self-management was being transitioned from her mother; the 'Queen' or 'God' of diabetes, having developed much knowledge. It was hard for her to manage with the competency of her mother, anxious for her to see 'bad' blood glucose results. However, she recognised two positive aspects of diabetes: meeting peers at camps and using diabetes as an excuse to not participate in an undesirable activity.

\subsection{Managing Your Way}

Participants wrote about current characteristics of their diabetes management and how they wished their management was, informing five categories.

\subsubsection{Developing diabetes knowledge}

Participants discussed developing diabetes knowledge in three ways: already having and attaining diabetes knowledge, desiring more diabetes knowledge, and wanting others, such as family and friends, to understand diabetes. Four participants were confident in their current levels of diabetes knowledge, learning from prior experiences and their diabetes team. Reliance on family for guidance varied depending on their family's knowledge and their independence.

Another three participants wanted greater diabetes knowledge. This would help in facing new challenges, managing proactively, developing independence, and understanding the consequences of specific actions (e.g., whether the sharpness of a needle affects absorption of insulin).

Six participants wanted others to have better diabetes knowledge. The benefits of friends, family, and teachers having a good understanding of diabetes would include noticing symptoms, offering advice, and helping in emergencies. Currently, people who were uninformed about diabetes made unhelpful comments and conflated different types of diabetes. Some participants would explain diabetes when noticed using medical technologies to minimise future misunderstandings. One participant suggested a resource for immediate family and friends be created that included medical information and tips for emotional and practical support, such 
as recipes. She felt this would reduce her burden of explaining and justifying her condition and actions to others.

\subsubsection{Balancing support and self-reliance}

Participants were divided in their desire for support networks or for self-reliance. The participants who favoured support networks were some of the young adults. Three participants wanted support from family, friends, and health professionals. This included talking openly about the frustrations of managing diabetes, particularly with friends with type 1 diabetes with first-hand understanding. One adolescent included her friends in management by creating a game described in Section 6.2.4.5. A suggestion was to form diabetes sports teams to foster safe, supportive environments, as is done with high performance teams currently (e.g., cycling Team Novo Nordisk) (“Home,” n.d.). One participant also felt positively about providing support by volunteering at diabetes camps, acting a role model for younger children.

Participants who favoured greater self-reliance championed not being reliant on health professionals for advice, being able to compile questions and only ask them at clinic visits, not having to deal with conflicting advice based on de-contextualised data, retaining privacy, and not frequently discussing diabetes with friends and family, as it highlighted their 'difference' and may burden their friends and family.

\subsubsection{Shrinking the role that diabetes plays}

A common desire related to shrinking the role that diabetes plays in adolescents' lives, and using technology to help do so. Desires included not having to stop an activity to manage diabetes, not frequently be thinking about, checking, or worrying about diabetes by knowing the correct insulin dosage had been administered, and not needing to plan food intake and activities in advance or remember to take medical equipment when going out. Other desires included having more confidence, independence, and better performance in school and sports.

Two participants suggested that reliable, closed-loop medical technologies could counter these issues. This technology would automatically test and sync blood glucose levels and administer insulin to suit, minimising the role of the adolescent in 
testing, insulin delivery, memory and decision-making, and reducing diabetes symptoms. In case of malfunction, they deemed it important that adolescents maintained sufficient diabetes knowledge.

\subsubsection{Focusing on positives or negatives}

Some participants discussed psychological aspects of diabetes management: worry, negative thinking when experiencing symptoms, and frustration at high or low blood glucose levels. One recognised the importance of having a positive attitude, noting the negative attitudes of others in social media support groups. While acknowledging the difficulties of self-management, and that effort did not always correspond with outcomes, she thought it best to focus on positives and not dwell on past or transitory issues. Another participant, along with health professionals (Chapter 4), suggested psychology support should be available.

\subsubsection{Accessing reliable technology and meaningful information}

The final desired aspect of self-management was access to technologies that were reliable and delivered meaningful information. Some participants were aware of technologies that were not accessible or affordable to them currently, and wanted access to these (e.g., CGMs, FreeStyle Libre Flash Glucose Monitoring System). Some had stopped using logbooks, as the laborious task outweighed the benefit received. While the blood glucose meter stored information, this was not commonly downloaded, and data access was unreliable. Other problems with the blood glucose meters were errors for too small a blood sample and not functioning when too hot or cold. One participant tried to heat her meter by putting it under blankets or putting it on her cat, but ultimately could not collect the data required.

\subsection{Love Letter, Break-up Letter}

Four break-up letters were written to blood glucose meters and one to a syringe holder. Two love letters were written to insulin pumps, one to a glucose monitoring system, one to a logbook, and one to an insulin pen. During the interviews, some participants talked about multiple devices, introducing more comparison within the information gathered. Three letters are included in full below. 
Dear My very problematic CareSens ${ }^{\mathrm{TM}} \mathrm{N}$.

Though you are a very permanent fixture in my life - often meeting 4 to six times a day, I wish I could let you go. Even though you are the cheapest way of monitoring my blood glucose we are only together because the government decided you were the best option. I don't ask much from you - just correct readings and that you stop deciding to pack it in if you get too hot or cold. Your predecessor was reliable, sleek and always functional, but alas you do not give me the same courtesy. You make me second guess myself and my health which I don't appreciate. Having an ongoing medical condition is hard enough without faulty devices and constant question marks. Sort it out and then we can talk. You're a bit outdated.

P.S. If only you were like the devices that didn't require pricks.

Dear insulin pump,

I love that you came into my life! You give me flexibility, confidence and happiness. Before you I did not realise how much I felt weighed down \& suffocated by the weight of Type One Diabetes. I love you because you enabled me to experience in many areas of my life. I felt the freedom and confidence to travel, to eat when and what I want, to play, run and jump. To step forward in life with greater independence. Although some days are frustrating and you seem to endlessly test my patience, overall I love what you have brought into my life. Whilst having type one diabetes is no "walk in the park," (and I would love a cure) I appreciate your presence in my life. I love how you tell me how much insulin I have onboard, can match my food to bolus and are discreet. I like no longer being restricted by 24 hour insulin, and can instantly drop or raise my basal rates. I am more confident to try new things, to be more active, and I love the support you offer me when I am unwell. Thanks pump, here's to a long and healthy future! (Unless a cure comes along!) Love [Name] $x$ 
Rocket - syringe holder to inject insulin

You came into my life at such a low point and I counted on you for support. I relied on you to stay healthy and happy, where most days you did the complete opposite. I started using you when I was first diagnosed, to ease the pain when giving my injections. At first I was relieved, as I didn't have to physically inject myself, I could simply push a button. Little did I know the noise of that button would continue to cause me grief, even up until today. Every meal time I felt terrified at the thought of it, blasting my headphones to my favourite songs through my walkman just to avoid that noise. The pain wasn't any easier, as you hurt me over and over with the bounce back spring that gave me the darkest bruises I've had from injecting. I'm glad I only had you in my life for a short period of time. Even looking at you a few years back brought tears to my eyes with the horrible memories flooding back.

Four categories were constructed in a combined analysis of the letters and interviews: acquiring and changing medical devices, requiring convenience and practicality for everyday contexts, collecting and using data, and corresponding with preferences and values.

\subsubsection{Acquiring and changing medical devices}

Adolescents desired access to affordable medical technologies that were reliable. They were frustrated by a lack of access to desirable devices, such as the Freestyle Libre, that they admired for its speed and fewer finger pricks. Following a change to Government subsidies, many participants felt frustrated using a meter some felt was less accurate and reliable. Although other meters were available, they were unaffordable. One participant, whose blood glucose meter was broken when a vehicle reversed over it, described another form of forced change. Her meter was replaced with a different model that she did not like.

In other instances, participants stopped using a medical device by choice. Reasons included moving to an insulin pen that could measure half units, and the insulin pump being described as an easier solution, minimising injections and being conceived of as a present. One participant described painful and noisy usage of a 
'rocket' device intended to make injections easier after diagnosis, hiding the needle from view and injecting with the push of a button. This example demonstrates the significance of considering the multi-sensory experience of using medical devices.

New devices could also help or hinder independence. For one adolescent, somewhat contrarily to previous research, his insulin pump temporarily reduced his independence as he had to learn how to use the new device, and was reliant on parents for site changes. Yet, like another participant in Chapter 6, the catalyst to do his own site changes happened at a diabetes camp.

\subsubsection{Requiring convenience and practicality for everyday contexts}

Adolescents discussed their functional demands of medical technologies within specific contexts, their preferences, and the inconvenience of carrying medical equipment. The functions available on medical devices affected how they were used in everyday contexts. For instance, while a blood glucose meter that did not make any beeping noises or have a light was discreet, it was also challenging to use at night, or when sleeping in a shared room. Convenience was important, with one lancing device described positively because it required only a one-button push to deploy. Helpful features of insulin pumps included showing how much insulin remained in the pump, calculating an insulin bolus to match food, and sites that worked for a week despite health professionals recommending they were changed every three days. Helpful features of insulin pens included cartridges that lasted 3-4 weeks, dosing half units, and showing last dosage and time since last injection.

There were mixed feelings about insulin pens versus insulin pumps. Pens do not require constant, invasive attachment, but must be carried and take time to use. Insulin pumps do not require multiple needle insertions and are easy to use in many situations (e.g., in a moving vehicle), but require backup equipment to be carried.

Participants described the inconvenience of carrying medical devices and consumables with them. Insulin pumps and blood glucose meters were described as "chunky" and "uncomfortable". While there was a desire for these devices to be smaller or more streamlined, the screen size needed to be legible. Adolescents stored devices in pockets, small bags or their bra, and could forget items if regularly switching bags. 
One participant described how she does not always take her medical equipment with her. She was aware that there may be emergency situations where she would need the devices, but had coincidentally been 'fine' in the past, happening to have her equipment when an earthquake struck. This behaviour typifies adolescents' propensity for risk-taking, one of the many features of normative adolescent development that interacts with managing a long-term condition (Warner \& Hauser, 2009). It reflects adolescent prioritising short-term benefits over possible consequences with a low-likelihood. Fear of medical devices breaking also arose, with one participant using disposable insulin pens rather than reusable ones while travelling, reasoning that she would have a stockpile of pens if one broke.

\subsubsection{Collecting and using data}

Participants discussed the accuracy of data and needing to trust them, a desire for meaningful information, and sharing data with other people. The poor reliability and accuracy of blood glucose meters was as a major frustration. YA1: "The amount of inaccurate numbers you've had, makes you second guess every single number, and so that really does play on your mind and you end up being a little bit more conservative with your corrections or your actions so then you run the risk of still being too high or too low." She (YA1) elaborated: "It scares me with how wrong you can be." Participants' confidence in the meter eroded over time, and led them to second-guess both the accuracy of the device and their own bodily feelings, making decision-making difficult.

When faced with a reading that did not match the way she felt, one participant would use a spare meter then calculate insulin dosage based on which reading best represented how she currently felt. Consecutive measurements could differ by up to $18 \mathrm{mmol} / \mathrm{L}$, which is clinically significant (Craig et al., 2011). While adolescents often did not wash their hands with soap and water before use as instructed, this could be challenging based on context, such as working on a farm or cycling, and other meters worked with hand sanitisers, which were more convenient.

Insulin pumps could also be unreliable, as insulin delivery lapsed if the insulin or battery ran out, although the pump alerted in advance. One participant found the correction calculation on his pump was inaccurate and resultantly he had 
to do this manually. In contrast, some adolescents did not experience reliability issues with their devices, and trusted them.

Adolescents described a desire for data to be transformed into meaningful information. One wanted a glucose monitoring system that would regularly collect and visualise her blood glucose levels. Seeing trends would help her understand how her blood glucose levels fluctuate overnight. Another participant felt features of her blood glucose meter, such as arrows for high and low levels, may be more helpful to people recently diagnosed with diabetes who did not have an insulin pump. This illustrates the importance of not only supplying data, but also transforming them into meaningful information. Others were pleased that their insulin pens and logbooks allowed them to check when they last injected.

Participants also discussed sharing blood glucose monitoring data with parents and health professionals. One participant thought a non-invasive blood glucose monitor would allow her parents to check her levels at night if concerned. While another participant wanted to gain insights by sharing a log of her blood glucose data with a health professional, frustratingly, her blood glucose meter stopped providing data for a day because it was not within the required functioning temperature range.

\subsubsection{Corresponding with preferences and values}

This category outlines two aspects: adolescents' thoughts about attention garnered by medical devices, and the level of fit between individuals and their medical devices. As medical devices for type 1 diabetes are used in many contexts, their appearances and discreetness were described as important factors. One participant valued her blood glucose meter not making any noise and drawing attention in particular contexts, while another described how her insulin pump was more discreet to use in public than injecting insulin using a pen. Participants voiced a preference for their medical devices to appear visually more like sleek, slim consumer devices. Disposable insulin pens and blood glucose meters and lancets were criticised as feeling "cheap" due to materials, screens, and faulty functions. Participants wanted functionality and aesthetics in tandem. 
When a medical technology is well-matched to the user's needs, it can facilitate positive feelings and life experiences. Writing about her insulin pump, one participant wrote, "You give me flexibility, confidence and happiness... I love you because you enabled me to experience in many areas of my life." Accordingly, this participant saw her device as not only administering insulin, but also allowing her the freedom to live her life unhampered by diabetes.

\subsection{Best and Worst}

Nine participants reviewed a range of medical devices, accessories and consumables (Table 9), attaching stickers to their devices to signify best, worst, and desired features (Figure 58). 
Table 9

Medical devices reviewed by participants.

\begin{tabular}{|c|c|}
\hline Type of device & Models reviewed \\
\hline \multirow[t]{4}{*}{ Blood glucose meter } & CareSens N POP \\
\hline & CareSens N \\
\hline & Freestyle Optium \\
\hline & Accu-Chek Performa \\
\hline \multirow[t]{2}{*}{ Lancet } & Accu-Chek Multiclix \\
\hline & Unable to identify model \\
\hline Test strips & CareSens \\
\hline Ketone meter & Optium Xceed \\
\hline \multirow[t]{5}{*}{ Insulin pen } & NovoPen Echo \\
\hline & NovoPen 4 \\
\hline & Novopen 3 \\
\hline & Sanofi Lantus SoloSTAR \\
\hline & Eli Lilly HumaPen Luxura \\
\hline \multirow[t]{2}{*}{ Insulin pump } & Animas Vibe \\
\hline & Medtronic Minimed \\
\hline \multirow[t]{2}{*}{ Pump sites/infusion sets } & Animas consumables \\
\hline & Medtronic consumables \\
\hline \multirow[t]{3}{*}{ Glucose } & Dextro Energy \\
\hline & Glucagon \\
\hline & Select juice box \\
\hline Kit bag & Freestyle \\
\hline
\end{tabular}

Data were sorted into existing categories of design considerations developed for medical devices (Carayon, Alvarado, \& Wiklund, 2010; Gardner-Bonneau, 2010; Stein \& Wiklund, 2010). These categories seek to consider aspects sensitive to patient use in everyday contexts. Three additional categories were created to represent findings that did not fit the existing considerations: reliability, waste, and comfort. 


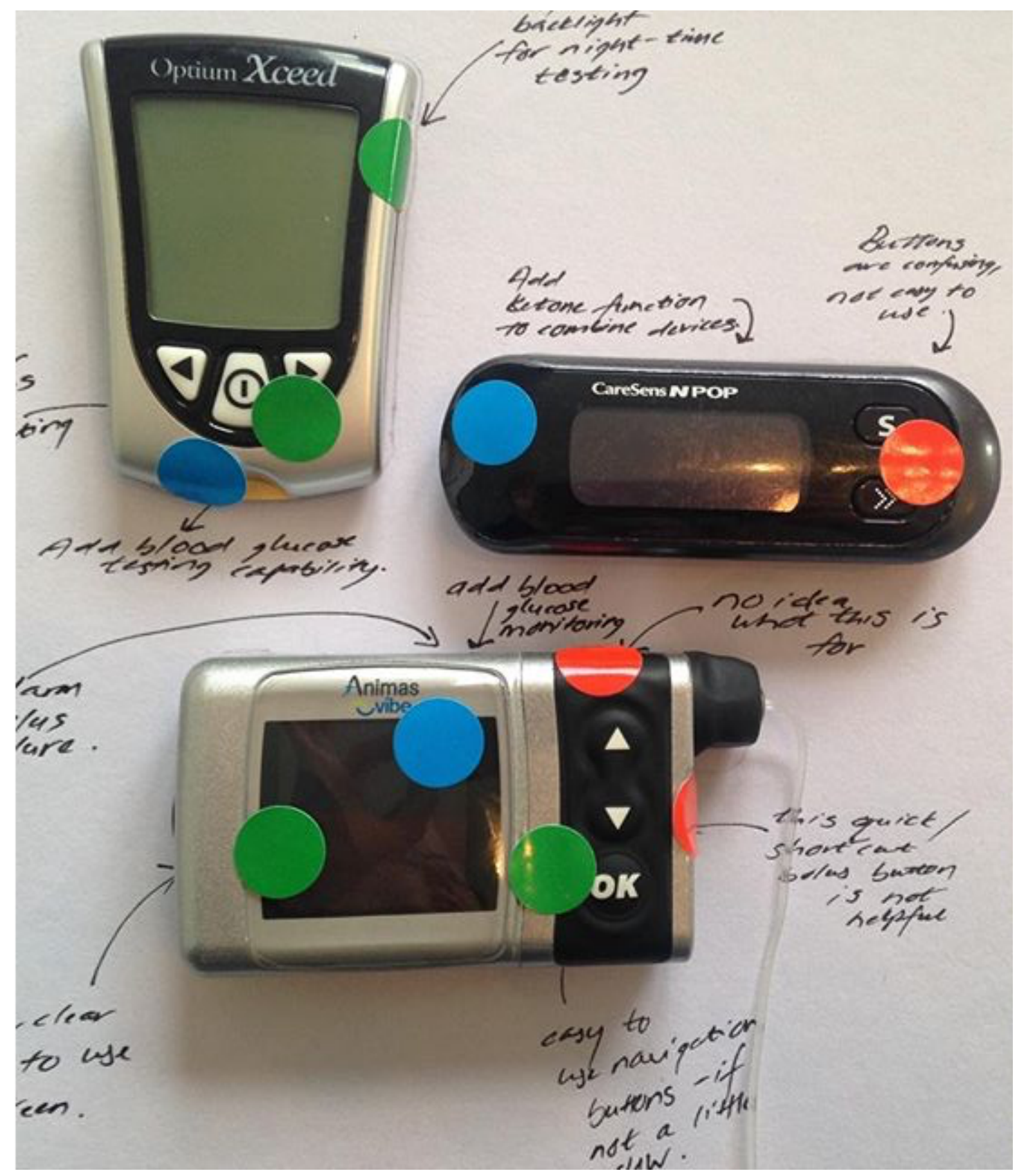

Figure 58. Best, worst, and suggested features of medical devices.

\subsubsection{Flexibility of user interfaces}

Medical devices needed to be flexible for varying adolescent needs, for instance, the NovoPen Echo could measure half units for small and accurate insulin dosages.

\subsubsection{Lighting}

Adolescents required device lights for use in dark environments, giving them enough light to take necessary actions without being overly bright. Participants were critical of blood glucose meters that did not have a backlit screen or a light near the test strip 
port. The Freestyle Optium meter was praised for its protruding button that controlled a soft orange light for use in dark environments.

\subsubsection{Noise}

There were issues both with medical devices making noises (e.g., an insulin pump alarming periodically for low insulin levels) and not making noises (e.g., no alarm for bolus failure). Adolescents wanted their medical devices to communicate important information to them, but not to draw unwanted attention. Alarms vibrating, rather than sounding, and having a silencing button (such as on a mobile phone) would be preferable.

\subsubsection{User fatigue}

Manually inputting data into a device, adjusting insulin rates (e.g., for illness or exercise), and filling pump cartridges were tedious. Participants wanted medical devices to sync to computers and phones, visualise data, calculate corrections, and prompt them of patterns in their blood glucose levels. Some also wanted a closedloop, artificial pancreas system, minimising their role.

\subsubsection{Size and shape}

Participants carried many devices, and thought making them smaller would allow them to be stored with other devices and draw less attention. However, they were wary that screen size and insulin capacity should not be reduced. The length of some insulin pens also made them difficult to store in kits designed to carry blood glucose testing equipment.

\subsubsection{Controls}

Buttons on some insulin pumps and blood glucose meters were intuitive and easy to use, with arrows commonly used for increasing and decreasing, or scrolling through options. However, the buttons on the CareSens N POP, one labelled with an arrow and one with an 'S', were criticised, with participants not understanding what these symbols represented. 
Both the Medtronic Minimed and Animas Vibe insulin pumps were criticised for slow speeds. Participants described slow data input and menu navigation, making them self-conscious and resulting in errors due to lag.

\subsubsection{Displays}

While screens were an appropriate size, they could be dull and difficult to read, particularly when outside in bright conditions. Adjustable screen brightness would be beneficial. Analogue displays such as the dial on an insulin pen could also be challenging to read, as the needle would settle between numbers. Overall though, displays were successful at providing accurate feedback. Examples included a screen on an insulin pen that displayed the dosage and duration since the previous injection, on-screen indicators for low battery and insulin on a pump, and a manual dial that showed how many lancets were available for use in a finger pricker.

\subsubsection{Learnability and intuitiveness}

Glucagon and refillable and disposable insulin pens were described as easy to administer. Instructions provided with the glucagon and syringe were essential, as they may need to be used in an emergency by another person. Pens being prefilled, or taking cartridges, also made them easier to use than a syringe.

\subsubsection{Adjustability}

Participants appreciated devices with adjustable settings, such as a lancet with variable depth settings and insulin pumps with the ability to reduce insulin for exercise.

\subsubsection{Portability and maneuverability}

Participants noted the advantages and disadvantages of carrying versus wearing medical devices. Being attached to a device could be "bulky" and visible, drawing attention. However, devices to be carried could be forgotten.

\subsubsection{Storage}

Participants used a kit bag to store their glucose meters and consumables and sometimes stored insulin delivery devices, other consumables, and glucose sources in 
these bags too. Some used bags supplied with devices and others bought their own. Complaints about these storage solutions included used test strips jamming the zip and the container used to store test strips making the kit bulky, though convenient as when down to the last strips in the pottle, a new container could be opened and the last strips added to it. The alternate flat packets of five test strips could also be mistakenly torn open. Compartmentalised containers for storing medical equipment were beneficial for organisation and compressing equipment, and there were opportunities for minimising packaging.

\subsubsection{Self-containment}

Some participants wanted medical devices to be integrated (e.g., combining a blood glucose meter with a ketone meter and lancet). While devices were individually small, cumulatively there was much equipment to be carried.

\subsubsection{Freedom from calibration, maintenance, and repair}

Cartridges and lancets were easy to replace, particularly if a drum of lancets was used rather than a single lancet. However, lancets were not frequently changed. One participant suggested the insulin pen have a rotatable drum of needles like some lancets.

\subsubsection{Climate (thermal environment, humidity, airflow, pressure)}

Using a blood glucose meter when swimming and an insulin pump in a humid climate were examples of environments making device use challenging. One participant had stopped going to the pool after becoming frustrated at repeatedly leaving the pool to test her blood glucose levels, and had also struggled to keep a pump site attached in humid weather, having to manually inject her short-acting insulin every few hours.

\subsubsection{Power requirements}

Medical technologies generally had a good battery life unless faulty, with batteries rarely needing to be changed. 


\subsubsection{Aesthetics and unobtrusiveness}

NovoPen and Sanofi Lantus SoloSTAR inulin pens were praised as 'sleek.' One participant wished her pen was smaller and discreet, though she was grateful someone recognised it was a medical device and stored it when she left it in a library. Another had previously used a sticker to personalise her pen, but had decided that these were 'ugly' and that she had grown out of them. Another participant described the challenge of finding medical technology that suited her aesthetic preferences when moving into young adulthood, no longer wanting items designed for children. Preferences change substantially early in life, with changes due to maturation common, although these are not yet fully understood (Loewenstein \& Angner, 2003).

Every few months, one participant bought herself a new bag to store her diabetes equipment, choosing to use consumer products rather than those supplied by device manufacturers to 'disguise' her test kit. In terms of discreetness, pumps were preferable to other insulin delivery devices when bolusing rather than injecting, however, they were often mistaken for mobile phones and users reprimanded, and were hard to hide when wearing swimwear or other outfits. Some adolescents were conscious of how peers might react if they noticed the insulin pump site protruding. While managing around complete strangers was fine, it could be awkward to do so in front of acquaintances.

\subsubsection{Reliability}

There were reliability issues with medical devices. Blood glucose meters sometimes did not recognise a test strip had been inserted, wasting blood and time, the date would reset, making it hard to look through past data, and readings were unreliable when the meter was hot or cold. With insulin pens, following injection insulin would squirt out of the pen, making the user unsure whether their entire dose had been delivered. With one adolescent's insulin pump, the feature to calculate corrections often did not work, suggesting a correction of 0 for a blood glucose level of $24.6 \mathrm{mmol} / \mathrm{L}$. While the adolescent could manually calculate his correction, the mathematics became difficult when also trying to bolus at the same time. 


\subsubsection{Waste}

Adolescents broadly objected to waste created through diabetes management. It was important for medical devices to correspond with people's values. For instance, four participants objected to disposable inulin pens, with one participant wanting to replace her disposable insulin pen with a reusable one to be more environmentally sustainable. Used consumables (e.g., test strips) could also cause hassle when strewn through test kits or households.

\subsubsection{Comfort}

Adolescents wanted fewer invasive and uncomfortable medical device interactions. Inserting an insulin pump site could be painful, but injecting insulin was more frequent. Participants also described not liking the marks, such as red dots and sticky residue, left on their skin by devices.

\subsection{Discussion}

\subsubsection{Psychosocial user requirements}

The 18 user requirements identified in this chapter largely supported and developed those constructed in earlier chapters.

1. Allow accessibility and affordability: Acquiring and changing medical devices, Accessing reliable technology and meaningful information

2. Foster trust in accuracy and reliability: Collecting and using data, Accessing reliable technology and meaningful information, Flexibility of user interfaces, Reliability

3. Provide usability: Controls, Learnability and intuitiveness

4. Facilitate comfortable and non-invasive use: Acquiring and changing medical devices, Requiring convenience and practicality for everyday contexts, Collecting and using data, Comfort

5. Minimise psychological distress: Experiencing psychosocial impacts, Experiencing social support and difficulties, Shrinking the role that diabetes plays, Focusing on the positives or negatives 
6. Align with values and preferences: Requiring convenience and practicality for everyday contexts, Aesthetics and unobtrusiveness, Waste

7. Provide choices, customisation or personalisation: Acquiring and changing medical devices, Adjustability

8. Perform in a life-proof manner: Collecting and using data, Climate

9. Enhance convenience: Requiring convenience and practicality for everyday contexts, Rushing to beat the clock, User fatigue, Size and shape, Controls, portability, and manoeuvrability, Storage, Self-containment, Freedom from calibration, Maintenance, and repair, Power requirements

10. Facilitate reliable and efficient night-time management: Requiring convenience and practicality for everyday contexts, Lighting, Displays

11. Communicate data and information to facilitate decision-making: Requiring convenience and practicality for everyday contexts, Collecting and using data, Developing diabetes knowledge, Noise, User fatigue, Displays

12. Couple behaviours with consequences: Rushing to beat the clock

13. Facilitate daily structure while allowing for spontaneity: Rushing to beat the clock, Reduce impact on life, Shrinking the role that diabetes plays

14. Facilitate realistic goals and responsiveness to get back on track: Managing a balancing act, Struggling for control

15. Minimise or eliminate feelings of stigma or embarrassment while using medical technology: Corresponding with preferences and values, Experiencing psychosocial impacts, Noise, Aesthetics and unobtrusiveness

16. Increase social awareness and normalise self-care actions and devices: Developing diabetes knowledge, Balancing support and self-reliance

17. Support transition of responsibility: Acquiring and changing medical devices, Collecting and using data, Balancing support and self-reliance

18. Facilitate interaction with diabetes peers and friends: Experiencing psychosocial impacts, Developing diabetes knowledge, Experiencing social support and difficulties 


\subsubsection{Discussion}

In the first exercise the metaphor of balancing, which was identified in previous studies, was supported (Hagey, 1984; Harris \& Pokorny, 2012; Huttlinger et al., 1992; Youngson et al., 2015). However, while the metaphors identified in the literature commonly related to glycaemic control, three participants in this study created metaphors about the psychosocial impacts of diabetes management: namely, an angel and devil, a shadow, aspects of worry, feeling left out, and hating diabetes. While metaphors pertaining to glycaemic control can be helpful in understanding the mechanics of diabetes, these metaphors provide a deeper understanding of how adolescents conceptualise diabetes management.

In the second exercise, participants discussed their current self-management, but also how they wished this to be. Often participants had differing views, some preferring social support and others self-reliance. The role of diabetes knowledge was important for participants, but they also desired better awareness from friends, family, and the public. A common desire was to shrink the role that diabetes played, looking toward automation and closed-loop systems of the near future. While some participants described negative psychosocial impacts of diabetes, others realised the need to develop a positive mindset for long-term management. Finally, there was a desire for better access to medical technologies that provided meaningful information to be implemented.

I was concerned that writing a break-up letter to a device that participants were required to use could be distressing. To alleviate this, participants were free to choose whether to write a love letter or a break-up letter to a device of their choice. No participants who completed the activity reported it as distressing. Adolescents wanted affordable access to reliable medical technologies, and were changing devices due to their features and their impact on quality of life. Although they had varying preferences, they wanted appropriate device functions for specific contexts, and found carrying medical equipment inconvenient. Current frustrations revolved around not trusting the accuracy of data, with considerations about providing more meaningful information and how data is shared with others. Adolescents also discussed eliminating medical device features that drew negative attention and 
improving the positive aspects of product design, along with the level of fit between their needs and devices.

In the final activity, where adolescents identified good, bad, and desired features of medical devices, their commentary focused largely on desiring intuitive and adjustable designs, reliability and resilience, comfort, minimising waste, and easing storage and portability. The context-dependent nature of evaluating features was also evident, with some features such as beeping noises being seen as positive or negative in different environments.

While 10 participants completed some or all of the activities, seven participants completed no activities. I hypothesised that having six activities to complete, and the timing of delivery of the probes near school and university exam periods and Christmas holidays, may have been problematic. Further, two participants chose to complete some of the activities during the follow-up interview, rather than at home as intended. Some participants described the activities as fun, and one asked that some of his activities be returned to him, as he was proud of what he had produced.

In addition to the analyses above, the data were also compared to the NADI model to determine what type of information was produced using each of the activities (Van Der Bijl Brouwer \& Dorst, 2014). The information gathered through the probes ranged from very specific (e.g., button noises) to broader feelings (e.g., confidence). The managing your way and letter writing activities resulted in insights at all four levels of the NADI model. For example, with blood glucose monitoring, a solution was a meter that did not require finger pricks, a scenario was the ability to function reliably at a range of temperatures, an important goal was to give users confidence to make diabetes-related decisions, and a broad theme was managing blood glucose effectively. As anticipated, the metaphor activity produced higher-level information, and the best and worst activity more specific lower-level information. As discussed previously, themes and goals are helpful for reframing design problems and opening up the 'solution space', while scenarios and solutions are helpful for incremental innovation and refining solutions (van der Bijl-Brouwer, 2016). Designers and suppliers of medical devices could apply a similar combination of 
methods to gain insight into the user experiences and requirements of people with health conditions.

Table 10

Levels of information elicited by cultural probe activities.

\begin{tabular}{lcccc}
\hline & Solutions & Scenarios & Goals & Themes \\
\hline Making a metaphor & & $\checkmark$ & $\checkmark$ \\
\hline Managing your way & $\checkmark$ & $\checkmark$ & $\checkmark$ & $\checkmark$ \\
\hline Love letter, break-up letter & $\checkmark$ & $\checkmark$ & $\checkmark$ & $\checkmark$ \\
\hline Best and worst & $\checkmark$ & $\checkmark$ & & \\
\hline
\end{tabular}

\subsection{Summary}

Cultural probes were an effective method of gathering information about adolescents' experiences of self-management and user requirements for medical technologies. This cultural probes study was successful in answering the four subquestions posed.

Firstly, how do adolescents conceptualise self-management of type 1 diabetes? Adolescents drew metaphors for diabetes self-management. These were grouped into four categories: managing a balancing act, struggling for control, rushing to beat the clock, and experiencing psychosocial impacts.

Secondly, how do adolescents want to manage diabetes? Adolescents' current concerns included developing diabetes knowledge, focusing on positives or negatives, and experiencing social support and difficulties. They were also conscious of balancing support and self-reliance, accessing reliable technology and meaningful information, and shrinking the role that diabetes played.

Thirdly, what relationships do adolescents have with medical devices? Adolescents described the reasons they love or hate certain medical devices. They discussed acquiring and changing medical devices, requiring convenience and practicality for everyday contexts, collecting and using data, and corresponding with preferences and values. 
Finally, what are the best and worst features of current medical devices, and what features do they lack? Adolescents identified medical device features that were or were not currently effective, and features they wished were available. These features could largely be categorised into existing categories for design consideration of portable medical devices used in the home or various environments. Additional categories were created to represent findings that did not fit the existing considerations: reliability, waste, comfort, and surplus features. 


\section{Addressing Medical Device-Related Attention Through Participatory Design}

Adolescents and young adults with type 1 diabetes are required to use medical devices to test their blood glucose levels regularly. However, using these devices can draw attention and fuel stigma in various everyday situations. This study describes the results of a participatory design workshop that explored six strategies for addressing product-related stigma with young people with type 1 diabetes and designers. The workshop followed participatory design principles, giving young people with type 1 diabetes a role in the design process, and allowing designers and users to collaborate and learn from each other (Simonsen \& Robertson, 2013). This study aimed to develop one psychosocial user requirement identified in previous chapters: to minimise or eliminate feelings of stigma or embarrassment while using medical technology. While it was clear from previous chapters that some adolescents find using medical devices embarrassing or stigmatising, it was unclear how medical devices might be designed to reduce this discomfort. In this chapter, strategies for eliminating or reducing medical device-related stigma are identified, assessed, and trialled through participatory design.

Adolescents' self-management of type 1 diabetes should be considered in a social context. Self-management in public spaces can be challenging, as it can draw unwanted attention and questions at a life stage when social acceptance is important (Y.-L. Wang et al., 2010). Social anxiety can result in poorer self-management and quality of life among adolescents with type 1 diabetes (Di Battista, Hart, Greco, \& Gloizer, 2009).

Shabert et al. (2013) proposed a thorough framework for diabetes stigma that recognises the complexities of experiencing and managing diabetes-related stigma. While the framework recognises needle use as a contributor to stigma, it could be extended to recognise the stigma-inducing impacts of adolescents with type 1 diabetes being overweight or having body image anxieties, and also the design of medical technologies as a possible stigma-reducing strategy. 
Product-related stigma around glucose monitoring aligns with Goffman's (1963) concept of the discreditable, whereby there is no noticeable stigmatising attribute until the person uses their medical equipment to test their blood glucose levels. The act of managing health using medical devices is the same act that can trigger perceptions of, or anxiety about, stigma.

The current study focuses solely on product-related stigma rather than, for example, stigma associated with a hypoglycaemic episode. While it is clear that medical devices can be stigmatising, it is less clear how to design devices that have more positive appraisals.

A number of medical and assistive device designs exist aimed at reducing the negative attention from using these devices in everyday contexts. Designs include colostomy bags modelled on lingerie or tattoos ("Stephanie Monty designs colostomy bag for intimate moments," 2016), a catheter set that appears as toiletries (Corydon, 2015), scarves and accessories for storing and concealing insulin pumps (“Core77 Design Award 2011,” 2011; Kinsman, 2012), and sleek, insulin delivery jewellery (Figure 59) (Heiss, 2013).

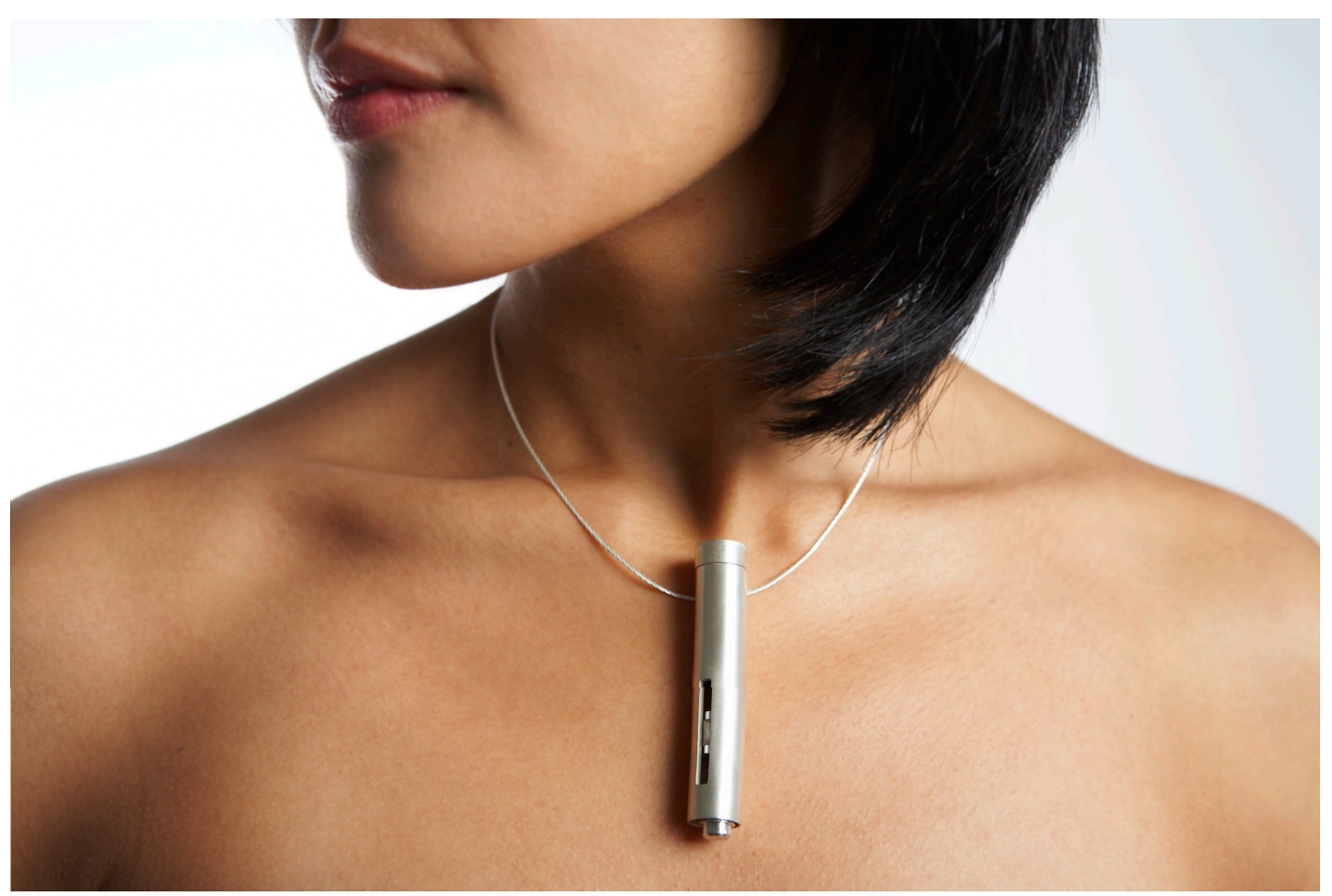

Figure 59. Jewellery for insulin delivery. Reproduced from "Diabetes Applicator Neckpiece," by L. Heiss with Nanotechnology Victoria, photo Narelle Portainer. Cast silver with stainless steel internal mechanisms. Copyright 2007. Reprinted with permission. 
These designs respond to stigma by hiding the medical device or disguising it as a consumer product. While some designs aimed to reduce negative attention, others increased positive attention. For instance, the Hue concept design uses boldly coloured inhalers (Zarki, 2017), another blood glucose meter draws on the design style of Marc Jacobs (Noe, 2017), the Google phone by Napka uses colourful, modular health tracking components (Howarth, 2015), and an online community share their decorated insulin pumps (Figure 60).

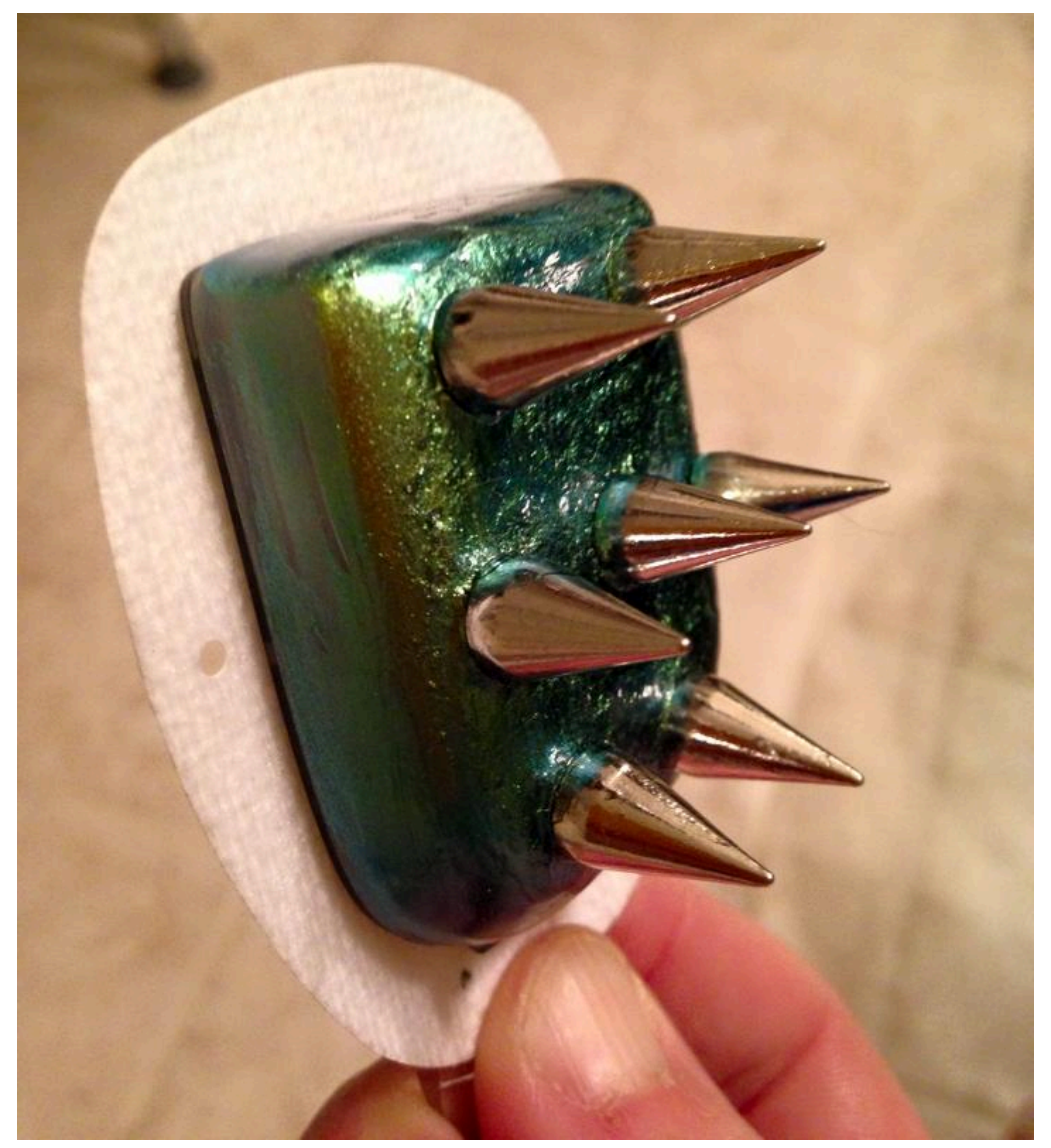

Figure 60. A 'pimped' insulin pump. Reproduced from "Pimp my pod," by C. Gladwell, 2015, Facebook. Copyright 2015 by Facebook. Reprinted with permission.

While medical and assistive devices are commonly designed to be discreet or unobtrusive, this may convey an inherent message of shame in these devices (Pullin, 2009). It has been suggested that the stigma associated with disability cannot be ameliorated by hiding the disability, and instead, symbols that contradict the stigma should be employed. For example, wheelchairs worn from collisions in wheelchair rugby indicate the intensity of the sport and contradict preconceptions of user frailty (Bispo \& Branco, 2011; Sansoni et al., 2016). Other strategies for addressing product- 
related stigma include education, technological advances, mainstream characteristics, positive association with existing brands, adoption of stigmatised characteristics, and other groups desiring assistive device functions (Stockton, 2009).

In addition, Jacobson (2010) proposed three strategies for addressing stigmatising features of assistive devices. These are to disguise, to shift attention away from, and to transform stigmatising features into prestigious features. This study draws on Vaes' (2014) Product Intervention Model for Stigma, which builds on the strategies outlined above, reporting 17 strategies for addressing stigma through 'product interventions', 'user interventions', and 'culture interventions'.

\subsection{Methods}

Adolescents can be active in research by taking on design roles and prototyping their ideas. A craft approach to participatory design can help young people to create personally meaningful health representations (Ananthanarayan, Siek, \& Eisenberg, 2016). Prototyping has been a common method used in participatory design workshops with adolescents (Fitton et al., 2016; Glasemann \& Kanstrup, 2011; Kanstrup, Bertelsen, \& Nøhr, 2015; van der Velden \& Machniak, 2014). Importantly, participatory workshops have demonstrated people with diabetes develop original, innovative, low-fidelity prototypes for medical technologies that address healthrelated emotions (Glasemann \& Kanstrup, 2010; Kanstrup, 2014). Further, narrowing the scope of the design workshop can give direction without limiting creativity for young people with type 1 diabetes (Glasemann \& Kanstrup, 2008).

\subsubsection{Participants}

Participants were two females and three males with type 1 diabetes aged 15-24 who had diabetes for 5-10 years, and five designers aged 21-29. Three of the designers had Master's degrees in design and were currently working in the industry, and the remaining two were currently completing a Master's or $\mathrm{PhD}$ designing assistive technologies. I facilitated the workshop. Blind portraits participants drew of each other during the workshop are pictured in Figure 61. Two workshops were run to accommodate participants' availability, with one designer participating twice. 

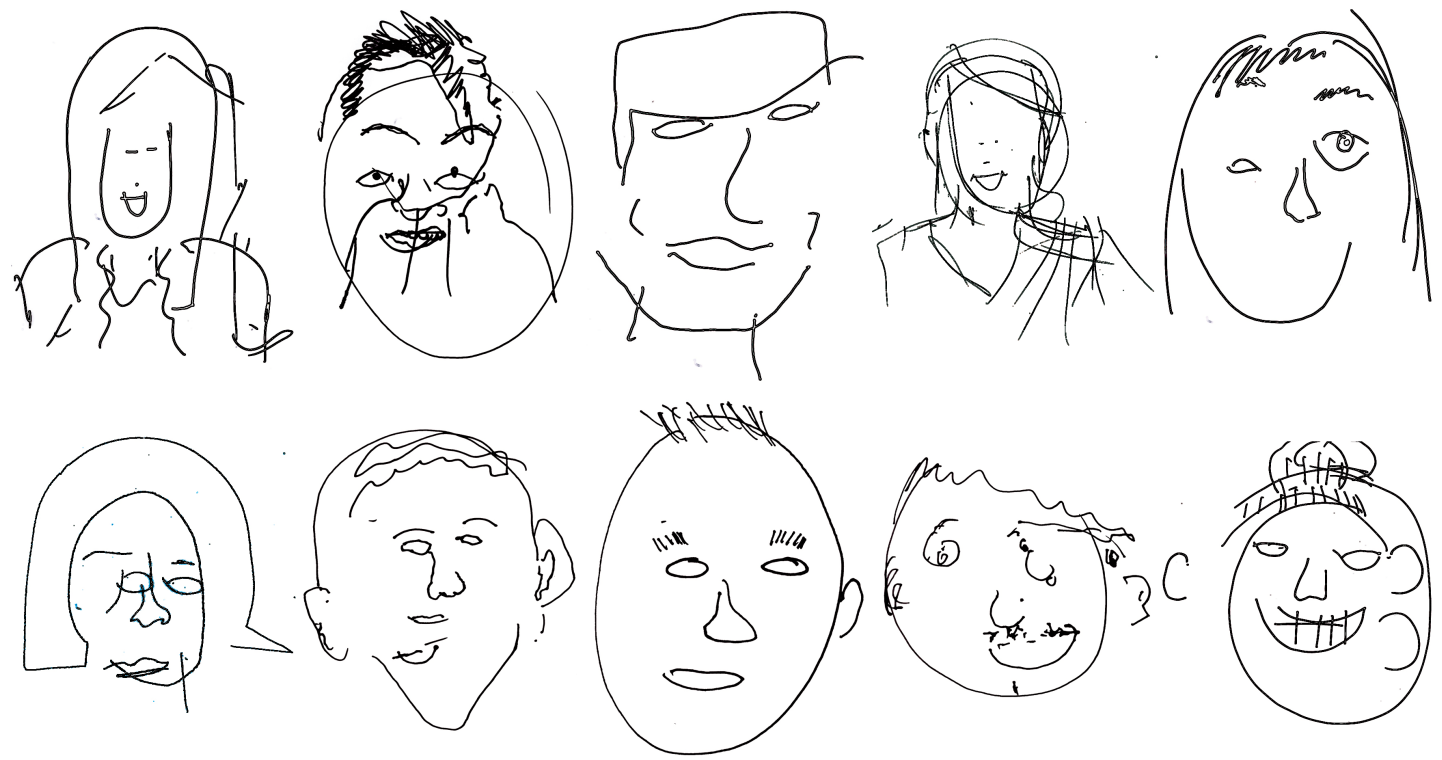

Figure 61. Blind portraits drawn to ready participants for drawing activities and partner interaction.

\subsubsection{Procedure}

The designers were given pre-workshop materials outlining the aetiology, treatment regimen, and medical devices used for type 1 diabetes management. Each of the participants with type 1 diabetes had met with the researcher prior to the workshop to take part in other research activities described in Chapters 6-9. As such, I had an established rapport with participants, and some had reviewed student designs from Chapter 8.

Before the workshop, participants perused quotes from prior interviews, such as "You're wondering what people think about what you're doing, and then it's also thinking, 'I'm not normal, I have to do this'." The aim of the workshop was to design medical technologies that help adolescents measure their blood glucose confidently in everyday situations. Participants were free to design medical devices or other products that would fulfil this aim. Blood glucose monitoring was selected for the workshop as it has been described as adolescents' least favourite part of managing type 1 diabetes, with some adolescents not testing in social environments due to stigma and peer influences (A. E. Carroll et al., 2007).

The workshop centred around six strategies for addressing stigma, adapted from 17 strategies by Vaes (2014). Strategies that did not pertain to the design of an object were eliminated from this workshop, and similar strategies were combined to 
limit the number of strategies to suit the participants' attention span and duration of the workshop. The six strategies used in the workshop were:

1. to strengthen the product's institutional identity by reinforcing the medical role played by the device;

2. to downplay stigma-sensitive features by disguising the product as an accepted non-medical item;

3. to minimise stigma-sensitive aspects of the device by making it invisible or less confronting and minimise its presence when not in use;

4. to strengthen the individual identity of the device by providing more choice and opportunities for personalisation, so users value it as an extension of their personality;

5. to strengthen the product's brand identity by creating or reinforcing positive brand associations; and

6. to increase the device's social power by making the user more visually assertive.

An image of a product that embodied each strategy from a non-diabetes context was provided to explain the strategies to participants (e.g., a miniaturised hearing aid for strategy three, and a sling with a pictogram showing how the user broke their arm for strategy six).

Participants brainstormed the advantages and disadvantages of each strategy in relation to blood glucose monitoring, and suggested how the strategy could be implemented, recording ideas individually on sticky notes then sharing these with the group as they affixed them to a wall (DiGiano, Tatar, \& Kireyev, 2006; Faste, Rachmel, Essary, \& Sheehan, 2013; Svanaes \& Seland, 2004). Next, participants voted for strategies that would be appropriate for different type of adolescents with type 1 diabetes by attaching stickers to them, similar to a method used in a previous design workshop (Glasemann \& Kanstrup, 2008).

Each adolescent participant then paired with one or two designers for the remainder of the workshop. They rapidly sketched ideas pertaining to blood glucose monitoring for three of the most popular strategies (Milton \& Rodgers, 2013). After sharing these with the whole group, each group completed a storyboard and sketch 
for one idea, then created a rapid prototype (Figure 62) (Houde \& Hill, 1997; Truong, Hayes, \& Abowd, 2006; Warfel, 2009). These were shared with the larger group, where each participant wrote one aspect they liked about each idea, and one aspect about which they were curious (D.school, n.d.). By phrasing their feedback as 'I like' and 'I wonder', the feedback was framed as an avenue for possible idea development rather than critique, so as to minimise distress. This was an adapted form of individual expert review (Wilson, 2014). Finally, the group had a debriefing session where they commented on the content and experience of the workshop. Breaks, music, and priming activities to make participants comfortable were also used during the three-hour workshop.

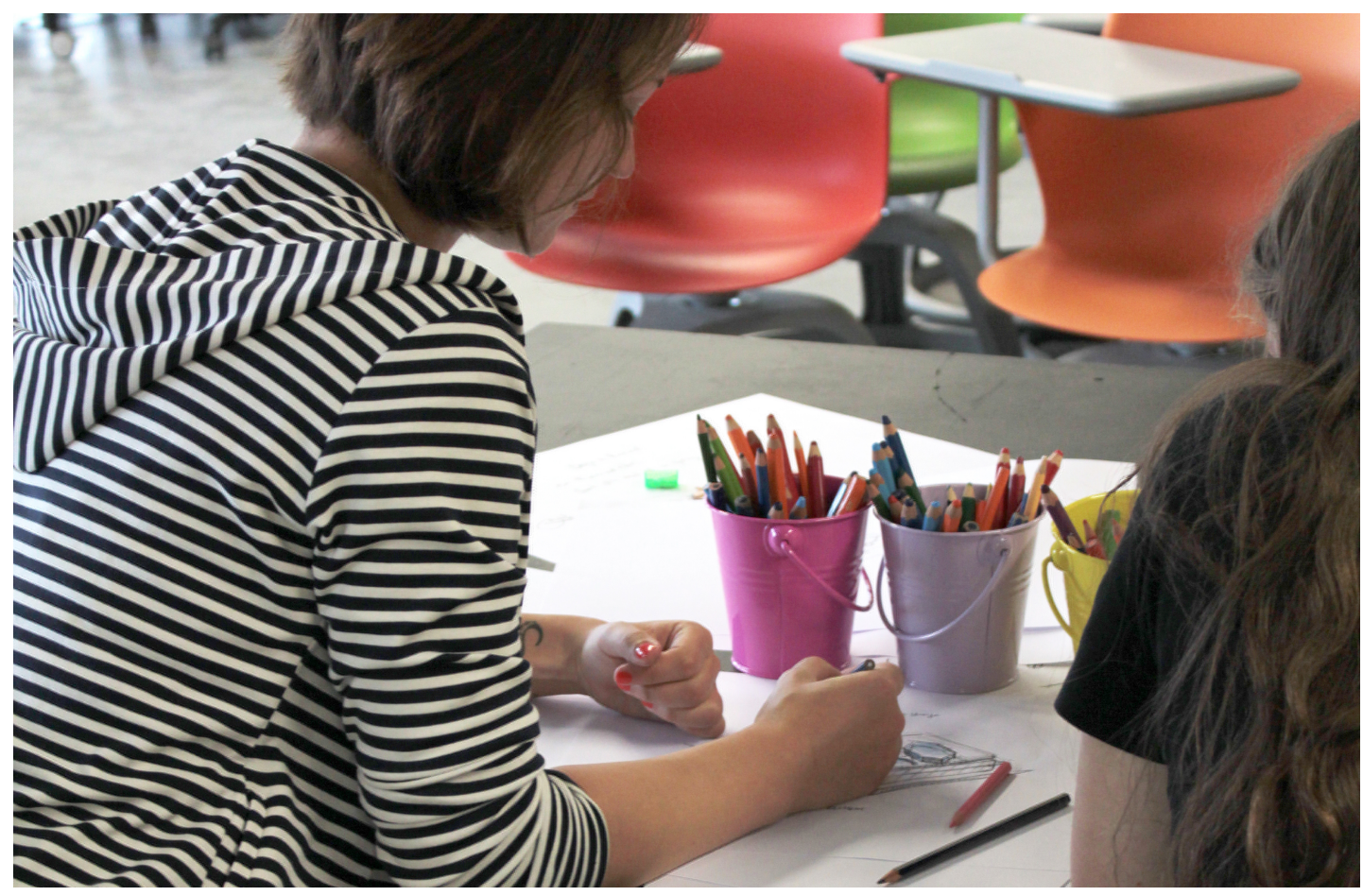

Figure 62. Participants sketching design concepts.

\subsection{Findings}

\subsubsection{Exploration of strategies to address stigma}

This section outlines participants' expected advantages and disadvantages of the strategies to address product-related stigma and areas of non-consensus. Participants 
also suggested how strategies could be applied in relation to blood glucose monitoring. A selection of rapid sketches is included.

\subsubsection{Strengthen the product's medical identity}

Participants felt the advantages of strengthening the product's medical identity were that devices would be recognisable as medical devices rather than drug paraphernalia, were easy and reliable to use, and that they would increase public awareness about type 1 diabetes. Disadvantages were the stigma of the device being associated with a 'disability' or medical condition, looking 'scary' and 'serious', not being user-friendly, and adding to misunderstandings and unwanted questions about self-care behaviours. Participants were divided over whether medical-looking devices were easy to use, and whether increased attention was advantageous. Features that made a device appear medical were white and grey colours, smooth plastic surfaces, exposed needles, medical wording and symbols, 'clunky' and plain design with basic shapes, LEP displays, a futuristic look, and having many unintuitive features.

\subsubsection{Disguise the product as an accepted non-medical item}

Benefits of disguising a medical device as a consumer device included making it less noticeable, allowing the user to hide their 'disability', and self-care behaviours not being discernible. This strategy might allow the device to be incorporated into accessories, allowing it to be worn rather than carried. The overriding negative aspects of this strategy were that onlookers might not know to help during an emergency, and non-standardised use. Looking unlike a medical device was thought to be positive for general use, but negative during emergencies. Participants also added that a disguised device might be difficult to find. Implementation ideas included blood glucose meters disguised as handbags, toys, smartphones, tablets, watches, clothing, or jewellery, or using materials and forms that reflect these items (Figure 63). This strategy is comparable to 'product metaphors', whereby a design evokes a metaphor of a different object (Hekkert \& Cila, 2015). 


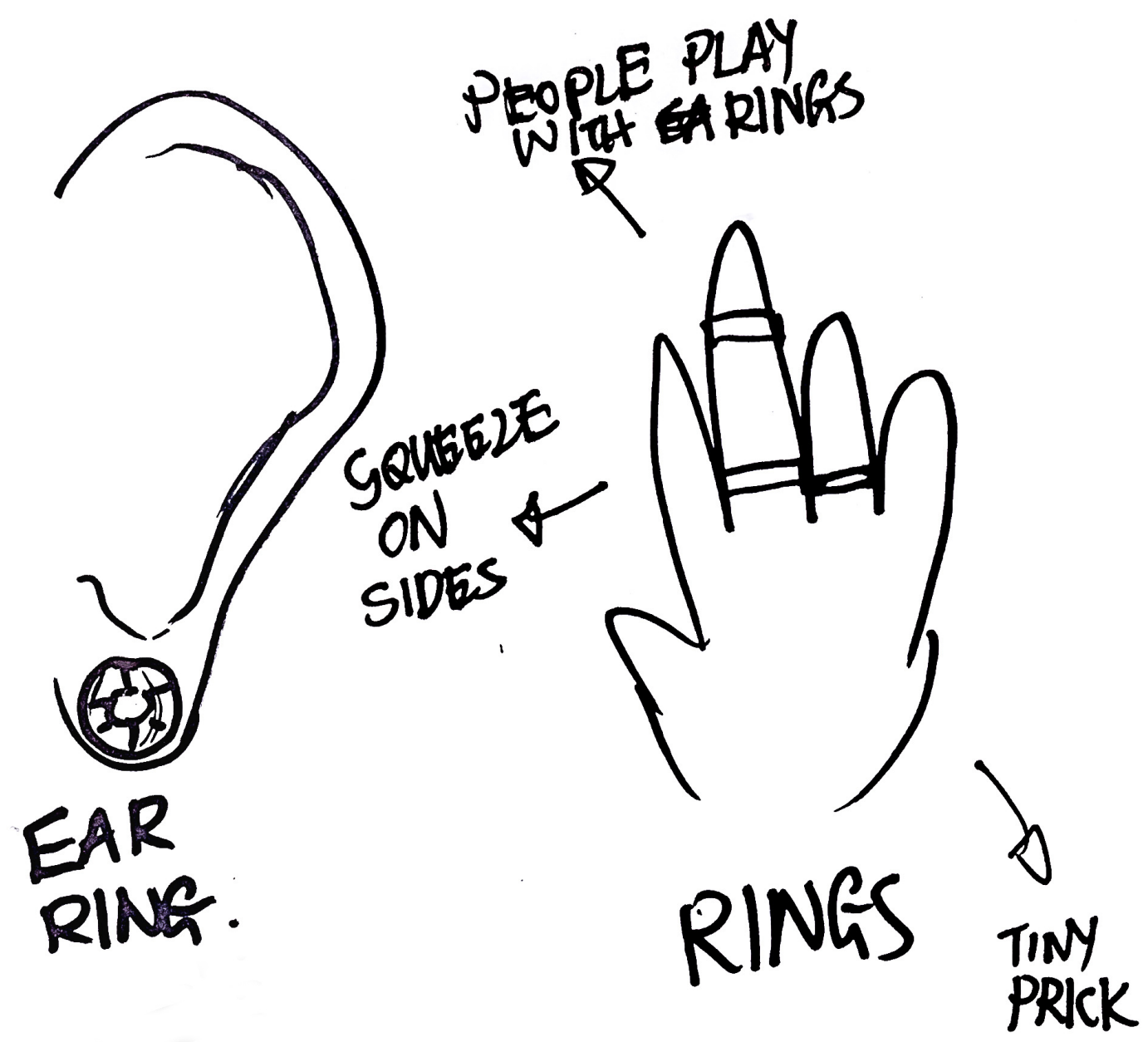

Figure 63. Earring and ring designs for glucose monitoring.

\subsubsection{Make the product invisible or less confronting and minimise its presence when not in use}

Advantages of making blood glucose meters invisible or less confronting included the device being unobtrusive and less embarrassing to use in public. Additionally, it might invite less 'social hassle' and unwanted questions. It could also be ergonomically designed to fit to the body. Again, while diminishing the attention devices generated was sometimes advantageous, this could also result in less awareness, peer support, and conversation about type 1 diabetes. There were also opposing views as to whether a device that applied this strategy would encourage testing as it was able to be worn, or discourage testing as the device's reduced visibility would no longer act as a visual prompt for testing. Participants felt that making the device less visible might make them feel more robotic, and as if the device ought to be hidden. If the device was slightly visible, this may provoke more 
questions. Devices being less visible may also be less intuitive to use and easier to lose. Ideas for implementation were through use of colours and transparent surfaces, miniaturising devices, or integrating devices into clothing. Other ideas included placing the device on unexposed parts of the body, or using a subdermal element that connected to a smartphone (Figure 64). Participants also considered using novel methods of testing glucose concentrations such as breath detection by animals, contact lenses, toilets, tattoos, or a human joey pouch.

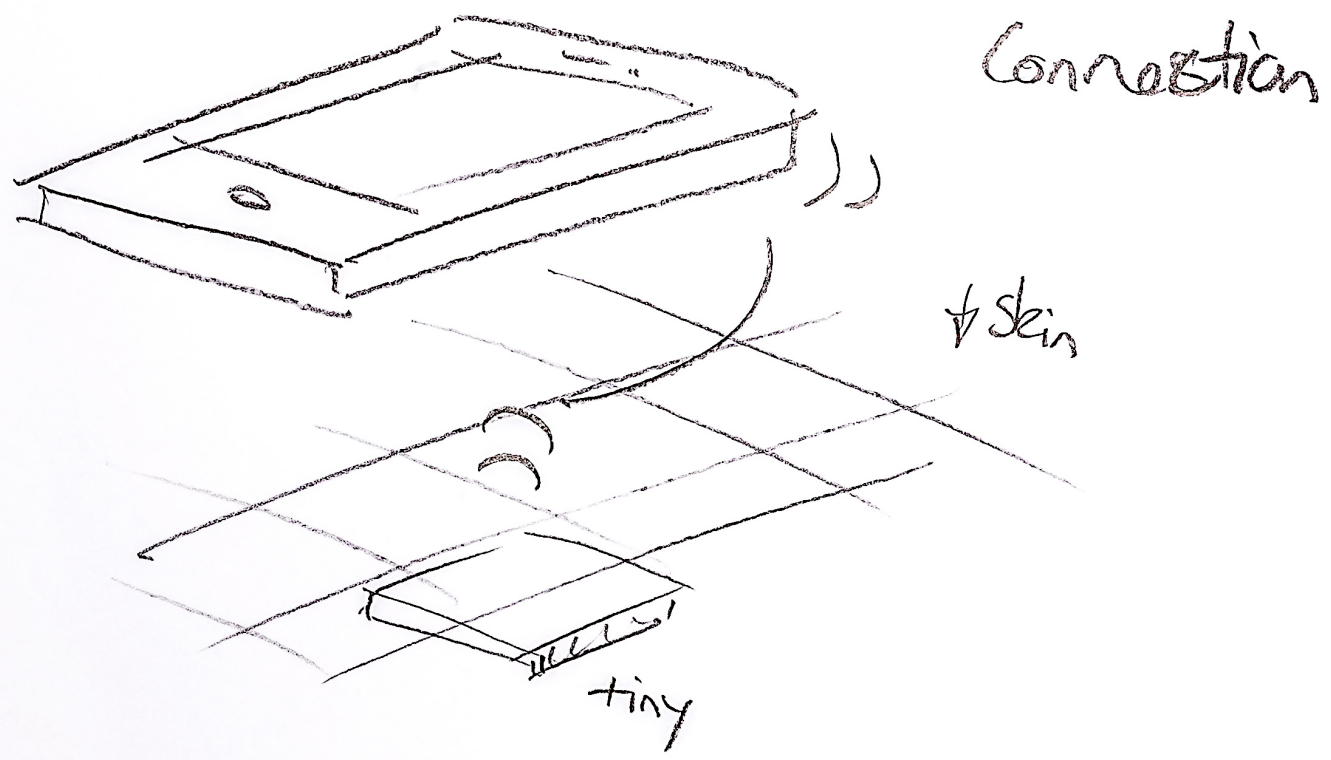

Figure 64. Sub-cutaneous chip and phone application for blood glucose testing, showing wireless connection to a chip implanted under the skin.

\subsubsection{Provide more choice and opportunities for personalisation}

Benefits of increased choice or personalisation of glucose monitoring equipment were having a sense of control, and feeling it was 'my device', reflecting the user's personal style and function as an extension of themselves. Participants felt they would have pride in a device that was personal and tailored to their condition. While some participants noted that this strategy gave users a choice to be discreet or flamboyant based on their preference, and thought it positive users would not be trying to hide the device, others were worried about the device drawing attention and that onlookers may not understand it was medical. The device could be 'trendy' or 'cool' and aid normalisation of frequent self-management, though participants were wary of struggling to stay aligned with fashion trends. The device could be 
customised for different environments and be comfortable to wear if it was made to measure. Participants worried this strategy might make devices expensive, be timeconsuming for the user, and encourage choosing a device based primarily on likability rather than efficiency. Strategies for personalisation included cases, accessories, attachments, stickers, programmable chameleon-like surfaces, colours, patterns, materials and skins (Figure 65).

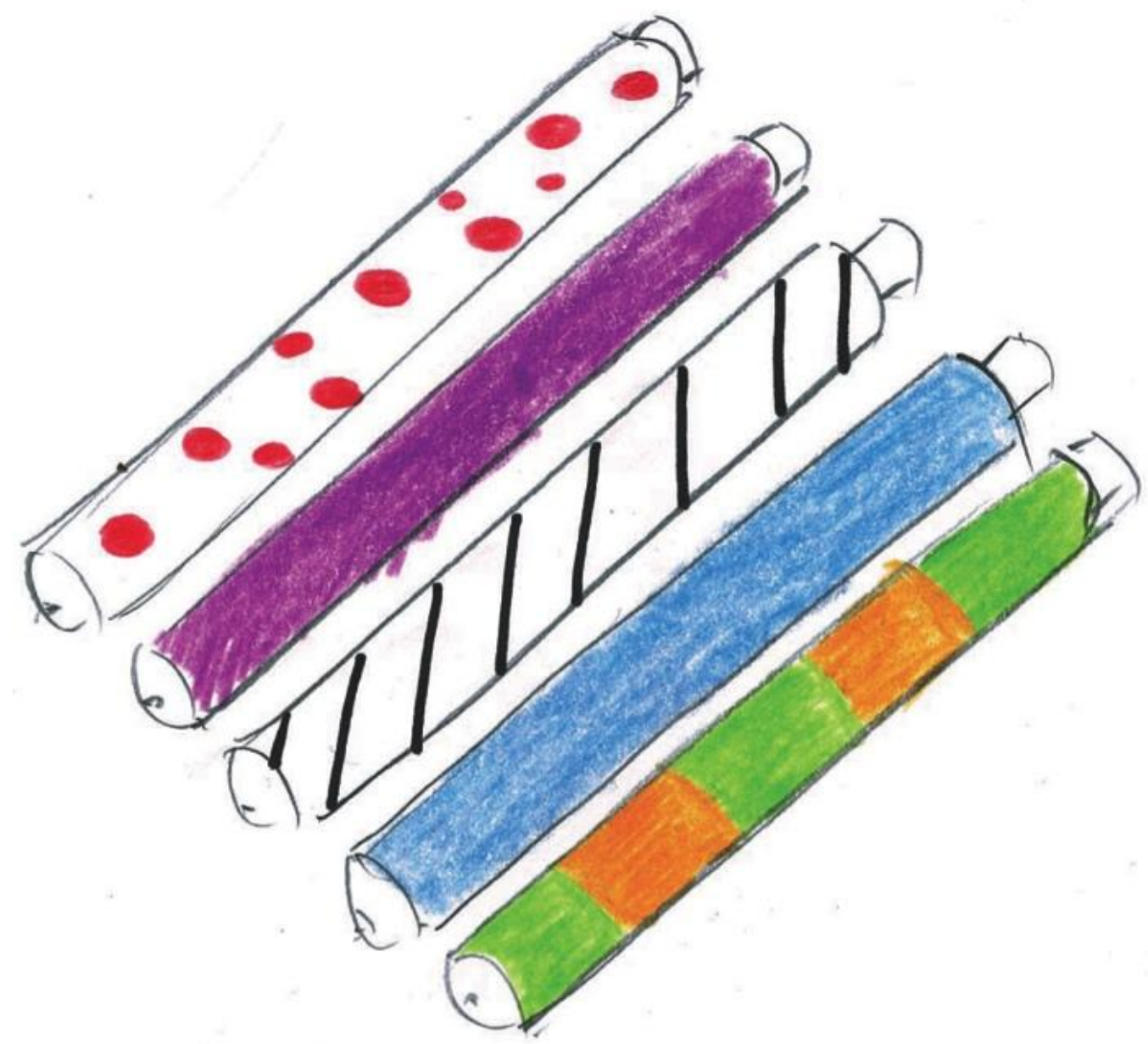

Figure 65. Personalising lancets with colourful skins.

Changes could be made frequently to suit an outfit, or to garner or diminish attention. Additionally, changes to the core functions could be made, similar to modular phones and computers. Participants also felt users could be given the option of wearing the device over or under clothing. Participants suggested customisable options could be supplied or created individually, and 3D printing could be used. One participant suggested the device making her look like a cyborg would be 'awesome', while others wanted to adapt elements from toys or movies. 


\subsubsection{Strengthen the product's brand identity}

Participants felt strengthening the blood glucose meter's brand identity could make it fashionable and desirable, which in turn could boost their self-confidence and pride, making public self-management more acceptable. The words 'luxury', 'cool', 'exclusivity', and 'loyalty' were associated with brands. Possible downsides could be pressure to replace the device frequently and not trusting the brand, and therefore the device. There was some concern that this strategy could commodify the medical condition, and consideration was given to whether people would notice product branding. Participants were divided whether branding and competition would increase or lower prices, and whether extra attention and associating type 1 diabetes to a brand would be positive or negative. Additionally, one participant felt that blood glucose technology needed to be functional but not appealing, whereas another participant disagreed, stating though devices must be functional, they could also be aesthetically appealing. Ways to associate the device with a brand would be to add a logo or to make the device look 'sporty' or 'classy' through the use of colours, materials, and patterns (Figure 66).

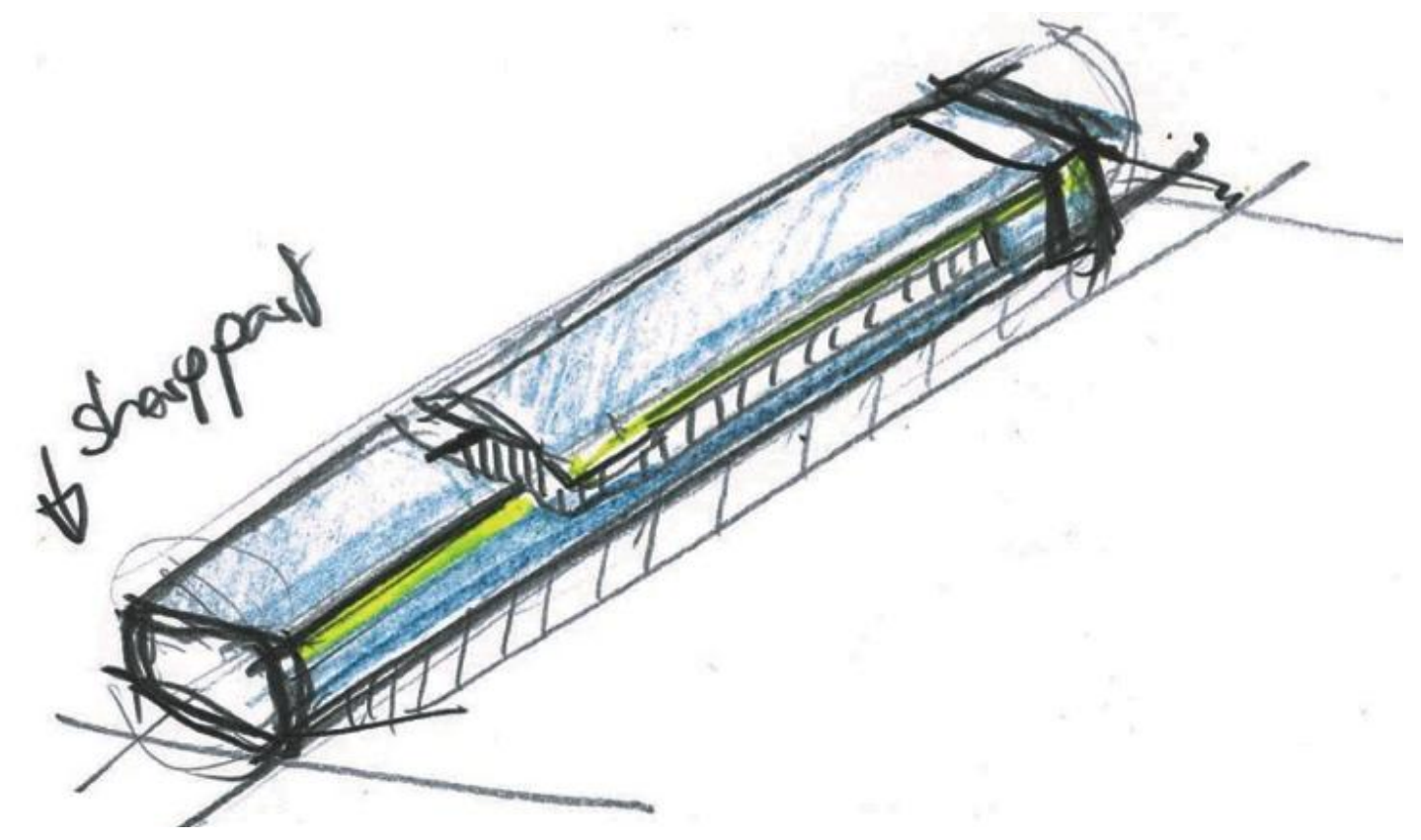

Figure 66. Lancet that borrows a 'sporty' aesthetic.

Participants suggested a new medical brand could be created with marketing and aesthetics akin to a consumer brand. The phrase 'dazzling camouflage' was used to describe the potential of a branded device. 


\subsubsection{Increase the product's social power}

Advantages of imbuing the device with greater social power would include avoiding repeated explanation of the device's purpose, and increasing awareness of diabetes and how to assist in an emergency. This strategy was thought to help in removing stigma, preventing users from hiding the condition and providing a talking point to evoke positive conversations. Some participants felt that it was helpful to focus on distinguishing type 1 from type 2 diabetes, and that humorous conversations about the condition and medical devices eased social interactions. While some adolescents thought this strategy would help communicate diabetes management in a straightforward manner, resulting in the user receiving fewer questions, others thought it might create further misunderstandings. Participants considered that this strategy might also draw attention to, and possibly make fun of, their condition. Again, participants did not want people to know they have diabetes in all situations, and this approach could highlight their vulnerability. Strategies for increasing the social power of blood glucose equipment included using social media, advertisements, public service announcements, flash mobs (choreographed public events), explanatory comic strips or descriptions on devices, QR codes to link to informative websites and diabetes awareness month, and having information printed on clothing or cards that could be given to people. A diabetes festival with themed rides and stalls was also proposed (Figure 67).

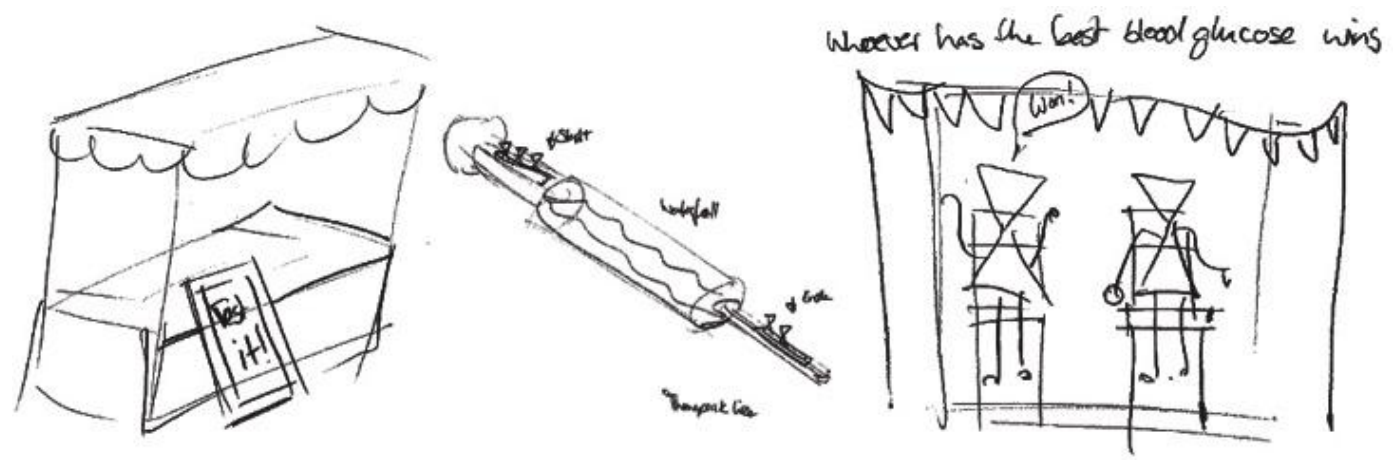

Figure 67. Diabetes festival with a stall with food shaped as a pancreas, syringe water slide, and blood glucose guessing competitions. 


\subsubsection{Selecting strategies to address stigma for various users}

Following the brainstorm detailed above, participants each voted for one strategy that would be appropriate for a young person who was embarrassed managing type 1 diabetes in public, one for someone who was confident in such circumstances, and one for someone wanting a game-changing technology (Table 11).

In previous interviews, participants had talked of their own feelings about being embarrassed or not being embarrassed, but also noted that they knew of others with type 1 diabetes who felt or acted differently from themselves. They had also read quotes by other research participants at the beginning of the workshop to increase their awareness of other people's opinions about device-related stigma and embarrassment. I chose to capitalise on their awareness of their own preferences and their knowledge of other individuals' preferences to ensure that the resulting designs catered for a wide spectrum of possible users. Further, this allowed participants to choose to design concepts that they would personally find helpful, akin to an 'autobiographical design' approach, or to design for others (Neustaedter \& Sengers, 2012).

Table 11

Voting by participants for strategies to address stigma for different types of users.

\begin{tabular}{l|lll|l} 
Strategy & Embarrassed user & Confident user & Game-changing user & Total \\
\hline Medical & 0 & 0 & 0 & 0 \\
\hline Disguise & 6 & 0 & 0 & 6 \\
\hline Invisible & 5 & 0 & 4 & 9 \\
\hline Personalise & 0 & 6 & 1 & 7 \\
\hline Brand & 0 & 3 & 1 & 4 \\
\hline Social power & 0 & 2 & 5 & 7
\end{tabular}

Votes were spread across strategies, with no strategy deemed appropriate for all user types and no user type limited to one strategy. This reflects the differing needs and preferences of adolescents with type 1 diabetes, but is not currently reflected by the 
minimal range available in medical technologies. Interestingly, no participants chose strategy one (strengthening medical identity), which is currently used for glucose monitoring equipment. This strategy was seen by some as synonymous with the status quo. The most popular strategies were making the device invisible, personalisation, adding social power, and disguising the device.

\subsubsection{Design concepts and reviews for glucose monitoring}

Participants designed five concepts during the workshop relating to the disguise, medical, and personalisation strategies. The designs and participants' reviews of them are described below. Interestingly, while the strategy to make the device less visible was the most popular, and the social power strategy also ranked highly, no groups chose to design for these strategies.

\subsubsection{Phone attachment and application}

The first group chose to use strategy two (disguise), to create a blood glucose meter to be attached to a smartphone and an app for viewing blood glucose and ketone levels (Figures 68-69). They produced a storyboard of desired interactions, including pressing buttons to measure blood glucose levels and ketones, inputting carbohydrate data, viewing data and graphs on the phone, having the phone communicate directly with an insulin pump, and being able to send data to a health professional (Figure 70). The group created different coloured and patterned attachments to demonstrate that the meter would come in a variety of styles. 


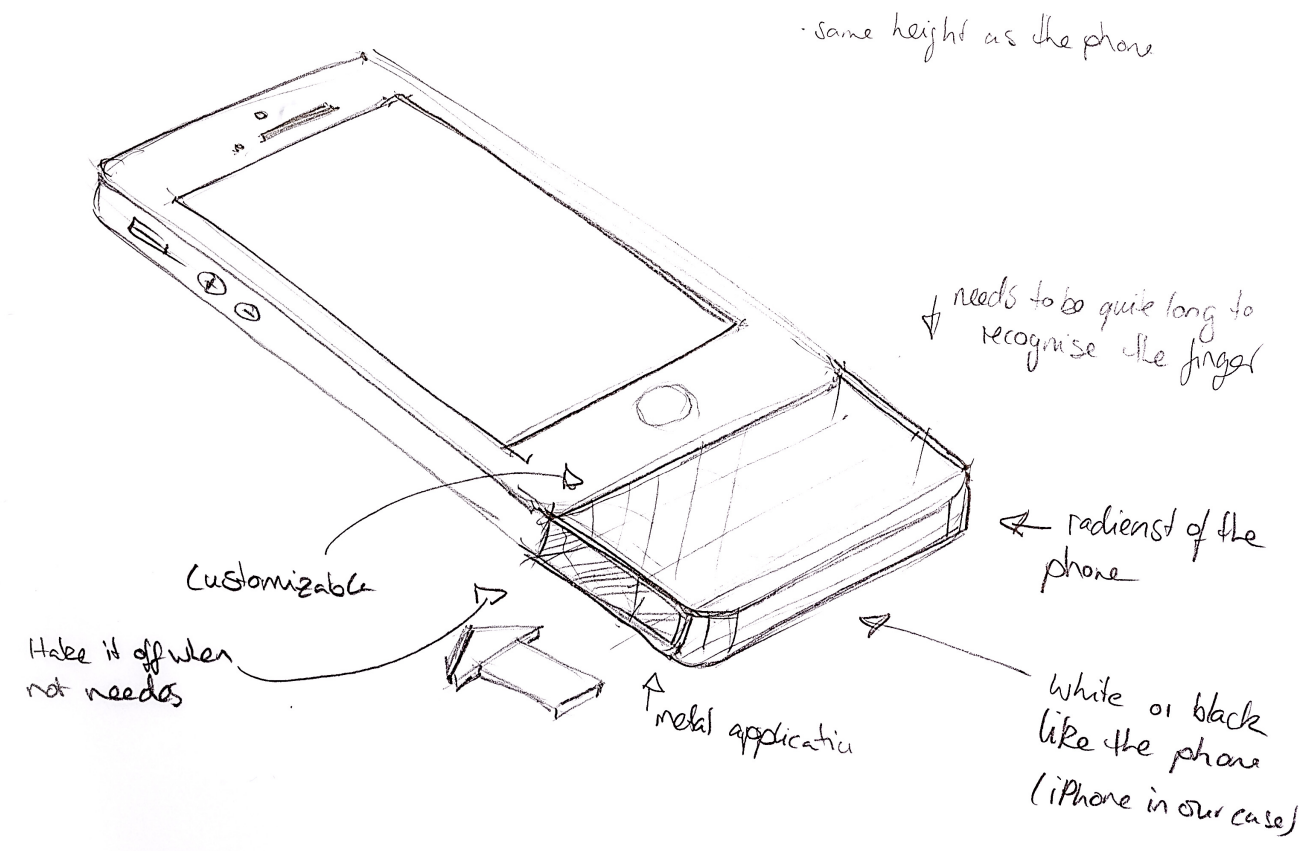

Figure 68. Smartphone attachment.

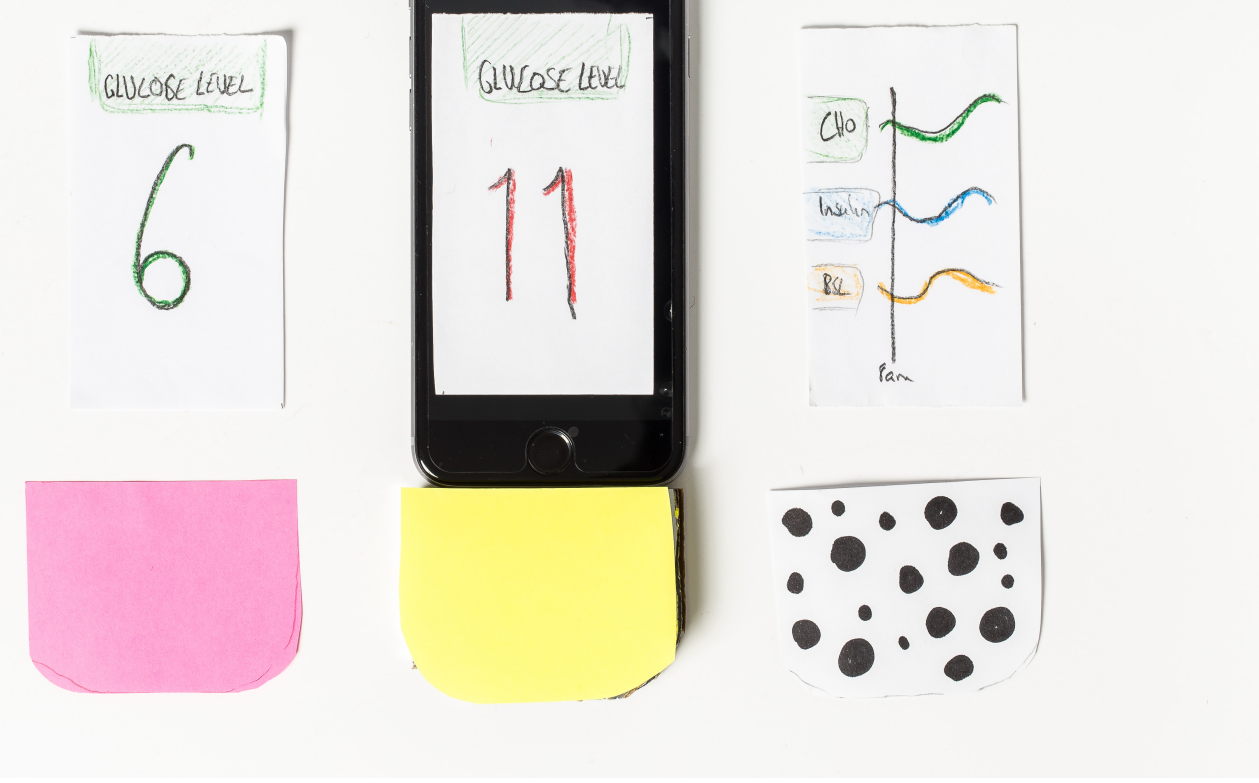

Figure 69. Smartphone attachment and user interface.

Participants commented positively about the variety of styles and wireless syncing, and that this concept used existing, frequently used technology, eliminating the need for an extra device. They also valued that the application would help educate new 
users and that it visualised data sets, allowing users to better understand trends in the data.

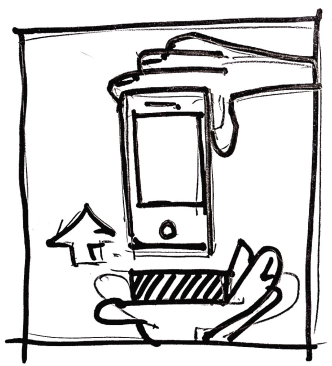

Aftechment

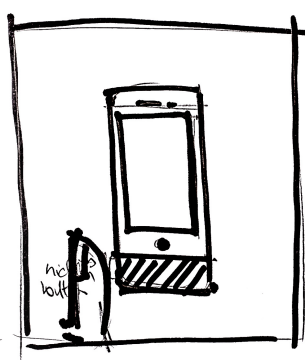

Buttononte \&do

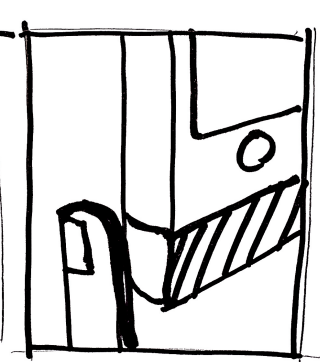

Butto Area

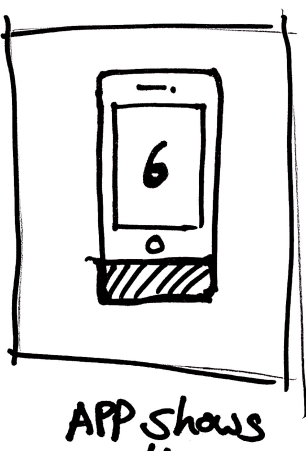

roults
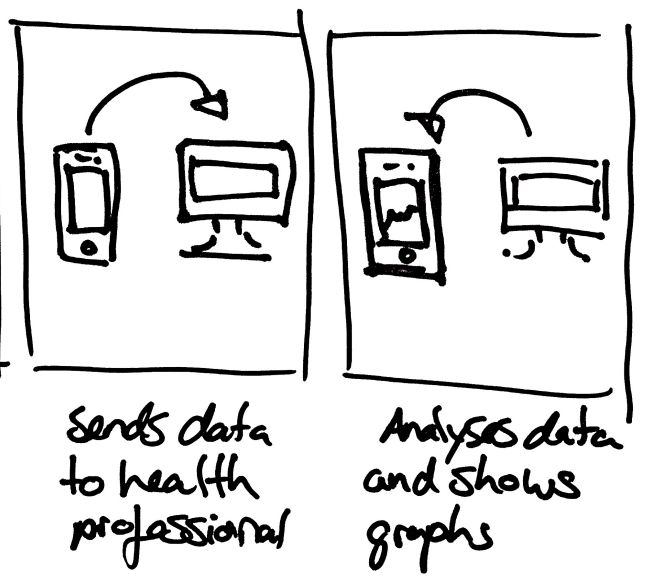

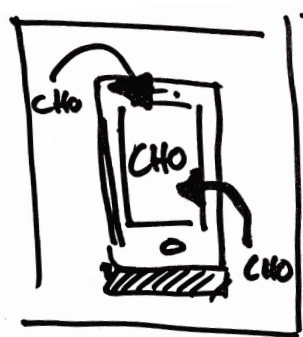

untering in

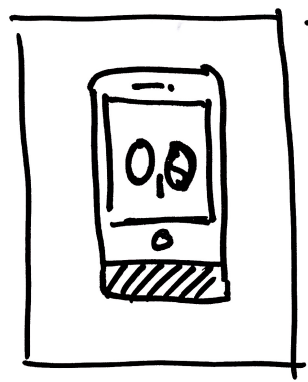

second sensor
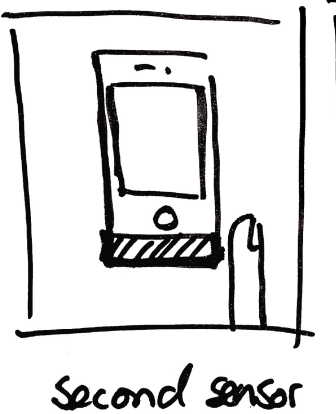

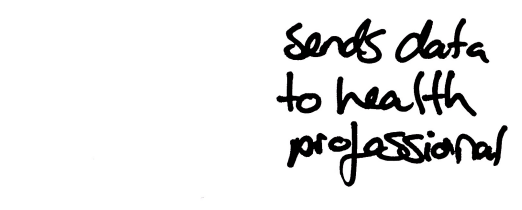

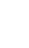


precaution, and commonly used SMS messaging to communicate blood glucose levels and insulin doses with parents while at school, before beginning to manage these decisions independently.

\subsubsection{Keychain glucose meter}

The second group also used strategy two (disguise) to create a small keychain glucose meter of approximately $2 \times 5 \mathrm{~cm}$ (Figures $71-72$ ). An enlarged prototype was created to represent the device, with a cartridge of 20 lancets for finger pricking and a double action switch to stop users being pricked unintentionally. Although the keychain had a small screen, it also paired with a smartphone app complete with reminders to test and maps for nearby food locations.

Participants liked the small size of the device and that it contained the blood glucose meter, lancets, and test strips within a single device. They questioned whether the screen was necessary, whether the casing could be customisable, how easy it would be to access the cartridge of lancets, and whether users might lose the small device.

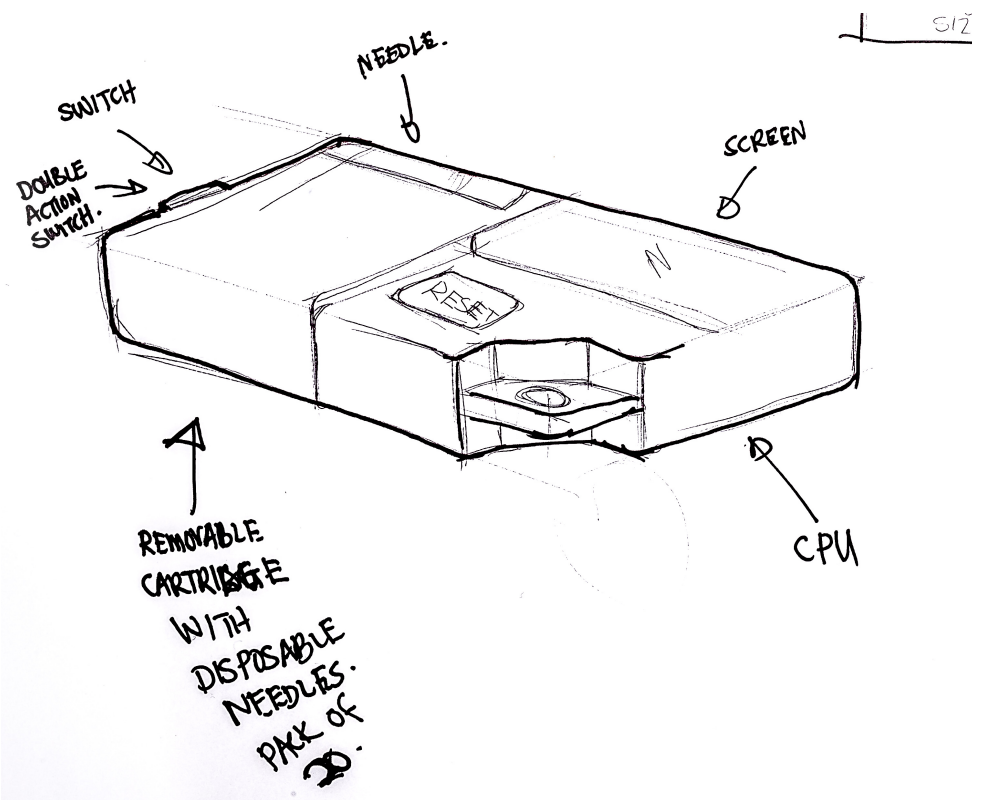

Figure 71. Miniature keychain blood glucose meter. 


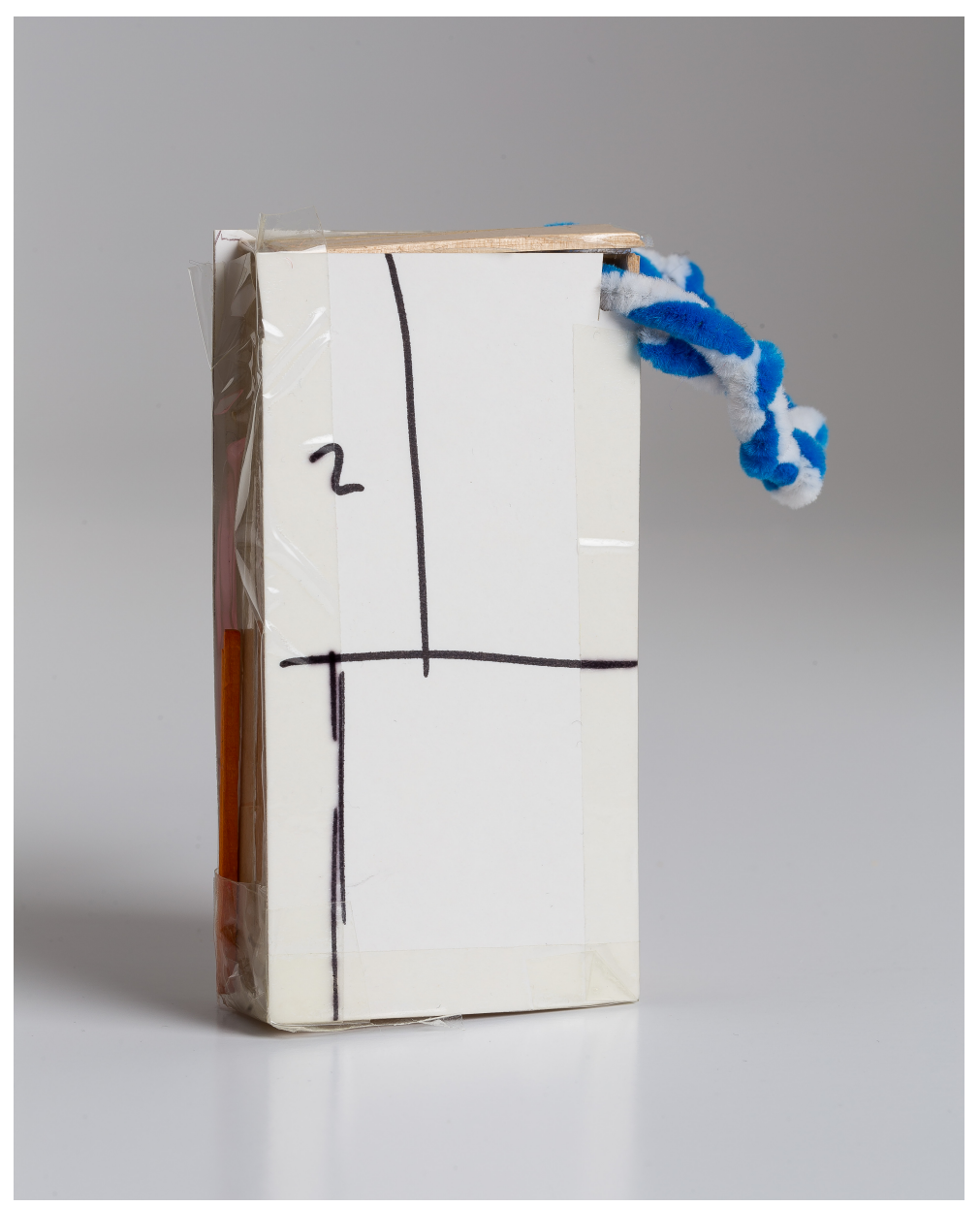

Figure 72. Keychain prototype at a larger scale.

\subsubsection{Hologram watch}

The third group also chose strategy two (disguise) to create a blood glucose meter that looked like a watch, but also had a hologram feature that embodied strategy one (medical identity), supplying clear medical information for emergencies and explaining the condition to others. Blood glucose level and target, time, and date were shown on the watch, and when a button was pushed, a hologram showing information from the insulin pump and basic information for people who do not have diabetes (e.g. 'type 1 diabetic', 'high blood sugar') was displayed. Test strips were housed in the watchstrap to be used with a traditional lancet (Figures 73-74). 


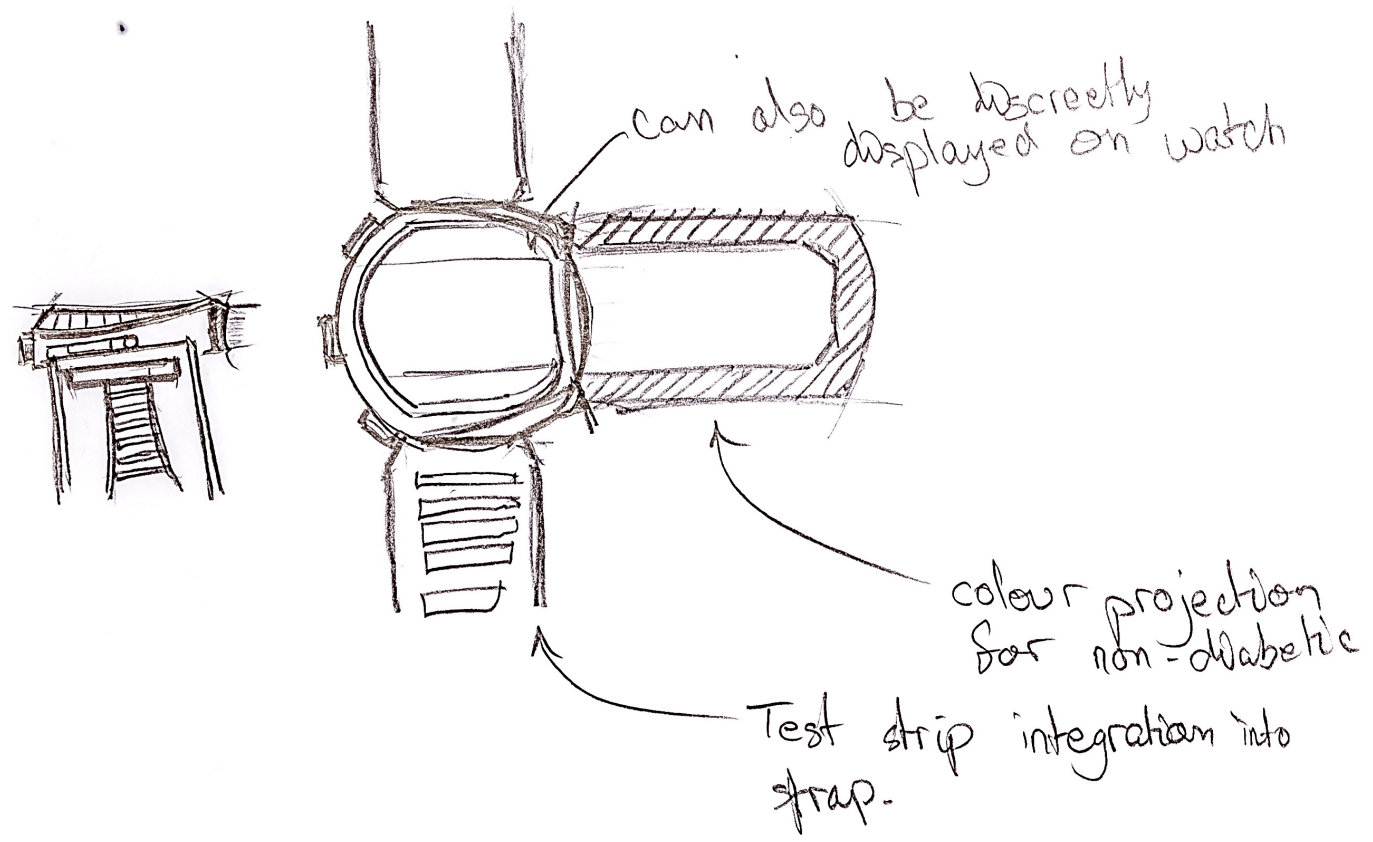

Figure 73. Hologram watch sketch.

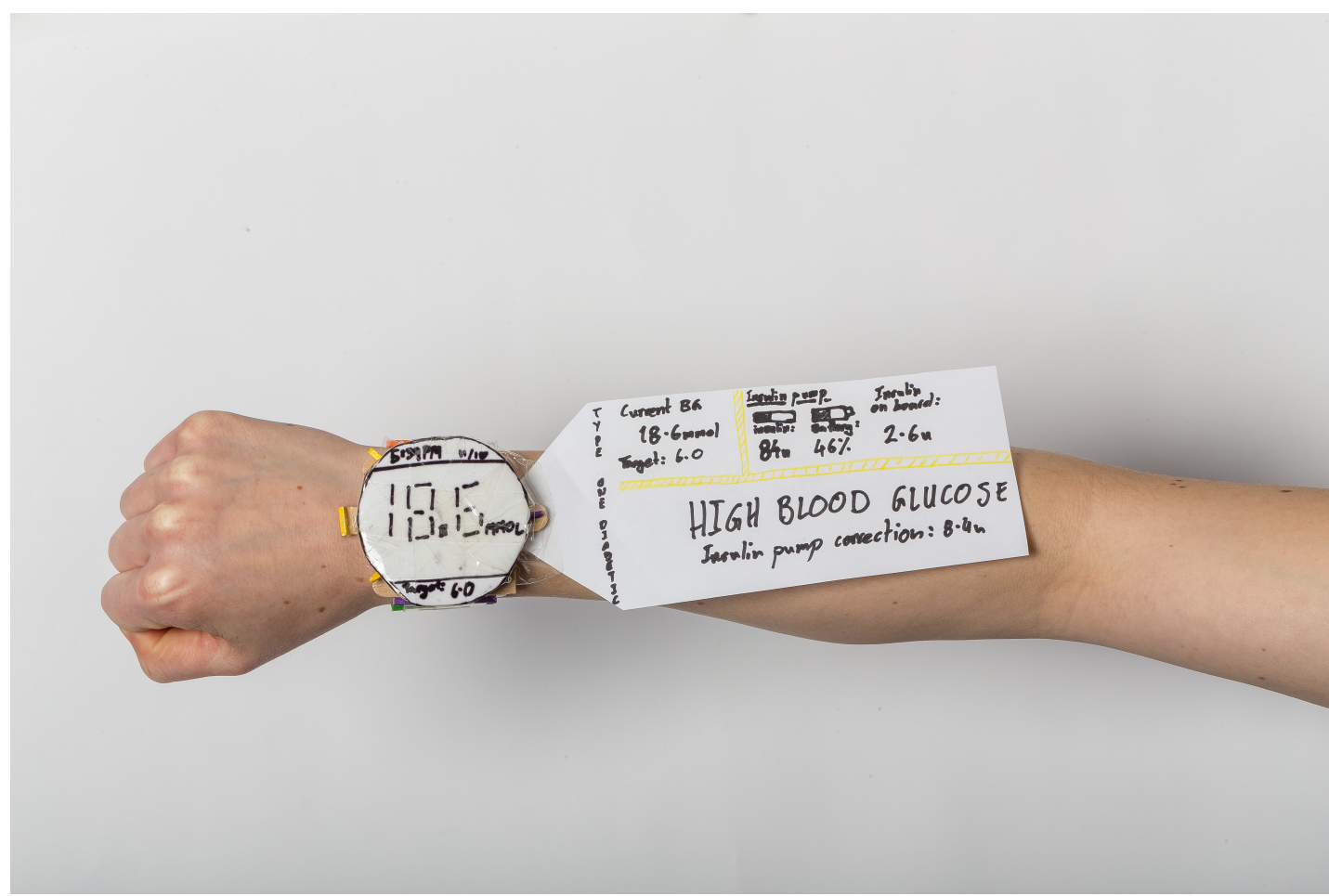

Figure 74. Hologram watch paper prototype.

Participants commented that using a watch made the device accessible and was convenient, as it was something people already wore. In addition, they liked that the watch projected detailed data and could be of help to people with diabetes while also educating others. They questioned whether the hologram would be legible, and if 
there might be a better way to display this information. They wanted to ensure the device was obvious in an emergency. Possible issues were the bulkiness of the watch, whether the test strips would be secure in the watchstrap, and if people might mistake it for a gaming device.

\subsubsection{3D-printed personalised blood glucose meter}

The fourth group chose strategy four (personalisation). They created a software concept for creating custom blood glucose meters using 3D-printed casing. The software would provide basic shapes that could be adapted by users, and would automatically generate connections for assembly. Users could also choose colours and materials in the $3 \mathrm{D}$-printing process (Figures 75-76). 

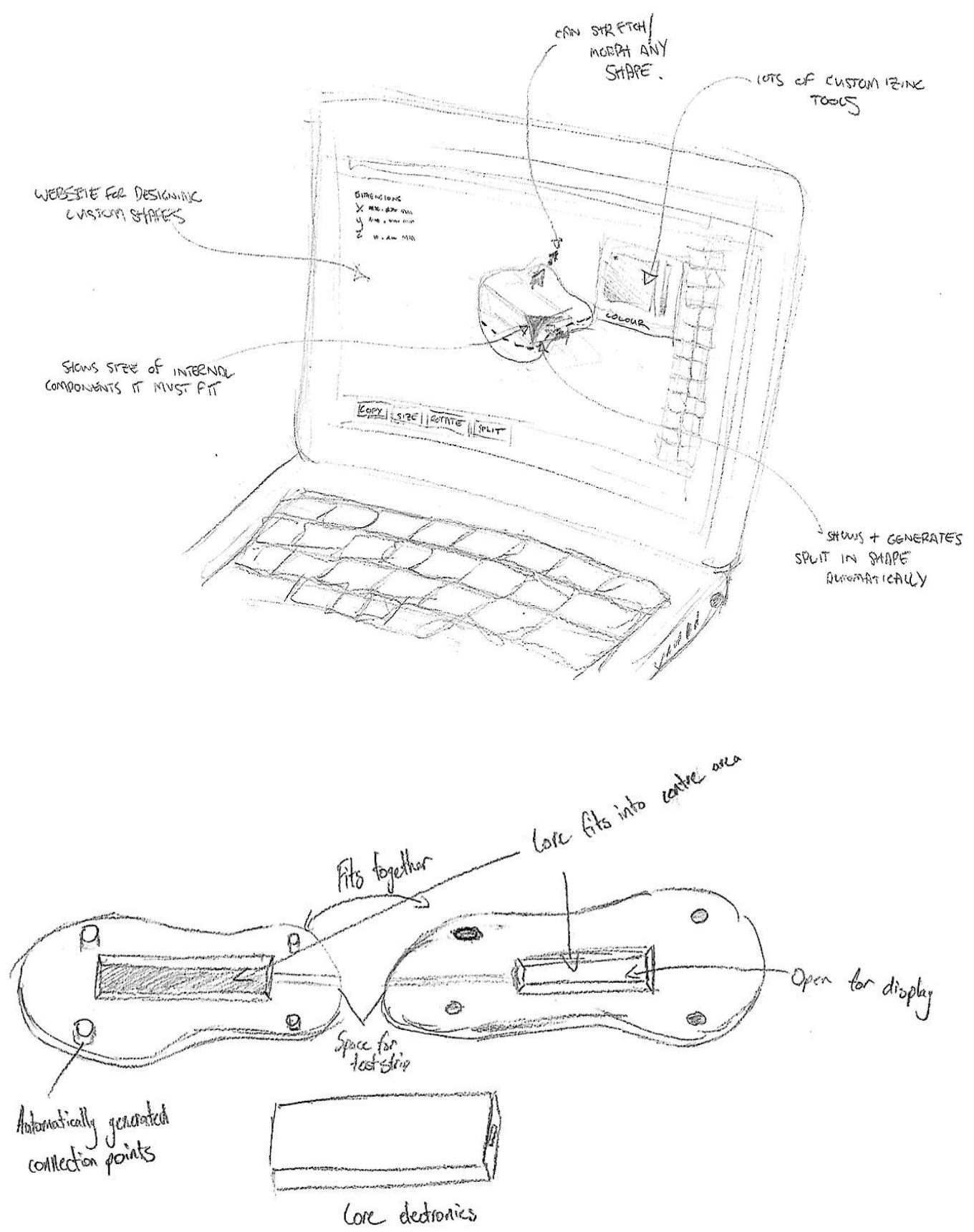

Figure 75. Sketch of software and 3D-printed blood glucose meters. 


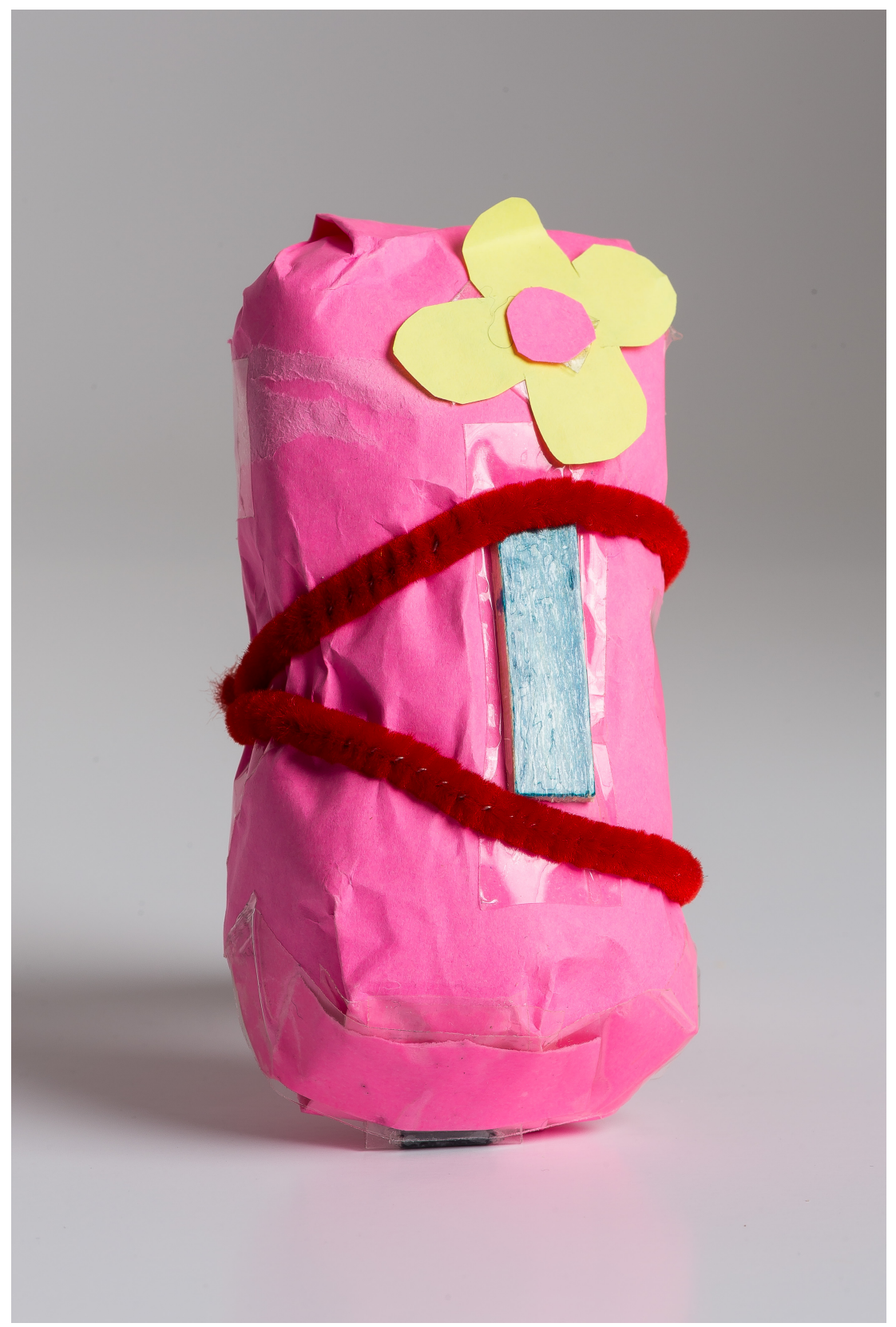

Figure 76. Prototype of a customised blood glucose meter.

Participants admired the simplicity of the idea, and that it introduced an element of fun. They thought it important that the software would simplify aspects of the manufacturing process, and thought personalisation might result in more positive connections with devices. Participants wondered if some customisations might affect the core functions of the device, how accessible it would be, if materials other than those used in 3D printers could be introduced for texture, and whether the software could aid the user to create interesting designs. 


\subsubsection{Modular testing kit}

The final group also used strategy four (personalisation), creating a modular test kit with a core compartment that fitted a lancet, blood glucose meter, and test strips, with extra modular storage for insulin pump site changes, insulin pens, snacks, rubbish, and such (Figures 77-78). They intended the core compartment and some extra storage to be used regularly, with more storage able to be added for occasions such as extended trips.

Participants liked the modular design, and how it had capacity for extra equipment and long items. They thought it would fit many purposes and environments, and would make transportation of equipment easier. They wondered if hexagons were the most efficient shape and whether this would make the kit frustrating to pack, though these did look appealing. They questioned whether the case had enough space for all the equipment people might want to carry, and whether having one larger case might be preferable for some people.

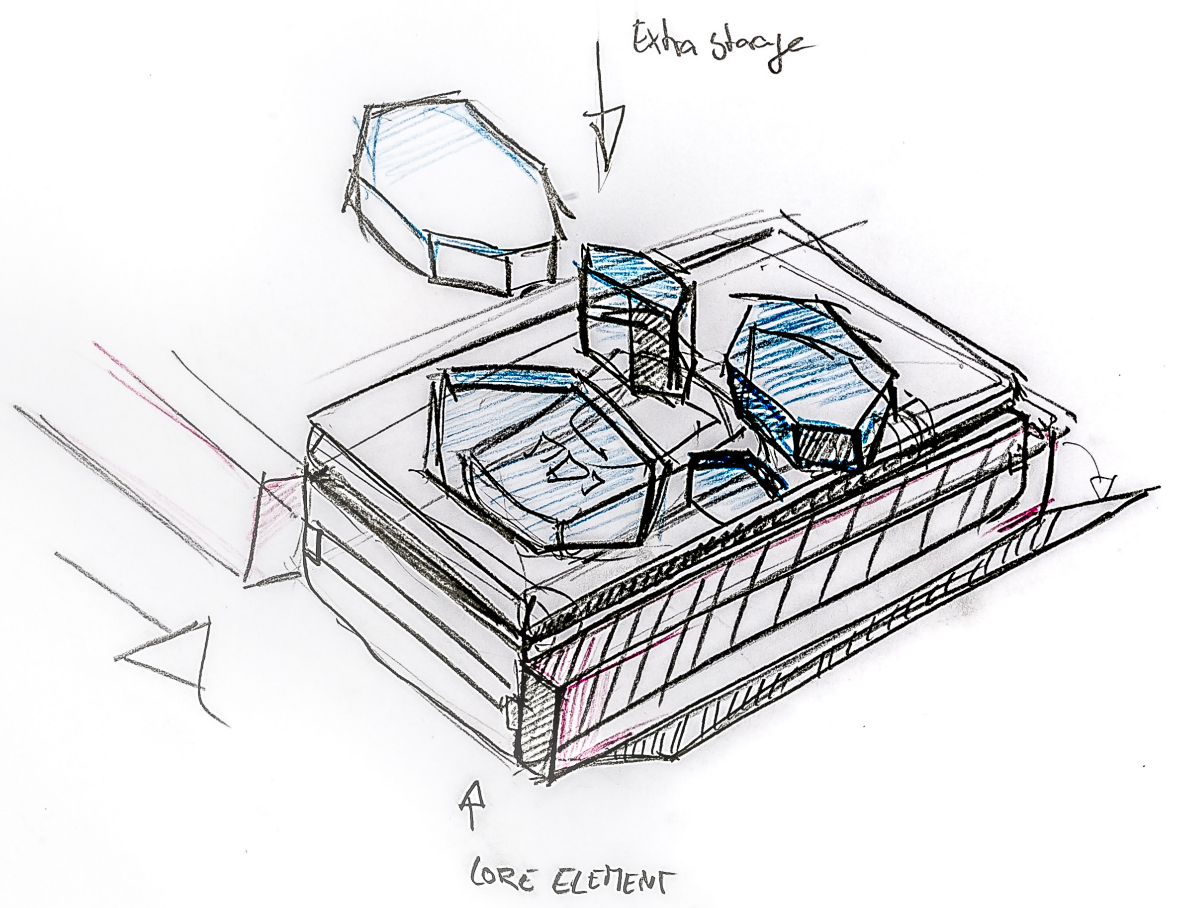

Figure 77. Sketch of a modular test kit with core element and extra storage. 


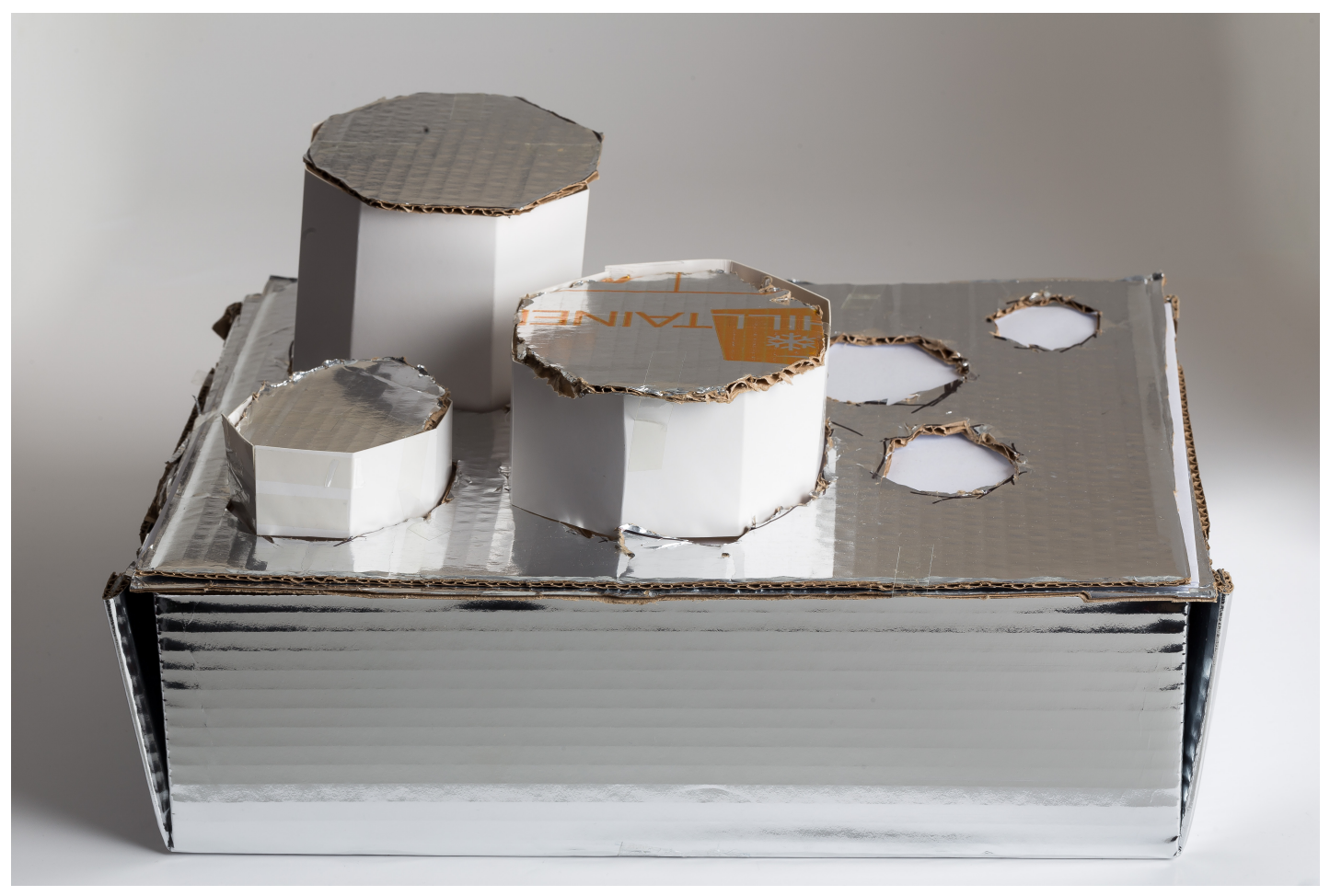

Figure 78. Prototype of a modular test kit.

\subsection{Discussion}

\subsubsection{Addressing stigma}

The workshop was themed around addressing medical-device related stigma, although participants with type 1 diabetes had varying experiences relating to this. While some preferred people not knowing about their condition and managing privately, others were more open to managing publicly. There was debate during the workshop, with one participant stating that devices need only be functional, while another thought while devices should be functional, any opportunity to make them aesthetically pleasing should be taken, as this would make using devices in public settings more enjoyable. This aligns with literature stating functionality and aesthetics need not be mutually exclusive, especially when addressing stigma (Bichard, Coleman, \& Langdon, 2007). As with previous literature, adolescents objected to unwanted attention and questions about their medical device or condition in certain contexts (Y.-L. Wang et al., 2010). These findings resulted in developing the user requirement, which previously centred on stigma and embarrassment, to focus on attention. While it was variable whether participants felt 
embarrassment or perceived or experienced stigma, all participants in this and previous chapters had some objection to moments of unwanted negative attention, some of which stemmed from the public use of medical devices. While this narrative was evident in the previous chapters, discussing and designing with the stigma strategies brought this nuance to the fore.

Diversity among participants allowed for a broad range of design outcomes, and was beneficial to the brainstorm activity. This diversity was demonstrated when there was no consensus for stigma-reducing strategies. In fact, this illustrated the requirement for multiple stigma-reducing strategies to be employed in the design of medical devices, as a single strategy will not suit all. This approach is contrary to currently accessible technologies. Further, participants expected that adolescents with different characteristics, such as being embarrassed, being confident, and wanting a game-changing device, would prefer blood glucose meters designed with different strategies, with no overlapping strategies chosen for embarrassed and confident users.

Another area of non-consensus raised across multiple strategies was the advantages and disadvantages of medical devices that attract attention. Participants felt it important that others knew they had type 1 diabetes in case of a medical emergency. They also felt that talking about diabetes and using devices in public spaces could increase awareness and discussion, aiding disclosure of diabetes and normalising self-care behaviours. Conversely, some described using medical devices publicly as embarrassing or stigmatising, and device use often resulted in people asking undesired questions about their actions, devices, and condition. Subsequently, there will be times when it is advantageous for medical devices to draw attention, and times when users prefer discretion. Additionally, for some users there is scope for devices to draw more positive forms of attention, even being desirable. This may be achieved through strategies such as strengthening the individual identity of the device, providing choice and opportunities for personalisation, strengthening the product's brand identity, and increasing the device's social power. These findings support previous literature, where recommendations caution concealment of medical and assistive devices as a signifier of shame (Bispo \& Branco, 2011; Pullin, 2009; Sansoni et al., 2016). 
While this workshop focused on stigma and embarrassment, a general frustration arose from repeatedly explaining diabetes devices over the course of a lifetime. Although this was related to the attention drawn by medical devices, it may need to be addressed through different strategies.

The user requirement that responds to these issues was initially 'to minimise or eliminate feelings of stigma or embarrassment while using medical technology'. Following this study, and the broader focus on attention, the user requirement was rephrased: 'to eliminate negative attention while using medical technology, and facilitate positive attention where appropriate'.

The workshop contributed to the development of two additional user requirements. Firstly, for helping to comfortably disclose and explain type 1 diabetes when appropriate, it became clear that there was a stark delineation between everyday and emergency situations, and that having pride in a medical device may encourage healthy disclosures. Secondly, for aligning with values and preferences, the importance of aesthetics varied between adolescents, with some only focused on traditionally functional elements of the devices and others valuing aesthetics in addition to basic functionality. Further, while desired aesthetics are often associated with consumer technologies, there was a reticence to associate medical technologies with brands.

\subsubsection{Participatory design approach}

Participants responded positively to the participatory approach, describing the workshop as 'fun' and suggesting that people with type 1 diabetes should be consulted in design processes. The workshop was also a helpful feature for participant recruitment, as adolescents thought they might enjoy participating.

While previous research had identified 17 strategies for reducing productrelated stigma, I limited this workshop to six strategies and limited the time available for each task, creating a pace that was rapid and engaging (Vaes, 2014). Sketching and prototyping designs resulted in additional, specific information being elicited. Examples include requirements for extended trips and what information needs to be communicated in medical emergencies. This points to the difference between this participatory approach in comparison to methods such as focus groups, whereby the 
act of designing can elicit knowledge (Keyson \& Bruns, 2009; Pedgley \& Wormald, 2007).

For most of the designers, designing with a user was a rare and positive occurrence. Some groups shared the ideation process equally, and in others the designers took a production role, leading drawing and prototyping activities but not contributing largely to ideation. However, such division in roles could make the tasks boring as pairs lacked synchrony in their activities. One designer conceptualised his role as helping to get his partner to articulate his needs, then collaboratively developing a concept based on these needs.

Although they were provided with pre-workshop materials, designers were nervous about their limited knowledge of diabetes and required participants to inform them of distinctions between more and less important aspects of selfmanagement and device functions. While it is clear to many experienced designers that they must interact with many participants to elicit varying needs and opinions, it struck one designer that had he only been working with one user, he would not have uncovered the variety of needs elicited in the group brainstorm.

Designers were hesitant to critique participants' ideas, though thought this workshop approach would be very helpful early in the research process to better understand optimal design direction and user requirements. Designers were concerned, however, that if they developed these ideas in isolation, the original participants may be upset by how much the concepts changed.

Designers remarked that participants were candid in discussing their personal experiences, and that this was beneficial to designing. My established rapport with the participants prior to the workshop may have facilitated this open sharing. Also, in one session some participants knew each other, and conversation flowed easily. Additionally, the walls of the workspace were covered in quotes about embarrassment and stigma taken from earlier interviews with workshop participants. These honest disclosures possibly seeded the frank discussions.

The designers had biases about the strategies. Strategies to make the device invisible or increase its social power were not selected for the final designs, although these both ranked highly in the voting task. Designers commented that these strategies were hard to implement. For instance, increasing social power may not 
always relate to a device, and designers did not want to sketch products that would be barely visible.

While the participatory design workshop helped to refine adolescents' requirements for blood glucose monitoring technology, the findings were based on a small number of participants, some of whom were quite confident about selfmanaging in public. It is likely that there is a participation bias, with young people with diabetes who participate in research experiencing less social discomfort.

\subsection{Summary}

This study aimed to develop the requirement for minimising or eliminating feelings of stigma or embarrassment while using medical technology. While it was clear from previous chapters that some adolescents find using medical devices embarrassing or stigmatising, it was unclear how medical devices might be designed to reduce this discomfort. Participants took part in a participatory design workshop based on six strategies for reducing stigma of blood glucose monitoring technologies. Participants valued different strategies for various types of users (e.g., embarrassed users, confident users). All stigma-reducing strategies had advantages and disadvantages, and all except for strengthening the medical identity of the product were thought to be valuable. Participants with diabetes were unsatisfied that strategy one (medical identity) was most commonly used, though new possibilities for this strategy were not largely explored.

Vaes' (2014) strategies were successful in stimulating discussion and ideation among adolescents and designers, though to be usable in a workshop setting, the number of strategies needed to be reduced. Addressing negative attention is a complex task, with differing opinions and experiences between users of medical devices. As such, this research suggests that a variety of stigma-reducing strategies must be employed to reflect the needs of differing users in various contexts. Further research is needed to determine how to design medical devices that do not create stigma for this group, and when different strategies may be appropriate. In particular, further research about context-based appraisals of whether it is advantageous for medical devices to draw attention should be conducted. 
The workshop method successfully facilitated open discussion of sensitive topics amongst adolescents and designers. This method resulted in detailed, contextual requirements, and original design concepts for blood glucose monitoring including a smartphone attachment, all-in-one keychain, holographic watch, system for 3D printing customised devices, and modular test kit.

While use of medical devices can result in perceived or enacted stigma for adolescents with type 1 diabetes, this research began to explore the benefits and drawbacks of six strategies for designing to minimise stigma in a diabetes-specific context. Primarily, though, adapting the stigma strategies for a workshop setting facilitated the development and reframing of a psychosocial user requirement developed earlier in the research. By researching through design, the intricacies of this requirement were elucidated. 


\section{Chapter 11 - Discussion}

The research documented in this thesis had two aims:

1. To describe adolescents' experiences of self-managing type 1 diabetes.

2. To construct psychosocial user requirements for the design of medical technology for adolescents' self-management of type 1 diabetes.

While previous chapters utilised differing methods to fulfil aspects of these aims, this chapter seeks to bring that information together in a summary of adolescents' experiences and user requirements, and how they can be mapped onto each other. I also compare and contrast the user requirements to a current process for selecting medical technologies to be subsidised in New Zealand, and report on the implications of and extensions to the research.

\subsection{Psychosocial User Requirements of Medical Technologies}

Throughout the thesis, psychosocial user requirements of medical technologies for adolescents with type 1 diabetes were constructed. In this final summary of the requirements, they have been categorised and edited to form a coherent list. Below are the final 20 user requirements that form nine categories.

\subsubsection{Allow accessibility and affordability}

Adolescents had access to subsidised medical technologies, however, in some instances were unaware of available subsidised technologies, were not eligible for subsidies, or desired unaffordable medical devices (Ministry of Health, 2017). Desired devices included an insulin pump, insulin pen with memory function, CGM, and FreeStyle Libre. Adolescents are often aware of medical technologies through peers with diabetes and the internet and media. Health professionals advocated increasing access to medical devices and consumables for adolescents with type 1 diabetes, and giving them greater access to services such as psychological therapy (Tanenbaum et al., 2017). Although health discourses commonly omit barriers of 
access (Prendiville, 2017), accessibility and affordability are issues which must be addressed, particularly for adolescents who have limited financial means.

\subsubsection{Foster trust in accuracy and reliability}

Accurate medical technology; fostering trust in reliability so adolescents can make informed self-management decisions without stress of uncertainty (Barnard et al., 2014; Chase et al., 2010). A frequent example was a blood glucose meter that gave widely varying results in everyday settings, resulting in adolescents acting in a riskaverse manner and administering less insulin than required, and experiencing reduced motivation to improve glycaemic control. Accuracy and reliability of medical technologies are pertinent in managing type 1 diabetes to inform decisionmaking, and the actions they facilitate need to be delivered accurately (Low et al., 2005). Glycaemic control is important, and medical technologies should enhance adolescents' motivation, confidence, and ability to improve and maintain this (Barnard et al., 2012).

\subsubsection{Manifest usability}

Self-care medical technologies demonstrating high levels of usability (Chase et al., 2010; Haak et al., 2007; Hanas et al., 2011; Perfetti, 2013), particularly as they need to be used during elevated and reduced levels of glycaemia where cognitive functioning may be impaired. Examples included the success of insulin pens when compared to the traditional vial and syringe methods, and poorly labelled buttons on a blood glucose meter contrasted with straightforward button labels on an insulin pump. For infrequent actions, such as changing the time on a meter, users needed to consult the instruction manual as devices were not intuitive. Usable technologies can reduce the burden of management (A. E. Carroll et al., 2007). Designers must consider interface design, being aware that these devices will be used in medical states with cognitive impairment and added stress.

\subsubsection{Affording comfort}

Medical technologies affording both physical and psychological comfort. 


\subsubsection{Allow comfortable and non-invasive use}

Medical technologies being comfortable and non-invasive where possible (Barnard et al., 2014; Chase et al., 2010; Davis \& Renda, 2006; Hanas et al., 2011; Korytkowski et al., 2005; Perfetti, 2013). Currently insulin injections could be painful and induce needle phobias, and pens and pumps left marks on the body, fuelling body image issues. Participants desired non-invasive technologies, though the technology behind these solutions is still being refined (Tura et al., 2007). For the one participant who had a CGM, she checked her glucose level more regularly as this did not require a finger prick every time. Similarly, some participants described insulin pumps as advantageous, as the wearer could avoid multiple daily injections, even though the pump site was invasive (Lindholm Olinder et al., 2007; Perfetti, 2013; Seereiner et al., 2010). Designers can develop concepts for the implementation of non-invasive technologies in development, ensuring that they are embedded in an appropriate manner.

\subsubsection{Minimise psychological distress}

Medical devices not causing psychological distress. The long-term and intensive nature of diabetes management can be psychologically challenging, with adolescents who have not reached a state of acceptance being aware of the unfairness of having to manage the condition, and feeling vulnerable (Richardson et al., 2001; Weijman et al., 2005). Some adolescents were fearful of hypoglycaemia, and required medical technologies that alleviated this fear (Barnard et al., 2014, 2012), and to minimise risk, some adolescents carried backup equipment; designers could make equipment more compact and portable.

Adolescents also experienced moods symptomatic of hypoglycaemia and hyperglycaemia, and undesirable feelings such as frustration, confusion, worry and stress. Adolescents' conceptualisation of effective diabetes management could affect how they felt about self-management, with some focused on effort and others more so on results; developing obsessive thinking about blood glucose results, frustration, or hiding results from parents or health professionals (Dweck, 2008). Although glycaemic control afforded by intensive management with accurate medical 
technologies could minimise symptoms, it could also be detrimental to wellbeing (Ritholz et al., 2007).

Medical technologies can relieve distress by giving adolescents 'peace of mind', quality of life, and facilitating acceptance (Barnard et al., 2008; McMahon et al., 2005), or could also be designed playfully, to draw positive attention to selfmanagement activities, putting peers at ease. Some parents, adolescents, researchers, and companies developed games around blood glucose testing and carbohydrate counting (Boyle, 2010; Glasemann et al., 2010; Kamel Boulos et al., 2015; Toscos et al., 2012), signalling a possible avenue for medical technologies that encourage selfmanagement behaviours while introducing elements of fun and achievement.

\subsubsection{Suitability and desirability}

Adolescents should have choices regarding medical technologies that meet their expectations, preferences, and values, and are adaptable.

\subsubsection{Inform realistic expectations}

Medical technologies, and their supporting services, should clearly communicate their advantages and disadvantages so adolescents have clear expectations when initiating use of a new device (Toscos et al., 2013). Expectations that differed from reality were in initiating insulin pump therapy, where adolescents expected the pump to manage for them, but discovered the pump required additional effort to afford greater freedom in eating and exercising (Low et al., 2005; Ritholz et al., 2007). That is, medical technologies did not match expectations and adolescents changed devices or behaviours to compensate. Designers should consider both medical devices and their support services, ensuring products' system images align with adolescents' mental models.

\subsubsection{Align with values and preferences}

Medical technology aligning with adolescents' values (Haak et al., 2007). Similar to the previous requirement, this can determine the suitability of the technology for the individual. While disposable insulin pens are convenient, participants were concerned about their environmental impact and switched to reusable insulin pens. 
By having medical technologies that align with values, states of cognitive dissonance can be avoided. Additionally, like consumer devices, adolescents wanted devices with quality product design and aesthetics, objecting to devices that felt 'cheap'. Health professionals recognised adolescents' preferences by communicating via text message rather than phone calls. Designers need to better understand adolescents' values and preferences, including them in the development of products, so their needs are represented. This could be achieved through extreme user approaches to design.

\subsubsection{Provide choices, customisation or personalisation}

Have choices of medical technologies, or being able to personalise or customise them (Glasemann et al., 2010; Kamel Boulos et al., 2015). This extra element of choice and control may improve self-management. Adolescents were frustrated by a change to a subsidised meter that felt forced, as although there were other options available, the ongoing cost of consumables was unaffordable and they perceived this meter to be worse than previous models. Current options for personalisation are aimed at children. Designers can explore not just minimising dissatisfaction with medical devices, but also creating desirable options in tandem with users, bringing participatory design to the fore. Modular designs may allow for the same mechanisms to be implemented in differing products.

\subsubsection{Integration into life}

Medical technologies being convenient and suiting use in everyday contexts, facilitating adolescents' freedom to participate in activities.

\subsubsection{Perform in a life-proof manner}

Medical technology being life-proof, allowing adolescents to use them in many contexts and trust in their durability (Low et al., 2005). A positive example was water-resistant devices. An example of where this requirement was not currently met is glucose meters that cannot function in a broad range of temperatures or cannot withstand being dropped (International Organization for Standardization, 2015). Designers must consider use in contextual extremes to create devices that perform 
more consistently. For instance, the idea of designing blood glucose meters able to be easily used while adolescents are intoxicated was mooted.

\subsubsection{Enhance convenience}

Medical technology minimising detracting aspects of management, including carrying several devices and manual data input from glucose meters to mobile apps (Barnard et al., 2014; Bergenstal et al., 2000; Lindholm Olinder et al., 2007). Successful examples include CGM-integrated insulin pumps and insulin pumps that automatically calculate insulin doses based on variables input by users, glucose meters that sync to smartphones, dual blood glucose and ketones meters, and blood glucose meters combined with lancets, increasing the efficiency of self-management. Designers can continue trends of automation for increased convenience, while facilitating engagement with meaningful information.

\subsubsection{Facilitate spontaneity and participation in everyday activities, and minimise impact on life}

Medical technologies facilitating adolescents' spontaneity and participation in everyday activities (Korytkowski et al., 2005). Insulin pumps afford spontaneous participation, allowing insulin administration to be easily halted or adjusted to suit exercise or food intake (Barnard et al., 2008; Lindholm Olinder et al., 2007). Adolescents noted the cumbersome nature of wearable devices, but also desired a closed-loop system that could be used during sports, eliminating the need for them to stop participating to self-manage (Iscoe et al., 2006). Designers must address the wearability of devices and the ability of technologies to predict blood glucose trends, facilitating proactive management and minimising the need to stop participating.

\subsubsection{Facilitate reliable and efficient night-time management}

Medical technology facilitating confident and efficient nocturnal management (Chase et al., 2010). Though it was uncommon, some adolescents were afraid of night-time hypoglycaemia, and found managing levels during the night frustrating (Barnard et al., 2014). Aspects that made this challenging were blood glucose meters with no lights, and an integrated CGM and insulin pump that alarmed every hour if 
the user's blood glucose levels were high. Adolescents wanted technologies with dim lights, backlights, noises to guide their use without needing to turn on lights, and straightforward information to treat hypoglycaemia. Adolescents generally preferred to manage in a drowsy state rather than waking, unless severely hypoglycaemic or new to diabetes management. Adolescents spend approximately a third of their time sleeping, and as such this context should be part of designers' considerations.

\subsubsection{Enhancement of self-management}

Medical technologies providing adolescents with meaningful information and facilitating their engagement in self-management that works for them.

\subsubsection{Provide meaningful information to facilitate decision-making}

While medical technologies currently collect data, devices should present users with meaningful information that is accurate and actionable to facilitate proactive selfmanagement (Snell-Bergeon, 2015). Adolescents did not commonly use physical logbooks or apps to record data (Perfetti, 2013; Toscos et al., 2012), as although useful to health professionals, these were considered laborious and did not provide sufficient personal benefit.

A device that did provide helpful situational awareness was an insulin pen that displayed the time since the last injection and the dose injected (Hanas et al., 2011), allowing adolescents to confirm they had injected or deliver a missed injection. Participants wanted to view blood glucose trends to better understand overnight fluctuations, anticipate their blood glucose levels, and respond proactively, rather than waiting for hypoglycaemia or hyperglycaemia to occur (Riddell \& Milliken, 2011). Designers visualising trends may also shift attention from 'good' and 'bad' individual data points, which can be distressing, to patterns that can be used to inform actions. Technology could also be better used for pattern recognition (e.g., high afternoon blood glucose levels) and accompanying behaviour change prompts, though contextual information would be important to ensure these are meaningful patterns and prompts (Glasemann et al., 2010; Toscos et al., 2011). While data can be useful for health professionals, data collected should also be made meaningful for adolescents, relevant to needs and preferences. 


\subsubsection{Couple behaviours with consequences}

Medical technology strengthening the link between behaviours and consequences, as the interconnectedness of diabetes and lifestyle elements can make this relationship unclear. An example is a CGM, which shows a line graph of blood glucose levels that allows users to see the impact of food, exercise, insulin, and illness. Currently, one participant found it challenging to understand why her night-time levels were not normoglycaemic, and was not able to determine the relationship between the time she ate dinner and nocturnal blood glucose levels with the current data and its reporting. Relationships between behaviours and consequences can be unclear in diabetes management, and where possible designers could better link these.

\subsubsection{Facilitate daily structure while allowing for spontaneity}

Adolescents agreed that daily structure improved their diabetes management, however, they also desired spontaneity. Structure included blood testing, delivering insulin, and eating known foods at regular times. For those who required reminders for self-care behaviours, subtle, context-aware prompts could be beneficial. Currently, many alarms were silenced on medical technologies as they alarmed at times that caused social distress. In situations other than emergencies, designers should create alerts that are subtle, customisable, and context-appropriate, so that they are used as intended.

\subsubsection{Facilitate realistic goals and responsiveness to get back on track}

Medical technologies aiding realistic goal setting and recovery from error and disengagement. Adolescents described the difficulty controlling diabetes, comparing it to a seesaw and a claw arcade game. These fluctuations could lead adolescents to give up in response to undesired results or motivate them to meet goals, however, many required better strategies for goal setting. Medical technologies should facilitate adolescents re-establishing glycaemic control after high or low blood glucose levels or periods of disengagement. Insulin pumps currently allow easy corrections to be made for high blood glucose levels, supporting adolescents' responsiveness. Designers can also utilise research about patient-led goal setting, using prompts to aid adolescents in setting better quality goals. 


\subsubsection{Social considerations}

Medical technologies being socially considerate, facilitating disclosure when appropriate and giving users power over the attention they garner.

\subsubsection{Help to comfortably disclose and explain type 1 diabetes when appropriate}

As diabetes is an invisible medical condition, medical technologies and their use commonly draw unwanted attention to their users, forcing or limiting choice to disclose diabetes in social contexts. While disclosure can be beneficial psychologically, technologies should facilitate adolescent choice about who they disclose to and not reveal by default (Hains et al., 2007; Thompson Jr. \& Gustafson, 1996; Warner \& Hauser, 2009; Wysocki \& Greco, 2006). Currently, onlookers might ask questions or make offensive comments, and adolescents were concerned what others think of them when managing in public settings (Y.-L. Wang et al., 2010). A participant described injecting with an insulin pen in public as "degrading". Designers could make desirable medical technologies that act as prompts for voluntary disclosure.

\subsubsection{Eliminate negative attention while using medical technology, and facilitate positive attention where appropriate}

Medical technologies suitable for a wide variety of contexts, and not inducing embarrassment or stigmatisation (Dickinson \& O’Reilly, 2004; Y. -L. Wang et al., 2010). Some medical devices such as syringes attract stigma, and needles can disturb onlookers with needle phobias, whereas a shift to more discrete insulin pens and pumps has reduced these feelings of stigma. Medical technology should not invoke negative connotations of drug paraphernalia. Adolescents had vastly differing opinions on whether using medical devices in public spaces was embarrassing, with both negative and positive aspects to devices drawing attention (A. E. Carroll et al., 2007; Low, Massa, Lehman, \& Olshan, 2005). Negative aspects included undesired questions about the medical device, the insulin pump making loud noises while in quiet spaces, or feeling too embarrassed to test their blood glucose or inject insulin in

public spaces (Barnard et al., 2008; Korytkowski et al., 2005; Seereiner et al., 2010). 
Positive aspects included medical devices drawing attention during emergencies, and public device use increasing awareness and discussion, aiding disclosure of diabetes and normalising self-care behaviours (e.g. Health tracking with the Fitbit) (Ritholz et al., 2007). Subsequently, there will be times when it is advantageous for medical devices to draw attention, and other times when users prefer discretion. In addition, for some users there is scope for devices to draw more positive forms of attention, even being desirable (Hanas et al., 2011; Perfetti, 2013). Designers may achieve this through strategies such as strengthening the individual identity of the device by providing more choice and opportunities for personalisation, strengthening the product's brand identity, and increasing the device's social power (Bispo \& Branco, 2011; Pullin, 2009, pp. 16-25; Sansoni et al., 2016). However, each strategy will not be appropriate for all users, due to their diverse preferences (Sansoni et al., 2016).

\subsubsection{Enhancement of support network}

Medical technologies could better support the transition of responsibility from parents to children and adolescents, and strengthen adolescents' interactions with friends and diabetes peers.

\subsubsection{Support transition of responsibility}

During the adolescent years, both parental support and the adolescent's growing independence with diabetes management are important. There was tension between parents and adolescents about diabetes knowledge, independence, privacy, and 'nagging' (Greca \& Bearman, 2002). Medical technologies should support the transition of responsibility by fostering clear expectations of information and data sharing and making use of adjustable settings (Y.-L. Wang et al., 2010). Some parents took active roles, copying blood glucose data into physical logbooks to encourage their adolescent to engage with their results, and other adolescents would tell their parent whether they had tested or what their blood glucose level was when prompted. Some adolescents, however, did not want their parent or health professional to know their blood glucose level or whether they had not tested it, and would fabricate results when questioned (A. E. Carroll et al., 2007), or felt they could not manage to the level their parents had achieved. Diabetes Nurse Specialists 
currently took on a role of encouraging parents to transition responsibility to adolescents, and negotiating interactions. Designers can ensure technology is implemented to better negotiate roles and data sharing between adolescents, parents and health professionals to ease tension, while ensuring the adolescent safely learns sufficient self-management skills to become independent.

\subsubsection{Facilitate interaction with diabetes peers and friends}

Medical technologies facilitating positive interactions among peers with diabetes and friends ( $\mathrm{Lu}$ et al., 2015; Wysocki \& Greco, 2006). Currently, questions from friends or peers about medical devices could be embarrassing or frustrating (Warner \& Hauser, 2009). Adolescents differed in whether they desired peer support and friendship form others with type 1 diabetes. At different extremes, one adolescent felt diabetes had a positive impact on her life due to the friends she had made, whereas another did not want to meet others with diabetes as he did not want his life to centre on diabetes. Where they did have diabetes peers, adolescents used social media to share information and joke or vent about diabetes-related experiences. While a Facebook page had been established up by health professionals, adolescents did not share openly on this more visible forum. Diabetes camps were a common way of meeting others with diabetes, though relationships had to be nurtured after returning home.

Table 12 below demonstrates the grouped psychosocial user requirements, and in which chapters they were identified. 
Table 12

Development of psychosocial user requirements.

Psychosocial User Requirements

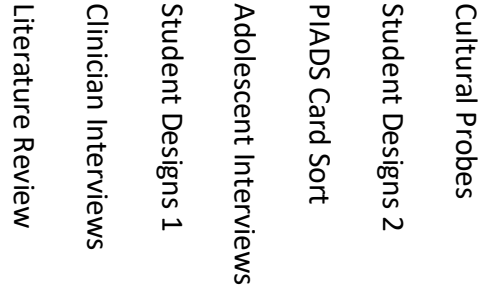

\begin{tabular}{|c|c|c|c|c|c|c|c|c|c|}
\hline $\begin{array}{l}\text { Access to } \\
\text { medical } \\
\text { technologies }\end{array}$ & Allow accessibility and affordability & & $\checkmark$ & & $\checkmark$ & $\checkmark$ & & $\checkmark$ & \\
\hline Reliability & Foster trust in accuracy and reliability & & $\checkmark$ & & $\checkmark$ & $\checkmark$ & & $\checkmark$ & \\
\hline Usability & Provide usability & $\checkmark$ & & & & $\checkmark$ & & $\checkmark$ & \\
\hline \multirow[t]{2}{*}{ Comfort } & $\begin{array}{l}\text { Facilitate comfortable and non-invasive } \\
\text { use }\end{array}$ & $\checkmark$ & & $\checkmark$ & & $\checkmark$ & & $\checkmark$ & \\
\hline & Minimise psychological distress & $\checkmark$ & $\checkmark$ & & $\checkmark$ & $\checkmark$ & $\checkmark$ & $\checkmark$ & \\
\hline \multirow[t]{3}{*}{$\begin{array}{l}\text { Suitability \& } \\
\text { desirability }\end{array}$} & $\begin{array}{l}\text { Communicate to inform realistic } \\
\text { expectations }\end{array}$ & $\checkmark$ & $\checkmark$ & & $\checkmark$ & & & & \\
\hline & Align with values and preferences & & $\checkmark$ & & & & & $\checkmark$ & $\checkmark$ \\
\hline & $\begin{array}{l}\text { Provide choices, customisation or } \\
\text { personalisation }\end{array}$ & & $\checkmark$ & & $\checkmark$ & & & $\checkmark$ & \\
\hline \multirow{4}{*}{$\begin{array}{l}\text { Integration into } \\
\text { life }\end{array}$} & Perform in a life-proof manner & $\checkmark$ & $\checkmark$ & $\checkmark$ & $\checkmark$ & $\checkmark$ & & $\checkmark$ & \\
\hline & Enhance Convenience & $\checkmark$ & $\checkmark$ & & $\checkmark$ & $\checkmark$ & & $\checkmark$ & \\
\hline & $\begin{array}{l}\text { Facilitate reliable and efficient night-time } \\
\text { management }\end{array}$ & $\checkmark$ & & & $\checkmark$ & $\checkmark$ & $\checkmark$ & $\checkmark$ & \\
\hline & $\begin{array}{l}\text { Facilitate spontaneity and participation in } \\
\text { everyday activities, and minimise impact } \\
\text { on life }\end{array}$ & $\checkmark$ & & $\checkmark$ & $\checkmark$ & $\checkmark$ & $\checkmark$ & $\checkmark$ & \\
\hline \multirow{4}{*}{$\begin{array}{l}\text { Enhancement of } \\
\text { self- } \\
\text { management }\end{array}$} & $\begin{array}{l}\text { Provide meaningful information to } \\
\text { facilitate decision-making }\end{array}$ & $\checkmark$ & $\checkmark$ & $\checkmark$ & $\checkmark$ & $\checkmark$ & $\checkmark$ & $\checkmark$ & \\
\hline & Couple behaviours with consequences & & $\checkmark$ & & $\checkmark$ & & & $\checkmark$ & \\
\hline & $\begin{array}{l}\text { Facilitate daily structure while allowing for } \\
\text { spontaneity }\end{array}$ & & $\checkmark$ & & $\checkmark$ & $\checkmark$ & $\checkmark$ & $\checkmark$ & \\
\hline & $\begin{array}{l}\text { Facilitate realistic goals and } \\
\text { responsiveness to get back on track }\end{array}$ & $\checkmark$ & $\checkmark$ & & $\checkmark$ & $\checkmark$ & & $\checkmark$ & \\
\hline $\begin{array}{l}\text { Social } \\
\text { considerations }\end{array}$ & $\begin{array}{l}\text { Help to comfortably disclose and explain } \\
\text { type } 1 \text { diabetes when appropriate }\end{array}$ & $\checkmark$ & $\checkmark$ & $\checkmark$ & $\checkmark$ & $\checkmark$ & $\checkmark$ & & $\checkmark$ \\
\hline
\end{tabular}




\begin{tabular}{|c|c|c|c|c|c|c|c|c|c|}
\hline & $\begin{array}{l}\text { Eliminate negative attention while using } \\
\text { medical technology, and facilitate positive } \\
\text { attention where appropriate }\end{array}$ & $\checkmark$ & $\checkmark$ & $\checkmark$ & $\checkmark$ & $\checkmark$ & $\checkmark$ & $\checkmark$ & $\checkmark$ \\
\hline \multirow{2}{*}{$\begin{array}{l}\text { Enhancement of } \\
\text { support network }\end{array}$} & Support transition of responsibility & $\checkmark$ & $\checkmark$ & & $\checkmark$ & $\checkmark$ & & $\checkmark$ & \\
\hline & $\begin{array}{l}\text { Facilitate interaction with diabetes peers } \\
\text { and friends }\end{array}$ & $\checkmark$ & $\checkmark$ & $\checkmark$ & $\checkmark$ & $\checkmark$ & & $\checkmark$ & \\
\hline
\end{tabular}

In Chapter 2, 11 relevant sets of user requirements for medical technologies were identified, with seven requirements identified in at least two. These were to:

- minimise impact on daily life and facilitate living a full life;

- facilitate extended engagement;

- facilitate support network and connective services;

- provide meaningful information;

- demonstrate usability;

- offer choice and personalisation;

- accommodate social considerations and attention preferences; and

- provide security

(Cafazzo et al., 2012; Glasemann \& Kanstrup, 2011; Kanstrup, 2014; Kanstrup et al., 2008; Lang, 2012; Machniak, 2013; J. L. Martin et al., 2008; Nordfeldt et al., 2010; Toscos et al., 2011, 2012).

Each of these requirements was also constructed in this research, demonstrating the replicated nature of the requirements across a selection of age groups and chronic diseases. This indicates that there may be grounds to generalise psychosocial user requirements of medical devices across larger populations than those studied (Lang, 2012). However, further research and testing would be required to compare requirements among groups.

The requirements also map to Norman's (2004) three-part model outlining interactions with designed objects: 'visceral', 'behavioural', and 'reflective'. This is similar to Walter's (2010) approach, acknowledging the importance of functionality, reliability, and usability, but also prioritising pleasure. Usable designs are not necessarily pleasurable, and vice versa. Although functionality and usability are extremely important to consider, focusing so closely on these factors can inhibit 
designers from thinking about the meanings their devices convey (Polgar, 2010). Usability should be addressed in concert with other user requirements.

\subsection{Pharmac Case Study}

The Pharmaceutical Management Agency (Pharmac) determine which medicines and related products are subsidised in New Zealand (Pharmac, 2014). This case study investigates the criteria used for Pharmac's recent evaluation of blood glucose meters, consumables, and associated products in relation to the findings of the current research (Pharmac, 2015b). The review of blood glucose meters covers New Zealanders of all ages with type 1 and type 2 diabetes, but encompasses the adolescent group with type 1 diabetes.

In addition to laboratory testing of proposed devices, Pharmac have composed usability criteria and evaluated devices against them. Criteria were composed through a public consultation stage, with 313 respondents, where people chose the device functions, associated services, and testing criteria that were relevant for them by choosing items from a list, or submitting their own items. Respondents were individual consumers, consumer organisations, health professionals, and suppliers (Pharmac, 2015a, 2015b). While the results of testing are not yet available, the criteria were:

- "Ability to provide test averages;

- backlighting and/or port lighting;

- size and clarity of date, time and glucose readings;

- testing time;

- memory capacity;

- accessories supplied with Products, such as lancing device and log books;

- ease of strip canister/packaging for opening;

- physical attributes, such as size and colour of Products;

- ability to download the Product's readings and the functionality of the program used for the download;

- integration with practice management systems in both hospital and community care, and 
- any other functions or attributes the Product may possess" (Pharmac, 2015c, pp. 5-6).

I have compared these criteria, which will be used to inform which blood glucose meters are subsidised in New Zealand, with the psychosocial user requirements developed in my research (Table 13). Where a Pharmac criterion overlapped with multiple psychosocial user requirements, I have duplicated it.

\section{Table 13}

Comparison of psychosocial user requirements and Pharmac usability criteria.

\begin{tabular}{|c|c|c|}
\hline $\begin{array}{l}\text { Psychosocial } \\
\text { user requirement }\end{array}$ & Sub-requirement & Pharmac usability criteria \\
\hline $\begin{array}{l}\text { Access to medical } \\
\text { technologies }\end{array}$ & Allow accessibility and affordability & $\begin{array}{l}\text { Accessories supplied with Products, } \\
\text { such as lancing device and log books }\end{array}$ \\
\hline Reliability & $\begin{array}{l}\text { Foster trust in accuracy and } \\
\text { reliability }\end{array}$ & \\
\hline Usability & Provide usability & $\begin{array}{l}\text { Size and clarity of date, time, and } \\
\text { glucose readings } \\
\text { Memory capacity } \\
\text { Ease of strip canister/packaging for } \\
\text { opening } \\
\text { Ability to download the Product's } \\
\text { readings and the functionality of the } \\
\text { program used for the download }\end{array}$ \\
\hline \multirow[t]{2}{*}{ Comfort } & $\begin{array}{l}\text { Facilitate comfortable and } \\
\text { non-invasive use }\end{array}$ & \\
\hline & Minimise psychological distress & \\
\hline \multirow[t]{3}{*}{$\begin{array}{l}\text { Suitability and } \\
\text { desirability }\end{array}$} & $\begin{array}{l}\text { Communicate to inform } \\
\text { realistic expectations }\end{array}$ & \\
\hline & Align with values and preferences & $\begin{array}{l}\text { Physical attributes, such as size and } \\
\text { colour of Products }\end{array}$ \\
\hline & $\begin{array}{l}\text { Provide choices, customisation or } \\
\text { personalisation }\end{array}$ & \\
\hline \multirow{2}{*}{$\begin{array}{l}\text { Integration into } \\
\text { life }\end{array}$} & Perform in a life-proof manner & \\
\hline & Enhance convenience & Testing time \\
\hline
\end{tabular}




\begin{tabular}{|c|c|c|}
\hline & $\begin{array}{l}\text { Facilitate reliable and efficient } \\
\text { night-time management }\end{array}$ & Backlighting and/or port lighting \\
\hline & $\begin{array}{l}\text { Facilitate spontaneity and } \\
\text { participation in everyday activities, } \\
\text { also minimise impact on life }\end{array}$ & $\begin{array}{l}\text { Physical attributes, such as size and } \\
\text { colour of Products }\end{array}$ \\
\hline \multirow[t]{4}{*}{$\begin{array}{l}\text { Enhancement of } \\
\text { self-management }\end{array}$} & $\begin{array}{l}\text { Provide meaningful information to } \\
\text { facilitate decision-making }\end{array}$ & $\begin{array}{l}\text { Ability to provide test averages } \\
\text { Size and clarity of date, time, and } \\
\text { glucose readings } \\
\text { Ability to download the Product's } \\
\text { readings and the functionality of the } \\
\text { program used for the download } \\
\text { Integration with practice } \\
\text { management systems in both } \\
\text { hospital and community care }\end{array}$ \\
\hline & $\begin{array}{l}\text { Couple behaviours with } \\
\text { consequences }\end{array}$ & \\
\hline & $\begin{array}{l}\text { Facilitate daily structure while } \\
\text { allowing for spontaneity }\end{array}$ & \\
\hline & $\begin{array}{l}\text { Facilitate realistic goals and } \\
\text { responsiveness to get back on track }\end{array}$ & \\
\hline \multirow[t]{2}{*}{$\begin{array}{l}\text { Social } \\
\text { considerations }\end{array}$} & $\begin{array}{l}\text { Help to comfortably disclose and } \\
\text { explain type } 1 \text { diabetes when } \\
\text { appropriate }\end{array}$ & \\
\hline & $\begin{array}{l}\text { Eliminate negative attention while } \\
\text { using medical technology, and } \\
\text { facilitate positive attention where } \\
\text { appropriate }\end{array}$ & \\
\hline \multirow{2}{*}{$\begin{array}{l}\text { Enhancement of } \\
\text { support network }\end{array}$} & Support transition of responsibility & \\
\hline & $\begin{array}{l}\text { Facilitate interaction with diabetes } \\
\text { peers and friends }\end{array}$ & \\
\hline
\end{tabular}

There are clear overlaps in the criteria. However, the psychosocial user requirements cover a broader spectrum, while the Pharmac usability criteria do not consider reliability, comfort, social considerations, or enhancing support networks. While the psychosocial user requirements were developed in response to adolescent and young adult users with type 1 diabetes, their needs may also be evident in users of other ages and with other types of diabetes. Further, adolescents with type 1 diabetes currently 
have poor glycaemic control compared to other groups, and as such, responding to their needs is pertinent (Borus \& Laffel, 2010; Hoffman, 2002; Wood et al., 2013).

Finally, elements in the categories that did not overlap with the Pharmac criteria do have impacts on health behaviours. For instance, when adolescents did not trust the accuracy of their blood glucose meter they lost motivation to improve glycaemic control, when they were worried about unwanted attention drawn by the device they delayed or skipped testing, and when data sharing was not wellconsidered adolescents' relationships with parents and health professionals suffered. While reliability may be covered in part by laboratory testing, current meters that were not trusted had also passed this testing, and impacted adolescents' selfmanagement. As such, these requirements reflect important impacts on adolescents' self-management, and should be considered when determining which medical devices are subsidised.

\subsection{Grounded Theory Summary of Adolescent Experiences}

Grounded theory was used to conduct and analyse much of the research throughout this thesis, with a focus on the experiences of adolescents. In each chapter, categories were reported as informed by the research to that point. In this section, the final, substantive grounded theory is articulated.

\subsubsection{Conceptualising type 1 diabetes and its management}

Conceptualising diabetes is about how adolescents come to understand diabetes and its place in their lives. Properties of this category include developing and applying diabetes knowledge, conceptualising diabetes and its management, developing acceptance, and engaging in self-management.

In terms of developing and applying diabetes knowledge, people with diabetes began by seeking knowledge, then became confident that they had sufficient knowledge to manage in most situations and experiment with changes in their regimen, while still feeling challenged at times by unusual situations. However, greater confidence was not always aligned with better self-management, as some knowledgeable adolescents were likely to test less regularly. The process of knowledge acquisition varied based on diagnosis age, with education aimed at 
parents for younger children, learning happening formally with the diabetes team, and through personal experience. Adolescents followed and created informal guidelines for self-management.

Medical technologies could increase adolescents' feelings of expertise over diabetes. Adolescents varied, however, in whether their medical technologies were empowering or reminded them of their vulnerability. Initially, complex technologies and manual calculations could be confusing, along with the variable nature of diabetes. While the acquisition of knowledge was positive, it did not always translate into corresponding actions, and could be manipulated for adolescents' goals such as weight loss, contrary to ideal diabetes management.

Although medical technologies could be used to transform data into more meaningful information, this did not currently happen effectively, with barriers around tedious data logging, data access, affordability, data syncing, and value judgments attached to blood glucose numbers. Adolescents wanted technology that allowed them to better visualise trends in blood glucose levels, to facilitate proactive management.

In addition to acquiring and applying diabetes knowledge, adolescents are conceptualising both diabetes and its management. Ultimately, managing wellness was key in managing this long-term condition, with much discussion of aiming to fit diabetes around the rest-of-life. This allowed adolescents to live how they wanted, whether taking part in activities or eating desirable food, adjusting their management to suit. Others desired minimising their role in diabetes management by acquiring closed-loop technologies. However, adolescents currently required a high level of self-management to allow their diabetes to fit predictably into their life. In reality, adolescents made lifestyle changes to accommodate diabetes management, such as changes to their diet, making sure they had the necessary technology and food on hand and pausing to establish blood glucose levels before exercise. Also, many aspects of adolescents' lifestyle, including fledgling independence, mental health issues, inflexible or unstructured schedules, and choices of diet, exercise, and drugs interacted negatively with diabetes management. Additionally, diabetes could negatively impact daily life, through hyperglycaemia or hypoglycaemia, tiredness, missing classes, and such. 
Adolescents used personification and metaphor to describe diabetes and its management, and some found labels such as 'diabetes' and 'diabetic' overwhelming. Metaphors used were about: managing a balancing act, maintaining blood glucose levels within range; struggling for control, taking actions but not being in total control of diabetes; rushing to beat the clock, aiming for daily structure but experiencing hurdles; and experiencing psychosocial impacts. These metaphors begin to explore the psychosocial effects of diabetes.

Adolescents varied in four domains as to their opinions of what constituted 'good' diabetes. Some judged management against guidelines provided by health professionals, some contextualised management within their daily lives, with adolescents on a continuum of prioritising diabetes management or lifestyle, some focused on results whereas others focused on effort, and finally, some focused holistically on physical and mental health.

Acceptance of type 1 diabetes and its required management was described as a process and a state, where from diagnosis onwards, acceptance could be worked towards and a state of acceptance achieved. In this state, the person could become engaged in their self-management, facilitating healthier self-management. While those diagnosed as young children or post-adolescence may find acceptance easier, not everybody reached a state of acceptance and there may be regression. A signal of acceptance was in taking a proactive rather than passive approach to selfmanagement, choosing to engage in self-management. Family played a role in developing acceptance, with the way parents felt about the condition affecting the adolescent's own feelings. Barriers to acceptance included adolescents feeling invincible or overwhelmed by feelings of vulnerability, or having an identity conflict around having a chronic disease, whereby a young person who has previously been fit and healthy struggles to balance the existing self-image of healthiness and of also having type 1 diabetes.

Building from acceptance, engagement is described as both a process and a state. The process of becoming engaged was predominantly described in one of three ways. Firstly, a gradual process that aligned with the individual maturing, secondly, a defining moment where some factor external to the person resulted in an epiphany, and thirdly, as being proactive and setting goals. Some adolescents, however, did not 
want to engage. They wanted to feel 'normal', and completing self-care behaviours shattered this illusion.

\subsubsection{Wanting to minimise the impact of type 1 diabetes}

Adolescents wanted to minimise the impact of diabetes on their lives, both physically and psychosocially, and engage in other aspects of life. Current diabetes management and technologies were inconvenient and impacted quality of life, ability to participate in everyday activities, and adolescents' sense of security. Medical devices contributed both positively and negatively to adolescents' efficiency, productivity, and eagerness to try new things, allowing them to manage their diabetes symptoms and giving them peace of mind, but taking time to use and find a private space to do so if desired. Some adolescents also had fluctuations in self-esteem and emotional reactions to blood glucose monitoring, feeling frustrated or unhappy with hyperglycaemic or hypoglycaemic levels and how this would impact their day, but feeling happy about normoglycaemic levels. Interestingly, while aiming for a narrow glycaemic range could be physically beneficial, it could be psychologically challenging. However, many recognised that not testing would result in increased frustrations. Currently, issues with medical technologies that impacted convenience and quality of life included portability, noises, inadequate lighting, non-intuitive controls, tedious use, malfunctions, limited performance in varying climates, pain and discomfort, and bleeding and bruising, impacting body image. Adolescents and health professionals suggested affordable closed-loop systems, integrated devices, syncing to smartphones, and insulin dose calculators.

While medical technologies reduced diabetes symptoms, allowing adolescents to participate in everyday activities and social situations, they could also negatively impact this ability. Participation in everyday activities and managing diabetes were sometimes in conflict, with each aspect prioritised on different occasions. Participation was a very important aspect of life. A8: "Participating means feeling included and that's one of the key things in life or in happiness."

Some adolescents withdrew from social activities, not wanting to inconvenience friends with diabetes-related delays. Some planned in advance and incorporated structured management; making self-management easier, yet reducing 
spontaneity. Spontaneity and flexibility, specifically with eating and exercising, largely increased when using insulin pumps, as they gradually released insulin. While participation was usually positive, diabetes was occasionally used as an excuse for not participating in an undesired activity.

Adolescents' sense of security was affected depending on whether they trusted their medical devices to be accurate and reliable. When blood glucose meters were trusted, they minimised adolescents' fear of exercise-induced and nocturnal hypoglycaemia, and facilitated trying new foods and experiences. However, many adolescents' trust in the accuracy of blood glucose meters eroded over time. Accordingly, they gave insulin conservatively, second-guessed the meter and their physiological symptoms, struggled to make diabetes-related decisions, took another measurement using the same or a different meter, or had decreased motivation to improve glycaemic control.

Although adolescents are commonly described as risk-takers, risk-averse behaviour was also evident in managing blood glucose levels. Adolescents predominantly acted to minimise short-term risks, such as hypoglycaemia, but less so for long-term risks such as running high. Adolescents ran high to avoid hypoglycaemia, avoid managing in public, or because they were not highly engaged in self-management and did the minimum to avoid acute illness. Forgetting to test blood glucose levels or inject insulin was a common feature of adolescent selfmanagement that often improved over time. While some had tried setting reminder alarms, often these did not work as daily schedules were changeable, particularly when at university or moving from weekdays to weekends.

\subsubsection{Having individual medical technology needs}

In addition to normative adolescent development, some characteristics that affect adolescents' self-management can be more individual. While adolescents are a small subset of people with type 1 diabetes, the group is described as "extremely heterogeneous" (SE2). Personality and intelligence affected how adolescents managed diabetes. Technology that suited adolescents could make them feel at peace, and as though a weight had been lifted. 
Adolescents were changing technologies in accordance with their individual needs and characteristics. Reasons for changing medical devices included a change dictated by others (e.g., Pharmac subsidy), technology suggested by health professionals, broken devices, desired features, and anticipated improvements in self-management or quality of life. Barriers to change were commonly to do with affordability.

Adolescents have varying compatibility with these devices, and there are limited choices and levels of adaptability in current medical technology. The first response to dissonant technology was to discard technologies, for reasons such as unmet expectations around the impact of the device, cumbersome and tedious user experience, and dissonance with values. The second response to experiencing dissonance with medical technology was for adolescents to change medical technologies and demonstrate a resulting change in behaviour, such as acquiring an insulin pump and becoming more engaged. Adolescents having choices about which medical technologies to use arose as a factor that could be important in affecting selfmanagement, but that is currently limited. This could be a way for the adolescent to establish control, and links back to normative adolescent development. There was also a desire for personalised medical technology, where the technology is adapted to the needs of the individual.

\subsubsection{Struggling for independence}

Adolescents with type 1 diabetes were developing independence as is common with normative adolescent development. Processes included getting accustomed to medical devices, and transitioning responsibility from health professionals and parents.

When initially diagnosed or supplied with a new device, adolescents went through a process of getting accustomed to devices. After diagnosis many did not initially want to use the devices, finding tasks overwhelming and fearing needles, but did so out of necessity. Some adolescents used the 'popper' or 'rocket' for injections to ease this process. Other adolescents found a change in medical devices could increase their independence. For a small group, the experience at diagnosis was more 
positive, and there were perks and bribes supplied by family and health professionals when initially using the devices.

Some adolescents felt independent from diagnosis onwards, whereas for others this feeling developed over time, taking years to become confident in decision-making and not being worried by others' judgements. However, a diagnosis during adolescence could initially lead the individual to feel less independent. Overall, medical technologies increased adolescents' sense of independence. For example, most adolescents viewed their blood glucose meter as essential, letting them self-manage and go about daily life independently, without being completely reliant on health professionals or physiological signs. Some adolescents found it crucial that they lead their diabetes management, as they were the only person with first-hand knowledge of their life. Feelings of independence and confidence garnered through self-management could also bolster self-efficacy in other areas of life.

Paired with developing independence was the transition of responsibility from health professionals and parents to adolescents. Initially, people with diabetes were heavily reliant on health professionals, but this diminished over time. Health professionals strove to facilitate adolescents' independent self-management, but also a strong support network from family and the clinic. As adolescents developed independence, tensions could develop between the personal, lived experience of the adolescent and the health professionals' guidelines, formed by more abstract medical knowledge. Participants who favoured more self-reliance championed not requiring advice when approaching new challenges, and being able to compile questions and only ask them at clinics, though health professionals played a role in negotiating the transition of responsibility, sometimes providing explicit strategies.

While there was commonly some transition of responsibility from parents to adolescents, this process was more pronounced for individuals diagnosed at a younger age. While some children were interested in taking on management activities from initiation, most were not. Slowly, adolescents became increasingly involved in appointments and independent with tasks. By the time they were in their late teens, it was common to be testing and self-administering insulin. Some adolescents were further along this transition process, at a stage where they kept 'in touch' with their parents about their diabetes but were largely responsible for 
management, viewing parental input as 'nagging'. Parents may advocate for their child's independence, recognising that only the adolescent had full knowledge of contextual details.

While parental support typically decreases over these years, this is not always the case, and parents were still undertaking more administrative tasks for younger adolescents. Variables in the process included the age at diagnosis, parental style, diabetes team approach, and the adolescent's individual characteristics. Some adolescents did not want to burden their family with frequent discussion about diabetes.

While adolescents were capable of tasks such as calculating dosage and drawing up insulin, some found it convenient to let their parents do this. Sometimes a change in medical device, such as getting an insulin pump, was a catalyst to shift responsibilities. Alternatively, some adolescents stopped using insulin pumps as they did not want to be attached to the device or were not skilled using it.

Transitioning responsibility for management was crucial, yet often the standard of management slipped during and after this process. Characteristics of a successful transition strategy included beginning at a young age, maintaining some support, and making the process a gradual transition.

\subsubsection{Managing identity}

This concept examines the ways in which adolescents seek to manage their diabetes while managing identity. Aspects include disclosing diabetes, managing attention, embarrassment and stigma, and attempting deception to convince others of 'good' self-management practices. In this sense, adolescents are managing their identities both as regular adolescents and as 'good' diabetics.

Initial disclosure practices varied based on the age of diagnosis, and adolescents were intermittently placed in situations where they could choose to disclose their diabetes. Disclosure was important for both safety and mental health. However, these benefits were balanced against a desire for privacy and convenience. A small proportion of adolescents found disclosure straightforward. They had a moral status that emboldened them. YA2: "I told everyone. I was never taught that it was something that should be hidden." Others had not informed people such as 
teammates or new flatmates about their condition, and there could be social anxiety about being viewed as different. Adolescents spoke as though they ought to inform people in case of emergency, but did not want to, and were thus conflicted.

Over time, adolescents shared and developed strategies for disclosure. Some would proactively inform people early in the relationship. Though using medical devices in public spaces sometimes removed adolescents' choice about whether to disclose, some used this phenomenon purposefully, waiting for onlookers to enquire about the devices. As adolescents left school, they tended to disclose to fewer people, and did not have the same structured opportunities for disclosure.

Adolescents were also dealing with stigma, embarrassment, body image and attention, in tandem with a strong awareness of others' perceptions of them. While adolescents were sometimes concerned about what others think, and this affected their behaviour, it was rare for strangers to enquire about their actions. While some adolescents took infrequent negative judgements personally, others thought it reflected poorly on those making the judgments, recognising their poor understanding of diabetes. Importantly, not all participants felt embarrassed or stigmatised, and many were confident managing in public spaces and around close friends, though less so in front of acquaintances. YA7: "You don't really get offended by somebody or what they say. You let it offend you."

While some relished attention, unwanted attention remained problematic, with adolescents frustrated in instances by questions about their devices and selfmanagement choices, and by having to explain diabetes. There were both advantages and disadvantages to medical devices that attract attention. Advantages were drawing attention in emergencies, increasing awareness, aiding disclosure of diabetes and normalising self-care behaviours. Disadvantages were feeling embarrassed or stigmatised, and receiving undesired questions. Subsequently, there will be times when it is advantageous for medical devices to draw attention, and other times when users prefer discretion. Additionally, for some users there is scope for devices to draw more positive forms of attention, even being desirable.

Childhood and young adulthood were easier times for initiating use of a new, possibly embarrassing device, such as an insulin pump. Adolescents developed coping strategies to deal with public self-management; skipping or delaying these 
activities until in private, moving to a private space such as a bathroom, and using devices discreetly. Devices resembling consumer devices could allow discretion, but also proved problematic when mobile phones or similar devices were prohibited, resulting in adolescents breaking social norms and having to advocate for themselves. Injections could also be stigmatising, mistaken for illicit drug use. Adolescents desired functionality and aesthetics in tandem, preferring devices that suit their taste and do not feel cheap. While managing identity was predominantly about perceived reactions to the use of medical devices, the effects diabetes has on mood could also affect the way others perceived the individual.

The final aspect of managing identity stemmed from parents and clinicians wanting to know about adolescents' self-management practices and results, and the ways in which adolescents handled these requests. Many adolescents anticipate negative reactions when their log shows either a low frequency of blood glucose tests or blood glucose measurements outside the recommended range. Two adolescent strategies for alleviating tensions in data sharing were attempting deception and interfering with data. Where it was historically easier to supplement a fabrication with a falsified physical logbook, adolescents' deceptions are now exposed through the presence of digital data. Additionally, some adolescents did not want to share data as they felt that it was decontextualised.

Adolescents' parents also had variable access to blood glucose data, spanning: carrying out blood glucose tests, enquiring about results, and looking at data but letting the adolescent make decisions, to helping troubleshoot. Adolescents were more reticent to share their data with parents than health professionals.

\subsubsection{Receiving and providing social support}

Adolescents experienced social support from friends, two-way support from peers with diabetes, and worked to educate others about diabetes. Some friends were receptive to learning about diabetes out of interest and in case of emergencies, others provided practical forms of support (e.g., carrying equipment while running, spotting symptoms), and others emotional support (e.g., making jokes to ease distress, being patient). Adolescents were divided about the value of input from friends. For those desiring involvement, they wanted to be more open with friends 
about feelings and struggles, and included friends in diabetes management by creating games. For those desiring less involvement, they found their friends, though well-intentioned, could be more dramatic than supportive, and that discussing diabetes made the adolescent feel different and requesting patience made them feel they were inconveniencing friends.

While many people with diabetes were initially reticent to meet peers with type 1 diabetes, by adolescence, many had developed and maintained friendships through social media and organised camps and events and viewed these relationships positively. However, fewer older adolescents and young adults had maintained these peer relationships. Having peer support from others with type 1 diabetes helped, both in terms of simply having extra friends, but also being able to talk about diabetes with someone with first-hand experience. In addition to feeling supported by these friends, adolescents also felt gratified by providing support by acting as a buddy for others who were struggling, volunteering at camps, and passing on knowledge and insights to younger people with diabetes. By taking part in online forums and groups, adolescents could also compare themselves to others with type 1 diabetes, sometimes realising they were coping well comparatively. However, some adolescents did not want to meet peers with diabetes, feeling they did not want their friendships to revolve around the condition.

Finally, adolescents were educating others about type 1 diabetes. While some felt general awareness of diabetes was improving, many recounted instances where people conflated type 1 and type 2 diabetes and assigned blame to adolescents for developing the condition, assuming that the adolescent ate unhealthy food. Some adolescents took this opportunity to educate people about type 1 diabetes, while others tried to ignore it after feeling negatively judged. Some adolescents felt by managing openly, they were increasing social acceptance and normalisation of diabetes one person at a time.

\subsubsection{Mapping requirements to grounded theory}

The psychosocial user requirements and grounded theory can be mapped onto each other (Figure 79). The first column lists the psychosocial user requirements, and the second, the grouped requirements. The third column lists the higher-level grounded 
theory concepts. These are the processes that adolescents are experiencing while managing diabetes with medical technologies. By considering these processes and requirements during the design of medical technologies, device designers and manufacturers may create technologies that are better aligned to adolescents' needs.

The user requirements correspond to every grounded theory category identified, and some groups of requirements map onto multiple grounded theory categories. This demonstrates the relationships between the high-level and more specific information collected throughout the research, with most research methods aiming to collect information at both levels. As discussed previously, these higher levels can be helpful for opening up 'solution spaces', and these lower-levels can produce more specific insights helpful for incremental innovation (van der BijlBrouwer, 2016). 


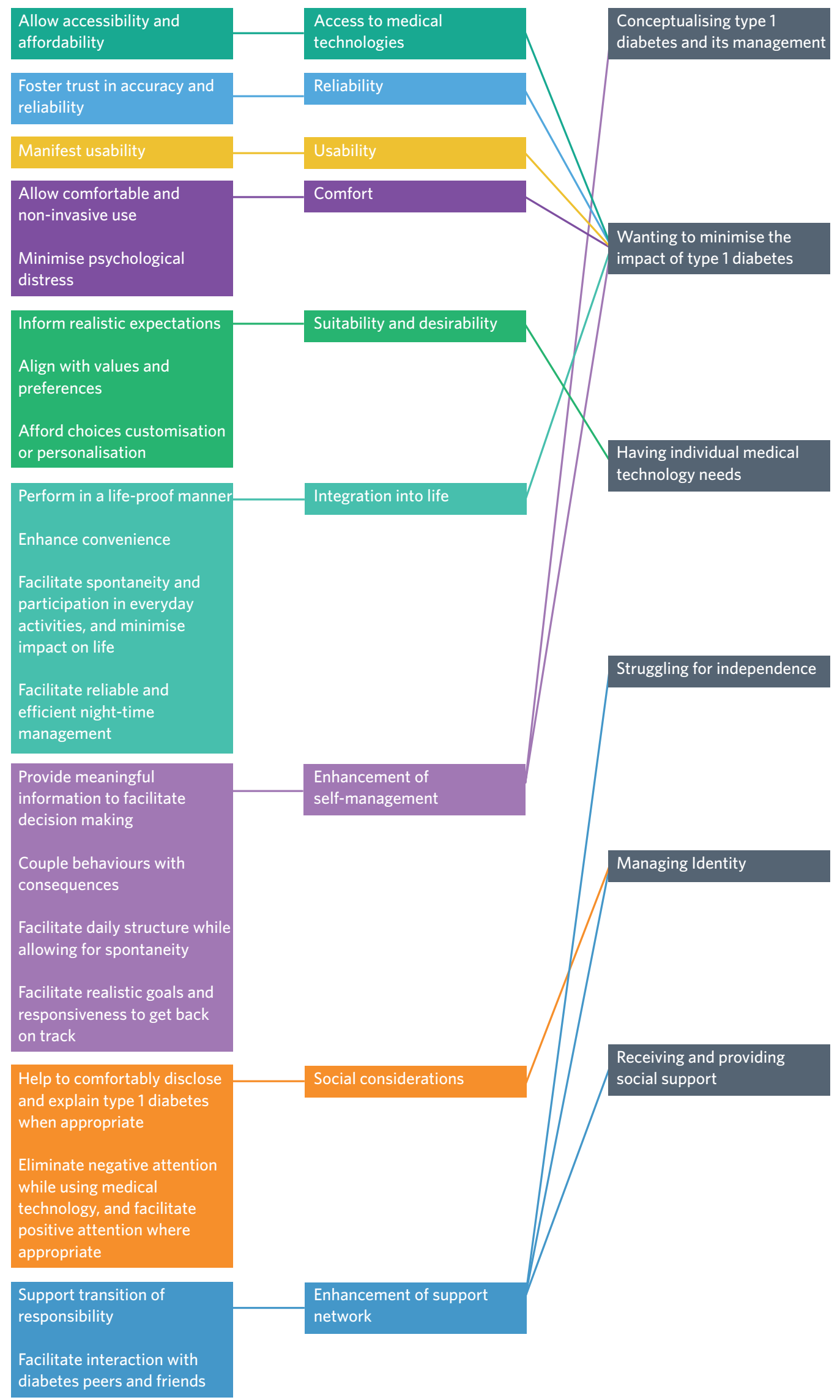

Figure 79. Mapping psychosocial user requirements to grounded theory categories. 
The grounded theory constructed also maps to Cronin's (2004, paragraph 33) metaanalysis of patient centred care, that listed six recurring aspects:

- "Education and shared knowledge;

- involvement of family and friends;

- collaboration and team management;

- sensitivity to nonmedical and spiritual dimensions;

- $\quad$ respect for patient needs and preference; and

- the free flow and accessibility of information"

The only aspect not addressed was 'spiritual dimensions'. In the preparation process, Màori spiritual factors such as tapu and noa were noted in case they arose in the research, however none of the participants identified as Māori or spoke about spirituality.

Further, the grounded theory reflects Kanstrup's (2014) two core tensions in diabetes management: tensions between ideal and actual self-management, and between the individual and their interactions with others. This was evident in adolescents' descriptions of self-management experiences and their conceptualisation of 'good' diabetes management, and how they negotiated interactions with parents, clinicians, and peers, managing their identity. This signalled that designers must consider users' situated problems and behaviours to create technology that responds to real issues, and not simply a medical condition (Papanek, 1984).

\subsection{Contributions and Implications}

Although almost every psychosocial and contextual aspect of an adolescent's life can correlate with their adherence (Williams et al., 2009), the impact of medical technologies on self-management of chronic conditions is not often considered (Wessels et al., 2003). Interactions with devices in realistic environments should be considered (Hgen, 2012; Turieo, 2011), and Barber (1996) hypothesised that many devices are not used effectively because they are created for the impairment or disease, rather than the user. 
As such, prior to this research, little was known about adolescent psychosocial user requirements of medical devices for self-managing type 1 diabetes. This section discusses the major contributions and implications of this research. The first major contribution was the development of adolescents' psychosocial user requirements of medical technologies for type 1 diabetes. Though some requirements for diabetes and other medical technologies exist, and were discussed in Chapter 2, no comprehensive set existed for this group. In creating the requirements it also became clear that there were similarities in requirements from other groups such as adolescents with cystic fibrosis, and as such, it is likely that with further research, some requirements may be able to be generalised across self-care medical devices for specific user groups. Through the development of requirements, the importance of values around environmental sustainability was discussed multiple times, and represents a requirement that was not found previously.

A second major contribution was the substantive grounded theory of adolescents' experiences of self-management. While there were existing qualitative descriptions of adolescents' self-management experiences, this research built upon these, and explored certain areas in greater depth. Areas of particular interest include the process of changing medical devices, exploring the suitability of devices for individuals, attempts at deception and uneasiness over data-sharing between adolescents and their healthcare professionals and parents, concepts of self, identity and acceptance, and the impacts of medical technologies on participating in everyday activities. This research also explored the concept of engagement in a real-life setting, investigating what being engaged might look like for adolescents.

This research has also advanced discussions about responding to attention, stigma, and embarrassment. In the initial interviews, health professionals suggested adolescents would like both 'cool' and 'discreet' medical devices. These tensions pervaded the research, culminating with the design workshop. While not all adolescents felt embarrassed or stigmatised using medical devices in social contexts, most experienced negative attention. This is consistent with previous research, where adolescents objected to unwanted attention and questions about their medical devices or condition (Y.-L. Wang et al., 2010). There will be times when it is advantageous for medical devices to draw attention, and other times when users 
prefer discretion, with adolescents having varying preferences and requiring choice. This may be achieved through strategies such as strengthening the individual identity of the device by providing choice and opportunities for personalisation, strengthening brand identity, and increasing social power. These findings support and expand on previous literature, where recommendations cautioning concealment of medical and assistive devices as signifiers of shame has been discussed (Bispo \& Branco, 2011; Pullin, 2009; Sansoni et al., 2016).

This research also provided contributions in its uncommon pairing of constructivist grounded theory and participatory design methodologies. These methodologies are both congruent and divergent. While largely compatible, tensions arose around balancing theory and design outputs, and reporting of grounded theory against reporting of findings from individual methods. The research also made contributions in modelling how psychosocial user requirements can be developed and tested through a range of methods and used to inform design. In particular, this work built from Lang's (2012) use of vignettes for member checking, using designed objects to test requirements and stimulate further discussion. Designing was used as a tool to both develop and test user-requirements, following a research through design approach. While this yielded positive results, it was necessary to make user requirements explicit to garner feedback not restricted to individual design concepts. Through designing, gaps where more information was necessary were identified. Additionally, while some user requirements were initially framed negatively (e.g., not being embarrassing or stigmatising), design explored the positive side of these requirements (e.g., what makes a desirable medical device). Further, the array of methods accommodated the variety of adolescents' preferred communication styles, with individual participants responding to discussions, writing, or drawing, resulting in a broad variety of data to be interpreted.

Participants responded positively to the participatory approach, describing the workshop as enjoyable and suggesting people with type 1 diabetes should be consulted in design processes. Sketching and prototyping designs, rather than simply discussing experiences, generated additional, specific information. This identifies the difference between this participatory approach compared to other methods, whereby 
the act of designing elicits knowledge (Keyson \& Bruns, 2009; Pedgley \& Wormald, 2007).

Novel methods included adapting the PIADS questionnaire into a card sort task and prompt for discussion, adapting the strategies for addressing stigma into a design workshop, writing letters to medical devices, drawing diabetes metaphors, and labelling the best, worst, and desired features of devices. The assortment of methods in this research provided information at many levels, spanning high-level ideas about what constitutes good diabetes management to specific flaws with individual medical devices. The methods also gave me the opportunity to develop rapport with participants over multiple sessions, facilitating open discussions.

Some qualifications should be made about this research. The research relied on a small sample of adolescent and young adult participants. There was likely a participation bias, with those who chose to participate largely managing their diabetes well. Young adult participants were included to minimise bias, as although currently managing well they could reflect on previous, more challenging years. Health professionals described a wide range of actual self-management practices, and this full range of behaviours was not evident within the sample of participants. Additionally, some aspects such as the transition of responsibility described by health professionals and in previous studies were less evident in this research, because of the older diagnosis age of participants.

Further, being conducted in New Zealand, the range of medical devices adolescents had access to differs from other countries. For instance, CGMs are more prevalent in the United Kingdom and United States of America, and concerns about the accuracy of devices largely stemmed from one brand of blood glucose meter for which New Zealand has a sole-supply agreement. Nevertheless, fundamental issues such as trust in a medical device are important regardless of brand or device.

The design activities throughout the thesis were also limited and used to develop and provide additional support for user requirements, rather than produce new products. Further research would need to be conducted to evaluate how medical technology designers may utilise psychosocial requirements in the development process. 
The research process could have been improved by gaining access to adolescent participants earlier. However, the ethics and locality approval processes made this challenging. Further, recruiting more culturally diverse participants would have better reflected New Zealand's current and indigenous population, and begun to indicate whether there are cultural dimensions that affect diabetes management or user requirements. No adolescent participants identified as Māori or Pasifika.

Extensions to the research include testing the final psychosocial user requirements more thoroughly with adolescents, and providing the requirements to medical technology designers to use. Health professionals and parents could be invited to participate in design workshops to further develop their insights into user requirements, and particularly the transition of responsibility. The research could also be extended to other age groups with type 1 diabetes, other types of diabetes, and medical device designers to determine the generalisability of experiences and requirements, and how they may inform the design process.

Further research is needed to determine how to design medical devices that avoid stigma and unwanted attention, and when different strategies may be appropriate. In particular, further research about context-based appraisals of whether it is advantageous for medical devices to draw attention should be conducted. Following this, there will be an opportunity to design medical devices that draw optional and more positive forms of attention, acting as prompts for disclosure and discussion about diabetes where the adolescent deems this suitable.

Throughout the research, unmet user requirements signalled opportunities for developing technologies. Though beyond the scope of the current research, possible avenues are outlined. Firstly, an easily accessible resource outlining subsidised technologies and their features could be designed to increase adolescents' awareness of affordable options, as currently their awareness of devices is limited, based primarily on interactions with diabetes peers and health professionals. This might help, for instance, those participants who desired a blood glucose meter with a backlight, or an insulin pen with a memory function.

Secondly, while there were many available apps for diabetes management, and some adolescents had tried them, they were unpopular with people with type 1 diabetes. While medical technologies that sync to apps are not subsidised, there is 
opportunity to design apps that work with existing blood glucose meters. Adolescents desire meaningful, visual information and smart prompts, without tedious data entry. Artificial intelligence could be harnessed to analyse data and prompt suggestions, providing safety was ensured. Further, adolescents' varying conceptualisation of what constitutes 'good' diabetes management could be considered, creating a customisable app that focuses on information pertinent to different adolescents such as efforts, results, physical and mental health, and life balance. As some parents and adolescents were already developing ad-hoc games, serious or persuasive games can be considered, introducing fun and achievement, and facilitating interactions with peers.

Finally, developing technologies in response to tensions in transitioning responsibility may be effective. Currently, health professionals were negotiating roles, and families dealing with negative tensions. Establishing explicit processes for transitioning responsibility based on successful strategies (i.e., beginning early, gradual transition, maintaining support) may ease this process. Data sharing technologies with customisable settings may be able to minimise parents' anxiety about management and lack of information, while also decreasing 'nagging' experienced by adolescents.

Future research must also consider how individuals manage their health as part of daily life. New Zealanders are living longer, but with long-term conditions. Designers have an opportunity to take this shift into consideration when creating new personal medical devices, considering user experiences and contexts. Device safety is regulated, and advice is available on usability, yet further considerations of how devices are integrated into daily life are not currently required. As part of the 'Smart Interactions' lab, I have begun investigating how medical technologies may increase engagement across a broad spectrum of conditions. As individuals increasingly manage their health outside of hospital environments, there is scope for investigating how to design technologies that are successfully integrated into daily life, realising the expected improvements in health outcomes while not compromising other goals.

There are a number of implications of the research. Firstly, medical technologies impact the self-management of adolescents with type 1 diabetes. 
Further, adolescents currently have unmet psychosocial user requirements of medical technologies. Based on publicly available information, it appears that Pharmac are not considering the full range of adolescents' psychosocial requirements when determining which devices are subsidised. Medical technologies are available that fulfilled some requirements, such as a blood glucose meter that synced with a smartphone. However, these technologies were not affordable for adolescents. Next, the research demonstrated that adolescents have different and dynamic needs and preferences. It is unlikely that a single medical technology option will suit all users in all situations. Finally, it is clear that cumulative knowledge across studies needs to be built to inform the design of these medical technologies in a rigorous manner.

Applications and recommendations from the research can be described for four groups: designers, health professionals, Pharmac, and adolescents with type 1 diabetes and their support people. Firstly, designers must be aware of the importance of the varying contexts in which personal medical technologies are used, and how device features that are positive in one context may be negative in another. As such, research, design and testing should involve a wide range of contexts where possible. Also, an extreme user approach to designing medical technology may result in products which are suitable for a wide variety of users. While the current research demonstrated a variety of methods for gathering and analysing data to inform design, research design should be tailored to suit the context of future designers and their participants, though can they draw from the insights garnered here.

Health professionals should continue to support adolescents' selfmanagement, and help their patients make informed choices around medical technologies. Currently, not all adolescents are aware of the full range of medical technologies that are subsidised or available to them. Secondly, health professionals should explore adolescents' self-management ideals and practices, ensuring an appropriate, informed balance between these preferences, practices and likely health outcomes. Finally, health professionals need to be mindful of unanticipated effects of medical technologies, for instance, of patients being caught out in acts of deception through downloadable blood glucose records. These instances have the possibility to negatively affect the patient clinician relationship. 
Pharmac have moved to a user-testing model for determining subsidies for diabetes medical technologies. While this is likely to result in helpful feedback from possible users, adolescents should be involved in this process. Through this research, it was clear that adolescents gave more detailed descriptions of the impacts on their daily lives of medical technologies than did proxies such as health professionals. As such, this group needs to be adequately represented in Pharmac's decision-making processes to ensure their needs are taken into account. Further, based on publicly available information, it is likely that Pharmac are not currently considering the full range of adolescents' psychosocial user requirements of medical technologies. As such, the parameters of their process for determining which technologies are subsidised could be widened to encompass these. Further, it may be valuable to gather information not only about adolescents' experiences with current technologies, but also their desires for technologies in the future.

Adolescents are self-managing diabetes in a changeable environment, undergoing a period of rapid development in combination with the advent of new technologies. It is clear that there can be unintentional misalignments between adolescents' needs and preferences and their medical technologies. As such, where desired, adolescents can engage with the range of individuals and organisations that facilitate health management (e.g., parents, health professionals, Pharmac, device manufacturers) to support the alignment process. 


\section{Conclusion}

Medical technologies are not commonly associated with karate, movie theatres, or cats, but this research was embedded in adolescents' daily lives, and all the incongruities that these hold. Accordingly, almost every psychosocial or contextual aspect of an adolescent's life impacts their self-management of type 1 diabetes (Williams et al., 2009).

I constructed adolescents' shared experiences of diabetes self-management, and how these differ between individuals. These processes were conceptualising type 1 diabetes and its management, wanting to minimise the impact of type 1 diabetes, having individual medical technology needs, struggling for independence, managing identity, and receiving and providing social support.

I also documented the psychosocial user requirements of medical technologies for adolescents with type 1 diabetes. These go beyond traditional human factors considerations and begin to examine how to not only reduce negative impacts of medical technologies, but also how to increase their desirability. These 20 requirements fell into nine categories: access to medical technologies, reliability, usability, comfort, suitability and desirability, integration into life, enhancement of self-management, social considerations, and enhancement of support network. These requirements can impact adolescents' self-management, for example, with adolescents who were conscious of a medical device drawing attention subsequently delaying or skipping use in social contexts.

This research drew from a range of existing, adapted, and novel participatory methods. These methods provided a successful approach to eliciting sensitive health information from adolescents, and involving them in the design of medical technologies. It also demonstrated how medical technologies can be designed to respond to user requirements, and how designing can be used to stimulate discussion and develop and provide additional support for user requirements with young participants. Further, the combination of these methods with grounded theory is uncommon, and demonstrates how grounded theory can be used in design research.

This research demonstrates the importance of designing personal medical technologies for people and the lives they lead, and not solely in response to a 
biomedical problem. Including adolescents in a participatory role gave light to their unique needs and experiences, and to the context-based nature of medical device appraisals. While the research focused only on adolescents and young adults with type 1 diabetes, there are similarities in the findings to existing research, suggesting some experiences and user requirements are shared across broader groups. 


\section{References}

American Chemical Society (2015, January 14). Tattoo-like sensor can detect glucose levels without a painful finger prick. Retrieved from www.acs.org/content/acs/en/pressroom/presspacs/2015/acs-presspacjanuary-14-2015/tattoo-like-sensor-can-detect-glucose-levels-without-apainful-finger-prick.html

Anagnostopoulou, E., Magoutas, B., Bothos, E., Schrammel, J., Orji, R., \& Mentzas, G. (2017). Exploring the links between persuasion, personality and mobility types in personalized mobility applications. In P. de Vries, H. Oinas-

Kukkonen, L. Siemons, N. Beerlage-de Jong, \& L. van Gemert-Pijnen (Eds.), Persuasive technology: Development and implementation of personalized technologies to change attitudes and behaviors (pp. 107-118). Amsterdam, The Netherlands: Springer. https://doi.org/10.1007/978-3-31955134-0_9

Ananthanarayan, S., Siek, K., \& Eisenberg, M. (2016). A craft approach to health awareness in children. In M. Foth (Chair), Proceedings of the 2016 ACM Conference on Designing Interactive Systems (pp. 724-735). Brisbane, Australia: ACM.

Anderson, B. J., Auslander, W. F., Jung, K. C., Miller, J. P., \& Santiago, J. V. (1990). Assessing family sharing of diabetes responsibilities. Journal of Pediatric Psychology, 15(4), 477-492. https://doi.org/10.1093/jpepsy/15.4.477

Anderson, B. J., \& Redondo, M. J. (2011). What can we learn from patient-reported outcomes of insulin pen devices? Journal of Diabetes Science and Technology, 5(6), 1563-1571.

Anderson, R. M., \& Funnell, M. M. (2000). Compliance and adherence are dysfunctional concepts in diabetes care. The Diabetes Educator, 26(4), 597604. https://doi.org/10.1177/014572170002600405

Anderson, R. M., \& Funnell, M. M. (2012). Negotiating behavior changes with patients who have diabetes: Negotiation or coercion? Diabetes Management, 2(1), 41-46. 
Anderson-Lister, G., \& Treharne, G. J. (2014). "Healthy" individuals’ perceptions of type 1 and type 2 diabetes cause and management: A "think-aloud", mixedmethods study using video-based vignettes. Journal of Health Psychology, 19(11), 1371-1381. https://doi.org/10.1177/1359105313490315

Angen, M. J. (2000). Evaluating interpretive inquiry: Reviewing the validity debate and opening the dialogue. Qualitative Health Research, 10(3), 378-395. https://doi.org/10.1177/104973230001000308

Aoki, N., Ohta, S., Masuda, H., Naito, T., Sawai, T., Nishida, K., . . \& Fukui, T. (2004). Edutainment tools for initial education of type-1 diabetes mellitus: Initial diabetes education with fun. Medinfo, 11(2), 855-859.

Archer, L. B. (1981). A view of the nature of the design research. In R. Jacques \& J. A. Powell (Eds.), Design: Science: Method (pp. 30-47). Surrey, United Kingdom: IPC Business Press Ltd.

Badlan, K. (2006). Young people living with cystic fibrosis: An insight into their subjective experience. Health \& Social Care in the Community, 14(3), 264270. https://doi.org/10.1111/j.1365-2524.2006.00619.x

Bainbridge, E., Bevans, S., Keeley, B., \& Oriel, K. (2011). The effects of the Nintendo Wii Fit on community-dwelling older adults with perceived balance deficits: A pilot study. Physical \& Occupational Therapy in Geriatrics, 29(2), 126-135. https://doi.org/10.3109/02703181.2011.569053

Balmain, N. (2017, June 23). The clothing range for diabetic women [video file]. Retrieved from http://www.bbc.com/news/av/health-40360398/meet-thedesigner-making-clothes-for-diabetic-women

Bandura, A. (2001). Social cognitive theory: An agentic perspective. Annual Review of Psychology, 52(1), 1-26.

Barber, J. (1996). The design of disability products: A psychological perspective. British Journal of Occupational Therapy, 59(12), 561-563.

Barnard, K. D., Parkin, C., Young, A., \& Ashraf, M. (2012). Use of an automated bolus calculator reduces fear of hypoglycemia and improves confidence in dosage accuracy in patients with type 1 diabetes mellitus treated with multiple daily insulin injections. Journal of Diabetes Science and Technology, 6(1), 144-149. 
Barnard, K. D., Speight, J., \& Skinner, T. C. (2008). Quality of life and impact of continuous subcutaneous insulin infusion for children and their parents. Practical Diabetes International, 25(7), 278-283. https://doi.org/10.1002/pdi.1280

Barnard, K. D., Wysocki, T., Allen, J. M., Elleri, D., Thabit, H., Leelarathna, L., . . \& \& Hovorka, R. (2014). Closing the loop overnight at home setting: psychosocial impact for adolescents with type 1 diabetes and their parents. BMJ Open Diabetes Research \& Care, 2(1), e000025. https://doi.org/10.1136/bmjdrc2014-000025

Barrett, C., \& Taylor, P. (2010). The effects of the odstock drop foot stimulator on perceived quality of life for people with stroke and multiple sclerosis. Neuromodulation: Technology at the Neural Interface, 13(1), 58-64.

Barrett, D. H., Davidson, P. C., Steed, L. J., Abel, G. G., Loman, K. E., \& Saudek, C. D. (1995). Evaluation of the psychosocial impact of the MiniMed variablerate implantable insulin pump. Southern Medical Journal, 88(12), 1226-1230.

Barter, C., \& Renold, E. (2000). "I wanna tell you a story": Exploring the application of vignettes in qualitative research with children and young people. International Journal of Social Research Methodology, 3(4), 307-323. https://doi.org/10.1080/13645570050178594

Bateman, T., \& Jacoby, B. (2017). EU medical device and diagnostic regulatory overhaul: A guide for novices (22). Oxford, England: Pharma Ventures.

Beard, R. L. (2004). In their voices: Identity preservation and experiences of Alzheimer's disease. Journal of Aging Studies, 18(4), 415-428.

Bellows, M., Kovacs Burns, K., Jackson, K., Surgeoner, B., \& Gallivan, J. (2015). Meaningful and effective patient engagement: What matters most to stakeholders. Patient Experience Journal, 2(1), 18-28.

Berge, J. M., Patterson, J. M., Goetz, D., \& Milla, C. (2007). Gender differences in young adults' perceptions of living with cystic fibrosis during the transition to adulthood: A qualitative investigation. Families, Systems \& Health, 25(2).

Bergenstal, R., Pearson, J., Cembrowski, G. S., Bina, D., Davidson, J., \& List, S. (2000). Identifying variables associated with inaccurate self-monitoring of 
blood glucose: Proposed guidelines to improve accuracy. The Diabetes Educator, 26(6), 981-989. https://doi.org/10.1177/014572170002600610

Bergold, J., \& Thomas, S. (2012). Participatory research methods: A methodological approach in motion. FQS, 13(1). Retrieved from http://www.qualitativeresearch.net/index.php/fqs/article/view/1801/3334\%202.11.2016

Berlin, K. S., Hobart, D. W., Jastrowski, K. E., Hains, A. A., Parton, E. A., \& Alemzadeh, R. (2006). Contextual assessment of problematic situations identified by insulin pump using adolescents and their parents. Families, Systems, \& Health, 24(1), 33-44.

Beshyah, S. A. (2009). Fasting during the month of Ramadan for people with diabetes: Medicine and Fiqh united at last. Ibnosina Journal of Medicine and Biomedical Sciences, 1(2), 58-60.

Bichard, J. -A., Coleman, R., \& Langdon, P. (2007). Does my stigma look big in this? Considering acceptability and desirability in the inclusive design of technology products. In C. Stephanidis (Ed.), Universal Access in Human Computer Interaction. Coping with Diversity (pp. 622-631). Berlin, Heidelberg: Springer. Retrieved from https://doi.org/10.1007/978-3-54073279-2_69

Bispo, R., \& Branco, V. (2011). Designing out stigma: A new approach to designing for human diversity. Retrieved from https://iconline.ipleiria.pt/handle/10400.8/456

Bluelarix (2010, June 9). GlucaShield: Diabetes emergency kit for children. Retrieved from http://boards.core77.com/viewtopic.php?t=21803\&p=151013

Bodenheimer, T., Wagner, E. H., \& Grumbach, K. (2002). Improving primary care for patients with chronic illness: The chronic care model, Part 2. Jama, 288(15), 1909-1914.

Boehner, K., Vertesi, J., Sengers, P., \& Dourish, P. (2007). How HCI interprets the probes. In M. B. Rosson (Chair), Proceedings of the SIGCHI conference on human factors in computing Systems (pp. 1077-1086). New York, NY: ACM. https://doi.org/10.1145/1240624.1240789

Boise, M. (2017, February 15). Jerry the Bear: A new-age T1D toy. Retrieved from https://beyondtype1.org/jerry-bear-new-age-t1d-toy/ 
Borus, J. S., \& Laffel, L. (2010). Adherence challenges in the management of type 1 diabetes in adolescents: Prevention and intervention. Current Opinion in Pediatrics, 22(4), 405-411. https://doi.org/10.1097/MOP.0b013e32833a46a7

Boss, P. (2009). Ambiguous loss: Learning to live with unresolved grief. Cambridge, USA: Harvard University Press.

Boyle, R. (2010, April 27). Bayer's didget blood-sugar monitor attaches to Nintendo DS, enticing kids to manage health | popular science. Retrieved from http://www.popsci.com/gadgets/article/2010-04/blood-sugar-monitorattaches-nintendo-ds-enticing-kids-manage-health\#page-2

Brand, T. (2016). The artificial pancreas you've never heard of: "Genesis." Retrieved from http://www.diabetesdaily.com/blog/please-do-not-touch-the-artificialpancreas-youve-never-heard-of-genesis-288891/

Bratteteig, T., \& Wagner, I. (2014). Disentangling participation. Heidelberg, Germany: Springer. https://doi.org/10.1007/978-3-319-06163-4_1

Braun, V., \& Clarke, V. (2006). Using thematic analysis in psychology. Qualitative Research in Psychology, 3(2), 77-101. https://doi.org/10.1191/1478088706qp063oa

Briere, R. (2001). Executive summary. In R. Briere (Ed.), Crossing the quality chasm: A new health system for the 21st century (pp. 1-22). Washington DC: National Academy Press. Retrieved from https://www.ncbi.nlm.nih.gov/books/NBK222271/

Bright, F. A. S., Kayes, N. M., Worrall, L., \& McPherson, K. M. (2015). A conceptual review of engagement in healthcare and rehabilitation. Disability and Rehabilitation, 37(8), 643-654. https://doi.org/10.3109/09638288.2014.933899

Brown, A., \& Wolf, A. (2015, July 9). Bionic pancreas dual-chamber pump unveiled at Friends For Life 2015. Retrieved from https://diatribe.org/bionic-pancreasdual-chamber-pump-unveiled-friends-life-2015

Brown, S. M., Rozenblum, R., Aboumatar, H., Fagan, M. B., Milic, M., Lee, B. S., . . . \& Frosch, D. L. (2015). Defining patient and family engagement in the intensive care unit. American Journal of Respiratory and Critical Care Medicine, 191(3), 358-360. https://doi.org/10.1164/rccm.201410-1936LE 
Buchbinder, M. H., Detzer, M. J., Welsch, R. L., Christiano, A. S., Patashnick, J. L., \& Rich, M. (2005). Assessing adolescents with insulin-dependent diabetes mellitus: A multiple perspective pilot study using visual illness narratives and interviews. Journal of Adolescent Health, 36(1), 71.e9-71.e13. https://doi.org/10.1016/j.jadohealth.2004.02.019

Bull, T. (2015). Anticipated telehealth device usage in younger adults. Florida: University of Central Florida. Retrieved from http://stars.library.ucf.edu/honorstheses1990-2015/1819

Burrows, A., Mitchell, V., \& Nicolle, C. (2015). Cultural probes and levels of creativity. In S. Boring \& E. Rukzio (Chairs), Proceedings of the 17th international conference on human-computer interaction with mobile devices and services adjunct (pp. 920-923). Copenhagen, Denmark: ACM. Retrieved from http://dl.acm.org/citation.cfm?id=2794302

Cafazzo, J. A., Casselman, M., Hamming, N., Katzman, D. K., \& Palmert, M. R. (2012). Design of an mHealth app for the self-management of adolescent type 1 diabetes: A pilot study. Journal of Medical Internet Research, 14(3), e70. https://doi.org/10.2196/jmir.2058

Cambridge Consultants (2014, February 10). Simplifying life with diabetes. Retrieved from https://www.cambridgeconsultants.com/media/pressreleases/simplifying-life-diabetes

Cameron, F. (2006, June). Teenagers with diabetes: Management challenges. Retrieved from

http://search.informit.com.au/documentSummary;dn=363237809511506;res $=\mathrm{IELHE}$

Carayon, P., Alvarado, C. J., \& Wiklund, M. (2010). Environment of use. In M. Weinger, M. Wiklund, \& D. Gardner-Bonneau (Eds.), Handbook of human factors in medical device design (pp.63-96). Boca Raton, Florida: CRC Press. https://doi.org/10.1201/b10439

Carroll, A. E., DiMeglio, L. A., Stein, S., \& Marrero, D. G. (2011). Using a cell phonebased glucose monitoring system for adolescent diabetes management. The Diabetes Educator, 37(1), 59-66. https://doi.org/10.1177/0145721710387163 
Carroll, A. E., Downs, S. M., \& Marrero, D. G. (2007). What adolescents with type I diabetes and their parents want from testing technology: A qualitative study. CIN: Computers, Informatics, Nursing January/February 2007, 25(1), 23-29.

Carroll, J. M. (Ed.). (1995). Scenario based design: Envisioning work and technology in system development. New York, NY: Wiley.

Carter, B. (2009). Tick box for child? The ethical positioning of children as vulnerable, researchers as barbarians and reviewers as overly cautious. International Journal of Nursing Studies, 46(6), 858-864. https://doi.org/10.1016/j.ijnurstu.2009.01.003

Carter, P. J., Cutfield, W. S., Hofman, P. L., Gunn, A. J., Wilson, D. A., Reed, P. W., \& Jefferies, C. (2008). Ethnicity and social deprivation independently influence metabolic control in children with type 1 diabetes. Diabetologia, 51(10), 1835-1842. https://doi.org/10.1007/s00125-008-1106-9

Casakin, H. (2012). An empirical assessment of metaphor use in the design studio: analysis, reflection and restructuring of architectural design. International Journal of Technology and Design Education, 22(3), 329-344.

Charmaz, K. (1995). The body, identity, and self: Adapting to impairment. The Sociological Quarterly, 36(4), 657-680.

Charmaz, K. (2002). Stories and silences: Disclosures and self in chronic illness. Qualitative Inquiry, 8(3), 302-328.

Charmaz, K. (2006). Constructing grounded theory: A practical guide through qualitative analysis ( $1^{\text {st }}$ ed.). London, United Kingdom; Thousand Oaks, CA: SAGE Publications Ltd.

Charmaz, K. (2014). Constructing grounded theory ( $2^{\text {nd }}$ ed.). London, United Kingdom: Sage.

Charmaz, K., \& Bryant, A. (2010). Grounded theory. In P. Peterson, E. Baker \& B. McGaw (Eds.), International encyclopedia of education ( $3^{\text {rd }}$ ed.) (pp. 406412). Oxford, United Kingdom: Elsevier. https://doi.org/10.1016/B978-0-08044894-7.01581-5

Chase, H. P., Beck, R. W., Xing, D., Tamborlane, W. V., Coffey, J., Fox, L. A., . . \& Ruedy, K. J. (2010). Continuous glucose monitoring in youth with type 1 diabetes: 12-month follow-up of the Juvenile Diabetes Research Foundation 
continuous glucose monitoring randomized trial. Diabetes Technology \& Therapeutics, 12(7), 507-515.

Cogen, F. R., Streisand, R., \& Sarin, S. (2002). Selecting children and adolescents for insulin pump therapy: Medical and behavioral considerations. Diabetes Spectrum, 15(2), 72-75.

Consolvo, S., Everitt, K., Smith, I., \& Landay, J. A. (2006). Design requirements for technologies that encourage physical activity. In R. Grinter, T. Rodden, P. Aoki, E. Cutrell, R. Jeffries, \& G. Olson (Eds.), Proceedings of the SIGCHI conference on human factors in computing systems (pp. 457-466). Montréal, Canada: ACM. Retrieved from http://dl.acm.org/citation.cfm?id=1124840

Core77 design award 2011: Hanky pancreas, notable for soft goods/apparel (n.d.). Retrieved from http://www.core77.com//posts/20185/Core77-Design-Award2011-Hanky-Pancreas-Notable-for-Soft-GoodsApparel

Corydon, M. (2015, June 22). SpeediCath compact set. Retrieved from http://red-dot21.com/design/speedicath-compact-set/speedicath-compact-set/

Coulter, A. (2011). Engaging patients in healthcare. New York, NY: Open University Press.

Craig, M. E., Twigg, S. M., Donaghue, K. C., Cheung, N. W., Cameron, F. J., Conn, J., .. \& \& Silink, M. (2011). National evidence-based clinical care guidelines for type 1 diabetes in children, adolescents and adults. Canberra, Australia: Australian Government Department of Health and Ageing. Retrieved from http://www.apeg.org.au/portals/0/guidelines1.pdf

Cronin, C. (2004). Patient-centered care: An overview of definitions and concepts. Washington, DC: National Health Council.

Cross, N. (2006). Designerly ways of knowing. London, United Kingdom: Springer. https://doi.org/10.1007/1-84628-301-9_1

Crouch, C., \& Pearce, J. (2013). Doing research in design. London, United Kingdom: Bloomsbury. Retrieved from https://ebookcentral.proquest.com/lib/vuw/detail.action?docID=1441495

D’Alberton, F., Nardi, L., \& Zucchini, S. (2012). The onset of a chronic disease as a traumatic psychic experience: A psychodynamic survey on type 1 diabetes in young patients. Psychoanalytic Psychotherapy, 26(4), 294-307. 
Davis, S. N., \& Renda, S. M. (2006). Psychological insulin resistance overcoming barriers to starting insulin therapy. The Diabetes Educator, 32(Supplement 4), $146 \mathrm{~S}-152 \mathrm{~S}$.

DAWN (2015a). Room for diabetes in your life (DAWN dialogue tools). Diabetes attitudes wishes \& needs. Retrieved from http://www.dawnstudy.com/content/dam/Dawnstudy/AFFILIATE/wwwdawnstudycom/Home/TOOLSANDRESOURCES/Documents/DAWN_Room_diabetes _in_your_life_example.pdf

DAWN (2015b). Your plans to change your way of life (DAWN dialogue tools).

Diabetes attitudes wishes \& needs. Retrieved from http://www.dawnstudy.com/content/dam/Dawnstudy/AFFILIATE/wwwdawnstudycom/Home/TOOLSANDRESOURCES/Documents/DAWN_Your\%20plans \%20to\%20change\%20your\%20way\%20of\%20life_example.pdf

Day, H., \& Jutai, J. (1996). Measuring the psychosocial impact of assistive devices. Canadian Journal of Rehabilitation, 9, 159-168.

DCCT Research Group (1994). Effect of intensive diabetes treatment on the development and progression of long-term complications in adolescents with insulin-dependent diabetes mellitus: Diabetes control and complications trial. The Journal of Pediatrics, 125(2), 177-188. https://doi.org/10.1016/S00223476(94)70190-3

Deacon, A. J., \& Edirippulige, S. (2015). Using mobile technology to motivate adolescents with type 1 diabetes mellitus: A systematic review of recent literature. Journal of Telemedicine and Telecare, 21(8), 431-438. https://doi.org/10.1177/1357633X15605223

Delamater, A. (2006). Improving patient adherence. Clinical Diabetes, 24(2), 71-77. Delamater, A., Patino-Fernandez, A. M., Pulgaron, E., \& Daigre, A. (2012a). Regimen adherence measures. In D. Christie \& C. Martin (Eds.), Psychosocial aspects of diabetes: Children, adolescents and their families (pp. 246-250). London, United Kingdom: Radcliffe. 
Delamater, A., Patino-Fernandez, A. M., Pulgaron, E., \& Daigre, A. (2012b).

Regimen adherence: Paradigms and approaches. In D. Christie \& C. Martin (Eds.), Psychosocial aspects of diabetes: Children, adolescents and their families (pp. 53-64). London, United Kingdom: Radcliffe.

Denzin, N. K. (1992). Symbolic interactionism and cultural studies: The politics of interpretation. Cambridge, Massachusetts: Blackwell.

Derraik, J. G. B., Reed, P. W., Jefferies, C., Cutfield, S. W., Hofman, P. L., \& Cutfield, W. S. (2012). Increasing incidence and age at diagnosis among children with type 1 diabetes mellitus over a 20-year period in Auckland (New Zealand). PLOS ONE, 7(2), e32640. https://doi.org/10.1371/journal.pone.0032640

Designbuzz (2012a, April 18). inTouch bracelet takes blood sugar and glucose readings in a non intrusive way. Retrieved September 21, 2017, from http://www.designbuzz.com/intouch-bracelet-takes-blood-sugar-glucosereadings-intrusive/

Designbuzz (2012b, April 30). Bracelet for diabetics keeps a tab on heartbeat and glucose levels. Retrieved September 21, 2017, from http://www.designbuzz.com/bracelet-diabetics-keeps-tab-heartbeat-glucoselevels/

Devers, K. J., \& Frankel, R. M. (2000). Study design in qualitative research--2: Sampling and data collection strategies. Education for Health, 13(2), 263-271.

Di Battista, A. M., Hart, T. A., Greco, L., \& Gloizer, J. (2009). Type 1 diabetes among adolescents. The Diabetes Educator, 35(3), 465-475. https://doi.org/10.1177/0145721709333492

Diabetes New Zealand (n.d.). Diabetes New Zealand. Retrieved from https://www.diabetes.org.nz/home

Diabetes Youth New Zealand (n.d.). Diabetes Youth New Zealand. Retrieved from http://www.diabetesyouth.org.nz/

Dickinson, J. K., \& O’Reilly, M. M. (2004). The lived experience of adolescent females with type 1 diabetes. The Diabetes Educator, 30(1), 99-107.

DiGiano, C., Tatar, D., \& Kireyev, K. (2006). Learning from the Post-It: Building collective intelligence through lightweight, flexible technology. In P. Hinds \& D. Martin (Chairs), Conference on computer supported cooperative work 
companion, Banff. Banf, Canada: ACM. Retrieved from

http://citeseerx.ist.psu.edu/viewdoc/download?doi=10.1.1.572.2707\&rep=rep $1 \&$ type $=$ pdf

Djajadiningrat, J. P., Gaver, W. W., \& Fres, J. W. (2000). Interaction relabelling and extreme characters: methods for exploring aesthetic interactions. In J. Karat \& J. Thakara (Chairs), Proceedings of the 3rd conference on designing interactive systems: Processes, practices, methods, and techniques (pp. 66-71). New York: ACM. Retrieved from http://dl.acm.org/citation.cfm?id=347664

Downton, P. (2003). Design research. Melbourne, Australia: RMIT University Press.

Druin, A. (1999). Cooperative inquiry: Developing new technologies for children with children. In M. G. Williams \& M. W. Altom (Chairs), Proceedings of the SIGCHI conference on human factors in computing systems (pp. 592-599).

Pittsburgh, Pensylvania: ACM. Retrieved from http://dl.acm.org/citation.cfm?id=303166

D.school. (n.d.). Bootcamp bootleg. Retrieved from https://dschool.stanford.edu/resources/the-bootcamp-bootleg

Du Pasquier-Fediaevsky, L., Chwalow, A., \& Tubiana-Rufi, N. (2005). Is the relationship between adherence behaviours and glycaemic control bidirectional at adolescence? A longitudinal cohort study. Diabetic Medicine, 22(4), 427-433.

Dweck, C. S. (2008). Mindset: The new psychology of success. New York, NY: Ballantine Books.

Elissa, K., Bratt, E. -L., Axelsson, Å. B., Khatib, S., \& Sparud-Lundin, C. (2017). Societal norms and conditions and their influence on daily life in children with type 1 diabetes in the West Bank in Palestine. Journal of Pediatric Nursing, 33, 16-22. https://doi.org/10.1016/j.pedn.2016.12.005

Elkind, D. (1998). All grown up and no place to go: Teenagers in crisis. Boston, Massachusetts: Da Capo Press.

Emerson, R. M., Fretz, R. I., \& Shaw, L. L. (2011). Writing ethnographic fieldnotes ( $^{\text {nd }}$ ed.). Chicago, IL: University of Chicago Press.

Essen, L. von. (2004). Proxy ratings of patient quality of life factors related to patient-proxy agreement. Acta Oncologica, 43(3), 229-234. 
Eysenbach, G. (2000). Consumer health informatics. BMJ : British Medical Journal, 320(7251), 1713-1716.

Farmer, A., Gibson, O., Hayton, P., Bryden, K., Dudley, C., Neil, A., \& Tarassenko, L. (2005). A real-time, mobile phone-based telemedicine system to support young adults with type 1 diabetes. Journal of Innovation in Health Informatics, 13(3), 171-177. https://doi.org/10.14236/jhi.v13i3.594

Farmer, T., Robinson, K., Elliott, S. J., \& Eyles, J. (2006). Developing and implementing a triangulation protocol for qualitative health research. Qualitative Health Research, 16(3), 377-394.

Faste, H., Rachmel, N., Essary, R., \& Sheehan, E. (2013). Brainstorm, chainstorm, cheatstorm, tweetstorm: New ideation strategies for distributed HCI design. In W. E. Mackay (Chair), Proceedings of the SIGCHI conference on human factors in computing systems (pp. 1343-1352). Paris, France: ACM. Retrieved from http://dl.acm.org/citation.cfm?id=2466177

Fitton, D., Bell, B. T., Little, L., Horton, M., Read, J. C., Rouse, M., \& Toth, N. (2016). Working with teenagers in HCI research: A reflection on techniques used in the Taking on the Teenagers Project. In L. Little, D. Firron, B. Bell, \& N. Tosh (Eds.), Perspectives on HCI Research with Teenagers (pp. 237-267).

Switzerland: Springer. Retrieved from http://link.springer.com/chapter/10.1007/978-3-319-33450-9_10

Fotopoulou, A., \& O'Riordan, K. (2017). Training to self-care: Fitness tracking, biopedagogy and the healthy consumer. Health Sociology Review, 26(1), 5468. https://doi.org/10.1080/14461242.2016.1184582

Frankel, L., \& Racine, M. (2010). The complex field of research: For design, through design, and about design. In D. Durling (Eds.), Proceedings of the Design Research Society (DRS) International Conference.

Franklin, V. L., Greene, A., Waller, A., Greene, S. A., \& Pagliari, C. (2008). Patients' engagement with "Sweet Talk" - A text messaging support system for young people with diabetes. Journal of Medical Internet Research, 10(2). https://doi.org/10.2196/jmir.962

Frøisland, D. H., Årsand, E., \& Skårderud, F. (2012). Improving diabetes care for young people with type 1 diabetes through visual learning on mobile phones: 
Mixed-methods study. Journal of Medical Internet Research, 14(4), e111. https://doi.org/10.2196/jmir.2155

Gabbay, R. A., \& Durdock, K. (2010). Strategies to increase adherence through diabetes technology. Journal of Diabetes Science and Technology, 4(3), 661665.

Gallivan, J., Kovacs Burns, K. A., Bellows, M., \& Eigenseher, C. (2012). The many faces of patient engagement. J Particip Med, 4, e32.

Gardner-Bonneau, D. (2010). Home health care. In M. Weinger, M. Wiklund \& D. Gardner-Bonneau (Eds.), Handbook of human factors in medical device Design (pp. 747-770). Boca Raton, Florida: CRC Press. https://doi.org/10.1201/b10439

Garmer, K., Ylven, J., \& Karlsson, I. M. (2004). User participation in requirements elicitation comparing focus group interviews and usability tests for eliciting usability requirements for medical equipment: A case study. International Journal of Industrial Ergonomics, 33(2), 85-98.

Gaver, B., Dunne, T., \& Pacenti, E. (1999). Design: Cultural probes. Interactions, 6(1), 21-29.

Gaver, W., \& Dunne, A. (1999). Projected realities: Conceptual design for cultural effect. In M. G. Williams \& M. W. Altom (Chairs), Proceedings of the SIGCHI conference on human factors in computing systems (pp. 600-607). Pittsburgh, Pennsylvania: ACM. Retrieved from http://dl.acm.org/citation.cfm?id=303168

Genteel (n.d.). Genteel - The gentle lancing device. Retrieved from https://www.mygenteel.com

GEreports (2009). Fun hospital visits \& MRI scans for kids. Retrieved from https://www.youtube.com/watch?v=nPlvBPtxEl4

Gerling, K. M., Schild, J., \& Masuch, M. (2010). Exergame design for elderly users: The case study of SilverBalance. In Proceedings of the 7th International Conference on Advances in Computer Entertainment Technology. Retrieved from http://dl.acm.org/citation.cfm?id=1971650 
Gheerawo, R., \& Bichard, J. -A. (2010, November). Digital barriers: Making technology work for people. Presented at the 3rd International Conference for Universal Design, Hamamatsu, Japan.

Gjengedal, E., Rustoen, T., Wahl, A. K., \& Hanestad, B. R. (2003). Growing up and living with cystic fibrosis: Everyday life and encounters with the health care and social services - A qualitative study. Advances in Nursing Science Childhood Health and Illness, 26(2), 149-159.

Glasemann, M., \& Kanstrup, A. M. (2008). Evoking creativity: Young diabetics design their own mobile diabetes supporter. In J. J. Jensen, K. L. Jensen, A. M. Kanstrup, L. B. Larsen, T. Nyvang, \& J. Stage (Eds.), Research symposium (pp. $\mathrm{X}-\mathrm{X})$. Retrieved from http://vbn.aau.dk/files/16183196/ElectronicProceedingsDHRS2008.pdf\#page $=48$

Glasemann, M., \& Kanstrup, A. M. (2010). User prototypes as partly unconscious communication: Understanding young diabetics' visions for mobile diabetes technology. Retrieved from http://vbn.aau.dk/en/publications/userprototypes-as-partly-unconscious-communication(8de033ce-55ce-44c9-afa28590fc709087).html

Glasemann, M., \& Kanstrup, A. M. (2011). Emotions on diabetes: A design case of user mock-ups by young people living with diabetes. CoDesign, 7(2), 123130. https://doi.org/10.1080/15710882.2011.609894

Glasemann, M., Kanstrup, A. M., \& Ryberg, T. (2010). Making chocolate-covered broccoli: Designing a mobile learning game about food for young people with diabetes. In O. W. Bertelson \& P. Krogh (Chairs), Proceedings of the 8th ACM conference on designing interactive systems (pp. 262-271). New York, NY: ACM. https://doi.org/10.1145/1858171.1858219

Glaser, B., \& Strauss, A. (1967). The discovery of grounded theory. Chicago, IL: Aldine.

Glasgow, R. E., \& Anderson, R. M. (1999). In diabetes care, moving from compliance to adherence is not enough. Diabetes Care, 22(12), 2090-2092.

Glesne, C. (2011). Becoming qualitative researchers: An introduction (4th ed.). Boston, MA: Allyn \& Bacon. 
Glucowise (n.d.). GlucoWise. Retrieved from http://www.gluco-wise.com/

Goffman, E. (1963). Stigma: Notes on the management of spoiled identity. Englewood Cliffs, NJ: Prentice-Hall.

Goffman, E. (1978). The presentation of self in everyday life. Harmondsworth, England: Penguin.

Goldstein, H. H. (2008). Pen devices to improve patient adherence with insulin therapy in type 2 diabetes. Postgrad Med, 120(3), 172-179.

Goodwin, K. (2009). Designing for the digital age: How to create human-centered products and services / Kim Goodwin. Indianapolis, IN: Wiley Pub.

Graue, M., Wentzel-Larsen, T., Bru, E., Hanestad, B. R., \& Søvik, O. (2004). The coping styles of adolescents with type 1 diabetes are associated with degree of metabolic control. Diabetes Care, 27(6), 1313-1317.

Greca, A. M. L. (1990). Social consequences of pediatric conditions: Fertile area for future investigation and intervention? Journal of Pediatric Psychology, 15(3), 285-307. https://doi.org/10.1093/jpepsy/15.3.285

Greca, A. M. L., Auslander, W. F., Greco, P., Spetter, D., Fisher, E. B., \& Santiago, J. V. (1995). I get by with a little help from my family and friends: Adolescents' support for diabetes care. Journal of Pediatric Psychology, 20(4), 449-476. https://doi.org/10.1093/jpepsy/20.4.449

Greca, A. M. L., \& Bearman, K. J. (2002). The diabetes social support questionnairefamily version: Evaluating adolescents' diabetes-specific support from family members. Journal of Pediatric Psychology, 27(8), 665-676. https://doi.org/10.1093/jpepsy/27.8.665

Haak, T., Edelman, S., Walter, C., Lecointre, B., \& Spollett, G. (2007). Comparison of usability and patient preference for the new disposable insulin device Solostar versus Flexpen, lilly disposable pen, and a prototype pen: An open-label study. Clinical Therapeutics, 29(4), 650-660.

Hagey, R. (1984). The phenomenon, the explanations and the responses: Metaphors surrounding diabetes in urban Canadian Indians. Social Science \& Medicine, 18(3), 265-272. https://doi.org/10.1016/0277-9536(84)90089-3

Hains, A. A., Berlin, K. S., Davies, W. H., Smothers, M. K., Sato, A. F., \& Alemzadeh, R. (2007). Attributions of adolescents with type 1 diabetes related to 
performing diabetes care around friends and peers: the moderating role of friend support. Journal of Pediatric Psychology, 32(5), 561-570.

Hanas, R., de Beaufort, C., Hoey, H., \& Anderson, B. (2011). Insulin delivery by injection in children and adolescents with diabetes. Pediatric Diabetes, 12(5), 518-526. https://doi.org/10.1111/j.1399-5448.2010.00731.x

Hanne, M., \& Hawken, S. J. (2007). Metaphors for illness in contemporary media. Medical Humanities, 33(2), 93-99. https://doi.org/10.1136/jmh.2006.000253

Harrington, J. W. (2008). People-first language. Consultant for Paediatricians, 7(8), 305.

Harris, S. T., \& Pokorny, M. E. (2012). Living with diabetes: What patients are saying. Care Management Journals; New York, 13(2), 46-50.

Haseman, B., \& Mafe, D. (2009). Acquiring know-how: Research training for practice-led researchers. In H. Smith \& R. T. Dean (Eds.), Practice-led Research, Research-led Practice in the Creative Arts (pp. 211-228). Edinburgh, United Kingdom: Edinburgh University Press.

Haskard, K. B., DiMatteo, M. R., \& Williams, S. L. (2009). Adherence and health outcomes: How much does adherence matter? In S. A. Shumaker, J. K. Ockene, \& K. Riekert (Eds.), Handbook of Health Behavior Change ( $3^{\text {rd }}$ ed), (pp. 771-784). New York, NY: Springer Publishing Company.

Hassling, L., Nordfeldt, S., Eriksson, H., \& Timpka, T. (2005). Use of cultural probes for representation of chronic disease experience: Exploration of an innovative method for design of supportive technologies. Technology \& Health Care, 13(2), 87-95.

Hatch, J. A. (2002). Doing qualitative research in education settings. Albany, New York: Suny Press. Retrieved from https://books.google.co.nz/books?hl=en\&lr=\&id=yWlGAAAAQBAJ\&oi=fnd \&pg=PR3\&dq=J.+Amos + Hatch,+ Doing + Qualitative + Research + in + Educatio n+Settings+\&ots=F87jzDFr_G\&sig=DEI47ZuZZxy1biNYzT64bYChLuM

Haynes, R. B., Taylor, W. D., \& Sackett, D. L. (1979). Compliance in healthcare. Baltimore, MD: Johns Hopkins University Press.

Heisler, M., Piette, J. D., Spencer, M., Kieffer, E., \& Vijan, S. (2005). The relationship between knowledge of recent $\mathrm{HbAlc}$ values and diabetes care understanding 
and self-management. Diabetes Care, 28(4), 816-822.

https://doi.org/10.2337/diacare.28.4.816

Heiss, L. (2013). Innovative forms of healing: New media art as a catalyst for lasting change in therapeutic settings. Retrieved from http://ses.library.usyd.edu.au/handle/2123/9737

Hekkert, P., \& Cila, N. (2015). Handle with care! Why and how designers make use of product metaphors. Design Studies, 40, 196-217. https://doi.org/10.1016/j.destud.2015.06.007

HelpAround (n.d.). Diabetes helpers answer each other on HelpAround. Retrieved from http://helparound.co/

Hester, C. J. (2004). Adolescent consent: Choosing the right path. Issues in Comprehensive Pediatric Nursing, 27(1), 27-37.

Hey, J., Linsey, J., Agogino, A. M., \& Wood, K. L. (2008). Analogies and metaphors in creative design. International Journal of Engineering Education, 24(2), 283.

Hgen, S. (2012). Enabling patient compliance-A synergy of human factors engineering and industrial design. Medical Design News. Cleveland, Ohio: Penton Media. Retrieved from http://search.proquest.com/docview/1019959368/fulltext/422204DF5C5F439 $\mathrm{BPQ} / 5$ ? accountid $=14782$

Hibbard, J. H., Stockard, J., Mahoney, E. R., \& Tusler, M. (2004). Development of the patient activation measure (PAM): Conceptualizing and measuring activation in patients and consumers. Health Services Research, 39(4), 1005-1026.

Hill, K. (2015, January 31). Saturday Morning, Saturday 31 January 2015 | Radio New Zealand National [audio file]. Retrieved from http://www.radionz.co.nz/national/programmes/saturday/20150131

Hilliard, M. E., Wu, Y. P., Rausch, J., Dolan, L. M., \& Hood, K. K. (2013). Predictors of deteriorations in diabetes management and control in adolescents with type 1 diabetes. Journal of Adolescent Health, 52(1), 28-34. https://doi.org/10.1016/j.jadohealth.2012.05.009

Hirose, M., Beverly, E. A., \& Weinger, K. (2012). Quality of life and technology: Impact on children and families with diabetes. Current Diabetes Reports, 12(6), 711-720. https://doi.org/10.1007/s11892-012-0313-4 
Ho, A. Y. K., Berggren, I., \& Dahlborg-Lyckhage, E. (2010). Diabetes empowerment related to Pender's Health Promotion Model: A meta-synthesis. Nursing \& Health Sciences, 12(2), 259-267. https://doi.org/10.1111/j.14422018.2010.00517.x

Hoey, H. (2009). Psychosocial factors are associated with metabolic control in adolescents: Research from the Hvidoere Study Group on Childhood Diabetes. Pediatric Diabetes, 10, 9-14. https://doi.org/10.1111/j.13995448.2009.00609.x

Hoffman, R. P. (2002). Adolescent adherence in type 1 diabetes. Comprehensive Therapy, 28(2), 128-133. https://doi.org/10.1007/s12019-002-0050-8

Holdich, P., Gillibrand, W., \& Rodriguez, A. (2016). How do people with Type 2 diabetes and healthcare professionals understand and manage decisionmaking involving risks. International Journal of Integrated Care, 16(6). https://doi.org/10.5334/ijic.2984

Holmbeck, G. N. (2002). A developmental perspective on adolescent health and illness: An introduction to the special issues. Journal of Pediatric Psychology, 27(5), 409-416. https://doi.org/10.1093/jpepsy/27.5.409

Holmquist, L. (2006). Inventing the future. Interactions, 13(2), 44-ff. https://doi.org/10.1145/1116715.1116749

Holmquist, L. E. (2004). User-driven innovation in the future applications lab. In E. Dykstra-Erickson \& M. Tscheligi(Eds.), CHI'04 Extended Abstracts on Human Factors in Computing Systems (pp. 1091-1092). Vienna, Austria: ACM. Retrieved from http://dl.acm.org/citation.cfm?id=985995

Home (n.d.). Team novo nordisk pro cycling. Retrieved from http://www.teamnovonordisk.com/

Houde, S., \& Hill, C. (1997). What do prototypes prototype? In M. Helander, T. K. Landauer \& P. Prabhu (Eds.), Handbook of human-computer interaction $\left(2^{\text {nd }}\right.$ ed), (pp. 367-381). Amsterdam, The Netherlands: Elsevier Science.

Howarth, D. (2015, January 22). Health-monitoring components for modular Google smartphone. Retrieved from https://www.dezeen.com/2015/01/22/lapkacomponents-heart-rate-monitor-google-project-ara-google-modularsmartphone/ 
Huber, J. G., Jutai, J., Strong, J. G., \& Plotkin, A. D. (2008). The psychosocial impact of closed-circuit televisions on persons with age-related macular degeneration. Journal of Visual Impairment \& Blindness, 102(11), 690-701.

Huebner, A. J. (2009). An introduction to adolescent development. In W. T. O’Donohue (Ed.), Behavioral approaches to chronic disease in adolescence (pp. 7-14). New York, NY: Springer. Retrieved from http://link.springer.com/chapter/10.1007/978-0-387-87687-0_2

Hunt, L. M., Arar, N. H., Larme, A. C., Rankin, S. H., \& Anderson, R. M. (1998). Contrasting patient and practitioner perspectives in type 2 diabetes management. Western Journal of Nursing Research, 20(6), 656-682. https://doi.org/10.1177/019394599802000602

Huttlinger, K., Krefting, L., Drevdahl, D., Tree, P., Baca, E., \& Benally, A. (1992). "Doing battle": A metaphorical analysis of diabetes mellitus among Navajo people. American Journal of Occupational Therapy, 46(8), 706-712.

iBGStar (2012, March 12). New app will allow diabetics to manage condition on their iPhone. Retrieved from http://www.dailymail.co.uk/health/article2113676/New-iPhone-app-iBGStar-device-allow-diabetics-managecondition.html

Ickovics, J. R., \& Meisler, A. W. (1997). Adherence in AIDS clinical trials: A framework for clinical research and clinical care. Journal of Clinical Epidemiology, 50(4), 385-391. https://doi.org/10.1016/S0895-4356(97)000413

Ingersoll, G. M., Orr, D. P., Herrold, A. J., \& Golden, M. P. (1986). Cognitive maturity and self-management among adolescents with insulin-dependent diabetes mellitus. The Journal of Pediatrics, 108(4), 620-623. https://doi.org/10.1016/S0022-3476(86)80852-6

Ingraham, N. (2014, January 16). Google X building "smart" contact lens to measure glucose levels for diabetics. Retrieved from https://www.theverge.com/2014/1/16/5317210/google-x-building-smartcontact-lens-to-measure-glucose-levels-for InsuJet (2017). InsuJet. Retrieved from http://insujet.com/ 
International Electrotechnical Commission (2007). IEC 62366, Ed.1: Medical devices - Application of usability engineering to medical devices. Geneva, Switzerland: International Electrotechnical Commission.

Inventor Spot (n.d.). See the innovative insulin delivery tools from winners of the “Ignite: Diabetes Challenge!" Retrieved from http://inventorspot.com/articles/see_innovative_insulin_delivery_tools_winn ers_ignite_diabetes_ch

Iscoe, K. E., Campbell, J. E., Jamnik, V., Perkins, B. A., \& Riddell, M. C. (2006). Efficacy of continuous real-time blood glucose monitoring during and after prolonged high-intensity cycling exercise: spinning with a continuous glucose monitoring system. Diabetes Technology \& Therapeutics, 8(6), 627-635.

International Organization for Standardization (2015). IEC 60601. Geneva, Switzerland: International Organization for Standardization.

ISPAD (2014). ISPAD clinical practice consensus guidelines 2014 - International Society for Pediatric and Adolescent Diabetes. Retrieved from http://www.ispad.org/?page=ISPADClinicalPract Jacobson, R. (1999). Information design. Massachusetts: MIT Press. Jacobson, S. (2010). Overcoming stigma associated with assistive devices. In J. Gregory (Chair), 7th Int'l Conf. on Design and Emotion. Chicago, Illinois: Illinois Institute of Technology.

Jordan, P. W. (1999). Pleasure with products: Human factors for body, mind and soul. In W. Green \& P. W. Jordan (Eds.), Human factors in product design: Current practice and future trends (pp. 206-217). London, United Kingdom: Taylor \& Francis.

Julius, R. J., Novitsky, M. A., \& Dubin, W. R. (2009). Medication ddherence: A review of the literature and implications for clinical practice: Journal of Psychiatric Practice, 15(1), 34-44. https://doi.org/10.1097/01.pra.0000344917.43780.77

Jupp, V. (2006). The SAGE dictionary of social research methods. London, United Kingdom: SAGE Publications, Ltd. https://doi.org/10.4135/9780857020116 Jutai, J., \& Day, H. (2002). Psychosocial impact of assistive devices scale (PIADS). Technology \& Disability, 14(3), 107-111. 
Jutai, J., Rigby, P., Ryan, S., \& Stickel, S. (2000). Psychosocial impact of electronic aids to daily living. Assistive Technology, 12(2), 123-131. https://doi.org/10.1080/10400435.2000.10132018

Juvenile Diabetes Research Foundation Continuous Glucose Monitoring Study Group (2010). Prolonged nocturnal hypoglycemia is common during 12 months of continuous glucose monitoring in children and adults with type 1 diabetes. Diabetes Care, 33(5), 1004-1008. https://doi.org/10.2337/dc09-2081

Kamel Boulos, M. N., Gammon, S., Dixon, M. C., MacRury, S. M., Fergusson, M. J., Miranda Rodrigues, F., . . \& Yang, S. P. (2015). Digital games for type 1 and type 2 diabetes: Underpinning theory with three illustrative examples. JMIR Serious Games, 3(1). https://doi.org/10.2196/games.3930

Kanstrup, A. M. (2012). A small matter of design: An analysis of end users as designers. In K, Halskov, H. Winschiers-Theophilus, Y. Lee, J. Simonsen, \& K. Bødker (Eds.), Proceedings of the 12th participatory design conference: Research papers - Volume 1 (pp. 109-118). New York, NY: ACM. https://doi.org/10.1145/2347635.2347651

Kanstrup, A. M. (2014). Design concepts for digital diabetes practice: Design to explore, share, and camouflage chronic illness. International Journal of Design, 8(3), 49-60.

Kanstrup, A. M., Bertelsen, P., Glasemann, M., \& Boye, N. (2008). Design for more: An ambient perspective on diabetes. In D. Hakken (Chair), Proceedings of the tenth anniversary conference on participatory design 2008 (pp. 118-127). Indianapolis, IN: Indiana University. Retrieved from http://dl.acm.org/citation.cfm?id=1795234.1795252

Kanstrup, A. M., Bertelsen, P., \& Nøhr, C. (2015). Patient innovation: An analysis of patients' designs of digital technology support for everyday living with diabetes. Health Information Management Journal, 44(1), 12-20.

Kanstrup, A. M., Madsen, J., Nøhr, C., Bygholm, A., \& Bertelsen, P. (2017). Developments in participatory design of health information technology: A review of PDC publications from 1990-2016. Participatory Design \& Health Information Technology, 233, 1-13 
Katz, S. (2010, August 24). Insulin pump manufacture is complicated, but making (iPhone) strides. Retrieved from http://www.healthline.com/diabetesmine/insulin-pump-manufacter-iscomplicated-but-making-iphone-strides

Keyson, D. V., \& Bruns, M. (2009). Empirical research through design. In K. Lee (Ed.), Proceedings of the 3rd IASDR conference on design research (pp. 45484557). Retrieved from http://www.iasdr2009.or.kr/Papers/Special\%20Session/Assessing\%20knowled ge\%20generated\%20by\%20research\%20through\%20design/Empirical\%20Res earch\%20Through\%20Design.pdf

Kim, D. -J., Hazlett-Knudsen, R., Culver-Godfrey, H., Rucks, G., Cunningham, T., Portee, D., .. \& B Behal, A. (2012). How autonomy impacts performance and satisfaction: Results from a study with spinal cord injured subjects using an assistive robot. IEEE Transactions on Systems, Man, and Cybernetics-Part A: Systems and Humans, 42(1), 2-14.

Kinsman, K. (2012). Physically challenged and fashionable. Retrieved from http://www.cnn.com/2012/08/03/living/disability-fashion/index.html

Kolko, J. (2012). Wicked problems: Problems worth solving - My epiphany. Austin, TX: Austin Center for Design. Retrieved from https://www.wickedproblems.com/read.php

Korytkowski, M., Niskanen, L., \& Asakura, T. (2005). FlexPen ${ }^{\oplus}$ : Addressing issues of confidence andconvenience in insulin delivery. Clinical Therapeutics, 27, S89-S100.

Kovacs Burns, K., Bellows, M., Eigenseher, C., \& Gallivan, J. (2014). 'Practical' resources to support patient and family engagement in healthcare decisions: A scoping review. BMC Health Services Research, 14, 175. https://doi.org/10.1186/1472-6963-14-175

KyngÄs, H. A., \& Barlow, J. (1995). Diabetes: An adolescent's perspective. Journal of Advanced Nursing, 22(5), 941-947. https://doi.org/10.1111/j.1365-

2648.1995.tb02646.x 
KyngÄs, H. A., Kroll, T., \& Duffy, M. E. (2000). Compliance in adolescents with chronic diseases: A review. Journal of Adolescent Health, 26(6), 379-388. https://doi.org/10.1016/S1054-139X(99)00042-7

La Greca, A. M., Auslander, W. F., Greco, P., Spetter, D., Fisher, E. B., \& Santiago, J. V. (1995). I get by with a little help from my family and friends: Adolescents' support for diabetes care. Journal of Pediatric Psychology, 20(4), 449-476. https://doi.org/10.1093/jpepsy/20.4.449

Lai, J. (2013). User experience for people with diabetes. Retrieved from http://johnlaidesign.com/user-experience-for-people-with-diabetes/ Lancaster, B. M., Pfeffer, B., McElligott, M., Ferguson, A. T., Miller, M., Wallace, D., \& Lane, J. T. (2010). Assessing treatment barriers in young adults with type 1 diabetes. Diabetes Research and Clinical Practice, 90(3), 243-249. https://doi.org/10.1016/j.diabres.2010.07.003

Lang, A., Martin, J., Sharples, S., \& Crowe, J. A. (2010, June). Adolescent participation in HTA: The identification of appropriate proxies for adolescent user needs of medical devices. Presentation at the 7th Annual Conference of the International Society for Health Technology Assessment, Dublin, Ireland. Retrieved from http://eprints.nottingham.ac.uk/1349/1/HTAi_posterAL_eprint.pdf

Lang, A. R. (2012, November 23). Medical device design for adolescents [eThesis]. Retrieved from http://eprints.nottingham.ac.uk/12501/

Lang, A. R., Martin, J. L., Sharples, S., \& Crowe, J. A. (2013). The effect of design on the usability and real world effectiveness of medical devices: A case study with adolescent users. Applied Ergonomics, 44(5), 799-810. https://doi.org/10.1016/j.apergo.2013.02.001

Lang, A. R., Martin, J. L., Sharples, S., Crowe, J. A., \& Murphy, E. (2012). Not a minor problem: Involving adolescents in medical device design research. Theoretical Issues in Ergonomics Science, 15(2), 181-192. https://doi.org/10.1080/1463922X.2012.678910

Lawrence, J. M., Laffel, L., Wysocki, T., Xing, D., Beck, R. W., Huang, E. S., . . \& Tamborlane, W. V. (2010). Quality of life measures in children and adults with type 1 diabetes: The juvenile diabetes research foundation continuous 
glucose monitoring randomized trial. Diabetes Care, 40(11), 2-6.

https://doi.org/10.2337/dc10-0331

Leedy, P. D., \& Ormrod, J. E. (2013). Practical research: Planning and design (10 ${ }^{\text {th }}$ ed.). Hoboken, New Jersey: Pearson.

Levich, B. R. (2007). Self-management in chronic illness. In J. Nuovo (Ed.), Chronic Disease Management (pp. 9-31). New York, NY: Springer. Retrieved from http://link.springer.com/chapter/10.1007/978-0-387-49369-5_2

Lin, L., Isla, R., Doniz, K., Harkness, H., Vicente, K. J., \& Doyle, D. J. (1998). Applying human factors to the design of medical equipment: Patientcontrolled analgesia. Journal of Clinical Monitoring and Computing, 14(4), 253-263. https://doi.org/10.1023/A:1009928203196

Lincoln, Y. S. (2013). The constructivist credo. Walnut Creek, CA: Left Coast Press, Inc. Retrieved from http://ebookcentral.proquest.com/lib/vuw/detail.action?docID=1187038

Lindholm Olinder, A., Kernell, A., \& Smide, B. (2007). Continuous subcutaneous insulin infusion in young girls: A two-year follow-up study. European Diabetes Nursing, 4(1), 34-39.

Loewenstein, G., \& Angner, E. (2003). Predicting and indulging changing preferences. In G. Loewenstein, D. Read \& R. Baumeister (Eds.), Time and decision: Economic and psychological perspectives on intertemporal choice (pp. 351-91). New York, NY: Russell Sage Foundation. Retrieved from http://www.andrew.cmu.edu/user/gl20/GeorgeLoewenstein/Papers_files/pdf/ PredictingIndulging.pdf

Low, K. G., Massa, L., Lehman, D., \& Olshan, J. S. (2005). Insulin pump use in young adolescents with type 1 diabetes: A descriptive study. Pediatric Diabetes, 6(1), 22-31. https://doi.org/10.1111/j.1399-543X.2005.00089.x

Lu, Y., Pyatak, E. A., Peters, A. L., Wood, J. R., Kipke, M., Cohen, M., \& Sequeira, P. A. (2015). Patient perspectives on peer mentoring type 1 diabetes management in adolescents and young adults. The Diabetes Educator, 41(1), 59-68. https://doi.org/10.1177/0145721714559133 
Machniak, M. (2013). Sprinklr: Designing a 'cool' health-oriented social networking site with and for teenagers. Retrieved from https://www.duo.uio.no/handle/10852/37422

Mann, G., Taylor, P., \& Lane, R. (2011). Accelerometer-triggered electrical stimulation for reach and grasp in chronic stroke patients: A pilot study. Neurorehabilitation and Neural Repair, 25(8), 774-780.

Martin, C., Nunez, J., \& Royo, D. (2012). The impact of diagnosis. In D. Christie \& C. Martin (Eds.), Psychosocial aspects of diabetes: Children, adolescents and their families (pp. 24-37). London, United Kingdom: Radcliffe.

Martin, J. L., Murphy, E., Crowe, J. A., \& Norris, B. J. (2006). Capturing user requirements in medical device development: The role of ergonomics. Physiological Measurement, 27(8), 49-62.

Martin, J. L., Norris, B. J., Murphy, E., \& Crowe, J. A. (2008). Medical device development: The challenge for ergonomics. Applied Ergonomics, 39(3), 271283.

Mattelmäki, T. (2006). Design probes. Helsinki, Finland: University of Art and Design Helsinki. Retrieved from https://aaltodoc.aalto.fi/handle/123456789/11829

Mattelmäki, T., Vaajakallio, K., \& Koskinen, I. (2013). What happened to empathic design? Design Issues, 30(1), 67-77. https://doi.org/10.1162/DESI_a_00249

Mattheiss, E., Hochleitner, C., Busch, M., Orji, R., \& Tscheligi, M. (2017). Deconstructing Pokémon Go - An empirical study on player personality characteristics. In P. de Vries, H. Oinas-Kukkonen, L. Siemons, N. Beerlage-de Jong, \& L. van Gemert-Pijnen (Eds.), Persuasive technology: Development and implementation of personalized technologies to change attitudes and behaviors (pp. 83-94). Amsterdam, The Netherlands: Springer. https://doi.org/10.1007/978-3-319-55134-0_7

Mawson, S., Nasr, N., Parker, J., Zheng, H., Davies, R., \& Mountain, G. (2014). Developing a personalised self-management system for post stroke rehabilitation; utilising a user-centred design methodology. Disability and Rehabilitation: Assistive Technology, 9(6), 521-528. 
May, B. (2009, February 18). Flex insulin pump overcomes diabetes, fear of leeches Yanko Design. Retrieved from http://www.yankodesign.com/2009/02/18/flexinsulin-pump-overcomes-diabetes-fear-of-leeches/

McCarthy, G. Type 1 Diabetes Experiences [video file] (2014). Retrieved from https://www.youtube.com/playlist?list=PLD19JkUchmHZ9Tx9f4ABjVV0iGG KPAHvt

McDonagh, D., \& Thomas, J. (2010). Disability + relevant design: Empathic design strategies supporting more effective new product design outcomes. The Design Journal, 13(2), 180-198. https://doi.org/10.2752/175470710X12735884220899

McMahon, S. K., Airey, F. L., Marangou, D. A., McElwee, K. J., Carne, C. L., Clarey, A. J., . . \& Jones, T. W. (2005). Insulin pump therapy in children and adolescents: improvements in key parameters of diabetes management including quality of life. Diabetic Medicine: A Journal of the British Diabetic Association, 22(1), 92-96. https://doi.org/10.1111/j.1464-5491.2004.01359.x

McPherson, K. M., Kayes, N. M., \& Kerston, P. (2014). MEANING as a smarter approach to goals in rehabilitation. In R. J. Siegert \& W. M. M. Levack (Eds.), Rehabilitation goal setting: Theory, practice and evidence (pp. 105-119). Hoboken, NJ: CRC Press.

Medsafe (2017, April 18). Current guidelines on the regulation of therapeutic products in New Zealand. Retrieved from http://www.medsafe.govt.nz/regulatory/current-guidelines.asp

Melles, M., \& Freudenthal, A. (2003). Next generation equipment in the intensive care unit: Data collection for design guidelines. Conference on human decision making and manual control, Linköping university, June 2-4, 2003 (pp. 65-73). Retrieved from http://repository.tudelft.nl/islandora/object/uuid:ce4adccc-c550-4595-995330148c3f4d0a/datastream/OBJ/view

Menichetti, J., Libreri, C., Lozza, E., \& Graffigna, G. (2014). Giving patients a starring role in their own care: A bibliometric analysis of the on-going literature debate. Health Expectations, 19(3), 516-526. https://doi.org/10.1111/hex.12299 
Michaud, P. -A., Suris, J. -C., \& Viner, R. (2007). The adolescent with a chronic condition: Epidemiology, developmental issues and health care provision/PA. Michaud, J. Suris and R. Viner. Retrieved from http://apps.who.int/iris/handle/10665/43775

Milewski, J., \& Parra, H. (2010, September). Gathering requirements for a personal health management system. In J. E. Bardram \& M. Langheinrich (Chairs), Proceedings of the 12th ACM international conference adjunct papers on ubiquitous computing - adjunct (pp. 415-416). New York, NY: ACM. https://doi.org/10.1145/1864431.1864464

Milton, A., \& Rodgers, P. (2013). Research methods for product design. London, United Kingdom: Laurence King.

Ministry of Health (2013). Health loss in New Zealand: A report from the New Zealand Burden of Diseases, Injuries and Risk Factors Study 2006-2016. Wellington, New Zealand: Ministry of Health. Retrieved from http://www.health.govt.nz/publication/health-loss-new-zealand-report-newzealand-burden-diseases-injuries-and-risk-factors-study-2006-2016 Ministry of Health (2016). Health Loss in New Zealand 1990-2013: A report from the New Zealand Burden of Diseases, Injuries and Risk Factors Study. Wellington, New Zealand: Ministry of Health.

Ministry of Health (2017). Special authority forms. Retrieved from http://www.pharmac.govt.nz/SAForms

Money, A. G., Barnett, J., Kuljis, J., Craven, M. P., Martin, J. L., \& Young, T. (2011). The role of the user within the medical device design and development process: Medical device manufacturers' perspectives. BMC Medical Informatics and Decision Making, 11(15). https://doi.org/10.1186/1472-6947$11-15$

Mudge, S., Kayes, N., \& McPherson, K. (2015). Who is in control? Clinicians' view on their role in self-management approaches: A qualitative metasynthesis. BMJ Open, 5. doi:10.1136/bmjopen-2014-007413

Muratovski, G. (2016). Research for designers: A guide to methods and practice. London, United Kingdom: SAGE Publications.

Murphy, R. F. (1987). The body silent. New York, NY: Henry Holt and Company. 
MySugr.com. (n.d.). mySugr. Retrieved from https://mysugr.com/

Neithercott, T. (2014, January). Top tech on the horizon 2014. Retrieved from http://www.diabetesforecast.org/2014/Jan/top-tech-on-the-horizon.html

Nejati, V., Zabihzadeh, A., Maleki, G., \& Tehranchi, A. (2012). Mind reading and mindfulness deficits in patients with major depression disorder. Procedia Social and Behavioral Sciences, 32(Supplement C), 431-437. Retrieved from https://doi.org/10.1016/j.sbspro.2012.01.065

Nemaura Medical (2016, March 21). New skin patch helps monitor glucose continuously. Retrieved from https://futurism.com/keeping-tabs-diabetessugarbeat-continuous-glucometer-cleared-europe/

Neugent, B. (2015, January 16). Sentry alerts dangerous nighttime lows. Retrieved from https://www.diabeteshealth.com/sentry-alerts-dangerous-nighttimelows/

Neustaedter, C., \& Sengers, P. (2012). Autobiographical design in HCI research: Designing and learning through use-it-yourself. In P. Oliver \& P. Wright (Eds.), DIS'12 Proceedings of the Designing Interactive Systems Conference (pp. 514-523). Newcastle Upon Tyne, United Kingdom: ACM. https://doi.org/10.1145/2317956.2318034

Noe, R. (2016, October 25). Brilliant, low-tech design for pain-free injections.

Retrieved from http://www.core77.com//posts/57320/Brilliant-Low-TechDesign-for-Pain-Free-Injections

Noe, R. (2017, April 17). Pensa designs sexy glucose monitoring devices. Retrieved from http://www.core77.com//posts/65281/Pensa-Designs-Sexy-GlucoseMonitoring-Devices

Nordfeldt, S., Hanberger, L., \& Berterö, C. (2010). Patient and parent views on a Web 2.0 diabetes portal-The management tool, the generator, and the gatekeeper: Qualitative study. Journal of Medical Internet Research, 12(2). https://doi.org/10.2196/jmir.1267

Norman, D. A. (2002). Emotion \& design: Attractive things work better. Interactions, $9(4), 36-42$.

Norman, D. A. (2004). Emotional design why we love (or hate) everyday things. New York, NY: Basic Books. 
O’Donohue, W. T., Benuto, L. T., \& Tolle, L. W. (2013). Handbook of adolescent health psychology. Berlin, Germany: Springer Science \& Business Media. O'Kane, A. A. (2016). Individual differences and contextual factors influence the experience and practice of self-care with type 1 diabetes technologies. London, United Kingdom: University College London.

One News (2014, November 2). Father adapts glucose monitor to help young diabetics and parents [video file]. Retrieved from https://www.tvnz.co.nz/onenews/new-zealand/father-adapts-glucose-monitor-to-help-young-diabeticsand-parents-6121190.

Orji, R., Nacke, L. E., \& Di Marco, C. (2017). Towards personality-driven persuasive health games and gamified systems. In G. Mark \& S. Fussell (Eds.), Proceedings of the 2017 CHI Conference on Human Factors in Computing Systems (pp. 1015-1027). Denver, Colorado: ACM. Retrieved from http://dl.acm.org/citation.cfm?id=3025577

Owen, T., Buchanan, G., \& Thimbleby, H. (2012). Understanding user requirements in take-home diabetes management technologies. Proceedings of the 26th Annual BCS Interaction Specialist Group Conference on People and Computers (pp. 268-273). Birmingham, United Kingdom: ACM. Retrieved from http://dl.acm.org/citation.cfm?id=2377950

Pańkowska, E., Blazik, M., Dziechciarz, P., Szypowska, A., \& Szajewska, H. (2009). Continuous subcutaneous insulin infusion vs. multiple daily injections in children with type 1 diabetes: A systematic review and meta-analysis of randomized control trials. Pediatric Diabetes, 10(1), 52-58.

Papanek, V. J. (1984). Design for the real world: Human ecology and social change. London, United Kingdom: Thames \& Hudson.

Pathi, T. (2011, December 22). Perfect kit for people suffering from diabetes: Dibikit. Retrieved from http://www.mrtechpathi.com/2011/12/perfect-kit-for-peoplesuffering-from.html

Pedgley, O., \& Wormald, P. (2007). Integration of design projects within a Ph. D. Design Issues, 23(3), 70-85. 
Perfetti, R. (2013). Comprehensive approach to the management of diabetes: Offering improved outcomes for diabetics and the healthcare system. Diabetes Manage, 3, 505-528.

Pfützner, A., Asakura, T., Sommavilla, B., \& Lee, W. (2008). Insulin delivery with FlexPen ${ }^{\oplus}$ : Dose accuracy, patient preference and adherence. Expert Opinion on Drug Delivery, 5(8), 915-925. https://doi.org/10.1517/17425247.5.8.915 Pharmac (2014). Introduction to PHARMAC. Retrieved from http://www.pharmac.health.nz/assets/factsheet-01-introduction-topharmac.pdf

Pharmac (2015a). Summary of responses to PHARMAC's consultation on future funding approach to blood glucose meters and test strips. Retrieved from https://www.pharmac.health.nz/assets/notification-2015-06-29-bloodglucose-meters.pdf

Pharmac (2015b, June 3). Future funding approach for blood glucose meters and test strips. Retrieved from http://www.pharmac.health.nz/assets/consultation2015-03-06-blood-glucose-meters.pdf

Pharmac (2015c, June 26). Invitation for expressions of interest for the supply of diabetes management products. Retrieved from http://www.pharmac.health.nz/news/rfp-2015-06-29-diabetes-managementproducts/

Polgar, J. M. (2010). The myth of neutral technology. In M. M. K. Oishi, I. M. Mitchell, \& H. F. Ven der Loos (Eds.), Design and use of assistive technology (pp. 17-31). New York, NY: Springer Publishing Company.

Prendiville, A. (2017). Communications: Communication design in chronic health. In E. Tsekleves \& R. Cooper (Eds.), Design for health (pp. 204-215). New York, NY: Routledge. Retrieved from http://ebookcentral.proquest.com/lib/VUW/reader.action?docID=4862641 Pullin, G. (2009). Design meets disability. London, United Kingdom: MIT Press.

Quirk, H., Blake, H., Dee, B., \& Glazebrook, C. (2014). You can't just jump on a bike and go: A qualitative study exploring parents' perceptions of physical activity in children with type 1 diabetes. BMC Pediatrics, 14, 313-324. https://doi.org/10.1186/s12887-014-0313-4 
Rados, C. (2004). Medical devices needed for children. FDA Consumer, 39(5), 16-17.

Ratner, C. (2008). Subjectivism. In L. M. Given (Ed.), Sage Encyclopedia of Qualitative Research Methods. Thousand Oaks, California: Sage.

Rich, M., Lamola, S., Gordon, J., \& Chalfen, R. (2000). Video intervention/prevention assessment: A patient-centered methodology for understanding the adolescent illness experience. Journal of Adolescent Health, 27(3), 155-165.

Richardson, A., Adner, N., \& Nordstrom, G. (2001). Persons with insulin-dependent diabetes mellitus: Acceptance and coping ability. Journal of Advanced Nursing, 33(6), 758-763. https://doi.org/10.1046/j.1365-2648.2001.01717.x

Riddell, M. C., \& Milliken, J. (2011). Preventing exercise-induced hypoglycemia in type 1 diabetes using real-time continuous glucose monitoring and a new carbohydrate intake algorithm: An observational field study. Diabetes Technology \& Therapeutics, 13(8), 819-825.

Rigby, P., Renzoni, A. M., Ryan, S., Jutai, J., \& Stickel, S. (2000). Exploring the impact of electronic aids for daily living upon persons with neuromuscular conditions. (Supplement 1), Canadian Journal of Occupational Therapy (pp. 24-27). Toronto, Canada: Sage.

Rigby, P., Ryan, S., Joos, S., Cooper, B., Jutai, J., \& DipOT, E. S. (2005). Impact of electronic aids to daily living on the lives of persons with cervical spinal cord injuries. Assistive Technology, 17(2), 89-97.

Ritholz, M. D., Smaldone, A., Lee, J., Castillo, A., Wolpert, H., \& Weinger, K. (2007). Perceptions of psychosocial factors and the insulin pump. Diabetes Care, 30(3), 549-554. https://doi.org/10.2337/dc06-1755

Rittel, H. W., \& Webber, M. M. (1973). Dilemmas in a general theory of planning. Policy Sciences, 4(2), 155-169.

Roberts, A. J., \& Taplin, C. E. (2015). Exercise in youth with type 1 diabetes. Current Pediatric Reviews, 11(2), 120-125.

Robertson, T., \& Simonsen, J. (2013). Participatory design: An introduction. In J. Simonsen \& T. Robertson (Eds.), Routledge international handbook of participatory design (pp. 1-17). New York, NY: Taylor \& Francis. 
Robertson, T., \& Wagner, I. (2013). Ethics: Engagement, representation and politicsin-action. In J. Simonsen \& T. Robertson (Eds.), Routledge International Handbook of Participatory Design (pp. 64-85). New York, NY: Taylor \& Francis.

Rockmore, T. (2005). On constructivist epistemology. Oxford, United Kingdom: Rowman \& Littlefield.

Rolfe, G. (2006). Validity, trustworthiness and rigour: Quality and the idea of qualitative research. Journal of Advanced Nursing, 53(3), 304-310. https://doi.org/10.1111/j.1365-2648.2006.03727.x

Rossi, M. G. (2016). Metaphors for patient education. A pragmatic-argumentative approach applying to the case of diabetes care. Rivista Italiana Di Filosofia Del Linguaggio, 10(2), 34-48. https://doi.org/10.4396/403

Rothbauer, P. (2008). Triangulation. In L. Given (Ed.), The SAGE Encyclopedia of Qualitative Research Methods (pp. 892-894). Thousand Oaks, CA: Sage. http://dx.doi.org.helicon.vuw.ac.nz/10.4135/9781412963909.n257

ROUND II: Nightscout Dexcom-Moto G (v1) Case (n.d.). Shapeways. Retrieved from http://www.shapeways.com/model/2616760/round-ii-nightscoutdexcom-moto-g-v1-case.html

Rozenblum, R., Miller, P., Pearson, D., \& Marelli, A. (2015). Patient-centered healthcare, patient engagement and health information technology: The perfect storm. In M. A. Grando, R. Rozenblum \& D. Bates (Eds.), Information technology for patient empowerment in healthcare (pp. 3-22). Boston, MA: De Gruyter. Retrieved from http://www.degruyter.com/viewbooktoc/product/211055

Russ, A. L., Fairbanks, R. J., Karsh, B. -T., Militello, L. G., Saleem, J. J., \& Wears, R. L. (2013). The science of human factors: Separating fact from fiction. BMJ Quality and Safety, 22, 802-808.

Saladin, S. P., \& Hansmann, S. E. (2008). Psychosocial variables related to the adoption of video relay services among deaf or hard-of-hearing employees at the Texas School for the Deaf. Assistive Technology, 20(1), 36-47. 
Sandelowski, M. (2008). Member check. In L. Given (Ed.), The SAGE Encyclopedia of Qualitative Research Methods (pp. 501-501). Thousand Oaks, CA: Sage. http://dx.doi.org.helicon.vuw.ac.nz/10.4135/9781412963909.n257

Sandelowski, M. (1993). Rigor or rigor mortis: The problem of rigor in qualitative research revisited. Advances in Nursing Science Methods of Inquiry, 16(2), 18.

Sansoni, S., Speer, L., Wodehouse, A., \& Buis, A. (2016). Aesthetic of prosthetic devices: From medical equipment to a work of design. In S. Fukuda (Ed.), Emotional Engineering Volume 4 (pp. 73-92). Berlin, Germany: Springer International Publishing. https://doi.org/10.1007/978-3-319-29433-9_5

Sawyer, S. M., \& Aroni, R. A. (2005). Self-management in adolescents with chronic illness. What does it mean and how can it be achieved. Medical Journal of Australia, 183(8), 405-409.

Sbarigia, G. (2009, October 8). Guessing game deuce [Yanko Design]. Retrieved from http://www.yankodesign.com/2009/10/08/guessing-game-deuce/

Schabert, J., Browne, J. L., Mosely, K., \& Speight, J. (2013). Social stigma in diabetes. The Patient - Patient-Centered Outcomes Research, 6(1), 1-10. Retrieved from https://doi.org/10.1007/s40271-012-0001-0

Schneider, B. (2007). Design as practice, science and research. In R. Michel (Ed.), Design Research Now (pp. 207-218). Basel, Switzerland: Birkhäuser Basel. https://doi.org/10.1007/978-3-7643-8472-2_12

Schwartz, P. (1992). The art of the long view. Director; London, 45(10), 78-82.

Seereiner, S., Neeser, K., Weber, C., Schreiber, K., Habacher, W., Rakovac, I., . . \& \& Pieber, T. R. (2010). Attitudes towards insulin pump therapy among adolescents and young people. Diabetes Technology \& Therapeutics, 12(1), 89-94.

Seligman, M. E., \& Maier, S. F. (1967). Failure to escape traumatic shock. Journal of Experimental Psychology, 74(1), 1-9.

Shaban, C. (2010). Body image, intimacy and diabetes. European Diabetes Nursing, 7(2), 82-86. https://doi.org/10.1002/edn.163

Shah, S. G. S., \& Robinson, I. (2006). User involvement in healthcare technology development and assessment: Structured literature review. International 
Journal of Health Care Quality Assurance; Bradford, 19(6), 500-515.

https://doi.org/http://dx.doi.org.helicon.vuw.ac.nz/10.1108/09526860610687 619

Shah, S. G. S., \& Robinson, I. (2007). Benefits of and barriers to involving users in medical device technology development and evaluation. International Journal of Technology Assessment in Health Care, 23(01), 131-137. https://doi.org/10.1017/S0266462307051677

Shah, S. G. S., Robinson, I., \& AlShawi, S. (2009). Developing medical device technologies from users' perspectives: A theoretical framework for involving users in the development process. International Journal of Technology Assessment in Health Care, 25(04), 514-521. https://doi.org/10.1017/S0266462309990328

Shiu, A. T. -Y., Kwan, J. J. Y. -M., \& Wong, R. Y. -M. (2003). Social stigma as a barrier to diabetes self-management: Implications for multi-level interventions. Journal of Clinical Nursing, 12(1), 149-150. https://doi.org/10.1046/j.1365-2702.2003.00735.x

Shogo, T. (2017). INS series. Retrieved from https://jamesdysonaward.org/enGB/projects/ins-series/

Simonsen, J., \& Robertson, T. (Eds.). (2013). Routledge international handbook of participatory design (hardback) - Routledge. New York, NY: Routledge. Retrieved from https://www.routledge.com/Routledge-InternationalHandbook-of-Participatory-Design/SimonsenRobertson/p/book/9780415694407

Six Until Me (n.d.). Six until me. Retrieved from http://sixuntilme.com/wp/ Skinner, T. C., John, M., \& Hampson, S. E. (2000). Social support and personal models of diabetes as predictors of self-care and well-being: A longitudinal study of adolescents with diabetes. Journal of Pediatric Psychology, 25(4), 257-267. https://doi.org/10.1093/jpepsy/25.4.257

Sklar, A., \& Naar, L. (2017). Foresight: The next big frontier in healthcare. In E. Tsekleves \& R. Cooper (Eds.), Design for Health (pp. 380-387). New York, NY: Routledge. Retrieved from http://ebookcentral.proquest.com/lib/VUW/reader.action?docID=4862641 
Smart Design (2010, May). Breakup letter creative meeting. Paper presented at the IIT Design Research Conference, IIT Institute of Design, Chicago, Illinois.

Snell-Bergeon, J. K. (2015). Assessing insulin delivery device satisfaction in patients with type 1 and type 2 diabetes. Diabetes Technology \& Therapeutics, 17(11), 759-762. https://doi.org/10.1089/dia.2015.0260

South Island Alliance (2013). The health of children and young people with chronic conditions and disabilities in the South Island 2013. New Zealand: South Island Alliance.

Spinuzzi, C. (2005). The methodology of participatory design. Technical Communication, 52(2), 163-174.

Stanton, A. L., Revenson, T. A., \& Tennen, H. (2007). Health psychology: Psychological adjustment to chronic disease. Annual Review of Psychology, 58(1), 565-592. https://doi.org/10.1146/annurev.psych.58.110405.085615

Starship Insulin Pump Program: Helping you transition to insulin pump therapy. (n.d.). Healthpoint. Retrieved from https://www.healthpoint.co.nz/download,361951.do

Stein, R., \& Wiklund, M. E. (2010). Mobile medical devices. In M. Weinger, M. Wiklund, \& D. Gardner-Bonneau (Eds.), Handbook of human factors in medical device design (pp. 715-745). Boca Raton, Florida: CRC Press. https://doi.org/10.1201/b10439

Steinhoff, C., \& Still, J. D. (2013). Priming categorization in a card sort. In M. Kurosu (Ed.), Human-computer interaction. Human-centred design approaches, methods, tools, and environments (pp. 265-272). Las Vegas, Nevada: Springer. https://doi.org/10.1007/978-3-642-39232-0_30

Stephanie Monty designs colostomy bag for intimate moments (2016, July 25). Retrieved from https://www.dezeen.com/2016/07/25/stephanie-montycolostomy-bag-intimate-moments-graduate-shows-2016-brunel-universitylondon/

Stickdorn, M., \& Schneider, J. (Eds.). (2011). This is service design thinking. New Jersey, NJ: Wiley.

Stockton, G. (2009). Stigma: Addressing negative associations in product design. The design society. Retrieved from http://eprints.hud.ac.uk/16636 
Strauss, A. L. (1969). Mirrors and masks: The search for identity. California: Sociology Press.

Straus, A., \& Corbin, J. (1990). Basics of qualitative research: Grounded theory procedures and techniques. Newbury Park, CA: Sage.

Strawhacker, M. (2001). Multidisciplinary teaming to promote effective management of type 1 diabetes for adolescents. Journal of School Health, 71(6), 213-217. https://doi.org/10.1111/j.1746-1561.2001.tb01318.x

Stylish Diabetes Clutch (n.d.). Retrieved from https://www.td2designs.com/products/stylish-tester-clutch-diabetics

Sullivan, M. (2014, June 12). iHealth's tiny new blood glucose monitor plugs into your headphone jack. Retrieved from https://venturebeat.com/2014/06/12/ihealths-tiny-new-blood-glucosemonitor-plugs-into-smartphone-headphone-jack/

Suls, J., \& Fletcher, B. (1985). The relative efficacy of avoidant and nonavoidant coping strategies: A meta-analysis. Health Psychology, 4(3), 249-288. https://doi.org/10.1037/0278-6133.4.3.249

Suris, J. -C., Michaud, P. -A., \& Viner, R. (2004). The adolescent with a chronic condition. Part I: Developmental issues. Archives of Disease in Childhood, 89(10), 938-942. https://doi.org/10.1136/adc.2003.045369

Svanaes, D., \& Seland, G. (2004). Putting the users center stage: Role playing and low-fi prototyping enable end users to design mobile systems. In E. DykstraErickson \& M. Tscheligi (Chairs), Proceedings of the SIGCHI conference on human factors in computing systems (pp. 479-486). Vienna, Austria: ACM. Retrieved from http://dl.acm.org/citation.cfm?id=985753

Swann, C. (2002). Action research and the practice of design. Design Issues, 18(1), 49-61.

Tanaka, K., Parker, J. R., Baradoy, G., Sheehan, D., Holash, J. R., \& Katz, L. (2012). A comparison of exergaming interfaces for use in rehabilitation programs and research. Loading..., 6(9). Retrieved from http://journals.sfu.ca/loading/index.php/loading/article/view/107

Tanenbaum, M. L., Adams, R. N., Hanes, S. J., Barley, R. C., Miller, K. M., Mulvaney, S. A., \& Hood, K. K. (2017). Optimal use of diabetes devices: Clinician 
perspectives on barriers and adherence to device use. Journal of Diabetes Science and Technology, 11(3), 484-492.

Tenderich, A. (2015, April 22). Amazing Afrezza - Non-invasive insulin that works! Retrieved from http://www.healthline.com/diabetesmine/amazing-afrezzanon-invasive-insulin-works

Thomas, A. M., Peterson, L., \& Goldstein, D. (1997). Problem solving and diabetes regiman adherence by children and adolescents with IDDM in social pressure situations: A reflection of normal development. Journal of Pediatric Psychology, 22(4), 541-561. https://doi.org/10.1093/jpepsy/22.4.541

Thomas, J., \& McDonagh, D. (2013). Empathic design: Research strategies. Australasian Medical Journal (Online); Floreat, 6(1), 1-6.

Thomas, R. J., Masthoff, J., \& Oren, N. (2017). Adapting healthy eating messages to personality. In P. W. de Vries, H. Oinas-Kukkonen, L. Siemons, N. Beerlagede Jong, L. van Gemert-Pijnen (Ed.), Persuasive technology: Development and implementation of personalized technologies to change attitudes and behaviors (pp. 119-132). Amsterdam, Netherlands: Springer. https://doi.org/10.1007/978-3-319-55134-0_10

Thompson Jr., R. J., \& Gustafson, K. E. (1996). Adaptation to chronic childhood illness (Vol. xii). Washington, DC: American Psychological Association.

Thoring, K., Luippold, C., \& Mueller, R. M. (n.d.). Opening the cultural probes box. Retrieved from http://www.researchgate.net/publication/252627658_Opening_the_Cultural_ Probes_Box_A_Critical_Reflection_and_Analysis_of_the_Cultural_Probes_ Method/file/3deec52344e0eb9eab.pdf

Thornberg, R., \& Charmaz, K. (2012). Grounded theory. In S. Lapan, M. Quartaroli \& F. Riemer (Eds.), Qualitative research: An introduction to methods and designs (pp. 41-67). San Francisco, CA: Jossey-Bass.

Timesulin (n.d.). Timesulin: A timer for the insulin pens you already use. Retrieved from https://timesulin.com/

Toscos, T., Connelly, K., \& Rogers, Y. (2011). What's your number?: A survey of how parents and teens cope with diabetes in the context of technology support. 2011 5th International Conference on Pervasive Computing Technologies for 
Healthcare (PervasiveHealth) (pp. 210-213). Dublin, Ireland: Institute for Computer Sciences, Social Informatics and Telecommunications Engineering.

Toscos, T., Connelly, K., \& Rogers, Y. (2012). Best intentions: Health monitoring technology and children. Austin, Texas: ACM.

https://doi.org/10.1145/2207676.2208603

Toscos, T., Connelly, K., \& Rogers, Y. (2013). Designing for positive health affect: Decoupling negative emotion and health monitoring technologies. In M. Czerwinski \& O. Mayora (Chairs), Proceedings of the 7th International Conference on Pervasive Computing Technologies for Healthcare (pp. 153160). Venice, Italy: Institute for Computer Sciences, Social-Informatics and Telecommunications Engineering. Retrieved from http://dl.acm.org/citation.cfm?id=2534537

Tran, L. (2009, July 16). Be flexible with diabetes | Yanko Design. Retrieved from http://www.yankodesign.com/2009/07/16/be-flexible-with-diabetes/ Treuhaft, T. (2015, April 10). Better living with uncertainty: Insulin angel releases medication tracker on Indiegogoenbsp. Retrieved from http://www.core77.com//posts/31369/Better-Living-with-UncertaintyInsulin-Angel-Releases-Medication-Tracker-on-Indiegogo

Truong, K. N., Hayes, G. R., \& Abowd, G. D. (2006). Storyboarding: An empirical determination of best practices and effective guidelines. In J. M. Carroll (Chair), Proceedings of the 6th conference on Designing Interactive systems (pp. 12-21). University Park, Pennsylvania: ACM. Retrieved from http://dl.acm.org/citation.cfm?id=1142410

Tsvyatkova, D., \& Storni, C. (2014). Investigating issues related to pediatric diabetes education: Problems and barriers. In A. Hein (Chair), Proceedings of the 8th International Conference on Pervasive Computing Technologies for Healthcare (pp. 191-194). Brussels, Belgium: Institute for Computer Sciences, SocialInformatics and Telecommunications Engineering. https://doi.org/10.4108/icst.pervasivehealth.2014.254935

Tura, A., Maran, A., \& Pacini, G. (2007). Non-invasive glucose monitoring: Assessment of technologies and devices according to quantitative criteria. 
Diabetes Research and Clinical Practice, 77(1), 16-40.

https://doi.org/10.1016/j.diabres.2006.10.027

Turieo, M. (2011, October 1). Industry viewpoint: Study shows users of combination devices respond to good design. Medical Design News. Retrieved from http://search.proquest.com/docview/898390106/fulltext/422204DF5C5F439B $\mathrm{PQ} / 12$ ? accountid $=14782$

Turner, T. (2012a, July 24). Blood buddy | Yanko Design. Retrieved from http://www.yankodesign.com/2012/07/24/blood-buddy/

Turner, T. (2012b, December 5). Smartphone diabetes management | Yanko Design. Retrieved from http://www.yankodesign.com/2012/12/05/smartphonediabetes-management/

Turner, T. (2013, June 26). Instant insulin you can wear | Yanko Design. Retrieved from http://www.yankodesign.com/2013/06/26/instant-insulin-you-canwear/

Turner, T. (2014, September 2). The sweetest smartwatch | Yanko Design. Retrieved from http://www.yankodesign.com/2014/09/02/the-sweetest-smartwatch/ Turner, T. (2015a, May 7). A future fit band for diabetes | Yanko Design. Retrieved from http://www.yankodesign.com/2015/05/07/a-future-fit-band-fordiabetes/

Turner, T. (2015b, November 24). Say peace out to painful pricking. Retrieved from http://www.yankodesign.com/2015/11/24/say-peace-out-to-painful-pricking/

Turner, T. (2016, June 6). The future of diabetes wearables | Yanko Design. Retrieved from http://www.yankodesign.com/2016/06/06/the-future-of-diabeteswearables/

Turtyl (2014). dShell - Waterproof continuous glucose monitor cases. Retrieved from https://www.kickstarter.com/projects/d-shell/dshell-waterproof-continuousglucose-monitor-cases

Type 1 Diabetes Network (n.d.). Retrieved from http://t1dn.org.au/

Vaes, K. R. V. (2014). Product stigmaticity: Understanding, measuring and managing product-related stigma. Delft, Netherlands: Delft University.

Van Der Bijl Brouwer, M., \& Dorst, K. (2014). How deep is deep? A four-layer model of insights into human needs for design innovation. In M. M. Echavarría 
(Chair), Design and emotion. Colombia: Ediciones Uniandes. Retrieved from https://opus.lib.uts.edu.au/handle/10453/33538

Van der Bijl-Brouwer, M. (2016). The challenges of human-centred design in a public sector innovation context. In P. Lloyd \& E. Bohemia (Eds.), DRS 2016 International Conference: Future-Focused Thinking, the Design Research Society (pp. 2149-2164). Retrieved from http://www.drs2016.org/s/294-vander-Bijl-Brouwer.pdf

Van der Velden, M., \& Machniak, M. (2014). Colourful privacy: Designing visible privacy settings with teenage hospital patients. ACHI 2014, The Seventh International Conference on Advances in Computer-Human Interactions (pp. 60-65). Barcelona, Spain: IARIA XPS Press. Retrieved from http://urn.nb.no/URN:NBN:no-45757

Van Voorden, M. (2013). Diabetes kit for kids. Retrieved from http://diabeteskit.melchiorvanvoorden.nl/

Varni, J. W., Seid, M., \& Rode, C. A. (1999). The PedsQL ${ }^{\mathrm{TM}}$ : Measurement model for the pediatric quality of life inventory. Medical Care, 37(2), 126-139.

Vaughan, L. (2017). Embracing the literacies of design as means and mode of dissemination. In L. Vaughan (Ed.), Practice-based Design Research (pp. 111118). London, United Kingdom: Bloomsbury Academic.

Velden, M. van der, \& Mörtberg, C. (2015). Participatory design and design for values. In J. van den Hoven, P. E. Vermaas, \& I. van de Poel (Eds.), Handbook of Ethics, Values, and Technological Design (pp. 41-66). Dordrecht, The Netherlands: Springer. https://doi.org/10.1007/978-94-007-6970-0_33

Vicente, K. J., Kada-Bekhaled, K., Hillel, G., Cassano, A., \& Orser, B. A. (2003). Programming errors contribute to death from patient-controlled analgesia: case report and estimate of probability. Canadian Journal of Anesthesia, 50(4), 328-332. https://doi.org/10.1007/BF03021027

Vieira, G. (2015, May 13). Interview: Life with chronic illness inspires elegant medical alert jewelry. Retrieved from http://www.diabetesdaily.com/blog/2015/05/interview-life-with-chronicillness-inspires-elegant-medical-alert-jewelry/ 
Visocky O'Grady, J., \& Visocky O’Grady, K. (2008). The information design handbook. Cincinnati: HOW Books.

Wallander, J. L., \& Varni, J. W. (1995). Appraisal, coping, and adjustment in adolescents with a physical disability. In J. L. Wallander \& J. Lawrence (Eds.) Adolescent Health Problems: Behavioral Perspectives (pp. 209-231). New York: Guilford Publications.

Waller, A., Franklin, V., Pagliari, C., \& Greene, S. (2006). Participatory design of a text message scheduling system to support young people with diabetes. Health Informatics Journal, 12(4), 304-318. https://doi.org/10.1177/1460458206070023

Walter, A. (2010). Designing for emotion. New York, NY: Book Apart.

Wang, R. H., Mihailidis, A., Dutta, T., \& Fernie, G. R. (2011). Usability testing of multimodal feedback interface and simulated collision-avoidance power wheelchair for long-term-care home residents with cognitive impairments. Journal of Rehabilitation Research and Development, 48(7), 801-821.

Wang, Y. -L., Brown, S. A., \& Horner, S. D. (2010). School-based lived experiences of adolescents with type 1 diabetes: a preliminary study. Journal of Nursing Research, 18(4), 258-265.

Ware, N. C., Hopper, K., Tugenberg, T., Dickey, B., \& Fisher, D. (2007). Connectedness and citizenship: Redefining social integration. Psychiatric Services, 58(4), 469-474. https://doi.org/10.1176/ps.2007.58.4.469

Warfel, T. Z. (2009). Prototyping. Brooklyn, NY: Rosenfeld.

Warner, D. E., \& Hauser, S. T. (2009). Unique considerations when treating adolescents with chronic illness. In W. T. O’Donohue (Ed.), Behavioral approaches to chronic disease in adolescence (pp. 15-28). New York, NY: Springer. Retrieved from http://link.springer.com/chapter/10.1007/978-0387-87687-0_3

Wasserman, R., Anderson, B. J., \& Schwartz, D. D. (2017). Illness-specific risk-taking in adolescence: A missing piece of the nonadherence puzzle for youth with type 1 diabetes? Diabetes Spectrum, 30(1), 3-10. https://doi.org/10.2337/ds150060 
Watts, S., O’Hara, L., \& Trigg, R. (2010). Living with Type 1 diabetes: A by-person qualitative exploration. Psychology \& Health, 25(4), 491-506. https://doi.org/10.1080/08870440802688588

"We Are Not Waiting" = Diabetes Data Innovation Now! (n.d.). Retrieved from http://www.diabetesmine.com/we-are-not-waiting

Weijman, I., Ros, W. J. G., Rutten, G. E. H. M., Schaufeli, W. B., Schabracq, M. J., \& Winnubst, J. A. M. (2005). The role of work-related and personal factors in diabetes self-management. Patient Education and Counseling, 59(1), 87-96. https://doi.org/10.1016/j.pec.2004.10.004

Wessels, R., Dijcks, B., Soede, M., Gelderblom, G., \& De Witte, L. (2003). Non-use of provided assistive technology devices, a literature overview. Technology \& Disability, 15(4), 231-238.

Whaley, B., Stone, A., Brady, S., \& Whaley, R. (2014). Explaining diabetes: Studying the effects of using analogies to talk about illness. Journal of Diabetes Nursing, $18(2), 72-76$.

World Health Organization (2002). Innovative care for chronic conditions: Building blocks for action. Geneva: Switzerland: World Health Organization. Retrieved from http://www.who.int/chp/knowledge/publications/icccreport/en/

World Health Organization (2017). Adolescent health. Retrieved from http://www.who.int/topics/adolescent_health/en/

Wiklund Axelsson, S., \& Melander Wikman, A. (2016). Ready for eHealth. Older Swedes' perceptions of eHealth services: Using the PIADS scale as a predictor for readiness. Technologies, 4(3), 29. https://doi.org/10.3390/technologies4030029

Wiklund, M. E. (2005a). The rising bar: Medical product design excellence. In M. E. Wiklund \& S. B. Wilcox (Eds.), Designing usability into medical products (pp. 3-10). Boca Raton, Florida: CRC Press.

Wiklund, M. E. (2005b). The vision statement for product design: In your mind's eye. In M. E. Wiklund \& S. B. Wilcox (Eds.), Designing usability into medical products (pp. 145-150). Boca Raton, Florida: CRC Press.

Williams, S. L., DiMatteo, M. R., \& Haskard, K. B. (2009). Psychosocial barriers to adherence and lifestyle change. In S. Shumaker, J. K. Ockene, \& K. A. Riekert 
(Eds.), Handbook of health behavior change ( ${ }^{\text {rd }}$ ed., pp. 445-461). New York, NY: Springer Publishing Company.

Wilson, C. (2014). User interface inspection methods. Waltham, MA: Elsevier.

Wood, J. R., Miller, K. M., Maahs, D. M., Beck, R. W., DiMeglio, L. A., Libman, I. M., . . \& Woerner, S. E. (2013). Most youth with type 1 diabetes in the T1D exchange clinic registry do not meet American Diabetes Association or International Society for Pediatric and Adolescent Diabetes Clinical Guidelines. Diabetes Care, 36(7), 2035-2037.

Wysocki, T., \& Greco, P. (2006). Social support and diabetes management in childhood and adolescence: Influence of parents and friends. Current Diabetes Reports, 6(2), 117-122. https://doi.org/10.1007/s11892-006-0022-y

Yoon, C. S., Wang, C., Ramachandran, H., \& Gunhee Lee, W. (n.d.). PEAL: A lifestyle transition system for patients recently diagnosed with diabetes. Retrieved from http://www.core77.com//projects/44299/PEAL-A-Lifestyle-TransitionSystem-for-Patients-Recently-Diagnosed-with-Diabetes

Youngson, A., Cole, F., Wilby, H., \& Cox, D. (2015). The lived experience of diabetes: Conceptualisation using a metaphor. British Journal of Occupational Therapy, 78(1), 24-32. https://doi.org/10.1177/0308022614561240

Youngson, A., Wilby, H., Cole, F., \& Cox, D. (2015). Battle and control: Conceptualising diabetes through metaphor. British Journal of Occupational Therapy, 78, 107-108.

Zarki, T. (2017). Tim zarki designs hue to aid people with asthma from being selfconscious. Retrieved from https://www.designboom.com/design/tim-zarkihue-inhaler-06-07-2017/ 


\section{Appendix A}

\section{Publications}

The following peer-reviewed publications were adapted to form sections of the thesis.

McCarthy, G. M., Rodríguez Ramírez, E. R., \& Robinson, B. J. (2017). Design experiments: Identifying areas for intervention and designing medical technology for adolescents and young adults with type 1 diabetes. The Design Journal, 20(Suppl. 1), 2044-2056.

McCarthy, G. M., Rodríguez Ramírez, E. R., \& Robinson, B. J. (2017). Letters to medical devices: A case study on the medical device user requirements of female adolescents and young adults with type 1 diabetes. In P. W. de Vries, H. Oinas-Kukkonen, L. Siemons, N. Beerlage-de Jong, \& L. van Gemert-Pijnen (Ed.), International Conference on Persuasive Technology (pp. 69-79). Amsterdam, The Netherlands: Springer. Retrieved from http://link.springer.com/chapter/10.1007/978-3-319-551340_6

McCarthy, G. M., Rodriguez Ramírez, E. R., \& Robinson, B. J. (2017). Participatory design to address stigma with adolescents with type 1 diabetes. In O. Mival (Chair), Proceedings of the 2017 Conference on Designing Interactive Systems (pp. 83-94). New York, NY: ACM. https://doi.org/10.1145/3064663.3064740

McCarthy, G. M., Rodríguez Ramírez, E. R., \& Robinson, B. J. (2016). Dissonant technologies: Health professionals' impressions of adolescents' interactions with medical technologies for managing type 1 diabetes. In R. Coles, S. Costa, \& S. Watson (Ed.), Well-being 2016: Co-creating Pathways to Well-Being (pp. 36-39). Birmingham, United Kingdom: Birmingham City University.

McCarthy, G. M. (2016). Design for type 1 diabetes: How adolescents' interactions with medical technology can inform design. In M. Foth (Chair), DIS 2016 Companion (pp. 35-36). Brisbane, Australia: ACM. 


\section{Appendix B}

\section{Information and Consent Form for Health Professionals}

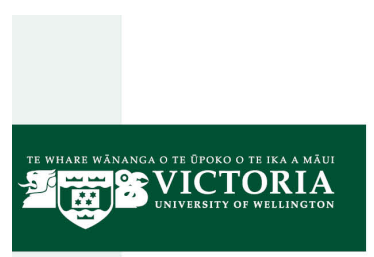

SCHOOL OF DESIGN Te Kura Hoahoa

FACULTY OF ARCHITECTURE \& DESIGN Te Wāhanga Waihanga-Hoahoa

VICTORIA UNIVERSITY OF WELLINGTON, PO Box 600, Wellington 6140, New Zealand

Phone + 64-4-463 6200 Fax +64-4-463 6204 Email architecture@vuw.ac.nz Web www.victoria.ac.nz/design

\section{Participant Information Sheet for a Study of Type 1 Diabetes Self- Management}

\section{Researcher: Gillian McCarthy, School of Design, Victoria University of Wellington}

I am a PhD student in the School of Design at Victoria University of Wellington. As part of this degree I am undertaking a research project leading to a thesis. The project I am undertaking is examining how designers might create medical technology that facilitates the self-management of adolescents with type 1 diabetes. This research project has received approval from the Victoria University of Wellington Human Ethics Committee.

I am asking healthcare professionals with experience regarding adolescents and type 1 diabetes to participate in this study. Participants will be interviewed about their professional experiences and opinions related to adolescent selfmanagement, and current and proposed medical technologies.

Should any participants feel the need to withdraw from the project, they may do so without question at any time before 18 June 2016, and any recordings of their participation will be deleted. Just let me know at the time.

Responses will form the basis of my research project and will be put into a written report on an anonymous basis. It will not be possible for you to be identified personally. All material collected from interviews will be kept confidential. No other person besides myself and my supervisors Dr. Edgar Rodriguez and Dr. Brian Robinson will see interview transcripts. The thesis will be submitted for marking to the School of Design and deposited in the University Library. It is intended that one or more articles will be submitted for publication in scholarly journals or at academic conferences. Interview recordings and transcripts will be destroyed two years after the end of the project.

If you have any further questions or would like to receive further information about the project, please contact me at gillian.mccarthy@vuw.ac.nz or my supervisors Dr. Edgar Rodriguez, at the School of Design at Victoria University (o4) 463 6245, edgar.rodriguez@vuw.ac.nz, and Dr. Brian Robinson, at the Graduate School of Nursing, Midwifery and Health (04) 4636155 ,

brian.robinson@vuw.ac.nz

Gillian McCarthy 


\section{CONSENT TO PARTICIPATE IN RESEARCH}

Designing for Self-management: Design Strategies for facilitating adolescents' self-management with type 1 diabetes self-care medical technology

I have been given and have understood an explanation of this research project. I have had an opportunity to ask questions and have them answered to my satisfaction. I understand that I may withdraw myself (or any information I have provided) from this project before 18 June 2016 without having to give reasons.

\section{Please tick the boxes below that apply:}

- I consent to information or opinions that I have given in an interview being used in any reports on this research (including for thesis, conference and journal publications).

- I understand that any information from our interview will not be attributed to me or identify me in any way.

- I understand that the tape recording and transcript of interviews will be destroyed within a two-year period following end of the project.

- I understand that the data I provide will not be used for any other purpose or released to others without my written consent.

- I understand that I will not receive any money for taking part in this research.

$\square$ I would like to receive a summary of the results of the research when it is completed.

$\square$ I would like to receive a summary of my interview once it has been transcribed.

\section{Signed:}

\section{Name of participant:}

Date: 


\section{Appendix C}

\section{Sample Information and Consent Form for Designers}

Variations of this form were used for the three groups of designers

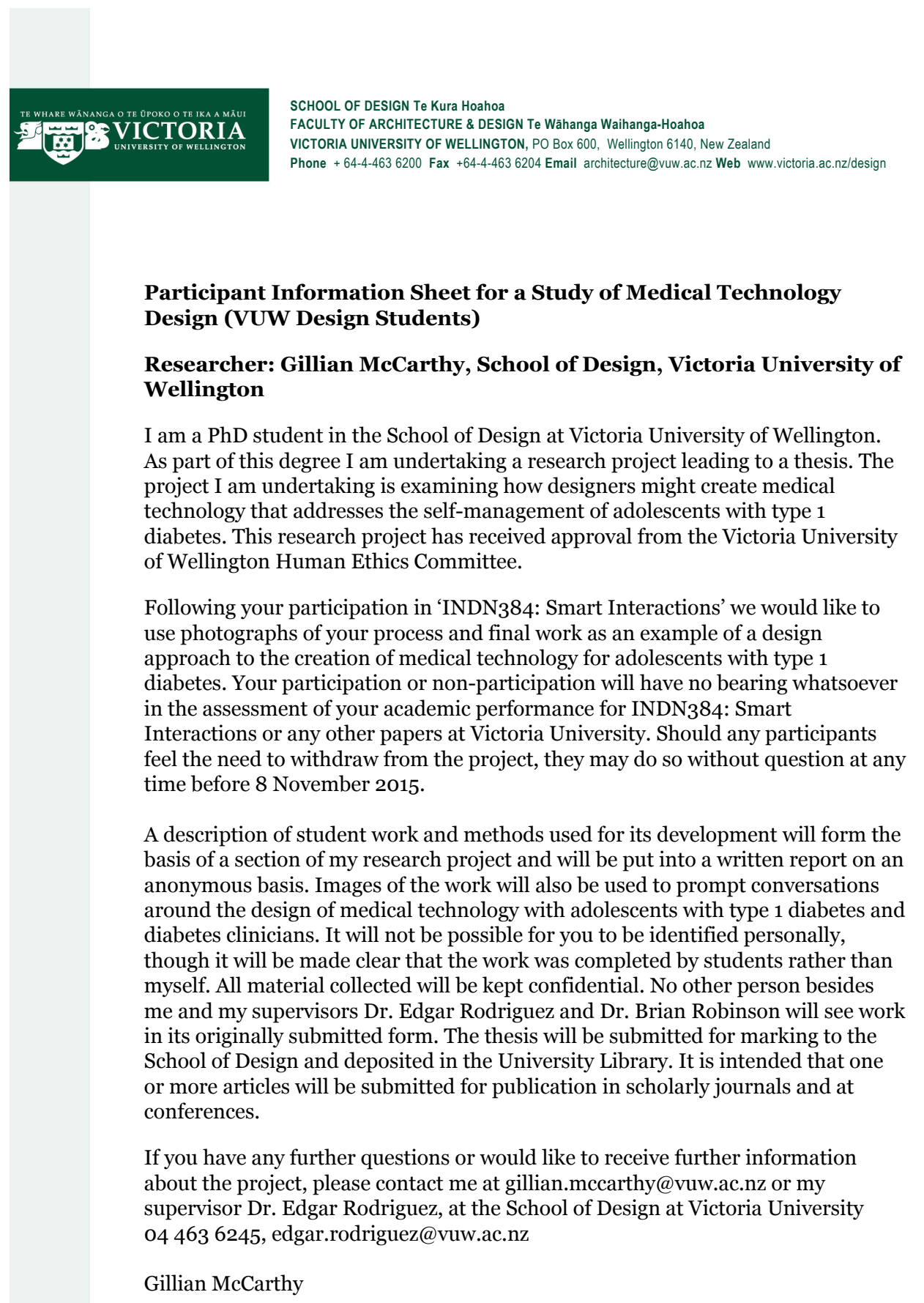


SCHOOL OF DESIGN Te Kura Hoahoa

FACULTY OF ARCHITECTURE \& DESIGN Te Wāhanga Waihanga-Hoaho

VICTORIA UNIVERSITY OF WELLINGTON, PO Box 600, Wellington 6140, New Zealand

Phone + 64-4-4636200 Fax +64-4-4636204 Email architecture@vuw.ac.nz Web www.victoria.ac.nz/design

\section{CONSENT TO PARTICIPATE IN RESEARCH (VUW Design Students)}

Designing for Adherence: Design Strategies for facilitating adolescents' adherence with Type 1 Diabetes Mellitus self-care medical devices

I have been given and have understood an explanation of this research project. I have had an opportunity to ask questions and have them answered to my satisfaction. I understand that I may withdraw myself (or any information I have provided) from this project before 8 November 2015 without having to give reasons. I understand that participation or nonparticipation will have no bearing whatsoever in the assessment of my academic performance for INDN384: Smart Interactions or any other papers at Victoria University.

\section{Please tick the boxes below that apply:}

- I consent to images of my work from 'INDN384: Smart Interactions' being used in any reports on this research (including for thesis, conference and journal publications) and as a prompt for interviews.

- I understand that the data I provide will not be used for any other purpose or released to others without my written consent.

- I understand that I will not receive any money for taking part in this research.

$\square$ I would like to receive a summary of the results of the research when it is completed.

\section{Signed:}

\section{Name of participant:}

Date: 


\title{
Appendix D
}

\section{Sample Information Sheet for Adolescents and Young Adults}

\section{Participant Information Sheet}

\author{
Study title: $\quad$ Designing for type 1 diabetes \\ Locality: Wellington Ethics committee ref.: 15/CEN/21/AM01 \\ Contact: $\quad$ www.designfortype1.org $\quad$ Lead investigator: Gillian McCarthy \\ gillian.mccarthy@vuw.ac.nz \\ 0273671540
}

\begin{abstract}
You are invited to take part in a study about the self-management of type 1 diabetes by young people. Whether or not you take part is your choice. If you don't want to take part, you don't have to give a reason, and it won't affect the care you receive. If you do want to take part now, but change your mind later, you can pull out of the study at any time.

This Participant Information Sheet will help you decide if you'd like to take part. It sets out why we are doing the study, what your participation would involve, what the benefits and risks to you might be, and what would happen after the study ends. We will go through this information with you and answer any questions you may have. Before you decide whether to participate you may want to talk about the study with other people, such as family, whānau, friends, or healthcare providers. Please feel free to do this.

If you agree to take part in this study, you will be asked to sign the Consent Form on the last page of this document. You may also choose whether you are happy for your health professional to pass on your contact details to the researcher so that they can contact you. If you do not want your contact details passed on, you will be able to initiate contact with the researcher by phone or email if you wish. You will be given a copy of both the Participant Information Sheet and the Consent Form to keep. This document is 5 pages long, including the Consent Form. Please make sure you have read and understood all the pages.
\end{abstract}

\section{WHAT IS THE PURPOSE OF THE STUDY?}

This study aims to understand more about the experiences of young people who are managing type 1 diabetes. We know that many young people find managing type 1 diabetes challenging, so we want to find out why this is, and what could be done to better match medical products and services to young people's needs and desires. This study has received approval from the Health and Disability Ethics Committee (Central Region (Ref. no 15/CEN/21/AM01).

\section{WHAT WILL MY PARTICIPATION IN THE STUDY INVOLVE?}

The study will take place during a 12-month period (January 2016 - January 2017). During the study you will be asked to share your experiences of managing type 1 diabetes with the 
researcher, and to explore how we could make this experience better or easier for you and other young people.

At the first meeting with the researcher you will be asked to chat about managing type 1 diabetes and to complete a brief card sort task, describing the effects your medical devices have on you. You will later be given a pack of research activities to take home with you and complete over a seven-day period. These activities will involve:

- Writing down diabetes-related moments that occur throughout the week.

- Answering some questions with written answers, drawings or collages.

- Taking photos of the important parts of your day and technology that you use.

- Letting us know a bit about your interests and daily life.

Following this, you will be invited to take part in a conversation to discuss the responses you created above, and to talk more about your experiences of managing diabetes. You will also be invited to take part in 1-2 focus groups, where other young people with type 1 diabetes and/or professional designers and design students studying the design of healthcare products and services from Victoria University of Wellington will be present. The researcher will not have access to your medical notes; the only details available will be those shared by you or your family/whānau. You can choose to take part in some or all of these stages of the study.

\section{WHAT ARE THE POSSIBLE BENEFITS AND RISKS OF THIS STUDY?}

There are no direct or immediate benefits of taking part in the study as the study is based on understanding how you currently go about managing your diabetes. However, your participation and the knowledge gained from this will hopefully benefit other young people in the future.

Should you decide to take part in this study, the researcher will schedule the interviews at times that minimise inconvenience for you and your family/whānau. The interviews will be arranged with you at the time of your choice. Any information you share during the duration of this study will be kept strictly confidential and not used for anything other than the research. It will not be shared with any other person. Research team members and focus group participants will only see de-identified information.

\section{WHO PAYS FOR THE STUDY?}

The study has been funded by a Victoria University of Wellington scholarship. By participating in this study you will not incur any costs. Please note, we are unable to offer you payment for your participation in this study.

WHAT IF SOMETHING GOES WRONG?

As this study is only concerned with documenting your experiences of managing type 1 diabetes, the risk of anything going wrong is unlikely. However, if you were injured in this study, you may be eligible for compensation from ACC just as you would be if you were injured in an accident at home or work. You would have to lodge a claim with ACC, which may take some time to assess. If your claim is accepted, you will receive funding to assist in your recovery. 


\title{
WHAT ARE MY RIGHTS?
}

Taking part in this research is entirely voluntary. You do not have to take part in this study, and if you choose not to take part this will not affect your medical care at all. If you do agree to take part you are free to withdraw from the study at any time, without having to give a reason and this will, in no way, affect your care now or in the future. The health professional who informed you of this study will not be informed whether you are participating or not by the researcher, though you are free to share whether you have chosen to take part with your health professional.

\section{WHAT HAPPENS AFTER THE STUDY OR IF I CHANGE MY MIND?}

- If you decide to participate you will be given the opportunity to receive a summary copy of the final report if you are interested.

- All notes taken whilst with you will be coded and kept secure in a locked environment in Victoria University of Wellington.

- After the study has been completed all data will be stored for five years in a locked environment then destroyed.

- Results of this study will be sent out in 2017 to those participants interested, to the Committee who gave ethical approval for this study, and to the primary health groups involved in the research. Publication will also occur in academic journals and conferences.

\section{WHO DO I CONTACT FOR MORE INFORMATION, TO SIGN UP, OR IF I HAVE CONCERNS?}

If you would like to sign up to participate, have any questions, concerns or complaints about the study at any stage, you can contact:

\author{
Gillian McCarthy \\ 0273671540 \\ gillian.mccarthy@vuw.ac.nz \\ www.designfortype1.org
}

If you want to talk to someone who isn't involved with the study, you can contact an independent health and disability advocate on:

$\begin{array}{ll}\text { Phone: } & 0800555050 \\ \text { Fax: } & 08002 \text { SUPPORT (0800 2787 7678) } \\ \text { Email: } & \quad \text { advocacy@hdc.org.nz }\end{array}$

For Maori health support please contact your health provider and they will refer you to the representative Maori health support group.

You can also contact the health care and disability ethics committee (HDEC) that approved this study on:
Phone:
08004 ETHICS
Email: hdecs@moh.govt.nz 


\section{Sample Assent Form for Adolescents Younger than 14 Years}

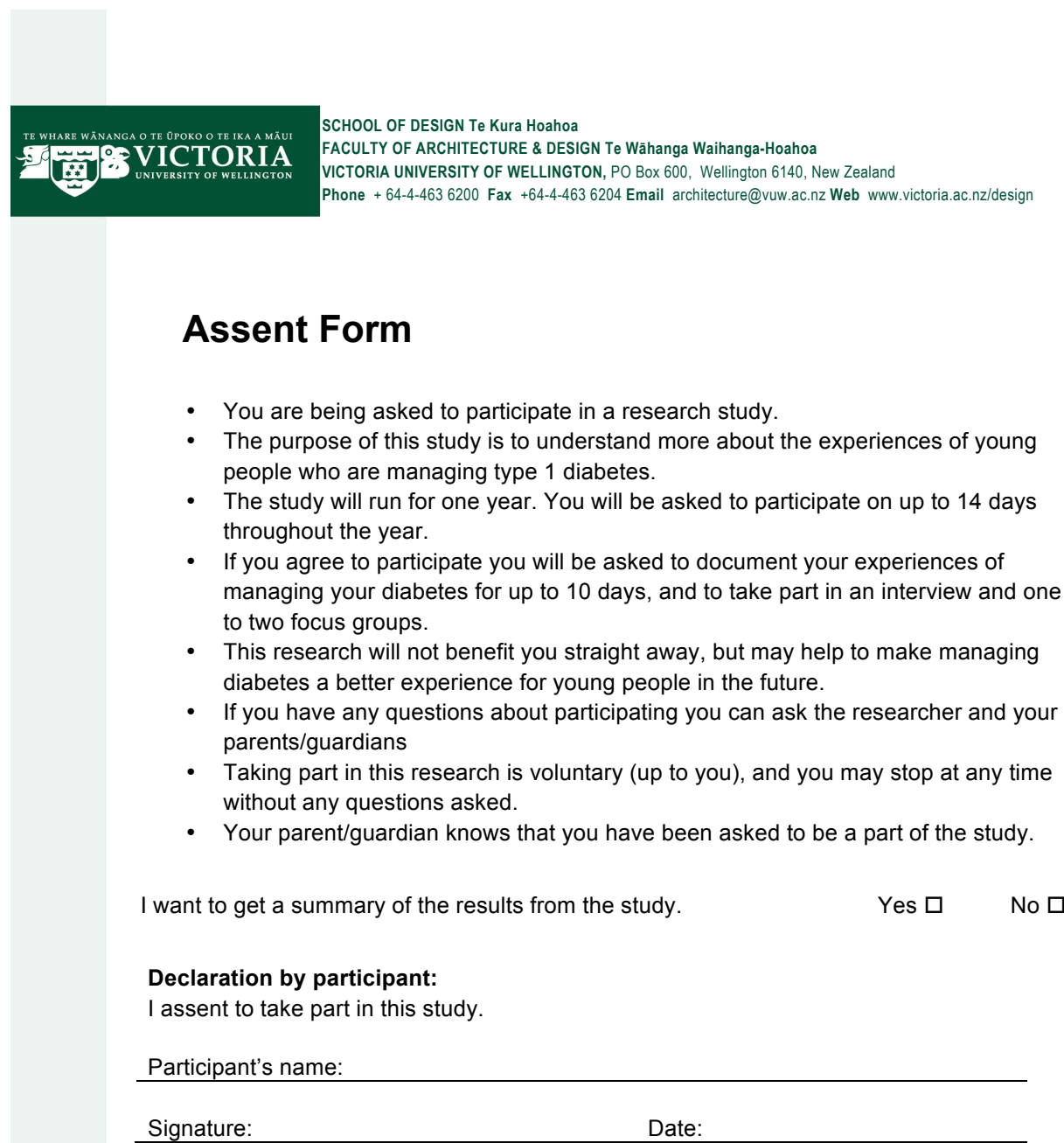

Declaration by member of research team:

I have given a verbal explanation of the research project to the participant, and have answered the participant's questions about it.

I believe that the participant understands the study and has given informed consent to participate.

Researcher's name:

Signature: Date: 
Sample Assent Form for Adolescents 14-15 Years

\section{Assent Form}

If you need an INTERPRETER, please tell us

- I have read, or have had read to me in my first language, and I understand the Participant Information Sheet.

- I have been given sufficient time to consider whether or not to participate in this study.

- I have had the opportunity to use a legal representative, whānau/ family support or a friend to help me ask questions and understand the study.

- I am satisfied with the answers I have been given regarding the study and I have a copy of this assent form and information sheet.

- I understand that taking part in this study is voluntary (my choice) and that I may withdraw from the study at any time without this affecting my medical care.

- If I decide to withdraw from the study, I agree that the information collected about me up to the point when I withdraw may continue to be processed.

- I understand that my participation in this study is confidential and that no material, which could identify me personally, will be used in any reports on this study.

- I understand that de-identified information I supply may be used in future teaching or research projects.

I wish to receive a summary of the results from the study.

Yes $\square$

No $\square$

Declaration by participant:

I hereby assent to take part in this study.

Participant's name:

Signature:

Date:

Declaration by member of research team:

I have given a verbal explanation of the research project to the participant, and have answered the participant's questions about it.

I believe that the participant understands the study and has given informed consent to participate.

Researcher's name:

Signature:

Date: 


\section{Parental Consent Forms for Adolescents 13 -15 Years}

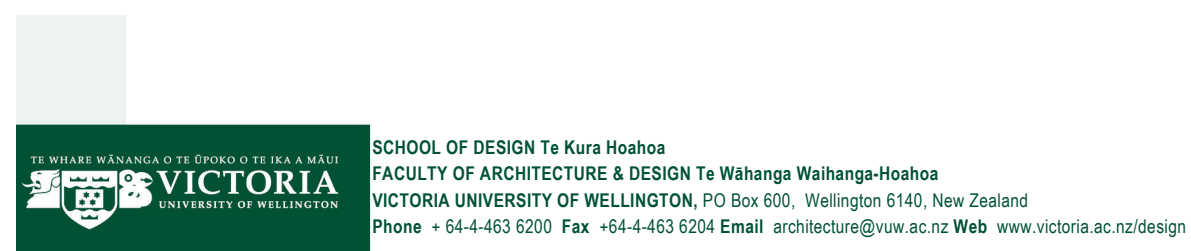

\section{Parent/Guardian Consent Form}

\section{If you need an INTERPRETER, please tell us}

- I have read, or have had read to me in my first language, and I understand the Participant Information Sheet.

- I have been given sufficient time to consider whether or not to allow my adolescent to participate in this study.

- I have had the opportunity to use a legal representative, whānau/ family support or a friend to help me ask questions and understand the study.

- I am satisfied with the answers I have been given regarding the study and I have a copy of this consent form and information sheet.

- I understand that my adolescent taking part in this study is voluntary and that he/she may withdraw from the study at any time without this affecting his/her medical care.

- If my adoelscent decides to withdraw from the study, I agree that the information collected about him/her up to the point when he/she withdraws may continue to be processed.

- I understand that my adolescent's participation in this study is confidential and that no material, which could identify him/her personally, will be used in any reports on this study.

- I understand that de-identified information he/she supplies may be used in future teaching or research projects.

I wish to receive a summary of the results from the study.

Yes $\square$

No $\square$

Declaration by parent/guardian:

I hereby consent for my adolescent to take part in this study.

Parent/Guardian's name:

Adolescent's Name:

Signature: Date:

Declaration by member of research team:

I have given an explanation of the research project to the participant, and have answered the participant's questions about it.

I believe that the participant understands the study and has given informed consent to participate.

Researcher's name:

Signature: Date: 


\section{Sample Consent Form for Adolescents and Young Adults 16}

\section{Years and Above}

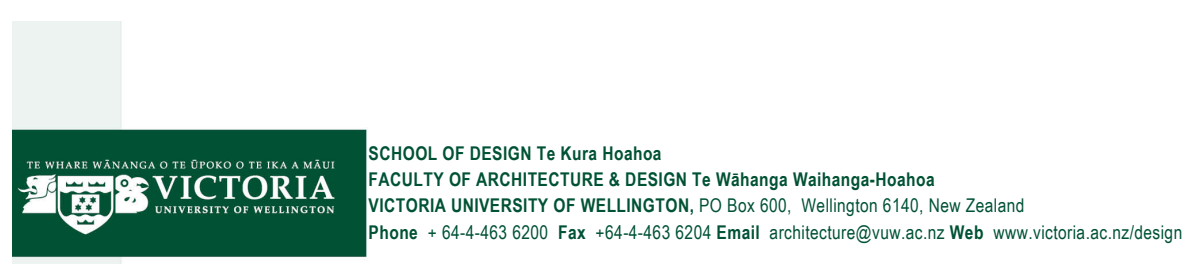

\section{Consent Form}

If you need an INTERPRETER, please tell us

- I have read, or have had read to me in my first language, and I understand the Participant Information Sheet.

- I have been given sufficient time to consider whether or not to participate in this study.

- I have had the opportunity to use a legal representative, whānau/ family support or a friend to help me ask questions and understand the study.

- I am satisfied with the answers I have been given regarding the study and I have a copy of this consent form and information sheet.

- I understand that taking part in this study is voluntary (my choice) and that I may withdraw from the study at any time without this affecting my medical care.

- If I decide to withdraw from the study, I agree that the information collected about me up to the point when I withdraw may continue to be processed.

- I understand that my participation in this study is confidential and that no material, which could identify me personally, will be used in any reports on this study.

- I understand that de-identified information I supply may be used in future teaching or research projects.

Declaration by participant:

I hereby consent to take part in this study.

Participant's name:

Signature:

Declaration by member of research team:

I have given a verbal explanation of the research project to the participant, and have answered the participant's questions about it.

I believe that the participant understands the study and has given informed consent to participate.

Researcher's name:

Signature:

Date: 